\title{
NBSIR $73-241(R)$
}

\section{Comparison of Photon Interaction Cross Section Data Sets. V11. Biggs - Lighthill (REV.) and ENDF/B.}

G. L. Simmons* and J. H. Hubbell*

*Science Applications. Inc. Huntsville, Alabama 35805

**Center for Radiation Research Institute for Basic Standards National Bureau of Standards

Washıngton. D. C. 20234

July 1973

Interim Report for Period April June 1973

Prepared for

Defense Nuclear Agency

Washington, D. C. 20305 

NBSIR 73-241

\section{COMPARISON OF PHOTON INTERACTION} CROSS SECTION DATA SETS.

\section{BIGGS-LIGHTHILL (REV.)}

AND ENDF/B.

G. L. Simmons* and J. H. Hubbell**

* Science Applications, Inc. Huntsville, Alabama 35805

**Center for Radiation Research Institute for Basic Standards National Bureau of Standards

Washington, D. C. 20234

July 1973

Interim Report for Period April-June 1973

This report is to be superseded by a future publication which will receive general distribution and should be cited as a reference. Please consult the NBS Office of Technical Information and Publications to obtain the proper citation.

Prepared for

Defense Nuclear Agency

Washington, D. C. 20305

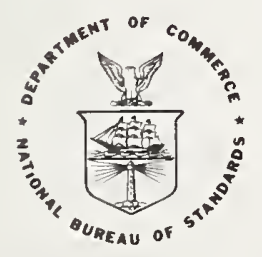

U. S. DEPARTMENT OF COMMERCE, Frederick B. Dent, Secretary 



\section{Abstract}

The Biggs and Lighthill photon cross section data set $(0.01 \mathrm{keV}$ to $>10 \mathrm{GeV}, Z=1$ to 100 ), previously compared in this report series with the $\mathrm{ENDF} / \mathrm{B}$ data set ( $1 \mathrm{keV}$ to $100 \mathrm{MeV}, 87$ elements with $Z=1$ to 94 ), has since been revised to provide explicit coherent and incoherent scattering cross sections, rather than the more approximate free-electron (KleinNishina) scattering cross section. In this report we present a comparison of this revised set with the ENDF/B set, again in the form of percent differences between the two data sets. These differences for total, coherent scattering, incoherent scattering, photoelectric absorption and pair production cross sections are tabulated for 87 elements with $z=1-83,86$, 90,92 and 94 at 41 energies between $1 \mathrm{keV}$ and $100 \mathrm{MeV}$. Coherent scattering differences below a few hundred keV are typically $1-5 \%$ and at higher energies are typically $10-30 \%$ for low $z$ and $2-10 \%$ for high $Z$. The incoherent scattering differences are generally less than $1 \%$ above $10 \mathrm{keV}$ and typically $5-30 \%$ at $1 \mathrm{keV}$. These differences are primarily in regions of negligible scattering contribution to the total cross section, for which the differences are dominated by differences in the photoelectric absorption. The resulting differences in the total cross section are typically $1-5 \%$ except near $1 \mathrm{keV}$ where differences are as high as $26 \%$ for $Z=68$ and 69 . In addition to the table of percent differences we include tables of the cross section values in barns/atom for these two sets, since they have not been generally available in this form. 



\section{INTRODUCTION}

In a previous report (II) of this series $1 /$ we compared a photon interaction cross section data set by Biggs and Lighthill $\frac{2,3 /}{3}$ wth the data in

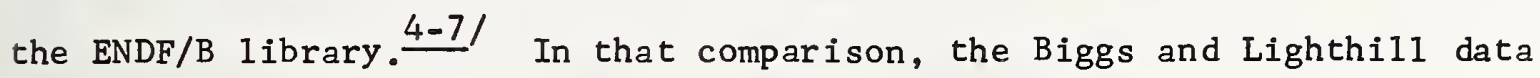
set consisted of parametric fits to empirical rational-function formulas for the atomic photoeffect, free-electron (Klein-Nishina) Compton scattering, and pair production cross sections. The coherent (Rayleigh) scattering cross section was not included.

Subsequent to the above comparison, to provide input to the DINT multigroup cross section preparation program 8 for photon transport calculations, Biggs and Lighthill modified their scattering cross sections to take into account electron binding. This was done by applying an additional rationàfunction factor with Z-dependent parameters to their Klein-Nishina rationalfunction approximation to give the incoherent scattering cross section including electron binding, and by also providing parameters for a rational-function approximation for the coherent scattering cross section.

As discussed in references (9) and (10) the source data for the hydrogen coherent and incoherent scattering cross sections were provided from nonrelativistic theory. $11 /$ The coherent scattering cross section source data were calculated from the relativistic Hartree-Fock results of Cromer and Waber. $12 /$ The incoherent scattering cross section source data for elements $z=2$ to 95 were calculated from the nonrelativistic Hartree-Fock incoherent scattering functions of Cromer and Mann $13 /$ and Cromer. $14 /$ For elements $z=96$ to 100 the incoherent scattering source data were calculated using Thomas-Fermi-Dirac nonrelativistic theory as described by Mendelsohn and Biggs. 15/ For both the coherent and incoherent scattering source data 
calculations, values of the momentum transfer variable $\sin (\theta / 2) / \lambda$ extending up to $80.0 \AA^{-1}$ were included.

Also, we have been informed by Biggs that for photon energies extending above $15 \mathrm{MeV}$, where the rational-function approximation 2 / to the KleinNishina cross section breaks down, the full Klein-Nishina formula, without electron-binding corrections, is used instead.

As a result of these modifications, the present Biggs and Lighthill photon cross section data set now corresponds directly to the ENDF/B library, with respect to the interaction types listed, and a more meaningful comparison can be made than that presented in report II.

\section{RESULTS OE TRE COMPARISON}

The present Biggs-Lighthill set, covering the photon energy range $10 \mathrm{eV}^{*}$ to beyond $10 \mathrm{GeV}$ for elements $\mathrm{Z}=1$ to 100 , is in the form of $\mathrm{Z}$-dependent coefficients of rational functions (polynomials or quotients of polynomials) fitted to the individual cross section types. These coefficients o1: parameters were supplied to us in the compact form of a card deck 1010 cards in length. Of this deck, 510 cards are occupied by photoeffect fit coefficients and.absorption edge information at three to six cards per element. The coherent and Incoherent scattering fit coefficients, each occupying two cards per element, account for 400 cards and the remaining 100 cards contain the patr poduction (pair and triplet combined) fit coefficients occupying a single cerd for each element.

In report II of this series $s^{1 /}$ we erroneously gave $100 \mathrm{eV}$ as the lower energy lingit Eor this set. 
Using this data deck and a program containing the fitting functions given by Biggs and Lighthill in references (2), (3) and (9), it is a straightforward task to compute the photoelectric, pair production and coherent and incoherent scattering cross sections for each element at each of the 41 principal (Grodstein grid) energies (1 $\mathrm{keV}$ to $100 \mathrm{MeV}$ ) for which the corresponding values are given on the ENDF/B tape. For each element in the ENDF/B set ( 87 elements over the range $Z=1$ to 94 ), the percentage deviation of the Biggs-Lighthill cross section, $\mu_{B L}$, from the ENDF/B cross section, $\mu_{E N D F / B}$, was calculated as

$$
\text { Percent Deviation }=100 \times\left(\frac{\mu_{B L}-\mu_{E N D F / B}}{\mu_{E N D F / B}}\right) \text {. }
$$

The resulting sign serves to indicate that the Biggs-Lighthill cross section value is greater $(+)$ or less $(-)$ than the corresponding ENDF/B value. In Table I we present the results of the comparison, with the percent deviations rounded off to the nearest one-tenth percent.

In Figure 1, we have indicated the combination of energy and atomic number for which the absolute deviation of the total cross section in the Biggs-Lighthill set exceeds the ENDF/B value by at least $3 \%$.

In addition, we also list in Tables II and III in barns/atom the Biggs-Lighthill (extended down to $10 \mathrm{eV}$ ) and ENDF/B cross section values, respectively, used in the above comparison, since they are not generally available in this tabular form. Values at the absorption-edge energies are omitted from Tables I - III. 
The Biggs-Lighthill photoeffect and pair production cross section values remain unchanged from those in references (2) and (3) used in the comparison given in report II in this series, $1 /$ hence the deviations listed in Table I in the present report for photoeffect and pair production are identical with the deviations Iisted in report II. As was discussed in report II, the BiggsLighthill photoeffect and pair production source data were taken primarily from Stom and Israel. 16/ Hence these deviations are similar in sign and marisude those Iisted in report I of this series l/ comparing the storm and Israel set with the ENDF/B values. For low Z's Storm and Israel did not Iist photoeffect values at the higher energies. Here the Biggs-Lighthill deviations from ENDF/B photoeffect values are similar in sign and magnitude to those Iisted in report III of this series $1 /$ comparing NSRDS-NBS 29 values $7 /$ with the ENDF/B values.

In Table I the Biggs-Lighthill coherent scattering cross sections are seen to differ from values in the ENDE/B set by typically 2 - 10\% for high-Z elements. For low -Z elements, particularly for energies above a few hundred keV, these deviations are typically $10-30 \%$ and as much as $75 \%$ for $\operatorname{Li}(Z=3)$ at $100 \mathrm{MeV}$. However, in the se regions of large differences the coherent scattering contribution to the total cross section is negligible.

The incoherent scattering differences, on the other hand, are largest at low energies, where the cross section is most sensitive to the atomic model used in generating the incoherent scattering function. Differences greater than $1 \%$, except for the highest $-Z$ elements, are confined to energies below $10 \mathrm{keV}$. The largest differences are found at I keV, typically 5 - 30\%, and as much as $103 \%$ for $\mathrm{Pu}(\mathrm{Z}=94)$ at $1 \mathrm{keV}$. As was the case for coherent 
scattering, the substantial deviations between Biggs-Lighthill and ENDF/B incoherent scattering cross sections fall in regions where this interaction contributes a negligible amount to the total cross section.

The differences exceeding $3 \%$ (as much as $26 \%$ at $1 \mathrm{keV}$ for $\operatorname{Er}(\mathrm{z}=68$ ) and $\operatorname{Tm}(Z=69))$ in total cross section values indicated in Fig. 1 are thus primarily differences in the photoeffect cross sections used except for the few isolated greater-than-3\% differences above $5 \mathrm{MeV}$ where the pair production cross section predominates. 



\section{$\underline{\text { References }}$}

1. G. L. Simmons and J. H. Hubbe11: "Comparisons of Photon Interaction Cross Section Data Sets. I. Storm-Israel and ENDF/B; II. Biggs-Lighthill and ENDF/B; III. NSRDS-NBS 29 and ENDF/B; IV. Kaman and ENDF/B; V. Photran and ENDF/B; VI. McGuire and Kaman Photoeffect Data," NBS Reports 10668, 10818 , $10842,10847,10848$ and NBSIR 73-176 (unpub1ished).

2. F. Biggs and R. Lighthill (Sandia Corp., Albuquerque, N.M.): "Analytical Approximations for X-Ray Cross Sections II," SC-RR-71 0507 (1971).

3. F. Biggs and R. Lighthi11: "Analytical Approximations for Total Pair Production Cross Sections," SC-RR-68-619 (1968).

4. The present ENDF/B photon interaction cross section library tape, also designated as RSIC (Radiation Shielding Information Center, Oak Ridge National Laboratory) tape DLC-7, became a part of the Evaluated Nuclear Data File System as a result of action taken by the Shielding Subcomittee of CSEWG (Cross Section Evaluation Working Group, AEC) at a meeting at Los Alamos May 12-13, 1970. This 1ibrary is documented in references 3,4 and 5 as discussed in reference 1 (I).

5. E. F. Plechaty and J. R. Terral1 (Lawrence Livermore Lab., Calif.): "Photon Cross Sections $1 \mathrm{keV}$ to $100 \mathrm{MeV}, "$ UCRL-50400, Vol. VI (1968).

6. W. H. McMaster, N. K. DelGrande and J. H. Mal lett (Lawrence Livermore Lab., Calif.) and J.H. Hubbell (National Bureau of Standards): "Compilation of X-Ray Cross Sections," UCRL-50174, Sec. I (1970), Sec. II, Rev. 1 (1969), Sec. III (1969) and Sec. IV (1969). These reports are available from the National Technical Information Service, Springfield, Virginia 22151.

7. J. H. Hubbe11: "Photon Cross Sections, Attenuation Coefficients, and Energy Absorption Coefficients from $10 \mathrm{keV}$ to $100 \mathrm{GeV}, "$ NSRDS-NBS 29 (1969).

8. K. G. Adams, F. Biggs and J. H. Renken: "DINT: A Computer Program which Prepares Multigroup Coherent-Incoherent Cross Sections for Photon Transport Calculations," SC-RR-72 0684 (1972).

9. F. Biggs and R. Lighthil1: "Analytical Approximations for Total and Energy Absorption Cross Sections for Photon-Atom Scattering, " SC-RR-72 0685 (1972).

10. F. Biggs and R. Lighthill: "Analytical Approximations for Photon-Atom Differential Scattering Cross Sections including Electron Binding Effects," SC-RR-72 0659 (1972).

11. M. H. Pirenne: The Diffraction of X-Rays and Electrons by Free Molecules, Cambridge Univ. Press, 1946.

12. D. T. Cromer and J. T. Waber: Scattering Factors Computed from Relativistic Dirac-Slater Wave Functions, Acta Cryst. 18,104-109 (1965); also private communication (1969) from Cromer to Biggs and Lighthill.

13. D. T. Cromer and J. B. Mann: "Compton Scattering Factors for Spherically Symmetric Free Atoms," J. Chem. Phys. 47, 1892-1893 (1967). 
14. D. T. Cromer: "Compton Scattering Factors for Aspherical Free Atoms," J. Chem. Phys. 50, 4857-4859 (1969).

15. L. B. Mendel sohn and F. Biggs: "Thomas-Fermi-Dirac Calculations of Incoherent-Scattering Factors," Phys. Rev. A $\underline{5}, 688-691$ (1972).

16. E. Storm and H. I. Israel (Los Alamos Sci. Lab., N. Mex.): "Photon Cross Sections from $1 \mathrm{keV}$ to $100 \mathrm{MeV}$ for Elements $Z=1$ to $Z=100$," Nuc1. Data 7A, 565-681 (1970). 
$Z=1$ HYOROGEN

$\begin{array}{crrrr}\begin{array}{c}\text { ENERGY } \\ \text { KEV }\end{array} & \text { TOTAL } & \text { SCATT, } & \begin{array}{r}\text { INCOH. } \\ \text { SCATT, }\end{array} & \begin{array}{r}\text { PHOTO } \\ \text { EFFECT }\end{array} \\ 1 & -2.6 & -.1 & 55.9 & -3.1 \\ 1.5 & -.0 & .5 & 30.0 & -1.8 \\ 2 & 1.7 & .5 & 14.8 & -.8 \\ 3 & .8 & -.8 & 2.7 & -.3 \\ 4 & .0 & -.6 & .3 & -.2 \\ 5 & .5 & 1.0 & .4 & .4 \\ 6 & .7 & .8 & .7 & 1.3 \\ 8 & .2 & -.7 & .3 & 2.9 \\ 10 & .2 & .1 & .1 & 4.0 \\ 15 & .0 & -1.1 & .1 & 4.4 \\ 20 & .1 & .1 & .1 & 3.2 \\ 30 & .2 & .2 & .2 & 1.9 \\ 40 & .2 & .4 & .2 & 1.5 \\ 50 & .2 & .2 & .2 & 1.1 \\ 60 & .1 & .2 & .1 & .1 \\ 80 & -.0 & .4 & -.0 & -1.0 \\ 100 & .0 & .7 & -.0 & -2.7 \\ 150 & .0 & 3.1 & .0 & -5.2 \\ 200 & .0 & 5.5 & .1 & -5.7 \\ 300 & .1 & 3.2 & .0 & -4.0\end{array}$

\begin{tabular}{|c|c|c|c|c|}
\hline \multicolumn{5}{|c|}{$2 H E L I \cup M$} \\
\hline ENERGY & & $\mathrm{COH}$ & INCOH. & РHOTO \\
\hline & TOTAL & SCATT. & SCATT. & EFFECT \\
\hline 1 & -6.7 & .3 & $-10 \cdot 7$ & -6.7 \\
\hline 1.5 & $-2 \cdot 0$ & .3 & -5.4 & $-2 \cdot 1$ \\
\hline 2 & -.2 & .2 & -1.5 & -.2 \\
\hline 3 & -.1 & .5 & $3 \cdot 1$ & -.3 \\
\hline 4 & .3 & 1.5 & $2 \cdot 5$ & -.5 \\
\hline 5 & 1.6 & 1.8 & 1.6 & $1 \cdot 3$ \\
\hline 6 & 2.1 & 1.6 & 1.0 & 3.8 \\
\hline 8 & $2 \cdot 1$ & $1 \cdot 1$ & .4 & 8.8 \\
\hline 10 & 1.9 & .9 & .6 & 13.7 \\
\hline 15 & .8 & .4 & .2 & 23.2 \\
\hline 20 & .3 & -.1 & -.1 & 29.5 \\
\hline 30 & -.0 & -.4 & -.1 & 39.3 \\
\hline 40 & -.3 & -.6 & -.2 & 47.8 \\
\hline 50 & -.5 & -.6 & -.5 & 54.9 \\
\hline 00 & -.8 & $-1 \cdot 0$ & -.9 & 60.7 \\
\hline 80 & .1 & -.8 & . 1 & 69.7 \\
\hline 100 & .0 & -.7 & .0 & 76.5 \\
\hline 150 & .1 & -.5 & .1 & 87.5 \\
\hline 200 & .1 & .1 & .1 & 93.2 \\
\hline 300 & .1 & .2 & .1 & 100.1 \\
\hline
\end{tabular}

\begin{tabular}{|c|c|c|c|c|}
\hline $\begin{array}{c}\text { ENERGY } \\
\text { KEV }\end{array}$ & TOTAL & $\begin{array}{l}\text { COH } \\
\text { SCATT. }\end{array}$ & $\begin{array}{l}\text { INCOH, } \\
\text { SCATT. }\end{array}$ & $\begin{array}{l}\text { PHOTO } \\
\text { EFFECT }\end{array}$ \\
\hline 1 & $12 \cdot 8$ & -2.3 & -7.3 & 12.8 \\
\hline 1.5 & 4.1 & -1.6 & $-5 \cdot 1$ & 4.2 \\
\hline 2 & -.2 & -.7 & -1.8 & -.2 \\
\hline 3 & -4.7 & -1.3 & .0 & -4.9 \\
\hline 4 & $-6 \cdot 1$ & -2.9 & .2 & -6.6 \\
\hline 5 & $-5 \cdot 2$ & -2.9 & .1 & -5.8 \\
\hline 6 & $-3 \cdot 5$ & $-2 \cdot 5$ & .1 & $-4 \cdot 2$ \\
\hline 3 & -.5 & $-2 \cdot 3$ & .1 & -.1 \\
\hline 10 & .9 & -2.5 & $\cdot 2$ & 4.0 \\
\hline 15 & $1 \cdot 3$ & $-1 \cdot 2$ & -.1 & 12.6 \\
\hline 20 & .4 & $-5 \cdot 2$ & .1 & 18.9 \\
\hline 30 & .1 & $-5 \cdot 4$ & .1 & $29 \cdot 2$ \\
\hline 40 & - 0 & $-6 \cdot 0$ & $\cdot 1$ & 37.0 \\
\hline 50 & . 0 & $-6 \cdot 2$ & . 2 & $42 \cdot 5$ \\
\hline 60 & -.1 & $-6 \cdot 3$ & .0 & 46.4 \\
\hline 80 & -.1 & -6.6 & .0 & $50 \cdot 8$ \\
\hline 100 & -.0 & $-6 \cdot 0$ & .0 & 53.3 \\
\hline 150 & .1 & $-5 \cdot 2$ & .1 & 58.0 \\
\hline 200 & $\cdot 1$ & $-7 \cdot 4$ & .1 & $61 \cdot 3$ \\
\hline 300 & $\cdot 1$ & $-4 \cdot 5$ & .1 & 65.5 \\
\hline
\end{tabular}

\begin{tabular}{ccccc}
$\begin{array}{c}\text { ENERGY } \\
\text { ENG }\end{array}$ & \multicolumn{5}{c}{ GERYLLIUM } \\
KEV & TOTAL & SCATT. & $\begin{array}{r}\text { INCOH. } \\
\text { SCATT. }\end{array}$ & $\begin{array}{r}\text { PHOTO } \\
\text { EFFECT }\end{array}$ \\
1 & 5.7 & -1.4 & 27.4 & 5.7 \\
1.5 & 2.8 & -.4 & 12.3 & 2.8 \\
2 & 1.0 & .3 & 4.9 & .9 \\
3 & -1.5 & -1.7 & -.7 & -1.5 \\
4 & -2.4 & -3.1 & -.9 & -2.4 \\
5 & -1.6 & -2.2 & -.1 & -1.6 \\
6 & -.1 & -1.9 & .4 & -.00 \\
8 & 2.6 & -2.7 & .6 & 3.8 \\
10 & 4.1 & -3.2 & .3 & 7.5 \\
15 & 3.6 & -4.6 & .0 & 14.8 \\
20 & 2.5 & -4.1 & .3 & 19.7 \\
30 & 1.1 & -3.6 & .3 & 26.9 \\
40 & .7 & -4.2 & .5 & 31.5 \\
50 & .7 & -4.6 & .7 & 34.1 \\
60 & .9 & -4.8 & .9 & 35.2 \\
80 & -.1 & -4.9 & -.0 & 35.0 \\
100 & -.0 & -4.4 & -.0 & 33.8 \\
150 & .0 & -4.4 & .1 & 33.1 \\
200 & .1 & -3.8 & .1 & 34.0 \\
300 & .1 & -3.1 & .1 & 36.9
\end{tabular}

\begin{tabular}{|c|c|c|c|c|c|}
\hline $\begin{array}{c}\text { ENERGY } \\
\text { KEV }\end{array}$ & & $\begin{array}{l}\mathrm{COH} \\
\mathrm{SCATT} .\end{array}$ & $\begin{array}{l}\text { INCOH. } \\
\text { SCATT. }\end{array}$ & $\begin{array}{r}\text { PHOTO } \\
\text { EFFECT }\end{array}$ & $\begin{array}{l}\text { PA IR } \\
\text { PROD. }\end{array}$ \\
\hline $\begin{array}{l}\text { KEV } \\
400\end{array}$ & $\begin{array}{c}\text { TOTAL } \\
.0\end{array}$ & $\begin{array}{c}\text { SCATT. } \\
=3.3\end{array}$ & $\begin{array}{c}\text { SCATT. } \\
.0\end{array}$ & .7 & PROD. \\
\hline 500 & .0 & $-2 \cdot 2$ & -.0 & $8 \cdot 3$ & \\
\hline 600 & -.0 & -.8 & -.0 & $18 \cdot 5$ & \\
\hline 800 & -.0 & -.6 & -.0 & 44.5 & \\
\hline 1000 & -.0 & -1.2 & -.0 & $76 \cdot 2$ & \\
\hline 1500 & -.0 & $-1 \cdot 5$ & -.0 & 69.3 & 1.1 \\
\hline 2000 & .0 & -1.7 & -.0 & 76.4 & 2.8 \\
\hline 3000 & -.0 & -1.8 & -.0 & $75 \cdot 4$ & .5 \\
\hline 4000 & -.1 & -1.9 & -.1 & $72 \cdot 7$ & $-2 \cdot 4$ \\
\hline 5000 & -.1 & -1.9 & -.0 & 73.4 & -2.6 \\
\hline 6000 & .0 & -2.0 & -.0 & 72.0 & $2 \cdot 3$ \\
\hline 8000 & .1 & -2.1 & -.0 & 71.8 & 3.7 \\
\hline 10000 & -.0 & -2.1 & $=.0$ & 73.0 & -.3 \\
\hline 15000 & .4 & $-1 \cdot 3$ & -.0 & 70.2 & 3.7 \\
\hline 20000 & .0 & .2 & -.0 & 69.4 & $\cdot 3$ \\
\hline 30000 & -.2 & 3.9 & -.0 & 69.4 & -.9 \\
\hline 40000 & -.1 & 8.3 & -.0 & 70.1 & -.4 \\
\hline 50000 & -.4 & 14.0 & -.0 & 70.9 & -1.0 \\
\hline 60000 & -.1 & 21.1 & -.0 & 71.6 & -.3 \\
\hline 80000 & -.2 & 38.2 & -.0 & 73.1 & -.04 \\
\hline 100000 & -.7 & 53.1 & -.0 & 74.3 & -1.2 \\
\hline
\end{tabular}

\begin{tabular}{|c|c|c|c|c|c|}
\hline ENERGY & & $\mathrm{COH}$ & INCOH. & PHOTO & PAIR \\
\hline KEV & TOTAL & SCATT . & SCATT. & EFFECT & PROD. \\
\hline 400 & .0 & -1.4 & .0 & 107.9 & \\
\hline 500 & .0 & $6 \cdot 3$ & .0 & 120.1 & \\
\hline 600 & -.0 & 7.9 & -.0 & 136.8 & \\
\hline 800 & -.0 & 12.8 & -.0 & 178.7 & \\
\hline 1000 & -.0 & $26 \cdot 3$ & -.0 & $226 \cdot 5$ & \\
\hline 1500 & -.0 & $25 \cdot 8$ & -.0 & 219.8 & 1.9 \\
\hline 2000 & -.0 & $25 \cdot 6$ & -.0 & 234.2 & -.3 \\
\hline 3000 & -.0 & $25 \cdot 3$ & -00 & 230.9 & .5 \\
\hline 4000 & -.0 & $25 \cdot 2$ & -.1 & 223.9 & $\cdot 3$ \\
\hline 5000 & -.0 & $25 \cdot 1$ & -.0 & 223.3 & .7 \\
\hline 6000 & .2 & $25 \cdot 1$ & -.0 & $218 \cdot 8$ & $4 \cdot 8$ \\
\hline 8000 & .3 & $25 \cdot 0$ & -.0 & 215.6 & 4.5 \\
\hline 10000 & -.1 & $24 \cdot 9$ & -.0 & 215.8 & -.6 \\
\hline 15000 & .2 & $25 \cdot 0$ & -.0 & 207.8 & 1.3 \\
\hline 20000 & -.1 & $26 \cdot 3$ & -.0 & 204.9 & -.1 \\
\hline 30000 & .5 & 29.5 & -.0 & 203.6 & 1.6 \\
\hline 40000 & . 9 & 33.4 & -.0 & 204.0 & $2 \cdot 1$ \\
\hline 50000 & .3 & 37.9 & -.0 & $205 \cdot 0$ & .6 \\
\hline 60000 & .2 & 43.6 & -.0 & 206.1 & .4 \\
\hline 80000 & -.1 & $58 \cdot 0$ & -.0 & 208.3 & -.1 \\
\hline 100000 & -.1 & $75 \cdot 6$ & -.0 & 210.3 & 0.1 \\
\hline
\end{tabular}

\begin{tabular}{|c|c|c|c|c|c|}
\hline $\begin{array}{c}\text { ENERGY } \\
\text { KEV }\end{array}$ & TOTAL & $\begin{array}{l}\text { COH } \\
\text { SCATT. }\end{array}$ & $\begin{array}{l}\text { INCOH. } \\
\text { SCATT. }\end{array}$ & $\begin{array}{r}\text { PHOTO } \\
\text { EFFECT }\end{array}$ & $\begin{array}{l}\text { PAIR } \\
\text { PROD. }\end{array}$ \\
\hline 400 & .1 & $-3 \cdot 3$ & $\cdot 1$ & 68.9 & \\
\hline 500 & .0 & $-3 \cdot 3$ & .0 & $72 \cdot 8$ & \\
\hline 600 & .0 & .8 & .0 & $78 \cdot 6$ & \\
\hline 800 & -.0 & $-2 \cdot 0$ & -.0 & 95.2 & \\
\hline 1000 & -.0 & 6.2 & -.0 & 114.0 & \\
\hline 1500 & -.0 & $5 \cdot 8$ & -.0 & 109.2 & $2 \cdot 4$ \\
\hline 2000 & -.0 & $5 \cdot 6$ & .0 & 118.5 & $-5 \cdot 2$ \\
\hline 3000 & -.0 & 5.4 & -.0 & 117.0 & $-1 \cdot 2$ \\
\hline 4000 & -.0 & $5 \cdot 3$ & -.0 & 112.9 & 1.0 \\
\hline 5000 & .1 & 5.2 & -.0 & 112.9 & $2 \cdot 5$ \\
\hline 6000 & .4 & $5 \cdot 2$ & -.0 & 110.1 & 6.6 \\
\hline 8000 & .5 & $5 \cdot 1$ & -.0 & 108.2 & $5 \cdot 6$ \\
\hline 10000 & -.1 & $5 \cdot 1$ & -.0 & $108 \cdot 4$ & -.6 \\
\hline 15000 & -.4 & 4.9 & -.0 & 103.3 & -1.9 \\
\hline 20000 & $-1 \cdot 1$ & $5 \cdot 6$ & -.0 & 201.5 & $-3 \cdot 7$ \\
\hline 30000 & .0 & 7.7 & $=.0$ & 100.8 & .1 \\
\hline 40000 & .8 & $10 \cdot 5$ & -.0 & $101 \cdot 2$ & 1.7 \\
\hline 50000 & .3 & 23.3 & -.0 & 101.9 & .5 \\
\hline 60000 & .1 & 17.1 & -.0 & 102.7 & .3 \\
\hline 80000 & -.5 & 26.2 & -.0 & 104.2 & -.8 \\
\hline 100000 & 0.7 & 38.1 & -.0 & 205.7 & $-1 \cdot 0$ \\
\hline
\end{tabular}

\begin{tabular}{|c|c|c|c|c|c|}
\hline ENERGY & & $\mathrm{COH}$ & INCOH. & PHOTO & PAIR \\
\hline & TOTAL & SCATT. & SCATT. & EFFECT & PROD. \\
\hline 400 & .0 & -2.8 & .0 & 39.9 & \\
\hline 500 & .0 & -6.5 & .0 & 43.0 & \\
\hline 600 & .0 & $-2 \cdot 5$ & -.1 & 47.3 & \\
\hline 800 & -.0 & 4.6 & -.0 & 61.5 & \\
\hline 1000 & -.0 & $8 \cdot 3$ & -.0 & 78.4 & \\
\hline 1500 & -.0 & 7.9 & -.0 & 74.1 & .9 \\
\hline 2000 & .0 & 7.7 & .0 & $81 \cdot 8$ & 2.8 \\
\hline 3000 & -.1 & 7.5 & .0 & 80.9 & -2.9 \\
\hline 4000 & -.2 & 7.3 & -.0 & 77.8 & -4.0 \\
\hline 5000 & -.2 & 7.3 & -.0 & 78.1 & $-3 \cdot 1$ \\
\hline 6000 & .1 & 7.2 & -.0 & 75.8 & 1.4 \\
\hline 8000 & .4 & 7.2 & .0 & 74.3 & 3.3 \\
\hline 10000 & .1 & 7.2 & -.0 & 74.5 & .5 \\
\hline 15000 & .8 & $7 \cdot 0$ & -.0 & 70.4 & 3.3 \\
\hline 20000 & .0 & $7 \cdot 3$ & -.0 & 69.0 & .1 \\
\hline 30000 & -.4 & 9.2 & -.0 & $68 \cdot 5$ & -.8 \\
\hline 40000 & -.2 & 11.5 & -.0 & 68.9 & -.4 \\
\hline 50000 & -.6 & $14 \cdot 3$ & -.0 & 69.5 & -.9 \\
\hline 60000 & -.2 & 17.2 & $=.0$ & 70.2 & -.3 \\
\hline 80000 & -.3 & 24.9 & -.0 & 71.6 & -.4 \\
\hline 100000 & -.9 & 35.0 & -.0 & $72 \cdot 8$ & $-1 \cdot 1$ \\
\hline
\end{tabular}




\begin{tabular}{|c|c|c|c|c|}
\hline ENERGY & & $\mathrm{COH}$ & INCOH. & PHOTO \\
\hline KEV & TOTAL & SCATT. & SCATT, & EFFECT \\
\hline$i$ & 5.0 & -.7 & 41.9 & 5.0 \\
\hline 1.5 & .3 & -.3 & 20.6 & .3 \\
\hline 2 & -1.1 & -.4 & 9.5 & -1.1 \\
\hline 3 & -.9 & -2.8 & 2.1 & -.9 \\
\hline 4 & .2 & -3.7 & 1.2 & .3 \\
\hline 5 & 1.6 & -2.7 & -4.0 & 1.8 \\
\hline 6 & 3.0 & -2.8 & -2.8 & 3.4 \\
\hline 8 & $5 \cdot 1$ & -3.6 & -1.5 & $6 \cdot 3$ \\
\hline 10 & 6.1 & -3.5 & .3 & 8.3 \\
\hline 15 & 4.7 & $-4 \cdot 3$ & -.0 & 10.7 \\
\hline 20 & 3.0 & $-4 \cdot 3$ & .4 & 11.8 \\
\hline 30 & $1 \cdot 3$ & -3.9 & .3 & 15.1 \\
\hline 40 & .6 & -4.6 & .2 & 18.5 \\
\hline 50 & .3 & $-5 \cdot 2$ & .1 & 21.2 \\
\hline 60 & .2 & $-5 \cdot 6$ & .1 & 23.0 \\
\hline 80 & -.1 & -5.8 & -.0 & 24.6 \\
\hline 100 & -.0 & -5.7 & .0 & 24.1 \\
\hline 150 & -.0 & $-5 \cdot 0$ & .0 & 21.7 \\
\hline 200 & .0 & -4.7 & .0 & 20.4 \\
\hline 300 & -.0 & -3.6 & .0 & 19.2 \\
\hline
\end{tabular}

$\begin{array}{ccrrr}\begin{array}{c}\text { ENERGY } \\ \text { KEV }\end{array} & \begin{array}{c}\text { 6 CARBON } \\ \text { TOTAL }\end{array} & \begin{array}{r}\text { SCATT } \\ \text { INCOH. }\end{array} & \begin{array}{r}\text { SCATT. } \\ \text { SCHOTO }\end{array} \\ 1 & .2 & -.5 & 31.6 & \text { EFFCT } \\ 1.5 & -2.4 & -.6 & 14.9 & -2.4 \\ 2 & -2.5 & -1.4 & 6.7 & -2.5 \\ 3 & -.7 & -3.5 & 2.8 & -.7 \\ 4 & 1.3 & -4.0 & 2.5 & 1.4 \\ 5 & 3.0 & -3.6 & 1.0 & 3.2 \\ 6 & 4.4 & -4.0 & .7 & 4.7 \\ 8 & 6.0 & -4.6 & .0 & 6.8 \\ 10 & 6.6 & -4.0 & 1.8 & 7.9 \\ 15 & 5.2 & -2.4 & .5 & 8.2 \\ 20 & 3.2 & -4.1 & .6 & 8.1 \\ 30 & 1.8 & -3.5 & .4 & 10.3 \\ 40 & 1.0 & -3.3 & .3 & 12.9 \\ 50 & .6 & -3.1 & .2 & 14.9 \\ 60 & .3 & -2.9 & .1 & 16.2 \\ 80 & .2 & -2.6 & .1 & 16.9 \\ 100 & .1 & -2.0 & .1 & 15.6 \\ 150 & .1 & -.8 & .1 & 11.8 \\ 200 & .2 & -.1 & .2 & 9.7 \\ 300 & .1 & 1.9 & .1 & 8.0\end{array}$

\begin{tabular}{|c|c|c|c|c|c|}
\hline $\begin{array}{c}\text { ENERGY } \\
\text { KEV }\end{array}$ & TOTAL & SCATT. & $\begin{array}{l}\text { INCOH. } \\
\text { SCATT. }\end{array}$ & $\begin{array}{l}\text { PHOTO } \\
\text { EFFECT }\end{array}$ & $\begin{array}{r}\text { PAIR } \\
\text { PROD. }\end{array}$ \\
\hline 400 & .0 & -3.4 & .0 & 20.0 & \\
\hline 500 & -.0 & -3.9 & -.0 & 22.9 & \\
\hline 600 & $=.0$ & 2.8 & -.0 & 27.6 & \\
\hline 800 & -.0 & 7.7 & -.0 & 40.5 & \\
\hline 1000 & -.1 & -3.4 & -.1 & 55.1 & \\
\hline 1500 & $-。 0$ & -3.8 & -.0 & $50 \cdot 3$ & 1.7 \\
\hline 2000 & -.0 & -3.9 & -.0 & $56 \cdot 3$ & $-4 \cdot 1$ \\
\hline 3000 & -.1 & $-4 \cdot 1$ & -.0 & 54.9 & -2.6 \\
\hline 4000 & -.1 & -4.2 & -.1 & $52 \cdot 2$ & -.9 \\
\hline 5000 & .0 & $-4 \cdot 3$ & -.0 & 52.5 & .8 \\
\hline 6000 & .4 & $-4 \cdot 3$ & -.0 & $50 \cdot 5$ & $5 \cdot 0$ \\
\hline 8000 & .6 & $-4 \cdot 3$ & -.0 & 49.3 & 4.6 \\
\hline 10000 & -.2 & -4.4 & -.0 & 49.6 & -.8 \\
\hline 15000 & -.6 & -4.5 & -.0 & 46.2 & $-2 \cdot 0$ \\
\hline 20000 & -1.7 & -4.4 & -.0 & 45.1 & $-4 \cdot 3$ \\
\hline 30000 & -.7 & -2.9 & -.0 & 44.8 & -1.4 \\
\hline 40000 & $\cdot 2$ & $-1 \cdot 1$ & -.0 & 45.3 & .3 \\
\hline 50000 & -.3 & $1 \cdot 1$ & -.0 & 45.9 & -.4 \\
\hline 60000 & -.2 & 3.3 & -.0 & 46.5 & -.2 \\
\hline 80000 & -.4 & 9.4 & -.0 & 47.8 & -.5 \\
\hline 00000 & -.2 & 17.0 & -.0 & 48.9 &.$-<$ \\
\hline
\end{tabular}

ENERGY COH INCOH. PHOTO PAIR KEV TOTAL SCATT. SCATT. EFFECT PROD.

$\begin{array}{rrrrr}.1 & 1.7 & -1 & 9.2 & \\ .1 & 5.4 & .1 & 13.0 & \\ .0 & 3.5 & .0 & 18.7 & \\ -.0 & 17.1 & -.0 & 32.6 & \\ -.0 & 17.8 & -.0 & 47.9 & \\ -.0 & 17.3 & -.0 & 42.5 & .6 \\ -.1 & 17.1 & -.0 & 47.5 & -1.4 \\ -.1 & 17.0 & -.0 & 45.8 & -2.5 \\ -.1 & 17.0 & -.0 & 43.3 & -1.8 \\ .3 & 17.0 & -.0 & 41.7 & -.6 \\ -.6 & 16.9 & -.0 & 40.9 & 3.5 \\ -.2 & 16.9 & -.0 & 41.2 & -.9 \\ -.3 & 16.8 & -.0 & 38.3 & -.8 \\ -1.3 & 16.7 & -.0 & 37.4 & -2.9 \\ -.7 & 18.3 & -.0 & 37.2 & -1.2 \\ -.1 & 20.3 & -.0 & 37.7 & -.1 \\ -.8 & 22.8 & -.0 & 38.4 & -1.1 \\ -.7 & 25.3 & -.0 & 39.0 & -.9 \\ -.8 & 31.8 & -.0 & 40.3 & -1.0 \\ -.0 & 40.0 & -.0 & 41.3 & -.0\end{array}$

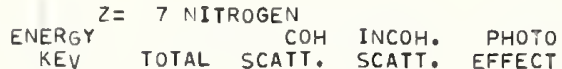

KEV TOTAL SCATT. SCATT: EFFECT

$\begin{array}{rrrrr}1 & -1.5 & -.2 & 14.0 & -1.5 \\ 1.5 & -2.4 & -.7 & 6.4 & -2.5 \\ 2 & -2.7 & -1.5 & 3.1 & -2.7 \\ 3 & -1.9 & -3.2 & 2.5 & -1.9 \\ 4 & -.8 & -3.4 & 1.7 & -.7 \\ 5 & .3 & -3.5 & 1.2 & .4 \\ 6 & 1.3 & -4.3 & .7 & 1.4 \\ 8 & 2.8 & -5.1 & .0 & 3.2 \\ 10 & 3.6 & -4.8 & .1 & 4.3 \\ 15 & 3.8 & -3.0 & .3 & 5.4 \\ 20 & 2.8 & -4.3 & .3 & 5.7 \\ 30 & 1.7 & -3.7 & .0 & 7.7 \\ 40 & 1.1 & -2.9 & .0 & 9.5 \\ 50 & .7 & -2.5 & .1 & 10.7 \\ 60 & .4 & -2.2 & .0 & 11.1 \\ 80 & .1 & -1.7 & -.0 & 10.4 \\ 100 & .1 & -1.1 & .0 & 8.4 \\ 150 & .1 & .2 & .0 & 4.3 \\ 200 & .1 & 1.1 & .0 & 2.6 \\ 300 & .0 & 2.7 & .0 & 1.9\end{array}$

ENERGY

KEV

400
500
600
800
1000
1500
2000
3000
4000
5000
6000
8000
10000
15000
20000
30000
40000
50000
60000
80000
00000

ENERGY ENERGY

KE
40
50
60
80
100
150
200
300
400
500
600
800
1000
1500
2000
3000
4000
5000
6000
8000

\begin{tabular}{|c|c|c|c|c|}
\hline TOTAL & $\begin{array}{l}\text { COH } \\
\text { SCATT. }\end{array}$ & $\begin{array}{l}\text { INCOH. } \\
\text { SCATT. }\end{array}$ & $\begin{array}{l}\text { PHOTO } \\
\text { EFFECT }\end{array}$ & $\begin{array}{l}\text { PAIR } \\
\text { PROD. }\end{array}$ \\
\hline .0 & 3.7 & .0 & 3.7 & \\
\hline-.0 & $5 \cdot 8$ & -.0 & 7.4 & \\
\hline-.0 & $6 \cdot 0$ & -.0 & $12 \cdot 8$ & \\
\hline-.1 & 6.4 & -.1 & 26.5 & \\
\hline-.0 & 11.8 & -.0 & 41.8 & \\
\hline-.0 & 11.5 & -.0 & 36.5 & -1.0 \\
\hline .0 & 11.3 & -.0 & 41.4 & 3.5 \\
\hline-.1 & 11.2 & -.0 & 40.0 & -3 \\
\hline-.3 & 11.1 & -.1 & 37.7 & -4.9 \\
\hline-.4 & 11.0 & -.0 & 38.1 & -4.2 \\
\hline-.0 & 11.0 & -.0 & 36.5 & .1 \\
\hline .3 & 11.0 & -.0 & 35.7 & 2.0 \\
\hline-.1 & 10.9 & -.0 & 36.2 & -.4 \\
\hline .7 & 10.8 & -.0 & 33.4 & 2.1 \\
\hline-.5 & 10.8 & -.0 & 32.6 & -1.0 \\
\hline$-1 \cdot 2$ & 12.0 & -.0 & 32.5 & $-2 \cdot 0$ \\
\hline-.9 & 13.7 & -.0 & 33.0 & $-1 \cdot 3$ \\
\hline$-1 \cdot 2$ & 15.9 & -.0 & 33.6 & -1.6 \\
\hline .6 & $18 \cdot 2$ & -.0 & $34 \cdot 3$ & -.7 \\
\hline .3 & 23.7 & -.0 & $35 \cdot 5$ & -.3 \\
\hline & 30.7 & -.0 & 36.6 & -。 \\
\hline
\end{tabular}

\begin{tabular}{|c|c|c|c|c|}
\hline ENERGY & & $\mathrm{COH}$ & INCOH. & РНOTO \\
\hline KEV & TOTAL & SCATT. & SCATT. & EFFECT \\
\hline 1 & -.4 & -.1 & 36.6 & -.3 \\
\hline 1.5 & $-2 \cdot 1$ & -.5 & 26.1 & $-2 \cdot 1$ \\
\hline 2 & -2.1 & $-1 \cdot 3$ & 19.3 & -2.1 \\
\hline 3 & -.4 & $-2 \cdot 7$ & 13.8 & -.4 \\
\hline 4 & .7 & -3.0 & 8.9 & .8 \\
\hline 5 & $1 \cdot 3$ & -3.5 & 8.3 & 1.3 \\
\hline 6 & 1.8 & $-4 \cdot 4$ & $5 \cdot 5$ & 2.0 \\
\hline 8 & 2.7 & $-5 \cdot 3$ & $2 \cdot 4$ & 3.0 \\
\hline 10 & 3.3 & -5.1 & 2.9 & 3.7 \\
\hline 15 & 3.2 & -4.0 & 1.1 & 4.2 \\
\hline 20 & 2.5 & -4.6 & .6 & $4 \cdot 3$ \\
\hline 30 & 1.9 & -3.7 & .3 & $5 \cdot 8$ \\
\hline 40 & 1.4 & -2.8 & .2 & 7.0 \\
\hline 50 & .9 & -2.2 & .2 & 7.6 \\
\hline 60 & .5 & -1.7 & .1 & 7.6 \\
\hline 80 & .2 & -.9 & .0 & 6.2 \\
\hline 100 & $\cdot 1$ & -.4 & .1 & 3.9 \\
\hline 150 & .1 & - 8 & .1 & -.6 \\
\hline 200 & .1 & 1.9 & $\cdot 1$ & -3.1 \\
\hline 300 & .1 & $3 \cdot 3$ & .0 & -4.9 \\
\hline
\end{tabular}

\begin{tabular}{|c|c|c|c|c|}
\hline & $\begin{array}{l}\mathrm{COH} \\
\mathrm{SCATT}\end{array}$ & $\begin{array}{l}\text { INCOH. } \\
\text { SCATT. }\end{array}$ & $\begin{array}{r}\text { PHOTO } \\
\text { EFFECT }\end{array}$ & $\begin{array}{r}\text { PAIR } \\
\text { PROD. }\end{array}$ \\
\hline $\begin{array}{c}.0 \\
.04\end{array}$ & 4.3 & . 0 & $-3 \cdot 3$ & \\
\hline .1 & $5 \cdot 1$ & .0 & $1 \cdot 3$ & \\
\hline-.0 & 7.6 & -.0 & 7.6 & \\
\hline-0 & 9.9 & -.0 & $21 \cdot 9$ & \\
\hline-.0 & 14.6 & -.0 & 37.3 & \\
\hline-.0 & 14.3 & -.0 & 31.7 & -.7 \\
\hline-.0 & 14.2 & . 0 & 35.9 & -2.9 \\
\hline-.1 & $14 \cdot 0$ & -.0 & 34.3 & $-3 \cdot 3$ \\
\hline-.2 & 14.0 & -.1 & 31.9 & -1.9 \\
\hline-.1 & 13.9 & -.0 & 32.3 & -.5 \\
\hline .5 & 13.9 & -.0 & $30 \cdot A$ & $3 \cdot 8$ \\
\hline .8 & $13 \cdot 8$ & -.0 & $30 \cdot 2$ & 4.1 \\
\hline-.1 & $13 \cdot 8$ & $=0$ & $30 \cdot 8$ & -.5 \\
\hline-.4 & 13.7 & -.0 & $28 \cdot 3$ & $-1 \cdot 0$ \\
\hline-1.6 & 13.6 & -.0 & 27.6 & -3.4 \\
\hline-.9 & 14.7 & -.0 & 27.6 & -1.4 \\
\hline .1 & $16 \cdot 3$ & -.0 & 28.2 & -.2 \\
\hline .8 & 18.4 & -.0 & $28 \cdot 8$ & $-1 \cdot 1$ \\
\hline .07 & 20.7 & -.0 & $29 \cdot 5$ & -.9 \\
\hline & $25 \cdot 8$ & -.0 & $30 \cdot 7$ & $-1 \cdot 0$ \\
\hline & $32 \cdot 4$ & $=.0$ & 31.8 & -.7 \\
\hline
\end{tabular}




\begin{tabular}{|c|c|c|c|c|}
\hline \multirow{2}{*}{$\operatorname{KENGY}^{\mathrm{ENER}}{ }^{\mathrm{K}=}$} & \multicolumn{2}{|c|}{9 FLUORINE } & \multirow[b]{2}{*}{$\begin{array}{l}\text { INCOH. } \\
\text { SCATT. }\end{array}$} & \multirow[b]{2}{*}{$\begin{array}{l}\text { PHOTO } \\
\text { EFFECT }\end{array}$} \\
\hline & TOTAL & SCATT & & \\
\hline 1 & 1.9 & -.2 & $18 \cdot 7$ & 1.9 \\
\hline $1 \cdot 5$ & -2.5 & -.6 & 17.5 & $-2 \cdot 5$ \\
\hline 2 & -4.1 & -1.3 & 15.5 & -4.1 \\
\hline 3 & $-4 \cdot 2$ & -2.4 & 13.3 & $-4 \cdot 2$ \\
\hline 4 & -3.6 & -2.7 & 8.9 & -3.6 \\
\hline 5 & -3.1 & -3.4 & 6.3 & -3.1 \\
\hline 6 & -2.6 & $-4 \cdot 2$ & 4.5 & -2.5 \\
\hline 8 & -1.4 & -4.8 & 2.5 & -1.4 \\
\hline 10 & -.6 & -4.8 & 2.8 & -.4 \\
\hline 15 & .5 & $-4 \cdot 6$ & 1.1 & .9 \\
\hline 20 & .7 & $-5 \cdot 5$ & .6 & 1.6 \\
\hline 30 & 1.0 & $-5 \cdot 8$ & .3 & $3 \cdot 3$ \\
\hline 40 & .7 & -6.4 & .3 & 4.8 \\
\hline 50 & .4 & -6.8 & .3 & 5.5 \\
\hline 60 & .1 & -7.0 & .2 & 5.6 \\
\hline 80 & -.2 & -6.9 & .0 & 4.5 \\
\hline 100 & -.2 & -7.0 & .1 & 2.3 \\
\hline 150 & -.1 & -6.6 & .1 & -1.6 \\
\hline 200 & -.0 & -6.1 & $\cdot 1$ & -3.4 \\
\hline 300 & -.0 & -5.1 & .0 & $-4 \cdot 2$ \\
\hline
\end{tabular}

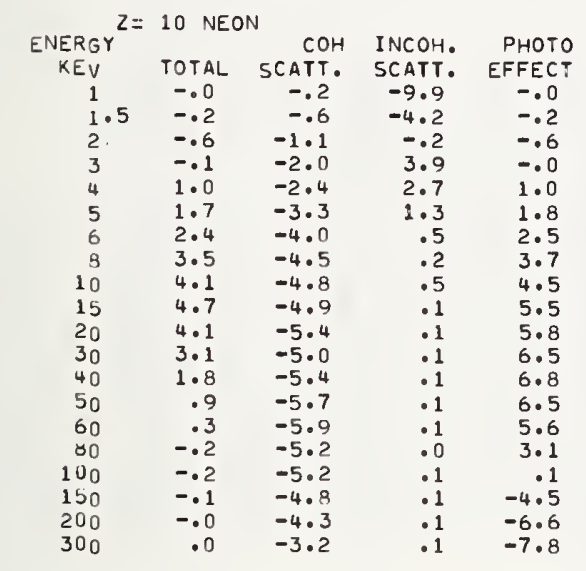

\begin{tabular}{|c|c|c|c|c|c|}
\hline $\begin{array}{c}\text { ENERGY } \\
\text { KEV }\end{array}$ & TOTAL & $\begin{array}{r}\mathrm{COH} \\
\text { SCATT. }\end{array}$ & $\begin{array}{l}\text { INCOH. } \\
\text { SCATT. }\end{array}$ & $\begin{array}{l}\text { PHOTO } \\
\text { EFFECT }\end{array}$ & $\begin{array}{l}\text { PAIR } \\
\text { PROD. }\end{array}$ \\
\hline 400 & .0 & $-4 \cdot 1$ & .0 & -2.4 & \\
\hline 500 & -.0 & $-3 \cdot 3$ & -.0 & 1.6 & \\
\hline 600 & -.0 & $-2 \cdot 5$ & -.0 & 7.0 & \\
\hline $\begin{array}{r}800 \\
1000\end{array}$ & $\begin{array}{l}-.0 \\
-.0\end{array}$ & $\begin{array}{l}-.3 \\
2.9\end{array}$ & $\begin{array}{l}=0 \\
-00\end{array}$ & $\begin{array}{l}19.7 \\
33.5\end{array}$ & \\
\hline 1500 & -.0 & 2.6 & -.0 & 27.7 & -.6 \\
\hline 2000 & .0 & $2 \cdot 4$ & .0 & 31.8 & -.2 \\
\hline 3000 & -.2 & $2 \cdot 2$ & -.0 & $30 \cdot 5$ & -3.8 \\
\hline 4000 & -.3 & $2 \cdot 1$ & -.1 & $28 \cdot 4$ & -3.5 \\
\hline 5000 & -.3 & $2 \cdot 1$ & -.0 & 28.9 & $-2 \cdot 3$ \\
\hline 6000 & .3 & $2 \cdot 0$ & -.0 & $27 \cdot 6$ & $2 \cdot 2$ \\
\hline 8000 & .7 & $2 \cdot 0$ & -.0 & $27 \cdot 2$ & 3.4 \\
\hline 10000 & -.0 & $2 \cdot 0$ & -.0 & 28.0 & -.1 \\
\hline 15000 & .2 & 1.9 & -.0 & 25.7 & .5 \\
\hline 20000 & $-1 \cdot 4$ & 1.8 & -.0 & 25.1 & -2.7 \\
\hline 30000 & $-1 \cdot 4$ & $2 \cdot 5$ & -.0 & $25 \cdot 2$ & -2.1 \\
\hline 40000 & -.6 & $4 \cdot 0$ & -.0 & 25.8 & -08 \\
\hline 50000 & -.9 & 5.7 & -.0 & $26 \cdot 5$ & $-1 \cdot 2$ \\
\hline 60000 & -.5 & $7 \cdot 7$ & $=.0$ & $27 \cdot 2$ & -.6 \\
\hline 80000 & -.6 & 11.9 & -.0 & $28 \cdot 4$ & -.7 \\
\hline 100000 & $-1 \cdot 0$ & 17.4 & -.0 & 29.5 & -1.1 \\
\hline
\end{tabular}

\begin{tabular}{|c|c|c|c|c|c|}
\hline ENERGY & & $\mathrm{COH}$ & I NCOH. & РHOTO & PAIR \\
\hline KEV & TOTAL & SCATT. & SCATT. & EFFECT & PROD。 \\
\hline 400 & .0 & -2.4 & .0 & -6.9 & \\
\hline 500 & .0 & $-1 \cdot 6$ & .0 & $-4 \cdot 4$ & \\
\hline 600 & -.0 & $-1 \cdot 1$ & .0 & -.1 & \\
\hline 800 & -.0 & 1.9 & -.0 & $12 \cdot 4$ & \\
\hline 1000 & -.0 & 1.0 & $=.0$ & $27 \cdot 6$ & \\
\hline 1500 & -.0 & .7 & -.0 & $24 \cdot 9$ & -.3 \\
\hline 2000 & -.0 & .6 & .0 & 30.7 & -3.0 \\
\hline 3000 & -.1 & .4 & -.0 & 31.0 & $-3 \cdot 1$ \\
\hline 4000 & -.2 & .3 & -.1 & $29 \cdot 2$ & -1.5 \\
\hline 5000 & -.0 & $\cdot 3$ & -.0 & 29.8 & -.2 \\
\hline 6000 & .6 & $\cdot 2$ & -.0 & $28 \cdot 5$ & 4.0 \\
\hline 8000 & .9 & .2 & -.0 & $27 \cdot 9$ & 4.0 \\
\hline 10000 & -.2 & .2 & -.0 & $28 \cdot 6$ & -.7 \\
\hline 15000 & -.7 & .1 & -.0 & 25.9 & -1.6 \\
\hline 20000 & -2.1 & .0 & -.0 & 25.0 & -3.8 \\
\hline 30000 & -1.0 & .6 & -.0 & 24.9 & -1.5 \\
\hline 40000 & -.1 & $2 \cdot 0$ & -.0 & $25 \cdot 4$ & -.1 \\
\hline 50000 & -.8 & $3 \cdot 5$ & -.0 & $26 \cdot 0$ & -1.0 \\
\hline 60000 & -.7 & $5 \cdot 3$ & -.0 & 26.6 & -.9 \\
\hline 80000 & -.9 & 9.2 & -40 & $27 \cdot 8$ & -1.0 \\
\hline 100000 & -.5 & $14 \cdot 4$ & -.0 & 28.8 & -.6 \\
\hline
\end{tabular}

$\begin{array}{ccccc}\begin{array}{c}\text { ENERGY } \\ \text { EN }\end{array} & 12 \text { MAGNESIUM } \\ \text { KEV } & \text { TOTAL } & \text { SCATT. } & \text { INCOH. } & \begin{array}{r}\text { PHOTO } \\ \text { SCATT. }\end{array} \\ 1 & -.4 & -1.2 & 7.9 & -.4 \\ 1.5 & 4.2 & -.9 & 2.5 & 4.2 \\ 2 & 1.3 & -.6 & 1.3 & 1.3 \\ 3 & -3.3 & -1.6 & -.1 & -3.3 \\ 4 & -5.4 & -2.7 & -.6 & -5.5 \\ 5 & -6.2 & -3.1 & -.5 & -6.2 \\ 6 & -6.3 & -3.2 & -.2 & -6.3 \\ 8 & -5.4 & -3.4 & .4 & -5.4 \\ 10 & -3.9 & -4.1 & .4 & -4.0 \\ 15 & -.4 & -4.9 & -.2 & -.2 \\ 20 & 2.1 & -4.3 & .1 & 2.7 \\ 30 & 3.8 & -5.6 & .2 & 6.3 \\ 40 & 3.1 & -8.9 & .1 & 8.1 \\ 50 & 1.5 & -11.7 & .1 & 8.5 \\ 00 & . .3 & -14.0 & .1 & 8.1 \\ 80 & -.6 & -13.4 & .0 & 5.8 \\ 100 & -.8 & -14.3 & .1 & 3.3 \\ 150 & -.6 & -15.5 & .1 & -.3 \\ 200 & -.4 & -15.7 & .0 & -1.8 \\ 300 & -. .2 & -25.5 & .0 & -2.9 \\ & & & & \end{array}$

ENERG

$\begin{array}{rrrrrr}\text { KEV } & \text { TOTAL } & \text { SCATT. } & \text { SCATT. } & \text { EFFECT } & \text { PROD. } \\ 400 & -.0 & -8.2 & .0 & -2.4 & \\ 500 & -.0 & -7.6 & .0 & -1.4 & \\ 600 & -.0 & -7.0 & .0 & .9 & \\ 800 & -.0 & -5.6 & -.0 & 10.1 & \\ 1000 & -.0 & -5.0 & -.0 & 21.7 & \\ 1500 & -.0 & -5.3 & -.0 & 19.2 & .0 \\ 2000 & .0 & -5.5 & -.0 & 25.1 & .9 \\ 3000 & -.2 & -5.7 & -.0 & 25.7 & -3.3 \\ 4000 & -.3 & -5.7 & -.1 & 24.1 & -3.1 \\ 5000 & -.3 & -5.8 & -.0 & 24.7 & -2.0 \\ 6000 & .4 & -5.8 & -.0 & 23.5 & 2.4 \\ 8000 & 1.0 & -5.9 & -.0 & 23.1 & 4.0 \\ 10000 & .3 & -5.9 & -.0 & 23.8 & .8 \\ 15000 & .8 & -5.9 & -.0 & 21.1 & 1.7 \\ 20000 & -.9 & -6.1 & -.0 & 20.3 & -1.5 \\ 30000 & -1.0 & -5.6 & -.0 & 20.1 & -1.5 \\ 40000 & -.4 & -4.4 & -.0 & 20.6 & -.5 \\ 50000 & -.8 & -3.0 & -.0 & 21.2 & -1.0 \\ 60000 & -.3 & -1.4 & -.0 & 21.8 & -.3 \\ 80000 & -.2 & 2.0 & -.0 & 22.9 & -.0 \\ 100000 & -.6 & 6.6 & -.0 & 23.9 & -.0\end{array}$


CROSS SECTION

C O MPAR I 50

$Z=13$ ALUMINUM

\begin{tabular}{|c|c|c|c|c|}
\hline $\begin{array}{c}\text { ENERGY } \\
\text { KEV }\end{array}$ & TOTAL & $\begin{array}{r}\mathrm{COH} \\
\text { SCATT. }\end{array}$ & $\begin{array}{l}\text { INCOH. } \\
\text { SCATY,. }\end{array}$ & $\begin{array}{r}\text { PHOTO } \\
\text { EFFECT }\end{array}$ \\
\hline 1 & 1.8 & -1.0 & 22.2 & 1.8 \\
\hline 1.5 & 1.9 & -.9 & 10.4 & 1.9 \\
\hline 2 & .4 & -.9 & 5.0 & .5 \\
\hline 3 & -1.6 & -2.0 & 2 & -1.6 \\
\hline 4 & -3.1 & -2.7 & -.7 & -3.2 \\
\hline 5 & -3.8 & -2.7 & 1.1 & -3.9 \\
\hline 6 & $-4 \cdot 0$ & -2.8 & $1 \cdot 0$ & -4.0 \\
\hline 8 & -3.5 & -3.0 & 1.1 & -3.5 \\
\hline 10 & -2.6 & -3.6 & -.0 & -2.5 \\
\hline 15 & -.2 & $-5 \cdot 3$ & -.1 & .0 \\
\hline 20 & 1.2 & -3.8 & .2 & 1.6 \\
\hline 30 & 1.7 & -5.7 & -.0 & 3.0 \\
\hline 40 & .8 & -9.6 & -.0 & 3.5 \\
\hline 50 & -.4 & $-13 \cdot 1$ & .0 & 3.2 \\
\hline 60 & -1.4 & -15.9 & .1 & 2.2 \\
\hline 80 & -1.8 & -15.4 & .0 & -.5 \\
\hline 100 & $-1 \cdot 7$ & $-16 \cdot 5$ & .1 & $-3 \cdot 5$ \\
\hline 150 & -1.1 & $-18 \cdot 0$ & .1 & -7.6 \\
\hline 200 & -.6 & $-18 \cdot 4$ & .1 & -9.2 \\
\hline 300 & -.3 & $-18 \cdot 3$ & .1 & -9.6 \\
\hline
\end{tabular}

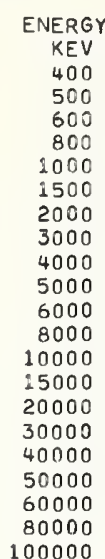

ENERGY

KEV

400
500
600
800
1000
1500
2000
3000
4000
5000
6000
8000
10000
15000
20000
30000
40000
50000
60000
80000
100000

100000

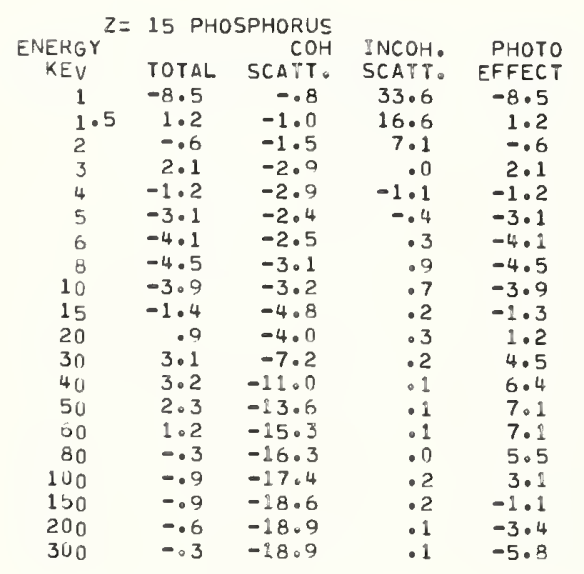

ENERGY

KEV

400
500
600
800
1000
1500
2000
3000
4000
5000
6000
8000
10000
15000
20000
30000
40000
50000
60000
80000
00000

$\$ 00000$
COH INCOH. PHOTO PAIR
TOTAL SCATT. SCATT. EFFECT PROD. $\begin{array}{rrrr}-.2 & -17.8 & 01 & -8.1\end{array}$ $\begin{array}{llll}-01 & -17.2 & -0 & -5.6\end{array}$ $\begin{array}{llll}-.1 & -16.3 & .0 & -1.6 \\ -.1 & -15.3 & -.0 & 10.0\end{array}$ $\begin{array}{llll}-.1 & -15.3 & -.0 & 10.0 \\ -.0 & -13.4 & -.0 & 23.9\end{array}$

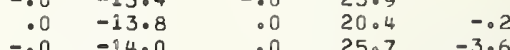

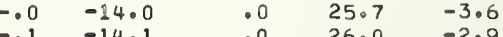
$\begin{array}{lllll}-.1 & -14.1 & .0 & 26.0 & -2.9 \\ -.1 & -14.2 & -.0 & 24.4 & -.7\end{array}$ $-11-14.2$ $.9-14.3$

$1.3-14.4$

$-.2-14.4$

$\begin{array}{rr}-.2 & -14.4 \\ -1.2 & -14.5\end{array}$

$-2.6 \quad-14.5$

$-1.0 \quad-24.3$

$.1-13.3$

$-.7 \quad-.2 .2$

$\begin{array}{ll}-.5 & -10.8 \\ -.7 & -8.0\end{array}$

$\begin{array}{ll}-.7 & -8.0 \\ -.1 & -4.2\end{array}$

$\begin{array}{ll}-.0 & 24.4 \\ -.0 & 25.0\end{array}$

$\begin{array}{rrr}.0 & .6 \\ .0 & 24.0 & 4.7\end{array}$

$\begin{array}{lll}.0 & 24.8 & -.5\end{array}$

$\begin{array}{lll}-.0 & 22.4 & -2.3\end{array}$

$\begin{array}{lll}-.0 & 21.7 & -4.4 \\ -.0 & 21.7 & -1.4\end{array}$

$\begin{array}{rl}-.0 & 22.3\end{array}$

$-.0 \quad 23.0$

$\begin{array}{lll}-.0 & 23.6 & -.8\end{array}$

$\begin{array}{lll}-.0 & 24.9 & -.8 \\ -.0 & 25.9 & -.1\end{array}$

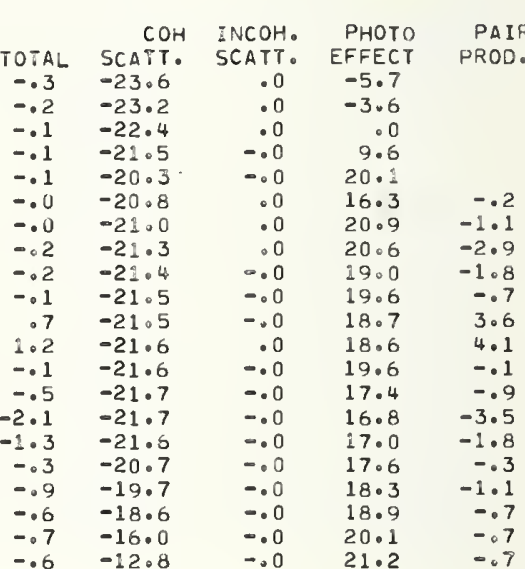

\begin{tabular}{|c|c|c|c|c|}
\hline ENERGY & & $\mathrm{COH}$ & $I \mathrm{NCOH}$ & PHOTO \\
\hline KEV & TOTAL & SCATT. & SCATT. & EFFECT \\
\hline 1 & $-6 \cdot 0$ & -.6 & $54 \cdot B$ & -6.1 \\
\hline 1.5 & -.4 & -1.0 & 27.7 & -04 \\
\hline 2 & 1.8 & -1.7 & 14.1 & 1.8 \\
\hline 3 & -.5 & -3.0 & 4.7 & -.5 \\
\hline 4 & -.8 & -2.9 & $2 \cdot 1$ & -.8 \\
\hline 5 & -1.3 & -2.4 & 1.5 & -2.3 \\
\hline 6 & $-1 \cdot 5$ & -2.6 & 1.4 & $-1 \cdot 5$ \\
\hline 8 & -1.2 & -3.2 & 1.0 & -1.2 \\
\hline 10 & -.5 & $-3 \cdot 1$ & -.3 & -.5 \\
\hline 15 & 1.7 & $-2 \cdot 9$ & -.1 & 2.9 \\
\hline 20 & $3 \cdot 1$ & -3.9 & .2 & 3.5 \\
\hline 30 & $3 \cdot 8$ & $-6 \cdot 6$ & .0 & $5 \cdot 1$ \\
\hline 40 & 3.0 & -9.7 & .0 & $5 \cdot 5$ \\
\hline 50 & $1 \cdot 8$ & $-11 \cdot 6$ & -.0 & $5 \cdot 0$ \\
\hline 60 & .5 & -12.8 & .0 & 401 \\
\hline 80 & $-1 \cdot 1$ & -23.8 & -.0 & $2 \cdot 3$ \\
\hline 100 & $-1 \cdot 5$ & $-14 \cdot 5$ & $\cdot 1$ & -1.7 \\
\hline 150 & $-1 \cdot 3$ & $-15 \cdot 3$ & - 1 & -6.7 \\
\hline 200 & -.9 & $-15 \cdot 5$ & $\cdot 0$ & -9.6 \\
\hline 300 & -.4 & $-15 \cdot 2$ & $\cdot 0$ & -12.2 \\
\hline
\end{tabular}

\begin{tabular}{|c|c|c|c|c|c|}
\hline ENERGY & & $\mathrm{COH}$ & INCOH. & PHOTO & PAI P \\
\hline KEV & TOTAL & SCATT. & SCATT. & EFFECT & PROD \\
\hline 400 & -.2 & $-14 \cdot 9$ & .0 & $-12 \cdot 7$ & \\
\hline 500 & -.2 & -14.3 & -.0 & -8.9 & \\
\hline 600 & -01 & $-13 \cdot 8$ & -.0 & $-4 \cdot 4$ & \\
\hline 800 & -.0 & $-12 \cdot 4$ & -.0 & $6 \cdot 3$ & \\
\hline 1000 & -.0 & -10.5 & -.0 & 18.4 & \\
\hline 1500 & .0 & $-11 \cdot 2$ & .0 & 13.8 & -.7 \\
\hline 2000 & -.0 & $-1: 3$ & .0 & $18 \cdot 0$ & -1.9 \\
\hline 3000 & -.2 & $-11 \cdot 6$ & .0 & 17.2 & -2.7 \\
\hline 4000 & -.1 & $-11 \cdot 8$ & -.0 & $15 \cdot 9$ & -1.0 \\
\hline 5000 & .0 & $-1: \cdot 8$ & -.0 & $16 \cdot 8$ & $\bullet$ \\
\hline 6000 & 1.0 & -11.9 & .0 & 16.0 & 4. \\
\hline 8000 & 1.6 & -12.0 & .0 & 26.2 & 4.8 \\
\hline 10000 & .1 & -12.0 & .0 & 27.4 & .4 \\
\hline 25000 & -.5 & $-12 \cdot 1$ & .0 & 15.5 & -.9 \\
\hline 20000 & $-2 \cdot 1$ & -12.1 & -.0 & $15 \cdot 1$ & -3.3 \\
\hline 30000 & -.6 & -12.1 & -.0 & 15.4 & -.8 \\
\hline 40000 & .8 & $-18 \cdot 2$ & -.0 & $16 \cdot 1$ & 2.0 \\
\hline 50000 & .2 & $-10 \cdot 2$ & .0 & 16.9 & .3 \\
\hline 60000 & .5 & -8.9 & .0 & 17.6 & .6 \\
\hline 80000 & .4 & $-6 \cdot 2$ & -.0 & $18 \cdot 9$ &. \\
\hline 00000 & .5 & -3.0 & -.0 & $20 \cdot 0$ & \\
\hline
\end{tabular}


$Z=17$ CHLORINE

$\begin{array}{crrrr}\begin{array}{c}\text { ENERGY } \\ \text { KEV }\end{array} & \text { TOTAL } & \text { SCATT. } & \begin{array}{r}\text { INCOH. } \\ \text { SCATT. }\end{array} & \begin{array}{r}\text { PHOTO } \\ \text { EFFECT }\end{array} \\ 1 & -2.8 & -.5 & 45.7 & -2.8 \\ 1.5 & -4.4 & -1.0 & 23.2 & -4.4 \\ 2 & -2.7 & -1.8 & 12.3 & -2.7 \\ 3 & 4.1 & -3.1 & 5.6 & 4.1 \\ 4 & .8 & -3.0 & 3.2 & .8 \\ 5 & -1.6 & -2.6 & 2.2 & -1.6 \\ 6 & -3.1 & -2.7 & 1.8 & -3.2 \\ 8 & -4.5 & -3.1 & 1.0 & -4.6 \\ 10 & -4.7 & -2.5 & -.2 & -4.7 \\ 15 & -3.1 & -2.4 & -.0 & -3.2 \\ 20 & -1.6 & -4.1 & .2 & -1.5 \\ 30 & -.5 & -3.4 & .1 & .8 \\ 40 & 1.2 & -4.5 & .1 & 2.1 \\ 50 & 1.2 & -5.1 & .1 & 2.5 \\ 00 & .7 & -5.3 & .1 & 2.4 \\ 80 & -.2 & -5.1 & .0 & 1.0 \\ 100 & -.6 & -5.1 & .1 & -.19 \\ 150 & -.5 & -4.8 & .2 & -4.2 \\ 200 & -.3 & -4.3 & .1 & -6.3 \\ 300 & -.1 & -3.5 & .1 & -8.4\end{array}$

$\begin{array}{ccccc}\text { ENERGY }^{Z}= & 18 \text { ARGON } \\ \text { KEV } & \text { TOTAL } & \text { SCATT. } & \begin{array}{r}\text { INCOH. } \\ \text { SCATT. }\end{array} & \begin{array}{r}\text { PHOTO } \\ \text { EFFECT }\end{array} \\ 1 & -6.5 & -.3 & 14.5 & -6.5 \\ 1.5 & -2.6 & -.8 & 6.2 & -2.6 \\ 2 & -.1 & -1.8 & 2.8 & -.1 \\ 3 & 1.3 & -3.1 & 2.3 & 1.3 \\ 4 & -2.6 & -3.0 & 1.5 & -2.6 \\ 5 & -3.3 & -2.7 & .9 & -3.3 \\ 6 & -3.7 & -2.9 & .5 & -3.7 \\ 8 & -3.7 & -3.1 & .2 & -3.7 \\ 10 & -3.1 & -2.6 & .3 & -3.1 \\ 15 & -.6 & -2.8 & .4 & -.6 \\ 20 & 1.3 & -4.2 & .3 & 1.5 \\ 30 & 3.5 & -3.7 & .1 & 4.2 \\ 40 & 4.0 & -5.1 & .1 & 5.5 \\ 50 & 3.5 & -5.8 & .1 & 5.9 \\ 60 & 2.7 & -6.2 & .1 & 5.7 \\ 80 & 1.0 & -6.2 & .1 & 4.2 \\ 100 & . .1 & -6.3 & .2 & 2.2 \\ 150 & -.4 & -6.1 & .2 & -1.9 \\ 200 & -.3 & -5.7 & .2 & -4.7 \\ 300 & -.1 & -4.9 & .2 & -8.2\end{array}$

\begin{tabular}{|c|c|c|c|c|}
\hline \multicolumn{5}{|c|}{$Z=19$ POTASSIUM } \\
\hline ENERGY & & $\mathrm{COH}$ & INCOH. & PHOTO \\
\hline KEV & TOTAL & SCATT. & SCATT. & EFFECT \\
\hline 1 & -3.0 & -.8 & & -3.0 \\
\hline 1.5 & $\begin{array}{l}-3.1 \\
-2.6\end{array}$ & $\begin{array}{l}-1.4 \\
-2.1\end{array}$ & $\begin{array}{r}-4.1 \\
-.6\end{array}$ & $\begin{array}{l}-3.1 \\
-2.6\end{array}$ \\
\hline $\begin{array}{l}2 \\
3\end{array}$ & $\begin{array}{l}-1.6 \\
-1.6\end{array}$ & -2.9 & 1.9 & -1.6 \\
\hline 4 & $2 \cdot 1$ & -2.9 & 1.7 & $2 \cdot 1$ \\
\hline 5 & -.2 & -2.8 & 1.0 & -.2 \\
\hline 6 & -1.9 & $-3 \cdot 0$ & .4 & $-1 \cdot 8$ \\
\hline 8 & -3.4 & -3.1 & -.1 & -3.4 \\
\hline 10 & -3.6 & $-3 \cdot 0$ & .1 & -3.6 \\
\hline 15 & $-2 \cdot 2$ & $-4 \cdot 6$ & .3 & $-2 \cdot 1$ \\
\hline 20 & -.4 & -4.1 & - 3 & -.3 \\
\hline 30 & $1 \cdot 9$ & $-3 \cdot 5$ & -1 & $2 \cdot 3$ \\
\hline 40 & $2 \cdot 9$ & $-4 \cdot 3$ & .1 & 4.0 \\
\hline 50 & 3.0 & $-4 \cdot 8$ & .1 & $4 \cdot 8$ \\
\hline 60 & $2 \cdot 6$ & $-5 \cdot 0$ & .1 & $5 \cdot 0$ \\
\hline 80 & $1 \cdot 3$ & $-5 \cdot 1$ & -.0 & $4 \cdot 2$ \\
\hline 100 & .4 & $-5 \cdot 0$ & $\cdot 2$ & $2 \cdot 7$ \\
\hline 150 & -.3 & $-4 \cdot 5$ & .1 & -.4 \\
\hline 200 & -.3 & $-4 \cdot 0$ & - 1 & -2.7 \\
\hline 300 & -.2 & -3.1 & .0 & $-5 \cdot 5$ \\
\hline
\end{tabular}

$\begin{array}{ccccc}\begin{array}{c}\text { ENERGY } \\ Z=\end{array} & 20 \text { CALCIUM } \\ \text { KEV } & \text { TOTAL } & \text { SCATT. } & \begin{array}{r}\text { INCOH. } \\ \text { SCATT. }\end{array} & \begin{array}{r}\text { PHOTO } \\ \text { EFFECT }\end{array} \\ 1 & -1.3 & -1.3 & -8.7 & -1.3 \\ 1.5 & -3.5 & -1.4 & -4.6 & -3.5 \\ 2 & -5.3 & -1.6 & -.9 & -5.3 \\ 3 & -5.2 & -2.4 & 1.8 & -5.2 \\ 4 & -1.5 & -2.8 & 1.7 & -1.4 \\ 5 & -1.5 & -2.9 & 1.1 & -1.5 \\ 6 & -1.6 & -2.9 & .4 & -1.7 \\ 8 & -1.7 & -2.7 & -.2 & -1.7 \\ 10 & -1.5 & -2.8 & .0 & -1.4 \\ 15 & -.0 & -2.8 & .4 & .0 \\ 20 & .9 & -3.8 & .3 & 1.1 \\ 30 & 1.4 & -3.2 & .1 & 1.7 \\ 40 & 1.0 & -3.9 & .1 & 1.5 \\ 50 & .3 & -4.3 & .1 & .8 \\ 60 & -.5 & -4.5 & .1 & -1.2 \\ 80 & -1.7 & -4.7 & .0 & -2.6 \\ 100 & -2.1 & -4.7 & .2 & -4.9 \\ 150 & -1.5 & -4.1 & .1 & -8.6 \\ 200 & -1.0 & -3.5 & .1 & -10.7 \\ 300 & -.4 & -2.6 & .1 & -12.4\end{array}$

\begin{tabular}{|c|c|c|c|c|c|}
\hline $\begin{array}{c}\text { ENERGY } \\
\text { KEV }\end{array}$ & TOTAL & $\begin{array}{l}\text { COH } \\
\text { SCATT. }\end{array}$ & $\begin{array}{l}\text { INCOH. } \\
\text { SCATT. }\end{array}$ & $\begin{array}{l}\text { PHOTO } \\
\text { EFFECT }\end{array}$ & $\begin{array}{l}\text { PAIR } \\
\text { PROD. }\end{array}$ \\
\hline 400 & -.0 & -2.7 & .1 & -8.2 & \\
\hline 500 & .0 & $-2 \cdot 1$ & .0 & -5.8 & \\
\hline 600 & -.0 & $-1 \cdot 3$ & .0 & $-2,0$ & \\
\hline 800 & .0 & .2 & -.0 & $7 \cdot 0$ & \\
\hline 1000 & .0 & 3.0 & -.0 & 16.8 & \\
\hline 1500 & .0 & 2.5 & .0 & 11.9 & .5 \\
\hline 2000 & -.1 & $2 \cdot 3$ & .0 & $15 \cdot 8$ & -6.5 \\
\hline 3000 & -.2 & $2 \cdot 0$ & .0 & 14.9 & -2.6 \\
\hline 4000 & .1 & 1.9 & -.0 & 13.8 & $1 \cdot 1$ \\
\hline 5000 & .5 & $1 \cdot 8$ & -.0 & 14.9 & $2 \cdot 8$ \\
\hline 6000 & 1.7 & 1.7 & .0 & $14 \cdot 3$ & $7 \cdot 2$ \\
\hline 8000 & 2.0 & 1.7 & .0 & $14 \cdot 7$ & 6.1 \\
\hline 10000 & -.1 & 1.6 & -.0 & $16 \cdot 0$ & -.1 \\
\hline 15000 & $-2 \cdot 3$ & 1.6 & -.0 & $14 \cdot 3$ & $-4 \cdot 1$ \\
\hline 20000 & $-4 \cdot 1$ & $1 \cdot 5$ & -.0 & 14.0 & -6.2 \\
\hline 30000 & $-1 \cdot 3$ & 1.5 & -.0 & 14.5 & -1.7 \\
\hline 40000 & .4 & 2.5 & -.0 & $15 \cdot 2$ & .5 \\
\hline 50000 &.- .5 & 3.7 & -.0 & $16 \cdot 0$ & -.5 \\
\hline 60000 & -.6 & $5 \cdot 0$ & -.0 & 16.8 & -.7 \\
\hline 80000 & -1.1 & $8 \cdot 1$ & -.0 & $18 \cdot 2$ & $-1 \cdot 2$ \\
\hline 00000 & -.4 & 11.6 & -.0 & 19.2 & -04 \\
\hline
\end{tabular}

\begin{tabular}{|c|c|c|c|c|c|}
\hline $\begin{array}{c}\text { ENERGY } \\
\text { KEV }\end{array}$ & TOTAL & $\begin{array}{r}\text { COH } \\
\text { SCATT. }\end{array}$ & $\begin{array}{l}\text { INCOH. } \\
\text { SCATT. }\end{array}$ & $\begin{array}{l}\text { PHOTO } \\
\text { EFFECT }\end{array}$ & $\begin{array}{l}\text { PAIR } \\
\text { PROD. }\end{array}$ \\
\hline 400 & -.0 & $-4 \cdot 2$ & .1 & -8.7 & \\
\hline 500 & -.0 & $-3 \cdot 5$ & .1 & $-6 \cdot 6$ & \\
\hline $\begin{array}{l}600 \\
800\end{array}$ & .0 & $-2 \cdot 8$ & .0 & $-2 \cdot 8$ & \\
\hline $\begin{array}{r}800 \\
1000\end{array}$ & .0 & $\begin{array}{r}-1 \cdot 1 \\
1 \cdot 3\end{array}$ & $\begin{array}{l}=0 \\
-.0\end{array}$ & $\begin{array}{r}6.0 \\
5.5\end{array}$ & \\
\hline 1500 & .0 & .7 & .0 & 10.8 & -.4 \\
\hline 2000 & .0 & .5 & .0 & 14.7 & -.5 \\
\hline 3000 & -.2 & .2 & .0 & 13.7 & -2.9 \\
\hline 4000 &.$- \overline{3}$ & .0 & -.1 & 12.6 & -1.9 \\
\hline 5000 & -.2 & -.0 & -.0 & 13.8 & -.9 \\
\hline 6000 & . 8 & -.1 & -.0 & 13.1 & 3.4 \\
\hline 8000 & 1.5 & -.2 & .0 & 13.5 & 4.2 \\
\hline 10000 & .2 & -.2 & -.0 & 14.8 & .4 \\
\hline 15000 & .0 & -.3 & -.0 & 13.1 & .0 \\
\hline 20000 & $-1 \cdot 8$ & -.3 & -.0 & $12 \cdot 8$ & -2.7 \\
\hline 30000 & $-1 \cdot 1$ & -.4 & -.0 & 13.3 & -1.5 \\
\hline 40000 & -.2 & .5 & -.0 & 14.0 & -.2 \\
\hline 50000 & -.7 & 1.7 & -.0 & 14.8 & -.9 \\
\hline 60000 & -.4 & 3.0 & -.0 & 15.6 & -.4 \\
\hline 80000 & -.3 & 6.0 & -.0 & 16.9 & $=$ \\
\hline 00000 & -.1 & 9.2 & -.0 & 18.0 & \\
\hline
\end{tabular}

\begin{tabular}{|c|c|c|c|c|c|}
\hline ENERGY & & $\mathrm{COH}$ & I NCOH. & РHOTO & PAIR \\
\hline $\begin{array}{l}\text { KEV } \\
400\end{array}$ & $\begin{array}{l}\text { TOTAL } \\
-.1\end{array}$ & $\begin{array}{l}\text { SCATT. } \\
=2.3\end{array}$ & $\begin{array}{r}\text { SCATT. } \\
.0\end{array}$ & $\begin{array}{c}\text { EFFECT } \\
-6.1\end{array}$ & PROD. \\
\hline 500 & -.0 & -1.7 & .0 & $-4 \cdot 5$ & \\
\hline 600 & -.0 & -1.1 & -.0 & $-1 \cdot 6$ & \\
\hline 800 & -.0 & .7 & -.0 & $5 \cdot 2$ & \\
\hline 1000 & .0 & 3.0 & -.0 & 12.4 & \\
\hline 1500 & .0 & $2 \cdot 4$ & .0 & $7 \cdot 3$ & -.8 \\
\hline 2000 & -.0 & 2.2 & .0 & $10 \cdot 8$ & -2.0 \\
\hline 3000 & -.2 & 1.8 & .0 & 9.5 & -3.1 \\
\hline 4000 & -.2 & 1.7 & -.0 & $8 \cdot 5$ & -1.5 \\
\hline 5000 & -.1 & $1 \cdot 6$ & -.0 & 9.7 & -.5 \\
\hline 6000 & 1.0 & 2.5 & .0 & 9.1 & 3.8 \\
\hline 8000 & 1.5 & 1.4 & .0 & 9.6 & 4.2 \\
\hline 10000 & -.1 & 1.4 & .0 & 11.0 & -.1 \\
\hline 15000 & -.8 & 3.4 & -.0 & 9.4 & -1.4 \\
\hline 20000 & -2.7 & 2.3 & -.0 & 9.2 & -3.9 \\
\hline 30000 & -1.5 & 1.2 & -.0 & 9.7 & -1.9 \\
\hline 40000 & -.4 & 2,1 & -.0 & $10 \cdot 5$ & -.5 \\
\hline 50000 & -1.1 & 3,2 & -00 & 11.3 & -1.2 \\
\hline 60000 & -.8 & 4.5 & -.0 & $12 \cdot 1$ & -.9 \\
\hline 80000 & -.7 & 7.4 & -.0 & 13.4 & -.8 \\
\hline 100000 & -.3 & 10.6 & -.0 & $14 \cdot 5$ & -.3 \\
\hline
\end{tabular}

\begin{tabular}{|c|c|c|c|c|c|}
\hline $\begin{array}{c}\text { ENERGY } \\
\text { KEV }\end{array}$ & TOTAL. & $\begin{array}{r}\mathrm{COH} \\
\text { SCATT. }\end{array}$ & $\begin{array}{l}\text { INCOH. } \\
\text { SCATT. }\end{array}$ & $\begin{array}{r}\text { PHOTO } \\
\text { EFFECT }\end{array}$ & $\begin{array}{l}\text { PAIR } \\
\text { PROD. }\end{array}$ \\
\hline 400 & -.2 & -1.8 & .0 & -11.6 & \\
\hline 500 & -.1 & -1.2 & .0 & -8.7 & \\
\hline 600 & -.0 & -.4 & .0 & $-4 \cdot 4$ & \\
\hline 800 & .0 & 1.0 & -.0 & 6.4 & \\
\hline 1000 & .0 & 2.6 & -.0 & 18.6 & \\
\hline 1500 & .0 & $2 \cdot 0$ & .0 & $13 \cdot 5$ & -.9 \\
\hline 2000 & -.1 & 1.7 & .0 & $17 \cdot 5$ & $-4 \cdot 3$ \\
\hline 3000 & -.3 & 1.4 & .0 & 16,0 & $-3 \cdot 5$ \\
\hline 4000 & -.2 & 1.2 & -.0 & 15.1 & -.9 \\
\hline 5000 & .1 & 1.2 & -.0 & $16 \cdot 4$ & .6 \\
\hline 6000 & 1.4 & 1.1 & -.0 & 15.7 & 5.2 \\
\hline 8000 & 2.1 & $1 \cdot 0$ & .0 & 16.1 & 5.6 \\
\hline 10000 & .4 & 1.0 & -.0 & 17.5 & .8 \\
\hline 15000 & -.9 & .9 & -.0 & 15.7 & $-1 \cdot 6$ \\
\hline 20000 & -3.1 & .9 & -.0 & $15 \cdot 4$ & $-4 \cdot 5$ \\
\hline 30000 & $-1 \cdot 5$ & .8 & -.0 & 15.9 & -2.0 \\
\hline 40000 & -.0 & 1.6 & -.0 & 16.7 & -.0 \\
\hline 50000 & -.5 & 2.7 & -.0 & 17.5 & -.6 \\
\hline 60000 & -.3 & 3.9 & -.0 & $18 \cdot 3$ & -.3 \\
\hline 80000 & -.4 & 6.8 & -.0 & 19.7 & -0 \\
\hline 100000 & -.2 & 9.9 & -.0 & 20.9 & -.2 \\
\hline
\end{tabular}




\begin{tabular}{|c|c|c|c|c|}
\hline ENERGY & & $\mathrm{COH}$ & I NCOH. & PHOTO \\
\hline KEV & TOTAL & SCATT. & SCATT. & EFFECT \\
\hline $1 \cdot 5$ & $\begin{array}{l}-5.4 \\
-7.6\end{array}$ & $\begin{array}{l}-1 \cdot 2 \\
-1 \cdot 2\end{array}$ & $\begin{array}{r}-5 \\
1.1\end{array}$ & $\begin{array}{l}-5.4 \\
-7.6\end{array}$ \\
\hline 2 & -7.8 & -1.4 & $3 \cdot 2$ & -7.8 \\
\hline 3 & $\begin{array}{l}-5.6 \\
-3.0\end{array}$ & $\begin{array}{l}-2.3 \\
-2.8\end{array}$ & $\begin{array}{l}4.0 \\
3.2\end{array}$ & $\begin{array}{l}-5.6 \\
-3.0\end{array}$ \\
\hline $\begin{array}{l}4 \\
5\end{array}$ & -.0 & -3.0 & $\begin{array}{l}3.2 \\
2.2\end{array}$ & -.0 \\
\hline 6 & -.6 & -3.1 & 1.4 & -.6 \\
\hline 8 & $-2 \cdot 1$ & $-2 \cdot 9$ & .4 & $-2 \cdot 1$ \\
\hline 10 & -3.0 & -2.9 & 1.2 & -3.0 \\
\hline 15 & $-3 \cdot 3$ & -.8 & .8 & -3.4 \\
\hline 20 & -2.6 & -3.2 & .5 & -2.6 \\
\hline 30 & -.4 & $-2 \cdot 0$ & .7 & -.3 \\
\hline 40 & $1 \cdot 3$ & -1.7 & .5 & 1.6 \\
\hline 50 & $2 \cdot 2$ & $-1 \cdot 4$ & .4 & 2.9 \\
\hline 60 & $2 \cdot 6$ & $-1 \cdot 2$ & $\cdot 3$ & 3.8 \\
\hline 80 & $2 \cdot 2$ & -.8 & .1 & $4 \cdot 2$ \\
\hline 100 & 1.5 & -.3 & .3 & 3.5 \\
\hline 150 & .4 & .9 & $\cdot 2$ & .9 \\
\hline 200 & .1 & 1.8 & .2 & -1.5 \\
\hline 300 & .0 & $3 \cdot 1$ & $\cdot 1$ & $-5 \cdot 5$ \\
\hline
\end{tabular}

\begin{tabular}{|c|c|c|c|c|c|}
\hline $\begin{array}{c}\text { ENERGY } \\
\text { KEV }\end{array}$ & TOTAL & $\begin{array}{l}\text { COH } \\
\text { SCATT. }\end{array}$ & $\begin{array}{l}\text { INCOH. } \\
\text { SCATT. }\end{array}$ & $\begin{array}{r}\text { РНOTO } \\
\text { EFFECT }\end{array}$ & $\begin{array}{l}\text { PAIR } \\
\text { PROD. }\end{array}$ \\
\hline 400 & .0 & 4.1 & .1 & -7.4 & \\
\hline $\begin{array}{l}500 \\
600\end{array}$ & .0 & $\begin{array}{l}4.9 \\
5.8\end{array}$ & .1 & $\begin{array}{l}-7.0 \\
-4.9\end{array}$ & \\
\hline 800 & .1 & 7.5 & .0 & 1.5 & - \\
\hline 1000 & .1 & 9.4 & .0 & 8.9 & \\
\hline 1500 & .0 & 8.8 & .0 & 5.6 & -1.9 \\
\hline 2000 & .1 & $8 \cdot 5$ & .0 & 10.2 & .1 \\
\hline 3000 & -.3 & 8.2 & .0 & 9.5 & -3.7 \\
\hline 4000 & -.5 & 8.1 & -.0 & $8 \cdot 8$ & -3.0 \\
\hline 5000 & -.4 & $8 \cdot 0$ & .0 & $10 \cdot 1$ & $-2 \cdot 0$ \\
\hline 6000 & .7 & 7.9 & .0 & 9.4 & $2 \cdot 5$ \\
\hline 8000 & 1.6 & 7.9 & .0 & 9.8 & 4.1 \\
\hline 10000 & .5 & $7 \cdot 8$ & $\cdot 0$ & 11.1 & 1.1 \\
\hline 15000 & .8 & $7 \cdot 8$ & -.0 & 9.2 & 1.4 \\
\hline 20000 & -1.4 & 7.7 & -.0 & 8.7 & $-2 \cdot 0$ \\
\hline 30000 & -1.6 & $7 \cdot 6$ & -.0 & $9 \cdot 0$ & -2.0 \\
\hline 40000 & -.8 & 8.4 & -.0 & 9.7 & -1.0 \\
\hline 50000 & -1.2 & 9.6 & -.0 & 10.5 & -1.4 \\
\hline 60000 & -.6 & 10.8 & -.0 & 11.2 & -.7 \\
\hline 80000 & -.04 & 13.8 & -.0 & 12.4 & -.4 \\
\hline 100000 & -.5 & 17.0 & -.0 & $13 \cdot 5$ & -.5 \\
\hline
\end{tabular}

\begin{tabular}{|c|c|c|c|c|}
\hline ENERGY & & $\mathrm{COH}$ & INCOH. & РHOTO \\
\hline KEV & TOTAL & SCATT. & SCATT. & EFFECT \\
\hline 1 & -7.2 & $-1 \cdot 1$ & 6.9 & -7.2 \\
\hline $1 \cdot 5$ & $-6 \cdot 0$ & $-1 \cdot 2$ & 6.1 & $\begin{array}{l}-6.0 \\
-6.0\end{array}$ \\
\hline 2 & -6.0 & $-1 \cdot 3$ & $6 \cdot 5$ & $-6 \cdot 0$ \\
\hline 3 & $-5 \cdot 3$ & -2.2 & $5 \cdot 6$ & -5.3 \\
\hline 4 & -3.7 & $-2 \cdot 8$ & $4 \cdot 2$ & -3.7 \\
\hline 5 & $-1 \cdot 2$ & $-3 \cdot 1$ & 3.1 & -1.2 \\
\hline 6 & -.2 & $-3 \cdot 2$ & $2 \cdot 1$ & -.2 \\
\hline 8 & -.2 & -3.1 & 1.0 & -.2 \\
\hline 10 & -.7 & -3.2 & $1 \cdot 3$ & -.7 \\
\hline 15 & $-1 \cdot 3$ & -3.1 & .8 & $-1 \cdot 3$ \\
\hline 20 & $-1 \cdot 2$ & -3.1 & .5 & -1.2 \\
\hline 30 & -.4 & $-2 \cdot 0$ & . 9 & -.4 \\
\hline 40 & $\cdot 2$ & $-1 \cdot 4$ & .7 & .2 \\
\hline 50 & .4 & -1.0 & .4 & .6 \\
\hline 60 & .4 & -.8 & .4 & .6 \\
\hline 80 & -.1 & -.2 & .2 & -.2 \\
\hline 100 & -.6 & .4 & .3 & -1.7 \\
\hline 150 & $-1 \cdot 0$ & 1.7 & $\cdot 1$ & -5.6 \\
\hline 200 & -.8 & $2 \cdot 7$ & .0 & -8.5 \\
\hline 300 & -.4 & 4.1 & .0 & $-12 \cdot 3$ \\
\hline
\end{tabular}

ENERGY
KEV
400
500
600
800
1000
1500
2000
3000
4000
5000
6000
8000
10000
15000
20000
30000
40000
50000
60000
80000
100000

$\begin{array}{rrrrr} & \text { COH } & \text { INCOH. } & \text { PHOTO } & \text { PAIR } \\ \text { TOTAL } & \text { SCATT. } & \text { SCATT. } & \text { EFFECT } & \text { PROD. } \\ -.2 & 5.1 & .0 & -13.2 & \\ -.1 & 6.0 & .0 & -11.4 & \\ -.0 & 6.9 & -.0 & -7.7 & \\ .0 & 8.5 & -.0 & 1.6 & \\ .1 & 10.6 & -.0 & 12.2 & \\ .0 & 10.0 & .0 & 8.7 & -2.4 \\ .0 & 9.8 & .0 & 13.1 & -1.1 \\ -.3 & 9.5 & .0 & 12.3 & -3.9 \\ -.5 & 9.3 & -.1 & 11.5 & -3.0 \\ -.4 & 9.2 & -.0 & 12.7 & -1.7 \\ .8 & 9.2 & -.0 & 12.1 & 2.7 \\ 1.6 & 9.1 & -.0 & 12.6 & 4.0 \\ .3 & 9.1 & -.0 & 13.9 & .7 \\ .4 & 9.0 & -.0 & 11.9 & .6 \\ -1.7 & 9.0 & -.0 & 11.5 & -2.4 \\ -1.2 & 8.8 & -.0 & 11.8 & -1.5 \\ -.0 & 9.6 & -.0 & 12.5 & -.0 \\ -.3 & 10.7 & -.0 & 13.3 & -.04 \\ .2 & 11.9 & -.0 & 14.0 & .3 \\ .3 & 14.9 & -.0 & 15.3 & .3 \\ .1 & 18.1 & -.0 & 16.5 & .1\end{array}$

$\begin{array}{ccccc}\text { ENERGY } & 23 \text { VANAOIUM } \\ \text { KEV } & \text { TOTAL } & \text { SCATT. } & \begin{array}{r}\text { INCOH. } \\ \text { SCATT. }\end{array} & \begin{array}{r}\text { PHOTO } \\ \text { EFFECT }\end{array} \\ 1 & -6.9 & -1.1 & 14.8 & -6.9 \\ 1.5 & -5.3 & -1.1 & 11.3 & -5.3 \\ 2 & -5.2 & -1.3 & 9.7 & -5.2 \\ 3 & -4.4 & -2.1 & 6.3 & -4.4 \\ 4 & -2.9 & -2.7 & 4.7 & -2.9 \\ 5 & -1.4 & -3.0 & 3.3 & -1.4 \\ 6 & -3.5 & -3.2 & 2.4 & -3.4 \\ 8 & -.4 & -3.2 & 1.3 & -.4 \\ 10 & .7 & -3.5 & 1.2 & .8 \\ 15 & 1.7 & -4.4 & .7 & 1.8 \\ 20 & 2.1 & -3.0 & .5 & 2.2 \\ 30 & 2.6 & -2.3 & .9 & 2.9 \\ 40 & 2.6 & -1.7 & .6 & 3.1 \\ 50 & 2.4 & -1.4 & .4 & 2.9 \\ 60 & 1.9 & -1.1 & .3 & 2.5 \\ 80 & . .7 & -.6 & .2 & 1.1 \\ 100 & -.2 & -.1 & .3 & -.8 \\ 150 & -.9 & 1.1 & .2 & -5.0 \\ 200 & -.8 & 2.2 & .1 & -7.9 \\ 300 & -.4 & 3.6 & .0 & -11.4\end{array}$

ENERGY
KEV
400
500
600
800
1000
1500
2000
3000
4000
5000
6000
8000
10000
15000
20000
30000
40000
50000
60000
$B 0000$
100000

\begin{tabular}{|c|c|c|c|c|}
\hline TOTAL & $\begin{array}{l}\mathrm{COH} \\
\text { SCATT. }\end{array}$ & $\begin{array}{l}\text { INCOH. } \\
\text { SCATT. }\end{array}$ & $\begin{array}{r}\text { PHOTO } \\
\text { EFFECT }\end{array}$ & $\begin{array}{r}\text { PAIR } \\
\text { PROD. }\end{array}$ \\
\hline-.2 & 4.5 & .0 & -12.1 & \\
\hline-.1 & $5 \cdot 4$ & .0 & $-10 \cdot 3$ & \\
\hline-.0 & 6.2 & -.0 & $-6 \cdot 6$ & \\
\hline .0 & 8.0 & -.0 & 3.1 & \\
\hline .1 & 9.5 & -.0 & 14.5 & \\
\hline .1 & 9.0 & .0 & $10 \cdot 1$ & $-1 \cdot 2$ \\
\hline-.1 & 8.7 & .0 & 14.4 & $-4 \cdot 7$ \\
\hline-.3 & 8.4 & .0 & 13.7 & $-3 \cdot 2$ \\
\hline-.1 & 8.2 & -.0 & 13.2 & -.5 \\
\hline .3 & 8.2 & -.0 & 14.8 & 1.3 \\
\hline 1.6 & 8.1 & -.0 & 14.4 & 5.4 \\
\hline 2.1 & 8.0 & .0 & $15 \cdot 2$ & $5 \cdot 2$ \\
\hline$\bullet 0$ & $8 \cdot 0$ & .0 & $16 \cdot 9$ & .0 \\
\hline-1.3 & 7.9 & -.0 & $15 \cdot 2$ & $-2 \cdot 1$ \\
\hline-3.1 & 7.9 & -.0 & 14.9 & $-4 \cdot 3$ \\
\hline-.9 & 7.7 & -.0 & $15 \cdot 5$ & -1.1 \\
\hline .5 & 8.4 & -.0 & $16 \cdot 3$ & .5 \\
\hline-.4 & 9.5 & -.0 & 17.2 & -.4 \\
\hline-.2 & 10.7 & -.0 & 18.0 & -.3 \\
\hline-.2 & $13 \cdot 6$ & -.0 & $19 \cdot 4$ & -.2 \\
\hline .9 & $16 \cdot 6$ & -.0 & $20 \cdot 6$ & .9 \\
\hline
\end{tabular}

$\begin{array}{crrrr}\begin{array}{c}\text { ENERGY } \\ \text { EN }\end{array} & 24 \text { CHROMIUM } \\ \text { KEV } & \text { TOTAL } & \text { SCATT. } & \begin{array}{r}\text { INCOH. } \\ \text { SCATT. }\end{array} & \begin{array}{r}\text { PHOTO } \\ \text { EFFECT }\end{array} \\ 1 & -4.3 & -.7 & 5.2 & -4.3 \\ 1.5 & -1.0 & -.9 & 2.5 & -1.0 \\ 2 & -1.7 & -1.2 & 1.8 & -1.7 \\ 3 & -3.0 & -2.1 & 1.3 & -3.0 \\ 4 & -2.5 & -2.7 & .7 & -2.5 \\ 5 & -.6 & -3.1 & .5 & -.6 \\ 6 & -3.8 & -3.4 & .3 & -3.8 \\ 8 & -.4 & -3.3 & .1 & -.4 \\ 10 & .7 & -3.3 & .1 & .7 \\ 15 & 1.3 & -3.2 & .2 & 1.4 \\ 20 & 1.5 & -4.0 & .4 & 1.6 \\ 30 & 1.6 & -6.2 & 2.1 & 1.9 \\ 40 & 1.3 & -7.9 & 1.4 & 1.9 \\ 50 & .9 & -9.2 & .9 & 1.7 \\ 60 & .4 & -10.1 & .8 & 1.4 \\ 80 & -.9 & -11.1 & .4 & .1 \\ 100 & -1.7 & -11.6 & .5 & -1.7 \\ 150 & -2.2 & -11.7 & .3 & -5.7 \\ 200 & -1.8 & -11.4 & .1 & -8.6 \\ 300 & -1.0 & -10.9 & .1 & -12.0\end{array}$

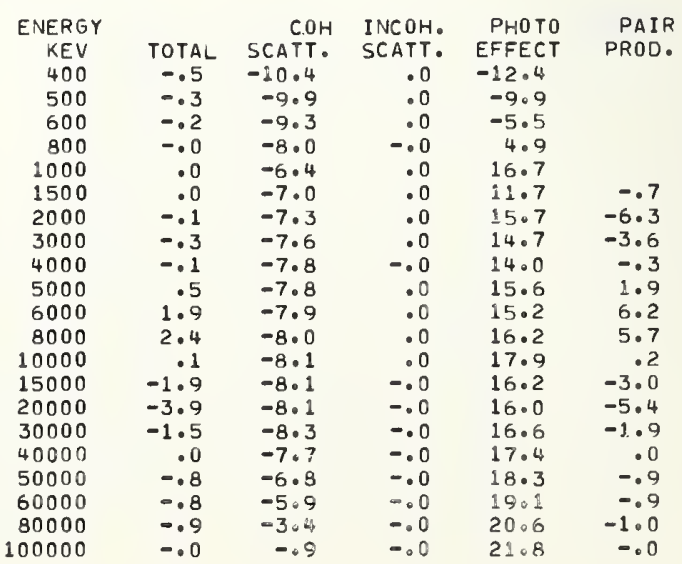




$\begin{array}{ccrrr}\text { ENERGY } & \text { 25 MANGANESE } \\ \text { ENEV } & \text { TOTAL } & \text { SCATT. } & \text { INCOH. } & \text { PHOTO } \\ \text { SEATT. } & \text { EFFECT } \\ 1 & -11.8 & -.9 & 8.2 & -11.8 \\ 1.5 & -3.8 & -1.0 & 3.3 & -3.9 \\ 2 & -3.1 & -1.2 & 2.0 & -3.2 \\ 3 & -3.8 & -1.9 & .8 & -3.8 \\ 4 & -3.4 & -2.5 & .2 & -3.4 \\ 5 & -1.6 & -2.9 & -.0 & -1.6 \\ 6 & 1.2 & -3.0 & .0 & 1.3 \\ 8 & -.4 & -3.0 & .2 & -.4 \\ 10 & 1.4 & -3.0 & .2 & 1.4 \\ 15 & 2.7 & -2.1 & .1 & 2.8 \\ 20 & 3.1 & -2.9 & .2 & 3.2 \\ 30 & 3.3 & -2.7 & .1 & 3.6 \\ 40 & 3.2 & -2.2 & .1 & 3.7 \\ 50 & 2.9 & -1.9 & .1 & 3.5 \\ 60 & 2.3 & -1.7 & .1 & 3.1 \\ 80 & 1.1 & -1.2 & -.1 & 1.8 \\ 100 & .0 & -.8 & .3 & .1 \\ 150 & -1.0 & . .3 & .0 & -4.0 \\ 200 & -1.0 & 1.4 & -.0 & -7.0 \\ 300 & -.6 & 2.7 & -.0 & -10.7\end{array}$

ENERGY
KEV
400
500
600
800
1000
1500
2000
3000
4000
5000
6000
8000
10000
15000
20000
30000
40000
50000
60000
80000
100000

$\begin{array}{rrrrr} & & & \\ \text { TOTAL } & \text { SCATT } & \text { INCOH. } & \text { PHOTO } & \text { PAIR } \\ -.3 & 3.6 & -.0 & -11.4 & \\ -.1 & 4.5 & -.0 & -9.5 & \\ -.0 & 5.3 & -.0 & -5.8 & \\ .1 & 6.8 & -.0 & 3.6 & \\ .1 & 8.5 & -.0 & 14.4 & \\ .1 & 8.0 & .0 & 9.7 & -1.2 \\ -.1 & 7.7 & .0 & 13.6 & -4.3 \\ -.3 & 7.4 & .0 & 12.7 & -3.4 \\ -.2 & 7.2 & -.0 & 12.1 & -.9 \\ .3 & 7.1 & -.0 & 13.6 & 1.3 \\ 1.7 & 7.1 & -.0 & 13.3 & 5.6 \\ 2.5 & 7.0 & -.0 & 14.2 & 5.8 \\ .6 & 7.0 & -.0 & 16.0 & 1.1 \\ -.5 & 6.9 & -.0 & 14.3 & -.7 \\ -2.5 & 6.9 & -.0 & 14.0 & -3.5 \\ -.8 & 6.7 & -.0 & 14.5 & -1.0 \\ .6 & 7.3 & -.0 & 15.3 & .7 \\ -.1 & 8.4 & -.0 & 16.2 & -.1 \\ .2 & 9.5 & -.0 & 17.0 & .2 \\ .2 & 12.2 & -.00 & 18.4 & .2 \\ .7 & 15.1 & -.0 & 19.6 & .7\end{array}$

\begin{tabular}{|c|c|c|c|c|}
\hline ENERGY & & $\mathrm{COH}$ & INCOH. & РНОTО \\
\hline KEV & TOTAL & SCATT. & SCATT. & EFFECT \\
\hline 1 & -6.9 & -.8 & $15 \cdot 6$ & -6.9 \\
\hline $1 \cdot 5$ & -2.6 & -1.0 & 8.9 & $-2 \cdot 6$ \\
\hline $\begin{array}{l}2 \\
3\end{array}$ & $\begin{array}{l}-3.4 \\
-4.9\end{array}$ & $\begin{array}{l}-1.1 \\
-1.8\end{array}$ & $\begin{array}{l}6.2 \\
3.2\end{array}$ & $\begin{array}{l}-3.4 \\
-4.9\end{array}$ \\
\hline 4 & $-4 \cdot 7$ & -2.4 & 1.6 & $-4 \cdot 8$ \\
\hline 5 & -3.4 & $-2 \cdot 8$ & $1 \cdot 1$ & -3.4 \\
\hline 6 & -1.4 & -3.0 & i. 0 & -1.4 \\
\hline 8 & -.6 & -3.0 & .9 & -.6 \\
\hline 10 & -.5 & $-3 \cdot 2$ & $1 \cdot 2$ & -.5 \\
\hline 15 & -1.1 & -2.9 &.$\overline{6}$ & -1.0 \\
\hline 20 & $-1 \cdot 2$ & -2.9 & .6 & $-1 \cdot 1$ \\
\hline 30 & -.5 & -2.8 & .7 & -.5 \\
\hline 40 & .4 & -2.4 & .5 & .5 \\
\hline 50 & 1.0 & -2.1 & .3 & 1.3 \\
\hline 60 & 1.4 & -1.9 & .3 & 1.9 \\
\hline 80 & 1.4 & -1.4 & $\cdot 0$ & $2 \cdot 2$ \\
\hline 100 & 1.0 & $-1 \cdot 0$ & .3 & 1.8 \\
\hline 150 & .0 & .1 & .1 & -.1 \\
\hline 200 & -.2 & 1.0 & .0 & -2.1 \\
\hline 300 & -.2 & $2 \cdot 3$ & -.0 & -5.1 \\
\hline
\end{tabular}

ENERGY
KEV
400
500
600
800
1000
1500
2000
3000
4000
5000
6000
8000
10000
15000
20000
30000
40000
50000
60000
80000
100000

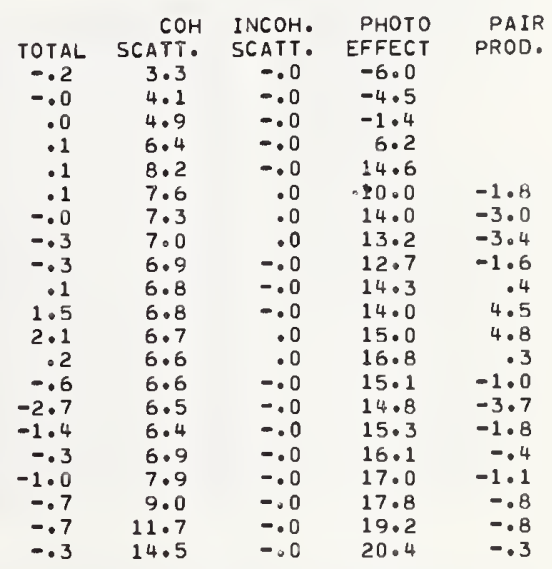

\begin{tabular}{|c|c|c|c|c|}
\hline $\begin{array}{c}\text { ENERGY } \\
\text { KEV }\end{array}$ & TOTAL & $\begin{array}{l}\text { COH } \\
\text { SCATT. }\end{array}$ & $\begin{array}{l}\text { INCOH. } \\
\text { SCATT. }\end{array}$ & $\begin{array}{l}\text { PHOTO } \\
\text { EFFECT }\end{array}$ \\
\hline 1 & $-14 \cdot 2$ & -.7 & 23.9 & $-14 \cdot 3$ \\
\hline 1.5 & -3.4 & -.9 & 14.8 & -3.5 \\
\hline 2 & $-1 \cdot 9$ & $-1 \cdot 1$ & 10.1 & -1.9 \\
\hline 3 & $-2 \cdot 5$ & $-1 \cdot 8$ & 4.7 & $-2 \cdot 5$ \\
\hline 4 & -3.1 & $-2 \cdot 3$ & $2 \cdot 3$ & $-3 \cdot 1$ \\
\hline 5 & -2.8 & -2.7 & 1.7 & -2.8 \\
\hline 6 & $-1 \cdot 8$ & -2.9 & 1.6 & -1.8 \\
\hline 8 & -4.6 & -2.9 & 1.5 & $-4 \cdot 6$ \\
\hline 10 & $-2 \cdot 5$ & -2.9 & $\therefore .5$ & $-2 \cdot 6$ \\
\hline 15 & -.9 & -1.9 & .7 &. .9 \\
\hline 20 & -.1 & -2.9 & .6 & -.1 \\
\hline 30 & 1.2 & -2.8 & .8 & 1.4 \\
\hline 40 & $2 \cdot 3$ & $-2 \cdot 6$ & .6 & 2.6 \\
\hline 50 & $2 \cdot 8$ & $-2 \cdot 3$ & .4 & 3.4 \\
\hline 60 & $3 \cdot 1$ & -2.2 & .4 & 3.9 \\
\hline 80 & $2 \cdot 7$ & $-1 \cdot 6$ & .1 & $4 \cdot 0$ \\
\hline 100 & 1.9 & $-1 \cdot 3$ & .3 & $3 \cdot 3$ \\
\hline 150 & $\cdot 3$ & -.4 & .1 & .7 \\
\hline 200 & -.2 & .5 & .1 & -1.7 \\
\hline 300 & -.4 & 1.8 & .0 & $-5 \cdot 4$ \\
\hline
\end{tabular}

ENERGY

\begin{tabular}{|c|c|c|c|c|c|}
\hline $\begin{array}{c}\text { ENERGY } \\
\text { KEV }\end{array}$ & TOTAL & $\begin{array}{r}\text { COH } \\
\text { SCATT. }\end{array}$ & $\begin{array}{l}\text { INCOH. } \\
\text { SCATT. }\end{array}$ & $\begin{array}{l}\text { PHOTO } \\
\text { EFFECT }\end{array}$ & $\begin{array}{r}\text { PAIR } \\
\text { PROD. }\end{array}$ \\
\hline 400 & -.2 & 2.8 & .0 & -6.9 & \\
\hline 500 & -.1 & 3.6 & .0 & -6.1 & \\
\hline 600 & .0 & 4.4 & .0 & $-3 \cdot 8$ & \\
\hline 800 & .1 & 5.8 & .0 & 2.5 & \\
\hline 1000 & .1 & 7.4 & -.0 & 9.8 & \\
\hline 1500 & .0 & 6.8 & .0 & $5 \cdot 3$ & -1.5 \\
\hline 2000 & -.1 & 6.6 & .0 & 9.1 & $-4 \cdot 3$ \\
\hline 3000 & -.3 & 6.3 & .0 & 8.5 & $-3 \cdot 3$ \\
\hline 4000 & -.2 & 6.1 & -.1 & 7.9 & -.9 \\
\hline 5000 & .3 & 6.0 & -.0 & 9.3 & 1.2 \\
\hline 6000 & 1.7 & 6.0 & -.0 & 9.0 & 5.2 \\
\hline 8000 & 2.1 & 5.9 & -.0 & 10.0 & 4.9 \\
\hline 10000 & -.1 & 5.9 & -.0 & 11.7 & -.2 \\
\hline 15000 & -1.5 & 5.8 & -.0 & 10.0 & -2.3 \\
\hline 20000 & -3.4 & 5.8 & -.0 & 9.8 & $-4 \cdot 6$ \\
\hline 30000 & -1.4 & 5.6 & -.0 & 10.3 & -1.7 \\
\hline 40000 & -.0 & 6.1 & -.0 & 11.1 & -.0 \\
\hline 50000 & -.9 & 7.1 & -.0 & 11.9 & $-1 \cdot 0$ \\
\hline 60000 & -.8 & 8.1 & -.0 & 12.7 & -.8 \\
\hline 80000 & $-1 \cdot 0$ & 10.7 & -.0 & 14.1 & $-1 \cdot 0$ \\
\hline 00000 & -.4 & 13.5 & -.0 & $15 \cdot 3$ & -.5 \\
\hline
\end{tabular}

\begin{tabular}{|c|c|c|c|c|}
\hline $\begin{array}{c}\text { ENERGY } \\
\text { KEV }\end{array}$ & TOTAL & $\begin{array}{r}\mathrm{COH} \\
\text { SCATT. }\end{array}$ & $\begin{array}{l}\text { INCOH. } \\
\text { SCATT. }\end{array}$ & $\begin{array}{r}\text { РHOTO } \\
\text { EFFECT }\end{array}$ \\
\hline 1 & -6.4 & -.7 & 30.4 & $-6 \cdot 3$ \\
\hline 1.5 & $-4 \cdot 6$ & -.8 & 19.4 & $-4 \cdot 6$ \\
\hline 2 & $-4 \cdot 2$ & -1.0 & 13.0 & $-4 \cdot 1$ \\
\hline 3 & -3.8 & -1.7 & 5.9 & -3.8 \\
\hline 4 & -2.9 & $-2 \cdot 2$ & 2.9 & -2.9 \\
\hline 5 & -1.9 & -2.6 & 2.0 & -1.8 \\
\hline 6 & -1.0 & $-2 \cdot 8$ & 1.8 & -1.0 \\
\hline 3 & -.6 & -2.8 & 1.8 & -.5 \\
\hline 10 & -.6 & -3.0 & 1.4 & -.6 \\
\hline 15 & -.9 & -2.2 & .6 & -.9 \\
\hline 20 & $-1 \cdot 0$ & $-2 \cdot 9$ & .6 & $-1 \cdot 0$ \\
\hline 30 & -.2 & -2.9 & .7 & -.2 \\
\hline 40 & .8 & -2.9 & .4 & 1.0 \\
\hline 50 & 1.7 & -2.8 & $\cdot 3$ & 2.1 \\
\hline 60 & $2 \cdot 3$ & -2.7 & $\cdot 3$ & 2.9 \\
\hline 80 & 2.6 & $-2 \cdot 1$ & $\cdot 0$ & 3.9 \\
\hline 100 & $2 \cdot 2$ & -1.9 & .3 & 3.9 \\
\hline 150 & .8 & $-1 \cdot 1$ & $\cdot 1$ & $2 \cdot 5$ \\
\hline 200 & .2 & -.3 & $\cdot 1$ & .7 \\
\hline 300 & -.1 & 1.0 & .0 & -2.6 \\
\hline
\end{tabular}

\begin{tabular}{|c|c|c|c|c|c|}
\hline $\begin{array}{c}\text { ENERGY } \\
\text { KEV }\end{array}$ & TOTAL & $\begin{array}{l}\mathrm{COH} \\
\text { SCATT. }\end{array}$ & $\begin{array}{l}\text { INCOH. } \\
\text { SCATT. }\end{array}$ & $\begin{array}{r}\text { PHOTO } \\
\text { EFFECT }\end{array}$ & $\begin{array}{l}\text { PAIR } \\
\text { PROD. }\end{array}$ \\
\hline 400 & -.1 & 1.9 & . 0 & -3.9 & \\
\hline 500 & -.0 & 2.7 & .0 & -2.9 & \\
\hline 600 & .1 & 3.5 & .0 & -.4 & \\
\hline 800 & .2 & 5.0 & .0 & $5 \cdot 5$ & \\
\hline 1000 & .1 & 6.4 & .0 & 11.7 & \\
\hline 1500 & .1 & 5.8 & .0 & 7.0 & -2.4 \\
\hline 2000 & .1 & $5 \cdot 6$ & .0 & 10.8 & .0 \\
\hline 3000 & -.3 & $5 \cdot 3$ & .0 & 10.2 & -3.2 \\
\hline 4000 & -.5 & 5.1 & -.1 & 9.4 & -2.6 \\
\hline 5000 & -.3 & 5.0 & -.0 & 10.7 & $-1 \cdot 1$ \\
\hline 6000 & $\$ .0$ & 5.0 & -.0 & 10.4 & 3.0 \\
\hline 8000 & 1.9 & 4.9 & .0 & 11.4 & $4 \cdot 2$ \\
\hline 10000 & .5 & 4.9 & -.0 & 13.2 & 1.0 \\
\hline 15000 & 1.1 & 4.8 & -.0 & 11.4 & 1.7 \\
\hline 20000 & -.8 & $4 \cdot 8$ & -.0 & $11 \cdot 2$ & -1.1 \\
\hline 30000 & -.7 & $4 \cdot 6$ & -.0 & 11.7 & -.8 \\
\hline 40000 & . 1 & $5 \cdot 1$ & -.0 & $12 \cdot 6$ & .1 \\
\hline 50000 & -.4 & 6.0 & -.0 & 13.4 & -.5 \\
\hline 60000 & .1 & 7.0 & -.0 & 14.2 & .1 \\
\hline 80000 & .4 & 9.6 & -.0 & 15.7 & .4 \\
\hline 100000 & .5 & $12 \cdot 3$ & -.0 & 16.9 & .6 \\
\hline
\end{tabular}


TABLE I - cont. PHOTON CROSS SECTION COMPARISON

\begin{tabular}{|c|c|c|c|c|}
\hline $\begin{array}{c}\text { ENERGY } \\
\text { KEV }\end{array}$ & TOTAL & $\begin{array}{r}\mathrm{COH} \\
\text { SCATT. }\end{array}$ & $\begin{array}{l}\text { INCOH. } \\
\text { SCATT. }\end{array}$ & $\begin{array}{r}\text { PHOTO } \\
\text { EFFECT }\end{array}$ \\
\hline$\stackrel{1}{1.5}$ & $\begin{array}{l}-17 \cdot 1 \\
-12.5\end{array}$ & $\begin{array}{l}-.5 \\
-.7\end{array}$ & $\begin{array}{r}15.3 \\
7.7\end{array}$ & $\begin{array}{l}-17 \cdot 1 \\
-12.5\end{array}$ \\
\hline 2 & -9.2 & -.9 & 3.9 & -9.2 \\
\hline 3 & $-6 \cdot 3$ & -1.5 & .7 & $-6 \cdot 3$ \\
\hline 4 & $-4 \cdot 4$ & $-2 \cdot 1$ & -.3 & -4.4 \\
\hline 5 & -2.8 & -2.6 & -.3 & -2.8 \\
\hline 6 & -1.4 & -2.9 & .1 & -1.4 \\
\hline 9 & .0 & -3.0 & .6 & .1 \\
\hline 10 & $-1 \cdot 3$ & -3.3 & .5 & $-1 \cdot 2$ \\
\hline 15 & -.4 & -3.4 & .1 & -.4 \\
\hline 20 & -.4 & -2.9 & .3 & -.4 \\
\hline 30 & -.2 & -3.2 & .2 & -.1 \\
\hline 40 & .2 & -3.6 & .1 & $\cdot 3$ \\
\hline 50 & .5 & -3.7 & - 1 & - 8 \\
\hline 60 & .7 & -3.7 & .1 & 1.1 \\
\hline 80 & .6 & $-3 \cdot 2$ & -.1 & 1.2 \\
\hline 100 & $\cdot 2$ & $-3 \cdot 1$ & .3 & .7 \\
\hline 150 & -.6 & -2.4 & .1 & -1.1 \\
\hline 200 & -.8 & -1.7 & .0 & -3.0 \\
\hline 300 & -.6 & -.7 & .0 & -5.9 \\
\hline
\end{tabular}

ENERGY
KEV
400
500
600
800
1000
1500
2000
3000
4000
5000
6000
8000
10000
15000
20000
30000
40000
50000
60000
80000
100000

\begin{tabular}{|c|c|c|c|c|}
\hline TOTAL & $\begin{array}{l}\text { COH } \\
\text { SCATT. }\end{array}$ & $\begin{array}{l}\text { INCOH. } \\
\text { SCATT. }\end{array}$ & $\begin{array}{l}\text { PHOTO } \\
\text { EFFECT }\end{array}$ & $\begin{array}{l}\text { PAIR } \\
\text { PROD. }\end{array}$ \\
\hline $\begin{array}{l}-.3 \\
-.1\end{array}$ & $\begin{array}{r}.4 \\
1.1\end{array}$ & $\begin{array}{r}.0 \\
.0\end{array}$ & $\begin{array}{l}-6.6 \\
-5.2\end{array}$ & \\
\hline-.0 & 1.8 & -.0 & -2.2 & \\
\hline .1 & 3.2 & -.0 & 5.4 & \\
\hline .1 & 4.7 & .0 & 14.0 & \\
\hline .1 & 4.2 & .0 & 9.5 & $-2,6$ \\
\hline . 1 & 3.9 & .0 & 13.5 & -.3 \\
\hline-.3 & 3.6 & .0 & 13.3 & $-3 \cdot 2$ \\
\hline-.5 & 3.4 & -.1 & 12.6 & $-2 \cdot 6$ \\
\hline-.3 & 3.4 & -.0 & 14.0 & -1.2 \\
\hline 1.0 & 3.3 & -.0 & 13.6 & 2.8 \\
\hline 1.6 & 3.2 & .0 & 14.7 & 3.5 \\
\hline-.0 & 3.2 & -.0 & 16.5 & -.1 \\
\hline-.1 & 3.1 & -.0 & 14.8 & -.1 \\
\hline-2.1 & 3.1 & -.0 & 14.5 & -2.7 \\
\hline-1.6 & 2.9 & -.0 & 15.1 & -1.9 \\
\hline-.5 & $3 \cdot 3$ & -.0 & 16.0 & -.5 \\
\hline-.9 & 4.2 & -.0 & 16.9 & $-1,0$ \\
\hline-.3 & $5 \cdot 3$ & -.0 & 17.7 & -.4 \\
\hline-.2 & 7.7 & -.0 & 19.2 & -.2 \\
\hline-.1 & 10.3 & -.0 & 20.5 & -.1 \\
\hline
\end{tabular}

\begin{tabular}{|c|c|c|c|c|}
\hline ENERGY & & $\mathrm{COH}$ & INCOH. & РНОTO \\
\hline KEV & TOTAL & SCATT. & SCATT. & EFFECT \\
\hline 1 & $-4 \cdot 7$ & -.6 & 17.3 & $-4 \cdot 8$ \\
\hline 1.5 & -7.9 & -.8 & 7.6 & -7.9 \\
\hline 2 & -3.7 & -1.0 & 3.5 & -3.7 \\
\hline 3 & $-1 \cdot 5$ & -1.5 & .4 & -1.5 \\
\hline 4 & $-1 \cdot 3$ & $-2 \cdot 0$ & -.5 & $-1 \cdot 3$ \\
\hline 5 & -1.4 & -2.4 & -.5 & -1.4 \\
\hline 6 & -1.9 & -2.6 & -.2 & -1.9 \\
\hline 8 & $-3 \cdot 2$ & -2.7 & .6 & $-3 \cdot 3$ \\
\hline 10 & -3.2 & -3.1 & .5 & -3.2 \\
\hline 15 & -2.7 & $-3 \cdot 3$ & .0 & -2.7 \\
\hline 20 & -2.7 & $-2 \cdot 8$ & $\cdot 2^{\circ}$ & -2.7 \\
\hline 30 & -1.9 & $-3 \cdot 3$ & .2 & -1.8 \\
\hline 40 & -.7 & -4.1 & .2 & -.6 \\
\hline 50 & . 2 & $-4 \cdot 4$ & .1 & .5 \\
\hline 60 & .9 & $-4 \cdot 6$ & .2 & 1.4 \\
\hline 80 & 1.4 & $-4 \cdot 1$ & -.0 & $2 \cdot 3$ \\
\hline 100 & $1 \cdot 1$ & -4.2 & .3 & 2.2 \\
\hline 150 & -.0 & -3.8 & .1 & .7 \\
\hline 200 & -.4 & -3.1 & .0 & $-1 \cdot 0$ \\
\hline 300 & -.4 & $-2 \cdot 0$ & .0 & -3.9 \\
\hline
\end{tabular}

ENERGY
KEV
400
500
600
800
1000
1500
2000
3000
4000
5000
6000
8000
10000
15000
20000
30000
40000
50000
60000
80000
100000

$\begin{array}{rrrrr} & & & \\ \text { TOTAL } & \text { SCATT. } & \text { INCOH. } & \text { PHOTO } & \text { PAIR } \\ -.3 & -1.2 & .1 & -5.2 & \\ -.1 & -.4 & .0 & -4.8 & \\ -.0 & .2 & .0 & -2.8 & \\ .1 & 1.5 & .0 & 2.6 & \\ .1 & 2.9 & .0 & 8.9 & \\ .1 & 2.3 & .0 & 4.8 & -2.1 \\ .0 & 2.0 & .0 & 8.8 & -.7 \\ -.3 & 1.8 & .0 & 8.7 & -3.0 \\ -.4 & 1.6 & -.0 & 7.9 & -2.0 \\ -.1 & 1.5 & -.0 & 9.2 & -.4 \\ 1.3 & 1.5 & -.0 & 8.9 & 3.8 \\ 2.2 & 1.4 & .0 & 9.8 & 4.7 \\ .6 & 1.3 & -.0 & 11.5 & 1.1 \\ .5 & 1.3 & -.0 & 9.7 & .7 \\ -1.8 & 1.3 & -.0 & 9.4 & -2.3 \\ -1.5 & 1.1 & -.0 & 9.8 & -1.8 \\ -. .4 & 1.5 & -.0 & 10.7 & -.5 \\ -.8 & 2.3 & -.0 & 11.5 & -.9 \\ -.0 & 3.3 & -.0 & 12.3 & -.02 \\ .2 & 5.6 & -.0 & 13.7 & .2 \\ .4 & 8.2 & -.0 & 14.9 & .5\end{array}$

$\begin{array}{ccccc}\text { ENERGY } & 31 \text { GALLIUM } \\ \text { KEV } & \text { TOTAL } & \text { SCATT. } & \begin{array}{r}\text { INCOH. } \\ \text { SCATT. }\end{array} & \begin{array}{r}\text { PHOTO } \\ \text { EFFECT }\end{array} \\ 1 . & -15.2 & -.6 & 22.5 & -15.3 \\ 1.5 & -13.3 & -.7 & 10.7 & -13.3 \\ 2 & -9.9 & -.9 & 5.1 & -9.9 \\ 3 & -7.2 & -1.5 & .5 & -7.2 \\ 4 & -5.5 & -2.0 & -.7 & -5.5 \\ 5 & -4.0 & -2.3 & -.6 & -4.0 \\ 6 & -2.7 & -2.4 & -.1 & -2.7 \\ 8 & -.8 & -2.6 & .8 & -.8 \\ 10 & -.4 & -3.0 & .5 & -.2 \\ 15 & 1.2 & -3.9 & -.1 & 1.3 \\ 20 & -.3 & -2.7 & .0 & -.3 \\ 30 & -.8 & -3.5 & .0 & -.7 \\ 40 & -.1 & -4.9 & .1 & .1 \\ 50 & .8 & -5.9 & .2 & 1.2 \\ 60 & 1.5 & -6.4 & .1 & 2.2 \\ 80 & 2.3 & -5.9 & -.0 & 3.6 \\ 100 & 2.1 & -6.1 & .2 & 4.0 \\ 150 & .8 & -6.1 & .1 & 3.2 \\ 200 & .1 & -5.6 & .2 & 1.7 \\ 300 & -.2 & -4.6 & .3 & -1.5\end{array}$

ENERGY
KEV
400
500
600
800
1000
1500
2000
3000
4000
5000
6000
8000
10000
15000
20000
30000
40000
50000
60000
80000
100000

\begin{tabular}{|c|c|c|c|c|}
\hline TOTAL & $\begin{array}{l}\mathrm{COH} \\
\text { SCATT. }\end{array}$ & $\begin{array}{l}\text { INCOH. } \\
\text { SCATT. }\end{array}$ & $\begin{array}{l}\text { PHOTO } \\
\text { EFFECT }\end{array}$ & $\begin{array}{r}\text { PAIR } \\
\text { PROD. }\end{array}$ \\
\hline-.2 & -3.8 & .1 & -3.5 & \\
\hline-.1 & $-3 \cdot 2$ & .1 & -3.6 & \\
\hline-.0 & -2.5 & .1 & -2.6 & \\
\hline .0 & $-1 \cdot 3$ & .0 & .5 & \\
\hline .1 & -.0 & .0 & 3.9 & \\
\hline .0 & -.6 & .0 & -.1 & -2.5 \\
\hline .0 & -.8 & .0 & 3.5 & -1.0 \\
\hline-.3 & -1.1 & .0 & $3 \cdot .3$ & -2.7 \\
\hline-.4 & -1.3 & -.1 & 2.6 & -1.6 \\
\hline$=.0$ & -1.4 & -.0 & 3.8 & -.0 \\
\hline 1.4 & $-1 \cdot 4$ & -.0 & 3.5 & 4.1 \\
\hline 2.2 & -1.5 & -.0 & $4 \cdot 4$ & 4.7 \\
\hline .5 & -1.5 & -.0 & 6.1 & .9 \\
\hline .5 & -1.6 & -.0 & 4.4 & .7 \\
\hline-1.5 & -1.6 & -.0 & 4.1 & -2.0 \\
\hline-1.0 & -1.8 & -.0 & 4.6 & -1.1 \\
\hline-.1 & -1.5 & -.0 & 5.4 & -.1 \\
\hline-.8 & -.6 & -.0 & 6.2 & -.9 \\
\hline-.4 & .3 & -.0 & 7.0 & -.5 \\
\hline-.2 & 2.5 & -.0 & 8.4 & -.3 \\
\hline .2 & 5.0 & -.0 & 9.5 & .2 \\
\hline
\end{tabular}

\begin{tabular}{|c|c|c|c|c|}
\hline ENERGY & & $\mathrm{COH}$ & INCOH. & РНOTO \\
\hline KEV & TOTAL & SCATT. & SCATT. & EFFECT \\
\hline 1 & $-7 \cdot 3$ & -.6 & 23.8 & -7.3 \\
\hline $1 \cdot 5$ & -16.9 & -.7 & 11.7 & -16.9 \\
\hline 2 & $-10 \cdot 0$ & -.9 & 5.6 & -10.0 \\
\hline 3 & $-5 \cdot 0$ & $-1 \cdot 5$ & 1.2 & -5.0 \\
\hline 4 & -3.2 & $-2 \cdot 0$ & .1 & -3.2 \\
\hline 5 & $-2 \cdot 2$ & -2.1 & .2 & $-2 \cdot 3$ \\
\hline 6 & -1.7 & -2.2 & .5 & -1.7 \\
\hline 8 & $-1 \cdot 3$ & $-2 \cdot 3$ & 1.0 & $-1 \cdot 3$ \\
\hline 10 & -1.7 & $-2 \cdot 5$ & $\cdot 3$ & $-1 \cdot 6$ \\
\hline 15 & 1.9 & $-2 \cdot 2$ & -.1 & $1 \cdot 9$ \\
\hline 20 & 1.0 & $-2 \cdot 6$ & $\cdot 1$ & 1.1 \\
\hline 30 & $1 \cdot 0$ & -3.5 & .1 & $1 \cdot 1$ \\
\hline 40 & 1.8 & $-5 \cdot 4$ & .2 & $2 \cdot 1$ \\
\hline 50 & $2 \cdot 6$ & -6.8 & .2 & 3.2 \\
\hline 60 & 3.2 & -7.6 & .1 & $4 \cdot 2$ \\
\hline 80 & $3 \cdot 6$ & -7.0 & .0 & 5.4 \\
\hline 100 & 3.1 & -7.4 & .2 & $5 \cdot 5$ \\
\hline 150 & $1 \cdot 2$ & -7.6 & .2 & 4.0 \\
\hline 200 & .2 & $-7 \cdot 3$ & .3 & 2.0 \\
\hline 300 & -.1 & $-6 \cdot 3$ & .6 & -1.7 \\
\hline
\end{tabular}

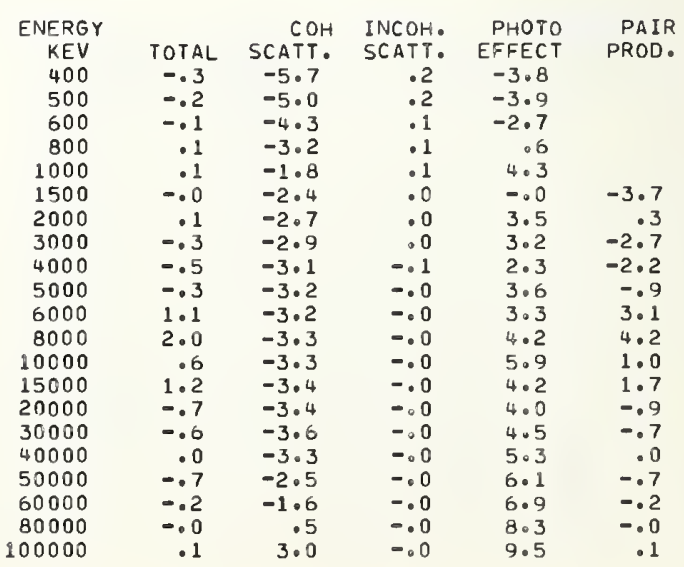




$\begin{array}{crrrr}\begin{array}{c}Z \\ \text { ENERGY }\end{array} & 33 \text { ARSENIC } \\ \text { KEV } & \text { TOTAL } & \text { SCATT. } & \begin{array}{r}\text { INCOH. } \\ \text { SCATT. }\end{array} & \begin{array}{r}\text { PHOTO } \\ \text { EFFECT }\end{array} \\ 1 & -22.7 & -.5 & 23.6 & -22.7 \\ 1.5 & -9.7 & -.7 & 11.1 & -9.7 \\ 2 & -6.8 & -1.0 & 4.6 & -6.8 \\ 3 & -5.0 & -1.6 & -.2 & -5.1 \\ 4 & -4.7 & -1.9 & -.9 & -4.8 \\ 5 & -4.4 & -2.0 & -.5 & -4.4 \\ 6 & -4.0 & -2.1 & .1 & -4.0 \\ 8 & -2.9 & -2.4 & .8 & -2.9 \\ 10 & -1.8 & -2.8 & .8 & -1.8 \\ 15 & 1.4 & -4.4 & .2 & 1.4 \\ 20 & -.2 & -2.5 & .1 & -.2 \\ 30 & -.8 & -3.4 & .1 & -.7 \\ 40 & -.1 & -5.6 & .2 & .2 \\ 50 & .9 & -7.2 & .2 & 1.4 \\ 60 & 1.8 & -8.3 & .1 & 2.6 \\ 80 & 2.9 & -7.6 & -.1 & 4.4 \\ 100 & 2.9 & -8.1 & .2 & 5.2 \\ 150 & 1.5 & -8.5 & .2 & 4.9 \\ 200 & .4 & -8.2 & .2 & 3.4 \\ 300 & -.3 & -7.5 & .1 & -. .2 \\ & & & & \end{array}$

ENERGY
KEV
400
500
600
800
1000
1500
2000
3000
4000
5000
6000
8000
10000
15000
20000
30000
40000
50000
60000
80000
100000

$\begin{array}{rrrrr} & \text { COH } & \text { INCOH. } & \begin{array}{r}\text { PHOTO } \\ \text { TOTAL }\end{array} \text { SCATT. } & \text { SCATR } \\ -.3 & -6.8 & .1 & -2.5 & \\ -.2 & -6.1 & .1 & -2.7 & \\ -.1 & -5.3 & .1 & -1.7 & \\ .0 & -4.4 & .1 & .7 & \\ -1 & -3.0 & .0 & 3.1 & \\ -.0 & -3.6 & .0 & -1.1 & -3.6 \\ .0 & -3.8 & .0 & 2.3 & .1 \\ -.4 & -4.1 & .0 & 2.0 & -3.0 \\ -.6 & -4.3 & -.1 & 1.2 & -2.5 \\ -.4 & -4.3 & -.0 & 2.5 & -1.2 \\ 1.1 & -4.4 & -.0 & 2.2 & 2.9 \\ 1.9 & -4.5 & -.0 & 3.1 & 3.9 \\ .3 & -4.5 & -.0 & 4.8 & .6 \\ .5 & -4.6 & -.0 & 3.2 & .7 \\ -1.8 & -4.6 & -.0 & 2.9 & -2.3 \\ -1.8 & -4.7 & -.0 & 3.5 & -2.1 \\ -.9 & -4.5 & -.0 & 4.3 & -1.0 \\ -1.3 & -3.7 & -.0 & 5.1 & -1.4 \\ -.7 & -2.8 & -.0 & 5.9 & -.7 \\ -.4 & -.8 & -.0 & 7.3 & -.4 \\ -.3 & 1.6 & -.0 & 8.5 & -.03\end{array}$

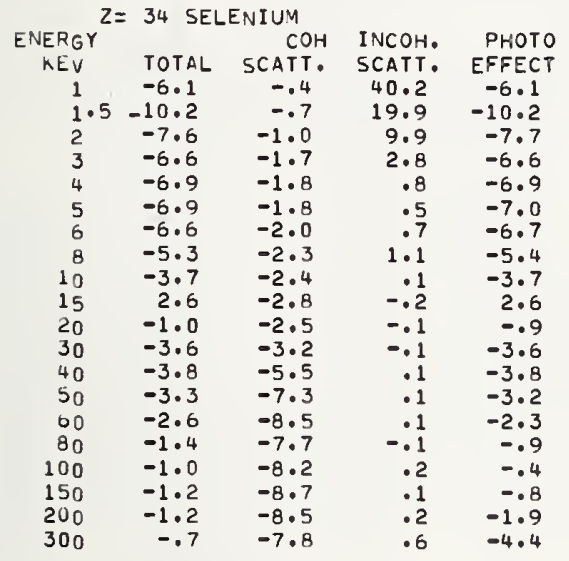

\begin{tabular}{|c|c|c|c|c|c|}
\hline $\begin{array}{c}\text { ENERGY } \\
\text { KEV }\end{array}$ & TOTAL & $\begin{array}{r}\mathrm{COH} \\
\text { SCATT. }\end{array}$ & $\begin{array}{l}\text { INCOH. } \\
\text { SCATT. }\end{array}$ & $\begin{array}{l}\text { PHOTO } \\
\text { EFFECT }\end{array}$ & $\begin{array}{l}\text { PAIR } \\
\text { PROD. }\end{array}$ \\
\hline 400 & -.6 & -6.9 & .1 & $-5 \cdot 6$ & \\
\hline 500 & -.3 & $-6 \cdot 3$ & .1 & $-4 \cdot 8$ & \\
\hline 600 & -.2 & $-5 \cdot 6$ & .1 & -2.9 & \\
\hline 800 & -.0 & -4.7 & .0 & $1 \cdot 1$ & \\
\hline 1000 & .1 & -3.3 & .0 & $5 \cdot 0$ & \\
\hline $\begin{array}{l}1500 \\
2000\end{array}$ & $\begin{array}{l}-0 \\
=1\end{array}$ & $\begin{array}{l}-3.9 \\
-4.1\end{array}$ & $\begin{array}{r}.0 \\
.0\end{array}$ & $\begin{array}{r}.8 \\
4.2\end{array}$ & $\begin{array}{l}-3.0 \\
-2.4\end{array}$ \\
\hline 3000 & -.3 & $-4 \cdot 4$ & .0 & 3.9 & $-2 \cdot 2$ \\
\hline 4000 & -.1 & $-4 \cdot 6$ & -.1 & $3 \cdot 0$ & -.3 \\
\hline 5000 & .4 & $-4 \cdot 6$ & -.0 & $4 \cdot 2$ & 1.4 \\
\hline 6000 & 2.0 & -4.7 & -.0 & 3.9 & 5.3 \\
\hline 8000 & 2.6 & $-4 \cdot 8$ & -.0 & $4 \cdot 8$ & $5 \cdot 3$ \\
\hline 10000 & .4 & $-4 \cdot 8$ & -.0 & 6.5 & .7 \\
\hline 15000 & -.4 & $-4 \cdot 9$ & -.0 & $4 \cdot 8$ & -.5 \\
\hline 20000 & $-2 \cdot 0$ & $-4 \cdot 9$ & -.0 & $4 \cdot 5$ & -2.5 \\
\hline 30000 & -.3 & -5.0 & -.0 & $5 \cdot 0$ & -.3 \\
\hline 40000 & .8 & -4.9 & -.0 & $5 \cdot 8$ & .9 \\
\hline 50000 & -.1 & -4.1 & -.0 & 6.7 & -.1 \\
\hline 60000 & .1 & -3.2 & -.0 & $7 \cdot 5$ & .1 \\
\hline 80000 & .1 & $-1 \cdot 2$ & -.0 & 8.9 & .1 \\
\hline 100000 & .9 & $1 \cdot 2$ & -.0 & $10 \cdot 1$ & . 9 \\
\hline
\end{tabular}

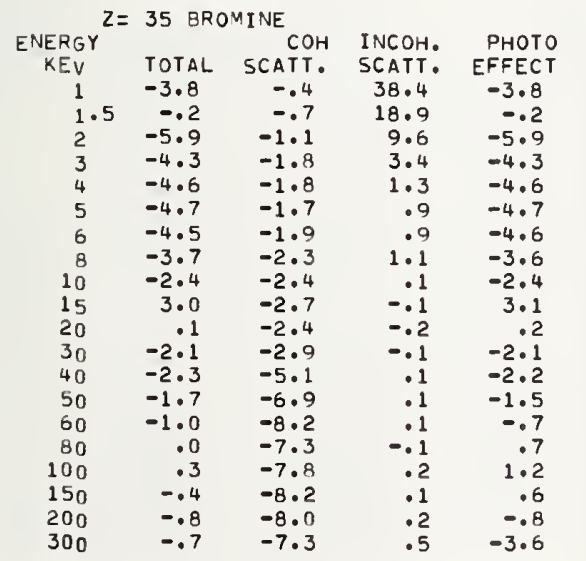

\begin{tabular}{|c|c|c|c|c|c|}
\hline $\begin{array}{c}\text { ENERGY } \\
\text { XEV }\end{array}$ & TOTAL & $\begin{array}{l}\mathrm{COH} \\
\text { SCATT. }\end{array}$ & $\begin{array}{l}\text { INCOH. } \\
\text { SCATT. }\end{array}$ & $\begin{array}{l}\text { РHOTO } \\
\text { EFFECT }\end{array}$ & $\begin{array}{l}\text { PAIR } \\
\text { PROD. }\end{array}$ \\
\hline 400 & -.6 & $-6 \cdot 5$ & .1 & -5.0 & \\
\hline 500 & -.3 & $-5 \cdot 8$ & .1 & $-4 \cdot 4$ & \\
\hline 600 & -.2 & $-5 \cdot 1$ & .1 & $-2 \cdot 6$ & \\
\hline 800 & .0 & $-4 \cdot 1$ & .1 & 1.2 & \\
\hline 1000 & .1 & $-2 \cdot 8$ & .0 & $4 \cdot 7$ & \\
\hline 1500 & -.0 & -3.4 & .0 & .5 & $-4 \cdot 1$ \\
\hline 2000 & .2 & -3.6 & . 0 & 3.9 & 3.8 \\
\hline 3000 & -.3 & -3.9 & .0 & 3.5 & -2.2 \\
\hline 4000 & -.8 & $-4 \cdot 1$ & -.1 & 2.6 & -3.2 \\
\hline 5000 & -.8 & $-4 \cdot 1$ & -.0 & 3.9 & $-2 \cdot 4$ \\
\hline 6000 & .6 & $-4 \cdot 2$ & -.0 & 3.5 & 1.6 \\
\hline 8000 & 1.7 & $-4 \cdot 3$ & -.0 & 4.5 & 3.4 \\
\hline 10000 & .8 & $-4 \cdot 3$ & -.0 & 6.2 & 1.3 \\
\hline 15000 & 2.5 & $-4 \cdot 4$ & -.0 & 4.5 & 3.5 \\
\hline 20000 & .3 & $-4 \cdot 4$ & -.0 & $4 \cdot 2$ & .4 \\
\hline 30000 & -.9 & $-4 \cdot 6$ & -.0 & 4.7 & -1.0 \\
\hline 40000 & -.4 & $-4 \cdot 4$ & -.0 & $5 \cdot 6$ & -.4 \\
\hline 50000 & -.7 & -3.6 & -.0 & 6.4 & -.8 \\
\hline 60000 & .2 & -2.7 & -.0 & $7 \cdot 2$ & .2 \\
\hline 80000 & .6 & -.8 & -.0 & $8 \cdot 7$ & .7 \\
\hline 100000 & .3 & $1 \cdot 6$ & -.0 & 9.9 & $\cdot 3$ \\
\hline
\end{tabular}

$\begin{array}{ccccc}\begin{array}{c}Z \\ \text { ENERGY }\end{array} & 36 \text { KRYPTON } \\ \text { KEV } & \text { TOTAL } & \text { SCATT. } & \begin{array}{r}\text { INCOH. } \\ \text { SCATT. }\end{array} & \begin{array}{r}\text { PHOTO } \\ \text { EFFECT }\end{array} \\ 1 & -2.8 & -.3 & 17.8 & -2.8 \\ 1.5 & -1.8 & -.7 & 7.6 & -1.8 \\ 2 & -7.3 & -1.2 & 3.2 & -7.3 \\ 3 & -3.6 & -1.8 & 1.2 & -3.7 \\ 4 & -3.8 & -1.7 & .4 & -3.9 \\ 5 & -4.4 & -1.7 & .2 & -4.4 \\ 6 & -4.8 & -1.9 & .3 & -4.8 \\ 8 & -4.7 & -2.4 & .7 & -4.7 \\ 10 & -3.7 & -2.3 & .7 & -3.8 \\ 15 & 1.5 & -2.3 & .3 & 1.5 \\ 20 & .1 & -2.3 & .1 & .1 \\ 30 & -1.0 & -3.2 & .1 & -1.9 \\ 40 & -.8 & -5.7 & .2 & -.5 \\ 50 & -.1 & -7.7 & .2 & .2 \\ 60 & .5 & -9.1 & .2 & 1.1 \\ 80 & 1.4 & -8.4 & -.0 & 2.5 \\ 100 & 1.5 & -8.9 & .3 & 3.0 \\ 150 & .4 & -9.5 & .2 & 2.3 \\ 200 & -.4 & -9.3 & .2 & .7 \\ 300 & -.8 & -8.7 & .3 & -2.6\end{array}$

\begin{tabular}{|c|c|c|c|c|c|}
\hline $\begin{array}{c}\text { ENERGY } \\
\text { KEV }\end{array}$ & TOTAL & $\begin{array}{l}\text { COH } \\
\text { SCATT. }\end{array}$ & $\begin{array}{l}\text { INCOH. } \\
\text { SCATT. }\end{array}$ & $\begin{array}{r}\text { PHOTO } \\
\text { EFFECT }\end{array}$ & $\begin{array}{l}\text { PAIR } \\
\text { PROD. }\end{array}$ \\
\hline 400 & -.6 & $-8 \cdot 0$ & .2 & -4.3 & \\
\hline 500 & -.4 & $-7 \cdot 3$ & .1 & $-4 \cdot 0$ & \\
\hline 600 & -.1 & -5.6 & .1 & $-2 \cdot 5$ & \\
\hline 800 & .0 & -5.7 & .1 & .8 & \\
\hline 1000 & .1 & $-4 \cdot 4$ & .1 & 3.8 & \\
\hline 1500 & -.0 & -5.0 & .0 & -.6 & $-4 \cdot 1$ \\
\hline 2000 & .1 & $-5 \cdot 3$ & .0 & $2 \cdot 7$ & \\
\hline 3000 & -.3 & $-5 \cdot 6$ & .0 & $2 \cdot 2$ & $-2 \cdot 2$ \\
\hline 4000 & -.5 & -5.7 & -.1 & $1 \cdot 3$ & -1.7 \\
\hline 5000 & -.2 & $-5 \cdot 8$ & -.0 & $2 \cdot 5$ & -.7 \\
\hline 6000 & 1.2 & $-5 \cdot 9$ & -.0 & $2 \cdot 2$ & 3.2 \\
\hline 8000 & $2 \cdot 0$ & $-5 \cdot 9$ & -.0 & 3.2 & 3.9 \\
\hline 10000 & $\cdot 3$ & $-6 \cdot 0$ & -.0 & 4.9 & .5 \\
\hline 15000 & .5 & -6.0 & -.0 & 3.2 & .7 \\
\hline 20000 & -1.4 & -6.1 & -.0 & 2.9 & -1.8 \\
\hline 30000 & $-1 \cdot 0$ & $-6 \cdot 2$ & -.0 & 3.4 & -1.2 \\
\hline 40000 & -.2 & -6.1 & -.0 & $4 \cdot 3$ & -.2 \\
\hline 50000 & -.8 & -5.3 & -.0 & $5 \cdot 1$ & -.9 \\
\hline 60000 & -.3 & -4.5 & -.0 & 6.0 & -.4 \\
\hline 80000 & -.0 & -2.6 & -.0 & 7.4 & -.0 \\
\hline 100000 & .4 & -.3 & -.0 & 8.6 & .4 \\
\hline
\end{tabular}




\begin{tabular}{|c|c|c|c|c|}
\hline \multicolumn{5}{|c|}{$Z=37$ RUBIDIUM } \\
\hline ENERGY & & $\mathrm{COH}$ & I NCOH. & РHOTO \\
\hline KEV & TOTAL & SCATT. & SCATT. & EFFECT \\
\hline 1 & $-2 \cdot 5$ & -.6 & $-3 \cdot 2$ & $-2 \cdot 5$ \\
\hline $1 \cdot 5$ & $1 \cdot 0$ & -.9 & .5 & 1.0 \\
\hline 2 & $-6 \cdot 5$ & $-1 \cdot 3$ & $1 \cdot 1$ & $-6 \cdot 5$ \\
\hline 3 & $-4 \cdot 1$ & $-1 \cdot 9$ & .9 & $-4 \cdot 0$ \\
\hline 4 & -4.0 & -1.7 & $\cdot 3$ & -4.0 \\
\hline 5 & $-4 \cdot 5$ & -1.6 & -.0 & $-4 \cdot 5$ \\
\hline 6 & -4.8 & -1.8 & .0 & -4.8 \\
\hline 8 & -4.7 & -2.4 & .4 & -4.8 \\
\hline 10 & -3.7 & -2.4 & .7 & -3.8 \\
\hline 15 & $1 \cdot 0$ & -1.8 & $\cdot 2$ & 1.2 \\
\hline 20 & 1.6 & $-2 \cdot 3$ & -.0 & 1.7 \\
\hline 30 & -.7 & -2.4 & -.0 &. .7 \\
\hline 40 & $-1 \cdot 2$ & $-4 \cdot 7$ & $\cdot 1$ & $-1 \cdot 0$ \\
\hline 50 & -.8 & $-6 \cdot 7$ & - 1 & -.6 \\
\hline 60 & -.4 & $-8 \cdot 1$ & .1 & $\bullet 1$ \\
\hline 80 & .6 & -6.9 & -.1 & $1 \cdot 3$ \\
\hline 100 & .9 & -7.3 & .2 & 1.9 \\
\hline 150 & .4 & $-7 \cdot 8$ & $\cdot 1$ & 1.9 \\
\hline 200 & -.2 & -7.6 & . 1 & .9 \\
\hline 300 & -.6 & -6.8 & . 1 & -1.7 \\
\hline
\end{tabular}

ENERGY
KEV
400
500
600
800
1000
1500
2000
3000
4000
5000
6000
8000
10000
15000
20000
30000
40000
50000
60000
80000
100000

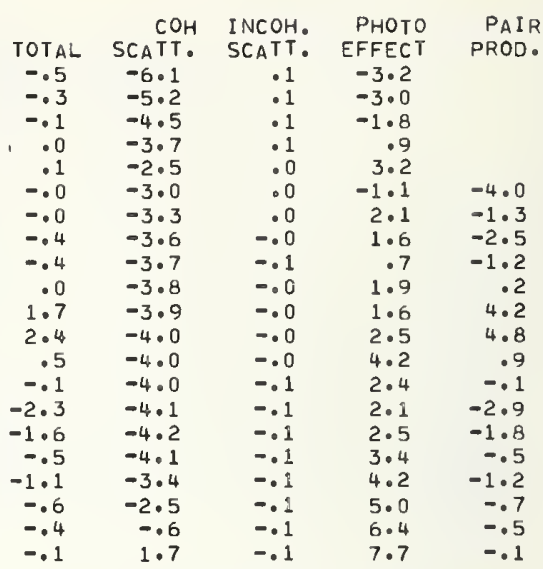

\begin{tabular}{|c|c|c|c|c|}
\hline ENERGY & & $\mathrm{COH}$ & INCOH. & PHOTO \\
\hline KEV & TOTAL & SCATT. & SCATT. & EFFECT \\
\hline 1 & $\cdot 8$ & -.9 & $-4 \cdot 9$ & .8 \\
\hline 1.5 & $-2 \cdot 6$ & $-1 \cdot 0$ & $-1 \cdot 5$ & $-2 \cdot 6$ \\
\hline 2 & -3.5 & $-1 \cdot 2$ & .5 & $-3 \cdot 5$ \\
\hline 3 & $-4 \cdot 0$ & -1.7 & .9 & -4.0 \\
\hline 4 & $-4 \cdot 6$ & -1.7 & $\cdot 5$ & $-4 \cdot 6$ \\
\hline 5 & -4.8 & -1.7 & .1 & -4.9 \\
\hline 6 & $-4 \cdot 7$ & -1.8 & -.0 & -4.7 \\
\hline 8 & $-3 \cdot 4$ & $-2 \cdot 2$ & $\cdot 2$ & -3.4 \\
\hline 10 & $-1 \cdot 5$ & -2.4 & $\cdot 5$ & -1.5 \\
\hline 15 & 3.8 & $-1 \cdot 5$ & $\cdot 3$ & 4.1 \\
\hline 20 & 3.0 & $-2 \cdot 3$ & .1 & $3 \cdot 1$ \\
\hline 30 & $1 \cdot 1$ & $-2 \cdot 2$ & -.1 & $1 \cdot 2$ \\
\hline 40 & $\cdot 3$ & -4.1 & $\cdot 1$ & .5 \\
\hline 50 & . 0 & -5.8 & .1 & .3 \\
\hline 60 & -.0 & -6.9 & $\cdot 1$ & .4 \\
\hline 80 & - 1 & $-5 \cdot 7$ & -.1 & .6 \\
\hline 100 & - 1 & -6.0 & $\cdot 2$ & .6 \\
\hline 150 & -.7 & $-6 \cdot 3$ & $\cdot 2$ & -.4 \\
\hline 200 & -1.2 & -5.9 & - 2 & $-2 \cdot 0$ \\
\hline 300 & $-1 \cdot 2$ & $-5 \cdot 1$ & .2 & $-4 \cdot 8$ \\
\hline
\end{tabular}

ENERGY

$K E V$
400
500
600
800
1000
1500
2000
3000
4000
5000
6000
8000
10000
15000
20000
30000
40000
50000
60000
80000
00000

ENERGY

\begin{tabular}{|c|c|c|c|c|}
\hline $\begin{array}{c}\text { ENEKGY } \\
\text { KEY }\end{array}$ & & $\mathrm{COH}$ & I NCOH. & PHOTO \\
\hline & TOTAL & SCATT. & SCATT. & \\
\hline 1 & -2.7 & -.9 & .8 & -2.7 \\
\hline 1.5 & .2 & $-1 \cdot 1$ & 2.9 & $\cdot 2$ \\
\hline 2 & -.2 & $-1 \cdot 2$ & 3.9 & -.1 \\
\hline 3 & $-4 \cdot 5$ & -1.7 & $3 \cdot 5$ & $-4 \cdot 5$ \\
\hline 4 & -3.4 & -1.9 & 2.6 & -3.4 \\
\hline 5 & -3.6 & -1.8 & 1.7 & -3.6 \\
\hline 6 & -3.9 & $-1 \cdot 8$ & $1 \cdot 1$ & -3.9 \\
\hline 8 & $-4 \cdot 2$ & $-2 \cdot 2$ & -2.4 & $-4 \cdot 2$ \\
\hline 10 & -3.8 & $-2 \cdot 3$ & .9 & -3.9 \\
\hline 15 & $-1 \cdot 5$ & -1.0 & .5 & -1.6 \\
\hline 20 & -.0 & -2.4 & . 2 & .0 \\
\hline 30 & -.9 & $-2 \cdot 2$ & .0 & -.8 \\
\hline 40 & $-1 \cdot 0$ & -4.2 & .2 & -.9 \\
\hline 50 & -.8 & -5.8 & $\cdot 2$ & -.7 \\
\hline 60 & -.5 & -6.8 & .1 & -.2 \\
\hline 80 & .2 & $-5 \cdot 7$ & -.1 & .6 \\
\hline 100 & .5 & $-6 \cdot 0$ & .2 & 1.2 \\
\hline 150 & .2 & -6.2 & .2 & 1.2 \\
\hline 200 & -.4 & $-5 \cdot 9$ & $\cdot 2$ & .2 \\
\hline 300 & -.9 & -5.0 & .0 & -2.4 \\
\hline
\end{tabular}

\begin{tabular}{|c|c|c|c|c|}
\hline $\begin{array}{c}\text { ENERGY } \\
\text { KEV }\end{array}$ & TOTAL & $\begin{array}{l}\text { COH } \\
\text { SCATT. }\end{array}$ & $\begin{array}{l}\text { INCOH. } \\
\text { SCATT. }\end{array}$ & $\begin{array}{r}\text { PHOTO } \\
\text { EFFECT }\end{array}$ \\
\hline 1 & $-4 \cdot 6$ & -.9 & 7.7 & -4.6 \\
\hline 1.5 & -.9 & $-1 \cdot 1$ & $7 \cdot 3$ & -.9 \\
\hline 2 & -.9 & $-1 \cdot 2$ & 6.7 & -.9 \\
\hline 3 & -6.0 & -1.7 & 4.8 & $-6 \cdot 0$ \\
\hline 4 & $-4 \cdot 7$ & $-1 \cdot 9$ & 3.4 & -4.7 \\
\hline 5 & -4.5 & -1.8 & $2 \cdot 3$ & $-4 \cdot 5$ \\
\hline 6 & -4.4 & -1.8 & 1.6 & $-4 \cdot 5$ \\
\hline 8 & -4.0 & $-2 \cdot 1$ & -3.8 & -4.0 \\
\hline 10 & -3.0 & $-2 \cdot 3$ & 1.2 & -3.0 \\
\hline 15 & $\cdot 1$ & -1.1 & .5 & .2 \\
\hline 20 & $2 \cdot 2$ & -2.4 & .1 & $2 \cdot 3$ \\
\hline 30 & -.6 & -2.2 & -.0 & -.6 \\
\hline 40 & -1.4 & -4.2 & $\cdot 1$ & $-1 \cdot 3$ \\
\hline 50 & $-1 \cdot 3$ & -5.8 & .1 & -1.1 \\
\hline O0 & -.8 & -6.8 & - 1 & -.6 \\
\hline 80 & .4 & $-5 \cdot 7$ & -.2 & .9 \\
\hline 100 & $1 \cdot 3$ & $-6 \cdot 0$ & .3 & 2.1 \\
\hline 150 & 1.5 & -6.2 & .1 & 3.3 \\
\hline 200 & .9 & -5.9 & .1 & 3.0 \\
\hline 300 & -.2 & -5.0 & -.1 & $1 \cdot 1$ \\
\hline
\end{tabular}

$$
\begin{array}{r}
\text { KEV } \\
400 \\
500 \\
600 \\
800 \\
1000 \\
1500 \\
2000 \\
3000 \\
4000 \\
5000 \\
6000 \\
8000 \\
10000 \\
15000 \\
20000 \\
30000 \\
40000 \\
50000 \\
60000 \\
80000 \\
100000
\end{array}
$$

\begin{tabular}{|c|c|c|c|c|}
\hline TOTAL & $\begin{array}{r}\mathrm{COH} \\
\text { SCATT. }\end{array}$ & $\begin{array}{l}\text { 1NCOH. } \\
\text { SCATT. }\end{array}$ & $\begin{array}{r}\text { PHOTO } \\
\text { EFFECT }\end{array}$ & $\begin{array}{l}\text { PAIR } \\
\text { PROD. }\end{array}$ \\
\hline-.1 & -4.2 & .1 & -00 & \\
\hline .0 & -3.5 & .1 & .5 & \\
\hline .1 & -2.6 & .1 & 1.8 & \\
\hline .2 & $-1 \cdot 8$ & .0 & $4 \cdot 3$ & \\
\hline .2 & -.6 & .0 & 6.2 & \\
\hline .0 & -1.2 & .0 & 1.7 & -3.7 \\
\hline .0 & $-1 \cdot 5$ & .0 & 4.9 & -1.2 \\
\hline-.3 & -1.8 & -.0 & $4 \cdot 3$ & $-2 \cdot 1$ \\
\hline-.2 & -1.9 & -.1 & 3.3 & -.6 \\
\hline .3 & $-2 \cdot 0$ & -.0 & 4.4 & .8 \\
\hline 2.0 & -2.1 & -.0 & 4.0 & 4.9 \\
\hline 2.9 & $-2 \cdot 2$ & -.0 & 4.9 & 5.5 \\
\hline 1.0 & $-2 \cdot 2$ & -.0 & 6.6 & 1.6 \\
\hline .4 & $-2 \cdot 3$ & -.1 & $4 \cdot 7$ & .5 \\
\hline-1.7 & $-2 \cdot 3$ & -.1 & $4 \cdot 3$ & $-2 \cdot 2$ \\
\hline-.8 & $-2 \cdot 5$ & -.1 & $4 \cdot 8$ & -.9 \\
\hline .6 & $-2 \cdot 4$ & -.1 & 5.6 & .6 \\
\hline .0 & -1.7 & -.1 & 6.5 & . 1 \\
\hline .5 & -.9 & -.1 & $7 \cdot 3$ & .6 \\
\hline .7 & 1.0 & -.1 & 8.8 & .7 \\
\hline 1.0 & $3 \cdot 3$ & $=.1$ & 10.1 & 1.0 \\
\hline
\end{tabular}

ENERGY

KEV
400
500
600
800
1000
1500
2000
3000
4000
5000
6000
8000
10000
15000
20000
30000
40000
50000
60000
80000
100000

COH INCOH. PHOTO PAIR TOTAL SCATT. SCATT. EFFECT PROD.

$\begin{array}{rr}-.8 & -4.3 \\ -.4 & -3.5 \\ -.1 & -2.7 \\ .1 & -1.9 \\ .2 & -.6 \\ -.0 & -1.2 \\ .1 & -1.5 \\ -.3 & -1.8 \\ -.4 & -1.9 \\ -.1 & -2.0 \\ 1.4 & -2.1 \\ 2.3 & -2.2 \\ .6 & -2.2 \\ .7 & -2.3 \\ -1.3 & -2.3 \\ -.7 & -2.4 \\ .3 & -2.3 \\ -.3 & -1.6 \\ .3 & -.8 \\ .4 & 1.2 \\ .5 & 3.5\end{array}$

$\begin{array}{rrr}.2 & -5.9 & \\ .1 & -4.8 & \\ .1 & -2.6 & \\ .1 & 1.9 & \\ .0 & 1.1 & \\ .0 & 4.6 & -4.2 \\ -.0 & 4.0 & -2.0 \\ -.1 & 3.0 & -1.6 \\ -.0 & 4.1 & -.4 \\ -.0 & 3.8 & 3.6 \\ -.0 & 4.7 & 4.4 \\ -.0 & 6.5 & 1.0 \\ -.1 & 4.6 & 1.0 \\ -.0 & 4.3 & -1.6 \\ -.1 & 4.8 & -.8 \\ -.1 & 5.7 & .3 \\ -.1 & 6.6 & -.3 \\ -.1 & 7.4 & .3 \\ -.1 & 8.9 & .4 \\ -.1 & 10.1 & .5\end{array}$

COH INCOH. PHOTO PAIR
TOTAL SCATT. SCATT. EFFECT PROD

$\begin{array}{rrrrr}-.5 & -4.2 & .2 & -3.7 & \\ -.2 & -3.5 & .2 & -3.2 & \\ -.1 & -2.8 & .1 & -1.8 & \\ .2 & -1.8 & .1 & 1.3 & \\ .1 & -. .6 & .1 & 4.2 & \\ -.0 & -1.2 & .0 & -.2 & -4.6 \\ .2 & -1.5 & .0 & 3.0 & 4.0 \\ -.2 & -1.8 & -.0 & 2.4 & -1.7 \\ -.7 & -1.9 & -.1 & 1.4 & -2.5 \\ -.7 & -2.0 & -.0 & 2.6 & -1.9 \\ .8 & -2.1 & -.0 & 2.2 & 2.0 \\ 2.0 & -2.1 & -.0 & 3.1 & 3.9 \\ 1.1 & -2.2 & -.0 & 4.8 & 1.8 \\ 2.7 & -2.2 & -.1 & 2.9 & 3.7 \\ .4 & -2.3 & -.1 & 2.5 & .5 \\ -. .9 & -2.4 & -.1 & 3.0 & -1.0 \\ -. .4 & -2.3 & -.1 & 3.8 & -.4 \\ -. .7 & -1.6 & -.1 & 4.7 & -.8 \\ .1 & -. .8 & -.1 & 5.5 & .2 \\ .6 & 1.1 & -.1 & 7.0 & .6 \\ .2 & 3.4 & -.1 & 8.2 & .2\end{array}$




\begin{tabular}{|c|c|c|c|c|}
\hline $\begin{array}{c}\text { ENERGY } \\
\text { KEV }\end{array}$ & TOTAL & $\begin{array}{l}\text { COH } \\
\text { SCATT. }\end{array}$ & $\begin{array}{l}\text { INCOH. } \\
\text { SCATT. }\end{array}$ & $\begin{array}{l}\text { PHOTO } \\
\text { EFFECT }\end{array}$ \\
\hline 1 & -1.9 & -.7 & 1.0 & -1.9 \\
\hline $1 \cdot 5$ & -.8 & $-1 \cdot 0$ & .3 & -.8 \\
\hline 2 & -.8 & $-1 \cdot 2$ & .4 & -.8 \\
\hline 3 & -3.7 & -1.8 & .7 & -3.7 \\
\hline 4 & -3.7 & $-2 \cdot 0$ & .8 & -3.7 \\
\hline 5 & -4.4 & -1.9 & .6 & $-4 \cdot 4$ \\
\hline 6 & -4.9 & -1.9 & .4 & -4.9 \\
\hline 8 & $-4 \cdot 9$ & -2.1 & -5.0 & $-4 \cdot 9$ \\
\hline 10 & -3.9 & $-2 \cdot 2$ & .9 & -3.9 \\
\hline 15 & .7 & $-1 \cdot 0$ & .4 & .8 \\
\hline 20 & .7 & -2.4 & -.0 & .8 \\
\hline 30 & -1.4 & $-2 \cdot 3$ & -.1 & -1.4 \\
\hline 40 & $-2 \cdot 3$ & $-4 \cdot 5$ & .1 & -2.2 \\
\hline 50 & $-2 \cdot 4$ & $-6 \cdot 1$ & .2 & $-2 \cdot 3$ \\
\hline 60 & $-2 \cdot 2$ & -7.2 & .1 & -2.1 \\
\hline 80 & -1.6 & $-6 \cdot 3$ & -.1 & -1.3 \\
\hline 100 & $-1 \cdot 1$ & -6.6 & .2 & -.8 \\
\hline 150 & -1.0 & -6.9 & .1 & -.7 \\
\hline 200 & $-1 \cdot 2$ & -6.6 & $\cdot 1$ & -1.6 \\
\hline 300 & -1.4 & $-5 \cdot 8$ & -.2 & -3.4 \\
\hline
\end{tabular}

\begin{tabular}{|c|c|c|c|c|}
\hline \multicolumn{5}{|c|}{ ADENUM } \\
\hline $\begin{array}{c}\text { ENERGY } \\
\text { KEV }\end{array}$ & TOTAL & $\begin{array}{l}\text { COH } \\
\text { SCATT. }\end{array}$ & $\begin{array}{l}\text { INCOH, } \\
\text { SCATT. }\end{array}$ & $\begin{array}{r}\text { PHOTO } \\
\text { EFFECT }\end{array}$ \\
\hline 1 & -8.0 & $=.6$ & 6.0 & -8.0 \\
\hline 1.5 & -1.7 & -.9 & 2.7 & -1.7 \\
\hline 2 & -.5 & -1.2 & 1.5 & -.5 \\
\hline 3 & $-5 \cdot 3$ & -1.8 & .9 & $-5 \cdot 3$ \\
\hline 4 & -3.8 & -1.9 & .7 & -3.8 \\
\hline 5 & -3.9 & -1.9 & .5 & -3.9 \\
\hline 6 & $-4 \cdot 1$ & -2.0 & .3 & $-4 \cdot 1$ \\
\hline 8 & $-4 \cdot 1$ & $-2 \cdot 1$ & -4.8 & $-4 \cdot 1$ \\
\hline 10 & $-3 \cdot 5$ & -2.2 & -2.1 & $-3 \cdot 5$ \\
\hline 15 & -.1 & -1.7 & -.8 & -.0 \\
\hline 20 & 1.1 & $-1 \cdot 2$ & -.6 & $1 \cdot 1$ \\
\hline 30 & -.8 & -2.4 & -.3 & -.8 \\
\hline 40 & -2.0 & -4.9 & -.0 & -1.9 \\
\hline 50 & $-2 \cdot 6$ & -6.9 & .1 & -2.5 \\
\hline 60 & -2.9 & -8.1 & .0 & -2.7 \\
\hline 80 & -2.9 & $-7 \cdot 3$ & -.2 & -2.9 \\
\hline 100 & -3.0 & $-7 \cdot 7$ & $\cdot 2$ & -3.0 \\
\hline 150 & -3.3 & $-8 \cdot 1$ & .1 & $-4 \cdot 0$ \\
\hline 200 & -3.3 & -7.9 & .1 & -5.4 \\
\hline 300 & -2.6 & -7.2 & .1 & -7.7 \\
\hline
\end{tabular}

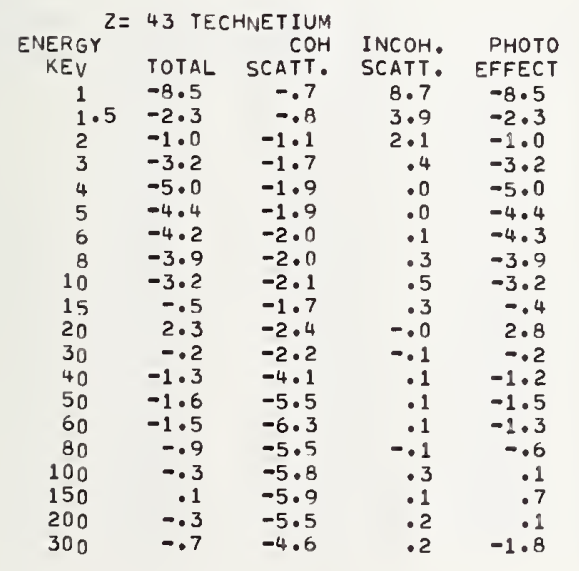

\begin{tabular}{|c|c|c|c|c|}
\hline \multicolumn{5}{|c|}{$\mathrm{Z}=44$ RUTHENIUM } \\
\hline $\begin{array}{c}\text { ENERGY } \\
\text { KEY }\end{array}$ & TOTAL & COH & $\begin{array}{l}\text { INCOH. } \\
\text { SCATT. }\end{array}$ & $\begin{array}{r}\text { РНOTO } \\
\text { EFFECT }\end{array}$ \\
\hline 1 & -7.0 & -.5 & 12.3 & -7.0 \\
\hline $1 \cdot 5$ & -1.6 & -.7 & 4.6 & -1.6 \\
\hline 2 & $-1 \cdot 8$ & $-1 \cdot 1$ & .3 & -1.9 \\
\hline 3 & $-4 \cdot 0$ & -1.7 & $-A$ & -4.0 \\
\hline 4 & $-4 \cdot 2$ & -1.9 & -.3 & -4.2 \\
\hline 5 & -3.7 & -2.0 & .1 & -3.7 \\
\hline 6 & -3.8 & $-2 \cdot 1$ & .4 & -3.8 \\
\hline$B$ & $-4 \cdot 0$ & -2.1 & 1.7 & -4.0 \\
\hline 10 & -3.7 & $-2 \cdot 2$ & 1.5 & -3.8 \\
\hline 15 & $-1 \cdot 3$ & -2.2 & .8 & $-1 \cdot 3$ \\
\hline 20 & 2.2 & -2.4 & .4 & 2.5 \\
\hline 30 & -.0 & $-2 \cdot 1$ & .2 & .0 \\
\hline 40 & -1.0 & -3.7 & .3 & -.9 \\
\hline 50 & $-1 \cdot 3$ & -4.6 & $\cdot 3$ & $-1 \cdot 1$ \\
\hline 60 & -1.2 & -5.1 & .2 & -2.0 \\
\hline 80 & -.6 & $-4 \cdot 5$ & -.1 & -.4 \\
\hline 100 & -.1 & $-4 \cdot 7$ & .3 & .1 \\
\hline 150 & .2 & -4.5 & .2 & .7 \\
\hline 200 & -.1 & -4.0 & .2 & .2 \\
\hline 300 & -.6 & -3.0 & .1 & -2.5 \\
\hline
\end{tabular}

\begin{tabular}{|c|c|c|c|c|c|}
\hline $\begin{array}{c}\text { ENERGY } \\
\text { KEV }\end{array}$ & TOTAL & SCATT. & $\begin{array}{l}\text { INCOH. } \\
\text { SCATT. }\end{array}$ & $\begin{array}{l}\text { РНOTO } \\
\text { EFFECT }\end{array}$ & $\begin{array}{l}\text { PAIF } \\
\text { PROD }\end{array}$ \\
\hline 400 & -.7 & -4.9 & .1 & -3.8 & \\
\hline 500 & -.3 & $-4 \cdot 3$ & - 1 & -2.5 & \\
\hline $\begin{array}{l}600 \\
800\end{array}$ & -.1 & $\begin{array}{l}-3.5 \\
-2.6\end{array}$ & $\begin{array}{l}.0 \\
.1\end{array}$ & $\begin{array}{r}-11 \\
4.9\end{array}$ & \\
\hline 1000 & .3 & -1.4 & . 0 . & 9.4 & \\
\hline 1500 & .1 & -2.0 & .0 & 5.3 & $-2 \cdot 5$ \\
\hline 2000 & -.0 & $-2 \cdot 3$ & .0 & $8 \cdot 6$ & -2.4 \\
\hline 3000 & -.3 & -2.6 & -.0 & 8.1 & -2.2 \\
\hline 4000 & -.1 & -2.8 & -.1 & 6.9 & -.0 \\
\hline 5000 & .6 & -2.9 & -.1 & 8.1 & 1.7 \\
\hline 6000 & 2.5 & -2.9 & -00 & 7.6 & 5.9 \\
\hline 8000 & 3.4 & -3.0 & -.0 & $8 \cdot 3$ & 6.3 \\
\hline 10000 & 1.2 & -3.1 & -.0 & 10.1 & $2 \cdot 0$ \\
\hline 15000 & -.1 & -3.1 & -.1 & 7.9 & -.2 \\
\hline 20000 & -2.5 & -3.2 & -.2 & 7.4 & -3.1 \\
\hline 30000 & -1.2 & -3.3 & -.1 & 7.8 & -1.4 \\
\hline 40000 & .4 & -3.2 & -0. & 8.6 & .4 \\
\hline 50000 & -.1 & -2.6 & -.1 & 9.5 & -.1 \\
\hline 60000 & .3 & -1.7 & -1 & 10.4 & .3 \\
\hline 80000 & .2 & .1 & -.1 & 11.9 & .2 \\
\hline 100000 & .3 & $2 \cdot 3$ & -.1 & 13.2 & .3 \\
\hline
\end{tabular}

$\begin{array}{lll}\text { ENERGY } & \text { COH INCOH. PHOTO } & \text { PAIR } \\ \text { KEV TOTAL SCATT. SCATT. EFFECT PROD. }\end{array}$

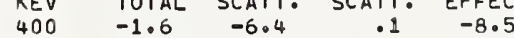

$\begin{array}{rrrrrr}400 & -1.6 & -6.4 & .1 & -8.5 & \\ 500 & -1.0 & -5.9 & .1 & -7.4 & \\ 600 & -.5 & -5.1 & .1 & -5.3 & \\ 800 & -.0 & -4.0 & .1 & -.1 & \\ 1000 & .2 & -3.0 & .1 & 5.3 & \\ 1500 & -.0 & -3.6 & .0 & 1.2 & -3.6 \\ 2000 & .2 & -3.9 & .0 & 4.4 & 2.4 \\ 3000 & -.3 & -4.2 & -.0 & 4.0 & -1.9 \\ 4000 & -.6 & -4.4 & -.1 & 3.1 & -2.2 \\ 5000 & -.5 & -4.4 & -.1 & 4.3 & -1.5 \\ 6000 & 1.0 & -4.5 & -.1 & 3.9 & 2.5 \\ 8000 & 2.1 & -4.6 & -.0 & 4.7 & 3.9 \\ 10000 & .9 & -4.6 & -.1 & 6.5 & 1.4 \\ 15000 & 2.0 & -4.7 & -.1 & 4.5 & 2.7 \\ 20000 & -1 & -4.7 & -.1 & 4.1 & .1 \\ 30000 & -.3 & -4.9 & -.1 & 4.5 & -.3 \\ 40000 & .4 & -4.8 & -.1 & 5.3 & .5 \\ 50000 & -.0 & -4.2 & -.1 & 6.2 & -.0 \\ 60000 & .7 & -3.4 & -.1 & 7.0 & .7 \\ 80000 & 1.1 & -1.6 & -.1 & 8.5 & 1.1 \\ 00000 & 1.0 & .5 & -.1 & 9.8 & 1.1\end{array}$

ENERGY COH INCOH P PHOTO PAIR KEV TOTAL SCATT. SCATT. EFFECT PROD.

$\begin{array}{rrrrrr}400 & -.5 & -3.8 & .1 & -2.9 & \\ 500 & -.3 & -3.2 & .2 & -2.5 & \\ 600 & -.0 & -2.4 & .1 & -1.2 & \\ 800 & .1 & -1.5 & .1 & 1.0 & \\ 1000 & .1 & -.2 & .1 & 2.8 & \\ 1500 & -.0 & -.0 & .0 & -1.4 & -3.0 \\ 2000 & .0 & -1.2 & .0 & 1.6 & -.6 \\ 3000 & -.3 & -1.5 & -.0 & .9 & -2.1 \\ 4000 & -.3 & -1.7 & -.1 & -.1 & -.8 \\ 5000 & .1 & -1.8 & -.1 & 1.0 & .5 \\ 6000 & 1.9 & -1.9 & -.1 & .6 & 4.5 \\ 8000 & 2.9 & -1.9 & -.0 & 1.5 & 5.3 \\ 10000 & 1.0 & -2.0 & -.1 & 3.3 & 1.6 \\ 15000 & .5 & -2.1 & -.1 & 1.3 & .8 \\ 20000 & -1.6 & -2.1 & -.1 & 1.0 & -1.9 \\ 30000 & -.8 & -2.2 & -.1 & 1.4 & -.8 \\ 40000 & .5 & -2.2 & -.1 & 2.3 & .5 \\ 50000 & -.1 & -1.6 & -.1 & 3.1 & -.1 \\ 60000 & .4 & -.8 & -.1 & 4.0 & .5 \\ 80000 & .6 & 1.0 & -.1 & 5.5 & .6 \\ 100000 & .8 & 3.2 & -.1 & 6.1 & .8\end{array}$

ENERGY COH INCOH. PHOTO PAIR

\begin{tabular}{|c|c|c|c|c|c|}
\hline $\begin{array}{l}\text { KERG } \\
\text { KEV }\end{array}$ & TOTAL & SCATT. & $\begin{array}{l}\text { INCOH. } \\
\text { SCATT. }\end{array}$ & $\begin{array}{l}\text { PHOTO } \\
\text { EFFECT }\end{array}$ & $\begin{array}{l}\text { PAIF } \\
\text { PROD. }\end{array}$ \\
\hline 400 & -.5 & -2.2 & .2 & -2.8 & \\
\hline $\begin{array}{l}500 \\
600\end{array}$ & $\begin{array}{l}-.3 \\
-.2\end{array}$ & $\begin{array}{r}-1.7 \\
-.9\end{array}$ & 1 & -2.9 & \\
\hline $\begin{array}{l}600 \\
800\end{array}$ & .0 & $\begin{array}{r}.09 \\
.2\end{array}$ & $\begin{array}{r}-1 \\
-0.0\end{array}$ & $\begin{array}{r}-2.1 \\
-.4\end{array}$ & \\
\hline 1000 & .1 & $1 \cdot 5$ & .1 & 1.2 & \\
\hline 1500 & -.1 & .8 & .0 & $-2 \cdot 5$ & -2.5 \\
\hline 2000 & .0 & .5 & .0 & .9 & .2 \\
\hline 3000 & -.3 & $\cdot 1$ & -.0 & $\cdot 3$ & $-1 \cdot 9$ \\
\hline 4000 & -.3 & -.1 & -.1 & -.5 & -.9 \\
\hline 5000 & .1 & -.2 & -.1 & .7 & - 3 \\
\hline 6000 & 1.9 & -.2 & -.1 & .4 & $4 \cdot 3$ \\
\hline 8000 & 2.9 & $=.3$ & -.1 & $1 \cdot 3$ & $5 \cdot 3$ \\
\hline 10000 & 1.1 & -.4 & -.1 & $3 \cdot 0$ & 1.8 \\
\hline 15000 & .5 & -.5 & -.1 & 1.0 & .7 \\
\hline 20000 & $-2 \cdot 0$ & -.5 & -.1 & .6 & -2.5 \\
\hline 30000 & -2.5 & -.6 & -.1 & 1.0 & -1.8 \\
\hline 40000 & -.3 & -.6 & -.1 & 1.8 & -.4 \\
\hline 50000 & -.7 & -.0 & -.1 & 2.7 & -.8 \\
\hline 60000 & -.1 & . 8 & -.1 & $3 \cdot 5$ & -.1 \\
\hline 80000 & $\cdot 1$ & $2 \cdot 6$ & -.1 & 5.0 & • \\
\hline 00000 & -.0 & $4 \cdot 8$ & -.1 & $6 \cdot 3$ & -.0 \\
\hline
\end{tabular}


ENERGY $Z=45$ RHODIUM

\begin{tabular}{crrrr} 
NERGY & \multicolumn{1}{c}{ COH } & INCOH. & PHOTO \\
KEV & TOTAL & SCATT. & SCATT. & EFFECT \\
1 & -16.6 & -.4 & 28.6 & -16.7 \\
1.5 & -3.8 & -.7 & 14.4 & -3.9 \\
2 & 1.9 & -1.1 & 5.7 & 2.0 \\
3 & 3.5 & -1.6 & .8 & 3.6 \\
4 & -4.1 & -1.8 & .5 & -4.1 \\
5 & -3.8 & -2.0 & .5 & -3.9 \\
6 & -4.0 & -2.1 & .8 & -4.0 \\
8 & -4.2 & -2.1 & 2.1 & -4.2 \\
10 & -3.8 & -2.2 & 1.0 & -3.9 \\
15 & -1.4 & -2.2 & .3 & -1.3 \\
20 & 1.8 & -2.4 & .0 & 2.2 \\
30 & 1.7 & -1.9 & .0 & 1.8 \\
40 & -.0 & -3.0 & .2 & .0 \\
50 & -.6 & -3.5 & .2 & -.5 \\
60 & -.7 & -3.7 & .1 & -.5 \\
80 & -.1 & -3.2 & -.2 & .0 \\
100 & .4 & -3.1 & .3 & .7 \\
150 & .3 & -2.7 & .0 & .7 \\
200 & -1.0 & -2.0 & .1 & -1.7 \\
300 & -.6 & -1.0 & .2 & -2.0
\end{tabular}

ENERGY
KEV
400
500
600
800
1000
1500
2000
3000
4000
5000
6000
8000
10000
15000
20000
30000
40000
50000
60000
80000
100000

\begin{tabular}{|c|c|c|c|c|}
\hline TOTAL & $\begin{array}{r}\text { COH } \\
\text { SCATT. }\end{array}$ & $\begin{array}{l}\text { INCOH. } \\
\text { SCATT. }\end{array}$ & $\begin{array}{r}\text { PHOTO } \\
\text { EFFECT }\end{array}$ & $\begin{array}{l}\text { PAI R } \\
\text { PROD. }\end{array}$ \\
\hline $\begin{array}{l}-.3 \\
-.1\end{array}$ & $\begin{array}{r}-.1 \\
.6\end{array}$ & $: 1$ & $\begin{array}{l}-1.9 \\
-1.2\end{array}$ & \\
\hline .1 & $1 \cdot 3$ & .1 & -.4 & \\
\hline $\begin{array}{r}-.2 \\
.1\end{array}$ & $\begin{array}{l}2.5 \\
3.7\end{array}$ & $\begin{array}{r}-.4 \\
.0\end{array}$ & $\begin{array}{l}1 \cdot 1 \\
2.0\end{array}$ & \\
\hline-.1 & 3.0 & .0 & $-2 \cdot 2$ & -4.0 \\
\hline .2 & 2.6 & .0 & .7 & 3.1 \\
\hline-.4 & 2.2 & -.0 & -.4 & -2.2 \\
\hline-.8 & $2 \cdot 1$ & -.1 & $-1 \cdot 3$ & -2.7 \\
\hline-.9 & 1.9 & -.1 & -.2 & $-2 \cdot 2$ \\
\hline .8 & 1.9 & -.1 & -.5 & 1.9 \\
\hline 2.1 & 1.8 & -.1 & .4 & 3.8 \\
\hline 1.1 & 1.7 & -.1 & 2.2 & 1.7 \\
\hline $2 \cdot 0$ & 1.6 & -.1 & .2 & 2.7 \\
\hline-.6 & 1.6 & -1 & -.1 & -.7 \\
\hline-1.5 & 1.5 & -.1 & .3 & -1.6 \\
\hline-.4 & 1.5 & -.1 & 1.2 & -.5 \\
\hline$\therefore 5$ & $2 \cdot 1$ & -.1 & $2 \cdot 1$ & -.5 \\
\hline .6 & 2.9 & -.1 & 2.9 & .6 \\
\hline 1.0 & $4 \cdot 7$ & -.1 & 4.4 & 1.0 \\
\hline .3 & 7.0 & -.1 & 5.7 & .3 \\
\hline
\end{tabular}

\begin{tabular}{|c|c|c|c|c|}
\hline ENERGY ${ }^{2}$ & $=46 \mathrm{PAL}$ & COH & I NCOH. & PHOT \\
\hline KEV & TOTAL & SCATT. & SCATT. & EFFEC \\
\hline 1 & -19.6 & -.2 & 31.5 & -19.6 \\
\hline $1 \cdot 5$ & -5.5 & -.6 & 14.4 & $-5 \cdot 5$ \\
\hline 2 & .5 & -1.0 & $5 \cdot 2$ & .6 \\
\hline 3 & .3 & -1.6 & -.3 & .3 \\
\hline 4 & $-4 \cdot 3$ & -1.8 & -.8 & $-4 \cdot 3$ \\
\hline 5 & -2.9 & $-2 \cdot 0$ & -.3 & -2.8 \\
\hline 6 & $-2 \cdot 8$ & $-2 \cdot 2$ & .2 & -2.8 \\
\hline 8 & -3.5 & $-2 \cdot 1$ & .7 & -3.5 \\
\hline 1 if & $-3 \cdot 8$ & $-2 \cdot 1$ & .7 & -3 \\
\hline 15 & -2.4 & -1.9 & $\cdot 2$ & -2.5 \\
\hline 20 & 1.0 & $-2 \cdot 4$ & .0 & $1 \cdot 3$ \\
\hline 30 & $-1 \cdot 3$ & $-1 \cdot 8$ & -.0 & $-1 \cdot 3$ \\
\hline 40 & -.3 & -2.4 & - 1 & -.2 \\
\hline 50 & .5 & -2.7 & .2 & .5 \\
\hline 60 & 1.0 & -2.7 & $\cdot 1$ & 1.2 \\
\hline 80 & 1.8 & $-2 \cdot 1$ & -.2 & 2.1 \\
\hline 100 & $2 \cdot 1$ & -2.0 & .3 & $2 \cdot 5$ \\
\hline 150 & $1 \cdot 5$ & $-1 \cdot 3$ & .1 & $2 \cdot 2$ \\
\hline 200 & .6 & -.5 & - 1 & $1 \cdot 0$ \\
\hline 300 & -.3 & .6 & .1 & $-1 \cdot 3$ \\
\hline
\end{tabular}

ENERGY

KEV
400
500
600
800
1000
1500
2000
3000
4000
5000
6000
8000
10000
15000
20000
30000
40000
50000
60000
80000
100000

$\begin{array}{rrrrr} & \text { COH } & \text { INCOH. } & \text { PHOTO } & \text { PAIR } \\ \text { TOTAL } & \text { SCATT. } & \text { SCATT. } & \text { EFFECT } & \text { PROD. } \\ -.4 & 1.5 & .2 & -2.6 & \\ -.2 & 2.1 & .2 & -2.5 & \\ .0 & 2.9 & .1 & -1.5 & \\ .2 & 4.1 & .0 & 1.0 & \\ .2 & 5.4 & .1 & 3.5 & \\ .0 & 4.7 & .0 & -.5 & -.9 \\ -.2 & 4.3 & .0 & 2.7 & -5.3 \\ -.4 & 3.9 & -. .0 & 1.5 & -2.4 \\ .3 & 3.7 & -.1 & .5 & 1.2 \\ 1.2 & 3.6 & -.1 & 1.7 & 3.2 \\ 3.3 & 3.6 & -.1 & 1.3 & 7.4 \\ 3.9 & 3.5 & -.0 & 2.2 & 7.0 \\ 1.0 & 3.4 & -.1 & 4.0 & 1.6 \\ -1.9 & 3.3 & -.1 & 1.9 & -2.5 \\ -4.0 & 3.3 & -.1 & 1.5 & -4.8 \\ -.9 & 3.2 & -.1 & 1.9 & -1.0 \\ 1.0 & 3.1 & -.1 & 2.7 & 1.1 \\ .2 & 3.8 & -.1 & 3.6 & .2 \\ .3 & 4.6 & -.1 & 4.5 & .3 \\ .2 & 6.4 & -. .1 & 6.0 & .2 \\ 1.1 & 8.6 & -. .1 & 7.3 & 1.2\end{array}$

\begin{tabular}{|c|c|c|c|c|}
\hline ENERGY & & $\mathrm{COH}$ & INCOH. & PHOT \\
\hline KEV & TOTAL & SCATT. & SCATT. & EFFEC \\
\hline 1 & $-11 \cdot 1$ & -.4 & 19.0 & $-11 \cdot 2$ \\
\hline $1 \cdot 5$ & $-2 \cdot 1$ & -.7 & 9.6 & $-2 \cdot 1$ \\
\hline 2 & $-1 \cdot 4$ & -1.0 & 4.2 & $-1 \cdot 4$ \\
\hline 3 & -1.9 & -1.5 & -.0 & -2.0 \\
\hline 4 & -3.9 & -1.7 & -.7 & -3.9 \\
\hline 5 & $-2 \cdot 5$ & $-2 \cdot 0$ & -.2 & $-2 \cdot 6$ \\
\hline 6 & $-2 \cdot 5$ & -2.1 & $\cdot 3$ & -2.5 \\
\hline 8 & -3.0 & $-2 \cdot 0$ & .8 & -3.1 \\
\hline 10 & -3.3 & $-2 \cdot 1$ & .7 & $-3 \cdot 3$ \\
\hline 15 & $-2 \cdot 2$ & -2.2 & $\cdot 2$ & $-2 \cdot 2$ \\
\hline 20 & .4 & $-2 \cdot 2$ & $\cdot 1$ & .6 \\
\hline 30 & .5 & -1.7 & - 1 & - 5 \\
\hline 40 & -.3 & $-2 \cdot 1$ & $\cdot 2$ & -.3 \\
\hline 50 & -.7 & $-2 \cdot 2$ & .2 & -.7 \\
\hline 60 & -.8 & $-2 \cdot 2$ & $\cdot 1$ & -.8 \\
\hline 80 & -.7 & -1.7 & -.1 & -.7 \\
\hline 100 & -.5 & $-1 \cdot 5$ & .3 & -.6 \\
\hline 150 & -.8 & -.7 & $\cdot 1$ & $-1 \cdot 0$ \\
\hline 200 & -1.0 & .1 & .2 & -1.9 \\
\hline 300 & -1.0 & 1.3 & $\cdot 2$ & -3.1 \\
\hline
\end{tabular}

ENERGY

\begin{tabular}{|c|c|c|c|c|c|}
\hline $\begin{array}{c}\text { ENERGY } \\
\text { KEV }\end{array}$ & TOTAL & $\begin{array}{l}\text { COH } \\
\text { SCATT. }\end{array}$ & $\begin{array}{l}\text { INCOH. } \\
\text { SCATT. }\end{array}$ & $\begin{array}{l}\text { PHOTO } \\
\text { EFFECT }\end{array}$ & PROD \\
\hline 400 & -.6 & 2.3 & .2 & -3.7 & \\
\hline 500 & -.2 & 2.8 & .1 & $-2 \cdot 6$ & \\
\hline 600 & - 1 & $3 \cdot 5$ & . 1 & -.6 & \\
\hline 800 & .4 & 4.7 & .1 & 3.8 & \\
\hline 1000 & .5 & 6.0 & .1 & $8 \cdot 0$ & \\
\hline 1500 & .1 & $5 \cdot 3$ & .0 & 3.9 & -2.2 \\
\hline 2000 & .1 & 4.9 & .0 & $7 \cdot 3$ & $-1 \cdot 5$ \\
\hline 3000 & -.3 & 4.5 & -.0 & $5 \cdot 8$ & -2 \\
\hline 4000 & -.1 & $4 \cdot 3$ & -.1 & 4.9 & -.3 \\
\hline 5000 & .4 & 4.2 & -.1 & 6.1 & 1.1 \\
\hline 6000 & 2.4 & $4 \cdot 1$ & -.1 & $5 \cdot 8$ & \\
\hline 8000 & $3 \cdot 3$ & $4 \cdot 0$ & -.0 & 6.7 & \\
\hline 10000 & $1 \cdot 3$ & 4.0 & -.1 & 8.6 & 1.9 \\
\hline 15000 & -.2 & 3.9 & -.1 & 6.5 &.- .2 \\
\hline 20000 & -2.5 & 3.9 & -.1 & 6.0 & -3.1 \\
\hline 30000 & -.8 & 3.7 & -.1 & 6.5 & $-1 \cdot c$ \\
\hline 40000 & 1.0 & 3.7 & -.1 & 7.4 & 1. \\
\hline 50000 & .6 & $4 \cdot 3$ & -.1 & $8 \cdot 3$ & 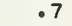 \\
\hline 60000 & 1.2 & $5 \cdot 1$ & -.1 & 9.2 & 1. \\
\hline 80000 & 1.2 & 6.9 & -.1 & $10 \cdot 8$ & \\
\hline 00000 & 1.1 & 9.1 & -.1 & $12 \cdot 2$ & \\
\hline
\end{tabular}

\begin{tabular}{ccccr} 
ENERGY & \multicolumn{5}{c}{ CADMIUM } \\
KEV & TOTAL & SCATT & INCOH. & PHOTO \\
1 & -21.7 & -.5 & 18.8 & -21.8 \\
1.5 & -2.8 & -.6 & 9.0 & -2.8 \\
2 & 4.5 & -.9 & 4.1 & 4.5 \\
3 & -1.9 & -1.4 & -.3 & -1.9 \\
4 & -8.0 & -1.7 & -1.2 & -8.0 \\
5 & -5.6 & -2.0 & -.7 & -5.6 \\
6 & -4.8 & -2.1 & .0 & -4.8 \\
8 & -4.3 & -1.9 & .9 & -4.3 \\
10 & -3.9 & -2.0 & .8 & -3.9 \\
15 & -2.4 & -2.0 & .1 & -2.4 \\
20 & -.5 & -2.2 & .0 & -.3 \\
30 & -.5 & -1.6 & .0 & -.5 \\
40 & -1.7 & -1.9 & .2 & -1.7 \\
50 & -2.0 & -1.9 & .2 & -2.0 \\
60 & -1.8 & -1.8 & .2 & -1.8 \\
80 & -1.1 & -1.3 & -.1 & -1.1 \\
100 & -.5 & -1.1 & .4 & -.06 \\
150 & -.2 & -.2 & .1 & -.3 \\
200 & -.4 & .17 & .1 & -.8 \\
300 & -.6 & 1.9 & .2 & -2.3
\end{tabular}

\begin{tabular}{|c|c|c|c|c|c|}
\hline $\begin{array}{c}\text { ENERGY } \\
\text { KEV }\end{array}$ & TOTAL & $\begin{array}{r}\text { COH } \\
\text { SCATT. }\end{array}$ & $\begin{array}{l}\text { INCOH. } \\
\text { SCATT. }\end{array}$ & $\begin{array}{l}\text { PHOTO } \\
\text { EFFECT }\end{array}$ & $\begin{array}{l}\text { PAIR } \\
\text { PROD. }\end{array}$ \\
\hline 400 & -.4 & 2.8 & .1 & -2.9 & \\
\hline 500 & -.2 & $3 \cdot 5$ & .1 & $-2 \cdot 4$ & \\
\hline 600 & .0 & $4 \cdot 3$ & . 1 & -1.2 & \\
\hline 800 & .3 & $5 \cdot 4$ & . 1 & 1.0 & \\
\hline 1000 & .3 & 6.6 & - 1 & $2 \cdot 6$ & \\
\hline 1500 & .0 & $5 \cdot 8$ & - 1 & $-1 \cdot 3$ & -2.8 \\
\hline 2000 & $\cdot 2$ & $5 \cdot 5$ & .0 & $1 \cdot 8$ & $2 \cdot 0$ \\
\hline 3000 & -.4 & $5 \cdot 1$ & -.0 & $\cdot 2$ & $-2 \cdot 0$ \\
\hline 4000 & -.6 & 4.9 & -.1 & -.7 & -1.8 \\
\hline 5000 & -.5 & $4 \cdot 8$ & -.1 & .4 & -1.2 \\
\hline 6000 & 1.2 & 4.7 & -.1 & $\cdot 0$ & 2.7 \\
\hline 8000 & $2 \cdot 3$ & 4.6 & -.0 & .9 & $4 \cdot 1$ \\
\hline 10000 & $1 \cdot 0$ & $4 \cdot 5$ & -.1 & $2 \cdot 7$ & $1 \cdot 5$ \\
\hline 15000 & $1 \cdot 3$ & 4.4 & -.1 & .7 & 1.7 \\
\hline 20000 & -1.0 & 4.4 & -1 & $\cdot 2$ & $-1 \cdot 2$ \\
\hline 30000 & -1.0 & 4.3 & -.1 & .7 & -1.2 \\
\hline 40000 & -.3 & $4 \cdot 2$ & -.1 & $1 \cdot 5$ & -.3 \\
\hline 50000 & -.8 & 4.8 & -.1 & $2 \cdot 4$ & -.9 \\
\hline 60000 & -.2 & $5 \cdot 6$ & -.1 & 3.2 & -.2 \\
\hline 80000 & -.1 & $7 \cdot 4$ & -.1 & $4 \cdot 8$ & -.1 \\
\hline 100000 & -.5 & $9 \cdot 6$ & -.1 & $6 \cdot 1$ & 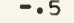 \\
\hline
\end{tabular}


ENERGY $2=49$ INDIUM

$\begin{array}{crrrr}\text { NERGY } & & \text { COH } & \text { INCOH. } & \text { PHOTO } \\ \text { KEV } & \text { TOTAL } & \text { SCATT. } & \text { SCATT. } & \text { EFFECT } \\ 1 & -19.8 & -.5 & 21.7 & -19.9 \\ 1.5 & -8.9 & -.6 & 10.8 & -8.9 \\ 2 & -4.7 & -.9 & 5.2 & -4.7 \\ 3 & -3.3 & -1.4 & -.0 & -3.4 \\ 4 & -10.0 & -1.7 & -1.2 & -10.1 \\ 5 & -7.6 & -1.9 & -.8 & -7.6 \\ 6 & -5.7 & -2.0 & .0 & -5.7 \\ 8 & -3.8 & -1.8 & 1.0 & -3.8 \\ 10 & -2.9 & -1.8 & -.6 & -2.9 \\ 15 & -1.1 & -1.7 & -.7 & -1.1 \\ 20 & .5 & -2.1 & -.5 & .7 \\ 30 & 2.9 & -1.7 & -.2 & 3.0 \\ 40 & .1 & -1.9 & .0 & .2 \\ 50 & -1.4 & -1.8 & .1 & -1.4 \\ 60 & -2.2 & -1.7 & -1 & -2.2 \\ 00 & -2.6 & -1.3 & -.3 & -2.7 \\ 100 & -2.4 & -1.0 & .3 & -2.7 \\ 150 & -2.0 & -.0 & -.0 & -2.7 \\ 200 & -1.8 & .9 & -.0 & -3.0 \\ 300 & -1.3 & 2.2 & .1 & -3.8\end{array}$

ENERGY
KEV
400
500
600
800
1000
1500
2000
3000
4000
5000
6000
8000
10000
15000
20000
30000
40000
50000
60000
80000
100000

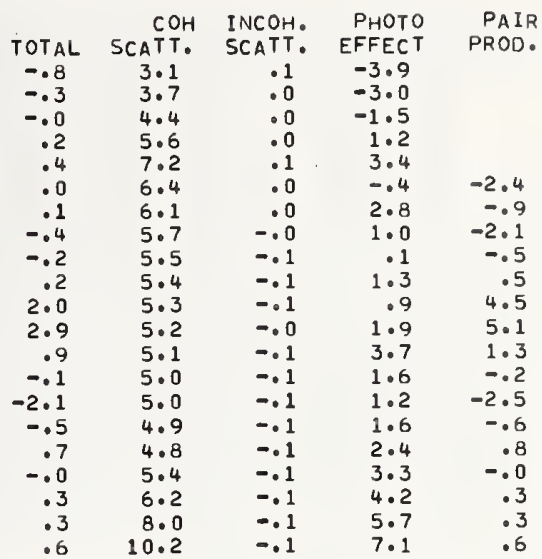

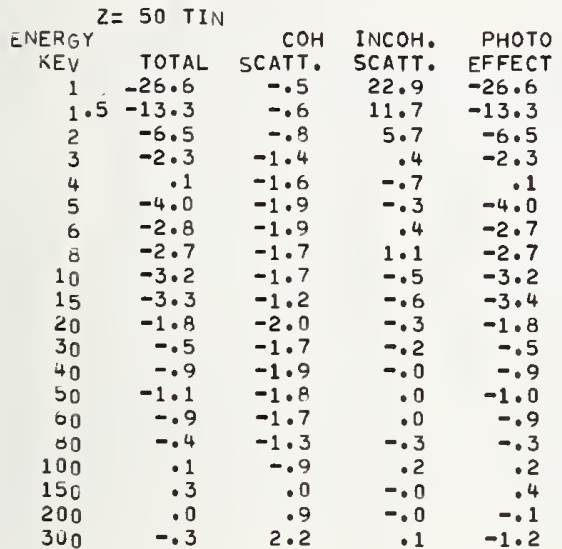

ENERGY

KEV

$\begin{array}{rrrrr} & \text { COH } & \text { INCOH. } & \text { PHOTO } & \text { PAIR } \\ \text { TOTAL } & \text { SCATT. } & \text { SCATT. } & \text { EFFECT } & \text { PROD. } \\ -.2 & 3.1 & .1 & -1.6 & \\ .1 & 3.9 & .1 & -.6 & \\ .3 & 4.5 & .1 & 1.0 & \\ .5 & 5.7 & .0 & 4.6 & \\ .6 & 6.8 & .1 & 7.7 & \\ .2 & 6.1 & .0 & 3.9 & -1.8 \\ .0 & 5.7 & .0 & 7.3 & -2.5 \\ -.3 & 5.3 & -.0 & 5.3 & -2.2 \\ .1 & 5.1 & -.1 & 4.3 & .3 \\ .7 & 4.9 & -.1 & 5.5 & 1.9 \\ 2.9 & 4.9 & -.1 & 5.1 & 6.1 \\ 3.9 & 4.8 & -.1 & 6.1 & 6.8 \\ 1.8 & 4.7 & -.1 & 8.0 & 2.7 \\ .2 & 4.6 & -.1 & 5.7 & .2 \\ -2.2 & 4.6 & -.1 & 5.3 & -2.6 \\ -.7 & 4.5 & -.1 & 5.7 & -.7 \\ .6 & 4.4 & -.1 & 6.6 & .6 \\ -.3 & 5.0 & -.1 & 7.5 & -.03 \\ .1 & 5.7 & -.1 & 8.4 & .1 \\ .2 & 7.5 & -.1 & 10.0 & .12 \\ .9 & 9.7 & -.1 & 11.4 & 1.0\end{array}$

$\begin{array}{ccccc}\text { ENERGY }^{2}= & 51 \text { ANTIMONY } \\ \text { KEV } & \text { TOTAL } & \begin{array}{c}\text { COH } \\ \text { SCATT. }\end{array} & \begin{array}{r}\text { INCOH. } \\ \text { SCATT. }\end{array} & \begin{array}{r}\text { PHOTO } \\ \text { EFFECT }\end{array} \\ 1 & -19.3 & -.5 & 24.3 & -19.3 \\ 1.5 & -9.2 & -.6 & 12.0 & -9.2 \\ 2 & -3.8 & -.8 & 5.1 & -3.8 \\ 3 & -.2 & -1.3 & -.5 & -.2 \\ 4 & -3.0 & -1.6 & -1.4 & -3.0 \\ 5 & -1.6 & -1.8 & -.3 & -1.5 \\ 6 & -2.2 & -1.9 & .1 & -2.1 \\ 8 & -3.6 & -1.8 & 1.1 & -3.6 \\ 10 & -4.3 & -2.0 & .9 & -4.3 \\ 15 & -3.8 & -3.2 & .1 & -3.8 \\ 20 & -1.3 & -1.9 & .1 & -1.3 \\ 30 & 4.6 & -1.8 & .0 & 5.5 \\ 40 & -2.4 & -1.8 & .1 & -2.4 \\ 50 & -4.6 & -1.7 & .1 & -4.7 \\ 60 & -5.3 & -1.6 & .1 & -5.5 \\ 80 & -4.8 & -1.2 & -.2 & -5.1 \\ 100 & -3.6 & -.9 & .4 & -4.0 \\ 150 & -1.2 & .1 & .1 & -1.5 \\ 200 & .0 & 1.0 & .1 & -.1 \\ 300 & . .5 & 2.3 & .1 & .1\end{array}$

ENERGY

$\begin{array}{ccccr} & & & \\ \text { TOTAL } & \text { SCATT. } & \text { INCOH. } & \text { PHOTO } & \text { PAIR } \\ .4 & 3.3 & .2 & .7 & \\ .4 & 4.0 & .1 & .8 & \\ .3 & 4.6 & .1 & .9 & \\ .2 & 6.1 & .1 & .3 & \\ .1 & 6.9 & .1 & -1.6 & \\ -.1 & 6.1 & .0 & -5.2 & -1.1 \\ -.2 & 5.7 & .0 & -2.0 & -3.3 \\ -.5 & 5.3 & -.0 & -3.8 & -2.3 \\ .1 & 5.1 & -.1 & -4.6 & .6 \\ .8 & 5.0 & -.1 & -3.4 & 2.1 \\ 2.9 & 4.9 & -.1 & -3.8 & 6.2 \\ 3.1 & 4.8 & -.0 & -2.8 & 6.3 \\ 1.1 & 4.7 & -.1 & -1.1 & 1.7 \\ -1.1 & 4.6 & -.1 & -3.1 & -1.4 \\ -3.0 & 4.6 & -.1 & -3.6 & -3.6 \\ -.4 & 4.5 & -.1 & -3.2 & -.4 \\ 1.1 & 4.4 & -.1 & -2.4 & 1.3 \\ .1 & 5.0 & -.1 & -1.5 & .2 \\ .3 & 5.7 & -.1 & -.7 & .4 \\ .1 & 7.5 & -.1 & .8 & .2 \\ .8 & 9.6 & -.1 & 2.1 & .8\end{array}$

\begin{tabular}{|c|c|c|c|c|}
\hline \multirow{4}{*}{$\begin{array}{c}\text { ENERGY } \\
\text { KEV } \\
1\end{array}$} & \multicolumn{2}{|c|}{ TELLURIUM } & \multirow[b]{2}{*}{ INCOH. } & \multirow[b]{2}{*}{ РНОTO } \\
\hline & $\pi-1$ & $\mathrm{COH}$ & & \\
\hline & TOTAL & SCATT. & SCATT. & EFFECT \\
\hline & $-24 \cdot 2$ & -.5 & $36 \cdot 0$ & $-24 \cdot 2$ \\
\hline $1 \cdot 5$ & -8.9 & -.6 & 18.0 & -8.9 \\
\hline 2 & $-4 \cdot 7$ & -.8 & 8.7 & -4.7 \\
\hline 3 & $-3 \cdot 3$ & -1.4 & 1.1 & -3.3 \\
\hline 4 & -3.7 & $-1 \cdot 6$ & -.5 & -3.7 \\
\hline 5 & $-5 \cdot 6$ & $-1 \cdot 7$ & -.3 & $-5 \cdot 6$ \\
\hline 6 & -3.6 & -1.8 & .4 & $-3 \cdot 6$ \\
\hline 8 & -2.7 & -1.7 & 1.1 & -2.7 \\
\hline 10 & -2.8 & -1.7 & -.2 & $-2 \cdot 8$ \\
\hline 15 & -2.9 & -2.1 & -.7 & -3.0 \\
\hline 20 & $-2 \cdot 0$ & -1.9 & -.5 & -2.0 \\
\hline 30 & $1 \cdot 8$ & -1.9 & -.2 & 2.3 \\
\hline 40 & .3 & -1.9 & .0 & $\cdot 3$ \\
\hline 50 & -.6 & $-1 \cdot 8$ & - 1 & -.6 \\
\hline 60 & -.9 & -1.7 & $\cdot 1$ & -.9 \\
\hline 80 & -.6 & $-1 \cdot 3$ & -.3 & -.6 \\
\hline 100 & .1 & -1.0 & 2.1 & .0 \\
\hline 150 & .7 & .0 & -.0 & 1.0 \\
\hline 200 & .7 & 1.0 & -.0 & 1.0 \\
\hline 300 & .2 & 2.3 & .1 & .2 \\
\hline
\end{tabular}

\begin{tabular}{|c|c|c|c|c|c|}
\hline $\begin{array}{c}\text { ENERGY } \\
\text { KEV }\end{array}$ & TOTAL & $\begin{array}{l}\text { COH } \\
\text { SCATT. }\end{array}$ & $\begin{array}{l}\text { INCOH. } \\
\text { SCATT. }\end{array}$ & $\begin{array}{r}\text { РHOTO } \\
\text { EFFECT }\end{array}$ & $\begin{array}{l}\text { PAIR } \\
\text { PROD. }\end{array}$ \\
\hline 400 & .1 & 3.2 & .1 & -.5 & \\
\hline 500 & . 1 & 3.9 & .0 & -.6 & \\
\hline 600 & .1 & $4 \cdot 6$ & .0 & -.1 & \\
\hline 800 & .2 & 5.7 & $\cdot 1$ & .4 & \\
\hline 1000 & .2 & $7 \cdot 1$ & - 1 & .4 & \\
\hline 1500 & -.1 & 6.4 & .0 & -3.3 & -2.5 \\
\hline 2000 & -.0 & 6.0 & .0 & -.2 & -.9 \\
\hline 3000 & -.5 & 5.6 & -.0 & -1.9 & -2.3 \\
\hline 4000 & -.4 & 5.4 & -.1 & -2.8 & -.9 \\
\hline 5000 & -.0 & $5 \cdot 3$ & -.1 & -1.6 & .0 \\
\hline 6000 & 1.8 & $5 \cdot 2$ & -.1 & -2.0 & 3.9 \\
\hline 8000 & $2 \cdot 6$ & $5 \cdot 1$ & -.1 & -1.0 & 4.5 \\
\hline 10000 & .6 & $5 \cdot 0$ & -.1 & $\cdot 8$ & 1.0 \\
\hline 15000 & -.3 & 4.9 & -.1 & $-1 \cdot 3$ & -.3 \\
\hline 20000 & $-2 \cdot 1$ & 4.9 & -.1 & -1.8 & -2.5 \\
\hline 30000 & -.7 & $4 \cdot 8$ & -.1 & -1.4 & -.8 \\
\hline 40000 & .4 & $4 \cdot 7$ & -.1 & -.6 & .4 \\
\hline 50000 & -.4 & $5 \cdot 2$ & -.1 & .3 & -.4 \\
\hline 60000 & -.0 & $6 \cdot 0$ & -.1 & $1 \cdot 1$ & -.0 \\
\hline 80000 & .1 & 7.7 & -.1 & $2 \cdot 6$ & .1 \\
\hline 100000 & $\cdot 5$ & $9 \cdot 8$ & -.1 & 3.9 & .5 \\
\hline
\end{tabular}




\begin{tabular}{|c|c|c|c|c|}
\hline $\begin{array}{c}\text { ENERGY } \\
\text { KEV }\end{array}$ & TOTAL & $\begin{array}{l}\text { COH } \\
\text { SCATT. }\end{array}$ & $\begin{array}{l}\text { INCOH. } \\
\text { SCATT. }\end{array}$ & $\begin{array}{r}\text { РНОTO } \\
\text { EFFECT }\end{array}$ \\
\hline $1_{1.5}$ & $\begin{array}{l}-20.4 \\
-13.1\end{array}$ & $\begin{array}{l}-.4 \\
=.6\end{array}$ & $\begin{array}{l}35.2 \\
17.7\end{array}$ & -20.4 \\
\hline 2 & -9.0 & -.9 & 8.9 & -9.0 \\
\hline 3 & -6.4 & -1.4 & 1.6 & $-6 \cdot 5$ \\
\hline 4 & $-8 \cdot 2$ & -1.5 & -.0 & $-8 \cdot 3$ \\
\hline 5 & $-5 \cdot 2$ & $-1 \cdot 6$ & -.0 & $-5 \cdot 2$ \\
\hline 6 & $-3 \cdot 5$ & -1.7 & .6 & -3.4 \\
\hline 8 & $-1 \cdot 9$ & -1.7 & 1.2 & -1.9 \\
\hline 10 & -1.4 & -1.6 & .6 & -1.4 \\
\hline 15 & -1.1 & -2.2 & -.1 & -1.0 \\
\hline 20 & -.7 & -1.8 & -.1 & -.6 \\
\hline 30 & .3 & -1.7 & -.1 & .5 \\
\hline 40 & -1.4 & -1.6 & .0 & -1.4 \\
\hline 50 & -2.3 & -1.4 & .1 & -2.3 \\
\hline 60 & -2.6 & $-1 \cdot 3$ & .1 & -2.7 \\
\hline 80 & -2.7 & -.8 & -.2 & $-2 \cdot 8$ \\
\hline 100 & -2.4 & -.6 & .3 & -2.7 \\
\hline 150 & -1.7 & .5 & .0 & -2.2 \\
\hline 200 & $-1 \cdot 3$ & 1.5 & -.0 & -2.2 \\
\hline 300 & -.9 & 2.8 & .1 & -2.5 \\
\hline
\end{tabular}

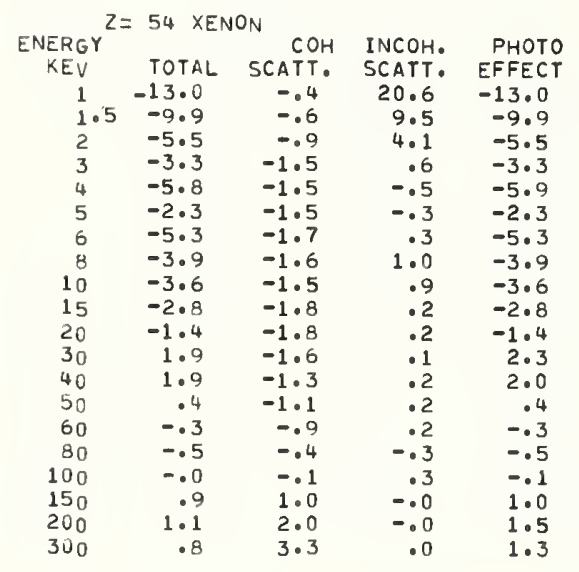

\begin{tabular}{|c|c|c|c|c|}
\hline $\begin{array}{c}\text { ENERGY } \\
\text { KEV }\end{array}$ & TOTAL & $\begin{array}{r}\mathrm{COH} \\
\text { SCATT. }\end{array}$ & $\begin{array}{l}\text { INCOH. } \\
\text { SCATT. }\end{array}$ & $\begin{array}{l}\text { PHOTO } \\
\text { EFFECT }\end{array}$ \\
\hline 1 & $-5 \cdot 4$ & -.5 & $1 \cdot 1$ & -5.4 \\
\hline $1 \cdot 5$ & $\begin{array}{l}=17.8 \\
=12.8\end{array}$ & -.8 & 3.1 & $\begin{array}{l}-17.8 \\
-12.8\end{array}$ \\
\hline $\begin{array}{l}2 \\
3\end{array}$ & & $\begin{array}{l}-1.2 \\
-1.5\end{array}$ & $\begin{array}{r}2 \cdot 5 \\
5\end{array}$ & $\begin{array}{r}12.0 \\
-9.0\end{array}$ \\
\hline $\begin{array}{l}3 \\
4\end{array}$ & $\begin{array}{l}-9.0 \\
-8.1\end{array}$ & $\begin{array}{l}-1.5 \\
-1.3\end{array}$ & -.6 & -8.2 \\
\hline $\begin{array}{l}4 \\
5\end{array}$ & -8.7 & $-1 \cdot 4$ & -.5 & -8.8 \\
\hline 6 & -6.0 & -1.6 & -.0 & -6.0 \\
\hline a & -2.4 & -1.7 & .8 & -2.4 \\
\hline 10 & $-1 \cdot 3$ & $-1 \cdot 5$ & .8 & -1.3 \\
\hline 15 & -.9 & -1.4 & $\cdot 1$ & -.9 \\
\hline 20 & -.8 & $-1 \cdot 7$ & .0 & -.8 \\
\hline 30 & .4 & -1.4 & .0 & .6 \\
\hline 40 & $1 \cdot 2$ & -1.0 & .2 & 1.2 \\
\hline 50 & .2 & -.7 & $\cdot 2$ & .2 \\
\hline 60 & -.2 & -.5 & .1 & -.2 \\
\hline 80 & -.2 & $\cdot 1$ & -.3 & -.2 \\
\hline 100 & $\cdot 3$ & .4 & $\cdot 3$ & .3 \\
\hline 150 & 1.0 & $1 \cdot 5$ & -.0 & $1 \cdot 2$ \\
\hline 200 & $1 \cdot 2$ & $2 \cdot 5$ & -.1 & 1.5 \\
\hline 300 & .8 & 4.0 & -.0 & $1 \cdot 2$ \\
\hline
\end{tabular}

\begin{tabular}{|c|c|c|c|c|}
\hline ENERGY ${ }^{Z}$ & $56 \mathrm{BA}$ & I UM $\mathrm{COH}$ & INCOH. & PHOTO \\
\hline KEV & TOTAL & SCATT. & SCATT. & EFFECT \\
\hline 1 & $-16 \cdot 8$ & -.7 & -4.1 & -16.8 \\
\hline 1.5 & -21.1 & -.9 & -.0 & $-21 \cdot 1$ \\
\hline 2 & -13.0 & -1.1 & $1 \cdot 1$ & $-\$ 3.0$ \\
\hline 3 & -5.8 & $-1,4$ & .7 & -5.9 \\
\hline 4 & -4.5 & -1.4 & -.2 & $-4 \cdot 5$ \\
\hline 5 & -6.8 & -1.4 & -.4 & -6.9 \\
\hline 6 & -6.1 & -1.6 & -.1 & -6.1 \\
\hline 8 & $-1 \cdot 6$ & -1.8 & .6 & $-1 \cdot 6$ \\
\hline 10 & -.5 & -1.8 & .7 & -.5 \\
\hline 15 & $-1 \cdot 0$ & $-2 \cdot 2$ & $\cdot 1$ & -1.0 \\
\hline 20 & -1.8 & -1.7 & -.0 & -1.8 \\
\hline 30 & -1.9 & -1.2 & -.1 & -2.0 \\
\hline 40 & .0 & -.7 & .1 & .1 \\
\hline 50 & -1.5 & -.3 & .1 & -1.6 \\
\hline 60 & $-2 \cdot 1$ & -.0 & .1 & $-2 \cdot 2$ \\
\hline 80 & -1.9 & .7 & -.3 & $-2 \cdot 1$ \\
\hline 100 & -1.1 & 1.0 & .4 & -1.3 \\
\hline 150 & .5 & 2.1 & -.0 & .6 \\
\hline 200 & 1.2 & 3.2 & -.0 & 1.4 \\
\hline 300 & 1.1 & 4.7 & .0 & 1.7 \\
\hline
\end{tabular}

\begin{tabular}{|c|c|c|c|c|c|}
\hline $\begin{array}{c}\text { ENERGY } \\
\text { KEV }\end{array}$ & TOTAL & $\begin{array}{l}\text { COH } \\
\text { SCATT. }\end{array}$ & $\begin{array}{l}\text { INCOH. } \\
\text { SCATT. }\end{array}$ & $\begin{array}{r}\text { РHOTO } \\
\text { EFFECT }\end{array}$ & $\begin{array}{l}\text { PAIR } \\
\text { PROD. }\end{array}$ \\
\hline 400 & -.6 & 3.8 & .1 & -2.6 & \\
\hline 500 & -.2 & 4.5 & .1 & $-2 \cdot 2$ & \\
\hline 600 & .0 & $5 \cdot 2$ & .1 & -1.4 & \\
\hline 800 & .2 & 6.3 & .0 & .4 & \\
\hline 1000 & .3 & $7 \cdot 8$ & .1 & 1.7 & \\
\hline 1500 & -.0 & 7.0 & .0 & -2.0 & -3.0 \\
\hline 2000 & .2 & 6.6 & .0 & 1.1 & 1.0 \\
\hline 3000 & -.4 & 6.2 & -.0 & -.5 & -2.0 \\
\hline 4000 & -.5 & 6.0 & -.1 & -1.4 & -1.4 \\
\hline 5000 & -.4 & 5.9 & -.1 & -.2 & -.8 \\
\hline 6000 & 1.4 & $5 \cdot 8$ & -.1 & -.5 & 3.1 \\
\hline 8000 & 2.5 & 5.7 & -.1 & .4 & $4 \cdot 2$ \\
\hline 10000 & .9 & 5.7 & -.1 & $2 \cdot 3$ & 1.4 \\
\hline 15000 & .8 & 5.6 & -.1 & $\cdot 1$ & 1.0 \\
\hline 20000 & $-1 \cdot 3$ & $5 \cdot 5$ & -.1 & -.4 & -1.5 \\
\hline 30000 & -.8 & 5.4 & $=.1$ & -.1 & -.9 \\
\hline 40000 & .1 & 5.4 & -.1 & .7 & .1 \\
\hline 50000 & -.5 & 5.8 & -.1 & 1.6 & -.5 \\
\hline 60000 & .2 & 6.6 & -.1 & 2.4 & .2 \\
\hline 80000 & .6 & $8 \cdot 3$ & -.1 & 4.0 & .6 \\
\hline 100000 & .8 & 10.4 & -.1 & $5 \cdot 3$ & - 8 \\
\hline
\end{tabular}

\begin{tabular}{|c|c|c|c|c|c|}
\hline $\begin{array}{c}\text { ENERGY } \\
\text { KEV }\end{array}$ & TOTAL & $\begin{array}{r}\mathrm{COH} \\
\text { SCATT. }\end{array}$ & $\begin{array}{l}\text { INCOH. } \\
\text { SCATT. }\end{array}$ & $\begin{array}{r}\text { PHOTO } \\
\text { EFFECT }\end{array}$ & $\begin{array}{r}\text { PAIR } \\
\text { PROD. }\end{array}$ \\
\hline 400 & .5 & 4.3 & .1 & .7 & \\
\hline 500 & .3 & $5 \cdot 1$ & .1 & .3 & \\
\hline 600 & $\cdot 2$ & 5.7 & .1 & .1 & \\
\hline 800 & .2 & 7.2 & .0 & .1 & \\
\hline 1000 & . 2 & 8.2 & .1 & -.4 & \\
\hline 1500 & -.1 & $7 \cdot 5$ & .0 & -3.8 & $-3 \cdot 5$ \\
\hline 2000 & .3 & 7.1 & .0 & -.6 & 4.0 \\
\hline 3000 & -.4 & 6.7 & -.0 & -2.1 & -1.8 \\
\hline 4000 & $-1 \cdot 0$ & $6 \cdot 5$ & -.1 & -2.9 & -2.7 \\
\hline 5000 & $-1 \cdot 2$ & 6.3 & -.1 & -1.7 & -2.7 \\
\hline 6000 & .4 & $6 \cdot 3$ & -.1 & $-2 \cdot 0$ & 1.0 \\
\hline 8000 & 1.6 & 6.2 & -.1 & $-1 \cdot 1$ & 2.7 \\
\hline 10000 & .7 & 6.1 & -.1 & .7 & 1.0 \\
\hline 15000 & 1.9 & $6 \cdot 0$ & -.1 & $-1 \cdot 6$ & 2.5 \\
\hline 20000 & -.3 & 6.0 & -.1 & $-2 \cdot 1$ & -.4 \\
\hline 30000 & $-1 \cdot 3$ & $5 \cdot 9$ & -.1 & -1.8 & -1.5 \\
\hline 40000 & -.8 & 5.8 & -.1 & -1.1 & -.9 \\
\hline 50000 & -1.1 & 6.3 & -.1 & -.2 & $-1 \cdot 1$ \\
\hline 60000 & .0 & 7.0 & -.1 & .6 & .0 \\
\hline 80000 & .9 & 8.7 & -.1 & $2 \cdot 0$ & 1.0 \\
\hline 100000 & 1.0 & 10.8 & -.1 & $3 \cdot 3$ & 1.1 \\
\hline
\end{tabular}

ENERGY COH INCOH. PHOTO PAIR

$\begin{array}{rrrrrr}\text { KEV } & \text { TOTAL } & \text { SCATT } & \text { SCATT } & \text { EFFECT } & \text { PROD } \\ 400 & .5 & 4.9 & .0 & .6 & \\ 500 & .3 & 5.7 & .0 & .3 & \\ 600 & .3 & 6.5 & .0 & .2 & \\ 800 & .3 & 7.4 & .1 & .3 & \\ 1000 & .2 & 8.8 & .1 & -.1 & \\ 1500 & -.1 & 8.0 & .0 & -3.6 & -4.1 \\ 2000 & .5 & 7.6 & .0 & -.5 & 6.0 \\ 3000 & -.3 & 7.2 & -.0 & -2.0 & -1.2 \\ 4000 & -1.1 & 7.0 & -.1 & -2.8 & -3.1 \\ 5000 & -1.5 & 6.9 & -.1 & -1.6 & -3.5 \\ 6000 & -.1 & 6.8 & -.1 & -2.0 & -.0 \\ 8000 & 1.2 & 6.7 & -.1 & -1.0 & 2.1 \\ 10000 & .7 & 6.7 & -.1 & .8 & 1.0 \\ 15000 & 3.0 & 6.6 & -.1 & -1.6 & 3.8 \\ 20000 & .9 & 6.6 & -.1 & -2.1 & 1.1 \\ 30000 & -.8 & 6.4 & -.1 & -1.8 & -.9 \\ 40000 & -.7 & 6.4 & -.1 & -1.0 & -.18 \\ 50000 & -1.1 & 6.8 & -.1 & -.2 & -1.2 \\ 60000 & .0 & 7.6 & -.1 & .1 .6 & .0 \\ 80000 & 1.0 & 9.3 & -.1 & 2.1 & 1.0 \\ 100000 & .9 & 11.3 & -.1 & 3.3 & .9\end{array}$

ENERGY COH INCOH. PHOTO PAIR

$\begin{array}{rrrrrr}\text { KEV } & \text { TOTAL } & \text { SCATT } & \text { SCATT. } & \text { EFFECT } & \text { PROD. } \\ 400 & .7 & 5.8 & .0 & 1.2 & \\ 500 & .5 & 6.4 & .1 & .9 & \\ 600 & .4 & 7.2 & .1 & .7 & \\ 800 & .3 & 8.5 & .1 & .1 & \\ 1000 & .1 & 9.6 & .1 & -1.2 & \\ 1500 & -.2 & 8.9 & .0 & -4.6 & -3.9 \\ 2000 & .4 & 8.5 & -.0 & -1.5 & 4.8 \\ 3000 & -.4 & 8.1 & -.1 & -2.9 & -1.6 \\ 4000 & -1.0 & 7.9 & -.2 & -3.7 & -2.8 \\ 5000 & -1.3 & 7.7 & -.1 & -2.5 & -2.9 \\ 6000 & .3 & 7.6 & -.1 & -2.9 & .8 \\ 8000 & 1.8 & 7.5 & -.1 & -1.9 & 3.0 \\ 10000 & 1.2 & 7.5 & -.1 & -.1 & 1.8 \\ 15000 & 3.2 & 7.4 & -.1 & -2.5 & 4.1 \\ 20000 & 1.0 & 7.4 & -.1 & -3.0 & 1.2 \\ 30000 & -.4 & 7.3 & -.1 & -2.8 & -.4 \\ 40000 & .0 & 7.2 & -.1 & -2.0 & .0 \\ 50000 & -.3 & 7.6 & -.1 & -1.2 & -.13 \\ 60000 & . .9 & 8.4 & -.1 & -. .4 & .9 \\ 80000 & 1.7 & 10.0 & -.1 & 1.0 & 1.8 \\ 100000 & 1.4 & 12.1 & -.1 & 2.3 & 1.5\end{array}$




\begin{tabular}{|c|c|c|c|c|}
\hline $\begin{array}{c}\text { ENERGY } \\
\text { KEV }\end{array}$ & TOTAL & $\begin{array}{r}\mathrm{COH} \\
\text { SCATT. }\end{array}$ & $\begin{array}{l}\text { INCOH. } \\
\text { SCATT. }\end{array}$ & $\begin{array}{r}\text { PHOTO } \\
\text { EFFECT }\end{array}$ \\
\hline 1 & $-17 \cdot 5$ & -.8 & -7.6 & -17.5 \\
\hline $1 \cdot 5$ & $\begin{array}{l}-24.9 \\
-16.5\end{array}$ & -.9 & -2.1 & -24.9 \\
\hline 2 & $\begin{array}{r}-16.5 \\
-7.4\end{array}$ & -1.1 & $\begin{array}{r}: 4 \\
1.2\end{array}$ & $\begin{array}{r}-16.6 \\
-7.5\end{array}$ \\
\hline $\begin{array}{l}3 \\
4\end{array}$ & $\begin{array}{l}-7.4 \\
-4.7\end{array}$ & $\begin{array}{l}-1.5 \\
-1.5\end{array}$ & $\begin{array}{r}1.2 \\
.8\end{array}$ & $\begin{array}{l}-1.5 \\
-4.7\end{array}$ \\
\hline 5 & -6.6 & -1.4 & .6 & -6.8 \\
\hline 6 & -1.1 & -1.5 & .8 & -1.1 \\
\hline 8 & -1.8 & -1.8 & 1.4 & -1.8 \\
\hline 10 & -1.9 & -1.9 & $4 \cdot 6$ & -1.9 \\
\hline 15 & $-2 \cdot 6$ & -2.4 & 2.9 & -2.6 \\
\hline 20 & -2.4 & -1.7 & 1.9 & -2.4 \\
\hline 30 & . 1 & $-1 \cdot 3$ & .7 & .2 \\
\hline 40 & -.1 & -.9 & .5 & -.1 \\
\hline 50 & -1.4 & -.6 & .4 & -1.4 \\
\hline 60 & -1.9 & -.3 & $\cdot 3$ & -1.9 \\
\hline 80 & -1.7 & .3 & -.2 & -1.8 \\
\hline 100 & -1.0 & .6 & .4 & -1.2 \\
\hline 150 & .1 & 1.7 & .0 & .1 \\
\hline 200 & .6 & 2.7 & -.0 & .6 \\
\hline 300 & .6 & 4.1 & .0 & .6 \\
\hline
\end{tabular}

\begin{tabular}{|c|c|c|c|c|}
\hline \multicolumn{5}{|c|}{$Z=58$ CER IUM } \\
\hline ENERGY & TOTAI & $\mathrm{COH}$ & I NCOH. & PHOTO \\
\hline & -19.3 & $\begin{array}{r}\text { SCATI } \\
-.9\end{array}$ & $\begin{array}{l}\text { SCATT. } \\
-12.9\end{array}$ & $\begin{array}{l}\text { EFFECT } \\
-19.3\end{array}$ \\
\hline $1 \cdot 5$ & $-21 \cdot 4$ & -1.2 & -6.4 & $-2 i \cdot 4$ \\
\hline 2 & $-14 \cdot 3$ & -1.7 & -2.5 & -14.3 \\
\hline 3 & $-8 \cdot 2$ & -2.4 & -.1 & $-8 \cdot 2$ \\
\hline 4 & -6.2 & -2.6 & $\cdot 3$ & $-6 \cdot 3$ \\
\hline 5 & $-6 \cdot 3$ & $-2 \cdot 5$ & .2 & -6.4 \\
\hline 6 & .4 & $-2 \cdot 5$ & .4 & .5 \\
\hline 8 & -2.6 & -2.6 & $1 \cdot 0$ & $-2 \cdot 6$ \\
\hline 10 & -1.4 & $-2 \cdot 3$ & $4 \cdot 5$ & -1.4 \\
\hline 15 & -1.9 & -1.9 & 3.2 & -1.9 \\
\hline 20 & -2.6 & -2.0 & $2 \cdot 1$ & -2.7 \\
\hline 30 & -2.1 & $-1 \cdot 7$ & .8 & -2.2 \\
\hline 40 & .7 & -1.4 & .5 & .9 \\
\hline 50 & -2.9 & $-1 \cdot 2$ & .4 & -2.9 \\
\hline 60 & $-3 \cdot 0$ & $-1 \cdot 0$ & .3 & -3.1 \\
\hline 80 & $-2 \cdot 3$ & -.4 & -.2 & $-2 \cdot 5$ \\
\hline 100 & $-1 \cdot 2$ & -.2 & .4 & -1.4 \\
\hline 150 & .7 & .8 & -.1 & .8 \\
\hline 200 & $1 \cdot 5$ & 1.7 & -.1 & 1.9 \\
\hline 300 & 1.5 & 3.1 & .0 & 2.5 \\
\hline
\end{tabular}

\begin{tabular}{|c|c|c|c|c|c|}
\hline $\begin{array}{c}\text { ENERGY } \\
\text { KEV }\end{array}$ & TOTAL & $\begin{array}{r}\text { COH } \\
\text { SCATT. }\end{array}$ & $\begin{array}{l}\text { INCOH. } \\
\text { SCATT. }\end{array}$ & $\begin{array}{l}\text { РHOTO } \\
\text { EFFECT }\end{array}$ & $\begin{array}{l}\text { PAIR } \\
\text { PROD. }\end{array}$ \\
\hline $\begin{array}{r}400 \\
500 \\
600 \\
800 \\
1000\end{array}$ & $\begin{array}{l}.4 \\
.3 \\
.3 \\
.2 \\
.2\end{array}$ & $\begin{array}{l}5.1 \\
5.9 \\
6.6 \\
7.8 \\
9.1\end{array}$ & $\begin{array}{r}.1 \\
.1 \\
.1 \\
.0 \\
.1\end{array}$ & $\begin{array}{r}.1 \\
.0 \\
.2 \\
.3 \\
-.0\end{array}$ & \\
\hline 1500 & -.2 & 8.3 & .0 & $-3 \cdot 4$ & $-4 \cdot 1$ \\
\hline 2000 & .5 & 7.9 & -.0 & -.3 & $\begin{array}{r}5.7 \\
-1.2\end{array}$ \\
\hline $\begin{array}{l}3000 \\
4000\end{array}$ & $=3$ & $\begin{array}{l}7.5 \\
7.3\end{array}$ & $\begin{array}{l}=.1 \\
=.2\end{array}$ & $\begin{array}{l}-1.6 \\
-2.4\end{array}$ & $\begin{array}{l}-1 \cdot 2 \\
-2 \cdot 8\end{array}$ \\
\hline 5000 & -1.5 & 7.2 & -01 & $-1 \cdot 1$ & -3.3 \\
\hline $\begin{array}{l}6000 \\
8000\end{array}$ & $1: 3$ & $\begin{array}{l}7.1 \\
7.0\end{array}$ & -1 & -.5 & $2 \cdot 3$ \\
\hline 10000 & .7 & 7.0 & -.1 & $1 \cdot 3$ & 1.1 \\
\hline 15000 & 2.7 & 6.9 &.- .1 & -1.1 & 3.5 \\
\hline 20000 & .6 & 6.8 & -.1 & -1.7 & .7 \\
\hline 30000 & -1.0 & 6.7 & -.1 & $-1 \cdot 4$ & $-1 \cdot 1$ \\
\hline 40000 & -.8 & 6.6 & -.1 & -.7 & -.8 \\
\hline 50000 & -1.1 & 7.0 & -.1 & .2 & $-1 \cdot 2$ \\
\hline 60000 & -.0 & 7.8 & -.1 & 1.0 & -.0 \\
\hline 80000 & .7 & 9.5 & -.1 & 2.4 & .7 \\
\hline$: 00000$ & .2 & $11 \cdot 5$ & -.1 & 3.7 & .2 \\
\hline
\end{tabular}

\begin{tabular}{|c|c|c|c|c|c|}
\hline $\begin{array}{c}\text { ENERGY } \\
\text { KEV }\end{array}$ & TOTAL & $\begin{array}{r}\mathrm{COH} \\
\text { SCATT. }\end{array}$ & $\begin{array}{l}\text { INCOH. } \\
\text { SCATT. }\end{array}$ & $\begin{array}{r}\text { PHOTO } \\
\text { EFFECT }\end{array}$ & PAIR \\
\hline 400 & 1.0 & $4 \cdot 2$ & .0 & 2.1 & \\
\hline 500 & .7 & 4.9 & .0 & 1.6 & \\
\hline 600 & .5 & $5 \cdot 6$ & .0 & 1.2 & \\
\hline 800 & $\cdot 3$ & $6 \cdot 8$ & .1 & .1 & \\
\hline 1000 & .2 & 7.9 & .0 & -1.7 & \\
\hline 1500 & -.3 & 7.1 & .0 & $-5 \cdot 0$ & -3.4 \\
\hline 2000 & .5 & 6.7 & -.0 & -1.8 & 6.3 \\
\hline 3000 & -.3 & 6.3 & -.1 & -2.9 & -.7 \\
\hline 4000 & $-1 \cdot 0$ & 6.1 & -.2 & -3.5 & -2.4 \\
\hline 5000 & $-1 \cdot 3$ & 6.0 & -.1 & -2.2 & -2.7 \\
\hline 6000 & .4 & 5.9 & -.1 & -2.5 & .9 \\
\hline 8000 & 1.9 & $5 \cdot 8$ & -.1 & $-1 \cdot 5$ & 3.2 \\
\hline 10000 & 1.5 & $5 \cdot 8$ & -.1 & .4 & $2 \cdot 2$ \\
\hline 15000 & 3.5 & 5.7 & -.1 & -1.9 & $4 \cdot 5$ \\
\hline 20000 & 1.1 & $5 \cdot 7$ & -.1 & $-2 \cdot 5$ & $1 \cdot 3$ \\
\hline 30000 & -.9 & $5 \cdot 6$ & -.1 & $-2 \cdot 2$ & -.9 \\
\hline 40000 & -.6 & $5 \cdot 5$ & -.2 & -1.4 & -.6 \\
\hline 50000 & -.8 & 5.8 & -.1 & -.6 & -.8 \\
\hline 60000 & .4 & 6.6 & -.1 & .2 & .4 \\
\hline 80000 & 1.1 & 8.2 & -.1 & 1.6 & 1.1 \\
\hline 100000 & .4 & 10.2 & -.2 & $2 \cdot 9$ & .4 \\
\hline
\end{tabular}

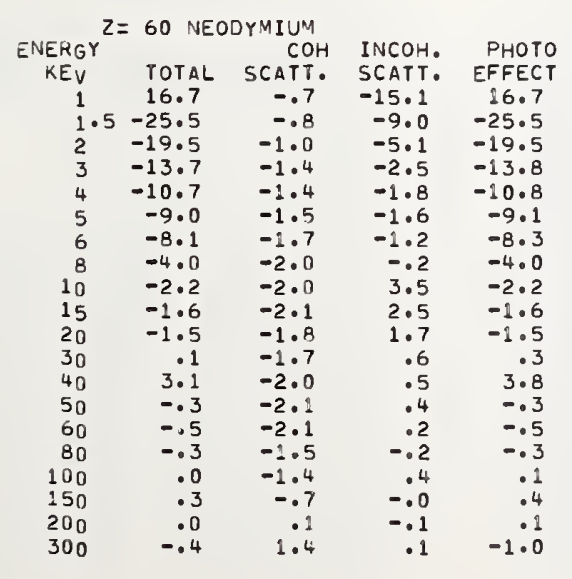

\begin{tabular}{rrrrrr} 
ENERGY & \multicolumn{1}{c}{ COH } & INCOH. & PHOTO & PAIR \\
KEV & TOTAL & SCATT. & SCATT. & EFFECT & PROD. \\
400 & .0 & 3.3 & -.0 & -.4 & \\
500 & .1 & 4.1 & .0 & -.6 & \\
600 & .1 & 4.8 & .0 & -.5 & \\
800 & .2 & 5.9 & .1 & -.2 & \\
1000 & .2 & 7.2 & .0 & -.5 & \\
1500 & -.2 & 6.4 & .0 & -3.7 & -3.9 \\
2000 & .5 & 6.0 & -.0 & -.5 & 6.4 \\
3000 & -.4 & 5.6 & -.1 & -1.7 & -1.3 \\
4000 & -1.3 & 5.4 & -.2 & -2.4 & -3.4 \\
5000 & -1.8 & 5.3 & -.1 & -1.2 & -4.0 \\
6000 & -.3 & 5.2 & -.1 & -1.6 & -.5 \\
8000 & 1.1 & 5.1 & -.1 & -.7 & 1.8 \\
10000 & .8 & 5.0 & -.1 & 1.1 & 1.2 \\
15000 & 3.4 & 5.0 & -.1 & -1.4 & 4.4 \\
20000 & 1.4 & 4.9 & -.1 & -2.0 & 1.7 \\
30000 & -.5 & 4.8 & -.2 & -1.8 & -.16 \\
40000 & -.4 & 4.7 & -.1 & -1.1 & -.4 \\
50000 & -.6 & 5.1 & -.2 & -.3 & -.7 \\
60000 &. .5 & 5.8 & -.2 & .5 & .6 \\
80000 & 1.2 & 7.4 & -.2 & 1.9 & 1.2 \\
100000 &. .4 & 9.4 & -.2 & 3.2 & .2
\end{tabular}

\begin{tabular}{|c|c|c|c|c|}
\hline ENERGY & & $\mathrm{COH}$ & INCOH. & PHOTO \\
\hline KEV & TOTAL & SCATT. & SCATT. & EFFECT \\
\hline 1 & $-19 \cdot 7$ & -.7 & $-15 \cdot 2$ & -19.7 \\
\hline $1 \cdot 5$ & $-22 \cdot 0$ & -.8 & -8.9 & $-22 \cdot 1$ \\
\hline 2 & -17.4 & $-1 \cdot 0$ & -5.0 & -17.4 \\
\hline 3 & $-12 \cdot 8$ & $-1 \cdot 4$ & -2.5 & -12.9 \\
\hline 4 & $-10 \cdot 6$ & -1.4 & -1.9 & -10.7 \\
\hline 5 & -9.2 & $-1 \cdot 5$ & -1.7 & -9.3 \\
\hline 6 & -.5 & -1.7 & $-1 \cdot 2$ & -.5 \\
\hline 8 & -3.8 & $-1 \cdot 8$ & -.2 & -3.9 \\
\hline 10 & -1.6 & -1.7 & 3.7 & -1.6 \\
\hline 15 & -.6 & -.8 & 2.7 & -.7 \\
\hline 20 & -.8 & -1.8 & 1.8 & -.8 \\
\hline 30 & -.2 & -1.6 & .6 & -.1 \\
\hline 40 & $1 \cdot 6$ & -1.7 & .5 & $2 \cdot 0$ \\
\hline so & $\cdot 2$ & $-1 \cdot 6$ & .4 & $\cdot 2$ \\
\hline 60 & -.2 & -1.5 & .3 & -.1 \\
\hline 80 & -.2 & -.9 & -.3 & -.2 \\
\hline 100 & .2 & -.7 & .4 & .2 \\
\hline 150 & .7 & .1 & -.1 & .8 \\
\hline 200 & .6 & 1.0 & -.1 & .8 \\
\hline 300 & $\cdot 3$ & 2.4 & .0 & $\cdot 2$ \\
\hline
\end{tabular}

$\begin{array}{rrrrrr}\begin{array}{c}\text { ENERGY } \\ \text { KEV }\end{array} & \text { TOTAL } & \begin{array}{r}\text { COH } \\ \text { SCATT. }\end{array} & \begin{array}{r}\text { INCOH. } \\ \text { SCATT. }\end{array} & \begin{array}{r}\text { PHOTO } \\ \text { EFFECT }\end{array} & \begin{array}{r}\text { PAIR } \\ \text { PROD. }\end{array} \\ 500 & -.4 & 2.4 & .1 & -1.5 & \\ 500 & -.2 & 3.1 & .1 & -1.4 & \\ 600 & .0 & 3.8 & .1 & -.8 & \\ 800 & .3 & 5.0 & .1 & .2 & \\ 1000 & -2 & 6.2 & .0 & .6 & \\ 1500 & -.2 & 5.4 & -.0 & -2.8 & -2.9 \\ 2000 & .1 & 5.0 & -.0 & .3 & 1.2 \\ 3000 & -.5 & 4.6 & -.1 & -1.0 & -2.1 \\ 4000 & -.7 & 4.4 & -.2 & -1.8 & -1.7 \\ 5000 & -.6 & 4.3 & -.1 & -.5 & -1.1 \\ 6000 & 1.3 & 4.2 & -.1 & -1.0 & 2.7 \\ 8000 & 2.5 & 4.1 & -.1 & -.0 & 4.1 \\ 20000 & 1.1 & 4.0 & -.1 & 1.8 & 1.7 \\ 15000 & 1.1 & 3.9 & -.2 & -.7 & 1.4 \\ 20000 & -1.2 & 3.9 & -.2 & -1.4 & -1.3 \\ 30000 & -.8 & 3.8 & -.2 & -1.2 & -.29 \\ 40000 & .4 & 3.7 & -.2 & -.5 & .5 \\ 50000 & .1 & 4.1 & -.2 & .3 & .2 \\ 60000 & 1.0 & 4.8 & -.2 & 1.1 & 1.1 \\ 80000 & 1.3 & 6.4 & -.2 & 2.6 & 1.4 \\ 100000 & 1.0 & 8.3 & -.2 & 3.9 & 1.1\end{array}$




\begin{tabular}{|c|c|c|c|c|}
\hline \multicolumn{5}{|c|}{$Z=61$ PROMETHIUM } \\
\hline $\begin{array}{c}\text { ENERGY } \\
\text { KEV }\end{array}$ & TOTAL & $\begin{array}{r}\text { COH } \\
\text { SCATT. }\end{array}$ & $\begin{array}{l}\text { INCOH. } \\
\text { SCATT: }\end{array}$ & $\begin{array}{r}\text { PHOTO } \\
\text { EFFECT }\end{array}$ \\
\hline 1 & -23.5 & -.6 & -13.2 & -23.5 \\
\hline 1.5 & -25.5 & -.8 & -7.6 & $-25 \cdot 5$ \\
\hline 2 & $-20 \cdot 0$ & $-1 \cdot 0$ & $-4 \cdot 2$ & $-20 \cdot 1$ \\
\hline 3 & -13.9 & $-1 \cdot 3$ & $-2 \cdot 0$ & -14.0 \\
\hline 4 & -10.9 & -1.4 & -1.4 & -12.0 \\
\hline 5 & -9.1 & -1.4 & $-1 \cdot 4$ & $-9 \cdot 3$ \\
\hline 6 & -8.1 & -1.6 & $-1 \cdot 1$ & -8.2 \\
\hline 8 & $-4 \cdot 5$ & -1.9 & -.2 & -4.6 \\
\hline 10 & -3.7 & -1.8 & 3.0 & -3.7 \\
\hline 15 & $-2 \cdot 5$ & $-1 \cdot 0$ & $2 \cdot 2$ & $-2 \cdot 6$ \\
\hline 20 & $-1 \cdot 3$ & $-1 \cdot 7$ & 1.5 & $-1 \cdot 3$ \\
\hline 30 & $1 \cdot 1$ & -1.8 & .5 & 1.3 \\
\hline 40 & 2.6 & $-2 \cdot 3$ & .4 & 3.3 \\
\hline 50 & -.2 & -2.6 & .3 & -.2 \\
\hline 60 & -.5 & -2.7 & .2 & -.4 \\
\hline 80 & -.5 & $-2 \cdot 1$ & -.3 & -.3 \\
\hline 100 & -.1 & -2.1 & .4 & .0 \\
\hline 150 & .7 & $-1 \cdot 5$ & -.1 & .9 \\
\hline 200 & .8 & -.8 & -.1 & $1 \cdot 2$ \\
\hline 300 & .5 & .5 & -.0 & .9 \\
\hline
\end{tabular}

ENERGY
KEV
400
500
600
800
1000
1500
2000
3000
4000
5000
6000
8000
10000
15000
20000
30000
40000
50000
60000
80000
100000

$\begin{array}{rc} & \text { COH } \\ \text { TOTAL } & \text { SCATT. } \\ .2 & 1.4 \\ .1 & 2.2 \\ .1 & 2.9 \\ .2 & 3.9 \\ .0 & 5.1 \\ -.3 & 4.4 \\ -.1 & 4.0 \\ -.6 & 3.6 \\ -.4 & 3.4 \\ .0 & 3.3 \\ 2.0 & 3.2 \\ 3.1 & 3.1 \\ 1.4 & 3.1 \\ .3 & 3.0 \\ -2.0 & 2.9 \\ -1.2 & 2.8 \\ .1 & 2.8 \\ -.4 & 3.1 \\ .1 & 3.7 \\ .2 & 5.3 \\ .1 & 7.2\end{array}$

$\begin{array}{rrr}\text { NCOH. } & \text { PHOTO } & \text { PAIR } \\ \text { SCATT. } & \text { EFFECT } & \text { PROD. } \\ -.0 & .3 & \\ -.0 & -.2 & \\ .0 & -.4 & \\ .2 & -.4 & \\ .0 & -.8 & \\ -.0 & -4.0 & -2.4 \\ -.0 & -.9 & -.6 \\ -.1 & -1.9 & -2.2 \\ -.2 & -2.5 & -.8 \\ -.1 & -1.3 & .2 \\ -.1 & -1.6 & 4.1 \\ -.1 & -.17 & 5.1 \\ -.1 & 1.2 & 2.0 \\ -.2 & -1.3 & .4 \\ -.2 & -2.0 & -2.3 \\ -.2 & -1.9 & -1.3 \\ -.2 & -1.2 & .1 \\ -.2 & -.4 & -.5 \\ -.2 & .4 & .1 \\ -.2 & 1.8 & .2 \\ -.2 & 3.1 & .1\end{array}$

\begin{tabular}{|c|c|c|c|c|}
\hline ENERGY & & $\mathrm{COH}$ & INCOH. & PHOTO \\
\hline KEV & TOTAL & SCATT. & SCATT. & EFFECT \\
\hline 1 & $-22 \cdot 8$ & -.6 & $-10 \cdot 5$ & -22.9 \\
\hline 1.5 & $-24 \cdot 1$ & -.7 & -5.5 & -24.1 \\
\hline 2 & -20.9 & -.9 & -2.6 & -20.9 \\
\hline 3 & -14.1 & $-1 \cdot 3$ & -.6 & -24.2 \\
\hline 4 & -10.7 & -1.4 & -.3 & -10.8 \\
\hline 5 & $-8 \cdot 6$ & -1.4 & -.5 & -8.7 \\
\hline 6 & -7.3 & -1.6 & -.5 & -7.4 \\
\hline 8 & $-5 \cdot 3$ & -1.8 & -.1 & $-5 \cdot 3$ \\
\hline 10 & $-2 \cdot 3$ & $-1 \cdot 8$ & $2 \cdot 5$ & $-2 \cdot 3$ \\
\hline 15 & -.9 & $-1 \cdot 3$ & $2 \cdot 0$ & -.9 \\
\hline 20 & $-1 \cdot 2$ & -1.8 & $1 \cdot 5$ & $-1 \cdot 1$ \\
\hline 30 & $-1 \cdot 0$ & -1.4 & .7 & $-1 \cdot 0$ \\
\hline 40 & $\cdot 3$ & -1.9 & .5 & .5 \\
\hline 50 & $-1 \cdot 1$ & -2.2 & .4 & $-1 \cdot 0$ \\
\hline 60 & -.7 & $-2 \cdot 2$ & $\cdot 3$ & -.7 \\
\hline 80 & .2 & $-1 \cdot 4$ & -.3 & $\cdot 3$ \\
\hline 100 & 1.1 & -1.4 & .4 & 1.2 \\
\hline 150 & 1.9 & -.8 & -.1 & $2 \cdot 3$ \\
\hline 200 & 1.6 & $\cdot 0$ & -.1 & $2 \cdot 3$ \\
\hline 300 & .8 & $1 \cdot 3$ & .0 & $1 \cdot 2$ \\
\hline
\end{tabular}

ENERGY
KEV
400
500
600
800
1000
1500
2000
3000
4000
5000
6000
8000
10000
15000
20000
30000
40000
50000
60000
80000
100000

\begin{tabular}{|c|c|c|c|c|}
\hline TOTAL & $\begin{array}{l}\text { COH } \\
\text { SCATT. }\end{array}$ & $\begin{array}{l}\text { INCOH. } \\
\text { SCATT. }\end{array}$ & $\begin{array}{r}\text { PHOTO } \\
\text { EFFECT }\end{array}$ & $\begin{array}{r}\text { PAIR } \\
\text { PROD. }\end{array}$ \\
\hline$\cdot 2$ & $2 \cdot 3$ & -.0 & .3 & \\
\hline 1 & 3.0 & .0 & -.2 & \\
\hline 12 & $3 \cdot 8$ & $\cdot 0$ & -.2 & \\
\hline .2 & 4.9 & 1 & $\begin{array}{l}-2 \\
-08\end{array}$ & \\
\hline-.3 & 5.3 & -0 & $-4 \cdot 0$ & -2.1 \\
\hline-.0 & 4.9 & -.0 & -.9 & . 1 \\
\hline-.6 & $4 \cdot 5$ & -.1 & -2.0 & -2.0 \\
\hline-.5 & 4.3 & -.2 & -2.7 & -.9 \\
\hline-.1 & 4.2 & -.1 & -1.4 & -.0 \\
\hline 1.9 & 4.1 & -.1 & -1.8 & 3.9 \\
\hline 3.1 & 4.0 & -.1 & -.9 & 5.0 \\
\hline 1.5 & 3.9 & -.1 & 1.0 & 2.2 \\
\hline .9 & $3 \cdot 8$ & -.2 & -1.6 & 1.2 \\
\hline $1 \cdot 2$ & $3 \cdot 8$ & -.2 & $-2 \cdot 3$ & $-1 \cdot 3$ \\
\hline-.3 & 3.7 & -.2 & $-2 \cdot 1$ & -.3 \\
\hline .9 & 3.6 & -.2 & -1.4 & 1.0 \\
\hline .4 & 3.9 & -.2 & -.6 & .5 \\
\hline $1 \cdot 0$ & 4.6 & -.2 & $\cdot 1$ & 1.1 \\
\hline $1 \cdot 1$ & 6.2 & -.2 & $1 \cdot 6$ & 1.1 \\
\hline .8 & $8 \cdot 1$ & -.2 & 2.8 & .8 \\
\hline
\end{tabular}

\begin{tabular}{|c|c|c|c|c|}
\hline $\begin{array}{c}\text { ENERGY } \\
\text { KEV }\end{array}$ & TOTAL & $\begin{array}{l}\text { COH } \\
\text { SCATT. }\end{array}$ & $\begin{array}{l}\text { INCOH. } \\
\text { SCATT. }\end{array}$ & $\begin{array}{r}\text { PHOTO } \\
\text { EFFECT }\end{array}$ \\
\hline 1 & $-23 \cdot 4$ & -.6 & -3.9 & -23.5 \\
\hline 1.5 & -25.9 & -.8 & -.7 & -26.0 \\
\hline 2 & $-23 \cdot 5$ & -.9 & .6 & -23.6 \\
\hline 3 & -16.1 & $-1 \cdot 3$ & .9 & $-16 \cdot 2$ \\
\hline 4 & $-12 \cdot 0$ & -1.4 & .5 & -12.1 \\
\hline 5 & -9.2 & -1.5 & . 1 & -9.4 \\
\hline 6 & -7.4 & -1.7 & $\cdot 0$ & $-7 \cdot 5$ \\
\hline 8 & -5.6 & -1.9 & $\cdot 3$ & -5.6 \\
\hline 10 & -3.7 & $-2 \cdot 2$ & .6 & -3.7 \\
\hline 15 & -1.5 & -3.1 & .5 & $-1 \cdot 5$ \\
\hline 20 & $-1 \cdot 2$ & -1.8 & .0 & -1.2 \\
\hline 30 & -1.0 & -1.9 & -.1 & -.9 \\
\hline 40 & -.3 & -3.0 & .1 & .0 \\
\hline 50 & $-1 \cdot 2$ & -3.7 & .2 & -8.1 \\
\hline 60 & $-1 \cdot 2$ & -4.1 & .1 & -1.1 \\
\hline 80 & -.7 & -3.4 & -.3 & -.06 \\
\hline 100 & -.0 & -3.6 & .4 & .1 \\
\hline 150 & .8 & $-3 \cdot 3$ & -.1 & 1.2 \\
\hline 200 & .8 & -2.7 & -.1 & 2.3 \\
\hline 300 & .3 & -1.6 & -.0 & .7 \\
\hline
\end{tabular}

ENERGY

KEV
400
500
600
800
1000
1500
2000
3000
4000
5000
6000
8000
10000
15000
20000
30000
40000
50000
60000
80000
100000

\begin{tabular}{|c|c|c|c|c|}
\hline TOTAL & $\begin{array}{r}\text { COH } \\
\text { SCATT. }\end{array}$ & $\begin{array}{l}\text { INCOH. } \\
\text { SCAT: }\end{array}$ & $\begin{array}{l}\text { PHOTO } \\
\text { EFFECT }\end{array}$ & $\begin{array}{r}\text { PAIR } \\
\text { PROD. }\end{array}$ \\
\hline-.0 & -.7 & .0 & .1 & \\
\hline-.1 & .0 & .0 & -.2 & \\
\hline .0 & .8 & .1 & -.2 & \\
\hline$\cdot 1$ & $1 \cdot 8$ & .1 & -.1 & \\
\hline .0 & 2.8 & .0 & -.5 & \\
\hline-.3 & $2 \cdot 0$ & -.0 & $-3 \cdot 8$ & -2.6 \\
\hline .1 & 1.7 & -.0 & -.7 & 1.4 \\
\hline-.5 & 1.3 & -.1 & -1.7 & -1.7 \\
\hline-.6 & $1 \cdot 1$ & -.2 & $-2 \cdot 5$ & -1.2 \\
\hline-.4 & $1 \cdot 0$ & -.2 & $-1 \cdot 2$ & -.6 \\
\hline 1.6 & .9 & -.1 & -1.5 & 3.2 \\
\hline 2.8 & . 8 & -.1 & -.6 & 4.6 \\
\hline 1.5 & .7 & -.2 & 1.3 & 2.2 \\
\hline 1.4 & .6 & -.2 & $-1 \cdot 3$ & 1.7 \\
\hline-.9 & .6 & -.2 & -2.0 & -1.1 \\
\hline-.9 & .5 & -.2 & -1.9 & -.9 \\
\hline$\cdot 2$ & .4 & -.2 & -1.2 & $\cdot 2$ \\
\hline-.2 & .7 & -.2 & -.5 & -.2 \\
\hline .5 & $1 \cdot 3$ & -.2 & .3 & .5 \\
\hline .7 & 2.8 & -.2 & 1.7 & .8 \\
\hline .4 & 4.7 & -.2 & 3.0 & .4 \\
\hline
\end{tabular}

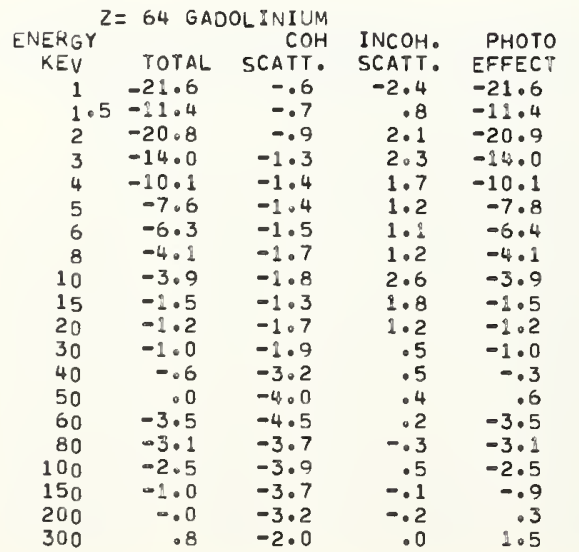

\begin{tabular}{|c|c|c|c|c|c|}
\hline $\begin{array}{c}\text { ENERGY } \\
\text { KEV }\end{array}$ & TOTAL & $\begin{array}{l}\text { COH } \\
\text { SCAPT. }\end{array}$ & $\begin{array}{l}\text { INCOH. } \\
\text { SCATT. }\end{array}$ & $\begin{array}{l}\text { PHOTO } \\
\text { EFFECT }\end{array}$ & $\begin{array}{l}\text { PAIR } \\
\text { PROD. }\end{array}$ \\
\hline 400 & .7 & -1.1 & $=0$ & 1.9 & \\
\hline 500 & .6 & -.5 & -.0 & 1.9 & \\
\hline 600 & .5 & .3 & -.0 & $1 \cdot 8$ & \\
\hline 800 & .3 & $1 \cdot 3$ & .1 & 1.0 & \\
\hline 1000 & -.0 & 2.2 & .0 & -.8 & \\
\hline 1500 & -.4 & 1.5 & -.0 & -4.1 & -2.7 \\
\hline 2000 & .0 & 1.1 & -.1 & -1.1 & 1.6 \\
\hline 3000 & -.6 & .7 & -.1 & -2.0 & -2.2 \\
\hline 4000 & -.9 & .5 & -.2 & $-2 \cdot 8$ & -2.0 \\
\hline 5000 & -.8 & .4 & -.2 & $-1 \cdot 5$ & -1.5 \\
\hline 6000 & $1 \cdot 1$ & .3 & -.2 & -1.9 & $2 \cdot 3$ \\
\hline 8000 & 2.3 & .2 & -.2 & -.9 & 3.8 \\
\hline 10000 & 2.2 & .2 & -.2 & .9 & 1.8 \\
\hline 15000 & 1.5 & .1 & -.2 & -1.7 & 2.0 \\
\hline 20000 & -.6 & .1 & -.2 & $-2 \cdot 5$ & -.7 \\
\hline 30000 & -.7 & -.0 & -.2 & -2.4 & -.7 \\
\hline 40000 & .3 & -.2 & -.2 & -1.7 & .4 \\
\hline 50000 & -.0 & .1 & -.2 & -.9 & -.0 \\
\hline 60000 & .8 & .8 & -.2 & -.2 & .8 \\
\hline 80000 & 2.0 & $2 \cdot 3$ & -.2 & $1 \cdot 3$ & 1.1 \\
\hline 00000 & .5 & 4.1 & -.2 & 2.5 & .5 \\
\hline
\end{tabular}


ENERGY $Z=65$ TERBIUM

$\begin{array}{ccccc}\begin{array}{c}\text { NERGY } \\ \text { KEV }\end{array} & \text { TOTAL } & \text { SCATT. } & \begin{array}{r}\text { INCOH. } \\ \text { SCATT. }\end{array} & \begin{array}{r}\text { PHOTO } \\ \text { EFFECT }\end{array} \\ 1 & -23.9 & -.6 & -3.5 & -24.0 \\ 1.5 & -10.4 & -.7 & .3 & -10.4 \\ 2 & -24.2 & -.8 & 2.0 & -24.2 \\ 3 & -16.3 & -1.2 & 2.7 & -16.4 \\ 4 & -12.1 & -1.4 & 2.2 & -12.2 \\ 5 & -9.2 & -1.5 & 1.7 & -9.4 \\ 6 & -7.3 & -1.6 & 1.4 & -7.4 \\ 8 & -4.5 & -1.8 & 1.4 & -4.5 \\ 10 & -5.5 & -2.0 & 2.1 & -5.6 \\ 15 & -2.4 & -1.7 & 1.6 & -2.4 \\ 20 & -1.9 & -1.8 & 2.2 & -1.9 \\ 30 & -1.6 & -1.9 & .5 & -1.6 \\ 40 & -1.1 & -3.4 & .5 & -.9 \\ 50 & -. .3 & -4.5 & .4 & .2 \\ 60 & -.4 & -5.1 & .3 & -.2 \\ 80 & .5 & -4.2 & -.3 & .7 \\ 100 & .9 & -4.5 & .4 & 1.2 \\ 150 & 1.1 & -4.5 & -.1 & 1.6 \\ 200 & .9 & -3.9 & -.2 & 1.6 \\ 300 & .4 & -2.9 & .0 & 1.0\end{array}$

ENERGY $=66$ DYSPROSIUM

\begin{tabular}{crrrr} 
ENERGY & \multicolumn{1}{c}{ COH } & INCOH. & PHOTO \\
KEV & TOTAL & SCATT. & SCATT. & EFFECT \\
1 & -23.7 & -.6 & -4.0 & -23.8 \\
1.5 & -16.3 & -.7 & -.6 & -16.3 \\
2 & -23.4 & -.8 & .9 & -23.4 \\
3 & -16.6 & -1.2 & 1.2 & -16.7 \\
4 & -12.3 & -1.3 & .9 & -12.4 \\
5 & -9.5 & -1.5 & .5 & -9.6 \\
6 & -7.4 & -1.6 & .5 & -7.5 \\
8 & -3.5 & -1.8 & .9 & -3.6 \\
10 & -5.7 & -2.0 & 2.1 & -5.8 \\
15 & -2.2 & -1.8 & .7 & -2.2 \\
20 & -1.4 & -1.8 & .5 & -1.3 \\
30 & -.7 & -1.9 & .3 & -.6 \\
40 & -.4 & -3.6 & .3 & -.1 \\
50 & -.3 & -4.8 & .3 & .3 \\
60 & -.3 & -5.6 & .2 & -.2 \\
80 & .5 & -4.7 & -.4 & .8 \\
100 & .8 & -5.0 & .4 & 1.1 \\
150 &. .9 & -5.1 & -.2 & 1.4 \\
200 & .6 & -4.6 & -.2 & 1.3 \\
300 & .1 & -3.5 & -.1 & .1
\end{tabular}

$\begin{array}{ccccc}\text { ENERGY }^{2=}=67 \text { HOLMIUM } \\ \text { KEV } & \text { TOTAL } & \text { SCATT. } & \begin{array}{r}\text { INCOH. } \\ \text { SCATT. }\end{array} & \begin{array}{r}\text { PHOTO } \\ \text { EFFECT }\end{array} \\ 1 & -24.4 & -.7 & -3.8 & -24.4 \\ 1.5 & -16.8 & -1.0 & -.6 & -16.8 \\ 2 & -24.6 & -1.3 & .9 & -24.6 \\ 3 & -16.9 & -1.9 & 1.2 & -17.0 \\ 4 & -11.8 & -2.2 & .8 & -11.9 \\ 5 & -8.5 & -2.3 & .5 & -8.6 \\ 6 & -6.1 & -2.2 & .5 & -6.2 \\ 8 & -3.0 & -2.4 & .9 & -3.0 \\ 10 & -7.8 & -2.5 & .4 & -7.8 \\ 15 & -2.1 & -2.4 & .3 & -2.1 \\ 20 & -1.1 & -2.0 & .1 & -1.1 \\ 30 & -1.5 & -2.1 & .1 & -1.6 \\ 40 & -2.5 & -4.0 & .3 & -2.4 \\ 50 & -3.2 & -5.3 & .3 & -3.1 \\ 60 & -2.8 & -6.2 & .2 & -2.7 \\ 80 & -1.2 & -5.3 & -.3 & -1.2 \\ 100 & -.4 & -5.6 & .4 & -.2 \\ 150 & .6 & -5.8 & -.1 & 1.1 \\ 200 & .9 & -5.3 & -.2 & 1.6 \\ 300 & .7 & -4.3 & -.0 & 1.7\end{array}$

\begin{tabular}{|c|c|c|c|c|c|}
\hline $\begin{array}{c}\text { ENERGY } \\
\text { KEV }\end{array}$ & TOTAL & $\begin{array}{r}\mathrm{COH} \\
\text { SCATT. }\end{array}$ & $\begin{array}{l}\text { INCOH. } \\
\text { SCATT. }\end{array}$ & $\begin{array}{r}\text { PHOTO } \\
\text { EFFECT }\end{array}$ & $\begin{array}{l}\text { PAIR } \\
\text { PROD. }\end{array}$ \\
\hline 400 & .1 & -2.0 & -.0 & .5 & \\
\hline 500 & .0 & $-1 \cdot 3$ & -.0 & .3 & \\
\hline 600 & .0 & -.6 & .0 & .2 & \\
\hline 800 & - 1 & .4 & .1 & -.0 & \\
\hline 1000 & -.1 & $1 \cdot 3$ & .0 & -1.0 & \\
\hline 1500 & -.4 & .6 & -.0 & $-4 \cdot 2$ & -3.5 \\
\hline 2000 & .2 & .2 & -.1 & -1.2 & 3.4 \\
\hline 3000 & -.5 & -.01 & -.1 & $-2 \cdot 1$ & -1.7 \\
\hline 4000 & -1.0 & -.3 & -.2 & $-2 \cdot 8$ & $-2 \cdot 3$ \\
\hline 5000 & $-1 \cdot 1$ & -.5 & -.2 & $-1 \cdot 5$ & $-2 \cdot 2$ \\
\hline 6000 & .6 & -.5 & -.2 & $-1 \cdot 9$ & 1.3 \\
\hline 8000 & 1.9 & -.6 & -.2 & -.9 & 3.2 \\
\hline 10000 & 1.0 & -.7 & -.2 & $1 \cdot 0$ & 1.5 \\
\hline 15000 & 2.0 & -.8 & -.2 & $-1 \cdot 7$ & 2.5 \\
\hline 20000 & $=0$ & $=.8$ & -.2 & $-2 \cdot 4$ & -.0 \\
\hline 30000 & -.4 & -.9 & -.2 & $-2 \cdot 3$ & -.5 \\
\hline 40000 & .6 & $-1 \cdot 0$ & -.2 & -1.7 & .6 \\
\hline 50000 & .4 & -.8 & -.2 & -.9 & .4 \\
\hline 60000 & 1.4 & -.1 & -.2 & -.1 & 1.4 \\
\hline 80000 & 1.5 & 1.3 & -.2 & 1.3 & 1.6 \\
\hline 100000 & .5 & 3.1 & -.2 & 2.6 & .5 \\
\hline
\end{tabular}

ENERGY

KEV

400

600

800
1000

1500

2000
3000

4000

5000

6000

8000
10000

15000

20000

30000

40000
50000

50000
60000

80000

100000

$\begin{array}{rrrrrr}\text { KEV } & \text { TOTAL } & \text { SCATT. } & \text { SCATT. } & \text { EFFECT } & \text { PROD. } \\ 400 & .4 & -3.5 & -.0 & 1.4 & \\ 500 & .3 & -2.8 & .0 & 1.2 & \\ 600 & .2 & -2.1 & -.0 & 1.0 & \\ 800 & .0 & -1.2 & .1 & -.2 & \\ 1000 & -.4 & -.3 & .0 & -2.5 & \\ 1500 & -.6 & -1.0 & -.0 & -5.4 & -3.9 \\ 2000 & -.1 & -1.4 & -.1 & -2.4 & 1.6 \\ 3000 & -.7 & -1.8 & -.2 & -3.0 & -2.1 \\ 4000 & -1.0 & -2.0 & -.2 & -3.7 & -2.0 \\ 5000 & -.9 & -2.1 & -.2 & -2.3 & -1.6 \\ 6000 & .9 & -2.2 & -.2 & -2.7 & 2.0 \\ 8000 & 2.1 & -2.3 & -.2 & -1.8 & 3.5 \\ 10000 & 1.0 & -2.3 & -.2 & .1 & 1.5 \\ 15000 & 1.5 & -2.4 & -.2 & -2.7 & 1.9 \\ 20000 & -.4 & -2.4 & -.2 & -3.5 & -.4 \\ 30000 & -.5 & -2.5 & -.2 & -3.4 & -.5 \\ 40000 & .5 & -2.6 & -.2 & -2.8 & .5 \\ 50000 & .1 & -2.4 & -.2 & -2.1 & .1 \\ 60000 & .9 & -1.8 & -.2 & -1.3 & 1.0 \\ 80000 & 1.3 & -. .4 & -.2 & .0 & 1.3 \\ 100000 & .8 & 1.3 & -.2 & 1.3 & .2\end{array}$

ENERGY COH INCOH. PHOTO PAIR KEV TOTAL SCATT. SCATT. EFFECT PROD.

$\begin{array}{rrrrrr}400 & .0 & -4.5 & .0 & .6 & \\ 500 & -.1 & -3.8 & .1 & .3 & \\ 600 & -.0 & -3.1 & .1 & .2 & \\ 800 & .0 & -2.3 & .1 & .0 & \\ 1000 & -.1 & -1.1 & .0 & -.0 & \\ 1500 & -.5 & -1.9 & -.0 & -3.7 & -3.9 \\ 2000 & .1 & -2.2 & -.1 & -.7 & 2.8 \\ 3000 & -.5 & -2.6 & -.1 & -1.3 & -1.5 \\ 4000 & -. .9 & -2.8 & -.3 & -2.0 & -2.0 \\ 5000 & -1.0 & -2.9 & -.2 & -.6 & -1.9 \\ 6000 & . .7 & -3.0 & -.2 & -1.0 & 1.6 \\ 8000 & 1.9 & -3.1 & -.2 & -.0 & 3.1 \\ 10000 & .9 & -3.2 & -.2 & 1.9 & 1.4 \\ 15000 & 1.9 & -3.2 & -.2 & -1.0 & 2.4 \\ 20000 & .1 & -3.3 & -.2 & -1.8 & .1 \\ 30000 & -.3 & -3.3 & -.2 & -1.8 & -.3 \\ 40000 & .4 & -3.4 & -.2 & -1.2 & .5 \\ 50000 & .0 & -3.3 & -.2 & -.5 & .0 \\ 60000 & .9 & -2.7 & -.2 & .3 & .9 \\ 80000 & 1.2 & -1.2 & -.2 & 1.7 & 1.3 \\ 100000 & .8 & .4 & -.2 & 2.9 & .8\end{array}$




$\begin{array}{ccrrr}\begin{array}{c}Z \\ \text { ENERGY }\end{array} & 69 \text { THULIUM } \\ \text { KEV } & \text { TOTAL } & \text { SCATT, } & \begin{array}{r}\text { INCOH. } \\ \text { SCATT, }\end{array} & \begin{array}{r}\text { PHOTO } \\ \text { EFFECT }\end{array} \\ 1 & -25.6 & -.5 & -3.4 & -25.6 \\ 1.5 & -19.6 & -.6 & -.7 & -19.6 \\ 2 & -21.5 & -.7 & .7 & -21.5 \\ 3 & -17.4 & -1.0 & 1.0 & -17.4 \\ 4 & -11.6 & -1.3 & .6 & -21.7 \\ 5 & -7.8 & -1.4 & .3 & -7.9 \\ 6 & -5.1 & -1.5 & .2 & -5.2 \\ 8 & -2.2 & -1.6 & .5 & -2.2 \\ 10 & -4.9 & -1.8 & -1.1 & -5.0 \\ 15 & -2.3 & -.9 & -.9 & -2.3 \\ 20 & -1.3 & -1.7 & -.8 & -1.3 \\ 30 & -1.7 & -2.1 & -.2 & -2.7 \\ 40 & -2.3 & -4.5 & .1 & -2.2 \\ 50 & -2.7 & -6.3 & .2 & -2.3 \\ 60 & -2.4 & -7.6 & .1 & -2.3 \\ 80 & -.3 & -6.6 & -.4 & -.0 \\ 100 & .6 & -7.1 & .4 & 1.0 \\ 150 & 1.2 & -7.5 & -.2 & 1.9 \\ 200 & 1.1 & -7.1 & -.2 & 2.0 \\ 300 & .4 & -6.3 & -.1 & 1.4\end{array}$

\begin{tabular}{|c|c|c|c|c|}
\hline $\begin{array}{c}\text { ENERGY } \\
\text { KEY }\end{array}$ & & $\mathrm{COH}$ & INCOH. & PHOTO \\
\hline $\begin{array}{r}\text { KEV } \\
1\end{array}$ & $\begin{array}{l}\text { TOTAL } \\
-20.8\end{array}$ & $\begin{array}{c}\text { SCATT. } \\
=.5\end{array}$ & $\begin{array}{c}\text { SCATT. } \\
-3.1\end{array}$ & $\begin{array}{l}\text { EFFECT } \\
=20.8\end{array}$ \\
\hline 1.5 & -9.0 & -.6 & -.7 & $-9 \cdot 1$ \\
\hline 2 & -20.6 & -.7 & .6 & -20.7 \\
\hline 3 & $-17 \cdot 6$ & $-1 \cdot 0$ & .9 & -17.7 \\
\hline 4 & -11.7 & $-1 \cdot 2$ & .5 & $-11 \cdot 8$ \\
\hline 5 & -7.8 & -1.4 & .1 & -7.8 \\
\hline 6 & $-5 \cdot 0$ & -1.4 & .0 & -5.1 \\
\hline 8 & -1.7 & -1.6 & .3 & -1.7 \\
\hline 10 & 1.2 & -1.9 & .5 & 1.2 \\
\hline 15 & -.2 & $-1 \cdot 3$ & $\cdot 5$ & -.2 \\
\hline 20 & -.7 & -1.7 & .1 & -.7 \\
\hline 30 & -1.4 & $-2 \cdot 2$ & -.1 & -1.4 \\
\hline 40 & -1.6 & -4.8 & .1 & -1.3 \\
\hline 50 & -1.6 & -6.9 & .2 & $-1 \cdot 0$ \\
\hline 60 & -1.7 & $-8 \cdot 3$ & $\cdot 1$ & -.8 \\
\hline 80 & -1.9 & -7.2 & -.5 & -1.7 \\
\hline 100 & $-3 \cdot 3$ & -7.8 & .4 & -3.1 \\
\hline 150 & $-4 \cdot 1$ & $-8 \cdot 3$ & -.2 & $-4 \cdot 2$ \\
\hline 200 & -3.8 & $-8 \cdot 0$ & -.3 & -4.1 \\
\hline 300 & -2.5 & $-7 \cdot 2$ & -.1 & -3.0 \\
\hline
\end{tabular}

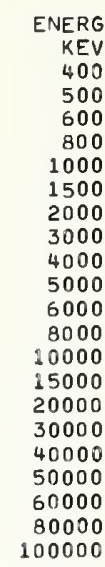

ENERG

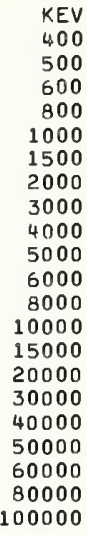

ENERGY

KEV

400
500
600
800
1000
1500
2000
3000
4000
5000
6000
8000
10000
15000
2000
30000
40000
5000
60000
8000
100000

E

$\begin{array}{ccccc}\text { ENERGY } & \text { T2 HAFNIUM } \\ \text { KEV } & \text { TOTAL } & \text { SCATT. } & \begin{array}{r}\text { INCOH. } \\ \text { SCATT. }\end{array} & \begin{array}{r}\text { PHOTO } \\ \text { EFFECT }\end{array} \\ 1 & -20.3 & -.5 & 1.5 & -20.3 \\ 1.5 & -9.1 & -.6 & 1.5 & -9.1 \\ 2 & -7.8 & -.7 & 2.0 & -7.8 \\ 3 & -17.9 & -1.0 & 1.7 & -18.0 \\ 4 & -12.4 & -1.3 & 1.1 & -12.5 \\ 5 & -8.2 & -1.4 & .7 & -8.3 \\ 6 & -5.0 & -1.4 & .5 & -5.1 \\ 8 & -1.8 & -1.5 & .5 & -1.8 \\ 10 & 8.7 & -1.8 & -1.3 & 8.9 \\ 15 & -.9 & -1.8 & -1.6 & 1.0 \\ 20 & -1.2 & -1.7 & -1.4 & -1.2 \\ 30 & -2.7 & -2.2 & -.4 & -2.7 \\ 40 & -2.1 & -5.2 & .0 & -1.9 \\ 50 & -. .9 & -7.6 & .1 & -.1 \\ 60 & . .6 & -9.2 & .1 & 2.1 \\ 80 & -1.4 & -8.2 & -.5 & -1.2 \\ 100 & -.9 & -8.8 & .4 & -.6 \\ 150 & . .4 & -9.5 & -.3 & 1.1 \\ 200 & 1.3 & -9.3 & -.3 & 2.4 \\ 300 & 1.7 & -8.6 & -.2 & 3.7\end{array}$
$\begin{array}{rrrr}\text { COH INCOH. PHOTO } & \text { PAIR } \\ \text { TOTAL SCATT. SCATT. EFFECT PROD. }\end{array}$

$\begin{array}{rrrrr}.0 & -5.5 & -.0 & .8 & \\ -.1 & -4.9 & -.0 & .4 & \\ -.1 & -4.1 & .0 & .2 & \\ -.1 & -3.3 & .1 & -.1 & \\ -.1 & -2.4 & .1 & -.8 & \\ -.5 & -3.1 & .0 & -3.8 & -3.5 \\ .2 & -3.4 & -.0 & -.8 & 2.9 \\ -.5 & -3.8 & -.1 & -1.3 & -1.6 \\ -.9 & -4.0 & -.2 & -1.9 & -2.1 \\ -1.0 & -4.1 & -.1 & -.5 & -2.0 \\ .0 & -4.2 & -.1 & -.9 & 1.3 \\ 1.7 & -4.3 & -.1 & .1 & 2.7 \\ .6 & -4.4 & -.1 & 2.0 & .9 \\ 1.2 & -4.4 & -.2 & -.9 & 1.5 \\ -.7 & -4.4 & -.2 & -1.7 & -.8 \\ -.09 & -4.5 & -.2 & -1.8 & -.9 \\ .3 & -4.6 & -.2 & -1.1 & .3 \\ .2 & -4.5 & -.2 & -.4 & .2 \\ 1.2 & -3.9 & -.2 & .4 & 1.3 \\ 1.7 & -2.5 & -.2 & 1.8 & 1.8 \\ 1.2 & -.9 & -.2 & 3.0 & 1.2\end{array}$

\begin{tabular}{|c|c|c|c|c|}
\hline TOTAL & $\begin{array}{r}\text { COH } \\
\text { SCATT. }\end{array}$ & $\begin{array}{l}\text { INCOH. } \\
\text { SCATT. }\end{array}$ & $\begin{array}{r}\text { PHOTO } \\
\text { EFFECT }\end{array}$ & $\begin{array}{r}\text { PA I } \\
\text { PROD }\end{array}$ \\
\hline 1.04 & -6.4 & -.1 & -1.9 & \\
\hline-.7 & -5.8 & -.0 & -.9 & \\
\hline-.2 & -5.1 & .0 & .0 & \\
\hline .0 & $-4 \cdot 2$ & .0 & .8 & \\
\hline-.0 & -3.4 & .1 & .1 & \\
\hline-.04 & $-4 \cdot 1$ & .0 & $-2 \cdot 9$ & -2.8 \\
\hline .2 & $-4 \cdot 5$ & -.0 & $\cdot 1$ & \\
\hline-.5 & $-4 \cdot 9$ & -.1 & -.5 & -1.4 \\
\hline-.7 & $-5 \cdot 1$ & -.2 & $-1 \cdot 1$ & $-1 \cdot 5$ \\
\hline-.6 & $-5 \cdot 2$ & -.1 & .2 & -1.2 \\
\hline 1.2 & $-5 \cdot 2$ & -.1 & -.2 & 2 \\
\hline 2.2 & $-5 \cdot 3$ & -.1 & .8 & 3. \\
\hline .9 & -5.4 & -.2 & $2 \cdot 8$ & $1 \cdot 3$ \\
\hline .9 & $-5 \cdot 5$ & -.2 & -.2 & 1.2 \\
\hline$-1 \cdot 0$ & $-5 \cdot 5$ & -.2 & $-1 \cdot 0$ & $-1 \cdot 2$ \\
\hline-.8 & -5.6 & -.2 & $-1 \cdot 1$ & - . \\
\hline .5 & $-5 \cdot 7$ & -.2 & -.4 & \\
\hline .4 & $-5 \cdot 5$ & -.2 & .3 & \\
\hline 1.3 & $-5 \cdot 0$ & -.2 & $1 \cdot 1$ & 1.4 \\
\hline 1.6 & -3.6 & -.2 & $2 \cdot 5$ & 1.7 \\
\hline 1.0 & $-2 \cdot 0$ & -.2 & 3.7 & 1. \\
\hline
\end{tabular}

COH INCOH. PHOTO PAIR TOTAL SCATT. SCATT. EFFECT PROD.

$\begin{array}{rrrrr}-.5 & -7.1 & -.0 & .0 & \\ -.3 & -6.5 & -.0 & .0 & \\ -.0 & -5.8 & .0 & .2 & \\ -.1 & -5.1 & .0 & .2 & \\ -.1 & -4.1 & .1 & -.4 & \\ -.5 & -4.8 & .0 & -3.5 & -3.3 \\ .1 & -5.2 & -.0 & -.6 & 2.7 \\ -.5 & -5.6 & -.1 & -1.1 & -1.3 \\ -.8 & -5.8 & -.2 & -1.7 & -1.7 \\ -.8 & -5.9 & -.2 & -.3 & -1.6 \\ .9 & -6.0 & -.1 & -.7 & 1.8 \\ 1.9 & -6.1 & -.1 & .3 & 3.0 \\ .8 & -6.1 & -.2 & 2.3 & 1.2 \\ 1.4 & -6.2 & -.2 & -.6 & 1.8 \\ -.3 & -6.2 & -.2 & -1.5 & -.3 \\ -.4 & -6.3 & -.2 & -1.5 & -.4 \\ .5 & -6.4 & -.2 & -.9 & .5 \\ .1 & -6.3 & -.2 & -.1 & .1 \\ .9 & -5.7 & -.2 & .6 & 1.0 \\ 1.4 & -4.4 & -.2 & 2.0 & 1.4 \\ 1.0 & -2.8 & -.2 & 3.3 & 1.1\end{array}$

ENERGY COH INCOH. PHOTO PAIR KEV TOTAL SCATT. SCATT. EFFECT PROD.

$\begin{array}{rrrrrr}400 & 1.4 & -7.8 & -.1 & 3.9 & \\ 500 & 1.0 & -7.2 & -.0 & 3.5 & \\ 600 & -5 & -6.5 & -.0 & 2.7 & \\ 800 & -.2 & -5.8 & .0 & .1 & \\ 1000 & -.8 & -4.7 & .0 & -3.8 & \\ 1500 & -. .9 & -5.5 & -.0 & -6.5 & -2.3 \\ 2000 & -. .4 & -5.8 & -.0 & -3.4 & -.8 \\ 3000 & -.7 & -6.2 & -.1 & -3.6 & -1.9 \\ 4000 & -. .4 & -6.4 & -.2 & -4.1 & -.4 \\ 5000 & .2 & -6.5 & -.2 & -2.7 & .6 \\ 6000 & 2.2 & -6.6 & -.2 & -3.1 & 4.3 \\ 8000 & 3.2 & -6.7 & -.2 & -2.0 & 5.0 \\ 10000 & 1.4 & -6.7 & -.2 & -.1 & 1.9 \\ 15000 & .3 & -6.8 & -.2 & -3.0 & .4 \\ 20000 & -1.4 & -6.8 & -.2 & -3.9 & -1.6 \\ 30000 & -.1 & -6.9 & -.2 & -4.0 & -.1 \\ 40000 & 1.1 & -7.0 & -.2 & -3.4 & 1.2 \\ 50000 & .3 & -6.9 & -.2 & -2.7 & .4 \\ 60000 & .8 & -6.3 & -.2 & -2.0 & .8 \\ 80000 & .9 & -5.0 & -.2 & -.06 & .9 \\ 100000 & 1.1 & -3.5 & -.2 & .2 & 1.1\end{array}$




\begin{tabular}{|c|c|c|c|c|}
\hline $\begin{array}{c}\text { ENERGY } \\
\text { KEY }\end{array}$ & TOTAL & SCATT. & $\begin{array}{l}\text { INCOH. } \\
\text { SCATT. }\end{array}$ & $\begin{array}{l}\text { PHOTO } \\
\text { EFFECT }\end{array}$ \\
\hline 1 & $-23 \cdot 4$ & -.5 & $1 \cdot 2$ & -23.4 \\
\hline 1.5 & & -.6 & .7 & -8.5 \\
\hline 2 & -5.0 & -.7 & 1.4 & -5.0 \\
\hline 3 & $-15 \cdot 9$ & $-1 \cdot 0$ & 1.9 & -15.9 \\
\hline 4 & -9.7 & $-1 \cdot 3$ & 1.5 & -9.8 \\
\hline 5 & $-6 \cdot 3$ & -1.4 & 1.0 & -6.3 \\
\hline 6 & -4.1 & -1.4 & .7 & -4.1 \\
\hline 8 & $-1,1$ & -1.5 & .5 & -1.1 \\
\hline 10 & 4.1 & -1.7 & -1.2 & 4.2 \\
\hline 15 & -.1 & -1.6 & -1.4 & -.1 \\
\hline 20 & .7 & -1.6 & -1.1 & .8 \\
\hline 30 & -.3 & -2.5 & -.3 & -.2 \\
\hline 40 & -1.4 & -5.9 & .1 & -1.0 \\
\hline 50 & -2.0 & $-8 \cdot 5$ & $\cdot 2$ & $-1 \cdot 3$ \\
\hline 60 & $-2 \cdot 1$ & -10.4 & .1 & -1.0 \\
\hline 80 & .9 & -9.4 & -.5 & 1.4 \\
\hline 100 & .9 & -10.2 & .4 & 1.4 \\
\hline 150 & .9 & -11.0 & -.3 & 1.8 \\
\hline 200 & 1.1 & -10.9 & -.3 & 2.2 \\
\hline 300 & 1.2 & $-10 \cdot 2$ & -.2 & 3.0 \\
\hline
\end{tabular}

\begin{tabular}{rrrrrr} 
ENERGY & \multicolumn{1}{c}{ COH } & INCOH. & PHOTO & PAIR \\
KEV & TOTAL & SCATT. & SCATT. & EFFECT & PROD. \\
400 & 1.2 & -9.5 & -.1 & 3.5 & \\
500 & 1.1 & -9.0 & -.0 & 3.9 & \\
600 & 1.0 & -8.3 & -.0 & 4.2 & \\
800 & .6 & -7.5 & -.0 & 3.7 & \\
1000 & .2 & -6.7 & -.0 & 2.0 & \\
1500 & -.3 & -7.4 & -.0 & -1.0 & -3.2 \\
2000 & .2 & -7.7 & -.0 & 2.1 & 1.3 \\
3000 & -.5 & -8.1 & -.1 & 1.9 & -1.8 \\
4000 & -.8 & -8.3 & -.2 & 1.3 & -1.7 \\
5000 & -.7 & -8.4 & -.2 & 2.9 & -1.4 \\
6000 & 1.0 & -8.5 & -.2 & 2.5 & 1.9 \\
8000 & 1.9 & -8.6 & -.2 & 3.6 & 2.9 \\
10000 & .4 & -8.7 & -.2 & 5.7 & .6 \\
15000 & .4 & -8.8 & -.2 & 2.6 & .5 \\
20000 & -1.2 & -8.8 & -.2 & 1.7 & -1.3 \\
30000 & -.4 & -8.8 & -.2 & 1.6 & -.4 \\
40000 & .9 & -9.0 & -.2 & 2.2 & .9 \\
50000 & .6 & -8.8 & -.2 & 3.0 & .6 \\
60000 & 1.4 & -8.3 & -.2 & 3.7 & 1.4 \\
80000 & 1.7 & -7.0 & -.2 & 5.2 & 1.7 \\
100000 & 1.4 & -5.5 & -.2 & 6.4 & 1.4
\end{tabular}

$\begin{array}{ccccr}\begin{array}{c}\text { ENERGY } \\ \text { EN }\end{array} & 74 \text { TUNGSTEN } \\ \text { KEV } & \text { TOTAL } & \text { SCATT. } & \begin{array}{r}\text { INCOH. } \\ \text { SCATT. }\end{array} & \begin{array}{r}\text { PHOTO } \\ \text { EFFECT }\end{array} \\ 1 & -20.2 & -.5 & 4.2 & -20.2 \\ 1.5 & -9.8 & -.6 & 1.9 & -9.8 \\ 2 & 1.4 & -.7 & 1.5 & 1.4 \\ 3 & -12.8 & -1.0 & 1.1 & -12.9 \\ 4 & -7.9 & -1.2 & .7 & -8.0 \\ 5 & -5.5 & -1.4 & .4 & -5.5 \\ 6 & -4.2 & -1.4 & .3 & -4.2 \\ 8 & -2.9 & -1.5 & .4 & -3.0 \\ 10 & -2.9 & -1.7 & -.9 & -2.9 \\ 15 & -3.5 & -1.7 & -1.4 & -3.6 \\ 20 & -3.0 & -1.6 & -1.3 & -3.1 \\ 30 & -2.7 & -2.3 & -.4 & -2.7 \\ 40 & -2.2 & -5.5 & -.0 & -1.9 \\ 50 & -1.5 & -8.2 & .1 & -.3 \\ 60 & -1.0 & -9.9 & .1 & .3 \\ 80 & -.0 & -8.9 & -.5 & .3 \\ 100 & -.5 & -9.6 & .4 & -.2 \\ 150 & -.7 & -10.4 & -.3 & -.1 \\ 200 & -.2 & -10.3 & -.3 & .3 \\ 300 & .7 & -9.6 & -.2 & 2.0\end{array}$

\begin{tabular}{|c|c|c|c|c|c|}
\hline $\begin{array}{c}\text { ENERGY } \\
\text { KEV }\end{array}$ & TOTAL & $\begin{array}{l}\text { COH } \\
\text { SCATT. }\end{array}$ & $\begin{array}{l}\text { INCOH. } \\
\text { SCATT. }\end{array}$ & $\begin{array}{r}\text { PHOTO } \\
\text { EFFECT }\end{array}$ & $\begin{array}{l}\text { PA IR } \\
\text { PROD. }\end{array}$ \\
\hline 400 & 1.2 & -8.8 & -.1 & 3.3 & \\
\hline 500 & 1.4 & $-8 \cdot 3$ & -.0 & 4.4 & \\
\hline 600 & 1.5 & $-7 \cdot 5$ & -.0 & 5.4 & \\
\hline 800 & 1.2 & -6.8 & -.0 & $6 \cdot 1$ & \\
\hline 1000 & .9 & $-5 \cdot 9$ & .0 & $5 \cdot 3$ & \\
\hline 1500 & .1 & -6.6 & -.0 & $2 \cdot 4$ & -2.7 \\
\hline 2000 & .5 & -7.0 & -.1 & $5 \cdot 8$ & $2 \cdot 2$ \\
\hline 3000 & -.1 & $-7 \cdot 3$ & -.1 & $5 \cdot 8$ & -.9 \\
\hline 4000 & -.3 & -7.5 & -.2 & $5 \cdot 3$ & -.8 \\
\hline 5000 & -.3 & $-7 \cdot 6$ & -.2 & 6.9 & -.6 \\
\hline 6000 & 1.4 & -7.7 & -.2 & 6.5 & 2.6 \\
\hline 8000 & 2.2 & -7.8 & -.2 & 7.7 & 3.4 \\
\hline 10000 & .7 & -7.9 & -.2 & 9.9 & 1.0 \\
\hline 15000 & .8 & $-8 \cdot 0$ & -.2 & 6.6 & 1.0 \\
\hline 20000 & -.5 & $-8 \cdot 0$ & -.2 & 5.6 & -.6 \\
\hline 30000 & .2 & $-8 \cdot 1$ & -.2 & $5 \cdot 5$ & .3 \\
\hline 40000 & 1.2 & $-8 \cdot 2$ & -.2 & 6.1 & 1.3 \\
\hline 50000 & .6 & $-8 \cdot 1$ & -.2 & 6.9 & $\cdot 6$ \\
\hline 60000 & 1.2 & $-7 \cdot 5$ & -.2 & 7.7 & $1 \cdot 3$ \\
\hline 80000 & 1.4 & -6.2 & -.2 & 9.2 & 1.4 \\
\hline 100000 & 1.2 & -4.7 & -.2 & 10.5 & 1.2 \\
\hline
\end{tabular}

\begin{tabular}{ccccr} 
ENERGY & \multicolumn{5}{c}{75 RHENIUM } \\
ENG & TOTAL & SCATT. & $\begin{array}{r}\text { INCOH. } \\
\text { SCATT. }\end{array}$ & $\begin{array}{r}\text { PHOTO } \\
\text { EFFECT }\end{array}$ \\
1 & -23.3 & -.4 & 6.7 & -23.4 \\
1.5 & -11.1 & -.6 & 2.7 & -11.2 \\
2 & -2.8 & -.7 & 1.5 & -2.8 \\
3 & -15.0 & -1.1 & .6 & -15.0 \\
4 & -9.6 & -1.2 & .2 & -9.6 \\
5 & -6.9 & -1.3 & .0 & -6.9 \\
6 & -5.2 & -1.3 & .1 & -5.3 \\
8 & -3.4 & -1.4 & .3 & -3.5 \\
10 & -2.6 & -1.6 & .6 & -2.7 \\
15 & -3.3 & -1.6 & .3 & -3.3 \\
20 & -1.0 & -1.6 & -.0 & -1.0 \\
30 & -.9 & -2.3 & -.1 & -.8 \\
40 & -2.0 & -5.6 & .2 & -1.7 \\
50 & -3.1 & -8.3 & .2 & -2.5 \\
60 & -4.0 & -10.2 & .1 & -3.3 \\
80 & -.6 & -9.1 & -.5 & -.3 \\
100 & -2.5 & -9.8 & .4 & -2.3 \\
150 & -3.1 & -10.6 & -.3 & -2.8 \\
200 & -2.0 & -10.5 & -.3 & -1.6 \\
300 & -.0 & -9.9 & -.2 & 1.1
\end{tabular}

\begin{tabular}{|c|c|c|c|c|c|}
\hline $\begin{array}{c}\text { ENERGY } \\
\text { KEV }\end{array}$ & TOTAL & $\begin{array}{l}\mathrm{COH} \\
\text { SCATT. }\end{array}$ & $\begin{array}{l}\text { INCOH. } \\
\text { SCATT. }\end{array}$ & $\begin{array}{r}\text { РHOTO } \\
\text { EFFECT }\end{array}$ & $\begin{array}{l}\text { PAIR } \\
\text { PROD. }\end{array}$ \\
\hline 400 & .7 & -9.1 & -.1 & 2.4 & \\
\hline 500 & .7 & -8.6 & -.0 & $2 \cdot 7$ & \\
\hline 600 & .4 & $-7 \cdot 8$ & .0 & $2 \cdot 3$ & \\
\hline 800 & -.4 & -7.2 & .1 & -.6 & \\
\hline 1000 & $-1 \cdot 3$ & -6.1 & .0 & $-5 \cdot 4$ & \\
\hline 1500 & -1.2 & -6.8 & -.0 & $-7 \cdot 7$ & -3.3 \\
\hline 2000 & -.3 & -7.2 & -.1 & $-4 \cdot 6$ & 2.5 \\
\hline 3000 & -.6 & $-7 \cdot 6$ & -.1 & $-4 \cdot 4$ & $-1 \cdot 1$ \\
\hline 4000 & -.9 & $-7 \cdot 8$ & -.3 & $-4 \cdot 8$ & $-1 \cdot 4$ \\
\hline 5000 & -.8 & $-7 \cdot 9$ & -.2 & $-3 \cdot 3$ & $-1 \cdot 3$ \\
\hline 6000 & .8 & -7.9 & -.2 & -3.7 & $1 \cdot 8$ \\
\hline 8000 & 1.7 & $-8 \cdot 0$ & -.2 & $-2 \cdot 7$ & 2.7 \\
\hline 10000 & .4 & $-8 \cdot 1$ & -.2 & -.8 & .7 \\
\hline 15000 & 1.2 & $-8 \cdot 2$ & -.2 & -3.8 & 1.5 \\
\hline 20000 & .2 & -8.2 & -.2 & -4.7 & .3 \\
\hline 30000 & .7 & $-8 \cdot 3$ & -.2 & $-4 \cdot 7$ & .8 \\
\hline 40000 & 1.4 & $-8 \cdot 4$ & -.2 & $-4 \cdot 1$ & 1.6 \\
\hline 50000 & .8 & $-8 \cdot 3$ & -.2 & -3.4 & $\cdot 8$ \\
\hline 60000 & 1.4 & $-7 \cdot 8$ & -.2 & $-2 \cdot 6$ & 1.4 \\
\hline 80000 & 1.6 & $-6 \cdot 5$ & -.2 & $-1 \cdot 2$ & 1.7 \\
\hline 100000 & 1.4 & $-5 \cdot 0$ & -.2 & .0 & 1.5 \\
\hline
\end{tabular}

$\begin{array}{ccccc}\begin{array}{c}\text { ENERGY } \\ \text { ENE }\end{array} & 76 \text { OSMIUM } \\ \text { KEV } & \text { TOTAL } & \text { SCATT. } & \begin{array}{r}\text { INCOH. } \\ \text { SCATT. }\end{array} & \begin{array}{r}\text { PHOTO } \\ \text { EFFECT }\end{array} \\ 1 & -22.3 & -.4 & 15.3 & -22.3 \\ 1.5 & -14.0 & -.6 & 8.3 & -14.0 \\ 2 & -7.8 & -.8 & 4.9 & -7.8 \\ 3 & -12.8 & -1.1 & 2.3 & -12.9 \\ 4 & -10.2 & -1.2 & 1.2 & -10.2 \\ 5 & -7.1 & -1.3 & 1.1 & -7.2 \\ 6 & -5.3 & -1.3 & .7 & -5.3 \\ 9 & -3.1 & -1.4 & .8 & -3.1 \\ 10 & -2.1 & -1.6 & -.7 & -2.2 \\ 15 & -1.0 & -1.6 & -1.6 & -1.0 \\ 20 & -.8 & -1.6 & -1.4 & -.8 \\ 30 & -1.7 & -2.3 & -.4 & -1.7 \\ 40 & -2.5 & -5.7 & -.0 & -2.2 \\ 50 & -2.8 & -8.3 & .1 & -2.3 \\ 60 & -3.0 & -10.3 & .0 & -2.2 \\ 80 & -2.5 & -9.2 & -.6 & -2.3 \\ 100 & -.2 & -9.9 & .4 & .2 \\ 150 & 1.3 & -10.7 & -.3 & 2.1 \\ 200 & 1.1 & -10.7 & -.4 & 2.2 \\ 300 & .1 & -10.0 & -.3 & 1.2\end{array}$

\begin{tabular}{|c|c|c|c|c|c|}
\hline $\begin{array}{c}\text { ENERGY } \\
\text { KEV }\end{array}$ & TOTAL & $\begin{array}{r}\mathrm{COH} \\
\text { SCATT. }\end{array}$ & $\begin{array}{l}\text { INCOH. } \\
\text { SCATT. }\end{array}$ & $\begin{array}{r}\text { PHOTO } \\
\text { EFFECT }\end{array}$ & $\begin{array}{l}\text { PAIR } \\
\text { PROD. }\end{array}$ \\
\hline 400 & -.5 & -9.3 & -.1 & .3 & \\
\hline 500 & -.6 & -8.7 & -.0 & -.2 & \\
\hline 600 & -.5 & $-8 \cdot 0$ & .0 & -.4 & \\
\hline 800 & -.5 & $-7 \cdot 3$ & -.1 & -.6 & \\
\hline 1000 & -.4 & -6.4 & .0 & -1.0 & \\
\hline 1500 & -.7 & $-7 \cdot 1$ & -.0 & -3.7 & -3.1 \\
\hline 2000 & .0 & $-7 \cdot 5$ & -.1 & -.6 & $2 \cdot 3$ \\
\hline 3000 & -.4 & -7.9 & -.1 & -.3 & -1.0 \\
\hline 4000 & -.6 & $-8 \cdot 1$ & -.3 & -.7 & $-1 \cdot 1$ \\
\hline 5000 & -.5 & $-8 \cdot 2$ & -.2 & .8 &. .9 \\
\hline 6000 & $1 \cdot 1$ & $-8 \cdot 3$ & -.2 & .4 & $2 \cdot 2$ \\
\hline 8000 & 1.8 & -8.4 & -.2 & $1 \cdot 3$ & 2.9 \\
\hline 10000 & .4 & $-8 \cdot 4$ & -.2 & 3.3 & .6 \\
\hline 15000 & .6 & -8.5 & -.2 & .3 & .8 \\
\hline 20000 & -.6 & -8.5 & -.2 &.- .5 & -.7 \\
\hline 30000 & -.3 & -8.6 & -.3 & -.5 & -.3 \\
\hline 40000 & .4 & -8.7 & -.2 & .3 & .4 \\
\hline 50000 & -.3 & $-8 \cdot 6$ & -.2 & $1 \cdot 1$ & -.3 \\
\hline 60000 & .2 & $-8 \cdot 1$ & -.2 & 1.9 & . 2 \\
\hline 80000 & .4 & -6.8 & -.3 & $3 \cdot 5$ & $\cdot 5$ \\
\hline 100000 & $\cdot 3$ & -5.4 & -.3 & 4.9 & \\
\hline
\end{tabular}




$\begin{array}{ccrrr}\text { ENERGY }^{2}= & 77 \text { IRIOIUM } & \text { COH } & \text { INCOH. } & \text { PHOTO } \\ \text { KEV } & \text { TOTAL } & \text { SCATT. } & \text { SCATT. } & \text { EFFECT } \\ 1 & -22.2 & -.3 & 21.9 & -22.2 \\ 1.5 & -7.4 & -.5 & 12.6 & -7.4 \\ 2 & .7 & -.8 & 7.5 & .7 \\ 3 & -14.6 & -1.1 & 3.5 & -14.6 \\ 4 & -11.4 & -1.2 & 1.9 & -11.4 \\ 5 & -7.9 & -1.3 & 1.8 & -8.0 \\ 6 & -6.0 & -1.3 & 1.3 & -6.1 \\ 8 & -3.8 & -1.4 & 1.2 & -3.8 \\ 10 & -2.3 & -1.6 & -.3 & -2.3 \\ 15 & -1 & -1.7 & -1.2 & .1 \\ 20 & -1.0 & -1.6 & -1.1 & -1.0 \\ 30 & -2.3 & -2.2 & -.4 & -2.3 \\ 40 & -2.1 & -5.6 & -.0 & -1.8 \\ 50 & -1.1 & -8.3 & .1 & -.4 \\ 60 & -.2 & -10.2 & .0 & 1.5 \\ 80 & -.8 & -9.2 & -.5 & -.5 \\ 100 & -.7 & -9.9 & .4 & 1.2 \\ 150 & 1.4 & -10.7 & -.3 & 2.2 \\ 200 & -.9 & -10.6 & -.4 & 1.9 \\ 300 & -.2 & -10.0 & -.3 & .9\end{array}$

\begin{tabular}{|c|c|c|c|c|}
\hline $\begin{array}{c}\text { ENERGY } \\
\text { KEV }\end{array}$ & TOTAL & $\mathrm{COH}$ & $I \mathrm{NCOH}$. & PHOTO \\
\hline $\begin{array}{r}\text { KEV } \\
1\end{array}$ & -20.0 & $\begin{array}{c}-.2 \\
-2\end{array}$ & $15.5^{\circ}$ & $=20.1$ \\
\hline $1 \cdot 5$ & -7.0 & -.5 & 6.8 & -7.0 \\
\hline 2 & -.5 & -.7 & 2.4 & -.5 \\
\hline 3 & $-14 \cdot 7$ & $-1 \cdot 1$ & -.3 & -14.8 \\
\hline 4 & $-15 \cdot 3$ & $-1 \cdot 2$ & -.6 & -15.4 \\
\hline 5 & -10.7 & $-1 \cdot 3$ & $\cdot 1$ & $-10 \cdot 8$ \\
\hline 6 & -7.5 & -1.4 & .2 & -7.6 \\
\hline 8 & $-3 \cdot 1$ & -1.5 & .9 & -3.1 \\
\hline 10 & -.1 & -1.6 & -.2 & -.0 \\
\hline 15 & -3.2 & -1.6 & -.9 & $-3 \cdot 3$ \\
\hline 20 & -.2 & -1.6 & -.9 & -.1 \\
\hline 30 & .2 & -2.2 & -.3 & .3 \\
\hline 40 & -.4 & -5.5 & .1 & -.0 \\
\hline 50 & -.6 & -8.1 & . 1 & .2 \\
\hline 60 & -.2 & -9.9 & .1 & 1.0 \\
\hline 80 & -.6 & -8.9 & -.5 & -.3 \\
\hline 100 & 1.8 & -9.6 & .5 & $2 \cdot 3$ \\
\hline 150 & 3.7 & -10.3 & -.3 & $4 \cdot 7$ \\
\hline 200 & 3.7 & -10.2 & -.3 & $5 \cdot 2$ \\
\hline 300 & 2.6 & -9.5 & -.3 & 4.6 \\
\hline
\end{tabular}

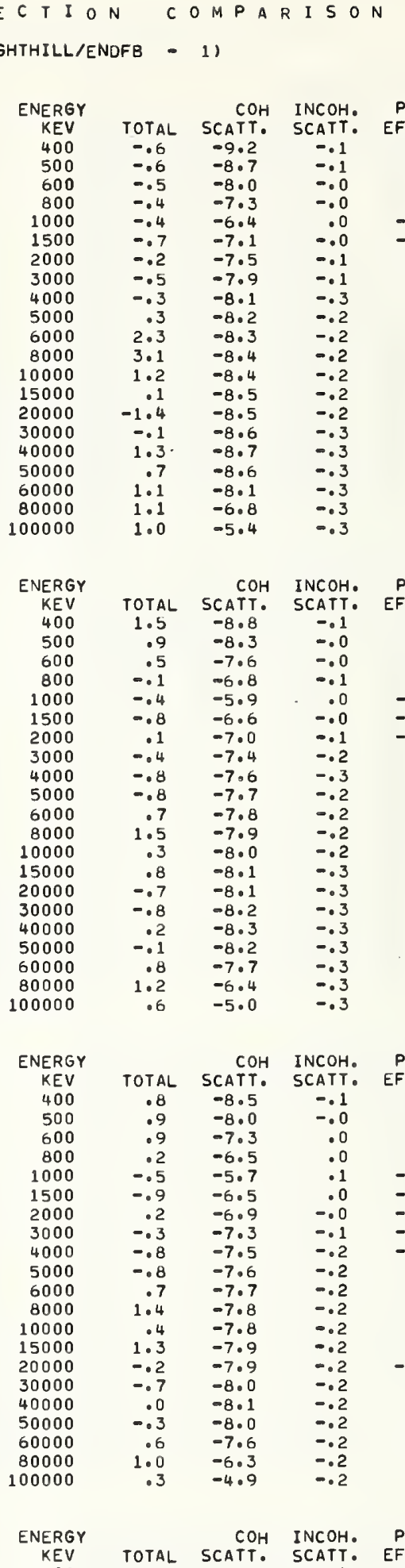

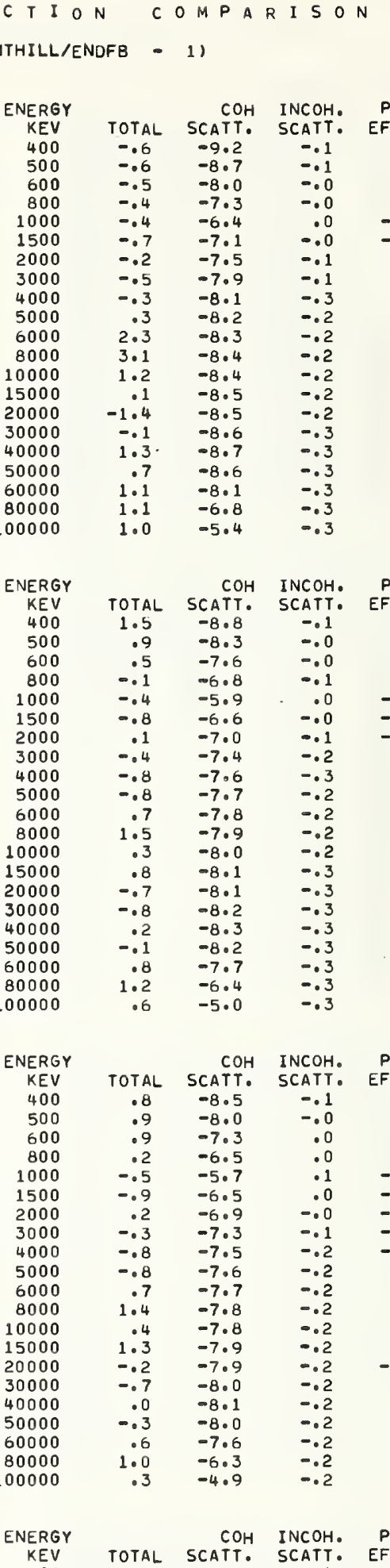

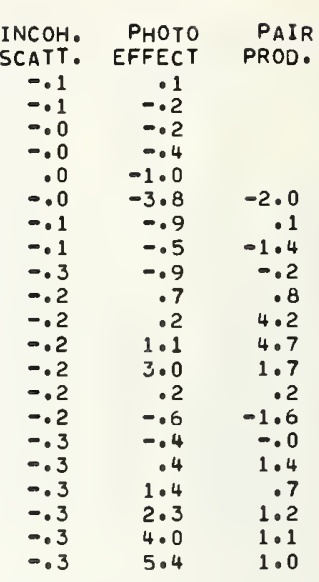

ENERGY KEV

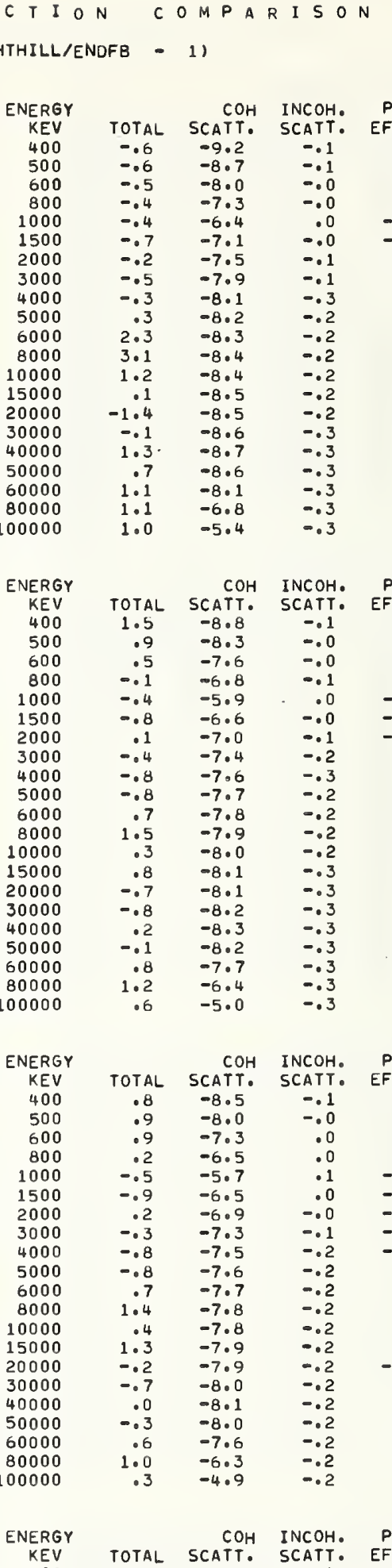

100000

ENERGY
KEV

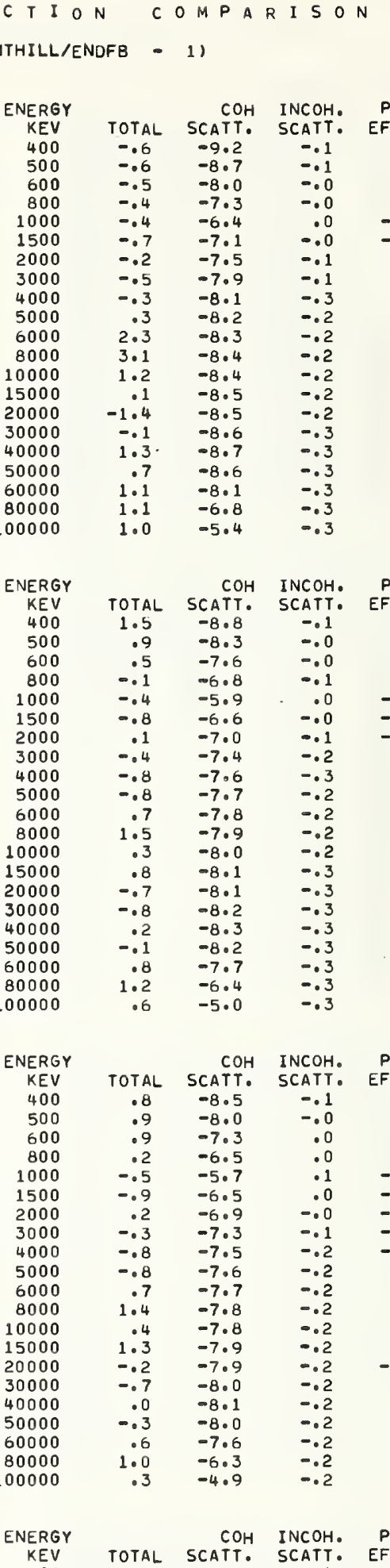

100000

ENERGY

\begin{tabular}{|c|c|c|c|c|c|}
\hline $\begin{array}{c}\text { ENERGY } \\
\text { KEV }\end{array}$ & TOTAL & $\begin{array}{r}\mathrm{COH} \\
\text { SCATT. }\end{array}$ & $\begin{array}{l}\text { INCOH. } \\
\text { SCATT. }\end{array}$ & $\begin{array}{l}\text { PHOTO } \\
\text { EFFECT }\end{array}$ & $\begin{array}{l}\text { PAIF } \\
\text { PROD. }\end{array}$ \\
\hline 400 & 2.2 & -8.5 & -.1 & 4.7 & \\
\hline 500 & 1.0 & $-8 \cdot 0$ & -.0 & 2.9 & \\
\hline 600 & .3 & $-7 \cdot 3$ & -.0 & $1 \cdot 6$ & \\
\hline 800 & -.3 & -6.6 & .0 & -.3 & \\
\hline 1000 & -.6 & $-5 \cdot 5$ & .1 & $-1 \cdot 8$ & \\
\hline 1500 & -.9 & $-6 \cdot 3$ & .0 & $-4 \cdot 6$ & -2.6 \\
\hline 2000 & .2 & $-6 \cdot 7$ & -.0 & $-1 \cdot 8$ & 4.4 \\
\hline 3000 & -.3 & -7.1 & -.1 & -1.1 & -0.6 \\
\hline 4000 & -.8 & $-7 \cdot 3$ & -.2 & $-1 \cdot 4$ & -1.5 \\
\hline 5000 & -.8 & -7.4 & -.2 & $\cdot 2$ & -1.3 \\
\hline 6000 & .8 & $-7 \cdot 5$ & -.2 & -.5 & 1.6 \\
\hline 8000 & 1.7 & -7.6 & -.2 & .2 & 2.7 \\
\hline 10000 & .8 & -7.7 & -.2 & 1.9 & $1 \cdot 1$ \\
\hline 15000 & 1.8 & -7.7 & -.2 & -.7 & $2 \cdot 3$ \\
\hline 20000 & .3 & $-7 \cdot 8$ & -.2 & -1.2 & .4 \\
\hline 30000 & -.3 & -7.8 & -.2 & -.7 & -.3 \\
\hline 40000 & .4 & -7.9 & -.2 & $\cdot 3$ & .5 \\
\hline 50000 & .1 & -7.9 & -.2 & 1.4 & .1 \\
\hline 60000 & 1.0 & -7.4 & -.2 & $2 \cdot 5$ & 1.0 \\
\hline 80000 & $1 \cdot 2$ & -6.1 & -.2 & 4.5 & 1.2 \\
\hline 00000 & .3 & -4.7 & -.2 & 6.1 & .4 \\
\hline
\end{tabular}




$\begin{array}{crrrr}\begin{array}{c}\text { ENERGY } \\ \text { ENER }\end{array} & 81 \text { THALLIUM } \\ \text { KEV } & \text { TOTAL } & \text { SCATT. } & \begin{array}{r}\text { INCOH. } \\ \text { SCATT. }\end{array} & \begin{array}{r}\text { PHOTO } \\ \text { EFFECT }\end{array} \\ 1 & -19.6 & -.3 & 23.7 & -19.7 \\ 1.5 & -8.6 & -.5 & 12.2 & -8.6 \\ 2 & -4.5 & -.7 & 5.6 & -4.5 \\ 3 & -7.6 & -1.0 & -.1 & -7.6 \\ 4 & -11.2 & -1.1 & -1.2 & -11.3 \\ 5 & -7.4 & -1.2 & -.7 & -7.5 \\ 6 & -5.7 & -1.3 & .1 & -5.7 \\ 8 & -4.0 & -1.4 & .9 & -4.1 \\ 10 & -3.0 & -1.5 & .8 & -3.1 \\ 15 & -1.9 & -1.4 & .5 & -1.9 \\ 20 & -.8 & -1.6 & .2 & -.8 \\ 30 & -.6 & -2.2 & .0 & -1.5 \\ 40 & -1.4 & -5.6 & .2 & -1.1 \\ 50 & -2.1 & -8.3 & .2 & -1.5 \\ 60 & -2.5 & -10.1 & .1 & -1.7 \\ 80 & -2.5 & -9.2 & -.5 & -1.6 \\ 100 & -1.7 & -9.9 & .5 & -1.4 \\ 150 & 1.3 & -10.7 & -.3 & 2.0 \\ 200 & 1.4 & -10.6 & -.4 & 2.4 \\ 300 & .3 & -10.0 & -.3 & 1.3\end{array}$

ENERGY
KEV
400
500
600
800
1000
1500
2000
3000
4000
5000
6000
8000
10000
15000
20000
30000
40000
50000
60000
80000
100000

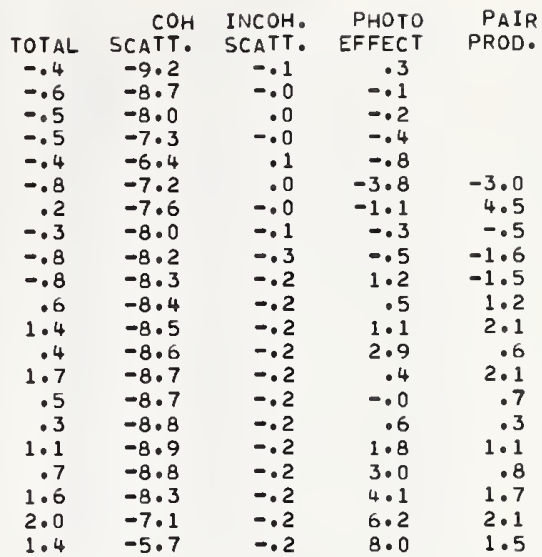

\begin{tabular}{|c|c|c|c|c|}
\hline ENERGY & & $\mathrm{COH}$ & INCOH. & РHOTO \\
\hline $\begin{array}{r}\text { KEV } \\
1\end{array}$ & $\begin{array}{l}\text { TOTAL } \\
-17.5\end{array}$ & $\begin{array}{c}\text { SCATT. } \\
-.4\end{array}$ & $\begin{array}{l}\text { SCATT. } \\
24.0\end{array}$ & $\begin{array}{l}\text { EFFECT } \\
-17.6\end{array}$ \\
\hline 1.5 & -7.2 & -.5 & 12.4 & -7.2 \\
\hline 2 & -3.1 & -.7 & 5.9 & -3.1 \\
\hline 3 & 6.2 & -1.0 & .3 & 6.2 \\
\hline 4 & -12.1 & $-1 \cdot 1$ & -.6 & $-12 \cdot 2$ \\
\hline 5 & -8.8 & $-1 \cdot 2$ & -.2 & -8.9 \\
\hline 6 & -6.9 & $-1 \cdot 3$ & .4 & -7.0 \\
\hline 8 & -4.8 & $-1 \cdot 3$ & 1.0 & -4.8 \\
\hline 10 & $-3 \cdot 5$ & -1.4 & 1.9 & -3.6 \\
\hline 15 & -.8 & -1.6 & 2.0 & -.8 \\
\hline 20 & -.3 & -1.5 & 1.5 & -.2 \\
\hline 30 & .8 & $-2 \cdot 3$ & .5 & 1.0 \\
\hline 40 & .5 & -5.7 & .4 & 9 \\
\hline 50 & .2 & $-8 \cdot 2$ & $\cdot 3$ & .9 \\
\hline 60 & .0 & -9.9 & .2 & 1.2 \\
\hline 80 & .6 & -9.1 & -.5 & $2 \cdot 1$ \\
\hline 100 & .8 & -9.8 & .5 & 1.2 \\
\hline 150 & .3 & -10.5 & -.3 & .9 \\
\hline 200 & -.1 & -10.4 & -.4 & .6 \\
\hline 300 & -.2 & -9.7 & -.3 & .6 \\
\hline
\end{tabular}

ENERGY

\begin{tabular}{|c|c|c|c|c|c|}
\hline $\begin{array}{c}\text { ENERGY } \\
\text { KEV }\end{array}$ & TOTAL & SCATT. & $\begin{array}{l}\text { INCOH. } \\
\text { SCATT. }\end{array}$ & $\begin{array}{l}\text { PHOTO } \\
\text { EFFECT }\end{array}$ & PROD. \\
\hline 400 & .1 & -9.1 & -.1 & 1.0 & \\
\hline 500 & .4 & $-8 \cdot 5$ & -.0 & 1.7 & \\
\hline 600 & .7 & $-7 \cdot 8$ & .0 & 2.4 & \\
\hline 800 & .6 & $-7 \cdot 1$ & .0 & $2 \cdot 8$ & \\
\hline 1000 & .4 & -6.2 & .1 & 2.2 & \\
\hline 1500 & -.3 & -6.9 & -.0 & -.9 & $-1 \cdot 6$ \\
\hline 2000 & .4 & $-7 \cdot 3$ & -.1 & 1.7 & 2.8 \\
\hline 3000 & -.2 & -7.7 & -.1 & 2.7 & -.8 \\
\hline 4000 & -.5 & -8.0 & -.3 & 2.5 & -.9 \\
\hline 5000 & -.1 & $-8 \cdot 1$ & -.2 & 4.3 & -.3 \\
\hline 6000 & 1.5 & -8.2 & -.2 & 3.6 & 2.6 \\
\hline 8000 & 2.0 & $-8 \cdot 3$ & -.2 & 4.2 & 3.1 \\
\hline 10000 & .6 & -8.3 & -.2 & 5.9 & .8 \\
\hline 15000 & .6 & -8.4 & -.2 & 3.5 & - 8 \\
\hline 20000 & -.7 & -8.5 & -.3 & 3.1 & -.8 \\
\hline 30000 & -.5 & $-8 \cdot 5$ & -.3 & $4 \cdot 0$ & -.6 \\
\hline 40000 & .3 & -8.6 & -.3 & $5 \cdot 2$ & .3 \\
\hline 50000 & -.2 & $-8 \cdot 6$ & -.3 & $6 \cdot 6$ & -.2 \\
\hline 60000 & .4 & -8.1 & -.3 & $7 \cdot 8$ & .5 \\
\hline 80000 & .8 & -6.9 & -.3 & 10.1 & .8 \\
\hline 00000 & .6 & $-5 \cdot 5$ & -.3 & 12.0 & .6 \\
\hline
\end{tabular}

\begin{tabular}{|c|c|c|c|c|}
\hline $\begin{array}{c}\text { ENEKGY } \\
\text { KEV }\end{array}$ & TOTAL & $\begin{array}{l}\text { COH } \\
\text { SCATT. }\end{array}$ & $\begin{array}{l}\text { INCOH. } \\
\text { SCATT. }\end{array}$ & $\begin{array}{r}\text { PHOTO } \\
\text { EFFECT }\end{array}$ \\
\hline 1 & $-22 \cdot 0$ & -.3 & 26.0 & -22.1 \\
\hline 1.5 & -9.3 & -.5 & 12.9 & -9.3 \\
\hline 2 & -3.8 & -.6 & $5 \cdot 3$ & -3.8 \\
\hline 3 & 8.7 & -1.0 & -1.0 & 8.8 \\
\hline 4 & -12.1 & $-1 \cdot 1$ & -1.8 & -12.1 \\
\hline 5 & -8.3 & $-1 \cdot 2$ & -1.0 & -8.4 \\
\hline 6 & -6.6 & $-1 \cdot 3$ & .1 & -6.7 \\
\hline 8 & $-5 \cdot 5$ & $-1 \cdot 3$ & 1.1 & -5.6 \\
\hline 10 & -5.0 & -1.4 & .9 & -5.1 \\
\hline 15 & 1.2 & -1.7 & -.1 & $1 \cdot 3$ \\
\hline 20 & -.1 & -1.5 & -.1 & -.0 \\
\hline 30 & -.1 & $-2 \cdot 2$ & .2 & -.0 \\
\hline 40 & $-1 \cdot 5$ & $-5 \cdot 3$ & .2 & -1.2 \\
\hline 50 & -2.5 & -7.6 & .2 & -2.1 \\
\hline 60 & -3.1 & -9.1 & $\cdot 1$ & -2.5 \\
\hline 80 & -2.9 & $-8 \cdot 3$ & -.5 & $-2 \cdot 2$ \\
\hline 100 & -2.4 & -8.9 & .5 & -2.2 \\
\hline 150 & -.7 & -9.5 & -.4 & -.3 \\
\hline 200 & -.4 & $-9 \cdot 3$ & -.4 & .1 \\
\hline 300 & -.4 & -8.5 & -.3 & .1 \\
\hline
\end{tabular}

ENERGY

\begin{tabular}{|c|c|c|c|c|c|}
\hline $\begin{array}{c}\text { ENERGY } \\
\text { KEV }\end{array}$ & TOTAL & $\begin{array}{l}\text { COH } \\
\text { SCATT. }\end{array}$ & $\begin{array}{l}\text { INCOH. } \\
\text { SCATT. }\end{array}$ & $\begin{array}{l}\text { PHOTO } \\
\text { EFFECT }\end{array}$ & $\begin{array}{l}\text { PAIR } \\
\text { PROD. }\end{array}$ \\
\hline 400 & -.3 & -7.8 & -.1 & .3 & \\
\hline 500 & -.0 & -7.2 & -.0 & .7 & \\
\hline 600 & .1 & -6.6 & -.0 & 1.1 & \\
\hline 800 & -.1 & -5.8 & .0 & .3 & \\
\hline 1000 & -.6 & $-4 \cdot 9$ & . 1 & $-1 \cdot 7$ & \\
\hline 1500 & $-1 \cdot 0$ & $-5 \cdot 7$ & -.0 & $-4 \cdot 5$ & -1.9 \\
\hline 2000 & .1 & $-6 \cdot 1$ & -.1 & -1.9 & 4.1 \\
\hline 3000 & -.3 & $-6 \cdot 5$ & -.1 & -1.2 & -.3 \\
\hline 4000 & -.6 & -6.7 & -.3 & -1.4 & -.9 \\
\hline 5000 & -.3 & -6.8 & -.2 & $\cdot 3$ & -.5 \\
\hline 6000 & 1.2 & -6.9 & -.2 & -.5 & 2.3 \\
\hline 8000 & $2 \cdot 0$ & -7.0 & -.2 & $\cdot 1$ & 3.1 \\
\hline 10000 & .9 & -7.1 & -.2 & 1.7 & 1.3 \\
\hline 15000 & 1.7 & $-7 \cdot 2$ & -.3 & -.8 & 2.1 \\
\hline 20000 & .4 & -7.2 & -.3 & $-1 \cdot 2$ & .5 \\
\hline 30000 & -.0 & $-7 \cdot 3$ & -.3 & -.6 & .0 \\
\hline 40000 & .6 & -7.4 & -.3 & .5 & .6 \\
\hline 50000 & .0 & -7.4 & -.3 & $1 \cdot 7$ & .0 \\
\hline 50000 & .7 & -6.9 & -.3 & 2.9 & . 8 \\
\hline 30000 & 1.0 & $-5 \cdot 6$ & -.3 & 4.9 & 1.0 \\
\hline 00000 & .5 & $-4 \cdot 3$ & -.3 & 6.7 & .3 \\
\hline
\end{tabular}

\begin{tabular}{|c|c|c|c|c|}
\hline $\begin{array}{c}\text { ENERGY } \\
\text { KEV }\end{array}$ & 86 RA & $\begin{array}{l}\text { ON COH } \\
\text { SCATT. }\end{array}$ & $\begin{array}{l}\text { INCOH. } \\
\text { SCATT. }\end{array}$ & $\begin{array}{r}\text { PHOTO } \\
\text { EFFECT }\end{array}$ \\
\hline 1 & $-11 \cdot 3$ & -.3 & 27.6 & -11.3 \\
\hline 1.5 & -5.6 & -.4 & $13 \cdot 5$ & $-5 \cdot 7$ \\
\hline 2 & -3.9 & -.6 & 5.7 & -3.9 \\
\hline 3 & $2 \cdot 3$ & -1.0 & -.6 & 2.3 \\
\hline 4 & -3.0 & -1.1 & -1.9 & -3.0 \\
\hline 5 & -9.8 & -1.1 & -1.1 & -9.9 \\
\hline 6 & -7.7 & $-1 \cdot 2$ & .1 & -7.7 \\
\hline 0 & -5.4 & $-1 \cdot 2$ & 1.3 & -5.5 \\
\hline 10 & $-4 \cdot 3$ & -1.2 & 1.0 & $-4 \cdot 4$ \\
\hline 15 & 2.7 & -1.5 & -.2 & 2.8 \\
\hline 20 & -1.3 & -1.5 & -.1 & -1.3 \\
\hline 30 & -.5 & $-2 \cdot n$ & .2 & -.4 \\
\hline 40 & $-1 \cdot 3$ & -4.4 & .3 & -1.1 \\
\hline 50 & -1.9 & -5.9 & .2 & -1.6 \\
\hline 20 & $-2 \cdot 0$ & $-6 \cdot 8$ & .2 & $-1 \cdot 6$ \\
\hline 80 & $-1 \cdot 2$ & -6.2 & -.6 & -.6 \\
\hline 100 & -2.6 & -6.6 & .5 & -2.4 \\
\hline 150 & .8 & $-6 \cdot 8$ & -.4 & 1.2 \\
\hline 200 & 1.0 & -6.3 & -.5 & 1.6 \\
\hline 300 & .1 & -5.4 & -.3 & .6 \\
\hline
\end{tabular}

\begin{tabular}{|c|c|c|c|c|c|}
\hline $\begin{array}{c}\text { ENERGY } \\
\text { KEV }\end{array}$ & TOTAL & $\begin{array}{r}\text { COH } \\
\text { SCATT. }\end{array}$ & $\begin{array}{l}\text { INCOH. } \\
\text { SCATT. }\end{array}$ & $\begin{array}{l}\text { PHOTO } \\
\text { EFFECT }\end{array}$ & $\begin{array}{l}\text { PAIR } \\
\text { PROD. }\end{array}$ \\
\hline 400 & -.4 & -4.7 & -.1 & -.2 & \\
\hline 500 & -.5 & $-4 \cdot 1$ & -.1 & -.5 & \\
\hline 600 & -.4 & -3.5 & -.0 & -.5 & \\
\hline 800 & -.3 & $-2 \cdot 6$ & .0 & -.5 & \\
\hline 1000 & -.3 & -1.6 & .0 & -.9 & \\
\hline 1500 & -.8 & $-2 \cdot 4$ & -.0 & -3.4 & $-1 \cdot 4$ \\
\hline 2000 & .3 & -2.9 & -.1 & -.6 & $4 \cdot 3$ \\
\hline 3000 & -.3 & $-3 \cdot 3$ & -.2 & -.5 & -.5 \\
\hline 4000 & -.8 & -3.5 & -.3 & -.6 & $-1 \cdot 3$ \\
\hline 5000 & -.7 & -3.7 & -.2 & $1 \cdot 1$ & -1.2 \\
\hline 6000 & .8 & $-3 \cdot 8$ & -.2 & .3 & 1.6 \\
\hline 8000 & $1 \cdot 6$ & -3.9 & -.2 & .8 & 2.5 \\
\hline 10000 & .7 & -4.0 & -.3 & $2 \cdot 3$ & 1.0 \\
\hline 15000 & $1 \cdot 8$ & $-4 \cdot 0$ & -.3 & -.6 & 2.2 \\
\hline 20000 & .8 & $-4 \cdot 1$ & -.3 & $-1 \cdot 3$ & .9 \\
\hline 30000 & .4 & $-4 \cdot 1$ & -.3 & $-1 \cdot 1$ & .5 \\
\hline 40000 & 1.0 & $-4 \cdot 3$ & -.3 & -.2 & 1.1 \\
\hline 50000 & .5 & $-4 \cdot 2$ & -.3 & - 8 & .6 \\
\hline 60000 & 1.4 & -3.8 & -.3 & 1.7 & 1.4 \\
\hline 80000 & 1.7 & $-2 \cdot 5$ & -.3 & $3 \cdot 5$ & 1.8 \\
\hline 100000 & 1.2 & $-1 \cdot 1$ & -.3 & $5 \cdot 1$ & $1 \cdot 2$ \\
\hline
\end{tabular}


$100 *($ BIGGS \& LIGHTHILL/ENOFB - 1)

\begin{tabular}{|c|c|c|c|c|}
\hline $\begin{array}{c}\text { ENERGY } \\
\text { KEV }\end{array}$ & TOTAL & $\begin{array}{r}\text { COH } \\
\text { SCATT. }\end{array}$ & $\begin{array}{l}\text { INCOH. } \\
\text { SCATT. }\end{array}$ & $\begin{array}{r}\text { PHOTO } \\
\text { EFFECT }\end{array}$ \\
\hline 1 & $2 \cdot 0$ & -.6 & 78.9 & 2.0 \\
\hline 1.5 & -.7 & -.6 & 45.8 & $=.7$ \\
\hline 2 & $-4 \cdot 5$ & -.7 & 28.8 & $-4 \cdot 5$ \\
\hline 3 & $-15 \cdot 0$ & $-1 \cdot 0$ & $10 \cdot 0$ & -15.2 \\
\hline 4 & $12 \cdot 3$ & $-1 \cdot 1$ & $2 \cdot 0$ & 12.4 \\
\hline 5 & -7.6 & -1.0 & .4 & -7.7 \\
\hline 6 & $-8 \cdot 5$ & -1.1 & .6 & -8.6 \\
\hline 8 & -6.8 & -1.1 & 1.7 & -7.0 \\
\hline 10 & $-6 \cdot 3$ & -1.0 & 10.2 & -6.5 \\
\hline 15 & -5.6 & -.6 & 5.6 & -5.9 \\
\hline 20 & -3.9 & -1.5 & $4 \cdot 6$ & -4.0 \\
\hline 30 & -.9 & -1.6 & 3.0 & -.9 \\
\hline 40 & -.3 & $-3 \cdot 1$ & 1.4 & -.1 \\
\hline 50 & -.4 & -3.9 & .8 & -.1 \\
\hline 60 & $=.6$ & $-4 \cdot 4$ & .7 & -.2 \\
\hline 80 & -1.0 & -3.7 & -.4 & -.8 \\
\hline 100 & -1.7 & -3.9 & .7 & -1.5 \\
\hline 150 & .7 & -3.6 & -.4 & .9 \\
\hline 200 & $1 \cdot 1$ & -3.0 & -.6 & 1.5 \\
\hline 300 & - 8 & -1.9 & 1.0 & .9 \\
\hline
\end{tabular}

$\begin{array}{crrrr}\text { ENERGY }^{Z}= & 92 \text { URANIUM } \\ \text { KEV } & \text { TOTAL } & \text { SCATT. } & \begin{array}{r}\text { INCOH. } \\ \text { SCATT. }\end{array} & \begin{array}{r}\text { PHOTO } \\ \text { EFFECT }\end{array} \\ 1 & -8.6 & -.5 & 85.4 & -8.7 \\ 1.5 & 2.9 & -.6 & 46.9 & 2.9 \\ 2 & 6.5 & -.7 & 29.4 & 6.5 \\ 3 & 3.3 & -1.0 & 8.4 & 3.3 \\ 4 & 20.7 & -1.1 & -1.6 & 20.9 \\ 5 & 5.7 & -1.1 & -3.5 & 5.7 \\ 6 & -3.0 & -1.2 & -3.2 & -3.0 \\ 8 & -1.2 & -1.3 & -1.1 & -1.2 \\ 10 & -.8 & -1.2 & 3.3 & -.8 \\ 15 & -.7 & -1.3 & 7.1 & -.7 \\ 20 & 2.1 & -1.4 & 6.3 & 2.3 \\ 30 & 1.0 & -1.5 & 4.2 & 1.1 \\ 40 & -.1 & -2.8 & 2.0 & .0 \\ 50 & -1.3 & -3.5 & 1.1 & -1.1 \\ 60 & -2.1 & -3.8 & .9 & -2.0 \\ 80 & -2.5 & -3.2 & -.3 & -2.5 \\ 100 & -1.9 & -3.3 & .7 & -1.9 \\ 150 & -.4 & -3.0 & -.4 & -.12 \\ 200 & 1.0 & -2.3 & -.6 & 1.3 \\ 300 & 1.5 & -1.2 & 1.7 & 1.6\end{array}$

ENERGY
KEV
400
500
600
800
1000
1500
2000
3000
4000
5000
6000
8000
10000
15000
20000
30000
40000
50000
60000
80000
100000

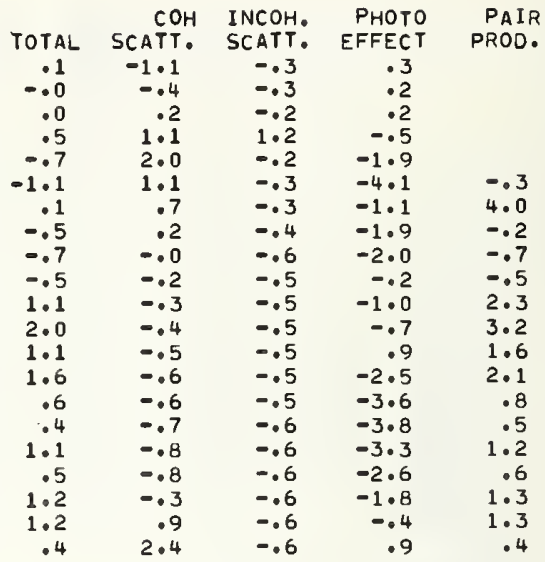

ENERGY

KEV
400
500
600
800
1000
1500
2000
3000
4000
5000
6000
8000
10000
15000
20000
30000
40000
50000
60000
80000
00000

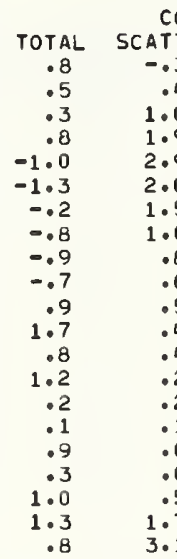

$\mathrm{COH}$ INCOH

\section{PHOTO PAIR} CATT. SCATT. EFFECT PROD. $\begin{array}{lll}-.3 & -.3 & 1.2\end{array}$

$\begin{array}{rrrr}.0 & -.3 & 1.0 & \\ 1.0 & -.3 & .8 & \\ 1.9 & 1.8 & -.5 & \\ 2.9 & -.3 & -2.5 & \\ 2.0 & -.4 & -4.8 & -.6 \\ 1.5 & -.4 & -1.9 & 3.4 \\ 1.0 & -.5 & -3.1 & -.5 \\ .8 & -.7 & -3.2 & -.9 \\ .6 & -.6 & -1.4 & -.8 \\ .5 & -.6 & -2.2 & 2.0 \\ .4 & -.6 & -1.8 & 2.8 \\ .4 & -.6 & -.3 & 1.2 \\ .2 & -.7 & -3.9 & 1.5 \\ .2 & -.7 & -5.1 & .3 \\ .1 & -.7 & -5.5 & .2 \\ .0 & -.7 & -5.1 & 1.0 \\ .0 & -.7 & -4.5 & .3 \\ .5 & -.7 & -3.8 & 1.1 \\ 1.7 & -.7 & -2.6 & 1.3 \\ 3.1 & -.7 & -1.4 & .8\end{array}$

ENERGY

\begin{tabular}{|c|c|c|c|c|c|}
\hline $\begin{array}{l}\text { ENERGY } \\
\text { KEV }\end{array}$ & TOTAL & $\begin{array}{r}\text { COH } \\
\text { SCATT. }\end{array}$ & $\begin{array}{l}\text { INCOH. } \\
\text { SCATT. }\end{array}$ & $\begin{array}{r}\text { PHOTO } \\
\text { EFFECT }\end{array}$ & $\begin{array}{l}\text { PAIR } \\
\text { PROD. }\end{array}$ \\
\hline 400 & .3 & .5 & -.4 & .5 & \\
\hline 500 & -.0 & 1.1 & -.5 & .2 & \\
\hline 600 & .0 & 1.8 & -.4 & .2 & \\
\hline 800 & $1 \cdot 3$ & 2.7 & 2.5 & -.2 & \\
\hline 1000 & -.5 & 3.6 & -.4 & -.8 & \\
\hline 1500 & $-1 \cdot 2$ & 2.7 & -.5 & $-3 \cdot 9$ & - 8 \\
\hline 2000 & -.4 & $2 \cdot 3$ & -.6 & -1.7 & 2.2 \\
\hline 3000 & -.8 & 1.8 & -.6 & $-2 \cdot 1$ & -.9 \\
\hline 4000 & -.8 & 1.6 & -.8 & $-3+0$ & -.6 \\
\hline 5000 & -.5 & 1.4 & -.7 & -1.7 & -.2 \\
\hline 6000 & 1.2 & 1.3 & -.7 & -2.5 & 2.7 \\
\hline 8000 & 2.0 & 1.2 & -.7 & $-2 \cdot 0$ & 3.3 \\
\hline 0000 & .8 & 1.1 & -.8 & -.4 & $1 \cdot 3$ \\
\hline 5000 & .9 & 1.0 & -.8 & $-3 \cdot 3$ & 1.2 \\
\hline 0000 & .5 & 1.0 & -.8 & $-4 \cdot 0$ & .6 \\
\hline 30000 & 1.4 & .9 & -.8 & $-3 \cdot 8$ & 1.6 \\
\hline+0000 & 2.4 & .8 & -.8 & -2.9 & 2.6 \\
\hline 50000 & 1.6 & $\cdot 8$ & -.8 & -1.9 & 1.7 \\
\hline 50000 & $2 \cdot 3$ & 1.2 & -.8 & -.9 & 2. \\
\hline 30000 & 2.4 & 2.4 & -.8 & .9 & 2.5 \\
\hline 00000 & $2 \cdot 2$ & 3.9 & -.8 & 2.4 & $2 \cdot 2$ \\
\hline
\end{tabular}




\begin{tabular}{|c|c|c|c|c|}
\hline ENERGY & $\begin{array}{l}1 \text { HYCR } \\
\text { HOTAL }\end{array}$ & COHER. & & \\
\hline (KEV) & & & SCATT. & EFFECT \\
\hline .010 & $\epsilon .652-01$ & $6.652-01$ & $2.124-05$ & 0.000 \\
\hline & & 6.65 & $4.7 \in S-C 5$ & $4.808+06$ \\
\hline & & $65=$ & $8.462-C 5$ & $2.195+06$ \\
\hline .030 & $\epsilon .566+c$ & $6.651-01$ & $1.897-04$ & $6.966+05$ \\
\hline & & 6.650 & & $.020+05$ \\
\hline & & $\epsilon . \in 4 S-C 1$ & & $4+05$ \\
\hline .060 & & 6.64 & & $5+04$ \\
\hline & & 6.64 & & $3.797+04$ \\
\hline & & 6.641 & 2.0 & $1.905+04$ \\
\hline & & & & \\
\hline & & 6.60 & & $5+03$ \\
\hline & & 6.55 & $1 . t$ & $5.951+02$ \\
\hline & & & & \\
\hline .5 & & 6.40 & & $9+02$ \\
\hline & & & & +01 \\
\hline & & & & \\
\hline $1 . C 00$ & 1.15 & $5.7 \mathrm{~s}$ & 1.3 & $2+01$ \\
\hline $1.50 \mathrm{C}$ & & & & \\
\hline & & & & \\
\hline 3.000 & 9.45 & 2.74 & 3.9 & $2.780-01$ \\
\hline & & $1 . \varepsilon$ & & \\
\hline & 7.05 & 1.35 & & \\
\hline 6.000 & 6.81 & $1.0 \mathrm{C}$ & 5.54 & -02 \\
\hline & & & & \\
\hline & & & & $75-03$ \\
\hline & 6.30 & 1.522 & 6.CSS- 01 & $1.133-03$ \\
\hline & 6.1 & 1. I 1 & & $58-04$ \\
\hline & & & & \\
\hline & & & & דת \\
\hline
\end{tabular}

\begin{tabular}{|c|c|c|c|c|}
\hline \multirow{2}{*}{ ENERGY ${ }^{2}$} & \multicolumn{2}{|c|}{$2 \mathrm{HEL}$ IUM } & \multirow{2}{*}{\multicolumn{2}{|c|}{$\begin{array}{l}\text { PHOTO } \\
\text { EFFECT }\end{array}$}} \\
\hline & TOTAL & $\begin{array}{l}\text { COHER. } \\
\text { SCATT. }\end{array}$ & & \\
\hline .010 & $.6 \in 1+00$ & $2 . \in \in 1+\infty 0$ & $t .125$ & c. 000 \\
\hline .C15 & & $2.661+C 0$ & $378-05$ & \\
\hline $.02 \mathrm{C}$ & & $1+00$ & & \\
\hline . $C \geq 0$ & & $2 . t \in 1+C O$ & & $5.402+06$ \\
\hline .040 & & & & 3.300 \\
\hline .050 & $14+C 6$ & & & \\
\hline .060 & $1.337 t$ & $2 . t \in 0+00$ & 2.2 & $1.337+c 6$ \\
\hline & & & & \\
\hline .100 & & & 6.1 & +05 \\
\hline .150 & 1.239 & $2.65 E+00$ & 1.38 & $1.239+05$ \\
\hline & & & & \\
\hline .300 & 1.644 & & & $1.644+04$ \\
\hline .400 & 6.877 & 2.63 & 9.9 & $6.874+03$ \\
\hline $.5 \mathrm{cc}$ & $3.4 \epsilon$ & & & \\
\hline$\cdot \in C 0$ & 1.57 & & & $1.967+03$ \\
\hline .800 & 8.083 & 2.5 & 4.C & $7+02$ \\
\hline $1 . \mathrm{COO}$ & & & & \\
\hline & 1.11 & & & $1.092+02$ \\
\hline $2 . c 00$ & 4.430 & 2.15 & 2.4 & 4.1 \\
\hline & 1.25 & & & \\
\hline & & 1.3 & & $3.783+00$ \\
\hline $\mathrm{coO}$ & 3.617 & $1 . \mathrm{C}$ & 7.7 & $9+00$ \\
\hline & & & & \\
\hline & & & & \\
\hline $1 \mathrm{C} .000$ & $1.651+$ & 3.52 & 1.0 & I. $653-01$ \\
\hline & $1.399+$ & & & \\
\hline & & & & \\
\hline & $1.224+00$ & $5.350-C 2$ & $1.166+C O$ & $4.222-03$ \\
\hline 40.000 & $1.171+0 \mathrm{C}$ & 3. $C E 2-C 2$ & $1.138+00$ & $1.634-03$ \\
\hline
\end{tabular}

\begin{tabular}{|c|c|c|c|c|}
\hline \multirow{2}{*}{ ENEREY ${ }^{2}$} & \multicolumn{2}{|c|}{3 LITHIUM } & & \\
\hline & TOTAL & $\begin{array}{l}\text { COHER } \\
\text { SCATT. }\end{array}$ & $\begin{array}{l}\text { INCOH. } \\
\text { SCATT. }\end{array}$ & $\begin{array}{l}\text { PHCTO } \\
\text { EFFECT }\end{array}$ \\
\hline.$C 10$ & I. $322+\mathrm{C} \epsilon$ & $5.587+00$ & $5.314-05$ & $1.322+06$ \\
\hline .015 & $8.27 \epsilon+05$ & S. $586+C C$ & $1.193-04$ & $8.276+05$ \\
\hline.$<20$ & $5.290+05$ & $5.586+00$ & $2.117-C^{4}$ & $5.290+05$ \\
\hline .030 & $2.518+C 5$ & $5.585+00$ & $4.743-04$ & $2.518+05$ \\
\hline .040 & $5+05$ & $5.583+0 C$ & $8.396-c 4$ & $1.385+05$ \\
\hline .050 & $8.313+C 4$ & $5.581+00$ & $1.306-03$ & $8.313+04$ \\
\hline $.0 \in 0$ & $4.137+06$ & $5.57 s+00$ & $1.873-03$ & $4.137+06$ \\
\hline & $2 . t \in 2+06$ & & & $2.662+06$ \\
\hline .100 & $1.64 t+0 t$ & S. SE $5+00$ & $5.116-03$ & $1.646+06$ \\
\hline & $6.005+05$ & $5.540+00$ & $1.126-02$ & $6.0 C 5+05$ \\
\hline .200 & $2.775+C E$ & $5.505+00$ & $1.959-02$ & $2.775+05$ \\
\hline .300 & $8.553+04$ & $5.811+00$ & $4.209-c 2$ & $8.952+04$ \\
\hline & $3.533+04$ & E. $\in 8 \varepsilon+00$ & $7.133-02$ & $3.933+04$ \\
\hline .500 & $2.061+c$ & $5.543+00$ & 1. $c \in O-C 1$ & $2.060+04$ \\
\hline & & & & +04 \\
\hline & $1+03$ & $5.028+0 c$ & $2.293-01$ & $5.156+03$ \\
\hline & $2.633+03$ & $4.673+0 \mathrm{C}$ & 3.147 & $2.628+03$ \\
\hline & $7.49 q+c$ & $3.535+$ & & $7.453+02$ \\
\hline 2.000 & $3.002+02$ & $3.4 C 4+00$ & $6.248-01$ & $2.961+02$ \\
\hline 3.000 & $8.101+01$ & $2 . t 86+0 C$ & 8. C72-C1 & $7.752+01$ \\
\hline & $3.267+01$ & & $9.549-01$ & $2.951+01$ \\
\hline 5.000 & $1.7 \mathrm{CO}+01$ & $1.882+c 0$ & 1. $C 87+00$ & $1.403+01$ \\
\hline & $1.048+01$ & $1.610+$ & $1.202+00$ & $7.663+00$ \\
\hline & & & & $2.955+00$ \\
\hline & $3.802+0 C$ & & 1.514 & 40 \\
\hline & & & & \\
\hline & $2.140+00$ & & $1.717+00$ & \\
\hline & $1.9 C 1+00$ & $1.387-c$ & $1.727+C 0$ & $3.550-02$ \\
\hline & $2+0$ & $8.074-02$ & $1.698+00$ & $1359-02$ \\
\hline
\end{tabular}

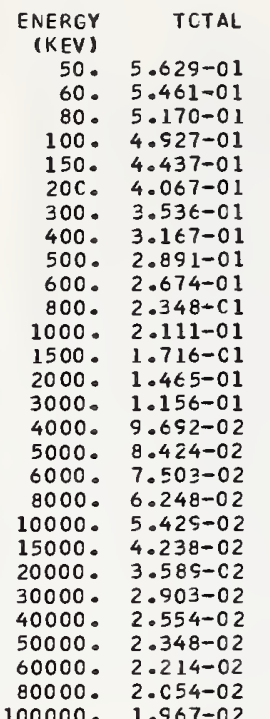
SCATT: $1.844-03$ $7.231-04$ $4.632-04$ $2.061-04$ $1.160-04$ $5.158-05$ $2.902-05$ $1.857-05$ $1.290-05$
$7.256-00$ $7.256-06$ $4.644-06$ $2.064-06$ $1.161-06$ $5.161-07$ 2.903-07 $1.858-07$ $1.290-07$ $7.257-08$ $4.645-08$ 2.064-08 $1.161-08$ $5.161-09$ 2.903-05 $1.858-0$ $1.290-09$ 7.257-10 100000 . 1.967-02
INCOH.
SCATT:
$5.610-01$
$5.448-01$
$5.163-01$
$4.523-01$
$4.435-01$
$4 . C 66-C 1$
$3.535-01$
$3.167-01$
$2 . E S 1-01$
$2.674-01$
$2.348-01$
$2.111-01$
$1.715-01$
$1.463-01$
$1.151-01$
$9.555-C 2$
$8.284-02$
$7.321-02$
$5.528-02$
$5 . C S 8-02$
$3.770-C 2$
$3 . C 24-02$
$2.159-02$
$1.746-C 2$
$1.456-02$
$1.254-02$
$9 . E 79-03$
$8196-03$

$8.156-03$
PHOTO EFFECT $1.77 t-05$
$9.524-06$ $3.5 \in C-06$ $1.655-06$ $4.225-07$ $1.64 \mathrm{C}-07$ $4.52 \mathrm{C}-08$ 1. $503-08$ $1 . C 1 E-08$ $6.332-09$ $3.2 C E-09$ $1.5 \varsigma \varepsilon-C s$ $9.428-1 C$ $5.93 \mathrm{C}-1 \mathrm{C}$ $3.315-10$ $2.277-1 \mathrm{C}$ $1.725-10$ $1.351-10$ $9.553-11$ $7.75 C-11$ $5 . C 1 E-11$ $3 . t 5 \varsigma-11$ $2.424-I 1$ $1 . \varepsilon C 3-11$ $1.435-11$ I $.15 \bar{z}-11$ 8. $9 \mathrm{CC}-12$ $7 \cdot 1<2-12$
PAIR PRCE.

$4.397-C 5$ $1.840-\mathrm{C} 4$ $5.548-04$
$9.708-C 4$ $1.396-03$ $1.815-C 3$ $2.6 C_{4}-03$ $4.682-03$ $5.650-C 3$ 7. $\mathrm{C} 36-\mathrm{C} 3$ 8. $083-03$ $8.917-C_{3}$ 5.559-C3 $1.147-02$

\begin{tabular}{|c|c|c|c|c|c|}
\hline $\begin{array}{r}\text { ENERGY } \\
\text { (KEV) } \\
50 . \\
60 \\
80 . \\
100 \\
150 . \\
200 \\
300 \\
400 . \\
500 . \\
600 \\
800 \\
1000 \\
1500 \\
2000 \\
3000 \\
4000 . \\
5000 \\
6000 \\
8000 \\
10000 \\
15000 \\
20000 \\
30000 \\
40000 \\
50000 \\
60000 \\
8000 \\
00000 \\
\end{array}$ & $\begin{array}{l}1.128+00 \\
1 . C 85+00 \\
1 . C 39+00 \\
5.883-01 \\
8.885-01 \\
2.143-C 1 \\
7.077-01 \\
6.337-01 \\
5.784-01 \\
5.349-C 1 \\
4.657-01 \\
4.223-01 \\
3.433-01 \\
2.934-01 \\
2.323-01 \\
1.556-01 \\
1.708-C 1 \\
1.529-01 \\
1.28 t-01 \\
1.128-01 \\
9.014-02 \\
7.831-02 \\
6.645-02 \\
6 . C 54-02 \\
5.656-02 \\
5.465-02 \\
5.220-02 \\
5.142-02\end{array}$ & $\begin{array}{l}\text { COHER. } \\
\text { SCATT } \\
1.988-02 \\
1.387-02 \\
7.836-03 \\
5.027-03 \\
2.240-03 \\
1.261-03 \\
5.609-04 \\
3.156-04 \\
2.021-04 \\
1.403-04 \\
7.895-05 \\
5.053-05 \\
2.246-05 \\
1.264-05 \\
5.616-06 \\
3.159-06 \\
2.022-06 \\
1.404-06 \\
7.898-07 \\
5.055-07 \\
2.247-07 \\
1.264-07 \\
5.616-08 \\
3.159-08 \\
2.022-08 \\
1.404-08 \\
7.898-09 \\
5.055-09\end{array}$ & $\begin{array}{r}\text { INCOH. } \\
\text { SCATT } \\
1.107+00 \\
1 . C 74+0 C \\
1 . C 31+O C \\
5.832-C 1 \\
8.867-C 1 \\
8.130-01 \\
7 . C 71-01 \\
6.334-01 \\
5.782-C 1 \\
5.34 E-01 \\
4.657-01 \\
4.222-01 \\
2.431-01 \\
2.527-01 \\
2.3 C 2-C 1 \\
1.519-01 \\
1.657-01 \\
1.464-01 \\
1.1 \subseteq 8-C 1 \\
1 . C 20-01 \\
7.540-C 2 \\
6 . C 48-02 \\
4.358-C 2 \\
3.452-02 \\
2.513-C 2 \\
2.508-C 2 \\
1.576-02 \\
1.639-02\end{array}$ & $\begin{array}{r}\text { PHOTO } \\
\text { EFFECT } \\
7.873-04 \\
4.352-04 \\
1.718-04 \\
8.4 C 4-C 5 \\
2.323-05 \\
5.41 S-06 \\
2.71 C-06 \\
1.167-06 \\
6.343-07 \\
4 . C C S-C 7 \\
2.084-07 \\
1.323-07 \\
6.365-08 \\
4 . C 2 C-08 \\
2.233-08 \\
1.521-C 8 \\
1.14 t-08 \\
5.164-09 \\
6.521-09 \\
5 . C 51-09 \\
3.222-05 \\
2.363-09 \\
1.535-09 \\
1.141-05 \\
5 . C 67-10 \\
7.521-10 \\
5.6 C E-1 C \\
4.470-10\end{array}$ & $\begin{array}{l}1.775-C 4 \\
7.138-04 \\
2.138-C 3 \\
3.659-c 3 \\
5.126-C 3 \\
6.490-03 \\
8.876-c 3 \\
1 . c 86-02 \\
1.473-02 \\
1.783-c 2 \\
2.251-02 \\
2.562-02 \\
2.783-c 2 \\
2.957-02 \\
3.244-C 2 \\
3.503-C 2\end{array}$ \\
\hline
\end{tabular}

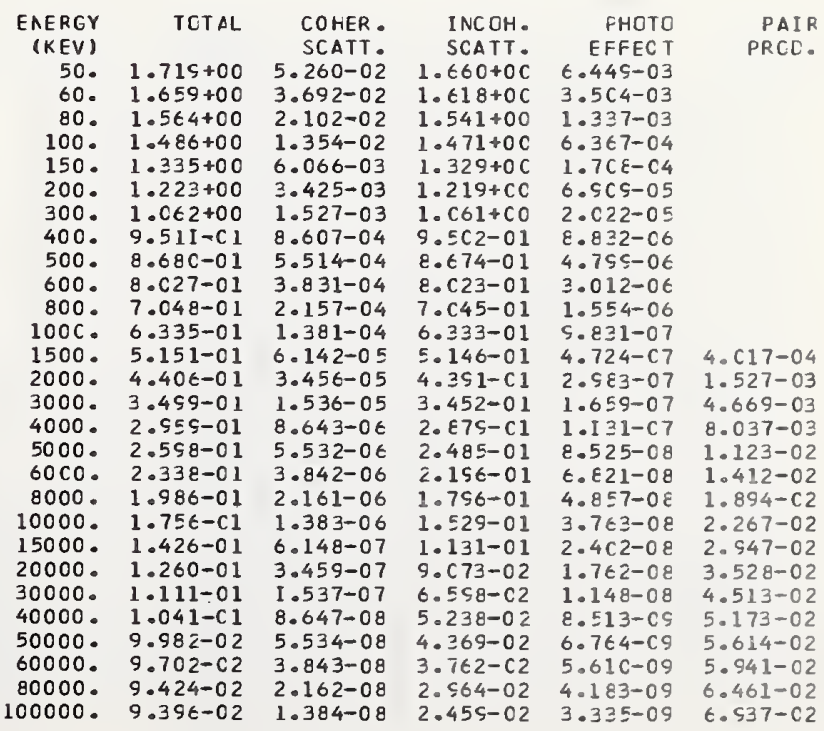




\begin{tabular}{|c|c|c|c|c|}
\hline \multicolumn{5}{|c|}{$z=48$ ERYLLILM } \\
\hline $\begin{array}{l}\text { ENEREY } \\
\text { (KEV) }\end{array}$ & TOTAL & $\begin{array}{l}\text { COHER } \\
\text { SCATT }\end{array}$ & $\begin{array}{l}\text { INCOH. } \\
\text { SCATT. }\end{array}$ & $\begin{array}{l}\text { PHOTO } \\
\text { EFFECT }\end{array}$ \\
\hline .010 & $1.4 \varepsilon S+C \epsilon$ & 1. $C \in 4+01$ & $8.756-c 5$ & $1.489+06$ \\
\hline . C15 & $1.697+06$ & 1. $c \in 4+01$ & 1. $575-C 4$ & $1.657+06$ \\
\hline .020 & $1.379+06$ & $1.064+01$ & $3.504-04$ & $1.379+06$ \\
\hline $.03 \mathrm{C}$ & $\varepsilon .248+C 5$ & $1 . C \in 4+C 1$ & $7.849-04$ & $8.248+05$ \\
\hline .040 & $5.201+05$ & $1.064+\mathrm{C} 1$ & 1.3 & $5.201+05$ \\
\hline $.05 \mathrm{C}$ & $3.455+C 5$ & 1. $C \in 4+C 1$ & & $3.495+05$ \\
\hline .060 & $2.470+05$ & $1.063+01$ & & $2.470+05$ \\
\hline & 1.3 & & & $1.373+05$ \\
\hline & $10+04$ & $1.062+$ & & $3+04$ \\
\hline .150 & 1.4 & 1. $c$ & & $9+06$ \\
\hline .200 & $7.129+05$ & 1. C54. & & +05 \\
\hline .300 & $2.454+05$ & $1.041+$ & 6.9 & $2.494+05$ \\
\hline .48 & $1.149+c$ & & & \\
\hline .500 & $6.240+04$ & $1.005+01$ & 1.7 & $6.239+04$ \\
\hline.$\in \mathrm{CO}$ & $3.773+c$ & & & \\
\hline & & 9.3 & & \\
\hline $1 . \mathrm{COC}$ & 8.5 & & & +03 \\
\hline & 2.6 & & & \\
\hline & $1 . c^{5}$ & 6.23 & 9.6 & \\
\hline 3 & 2.0 & 4.4 & & \\
\hline & & & & \\
\hline & $5.956+c$ & $2.8 \varepsilon$ & 1.4 & 5.5 \\
\hline & & & & \\
\hline & $1.567+0$ & $1.871+$ & & \\
\hline $\mathrm{C} . \mathrm{CCO}$ & $S .175+C$ & & & \\
\hline & 4.487 & 8.2 & & \\
\hline & & & & \\
\hline & $2 \cdot t 91+0 \mathrm{C}$ & $2.659-01$ & $2.273+C C$ & $1.518-01$ \\
\hline 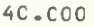 & $2.4 \epsilon E+C C$ & $1.574-C 1$ & $2.250+C C$ & $5.819-02$ \\
\hline
\end{tabular}

\begin{tabular}{|c|c|c|c|c|}
\hline & & & & \\
\hline $\begin{array}{l}\text { EAEREY } \\
\text { (KEV) }\end{array}$ & TOTAL & $\begin{array}{l}\text { COHER } \\
\text { SCATT }\end{array}$ & $\begin{array}{l}\text { INCOH. } \\
\text { SCATT. }\end{array}$ & $\begin{array}{r}\text { PHCTC } \\
\text { EFFECT }\end{array}$ \\
\hline . CIO & $6.303+06$ & $1.663+01$ & $8.355-c 5$ & $6.303+06$ \\
\hline & $4 \cdot t 22+0 t$ & $1 . t \in 3+01$ & $1.876-c 4$ & $4.622+06$ \\
\hline .020 & $3.524+C t$ & $1.663+01$ & $3.329-C 4$ & $3.524+06$ \\
\hline .030 & $2.077+06$ & $1.663+C 1$ & $7.459-C 4$ & $2.077+06$ \\
\hline .040 & $1.321+0 t$ & I. $.663+01$ & $1.321-c 3$ & $1.321+06$ \\
\hline .050 & $5.000+05$ & $1.662+01$ & $2 . C 55$ & $5.000+05$ \\
\hline.$c \in 0$ & $6.4 t 4+C 5$ & $1.6 \in 2+C 1$ & & \\
\hline .080 & $3.725+C E$ & $1.661+01$ & $5.157-03$ & $3.725+C 5$ \\
\hline .100 & $2.373+05$ & $1.66 \mathrm{C}+\mathrm{C} 1$ & $8.054-$ & $2.373+05$ \\
\hline & & & & $9.873+04$ \\
\hline $.2 \mathrm{CO}$ & $1.413+c t$ & $1.651+01$ & 3.08 & $1.413+0 t$ \\
\hline .300 & 5.257 & $1.637+01$ & & $5.257+05$ \\
\hline .400 & $2.573+05$ & $1.61 s+C 1$ & 1.12 & $2.573+05$ \\
\hline .500 & $1.4 \epsilon \bar{Z}+C E$ & $1.5 S_{t}+\mathrm{Cl}$ & 1.67 & $1.462+05$ \\
\hline .600 & $9.112+04$ & $1.570+01$ & & \\
\hline .800 & $4.1 \in 8+c 4$ & 1. $5 C \varepsilon+C 1$ & 3.66 & $4.167+04$ \\
\hline 1.000 & $2.2 \mathrm{C} 2+\mathrm{C}_{4}$ & $1.439+01$ & 5.05 & $00 \div 04$ \\
\hline & $6.7 c_{3}+c_{3}$ & $1.252+$ & 8.67 & $0+03$ \\
\hline 2.000 & $2.830+03$ & 1. $c \in 3+c 1$ & 1. $C 47+C 0$ & $2.818+03$ \\
\hline 3.000 & $8.211+c z$ & $7.527+$ & $1.394+0 \mathrm{C}$ & \\
\hline 4.000 & $3.374+02$ & $5 . t 54+$ & $1.649+00$ & $3.301+02$ \\
\hline 5. COC & $1.694+C 2$ & $4.545+C 0$ & $1.844+$ & $1.630+02$ \\
\hline$\epsilon . \mathrm{COO}$ & 5.68 & 3.7 & & $9.112+01$ \\
\hline E.c & $4 \cdot 102+C I$ & $2.7 \varepsilon 5+$ & $2.1 \subseteq 3+00$ & $3.605+01$ \\
\hline & & & & $1.737+01$ \\
\hline . 0 & $\varepsilon .395+$ & 1.305 & $2.596+$ & $4.454+00$ \\
\hline & $5.277+0 \mathrm{C}$ & $8.455-$ & $2.728+C O$ & \\
\hline & & & $2.755+C C$ & $4.376-\mathrm{Cl}$ \\
\hline 40.000 & $3.2 C E+C C$ & $2.61 C-C 1$ & $2.776+C C$ & $1.691-01$ \\
\hline
\end{tabular}

\begin{tabular}{|c|c|}
\hline & $\epsilon$ CAR $8 C$ \\
\hline $\begin{array}{l}\text { ENEREV } \\
\text { (KEV) }\end{array}$ & TOTAL \\
\hline.$C 10$ & $1.474+07$ \\
\hline .015 & $8.023+C \epsilon$ \\
\hline .020 & $C .444+C 6$ \\
\hline .030 & $4.117+C \epsilon$ \\
\hline .040 & $2.723+C 6$ \\
\hline $.05 \mathrm{C}$ & $1.85 t+C t$ \\
\hline .060 & $1.3 \varepsilon 0+0 E$ \\
\hline .080 & $8.068+05$ \\
\hline .100 & $5.177+05$ \\
\hline .150 & $2.1 \in 2+05$ \\
\hline $.2 \mathrm{CO}$ & $1.086+05$ \\
\hline .300 & S. $129+05$ \\
\hline $.4 \mathrm{CO}$ & $4.795+05$ \\
\hline .500 & $2.788+c 5$ \\
\hline .600 & $1.75 E+C E$ \\
\hline .800 & $8.1 \in 2+04$ \\
\hline $1 . \mathrm{COO}$ & $4.392+C 4$ \\
\hline 1.500 & $1.381+04$ \\
\hline 2.000 & $5.570+C 3$ \\
\hline $3 . \mathrm{CCO}$ & $1.794+C 3$ \\
\hline 4.000 & $7.525+C 2$ \\
\hline 5.000 & $3.811+02$ \\
\hline $6 . \mathrm{CCO}$ & $2.180+02$ \\
\hline $8 . \operatorname{CcC}$ & $9.05 s+01$ \\
\hline 10.000 & $4.6 \in 1+01$ \\
\hline $15 . \mathrm{COC}$ & $1.560+01$ \\
\hline $2 C .000$ & $8.520+C O$ \\
\hline 30.000 & 5. $C 37+00$ \\
\hline $4 C . C O O$ & $4.125+C \mathrm{C}$ \\
\hline
\end{tabular}

COHER.
SCATT:

$\begin{array}{lll}2.355+C 1 & 1.359-04 & 8.023+06\end{array}$

$2.3 c 4+C 1 \quad \begin{array}{lll}2.411-C 4 & 6.444+06 \\ 5.407-04 & 4.117+06\end{array}$

$2.394+019.578-C 4 \quad 2.723+06$

$2.394+C 1 \quad 1.451-C 3 \quad 1.896+06$

$2.354+01 \quad 2.140-03 \quad 1.380+06$

$\begin{array}{lll}2.3 c 3+C 1 & 3.778-03 & 8.068+05\end{array}$

$2.392+C 1 \quad 5 . \varepsilon \in 1-C 3 \quad 5.176+05$

$\begin{array}{lll}2.388+01 & 1.255-C 2 & 2.161+05\end{array}$

$\begin{array}{lll}2.383+01 & 2.261-02 \quad 1.086+05\end{array}$

$\begin{array}{lll}2.368+C 1 & 4.859-02 & 9.129+05\end{array}$

$2.349+01 \quad 8.375-02 \quad 4.795+05$

$\begin{array}{lll}2.325+C 1 & 1.256-C 1 & 2.788+05\end{array}$

$2.2 \subseteq 7+C 1 \quad 1.734-01 \quad 1.755+C 5$

$2.229+01 \quad 2.817-01 \quad 8.160+04$

$2.150+01 \quad 3.985-01 \quad 4.390+04$

$\begin{array}{lll}1.917+C 1 & 6.883-C 1 & 1.379+04 \\ 1.662+C 1 & 0.644-C 1 & 5.953+03\end{array}$

$1.215+01 \quad 1.453+C C \quad 1.780+03$

$5.17 \mathrm{C}+\mathrm{CC} \quad 1.829+00 \quad 7.41 \mathrm{~s}+02$

$\begin{array}{lll}7.221+C O & 2.1 C 6+O C & 3.718+02\end{array}$

$\begin{array}{lll}5.841+C \mathrm{C} & 2.3 \mathrm{C} 6+0 \mathrm{C} & 2.098+02\end{array}$

$4.15 S+C O \quad 2.562+C O \quad 8.387+01$

$3.23 \mathrm{C}+00 \quad 2.744+\mathrm{CC} \quad 4.064+01$

$1.581+C 0 \quad 3.045+00 \quad 1.058+01$

$1.28 \varepsilon+0 \mathrm{C} \quad 3.207+\mathrm{CO} \quad 4.025+00$

$6.758-C 1 \quad 3.3 C 9+00 \quad 1.052+00$

\begin{tabular}{|c|c|}
\hline ENERGY & TCTAL \\
\hline & \\
\hline 50. & $2.342+00$ \\
\hline 60. & $2.254+00$ \\
\hline 80. & $2.096+00$ \\
\hline 100. & $1.587+00$ \\
\hline 150. & $1.782+00$ \\
\hline 200 . & $1.631+00$ \\
\hline 300 . & $1.416+00$ \\
\hline 400 . & 1. $2 \epsilon E+00$ \\
\hline 500. & $1.157+00$ \\
\hline 600 . & $1.070+0 \mathrm{C}$ \\
\hline 800 & $9.357-01$ \\
\hline 1000 & $8.446-C 1$ \\
\hline 1500. & $6.87 C-C 1$ \\
\hline 2000 . & $5.884-\mathrm{C} 1$ \\
\hline 3000 . & $4.684=01$ \\
\hline $40 \mathrm{CO}$. & $3.972-01$ \\
\hline 5000 . & $3.49 \varepsilon-01$ \\
\hline 6000 . & $3.161-01$ \\
\hline 8000 & $2.714-\mathrm{Cl}$ \\
\hline 10000 . & $2.431-01$ \\
\hline 15000 . & $2.035-01$ \\
\hline 20000 . & $1.830-C_{1}$ \\
\hline 30000 . & $1.632-01$ \\
\hline 40000 . & $1.548-01$ \\
\hline 50000 . & $1.508-\mathrm{Cl} 1$ \\
\hline 60000 . & $1.487-01$ \\
\hline 80000 . & $1.473-01$ \\
\hline .00000 & $1.47 t-01$ \\
\hline
\end{tabular}

COHER.
SCATT.
$1.035-01$
$7.310-02$
$4.194-02$
$2.714-02$
$1.224-02$
$6.930-03$
$3.100-03$
$1.750-03$
$1.122-03$
$7.800-04$
$4.395-04$
$2.815-04$
$1.253-04$
$7.051-05$
$3.136-05$
$1.764-05$
$1.129-05$
$7.844-06$
$4.413-06$
$2.825-06$
$1.256-06$
$7.063-07$
$3.139-07$
$1.766-07$
$1.130-07$
$7.848-08$
$4.415-08$
$2.825-08$

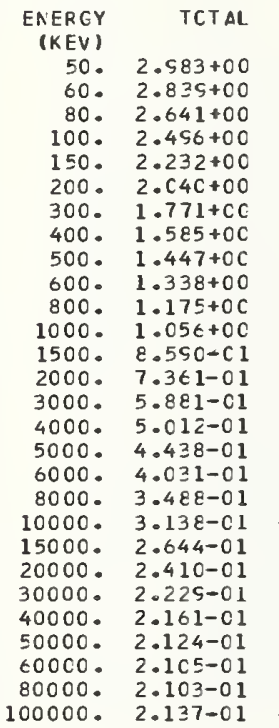

COHER :
SCATT:
$1.728-01$ $1.728-01$
$1.226-01$ $7.074-02$ $4.595-02$ $2.081-02$ $1.182-02$ $5.300-03$ $2.994-03$ $1.922-03$ $1.337-03$
$7.536-04$ $7.536-04$ $2.150-04$ $1.210-04$ 5.385-05 $3.030-05$ $1.940-05$ $1.347-05$ $7.580-06$ $4.852-06$ $2.157-0 t$ $1.213-0 t$ 5.393-07 3.034-07 $1.942-07$ $1.348-07$ $7.585-08$ $4.854-08$
INCOH.
SCATT:

$2.211+0 \mathrm{C}$
$2.16 \epsilon+0 \mathrm{C}$

2. $C 48+00$

1. $557+O C$

$1.7 E 9+O C$

$1.624+0 C$

$1.266+00$

$1.15 t+0 C$

$1.065+00$

$8.444-01$

$6.8 \in 1-01$

$5.854-01$

$4 . \in C 3-01$
$3.838-C 1$

3.314-01

2. $525-01$

$2.395-01$

2. $C 35-01$

$1.5 \mathrm{C} 8-\mathrm{Cl}$

$8.797-c 2$

$6.584-02$

5. $825-02$

5.C16-02

$2.552-02$
$3.279-02$
FHCTO

EFFECT $2.75 t-C 2$ $5.6 \geq t-03$

$2.65 t-03$

7.041-04 $2.84 \varepsilon-C 4$ $3,652-05$ $2.0 C_{4}-05$ $1.253-05$ $6.4 \equiv 2-06$

1. $548-06 \quad 7.044-C_{4}$

1. $23 C-06 \quad 2.543-03$

E. $84 t-C 7 \quad 8.1 \mathrm{C5}-\mathrm{C} 3$

4.67C-07 1.338-02

3.5ट2-07 1.847-C2

$2 . \varepsilon 15-07 \quad 2.326-C 2$

2.CCE-07 3.186-C2

1.557-07 3.516-02

$5.53 \varepsilon-08 \quad 5.272-02$

$7.291-08 \quad 6.2 \mathrm{Cl} 1-02$

$4.753-08 \quad 7.525-C 2$

3.525-08 8.499-02

$\begin{array}{ll}2.8 C C-08 & 5.252-C 2 \\ 2.323-08 & 9.855-C 2\end{array}$

$1.7=2-08 \quad 1.078-01$

1. $3 \varepsilon 1-0 \varepsilon \quad 1.149-C 1$

\begin{tabular}{|c|c|c|}
\hline (KEV) & $A$ & \\
\hline 50. & $3.72 s+c 0$ & $2.767-01$ \\
\hline 60. & $3.455+00$ & $1.983-01$ \\
\hline 80. & $3.214+00$ & \\
\hline 100. & $3.021+00$ & $7.595-C 2$ \\
\hline & $2.650+00$ & $3.480-02$ \\
\hline & $456+0 C$ & \\
\hline 300. & $2.125+00$ & \\
\hline 400. & $1.5 C 5+C O$ & 02 \\
\hline 500 & $1.738+0$ & \\
\hline & $1.6 \mathrm{c} \epsilon+00$ & \\
\hline 80 & 1.4 & 1.2 \\
\hline 1000 & $1.267+$ & \\
\hline 1500. & $1.031+00$ & $3.674-04$ \\
\hline 100. & $8.847-01$ & 2.0 \\
\hline 3000 . & $7 . c 8$ & 5.2 \\
\hline 4000 & 6.06 & $5.187-05$ \\
\hline & $5 \cdot 3 \xi$ & 3.3 \\
\hline & & \\
\hline 100 & $4.287-01$ & 1.29 \\
\hline & & 8.3. \\
\hline & & \\
\hline 20000 & 3.0 & 2.0 \\
\hline & & \\
\hline & & \\
\hline 500 & 2.81 & $3.326-07$ \\
\hline & $2-01$ & $2.310-07$ \\
\hline & & $1.299-07$ \\
\hline & $.879-01$ & $8.316-08$ \\
\hline
\end{tabular}

100000 .

INCOH.
SCATT:
$2.72 S+C C$
$2.672+O C$
$2.553+C C$
$2.442+C C$
$2.2 C 9+O C$
$2 . C 28+O O$
$1.765+O C$
$1.582+C C$
$1.445+O C$
$1.336+C C$
$1.174+O C$
$1 . C 55+O C$
$8.57 t-C 1$
$7.317-C 1$
$5.754-C 1$
$4.7 \subseteq 7-01$
$4.142-01$
$3.6 \in 1-01$
$2.5 S 4-C 1$
$2.54 S-C 1$
$1.885-C 1$
$1.512-C 1$
$1.1 C 0-01$
$\varepsilon .73 C-C 2$
$7.281-02$
$\epsilon .26 S-02$
$4.540-C 2$
$4 . C S 8-02$

(1)

$8.117-02$

$4.455-02$

$\varepsilon .252-03$

2. $1 \varepsilon \epsilon-03$

$8.753-04$

2.527-04

1. $c \subseteq 8-04$

$5.5 \subseteq 1-05$

$3.77 \varepsilon-C 5$

$1.551-05$

$1.231-05$

$5.8 \mathrm{E} E-\mathrm{C} \epsilon$

$3 \cdot t \varepsilon \varepsilon-\mathrm{C} 6$

$2.044-06$

1. $3 \subseteq \overline{2}-06$

1. C5C-06

$8.4 \mathrm{C} 4=07$

$5.585-07$

4.644-07

2. $568-07$

$2.17 \varepsilon-07$

$1.054-07$

E. $3 \varepsilon C-08$

$\epsilon .5 E 2-08$

$5.18 t-08$

$4.135-08$
$1.110-\mathrm{C}_{3}$ $4.253-C 3$ $1.264-02$ $2.137-\mathrm{C2}$ $3.702-\mathrm{C} 2$ 4. $\subseteq 38-02$ $5.893-\mathrm{C}^{2}$ $7.594-02$ $1.12 S \rightarrow C 1$ $1.288-01$ $1.396-C$ $1.478-\mathrm{Cl}$

1. $727-C 1$

\begin{tabular}{|c|c|c|}
\hline INCOH. & FHCTO & PAIF \\
\hline SCATT & EFFECT & PRCL. \\
\hline $3.254+0 \mathrm{C}$ & $1.58 z-C 1$ & \\
\hline $3.187+0 \mathrm{C}$ & 1.CS3-01 & \\
\hline $3 . C 55+0 C$ & $4.2 \in 1-02$ & \\
\hline $2 . \subseteq 25+0 C$ & $2.035-02$ & \\
\hline $2.650+0 \mathrm{C}$ & $5.371-03$ & \\
\hline $2.434+C C$ & $2.14 z-0 z$ & \\
\hline $2.11 s+0 \mathrm{C}$ & $6.15 t-04$ & \\
\hline 1. $\varepsilon s s+C C$ & $2 \cdot t 7 \varepsilon-04$ & \\
\hline $1.734+00$ & $1.4 \in \varepsilon-04$ & \\
\hline $1 . \in C 4+O C$ & S.2SS-05 & \\
\hline $1.4 \mathrm{Cg}+\mathrm{CC}$ & $4.8 C 5-C 5$ & \\
\hline $1.267+C C$ & $3 . C 27-c 5$ & \\
\hline 1. $\mathrm{C} 2 \mathrm{~S}+\mathrm{CC}$ & $1.435-05$ & $1.582-C 3$ \\
\hline $8.781-\mathrm{Cl}_{1}$ & $\varepsilon . \varsigma \varepsilon 2-C \epsilon$ & $6.357-c 3$ \\
\hline $6.5 C 5-C 1$ & 4. $S \in 2-C E$ & $1.817-c 2$ \\
\hline $5.757-01$ & $3.378-0 t$ & $3 . C 27-02$ \\
\hline $4.971-C_{1}$ & $2.547-0 t$ & $4.163-c 2$ \\
\hline $4: 393-01$ & $2 \cdot C 4 c-06$ & $5.156-02$ \\
\hline $3.553-01$ & $1.455-06$ & $6.942-c 2$ \\
\hline $3 . C 59-01$ & $1.125-06$ & $8.327-02$ \\
\hline $2.2 \in 2-C_{1}$ & $7.223-07$ & $1 . \mathrm{C} 85-\mathrm{C} 1$ \\
\hline $1 . \varepsilon 15-01$ & $5.3 c \in-07$ & $1.282-01$ \\
\hline $1.320-C_{1}$ & $3.4 \epsilon 5-07$ & $1.58 \mathrm{~s}-\mathrm{C} 1$ \\
\hline 1. $C 48-01$ & $2.571-c 7$ & $1.796-C_{1}$ \\
\hline $8.738-C 2$ & $2.044-07$ & $1.940-C_{1}$ \\
\hline $7.523-02$ & $1 . t S t-07$ & 2. $050-c 1$ \\
\hline $5.527-C_{2}$ & $1.2 t \in-07$ & $2.228-\mathrm{C} 1$ \\
\hline 4. $\subseteq 18-02$ & 1. CCS -07 & $2.387-\mathrm{Cl}$ \\
\hline
\end{tabular}




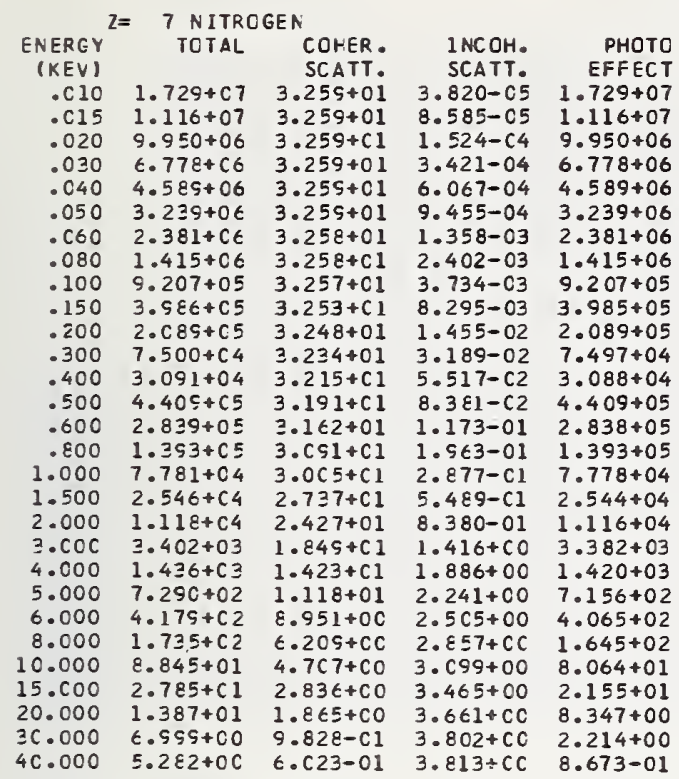

\begin{tabular}{|c|c|c|c|c|}
\hline \multirow{2}{*}{ ENEREY } & 8 GXYGEN & & & \\
\hline & TOTAL & $\begin{array}{l}\text { COFER } \\
\text { SCATT. }\end{array}$ & $\begin{array}{l}\text { INCOH. } \\
\text { SCATT. }\end{array}$ & $\begin{array}{r}\text { PHCTC } \\
\text { EFFECT }\end{array}$ \\
\hline & $4.747+c 7$ & $4.257+C i$ & $2.371-C 5$ & $4.747+07$ \\
\hline .015 & $1.021+07$ & $4.257+01$ & $5.331-C 5$ & $1.021+07$ \\
\hline & 1. $C 4 C+07$ & $4 \cdot 257+01$ & $5.474-05$ & $1.039+07$ \\
\hline $.0 \equiv c$ & $\varepsilon \cdot \varepsilon 71+C \epsilon$ & $4.257+C 1$ & $2.130-c 4$ & $8.871+06$ \\
\hline .040 & $6.552+c 6$ & $4.257+C 1$ & $82-c 4$ & $.592+06$ \\
\hline $.05 C$ & $4.850+C 6$ & $4.257+01$ & $5.5 C 4-c 4$ & $.890+06$ \\
\hline & & & & \\
\hline . 180 & & & $1.5 \mathrm{C} 7=$ & $2.255+06$ \\
\hline .100 & $2+c \epsilon$ & 4.25 & & $1.532+06$ \\
\hline & & & & \\
\hline .200 & & & & +05 \\
\hline & $1.4 \in 6$ & & & $1.466+05$ \\
\hline .400 & & & & \\
\hline $.50 \mathrm{C}$ & & & 5.6 & $3.352+04$ \\
\hline & & & & $4.341+05$ \\
\hline & & & & \\
\hline $1.0 \mathrm{CC}$ & 1.2 & $3.596+01$ & 2.1 & $1.217+05$ \\
\hline & $4 \cdot C$ & 3.70 & & $4.081+04$ \\
\hline 2. & 1.8 & & & $1.834+04$ \\
\hline & & $2.648+01$ & $1.388+$ & $5.772+03$ \\
\hline 4.000 & 2.49 & & & $2.471+03$ \\
\hline & & & & $1.256+03$ \\
\hline & 7.343 & & & $7.184+02$ \\
\hline & & & & $2.937+02$ \\
\hline & & & & $1.452+02$ \\
\hline & & & & \\
\hline & $2.21 \varepsilon$ & $2.615+$ & $4.111+C O$ & $1.545+01$ \\
\hline 30.000 & S. $842+C$ & $1.4 C 3+$ & $4.252+C C$ & $4.147+00$ \\
\hline & $6.8 \approx 2+C C$ & $\varepsilon, € \subsetneq 1-C 1$ & $4 \cdot 320+C C$ & $1.633+00$ \\
\hline
\end{tabular}

\begin{tabular}{|c|c|c|c|c|}
\hline \multicolumn{5}{|c|}{$Z=\varsigma$ FLUCR INE } \\
\hline $\begin{array}{l}\text { ENERGY } \\
\text { (KEV) }\end{array}$ & TOTAL & COHER - & I NCOH. & PHCTC \\
\hline .010 & $1.425+c-7$ & 5.388 & & \\
\hline & $267+07$ & & & \\
\hline & & $300+01$ & & \\
\hline $.0<0$ & $1.0=31+c 7$ & $5.388+01$ & $-1.837-0 \varepsilon$ & $1.531+07$ \\
\hline .030 & $6+07$ & & $-4.148-08$ & $1.226+07$ \\
\hline $.04 \mathrm{C}$ & $8.770+C 6$ & $5.388+01$ & $-7.4 \mathrm{Cl}-08$ & $8.770+06$ \\
\hline .050 & 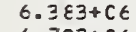 & $5.387+01$ & $-1.161-07$ & $6 \cdot 383+06$ \\
\hline .060 & 4.7 & $+\mathrm{C} 1$ & & $793+06$ \\
\hline .080 & $2.541+0 t$ & $5.386+01$ & $-3 \cdot \mathrm{CO}_{4}-\mathrm{C} 7$ & $2.940+06$ \\
\hline .100 & $1.5 \in 4+06$ & $E .38 E+C 1$ & -4.727 & $1.964+06$ \\
\hline & & & $-1 \cdot c 83$ & $9.022+05$ \\
\hline .200 & 5.0 & $5.377+01$ & $-1.559-06$ & $5.031+05$ \\
\hline .300 & $2.1 C_{1}+05$ & $5 \cdot 362+C 1$ & $-4.55 s-c 6$ & $2.100+05$ \\
\hline & & & & \\
\hline .500 & $6.163+04$ & 5.3 & $-1.351-05$ & $6.157+04$ \\
\hline$\bullet ૯ O C$ & $3 \cdot 743+C 4$ & 5.2 & $-2 \cdot c$ & $.738+04$ \\
\hline & & & & \\
\hline & $1.827+C 5$ & 01 & -6.24 & $1.827+05$ \\
\hline 1.500 & $6.232+C 4$ & 4.756 & -3.22 & $6.255+04$ \\
\hline 2.000 & 2.5944 & $4.408+$ & -2.5 & \\
\hline & $-1.136+04$ & $3.6 C 5+01$ & $-2 . C 50+c 4$ & $5.109+03$ \\
\hline 4.000 & & & 2.40 & \\
\hline & & & 2.30 & \\
\hline & & & 7.9 & 1.176 \\
\hline 000 & $3.724 t$ & $1.3 C E t$ & $3.724+C 7$ & $4.874+02$ \\
\hline & & & 1.01 & $35+02$ \\
\hline & & & & \\
\hline 000 & $1.417+05$ & 3.5 & $1.417+09$ & $2.668+01$ \\
\hline & & & & \\
\hline & & & & \\
\hline
\end{tabular}

\begin{tabular}{|c|c|c|c|c|c|}
\hline ENERGY & TOTAL & & INCOH. & PHOTC & \\
\hline KEVI & & & SCATT. & EFFECT & \\
\hline 50. & $4.553+00$ & $4.058-01$ & $3.768+O C$ & $4.185-01$ & \\
\hline 60. & & $.916-01$ & $3.700+0 C$ & $2.3<7-01$ & \\
\hline 80. & & $1-01$ & $3.549+00$ & $8.547-02$ & \\
\hline 100. & & & $3.403+0 C$ & $4.263-02$ & \\
\hline & $47+00$ & 5.16 & 3. C $C 84+C C$ & $1.127-02$ & \\
\hline 200 & & & $2.834+00$ & $4.515-03$ & \\
\hline & & & $2.465+00$ & $1.3 C 5-03$ & \\
\hline & & 7.5 & $2.214+00$ & 5.721 & \\
\hline & & & c & & \\
\hline 60 & & $3.392-$ & 1.870 & 1.980 & \\
\hline & 1.645 & 1.9. & 1.6 & 1.0 & \\
\hline & & & & & \\
\hline & $1.2 \mathrm{C}$ & 5.48 & $1.2 \mathrm{C} 1+O \mathrm{C}$ & $\epsilon-C 5$ & $2.125-0$ \\
\hline & & 3.08 & 1. $C 24+00$ & -05 & \\
\hline & & & & & \\
\hline 400 & 7.11 & 7.74 & c. $716-\mathrm{cl}$ & $7.15 C-0 E$ & 3. \\
\hline & & 4.99 & $9-\mathrm{Cl}$ & & \\
\hline & & 3.42 & & $4.31 \mathrm{~s}$ & \\
\hline 8000 & 5.11 & 1.9 & 4.1 & $3.081-06$ & 9.12 \\
\hline & & & & & \\
\hline & $4.137-01$ & $5.515-c$ & $2.635-01$ & $1.53 c-$ & \\
\hline & $3.86 t-c 1$ & $3.102-06$ & $2.117-01$ & $1.124-06$ & $1.749-$ \\
\hline & & 1.37 & 1.5 & & $2.104-$ \\
\hline & & $7.758=0$ & & $5.45 \mathrm{C}-$ & \\
\hline 50000 & $3.552-01$ & $4.965-0$ & 1.C19-01 & $4.3 \geq 3-$ & $2.573-$ \\
\hline & & 3.448 & $7-c 2$ & $c \in-C 7$ & $2.737-$ \\
\hline & & & & -07 & \\
\hline & & & & $2.135-07$ & \\
\hline
\end{tabular}

\begin{tabular}{|c|c|c|c|c|c|}
\hline $\begin{array}{l}\text { ENEREY } \\
\text { (KEV) }\end{array}$ & TCTAL & $\begin{array}{l}\text { COHER } \\
\text { SCATT. }\end{array}$ & $\begin{array}{l}\text { INCOH. } \\
\text { SCATT, }\end{array}$ & $\begin{array}{l}\text { PHOTO } \\
\text { EFFECT }\end{array}$ & $\begin{array}{l}\text { PAIR } \\
\text { PRCD. }\end{array}$ \\
\hline & $5.65 s+00$ & $5.895-\mathrm{Cl}$ & $4.275+0 C$ & 7. $51 \mathrm{c}-01$ & \\
\hline 60. & 5. $c \in \varepsilon+00$ & $4.255-01$ & $4.2 \mathrm{C} 6+0 \mathrm{C}$ & $4.363-01$ & \\
\hline 80. & $4.464+00$ & $2.512-01$ & $4 . C 43+00$ & $1.6 \subseteq 4-01$ & \\
\hline 100. & $4.127+00$ & $1.654-01$ & 3. $\varepsilon 80+c c$ & 8. C8 $8-02$ & \\
\hline 150. & $3.619+00$ & $7.640-02$ & $3.521+C 0$ & $2.14 C-02$ & \\
\hline 200. & $3.2 \varepsilon s+00$ & $4.380-02$ & $3.237+C C$ & $\varepsilon .5 \equiv 4-03$ & \\
\hline 300. & $2.843+00$ & $1.984-02$ & $2 . \varepsilon 21+0 \mathrm{C}$ & $2.455-03$ & \\
\hline 400. & $2.542+00$ & $1.127-02$ & $2.530+00$ & $1.072-03$ & \\
\hline 500. & $2.318+00$ & $7.252-03$ & $2.310+c C$ & $5.53 t-04$ & \\
\hline 600 . & $2.143+O C$ & $5.055-03$ & $2.137+0 \mathrm{C}$ & $3.791-04$ & \\
\hline 800 & $1.881+00$ & $2.857-03$ & 1. $\varepsilon 78+O C$ & $1.970-04$ & \\
\hline 1000 & $1.650+00$ & $1.834-03$ & $1.688+0 C$ & $1.235-04$ & \\
\hline 1500 & $1.376+00$ & $8.180-04$ & $1.372+O C$ & $5.835-05$ & $2.792-C_{3}$ \\
\hline 2000 . & $1.182+00$ & $4.610-04$ & $1.171+C C$ & $3.64 C-05$ & $1.116-C^{2}$ \\
\hline 3000 . & $9.528-C 1$ & $2.053-04$ & $9.2 C 6-C 1$ & 2.CC4-C5 & $3.158-C^{2}$ \\
\hline 4000 & $8.21 \mathrm{C}-01$ & $1.156-04$ & $7.676-01$ & $1.362-05$ & $5.332-02$ \\
\hline & $7.3 \in 1-\mathrm{Cl}$ & $7.401-05$ & $6.627-0 I$ & $1.027-05$ & $7.329-C 2$ \\
\hline $60 \mathrm{co}$. & $6.771-01$ & $5.142-05$ & $5.857-01$ & $\varepsilon .22 \epsilon-c \sigma$ & $9.134-02$ \\
\hline 8000 & $6 . C 0 t-01$ & $2.894-05$ & $4.750-C 1$ & $5 . \varepsilon \in 5-C 6$ & $1.21 \epsilon-\mathrm{Cl}$ \\
\hline & $5.531-01$ & $1.852-05$ & 4. $C 78-01$ & $4.557-06$ & $1.452-01$ \\
\hline 15000. & $4.885-C 1$ & $8.236-0 t$ & 3.c16-01 & $2.515-0 t$ & $1.873-\mathrm{Cl}$ \\
\hline 000 & $4.620-01$ & $4.634-06$ & $2.419-01$ & $2.14 t-06$ & $2.200-c 1$ \\
\hline & & $2.060-06$ & $1.759-01$ & $1.4<2-06$ & $2.724-\mathrm{Cl}$ \\
\hline & $4.47 t-01$ & $1.159-06$ & $1.357-01$ & $1.041-06$ & 3. $\mathrm{C} 79-\mathrm{Cl}$ \\
\hline 50000 . & $4.488-C 1$ & $7.416-07$ & $1.1 \in 5-C_{1}$ & $\varepsilon .27 \varepsilon-c 7$ & $3.323-C 1$ \\
\hline & $4.512-c 1$ & $5.150-07$ & $1 . C 03-01$ & $6.871-07$ & $3.508-01$ \\
\hline & & $2.897-07$ & & $5.127-07$ & $3.803-\mathrm{Cl}$ \\
\hline & $4.723-01$ & $1.854-07$ & $6.557-02$ & $4.0 \subseteq c-c 7$ & 4. $\mathrm{C} 68-\mathrm{Cl}$ \\
\hline
\end{tabular}

INCCH . SCATT $2.726+1 \mathrm{C}$ $2 \cdot 726+1 C$
$4.727+1 C$ $4.654+O C$ $4.434+0 C$ $3.554+0 C$

$3.661+00$ $3.183+C C$ $2.851+C C$ $2.6 C 2+0 C$ $2.4 C 7+00$ $2.114+00$ $1.5 C O+O C$ $1.544+C C$ $1.317+0 C$ ع.636-0 $7.456-01$ $7.456-01$
$6.585-C 1$ $6.585-C 1$
$5.389-01$ $4.5 \varepsilon 8-C 1$ 3.353-01 2.722-01 1.579-01 1.571-C1 $1.311-c 1$ 1. 129-01 $8.891-C 2$ 7.377-02
PHCTO

EFFECT $1.384+C C$ $7.644-01$
$2.572-01$ $1.41 s-01$ 3. $772-\mathrm{C} 2$ 1. 5 I 4-02 4. $358-03$ 1. $528-03$ 1. $\left\{\in 3-C_{3}\right.$ 6.745-04 $3.475-04$ 2.175-04 1. $023-0$ $6.37 t-05$ 3.512-05 $2.35 C-05$ 1. $8 \mathrm{C} 4-05$ $1.445-05$ 1. $032-05$ E.c21-06 5.142-06 3. $7 \& 2-C E$ 2. $473-0$ $1.837-06$ $1.461-06$ $1.213-0$ 9.051-07 $7.22 \mathrm{C}-07$ PRCE.

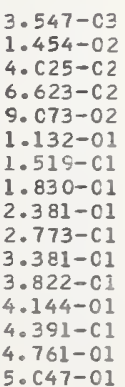




\begin{tabular}{|c|c|c|c|c|}
\hline \multicolumn{5}{|c|}{$Z=10 \mathrm{NEON}$} \\
\hline ENEREY & TOTAL & COHER. & $1 \mathrm{NCOH}$. & PHOTO \\
\hline (KEV) & & & & \\
\hline & $t \cdot t 52+01$ & $6.652+01$ & $1.565-c 5$ & 0.000 \\
\hline & t. $652+01$ & $c .652+c 1$ & & \\
\hline & $7.28 S+0 E$ & $6.652+01$ & $6.263-05$ & $7.288+06$ \\
\hline .030 & $75+C t$ & $6.652+01$ & & $8.875+06$ \\
\hline & $1+06$ & 6.65 & & +06 \\
\hline - $C 50$ & $75+c 6$ & 6.6 & & +06 \\
\hline .060 & $6.632+06$ & $\epsilon \cdot \epsilon$ & .04 & 6.6 \\
\hline .080 & $4 \cdot 550+c t$ & 6.6 & 1. $\mathrm{Cr}$ & 06 \\
\hline .100 & $t+C t$ & 6.64 & 1. & +06 \\
\hline .150 & $92+c 6$ & $6.645+0$ & -13 & $1.5 s_{2}+06$ \\
\hline .200 & $69+05$ & 6.64 & -03 & +05 \\
\hline .300 & & $t \cdot t_{2}$ & 1 & +05 \\
\hline .400 & & 6.60 & & \\
\hline .500 & 05 & 6.57 & & +05 \\
\hline & & $\epsilon .5$ & & .04 \\
\hline & & 6.4 & & 04 \\
\hline & & 6.3 & 1 & 05 \\
\hline 1.500 & 9.0 & 6.0 & & +04 \\
\hline $2 . c 00$ & $4.154+C 4$ & 5.62 & 6.4 & +04 \\
\hline $3 . c 00$ & $1.363+04$ & 4.72 & 1.2 & +04 \\
\hline 4.000 & $6.0 \mathrm{CS}+\mathrm{C3}$ & 3.85 & 1.842 & $5.969+03$ \\
\hline 5.00 & $3.140+03$ & 3.16 & 2.3 & $3.106+03$ \\
\hline & $35+03$ & 2.5 & 2. & $7+03$ \\
\hline & $7.793+02$ & $1.813+$ & 3.4 & 7.577 \\
\hline & $3.993+02$ & $1.336+C 1$ & $3.575+C C$ & $3.819+02$ \\
\hline & $1.1 \varsigma 5+02$ & $7.4 \in 1+C 0$ & $4.618+00$ & $1.074+02$ \\
\hline & $5.279+01$ & 4. $\varepsilon 47+C C$ & $4.545+C C$ & $4.300+01$ \\
\hline & $56+01$ & $2.584+\mathrm{CO}$ & $8+0 C$ & $1.175+01$ \\
\hline & $5 t+C 1$ & 1. $5 s t+C C$ & $5.3 C C+C C$ & $4 \cdot 662+0$ \\
\hline
\end{tabular}

\begin{tabular}{|c|c|c|}
\hline \multicolumn{3}{|c|}{$2=11$ SODIUM } \\
\hline $\begin{array}{r}\text { ENERGY } \\
\text { (KEV) }\end{array}$ & TOTAL. & $\begin{array}{l}\text { COHER } \\
\text { SCATT. }\end{array}$ \\
\hline .010 & $8.32 s+c 4$ & 8.C4S+C1 \\
\hline . C I5 & $1.059+05$ & $\varepsilon . C 4 c+c 1$ \\
\hline .020 & S. $343+C 4$ & $8.048+C 1$ \\
\hline .030 & $6.158+04$ & $\varepsilon . C 48+01$ \\
\hline .040 & $8 \cdot t 18+0 t$ & $8 . C 47+01$ \\
\hline .050 & $8.430+C 6$ & $8 \cdot C 46+C 1$ \\
\hline.$c \in 0$ & $7.3 c t+c 6$ & $8.045+01$ \\
\hline .080 & $5.175+C 6$ & $8.042+01$ \\
\hline .100 & $3.723+c t$ & $8 . c 38+01$ \\
\hline .150 & $1.877+06$ & $8.024+01$ \\
\hline .200 & $1.100+0 t$ & $\varepsilon \cdot c c t+c 1$ \\
\hline .300 & $4.905+05$ & $7.555+C 1$ \\
\hline .400 & $2.657+C 5$ & $7.885+c 1$ \\
\hline .500 & 1. $606+05$ & $7.805+C 1$ \\
\hline $.60 \mathrm{C}$ & $1.040+05$ & $7.715+C 1$ \\
\hline $.80 \mathrm{C}$ & $4.510+C 4$ & $7.515+C 1$ \\
\hline 1.000 & $2.505+04$ & $7.298+01$ \\
\hline 1.500 & $1.271+05$ & $6.774+C 1$ \\
\hline 2.000 & $64+C 4$ & $.282+01$ \\
\hline 3.000 & $1.552+04$ & $5 \cdot 361+01$ \\
\hline 4.000 & $8.578+c 3$ & $4.517+C 1$ \\
\hline $5 . \mathrm{CCC}$ & $4.485+c 3$ & $3.778+C 1$ \\
\hline 6.000 & $2 \cdot \in 27+03$ & $3.166+C 1$ \\
\hline $8 \cdot C 00$ & $1.1<2+03$ & $2.268+C 1$ \\
\hline 10.000 & $5.783+02$ & $1.675+01$ \\
\hline $15.0 \mathrm{CC}$ & $1.746+C 2$ & $9 \cdot 387+C 0$ \\
\hline 20.000 & $7.681+01$ & $6.160+00$ \\
\hline $30 \cdot c 00$ & $2.712+C 1$ & $3.274+C 0$ \\
\hline $4 \mathrm{C} .000$ & $1.503+C 1$ & 2. $C 17+C C$ \\
\hline
\end{tabular}

\begin{tabular}{|c|c|}
\hline $\begin{array}{r}\text { ENERG } \\
\text { (KEV }\end{array}$ & TOTAL \\
\hline 50 & $8.621+00$ \\
\hline 60 & $7.235+00$ \\
\hline 80 & $5 . S \in 2+00$ \\
\hline 100 & $5.36 \mathrm{C}+00$ \\
\hline 150 & $4.552+00$ \\
\hline 200 & $4.144+00$ \\
\hline 300 & $3.567+00$ \\
\hline 400 & $3.184+00$ \\
\hline 500 & $2.502+00$ \\
\hline 600 & $2.682+00$ \\
\hline 800 & $2.353+00$ \\
\hline 1000 & $2.114+00$ \\
\hline 1500 & $1.721+00$ \\
\hline 2000 & $1.482+00$ \\
\hline 3000 & $1.201+00$ \\
\hline 4000 & $1.043+00$ \\
\hline 5000 & $9.42 t-01$ \\
\hline 6000 & $8.740-01$ \\
\hline 8000 & $7.8 \in 4-01$ \\
\hline 10000 & $7.328-\mathrm{Cl}$ \\
\hline 15000 & $6.625-01$ \\
\hline 20000 & $6.375=01$ \\
\hline 30000 & $6.35 \mathrm{c}-\mathrm{Cl}$ \\
\hline 40000 & $6.447-01$ \\
\hline 50000 & $6.524-01$ \\
\hline 60000 & $6.556-01$ \\
\hline 80000 & $6.76 \varsigma-\mathrm{C} 1$ \\
\hline 100000 & $7.001-01$ \\
\hline
\end{tabular}

\begin{tabular}{|c|c|}
\hline $\begin{array}{r}\text { ENEREY } \\
\text { (KEV) }\end{array}$ & TCT AL \\
\hline 50. & 1. $064+01$ \\
\hline 60. & $8.605+00$ \\
\hline 80. & $6.82 t+C 0$ \\
\hline 100. & $6.032+00$ \\
\hline 150. & $5.0 \leqq 4+00$ \\
\hline 200 . & $4.57 s+c 0$ \\
\hline 300 . & $3.9 \geq 1+00$ \\
\hline 400. & $3.5 \mathrm{ct}+00$ \\
\hline 500. & $3.155+00$ \\
\hline 600. & $2 . \$ 51+00$ \\
\hline 800. & $2.585+\mathrm{Co}$ \\
\hline 1000. & $2.326+c 0$ \\
\hline 1500. & $1.854+0 C$ \\
\hline 2000 . & $1.633+00$ \\
\hline 3000 . & $1 \cdot 327+00$ \\
\hline 4000. & $1.155+0 \mathrm{C}$ \\
\hline 5000. & $1.047+00$ \\
\hline 6000. & $S .739-C 1$ \\
\hline 8000 . & $8.846-01$ \\
\hline 10000 & $8.331-01$ \\
\hline 15000 & $7.653-01$ \\
\hline 20000 . & $7.444-01$ \\
\hline 30000. & $7.40 \geq-01$ \\
\hline 40000 & $7.533-01$ \\
\hline 50000 & $7.678-01$ \\
\hline 60000 & $7.814-\mathrm{Cl}$ \\
\hline 80000 . & $8.060-01$ \\
\hline & $8.251-C_{1}$ \\
\hline
\end{tabular}

COHER.
SCATT.
$1.080+00$
$7.787-01$
$4.588-01$
$3.019-C 1$
$1.392-01$
$7.977-02$
$3.611-02$
$2.050-02$
$1.319-02$
$9.194-03$
$5.195-03$
$3.334-03$
$1.487-03$
$8.381-04$
$3.722-04$
$2.101-04$
$1.345-04$
$9.346-05$
$5.259-05$
$3.367-05$
$1.497-05$
$8.422-06$
$3.744-06$
$2.106-06$
$1.348-06$
$9.361-07$
$5.266-07$
$3.370-07$

INCOH.
SCATT.
$5.276+C C$
$5.2 C 6+C C$
$5 . C 17+C C$
$4.826+C C$
$4.351+O C$
$4 . C 4 C+C C$
$3 \cdot 524+C C$
$3.161+C C$
$2.887+O C$
$2.671+O C$
$2.347+C C$
$2.11 C+C C$
$1.715+C C$
$1.463+0 C$
$1.151+C C$
$9.555-01$
$8.284-01$
$7.321-01$
$5.588-01$
$5.0 S 8-01$
$3.770-01$
$3 . C 24-01$
$2.15 S-C 1$
$1.746-O 1$
$1.456-01$
$1.254-01$
$5.875-C 2$
$8.156-02$

PHCTO EFFEC T

$2.265+00$

1. $25 \mathrm{C}+0 \mathrm{C}$

4. $\varepsilon 57-C 1$

$6.2 \mathrm{C} 2-02$

2. 4 ऽ -02

$7.2 \in 8-03$

$3.1 \in 2-03$

$1.077-03$

5. $54 \varepsilon-04$

3. $5 \mathrm{CE}-\mathrm{C}$

1. $\in \varepsilon 3-C$

1. $c \in 2-04$

5. $\$ 1 \epsilon-05$

4. $035-05$

3. $C 4 \varepsilon-C$

2. $441-05$

$1.74 c-05$

$8.625-06$

$6.331-06$

4. $13 C-C E$

3. $063-06$

2. 434-06

$2.02 \mathrm{C}-06$

$1.5 \mathrm{C} E-\mathrm{C}$

1. $2 \mathrm{C} 1-0 \mathrm{t}$
PAIF
PRCE.

4. 401-03

1. $748-C 2$

4. $555-C 2$

8. 317-C 2

1. $417-01$

$1.876-C_{1}$

2. 229-C 1

2. $855-\mathrm{Cl}$

3. 351-01

$4.159-C 1$
$4.701-C 1$

5. $c 68-C 1$

5. 342-01

$5.781-C 1$

$6.181-C 1$

\begin{tabular}{|c|c|c|c|c|}
\hline $\begin{array}{r}\text { ENEREY } \\
\text { (KEV) }\end{array}$ & TOTAL & $\begin{array}{l}\text { COHER. } \\
\text { SCATT. }\end{array}$ & $\begin{array}{l}\text { INCOH. } \\
\text { SCATT. }\end{array}$ & $\begin{array}{r}\text { PHCTC } \\
\text { EFFECT }\end{array}$ \\
\hline . $C 10$ & $3.602+04$ & c. $579+C 1$ & 1. $C 58-c 4$ & $3.593+04$ \\
\hline .015 & 1. $C 18+C 5$ & $c .579+C 1$ & & $1.017+05$ \\
\hline .020 & 1. $642+05$ & $S .578+C 1$ & 4.21 & 05 \\
\hline .030 & $24+C$ & $S .578+c$ & $9.443-04$ & 1.834 \\
\hline .040 & 1. $67 \varepsilon+05$ & S. $577+c$ & $1 . \in 7$ & 1.6 \\
\hline .050 & $1.490+C 5$ & $S .575+C 1$ & $2 . \in C 2-C 3$ & $1.489+05$ \\
\hline .060 & 8. $C E 3+0 t$ & $5.574+01$ & $3.732-C 3$ & $8 . C 53+06$ \\
\hline .080 & t. $\$ 43+0 t$ & $5.570+$ & $D-c 3$ & $+3+06$ \\
\hline .10 & 5. & & & \\
\hline & $31+$ & & & \\
\hline .200 & $1.530+06$ & S. $525+c$ & 3.51 & +06 \\
\hline .200 & $6.772+C 5$ & $5.461+C$ & 8.41 & 6.7 \\
\hline .400 & $3.678+05$ & S. $377+01$ & $1.429-\mathrm{C} 1$ & $3.677+05$ \\
\hline .500 & $2.245+C 5$ & S. $276+C 1$ & $2.127-01$ & $2.244+05$ \\
\hline .600 & 1. & 9.16 & 2 . & +05 \\
\hline & & & & \\
\hline & 3. & & & \\
\hline & 1. & 7.51 & 1.0 & \\
\hline 2.000 & 7. & $7.241+$ & 1.33 & +04 \\
\hline 3.000 & $2.656+0$ & $6.068+C 1$ & 1.83 & $2.650+04$ \\
\hline 4.000 & $1.181+0$ & $5.137+01$ & $2 \cdot 30$ & $1 \cdot 176+04$ \\
\hline 5. & $6.231+0$ & $4.384+0$ & 2.75 & $6.184+03$ \\
\hline $6 . \mathrm{coc}$ & $3.675+0$ & $3.75 C+C 1$ & $3.188+$ & $3.634+03$ \\
\hline 8.000 & $1.587+0$ & $2.761+C 1$ & $3.942+$ & $1.555+03$ \\
\hline 10.000 & e. $244+0$ & Z. CE & $4.5 \mathrm{C7t}$ & $2+02$ \\
\hline & & & & \\
\hline & $1.0 s s+c 2$ & $7 \cdot \in C C+C C$ & & $9.656+01$ \\
\hline & $28+01$ & $4.022+00$ & 6.14 & $2.712+01$ \\
\hline $0 \Omega 0$ & $58+C 1$ & $2.4 \epsilon \varepsilon+C 0$ & $6.251+C C$ & $1.086+01$ \\
\hline
\end{tabular}

\begin{tabular}{|c|c|}
\hline $\begin{array}{l}\text { COHER . } \\
\text { SCATT. }\end{array}$ & $\begin{array}{l}\text { 1NCOH. } \\
\text { SCATT. }\end{array}$ \\
\hline $.363+00^{\circ}$ & 5. $7 \in C+C C$ \\
\hline $.814-01$ & $5.652+C C$ \\
\hline $.775-\mathrm{cl}$ & $5.5 C C+C O$ \\
\hline $.797-01$ & $5.255+0 \mathrm{C}$ \\
\hline $.749-01$ & 4. $\varepsilon 23+C C$ \\
\hline $.001-01$ & $4.44 C+00$ \\
\hline $.530-c 2$ & $3.875+0 \mathrm{C}$ \\
\hline $.571-c 2$ & $3.476+0 \mathrm{C}$ \\
\hline $.654-02$ & $3.175+0 \mathrm{C}$ \\
\hline & $2.538+0 \mathrm{C}$ \\
\hline $.513-03$ & $2 \cdot 5 \varepsilon 1+C C$ \\
\hline $.179-03$ & $2.321+0 \mathrm{C}$ \\
\hline $.864-03$ & 1. $\varepsilon \varepsilon 7+D C$ \\
\hline $.050-03$ & $1 . \in 10+O C$ \\
\hline $.677-04$ & $1.266+00$ \\
\hline & $1 . C 55+0 C$ \\
\hline $.686-04$ & $9.113-01$ \\
\hline $.171-04$ & $\varepsilon . C 53-01$ \\
\hline & $\epsilon=587-01$ \\
\hline $.215-05$ & $5 . \in C 8-01$ \\
\hline $.876-05$ & $4.147-01$ \\
\hline $.055-05$ & $3.227-01$ \\
\hline $.691-06$ & $2.419-01$ \\
\hline $.639-06$ & $1.521-01$ \\
\hline & $1.602-01$ \\
\hline $.173-06$ & $1.279-01$ \\
\hline $.599-07$ & $1.087-01$ \\
\hline & $5 . C 1 t-0 z$ \\
\hline
\end{tabular}

FHOTO

EFFEC

$3.515+0 C$

$1.537+00$

7. $4 \& 4-01$

$3.57 C-01$

S. $\in$ C1-02

3. 5 CS -02

$1.1 \leq 8-02$

$5.077-03$

$2.744-03$
$1.7 C 5-03$

$\varepsilon .74 \varepsilon-C 4$

5. $51 \varepsilon-04$

2.651-04

1.677-04

c. $354-05$

t. $38 \subseteq-05$

$4.823-C 5$

$3.862-05$

$2.753-05$

$2.135-05$

$1.364-05$

1.CC1-C5

t.524-06

$4 . \varepsilon \geq 5-C 6$

2. $845-06$

$3.1 \varepsilon \varsigma-0 t$

$2.375-06$
$1.85 t-C 6$

\begin{tabular}{|c|c|c|}
\hline ENERGY & TETAL & COHER - \\
\hline & & \\
\hline 50. & $1 \cdot 320+01$ & \\
\hline 60 . & $2 s+01$ & $1.196+00$ \\
\hline 80 & $811+00$ & $7.019-01$ \\
\hline 100. & $6.764+00$ & $4.608-01$ \\
\hline 150. & $5.610+00$ & $2.118-01$ \\
\hline 200 . & $5 . C 17+00$ & $1.212-01$ \\
\hline 300 . & $4.295+00$ & $5.476-02$ \\
\hline 400. & $3.82 \varepsilon+c 0$ & $2.10 \epsilon-02$ \\
\hline 500. & $3.48 t+00$ & $1.998-02$ \\
\hline 600. & $3.22 \mathrm{C}+00$ & $1.392-02$ \\
\hline 800. & $2.825+00$ & $7.862-03$ \\
\hline & & \\
\hline 1500 . & $2.067+00$ & $2.249-03$ \\
\hline 2000 . & $1.7 \varepsilon 3+00$ & $1.267-03$ \\
\hline & $1.454+$ & $5.642-04$ \\
\hline 4000 . & $1.271+00$ & $3.17 t-04$ \\
\hline 5000 & $1.158+0 C$ & $2.034-04$ \\
\hline & & $1.413-04$ \\
\hline 8000 . & $9.868-$ & $7.950-05$ \\
\hline 1000 & 9.29 & $5.090-05$ \\
\hline & & $2.263-05$ \\
\hline & 27 & $1.273-c$ \\
\hline & $8.5 C 5-c$ & $5.659-0 t$ \\
\hline & $8.722-c$ & $3.183-06$ \\
\hline & $8.89 c-c$ & $2.037-06$ \\
\hline & S.C34-c & $1.415-06$ \\
\hline & הכני & $7.959-07$ \\
\hline & & \\
\hline
\end{tabular}

100000 .

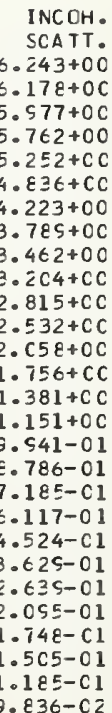

FHCTO EFFECT E. $2 \subseteq 1+O C$ $2.52 \mathrm{C}+00$ $1.132+00$ 5.417-01 $1.45 \subseteq-01$ $5.525-02$ 1.744-02 $7.644-03$ $4.155-03$ $2 . \in C \varepsilon-03$ $1.340-03$ $\varepsilon .44 C-04$ $2.536-04$
$1.4 C 5-04$ 5. $615-05$ 7.257-05 5.813-05 $4.146-05$ 3.217-05 2.C $57-05$ 1.511-05 5. $855-06$ 7.315-06 5.814-C6 4. 824-06 $3.555-06$ 2. $87 \mathrm{C}-06$
$5.357-\mathrm{C}^{3}$ $2.2 \mathrm{C3}-\mathrm{C} 2$ c. $883-c 2$ $1.351-C 1$ 1. $684-C$ 2. 258-01 3.545-01 $4.118-\mathrm{Cl}$ $4.584-C 1$
$5.612-C 1$ 6.076-01 6.435-01 6. $574-01$ $7.350-C 1$
PAIR
PRCL

$6.420-C 3$

$2.543-C 2$

$7.218-02$

$1.196-C 1$
$1.636-C 1$

2. $030-01$

2.681-01

3.180-01

$4 . C 54-C 1$
$4.740-C 1$

5. $8 \in 6-C 1$

6.626-01

$7.143-\mathrm{Cl}$

7. $530-01$

8.146-01 


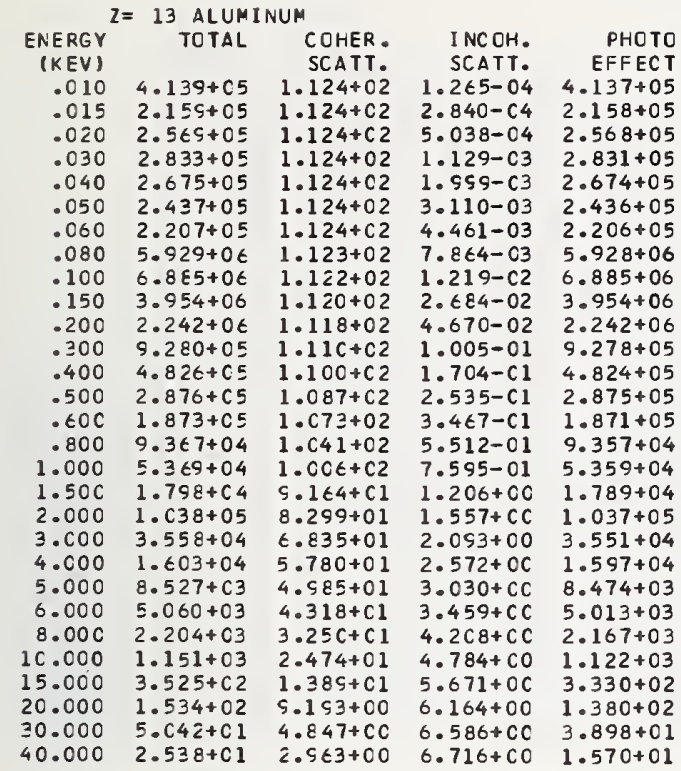

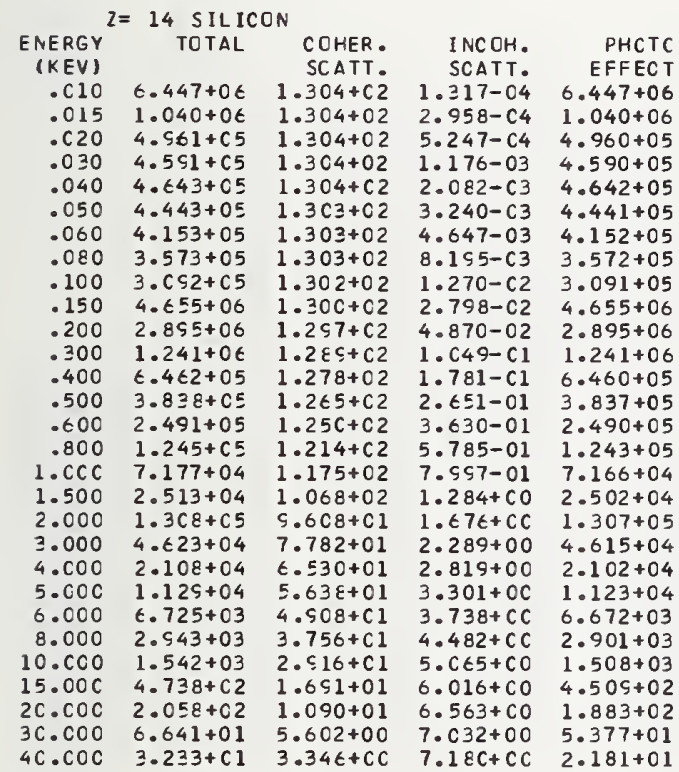

\begin{tabular}{|c|c|c|}
\hline $\begin{array}{l}\text { ENEREY } \\
\text { (KEV) }\end{array}$ & TCTAL & $\begin{array}{l}\text { COHER . } \\
\text { SCATT. }\end{array}$ \\
\hline 50. & 2. $\mathrm{C}_{14}+\mathrm{O} 1$ & $\begin{array}{l}\text { SCATT. } \\
2.212+00\end{array}$ \\
\hline 60 . & $1.4 \epsilon 8+01$ & $1.568+00$ \\
\hline 80. & $1.018+01$ & $9.033-01$ \\
\hline 100. & $8.4 \mathrm{C6}+00$ & $5.861-01$ \\
\hline & $6.682+00$ & $2.651-01$ \\
\hline 200 . & $5.5 C 7+00$ & $1.504-01$ \\
\hline & $5.026+00$ & $6.740-02$ \\
\hline & $4.472+0 C$ & $3.807-c 2$ \\
\hline 500. & $4.071+c 0$ & $2.442-C 2$ \\
\hline 600 . & $3.7 \in C+00$ & $1.699-02$ \\
\hline 800 . & $3.257+00$ & $9.574-03$ \\
\hline 1000. & $2 . s \in 1+00$ & $6.135-03$ \\
\hline 1500 . & $2.413+00$ & $2.731-03$ \\
\hline 2000 . & 2. CE $6+00$ & $1.537-03$ \\
\hline 3000 . & $1.71 C+C C$ & $6.838-04$ \\
\hline 4000 . & $1.5 \mathrm{Co}+\mathrm{co}$ & $3.848-04$ \\
\hline 5000 . & $1 . \lambda \varepsilon 1+C C$ & $2.463-04$ \\
\hline 6000 . & $1.295+00$ & $1.711-04$ \\
\hline 8000 . & $1.2 C_{1}+O C$ & $9.625-05$ \\
\hline 10000 . & $1.145+00$ & $6.161-05$ \\
\hline 15000. & 1.c $78+0 c$ & $2.739-05$ \\
\hline & $1.065+00$ & $1.541-05$ \\
\hline & 1. $\operatorname{cs} 7+00$ & $6.848-06$ \\
\hline 40000 & $1.135+0 \mathrm{C}$ & $3.852-06$ \\
\hline & $1.1 \in 5+O C$ & $2.465-06$ \\
\hline & $1.18 S+0 C$ & $1.712-06$ \\
\hline 80000 . & $1.233+00$ & $5.630-07$ \\
\hline & $1.279+00$ & $6.164-07$ \\
\hline
\end{tabular}
100000 .

\begin{tabular}{|c|c|}
\hline $\begin{array}{l}\text { ENERGY } \\
\text { (KEV) }\end{array}$ & TCTAL \\
\hline 50. & $1 \cdot t 4 C+01$ \\
\hline 60. & $\begin{array}{l}1.23 t+01 \\
8.955+00\end{array}$ \\
\hline 100. & $7.573+00$ \\
\hline 150. & $6.151+00$ \\
\hline 200 . & $5.469+00$ \\
\hline 300. & $4.665+00$ \\
\hline 400. & $4.153+00$ \\
\hline 500. & $3.781+00$ \\
\hline 600 . & $3.492+00$ \\
\hline 800. & 3. $c \in 2+00$ \\
\hline 1000 . & $2.750+00$ \\
\hline 1500. & $2.24 C+00$ \\
\hline 2000 . & $1.534+00$ \\
\hline 3000 . & $1.582+C C$ \\
\hline 4000 . & $1.389+00$ \\
\hline 5000 . & $1.270+0 C$ \\
\hline 6000 . & $1.1 S 1+O C$ \\
\hline 8000 . & $1.053+00$ \\
\hline 10000 . & $1.034+00$ \\
\hline 15000. & $9 . \in 00-01$ \\
\hline 20000 . & $5.437-01$ \\
\hline 30000 . & $9.722-\mathrm{Cl}$ \\
\hline 40000. & $1.0 C 4+0 \mathrm{C}$ \\
\hline & $1.025+00$ \\
\hline 60000 . & $1.042+00$ \\
\hline 80000 & $1.079+00$ \\
\hline
\end{tabular}
1. 99 CATT $1.429+00$ 8.372-01 5. $488-01$ 2.518-01 $1.439-01$ 6.495-02 3.682-02 2.368-02 1.649-02 $9.313-03$ 5.974-03 2.501-03 6.679-04 $3.760-04$ 2.408-04 1.672-04 9.411-05 6.024-05 $2.678-05$ $1.507-C_{5}$ 6.698-0t $3.768-0 t$ 2.412-06 $1.675-06$ 9.421-07 6.030-07

INCOH.
SCATT.
$6.720+C C$
$6.664+0 C$
$6.454+0 C$
$6.227+O C$
$5.684+0 C$
$5.238+0 C$
$4.575+C C$
$4.105+00$
$3.751+C C$
$3.471+0 C$
$3 . C 50+0 C$
$2.743+C C$
$2.229+C C$
$1.5 C 2+0 C$
$1.456+0 C$
$1.247+C C$
$1 . C 77+0 C$
$5.51 E-C 1$
$7.784-01$
$6.627-01$
$4.901-01$
$3.531-01$
$2.85 S-01$
$2.27 C-01$
$1.853-01$
$1.630-01$
$1.284-01$
$1 . C E 6-C 1$

1. $C \in t-C 1$
PHCTO 7. $E \& S+00$ $4.264+00$ $1.664+00$ 7. $S E C-01$ 2.1 $51-C 1$ $\varepsilon .729-02$ $1.124-02$ $t .11 t-03$ 2. $8 \equiv 3-03$ 1. $5 t \in-03$ $1.23 t-03$ $5.8 \varepsilon \mathrm{C}-04$ $3.6 \$ 7-04$ 2.053-04 $1.4 \mathrm{C} 1-\mathrm{C}_{4}$ $1.058-04$ $8.475-05$ 6. $C 4 \varepsilon-05$ $4 . t \subseteq 4-05$ $3.004-05$ $2.2 \mathrm{CE}-\mathrm{C} 5$ $1.44 C-C 5$ 1. $C E S-C 5$ $8.45 \varepsilon-06$ 7. $C 52-06$ 4.1 SE-CE
PAIF

$7.509-\mathrm{C} 3$

$8.472-C 2$

$1.410-\mathrm{C} 1$

1. $929-C 1$

. $391-01$

$3.145-C 1$

4.698-C

5.5C5-C 1

$6.863-C$

$7.766-C 1$
$8.361-C 1$

$8.801-C 1$

$9.508-01$

1. $\mathrm{C} 17+\mathrm{CC}$

$Z=15$ PHOSPHCRUS

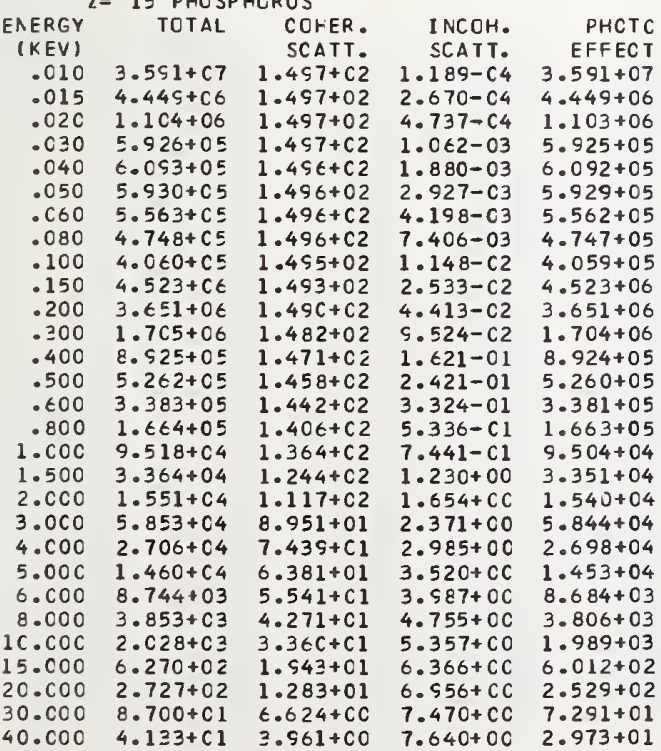

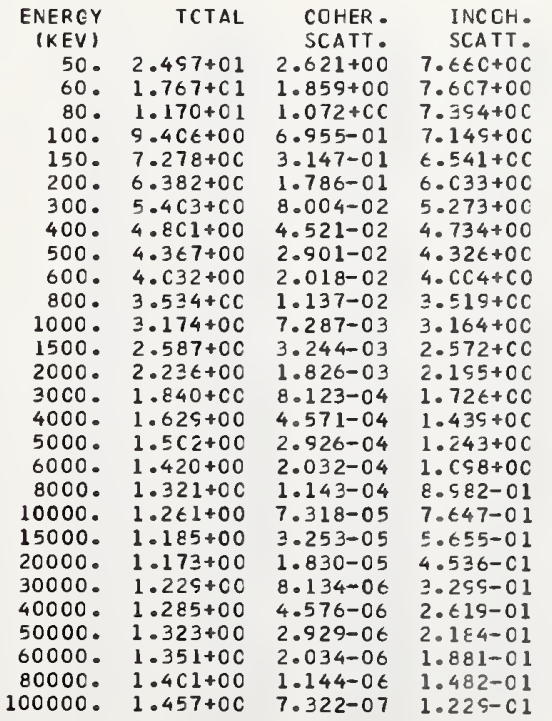

FHOTO FHOT $\begin{array}{ll}7.1 \varepsilon S+C C & 1 . C 7 z+01 \\ 7.121+00 & 5.97 \varepsilon+0 C\end{array}$ $\begin{array}{ll}7.121+00 & 5.57 \varepsilon+0 C \\ 6.525+0 C & 2.34 \varepsilon+00\end{array}$ $6.690+0 \mathrm{C} \quad 1.12 \mathrm{C}+0 \mathrm{C}$ $E .112+C C \quad 3 . C 4 \varepsilon-01$ $5.634+C C \quad 1.232-C 1$ $4.523+0 \mathrm{C} \quad 3.55 t-\mathrm{C} 2$ $4.415+0 C \quad 1.575-02$ 4. $C \geq 8+00 \quad \varepsilon \cdot 647-03$ $.737+C C \quad 5.474-03$ $3.284+O C \quad 2.82 \varepsilon-C 3$ $2 . \subseteq 53+C C \quad 1.77 \varepsilon-03$ $2.4 C 1+O C \quad \varepsilon .417-04$ 2. $C 4 S+C C \quad 5.2 E S-04$ $1.611+0 \mathrm{C}$ $1.343+C C$ $1.160+00$ $1.025+0 \mathrm{C}$ $\varepsilon \cdot 383-\mathrm{Cl}$ 7.137-01 $5.27 \varepsilon-01$ $4.234-C 1$ 3. $C 75-01$ 2.444-01 2. $C 39-C 1$ 1. $755-\mathrm{Cl}$ $1.383-01$ $2.512-04$ $1.584-04$ $1.457-04$ $1.1 \subseteq 5-04$ $8.557-C 5$ $6.643-05$ $4.254-05$ $3.12 t-05$ 2.042-05 $1.51 \epsilon-05$ (5)-05 $1.0 \mathrm{CC}-05$ $7.4 € 5-06$ $5.553-06$
PRCE.

$8.750-c 3$ $3.518-C 2$
$c .827-c 2$ $1.617-01$ $2.2 \mathrm{C} 6-\mathrm{Cl}$ $2.736-\mathrm{Cl}$ $3.621-\mathrm{Cl}$ $4.307-01$ $5.5 \mathrm{C} 5-\mathrm{C}$ 6.416-01 $7.887-C 1$ S. $608-C 1$ $1.014+C C$ $1.014+C C$ $1.164+C C$

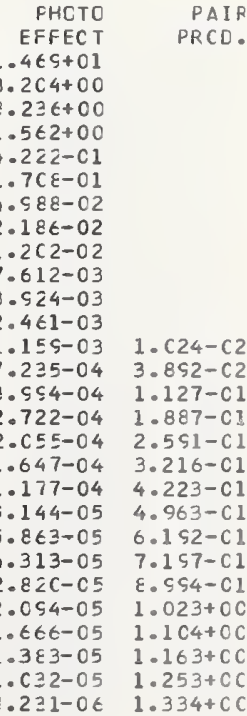




\begin{tabular}{|c|c|c|c|c|}
\hline \multicolumn{5}{|c|}{$Z=16$ SULFUR } \\
\hline $\begin{array}{r}\text { ENERGY } \\
\text { (KEV) }\end{array}$ & TOTAL & $\begin{array}{l}\text { COHER . } \\
\text { SCATT. }\end{array}$ & $\begin{array}{l}\text { INCOH. } \\
\text { SCAT }\end{array}$ & $\begin{array}{r}\text { PHOTO } \\
\text { EFFEC }\end{array}$ \\
\hline .010 & $8.310+07$ & $1.703+C 2$ & $1.055-c_{4}$ & 8.31 \\
\hline 15 & $1.353+07$ & $1.703+02$ & $2.370-04$ & $53+07$ \\
\hline & 3 & $1.703+c 2$ & $4.2 \mathrm{C6}=\mathrm{c}$ & +06 \\
\hline . $\mathrm{C} 30$ & $44+0$ & $1.703+c 2$ & $0-04$ & $8.043+05$ \\
\hline .040 & $58+0$ & $1.7 C 3+02$ & $.670-03$ & $6.656+05$ \\
\hline 50 & 6.8 & $1.7 \mathrm{C} 2+\mathrm{C}_{2}$ & 2.6 & $6.89 c+05$ \\
\hline .060 & $84+05$ & $1.702+02$ & $2-03$ & $6.982+05$ \\
\hline. $\mathrm{C} 8 \mathrm{C}$ & & 1.70 & $6-C_{3}$ & $6.670+05$ \\
\hline .100 & & 1.70 & $1.022-C 2$ & $6.124+05$ \\
\hline & 4. & 1.65 & & \\
\hline & & & & \\
\hline .300 & 2. & & & \\
\hline .400 & 1.1 & 1.67 & 1.4 & \\
\hline .500 & 6.8 & 1.66 & 2.1 & 6.8 \\
\hline.$€ 00$ & 4.4 & 1.645 & -01 & 4.4 \\
\hline .800 & 2. & $1.612+02$ & 4.8 & 2.212 \\
\hline. $\mathrm{COO}$ & $7 t+C$ & $1.568+C 2$ & $6.876-01$ & $1.275+05$ \\
\hline 500 & $64+0$ & $1.437+C 2$ & $1 \cdot 177+00$ & $4.549+04$ \\
\hline $\mathrm{COO}$ & $5+c$ & $1.293+C 2$ & $1.639+C C$ & $2 \cdot 1$ \\
\hline bo & 7 & $1.034+02$ & 2.4 & 7.2 \\
\hline 0 & & 8.524 & 3.1 & $3 \cdot 381+04$ \\
\hline & & & & $3+04$ \\
\hline $6 . \mathrm{C}$ & & & & \\
\hline 8.000 & $4.938+c$ & $4.824+$ & $5 . \mathrm{C}$ & 4.8 \\
\hline 0.000 & $2 . t$ & $3.844+c$ & 5.63 & $2.569+03$ \\
\hline & $41+$ & 2.303 & 6.69 & $7.844+02$ \\
\hline & 3. & $1.45 E+$ & $8+00$ & $3.321+02$ \\
\hline & 1.1 & 7.75 & 7.9 & $9.631+01$ \\
\hline & $16+C_{1}$ & $4.64 \mathrm{C}+\mathrm{CC}$ & 8. $c 53+c c$. & $3.942+01$ \\
\hline
\end{tabular}

\begin{tabular}{|c|c|c|c|c|}
\hline $\begin{array}{l}\text { ENEREY } \\
\text { (KEV) }\end{array}$ & TOTAL & $\begin{array}{l}\text { COHER. } \\
\text { SCATT. }\end{array}$ & $\begin{array}{l}\text { INCOH. } \\
\text { SCATT. }\end{array}$ & $\begin{array}{r}\text { PHOTO } \\
\text { EFFECT }\end{array}$ \\
\hline .010 & $1.1 C \varepsilon+C \varepsilon$ & $1.522+c 2$ & $8.540=05$ & $1.108+08$ \\
\hline .015 & 1. $\leftarrow 0 s+07$ & $1.922+c 2$ & 2. $\mathrm{COB}-04$ & $1.609+07$ \\
\hline 20 & $50+C t$ & 1. $S \leq 2+C 2$ & $3 \cdot 5$ & $250+06$ \\
\hline 30 & $32+0 t$ & 1. & & $1.432+06$ \\
\hline .040 & $10+0$ & $1.522+02$ & $7-03$ & $10+06$ \\
\hline .050 & $27+0 t$ & $1.522+c 2$ & $2.207-03$ & $1.127+06$ \\
\hline - $C \in C$ & 1. & 1.92 & $3.167-C 3$ & $1.045+06$ \\
\hline & 8 . & & & $8.894+05$ \\
\hline .100 & 7. & 1. & 8.6 & +05 \\
\hline & 5. & & & \\
\hline .200 & $4.261+$ & 2 & & \\
\hline .300 & $2.567+C_{6}$ & +02 & 7 & +06 \\
\hline .400 & $440+C 6$ & $1.898+C 2$ & $1.253-01$ & $1.440+06$ \\
\hline .500 & 8. & $1.885+02$ & $1.888-\mathrm{Cl}$ & $8.684+05$ \\
\hline .600 & & $1 . \varepsilon 7$ & $2.616-\mathrm{Cl}$ & $5.629+05$ \\
\hline & & 1.83 & 4.2 & +05 \\
\hline & & & 6.1 & 1.5 \\
\hline & $5 \cdot$ & 1.64 & & \\
\hline 2.000 & 2. & 2 & 1. & 2.6 \\
\hline 3.000 & $8.778+C$ & 1.19 & $\mathrm{CC}$ & 8.7 \\
\hline 4. $\mathrm{COO}$ & $4.18 s+0$ & $5.784+C 1$ & $3.313+0 \mathrm{C}$ & $s+04$ \\
\hline 5.000 & $.295+0$ & $8.234+$ & $3.556+C C$ & $2.291+04$ \\
\hline 6. 000 & $393+0$ & $7.055+C 1$ & $4.482+0 C$ & $1.385+04$ \\
\hline $8 . \mathrm{COO}$ & $.226+C$ & $5.443+C 1$ & $5.291+\mathrm{CO}$ & $6.167+03$ \\
\hline & $08+1$ & $4.378+C 1$ & $5.527+C 0$ & $3.258+03$ \\
\hline & & $2 \cdot \in \in 4+C_{1}$ & $7.045+00$ & $1.003+03$ \\
\hline & & $1.727+C 1$ & & $.269+02$ \\
\hline & $1.424+C 2$ & c. $286+C 0$ & $8.336+C C$ & $1.248+02$ \\
\hline & $6.556+C 1$ & $5.6 \mathrm{~s} 9+\mathrm{co}$ & $8 \cdot 551+c 0$ & $5.131+01$ \\
\hline
\end{tabular}

\begin{tabular}{|c|c|c|c|}
\hline $\begin{array}{r}\text { ENERGY } \\
\text { (KEV) } \\
50 . \\
60 . \\
80 . \\
100 \\
150 . \\
200 . \\
300 . \\
400 . \\
500 . \\
600 . \\
800 \\
1000 \\
1500 \\
2000 \\
3000 \\
4000 \\
5000 \\
6000 \\
8000 \\
10000 \\
15000 \\
20000 \\
30000 \\
40000 \\
50000 \\
60000 \\
80000\end{array}$ & $\begin{array}{l}3 \cdot 073+01 \\
2 \cdot 120+01 \\
1 \cdot 345+01 \\
1 \cdot 052+01 \\
7 \cdot 5 C 5+00 \\
6 \cdot 865+00 \\
5 \cdot 779+00 \\
5 \cdot 128+00 \\
4 \cdot 662+00 \\
4 \cdot 3 C 3+00 \\
3 \cdot 771+00 \\
3 \cdot 387+00 \\
2 \cdot 760+00 \\
2 \cdot 35 C+00 \\
1 \cdot 971+00 \\
1 \cdot 748+00 \\
1 \cdot 616+00 \\
1 \cdot 531+00 \\
1 \cdot 432+00 \\
1 \cdot 377+00 \\
1 \cdot 317+00 \\
1 \cdot 317+00 \\
1 \cdot 382+0 C \\
1 \cdot 445+00 \\
1 \cdot 45 C+00 \\
1 \cdot 525+00 \\
1 \cdot 586+00 \\
1 \cdot 649+00\end{array}$ & $\begin{array}{r}\text { COHER. } \\
\text { SCATT } \\
3.079+0 C \\
2.185+00 \\
1.261+00 \\
8.187-01 \\
3.707-01 \\
2.104-01 \\
9.433-02 \\
5.329-02 \\
3.419-02 \\
2.378-02 \\
1.341-02 \\
8.591-03 \\
3.825-03 \\
2.153-03 \\
9.578-04 \\
5.390-04 \\
2.450-04 \\
2.396-04 \\
1.348-04 \\
8.630-05 \\
3.836-05 \\
2.158-05 \\
9.592-06 \\
5.396-06 \\
3.453-06 \\
2.398-06 \\
1.349-06 \\
8.634-07\end{array}$ & $\begin{array}{r}\text { INCOH. } \\
\text { SCATT. } \\
\varepsilon .122+C C \\
8 . C 74+O C \\
7 . E 57+O C \\
7.605+0 C \\
6.5 \in 2+O C \\
6.423+O C \\
5.617+0 C \\
5 . C 45+C C \\
4.612+0 C \\
4.26 S+C C \\
3.752+0 C \\
3.375+0 C \\
2.744+C C \\
2.341+0 C \\
1.841+00 \\
1.535+00 \\
1.325+0 C \\
1.171+0 C \\
5.580-C 1 \\
8.157-C 1 \\
6 . C 32-01 \\
4.835-01 \\
3.515-C 1 \\
2.754-C 1 \\
2.330-C 1 \\
2 . C 06-01 \\
1.581-C 1 \\
1.311-01\end{array}$ \\
\hline
\end{tabular}
100000 .

\begin{tabular}{|c|c|}
\hline $\begin{array}{c}\text { ENERGY } \\
\text { (KEV) }\end{array}$ & $\mathrm{TCT} A \mathrm{~L}$ \\
\hline 50 & $3.793+01$ \\
\hline 60. & $2.562+01$ \\
\hline 80. & $1.564+01$ \\
\hline 100 & $1.189+01$ \\
\hline 150. & $8.637+00$ \\
\hline 200 . & $7.4 C S+00$ \\
\hline 300 . & $6.185+00$ \\
\hline 400. & $5.472+00$ \\
\hline 500. & $4.568+00$ \\
\hline 600 . & $4.582+00$ \\
\hline 800 . & $4.01 \overline{2}+00$ \\
\hline 1000 & $3.6 C 2+00$ \\
\hline 1500 . & $2.535+0 C$ \\
\hline 2000 . & $2.541+\mathrm{CO}$ \\
\hline 3000 & $2.104+00$ \\
\hline 4000 & $1.877+00$ \\
\hline 5000 . & $1.745+00$ \\
\hline 6000 & $1.660+00$ \\
\hline 8000 & $1.558+C 0$ \\
\hline 10000 & $1.455+00$ \\
\hline 15000 & $1.417+0 \mathrm{C}$ \\
\hline 20000 . & $1.422+00$ \\
\hline 30000 & $1.522+\mathrm{CO}$ \\
\hline 40000 & $1.6 \mathrm{Cl}+00$ \\
\hline 50000 . & $1.648+0 C$ \\
\hline 60000 . & $1.681+00$ \\
\hline 80000 & $1.745+C 0$ \\
\hline 100000 & $1.827+00$ \\
\hline
\end{tabular}

COHER SCATY.
$3.8 \geq 4+C O$ $3.824+C 0$ $1.610+00$ $1.055+00$ 4. $832-01$ 2.759-01 1. 245-01 7.055-02 4. 535-02 3.159-02 1.783-02 1. 144-02 5. $099-03$ 2. $872-03$ $2.872-03$
$1.278-03$ 7. 196-04 4. $608-04$ 3. $201-04$ 1. $801-04$ 1. $153-04$ $5.126-05$ 2. $884-05$ 1. $282-05$ 7. $210-06$ 4.615-06 3. 205-06 1. $803-06$ 1.154-06

INCCH.
SCATT.
$\varepsilon .5 C 1+C C$
$8.54 \epsilon+C C$
$\varepsilon .325+0 C$
$8 . C \epsilon 2+0 C$
$7.352+0 C$
$6 . \varepsilon 24+0 C$
$5.570+0 C$
$5.362+00$
$4.5 C 1+C C$
$4.536+C C$
$3.5 \varepsilon 7+O C$
$3.58 t+C C$
$2.515+0 C$
$2.487+0 C$
$1.556+C C$
$1.631+0 C$
$1.4 C 8+C C$
$1.245+00$
$1 . C 18+C C$
$8.666-01$
$6.4 C S-01$
$5.141-C 1$
$3.739-01$
$2.568-01$
$2.476-01$
$2.132-01$
$1.675-01$
$1.353-01$

1. $353-01$

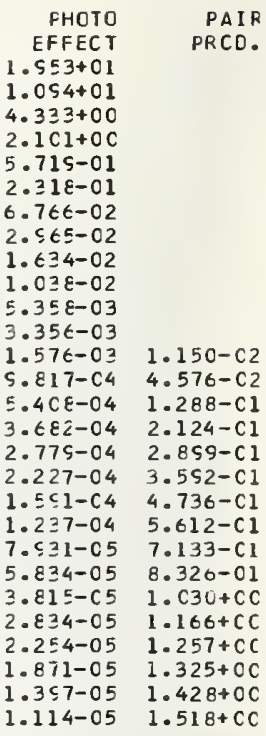

FHCTO EFFECT 2. $551+01$ $1.432+01$ 5. $7 \mathrm{C} 2+C \mathrm{C}$ $2.777+\mathrm{CC}$ 7.614-01 3. $C \leqq 7-01$ s. $C \in 4-02$ 2. $571-02$ 2. $1 \varepsilon t=02$ 1. $38 t-02$ $7.124-03$ 4. $448-03$ 2. $077-03$ $1.252-03$ 7. $111-04$ 4. $845-04$ 2. 6 55-04 2. $535-04$ $2.1 \mathrm{CC}-\mathrm{C}_{4}$ 1. $633-04$ 1. $C 45-04$ 7. $721-05$ 5. $C 53-05$ $3.755-05$ 2. $\varsigma \varepsilon \varepsilon-05$ $2.481-05$ 1. $\varepsilon 52-05$

1. $32 \mathrm{C}-\mathrm{C}_{2}$ 4 . $538-C 2$ $1.456-01$ 2. $447-C 1$ 3. 356-Cl 4. 150-C1 $5.358-C_{1}$ 6. $284-C 1$ 7. $759-C 1$ S. $C 77-C 1$ $1.148+C C$ 1. $304+C C$ 1. $400+O C$ 1. $468+C C$ 1. $687+O C$

A I R

PAIF

RCC.

$1.47 \varepsilon-05$

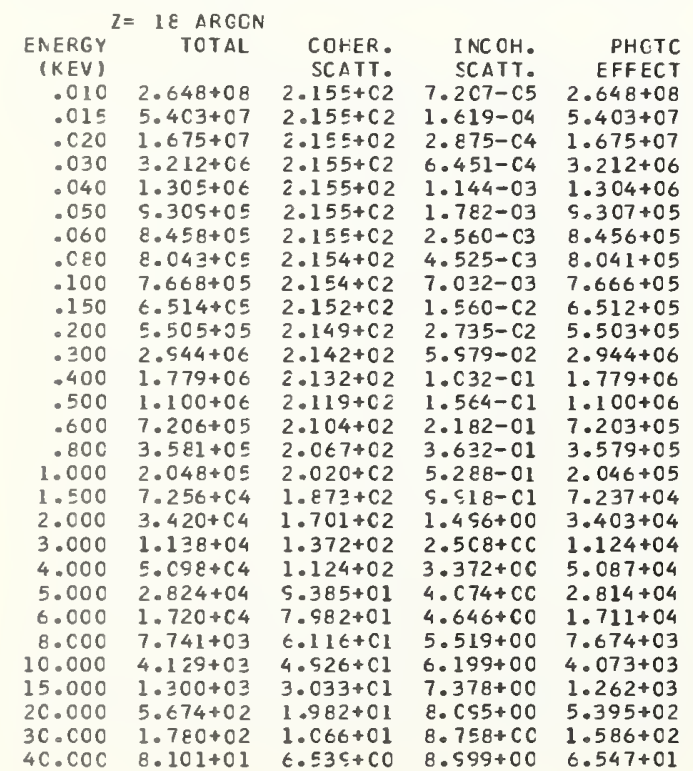

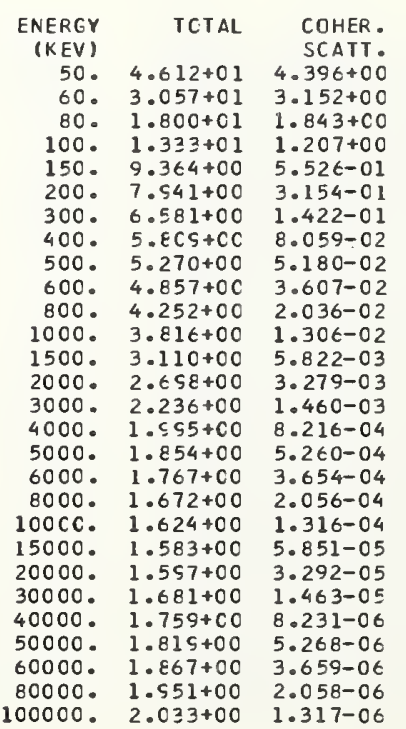

INCOH

SCATT.

S. $C 5 C+C C$

C. $\mathrm{CC} 7+0 \mathrm{C}$

8. $787+00$

8. $516+C C$

7. $\varepsilon 18+C C$

7. $221+O C$

6. $321+0 \mathrm{C}$

5. $677+C C$

$5.189+0 \mathrm{C}$

4. $\varepsilon C 3+C C$

4. $222+0 \mathrm{C}$

$3.757+C$

3. $\mathrm{C} 87+0 \mathrm{C}$

$2.634+0 C$

2. $\mathrm{C} 71+\mathrm{OC}$

1. $727+C$

1. $491+00$

1. $=18+C$

5. $176=01$

6.786-01

5. $443-01$

3. $559-01$

2. 143-0

2. $257-C$

2. $257-C 1$
$1.778-C 1$

1. $475-01$ 
$I=19$ POTASSILM

\begin{tabular}{|c|c|c|c|c|}
\hline $\begin{array}{l}\text { ENERGY } \\
\text { (KEV) }\end{array}$ & TOTA & $\begin{array}{l}\text { COHER. } \\
\text { SCATT. }\end{array}$ & $\begin{array}{l}\text { INCOH. } \\
\text { SCATT. }\end{array}$ & $\begin{array}{l}\text { PHDT } \\
\text { EFFEC }\end{array}$ \\
\hline. $\mathrm{ClO}$ & $6.141+04$ & $2.401+C 2$ & $1.001-04$ & \\
\hline . c15 & $5.7 \in 4+C 4$ & $2.401+02$ & $2.249-04$ & \\
\hline $.0<0$ & $7.145+07$ & $2.401+02$ & $3.992-04$ & \\
\hline .030 & $5.365+0 t$ & $2.401+C 2$ & $8.953-04$ & 365 \\
\hline .040 & $1.569+\mathrm{CE}$ & $2.4 C 1+C 2$ & & \\
\hline .050 & $1 . t \in O+O E$ & $2.401+02$ & -03 & \\
\hline . $c \in 0$ & $1.8 \mathrm{C} 2+0 \mathrm{E}$ & $2.4 \mathrm{Cc}+02$ & 3.546 & \\
\hline & $1.683+0 t$ & $2.355+02$ & & \\
\hline .100 & $1.402+0 t$ & $2.39 \varepsilon+02$ & & \\
\hline .150 & $8.437+05$ & $2.354+02$ & 2.151 & 8 \\
\hline .200 & $5.393+05$ & $.389+02$ & & \\
\hline .300 & $3.253+06$ & $2.374+02$ & & \\
\hline .400 & $2.1 \leq 3+0 t$ & $2.354+C 2$ & 1.4 & \\
\hline .500 & $1.3 \epsilon 7+0 \epsilon$ & $2.330+02$ & & \\
\hline & $9 . C 38+05$ & $2.303+02$ & 2.5 & \\
\hline .800 & $4.508+C 5$ & $2.240+c 2$ & 4.7 & \\
\hline 1.000 & $2.573+C 5$ & & & \\
\hline 1.500 & $9.044+C 4$ & $1.594+C 2$ & 1.2 & 9.0 \\
\hline 2.000 & $4.24 S+04$ & $820+02$ & & \\
\hline $3 . \mathrm{cc}$ & $1.432+$ & & & \\
\hline 4.000 & $6.070+04$ & $1.245+c 2$ & 3.6 & \\
\hline $5.00 \mathrm{C}$ & 3.3 & 1.04 & $4 \cdot 3$ & \\
\hline & & $8.837+$ & & \\
\hline & & & & \\
\hline o. & 5.061 & $5.41 \mathrm{st}$ & 6.51 & \\
\hline & & $3.337+c 1$ & & \\
\hline & & & & \\
\hline 30.000 & $2.1 \varsigma 9+C 2$ & $1.2 \mathrm{C} 8+\mathrm{Cl}$ & $9.183+C C$ & 1 \\
\hline & & & & \\
\hline
\end{tabular}

\begin{tabular}{|c|c|c|c|c|}
\hline \\
\hline $\begin{array}{l}\text { ENEREY } \\
\text { (KEV) }\end{array}$ & TOTAL & COHER . & $1 \mathrm{NCOH}$. & PHOTC \\
\hline .010 & $5.989+03$ & $\begin{array}{r}\text { SCATT: } \\
2.661+0\end{array}$ & $\begin{array}{r}\text { SCATT. } \\
344-04\end{array}$ & $\begin{array}{l}\text { EFFECT } \\
723+03\end{array}$ \\
\hline .015 & s. $4 \in 3+c 4$ & $2.661+C 2$ & $3.019-04$ & $5.437+04$ \\
\hline $.02 \mathrm{C}$ & $1.275+C E$ & $2 . \in \in 1+c 2$ & $5.358-04$ & $1.277+05$ \\
\hline .030 & $.585+06$ & $2.660+02$ & $1.2 \mathrm{Cl}-\mathrm{C} 3$ & $8.985+06$ \\
\hline .040 & $.573+c 6$ & $2.660+02$ & $2.128-c 3$ & $1.573+06$ \\
\hline .050 & $1.421+c \epsilon$ & $2.660+02$ & $3.312-03$ & $1.421+06$ \\
\hline .060 & $1.686+c \varepsilon$ & $2.65 s+02$ & $4.7 E 2-c 3$ & $1.686+06$ \\
\hline.$c 80$ & 1. $7 \in 2+C \epsilon$ & & $8.386-03$ & $1.761+06$ \\
\hline .100 & $1.550+06$ & $2.657+02$ & $1.3 \mathrm{Cl}-02$ & $1.549+06$ \\
\hline .150 & S. $s \in 9+C E$ & $2.651+02$ & $2.872-\mathrm{C} 2$ & S. $566+05$ \\
\hline & & & & \\
\hline .300 & $3.437+C E$ & $2.625+c 2$ & $1.084-01$ & $3.434+05$ \\
\hline .400 & $2.004+06$ & $2.60 c+02$ & $-\mathrm{Cl}$ & $2.004+06$ \\
\hline .500 & $1.540 t$ & 2.57 & & \\
\hline.$\in 00$ & $1 . c s 1+c 6$ & $2.535+02$ & $3.814-01$ & $1.091+06$ \\
\hline .800 & $5.679+05$ & $2.457+02$ & $-\mathrm{Cl}$ & $5.677+05$ \\
\hline 1.000 & 3.25 & 2.37 & & $s+0,-5$ \\
\hline 1.500 & $1.114+05$ & $2.167+C 2$ & $1.472+C C$ & $1.112+05$ \\
\hline $2.00 \mathrm{C}$ & $5.109+04$ & $1.974+02$ & & $5.089+04$ \\
\hline 3.000 & $1.708+$ & $1 . \in 34+$ & & $1.691+04$ \\
\hline 4.000 & $8 \cdot C 32+03$ & $1.3 \in 1+C 2$ & $3.868 t$ & $7.892+03$ \\
\hline $5 . c C C$ & $4.035+C 4$ & $1.145+02$ & $4.574+C 0$ & $4.027+04$ \\
\hline & & $9.758+$ & $5.167+\mathrm{CO}$ & $2.478+04$ \\
\hline & & & & $1.129+04$ \\
\hline 10.000 & $6.125+03$ & $5.952+01$ & $6.840+C C$ & $6.058+03$ \\
\hline 15000 & $1.958+C 3$ & $3.771+C 1$ & $8.051+0 c$ & $1.912+03$ \\
\hline & & & & \\
\hline 30.000 & $2.691+02$ & $1.35 s+C 1$ & $S . \in 11+O C$ & $2.459+02$ \\
\hline $4 \mathrm{C} \cdot \mathrm{COO}$ & $1.2 C 5+C 2$ & $8.323+0 \mathrm{C}$ & $5.896+C C$ & $1.022+02$ \\
\hline
\end{tabular}

\begin{tabular}{|c|c|c|c|c|}
\hline \multirow{2}{*}{ EAERGY } & \multicolumn{4}{|c|}{ ANDIUM } \\
\hline & TOTAL & COHER. & $1 \mathrm{NCOH}$. & PHCTO \\
\hline & $4 E 0+0 t$ & SCATT: & SCATT. & $\begin{array}{l}\text { EFFECT } \\
450+06\end{array}$ \\
\hline & $337+0 t$ & $2.533+c 2$ & $3.092-04$ & $3.337+06$ \\
\hline.$c 20$ & $3.219+C t$ & $2.533+02$ & $5.4 \varepsilon \varepsilon-C 4$ & $3.219+06$ \\
\hline.$c 30$ & $2.538+c t$ & $2.933+C 2$ & $1.230-\mathrm{C} 3$ & $2.538+06$ \\
\hline .040 & $4.123+06$ & $2.933+02$ & $2.179-C_{3}$ & $4.123+06$ \\
\hline .050 & $3.181+C \epsilon$ & $2.532+02$ & $3.392-03$ & $3.181+06$ \\
\hline & $2.8 \epsilon 1+c \epsilon$ & $\hat{L} .532+C 2$ & $4.8 \in 7-03$ & $2.861+06$ \\
\hline .080 & $2.329+0 \epsilon$ & $2.531+C 2$ & $8.589-03$ & $2.329+06$ \\
\hline & $1.8 \epsilon 2+C \epsilon$ & & & $1.862+06$ \\
\hline & & 2 & & $1.106+06$ \\
\hline .200 & $7.198+05$ & C.S17+C2 & $5.129-02$ & $7.195+05$ \\
\hline & $744+C 5$ & $2.898+c 2$ & $9-C 1$ & $3.741+05$ \\
\hline & & & & \\
\hline .500 & $1.773+06$ & $2.842+02$ & $2.833-\mathrm{cl}$ & $1.772+06$ \\
\hline.$\in 00$ & $1.238+06$ & $807+c 2$ & $3.901-01$ & $1.238+06$ \\
\hline & & & & \\
\hline 1.000 & $3.770+C 5$ & $2.642+C 2$ & $8.855-\mathrm{Cl}$ & $3.768+05$ \\
\hline 1.500 & $1.349+05$ & $2.423+C 2$ & $1.499+00$ & $1.347+05$ \\
\hline & & & & \\
\hline $3 . c 00$ & $2.177+04$ & $1.833+02$ & 3.073 & $2.158+04$ \\
\hline $4 . C O O$ & $9.521+03$ & $1.527+C 2$ & 3. $536+O C$ & $9.764+03$ \\
\hline & & & & $4.734+04$ \\
\hline 6.000 & $2.579+c$ & $1.092+02$ & $5.3 C 5+O C$ & $2.968+04$ \\
\hline & $1.378+04$ & $8.273+\mathrm{Cl}$ & $6.320+00$ & $1.369+04$ \\
\hline & & & & $7.358+03$ \\
\hline & & & & \\
\hline & 3 & & s.2 2 & $926+0$ \\
\hline & & & & $2.970+02$ \\
\hline & & & & \\
\hline
\end{tabular}

\begin{tabular}{|c|c|c|}
\hline $\begin{array}{l}\text { ENERGY } \\
\text { (KEVV) }\end{array}$ & TOTAL & $\begin{array}{l}\text { COHER } \\
\text { SCATT }\end{array}$ \\
\hline 50. & $5.5 \epsilon \varepsilon+01$ & $4.972+00$ \\
\hline 60. & $3.631+01$ & $3.563+00$ \\
\hline 80. & $2.068+01$ & $2.082+00$ \\
\hline 100. & $1.492+01$ & $1.363+00$ \\
\hline 150. & 1. $C 13+c 1$ & $6.236-01$ \\
\hline 200. & $8.481+00$ & $3.558-01$ \\
\hline & $6.574+0 \mathrm{C}$ & $1.604-01$ \\
\hline 400 . & $6.143+00$ & $9.086-02$ \\
\hline 500. & $5.568+00$ & $5.840-02$ \\
\hline 600 & $5.131+00$ & $4.067-02$ \\
\hline 800 . & $4.450+00$ & $2.296-02$ \\
\hline 1000 . & $4.029+00$ & $1.472-02$ \\
\hline 1500. & $3.284+00$ & $6.561-03$ \\
\hline 2000 . & $2.851+00$ & $3.696-03$ \\
\hline 3000 . & $2.37 \mathrm{C}+0 \mathrm{C}$ & $1.645-03$ \\
\hline 4000. & $2.122+00$ & \\
\hline 5000 . & $1.580+00$ & $5.928-04$ \\
\hline & $1.8 \varsigma 3+00$ & $4.118-04$ \\
\hline 8000 . & $1.757+00$ & $2.317-04$ \\
\hline 10000 & $1.745+00$ & $1.483-04$ \\
\hline & & $6.594-05$ \\
\hline 20000 . & $1.72 \mathrm{~s}+\mathrm{CO}$ & $3.710-05$ \\
\hline & $1.837+00$ & $1.649-05$ \\
\hline & $1.531+00$ & $9.276-06$ \\
\hline 50000 & $1.597+00$ & $5.937-06$ \\
\hline & $2 \cdot C 50+C 0$ & $4.123-06$ \\
\hline & $2.144+00$ & $2.319-06$ \\
\hline & $2+00$ & $1.484-06$ \\
\hline
\end{tabular}

ENERG

(KEV)$$
60
$$$$
100
$$$$
150
$$$$
\begin{array}{ll}
150 . & 1.057+01 \\
200 . & 9.063+00
\end{array}
$$

$300.7 .386+00$

400 . $6.488+00$

$500.5 .873+00$

$5.4 \mathrm{CS}+0 \mathrm{C}$

$1000.4 .244+00$

1500 . $3.459+00$

$2000 \cdot 3 . C C 4+00$

3000 - $2.5 \mathrm{C} 5+0 \mathrm{C}$

$4000.2 .253+00$

$\begin{array}{ll}5000 \cdot 2.112+00 \\ 6000 & 2.027+00\end{array}$

$\begin{array}{ll}6000 \cdot & 2.027+C 0 \\ 8000: & 1.5537+C 0\end{array}$

10000 - $1.851+00$

15000 . $1.847+00$

20000 . $1.872+0 \mathrm{C}$

30000 . $2.0 C 7+00$

40000 . $2.122+C 0$

50000 . $2.2 \mathrm{C} 1+00$

60000 . $2.2 \epsilon 1+00$

80000 . $2.262+00$

100000 .

$2.362+00$
$2.465+C 0$

COHER.
SCATT:
$.590+00$
$4.004+00$
$.339+00$
$.530+00$
$.000-01$
$.993-01$
$.800-01$
$.019-01$
$.551-02$
$.562-C 2$
$.575-02$
$.651-02$
$.359-03$
$.145-03$
$.845-03$
$.038-03$
$.649-04$
$.618-04$
$.599-04$
$.663-04$
$.395-05$
$.160-05$
$.849-05$
$.040-05$
$.658-06$
$4.624-06$
$.601-06$

SCATT:

s. $931+C C$

S. $697+O C$

S. $416+0 \mathrm{C}$

$8.00 \mathrm{C}+0 \mathrm{C}$

7. $\mathrm{CCS}+\mathrm{CC}$

$6.259+00$

$5.76 \mathrm{C}+00$

4. $t 8 s+0 C$

$4.217+0 C$

$3.429+O C$
$2.526+C C$

$2.3 \mathrm{Cl}+\mathrm{OC}$

$1.519+00$

$1.657+0 \mathrm{C}$

$1.4 E 4+0 C$

$1.158+00$

1. $C 20+C C$

t. $C 48-C 1$

$4.398-01$

2. 4 S2-C1

$2.5 C 8-C 1$

1. $57 t-01$

1. $\epsilon \exists S-01$

EFFEC T

2. $\mathrm{SCC}+01$

$1.17 \mathrm{C}+\mathrm{Cl}$

$5.7 t 5+0 C$
$1 . t 12+00$

$6.6 \equiv 5-01$

1. $5 t t-01$

$8.657-02$

$4.7 E 5-02$
$3 . C 15-C 2$

$1.54 t-02$

S. $633-03$

$4.4 \varepsilon 2-03$
$2.7 \varepsilon c-03$

$1.5<t-03$

1. $C \equiv 7-C 3$

7. $823-04$

t. $2 \epsilon \subseteq-C_{4}$

$4.478-04$
$3.48 C-04$

2.233-04

$1.643-04$

1. $074-04$

7. $5 \varepsilon 2-C$ s

C. $35 \mathrm{C}-05$

$3.535-05$

$3.135-05$

$1.832-\mathrm{C}_{2}$

$2 . \mathrm{CCO}-\mathrm{Cl}$

$3.317-\mathrm{Cl}$

$4.534-\mathrm{Cl}$

$5.619-01$
$7.386-C 1$

$8.7 \mathrm{CS}-\mathrm{Cl}$

1. $C 92+C$

$1.267+C C$
$1.567+C C$

$1.773+C C$

$1.910+C C$

$2 \cdot C 10+C$

$2.164+C C$
$2.3 \mathrm{Cl}+O C$

\begin{tabular}{|c|c|c|}
\hline $\begin{array}{l}\text { ENEREY } \\
\text { (KEV) }\end{array}$ & TCTAL & $\begin{array}{l}\text { COHER - } \\
\text { SCATT. }\end{array}$ \\
\hline 50. & $8 . C C C+C 1$ & $6.473+0 \mathrm{C}$ \\
\hline 60. & $5.108+01$ & $4.662+00$ \\
\hline 80. & $2.764+01$ & $2.743+00$ \\
\hline 100. & $1.899+01$ & $1.803+00$ \\
\hline 150. & $1.19 t+01$ & $8.298-01$ \\
\hline 200. & $9.714+00$ & $4.750-01$ \\
\hline 300. & $7.82 c+00$ & $2.148-01$ \\
\hline 400 . & $6.843+00$ & $1.218-01$ \\
\hline 500. & $6.185+00$ & $7.838-02$ \\
\hline 600 . & $5.651+00$ & $5.462-02$ \\
\hline 800. & $4.573+O C$ & $3.085-02$ \\
\hline 1000 . & $4.460+C 0$ & $1.980-02$ \\
\hline 1500. & $3.635+00$ & $8.828-03$ \\
\hline 100. & $3.1 \in 2+00$ & $4.974-03$ \\
\hline 3000 . & $2 . \in 41+O C$ & $2.215-03$ \\
\hline 4000. & $2.375+00$ & $1.247-03$ \\
\hline 5000 & $2.227+0 \mathrm{C}$ & $7.983-04$ \\
\hline & $2.141+00$ & $5.546-04$ \\
\hline 8000 . & $2.060+00$ & $3.121-04$ \\
\hline 10000 . & $2.031+00$ & $1.998-04$ \\
\hline 15000 . & $2 . C 2 S+00$ & $8.882-05$ \\
\hline 20000 . & $2.0 \notin 4+00$ & $4.997-05$ \\
\hline 30000. & $2.181+$ & $2.221-05$ \\
\hline & $2.2 \subseteq 5+C 0$ & $1.250-05$ \\
\hline 50000 . & $2.387+00$ & $7.998-06$ \\
\hline & $2.462+00$ & $5.554-06$ \\
\hline & $2.584+00$ & $3.124-06$ \\
\hline & $\in \mathrm{SC}+0$ & 2,000 \\
\hline
\end{tabular}

INCOH.

SCA TT .

FHOTC

EFFEC T

1. $C 42+C 1 \quad 6.311+C 1$

1. $C 39+01 \quad 3.6 C 3+01$

S. $8 E 5+0 C \quad 7.325+00$

S. $C E 1+O C \quad 2 . C 54+0 C$

E. $357+0 \mathrm{C} \quad 8.41 \mathrm{~s}-01$

7. $3 E S+O C \quad 2.4 \epsilon 1-C I$

$6 . E 14+O C \quad 1 . C 7 C-01$

t. $C 48+0 C \quad 5.84 t-02$

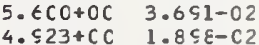

$\begin{array}{ll}4.523+C C & 1.8 \subseteq \varepsilon-C 2 \\ 4.428+00 & 1.1 \subseteq C-02\end{array}$

$3.6 C 1+00 \quad 5 . \leftarrow 05-03$

$2.41 \epsilon+O C$ 1. $93 C-03$

2. $C 15+C C$ 1.313-03

$1.740+C C$ S. SC $3-04$

$1.537+00 \quad 7.531-04$

1. $C 71+0 C$ 4.351-0 4

7. $117-01$ 2.E11-C4

t.351-01 2.06t-04

$4.618-01$

$2.0 t t-04$
$1.34 \mathrm{~s}-04$

3. $666-01$ 1. $\mathrm{CC}-04$

3. $C 58-01$

7. $9 \in 4-05$

$2.633-01 \quad 6.6 \mathrm{Cs}=05$

$\begin{array}{ll}2.075-01 & 4.532-C 5 \\ 1.721-01 & 3.5 \equiv 3-05\end{array}$

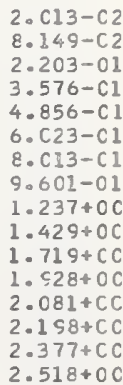


C D MPAR ISON

BIGGS AND LIGHTHILL IN BARAS/ATOM

\begin{tabular}{|c|c|c|c|c|}
\hline $\begin{array}{r}\text { ENERGY } \\
\text { (KEV) }\end{array}$ & TOTAL & $\begin{array}{l}\text { COHER. } \\
\text { SCATT. }\end{array}$ & $\begin{array}{l}\text { I NCOH. } \\
\text { SCATT. }\end{array}$ & $\begin{array}{r}\text { PHCTO } \\
\text { EFFECT }\end{array}$ \\
\hline.$C 10$ & $2.251+C \epsilon$ & $3.220+02$ & 1. $321-04$ & $2.251+06$ \\
\hline .015 & 4. $563+06$ & $3.215+02$ & $3.102-04$ & $4.962+06$ \\
\hline .020 & $3 \epsilon \varepsilon+06$ & $3.219+C 2$ & $C 5-C 4$ & $7+06$ \\
\hline .030 & $430+06$ & $3.215+02$ & 1.2 & 4.425 \\
\hline .040 & $02+C t$ & $3.21 s+02$ & $2.186-03$ & $2+06$ \\
\hline .050 & $+0 t$ & $3.21 \varsigma+02$ & $403-03$ & $2.839+06$ \\
\hline .060 & $=7+06$ & $18+02$ & $4.882-C_{3}$ & $.367+06$ \\
\hline.$C 80$ & $E S+C E$ & $2.217+C 2$ & $8.615-03$ & $759+06$ \\
\hline .100 & $91+06$ & $3.215+C 2$ & $1.336-02$ & $1.391+06$ \\
\hline .150 & $62+C 5$ & $3.21 C+02$ & $2.550-c 2$ & S. $059+05$ \\
\hline 00 & & & -02 & $6.691+05$ \\
\hline .200 & 4. & & $-\mathrm{Cl}$ & +05 \\
\hline .400 & 105 & & 1. & 05 \\
\hline 00 & 1. & $E+C 2$ & -01 & 1.8 \\
\hline .600 & 1. & 3. & $-C_{1}$ & 06 \\
\hline .800 & 7. & 02 & $-\mathrm{Cl}$ & +05 \\
\hline $1 . C 00$ & $4.573+c 5$ & $\mathrm{C} 2$ & $-\mathrm{Cl}$ & +05 \\
\hline 1.500 & $1.632+05$ & $2.655+02$ & $1.503+C C$ & $630+05$ \\
\hline 2.000 & $7.647+04$ & $2.4 \epsilon 7+C 2$ & $2.071+\mathrm{Co}$ & $7.622+04$ \\
\hline $3 . \mathrm{COC}$ & $2.584+04$ & $2.052+02$ & $3.0 \varepsilon 7+C O$ & $2.563+04$ \\
\hline $4 . \mathrm{COO}$ & $1.1 S_{1}+C_{4}$ & $1.712+02$ & 3. $571+\mathrm{CO}$ & $1.174+04$ \\
\hline $\mathrm{OOC}$ & $5.498+04$ & $1.440+02$ & $4.740+C C$ & $5.483+04$ \\
\hline 000 & $3+c 4$ & $1.224+C 2$ & $5.4 C 8+00$ & $3.470+04$ \\
\hline \&.cco & $8+c 4$ & $S .227+C 1$ & $6.458+C 0$ & $1.618+04$ \\
\hline$C \cdot \mathrm{COO}$ & $.824+03$ & $7.257+C 1$ & $7.340+C C$ & $8.744+03$ \\
\hline $\mathrm{co}$ & $2.815+C 3$ & $4.63 c+C 1$ & $8.725+C C$ & $1+03$ \\
\hline 000 & $1.22 t+c 3$ & $3.17 s+01$ & $9.577+O C$ & $1.195+03$ \\
\hline $\mathrm{COO}$ & $3.875+c 2$ & $1.732+C_{1}$ & 1. $c 44+01$ & $3.597+02$ \\
\hline $4 \mathrm{C} . \mathrm{COO}$ & $32 z+c 2$ & 1. $C 7 t+C 1$ & 1. $C 78+C 1$ & $1.517+02$ \\
\hline
\end{tabular}

\begin{tabular}{|c|c|c|}
\hline $\begin{array}{l}\text { ERGY } \\
\text { (KEY) }\end{array}$ & TOTAL & COHER - \\
\hline & $9.527+01$ & 7. $297+0$ C \\
\hline 60. & & $265+00$ \\
\hline 80. & $3.187+01$ & $105+00$ \\
\hline 100 & $2.140+01$ & $2.044+00$ \\
\hline & $1.297+01$ & $9.426-01$ \\
\hline 200. & 1. $C 3 \epsilon+01$ & $5.400-01$ \\
\hline & & $2.444-01$ \\
\hline & & $1.387-01$ \\
\hline 500. & $6.453+00$ & $8.928-02$ \\
\hline & & \\
\hline & & $3.516-c 2$ \\
\hline 1000 . & $4.67 t+00$ & $6-02$ \\
\hline 1500 & 3.81 & \\
\hline & & $5.671-03$ \\
\hline & & \\
\hline 4000 . & $2.506+c$ & $1.422-03$ \\
\hline 5000 . & & $9.103-04$ \\
\hline & & \\
\hline & 2.15 & $3.559-04$ \\
\hline & 2.17 & \\
\hline & & \\
\hline 20 & $2 . \bar{c} 23+$ & \\
\hline & & 2.53 \\
\hline & & \\
\hline & $2.618+0$ & $9.121-06$ \\
\hline & & \\
\hline & $2.834+0$ & \\
\hline & $2.548+00$ & $2.280-06$ \\
\hline
\end{tabular}

100000 .

\begin{tabular}{|c|c|c|}
\hline $\begin{array}{l}\text { AEREY } \\
\text { (KEV) }\end{array}$ & CTAL & $\begin{array}{l}\text { COHER } \\
\text { SCATT. }\end{array}$ \\
\hline 50. & $1.124+02$ & $8.154+0$ \\
\hline & & \\
\hline 80. & $3.651+01$ & $3.474+00$ \\
\hline & $4 c_{3}+c 1$ & $.288+00$ \\
\hline & & \\
\hline 200 & $1.10 \epsilon+01$ & \\
\hline & & \\
\hline & 7.5541 & \\
\hline $5 c$ & $6.8 \mathrm{C}$ & 1.0 \\
\hline & & \\
\hline & & \\
\hline 100 & 4.85 & 2.5 \\
\hline & & \\
\hline & 3 & \\
\hline & ci & \\
\hline & 2.65 & \\
\hline & & 1.0 \\
\hline & & \\
\hline & & \\
\hline & 2.30 & \\
\hline & & \\
\hline & & \\
\hline & $2.573 t$ & 2.84 \\
\hline & & \\
\hline & & \\
\hline & & \\
\hline & & \\
\hline & $.227+$ & $.558-0$ \\
\hline
\end{tabular}

$$
\text { COHER. }
$$
SCATT. $3.51 s+c 2$ $3.51 s+c 2$ $3.519+02$ $3.518+C 2$ $3.518+C 2$ $3.517+C 2$ $3.51 t+C 2$ $3.515+02$ $3.51 \mathrm{C}+\mathrm{C}_{2}$ $3.503+C 2$ $3.484+02$ $3.428+02$ $3.393+c 2$ $3.312+C 2$ $2.222+\mathrm{C} 2$ $2.584+C 2$ $2.741+02$ $2.291+02$ $1.917+\mathrm{C}^{2}$ $1.614+02$ $1.272+C$ $1.030+02$
$\varepsilon .088+01$ $5.066+C 1$ $3.53 s+C 1$ $1.201+C 1$
INCOH. PHCTC SCATT. EFFECT $1.380-C 4 \quad 1.472+06$ $3.058-04 \quad 5.716+06$ $\begin{array}{ll}.458-C 4 & 6.294+06\end{array}$ $2.183-03 \quad 4.180+06$ $3.359-C_{3} \quad 3.411+06$ $4.876-C 3 \quad 2.860+06$ $8.6 \mathrm{C} 5-03 \quad 2.142+06$ $1.334-02 \quad 1.704+06$ $2.946-C 2 \quad 1.121+06$ $5.138-\mathrm{C} 2 \quad 8.323+05$ $1.111-01 \quad 5.484+05$ $.895-C 1 \quad 4.085+05$ $\begin{array}{ll}2.836-01 & 3.254+05 \\ 3.505-C 1 & 1.561+06\end{array}$ 6.304-01 $8.855+05$ $8.854-C 1 \quad 5.296+05$ $1.457+C 0 \quad 1.923+05$ 2. $C E 1+C 0$ S. $070+04$ $3.074+C O \quad 3.076+04$ $3.570+C C \quad 1.411+04$ $4.744+C 0 \quad 7.649+03$ $6.631+00 \quad 1.891+04$ $7.533+$ CC $1.031+04$ $9.0 C 8+C 0 \quad 3.287+03$ $9.515+00 \quad 1.429+03$ $1.084+01 \quad 4.321+02$ $1.121+01 \quad 1.826+02$

\begin{tabular}{|c|c|c|}
\hline \\
\hline & TOTAL & COF'ER. \\
\hline (KEV) & & \\
\hline .010 & $5.523+0 t$ & $3.832+C 2$ \\
\hline & $1.107+C 7$ & $3.831+C 2$ \\
\hline .020 & $1.125+07$ & $3.831+02$ \\
\hline $.0 \geqq 0$ & $79+06$ & $3.831+C 2$ \\
\hline & 6.4 & \\
\hline .050 & $77+$ & $3.231+c 2$ \\
\hline & & \\
\hline & & \\
\hline $.1 \mathrm{CO}$ & $4 E+$ & $3.82 \varepsilon+c 2$ \\
\hline & & \\
\hline & & 3.81 \\
\hline $.3 c$ & & $+C 2$ \\
\hline & & \\
\hline .500 & 3.3 & $3.754+02$ \\
\hline & & \\
\hline & & \\
\hline 1.000 & $6.469+$ & 2.56 \\
\hline 1.50 & & \\
\hline & & \\
\hline 3.000 & $3 . t 82$ & 2.6 \\
\hline 4. & & \\
\hline & & \\
\hline $6 . c 0$ & & \\
\hline & & \\
\hline & & \\
\hline & & \\
\hline & 1 . & \\
\hline & & \\
\hline & & \\
\hline
\end{tabular}

I NCOH. SCATT. $1.135-C$ 2. 549-04 4. $524-C 4$ $1.014-03$ $1.797-C_{3}$ 2.798-03 4. $\mathrm{Cl}_{16-\mathrm{C}_{3}}$ 7. $089-03$ $1.100-02$ 2. $431-02$

4.244-02

S. 1 s -02

1. $573-\mathrm{Cl}_{1}$ $3.260-01$ $5.3 \mathrm{Cl}-\mathrm{Cl}$ 7.509-01 $1.303+\mathrm{CO}$ $1.841+00$ $2.862+C 0$ $3.792+C O$ $4.630+C C$ $5.381+00$ $6.646+C 0$ $7.629+00$ 1. $\mathrm{C} 22+01$ 1. $122+C 1$ $1.122+C 1$
$1.1 \in 3+C 1$
PHCTO

EFFECT

$1.107+07$

$1.125+07$

$8.579+06$

$6.411+06$

$4.977+06$

$4.007+06$

2. $823+06$

$2.148+06$

1. $354+05$

$5.871+05$

$4.258+05$

$3.335+05$

$1.631+06$

$6.465+05$

$2.373+05$

$1.106+05$

$1.656+04$

$9.002+03$

$4.540+04$

$2.187+04$

$.202+04$

$1.690+03$

$5.139+02$ $2.180+02$

\begin{tabular}{|c|c|}
\hline $\begin{array}{l}\text { ENERG } \\
\text { (KEV) }\end{array}$ & TCTAL \\
\hline 50 & $1.315+02$ \\
\hline 60. & $8.175+01$ \\
\hline 80 & $4.150+01$ \\
\hline 100 & $2.676+01$ \\
\hline 150. & $1.514+01$ \\
\hline 200. & $1.171+01$ \\
\hline 300 . & $9.115+00$ \\
\hline 400 . & $7.898+00$ \\
\hline 500 . & $7.112+O C$ \\
\hline 600. & $6.532+c 0$ \\
\hline 800 . & $5.698+00$ \\
\hline 1000 . & $5.1 C \epsilon+00$ \\
\hline 1500. & $4.163+00$ \\
\hline 2000 . & $3.624+00$ \\
\hline 3000 . & $3.05 t+00$ \\
\hline 4000 . & $2.787+00$ \\
\hline 5000 . & $2 . \in 47+0 C$ \\
\hline 6000 . & $2.56 \varsigma+00$ \\
\hline 8000 . & $2.455+00$ \\
\hline 10000 . & $2.460+00$ \\
\hline 15000 . & $2.440+O C$ \\
\hline 20000 . & 2. $512+\mathrm{CO}$ \\
\hline 30000 . & $2.753+0 \mathrm{C}$ \\
\hline 40000 . & $2.5 \geqslant 3+c c$ \\
\hline & 3. $C 4 \varepsilon+00$ \\
\hline 60000 . & $3.133+00$ \\
\hline 80000 . & $3.28 t+0 \mathrm{C}$ \\
\hline 100000 . & $3.459+0 \mathrm{C}$ \\
\hline
\end{tabular}

COHER. $8.432+00$ $6.018+00$ $3.498+00$ $2.282+0 \mathrm{C}$ $1.039+00$ 5.918-01 2.662-0 1.506-01 $9.672-02$ 6.732-02 3.798-02 $2.435-C 2$
$1.085-02$ 1.085-02 6.108-03 1.530-03 $0.730-03$ 6.802-04 3.827-04 2.450-04 1.089-04 6.127-0 2.723-05 $1.532-05$ 9.805-06 6.809-06 $3.830-06$
$2.451-06$
INCOH. FHOTO EFFEC ? 1. C87+01 $7.71 \mathrm{C}+01$ 1. $C 84+C 1 \quad 4.417+01$ 1. $C \in 0+01 \quad 1.817+01$ 1. $C 31+01$ S. $C 4 \varepsilon+00$ $9.451+C C \quad 2.541+0 C$ $8.777+C C \quad 1.041+00$ $7.656+0 C \quad 3.042-01$ t. $521+C C \quad 1.325-01$ $6.331+00 \quad 7.271-02$ $5.156+0 \mathrm{C} \quad 2.380-02$ $3.772+00 \quad 7 . \mathrm{CC}_{3}-03$ $3.219+0 \mathrm{C} \quad 4.261-03$ $2.111+00 \quad 1 . \in<5-03$ $1.822+O C \quad 1.227-03$ $1.611+O C$ S. $822-04$ $1.317+0 \mathrm{C} \quad 7.0 \mathrm{C} 3-04$ $1.122+0 \mathrm{C} \quad 5.435-04$ ع.2\$4-01 $3.47 \varepsilon-04$ $6.652-01 \quad 2.556-C$ $\begin{array}{ll}4 . \varepsilon 33-01 & 1.6 \varepsilon S-04 \\ 3.841-C 1 & 1.235-04\end{array}$ $\begin{array}{ll}3.841-C 1 & 1.235-04 \\ 3.204-01 & 5.854-C 5\end{array}$ $2.755-c 1$ $8.17 \varepsilon-05$ 2.173-01 $6.1<2-05$ 1. EC 3-C1 $4.867-05$

$2.211-C 2$ $8.869=\mathrm{C}$ 2.412-01 3. $527-01$ 6.612-01 $8.772-\mathrm{Cl}$ 1. $C 48+O C$ $1.344+O C$ $1.890+C C$ $2.128+C C$ $2.257+O C$ $2.425+C C$ $2.768+C C$

PAIR

PRCL. 5. $\varepsilon \in 3+00 \quad 4 . \in 13 z-02$ $4.628+00 \quad 1.4 \div 1-C 2$ 2. $531+0 C \quad 2.3 \subseteq 7-03$

\begin{tabular}{|c|c|c|}
\hline INCOH . & PHCTO & \\
\hline $\begin{array}{l}\text { SCATT. } \\
1 \geq 0+01\end{array}$ & EFFECT & \\
\hline $\begin{array}{l}1.120+01 \\
125+01\end{array}$ & $5.2 \varsigma 4+01$ & \\
\hline $1.12 s+01$ & $5 \cdot 3 \geq c+01$ & \\
\hline $\begin{array}{l}\text { 1. } 1 C E+C I \\
\text { 1. } C 7 t+01\end{array}$ & $2.1 s \varsigma+C 1$ & \\
\hline $\begin{array}{l}\text { 1. } C 7 t+01 \\
\text { s. } S 13+0 C\end{array}$ & 1. $C \varsigma \varepsilon+01$ & \\
\hline S. $S 13+0 \mathrm{C}$ & $3.111+00$ & \\
\hline $5.170+0 \mathrm{C}$ & $1.2 E 2+O C$ & \\
\hline E. C $44+C c$ & $2.77 t-01$ & \\
\hline $7.234+c 0$ & $1.648-01$ & \\
\hline $6 . \in 1 \varepsilon+0 C$ & $9.020-02$ & \\
\hline $6.12 \mathrm{~s}+0 \mathrm{C}$ & $5.6 S_{1}-02$ & \\
\hline $5.3 S C+C C$ & $2.51 C-02$ & \\
\hline 4. $E 4 S+C C$ & $1.812-02$ & \\
\hline 2. $543+C C$ & $8.428-03$ & $2.464-$ \\
\hline $3.365+C 0$ & $5.241-03$ & \\
\hline $2.646+0 \mathrm{C}$ & $2.8 \varepsilon 1-c 3$ & $660-$ \\
\hline $2.2 \mathrm{C7}+\mathrm{CC}$ & $1.5 \in 1-03$ & \\
\hline 1. $S C 5+0 C$ & $1.480-03$ & \\
\hline 1. $\in 84+0 C$ & $1.1 \& 7-C_{3}$ & 7.40 \\
\hline $1.377+O C$ & $\varepsilon .4 \varepsilon 3-04$ & \\
\hline $1.173+0 \mathrm{C}$ & $6.5 \leqq 4-04$ & 1.136 \\
\hline$\varepsilon .671-01$ & $4.2 \equiv 2-04$ & 1.425 \\
\hline $6.556-01$ & $3.115-04$ & \\
\hline 5. $C 58-C 1$ & 2. $C \geq \varepsilon-C 4$ & \\
\hline $4 . C 16-C 1$ & $1.514-04$ & \\
\hline $3.345-01$ & $1.2 C 4-C 4$ & \\
\hline 2. $\varepsilon 84-C_{1}$ & S.S૬S-C5 & \\
\hline $2.272-01$ & $7.465-05$ & \\
\hline & & \\
\hline
\end{tabular}

$5.555-05$

\begin{tabular}{|c|c|c|}
\hline INCOH. & FHOTO & PAIF \\
\hline SCATT. & EFFECT & \\
\hline $1.174+01$ & $1.113+02$ & \\
\hline $1.173+C 1$ & $6.40 \mathrm{C}+01$ & \\
\hline $1.149+01$ & $2.651+c 1$ & \\
\hline $1.115+01$ & $1.32 s+01$ & \\
\hline 1. $C 33+01$ & $3.77 t+O C$ & \\
\hline c. $56 \mathrm{C}+\mathrm{CC}$ & $1.557+00$ & \\
\hline $8.350+0 C$ & $4.5 \varepsilon 7-C 1$ & \\
\hline $7.546+C C$ & 2. $C C \varepsilon-01$ & \\
\hline $6 . S C 5+0 C$ & $1.1 C \leq-01$ & \\
\hline$E .3 \$ 4+O C$ & $7.011-02$ & \\
\hline $5 . t 24+0 C$ & $3.555-02$ & \\
\hline $5 . C 59+C C$ & $2.23 t-02$ & \\
\hline $4.114+00$ & $1.035-02$ & $2.716-C_{2}$ \\
\hline $3.511+C C$ & $6.4 C \geq-03$ & 1. $C C 7-C 1$ \\
\hline $2.7 \in 1+00$ & $3.5 C 5-03$ & $2.885-01$ \\
\hline $2.3 C 3+C C$ & $2.3 \varepsilon 1-03$ & $4.8 \mathrm{CO}-01$ \\
\hline 1. $S 8 \varepsilon+O C$ & $1.75 t-c_{3}$ & $6.557-C_{1}$ \\
\hline $1.757+O C$ & $1.4 \equiv 5-03$ & $8.1 C 2-c 1$ \\
\hline $1.437+c c$ & $1.028-03$ & 1. C $C 57+C C$ \\
\hline $1.223+0 C$ & $7.553-04$ & $1.236+C C$ \\
\hline 9. $C 48-C 1$ & $5.13 c-04$ & $1.535+O C$ \\
\hline $7.258-01$ & $3.77 t-c 4$ & $1.785+C C$ \\
\hline $5.278-01$ & $2.471-04$ & $2.225+0 C$ \\
\hline $4.150-C 1$ & 1. $\varepsilon \equiv \varepsilon-C 4$ & $2.514+C C$ \\
\hline $3.455-C 1$ & $1.461-04$ & $2.699+0 \mathrm{C}$ \\
\hline 3. $C C S-01$ & $1.213-04$ & $2.832+C C$ \\
\hline $2.371-01$ & S. C5t-05 & 3. $C 49+O C$ \\
\hline $1.567-01$ & $7.225-05$ & $3.263+C C$ \\
\hline
\end{tabular}




\begin{tabular}{|c|c|c|c|c|}
\hline ARGYY & TOTAL & COFER. & INCOH. & PHOTO \\
\hline (KEV) & & SCATT. & SCATT. & EFFECT \\
\hline .010 & $5.254+C 6$ & $4.157+C 2$ & & \\
\hline & $6.812+0 t$ & $4.157+02$ & $3.050-04$ & $6.812+06$ \\
\hline .020 & $6.572+06$ & $4.157+C 2$ & $.483-04$ & $6.972+06$ \\
\hline .030 & $7.195+06$ & $4.157+02$ & $229-03$ & $7.194+06$ \\
\hline .040 & $\epsilon .898+0 \epsilon$ & $4.157+C 2$ & $2.177-03$ & $6.898+06$ \\
\hline & $6.413+06$ & $4.156+02$ & $3.389-C_{3}$ & $6.413+06$ \\
\hline .060 & $08+06$ & $6+02$ & $4.8 \in 2-C 3$ & $5.508+06$ \\
\hline .080 & 5. $C 2 O+C E$ & $4.155+C 2$ & $8.578-03$ & $5.019+06$ \\
\hline & $=6+c t$ & $4.153+c 2$ & $1.330-02$ & $4.325+06$ \\
\hline .150 & $5+c 6$ & & & $2.774+06$ \\
\hline .200 & $1.754+06$ & $4.142+02$ & $5.119-02$ & $1.794+06$ \\
\hline .300 & $8.818+C 5$ & $4.123+02$ & $1.106-\mathrm{Cl}$ & $8.814+05$ \\
\hline .400 & & $4.098+02$ & & \\
\hline $.5 \mathrm{CO}$ & $3.207+C 5$ & $4.068+c 2$ & $2.822-01$ & $3.203+05$ \\
\hline.$\in 00$ & $2.1 \in \varepsilon+05$ & $4 \cdot C 33+02$ & $-C 1$ & $2.164+05$ \\
\hline .800 & $1.044+06$ & & & $1.043+06$ \\
\hline 1.000 & $6.947+05$ & $3.85 s+c 2$ & $8.776-\mathrm{Cl}$ & $6.943+05$ \\
\hline 1.500 & $2.705+C 5$ & $3.605+C 2$ & 1.4 & $2.702+C 5$ \\
\hline 2.000 & & & & $1.283+05$ \\
\hline 3.000 & $4.314+04$ & $2.821+c 2$ & $3.011+00$ & $4.286+04$ \\
\hline $4.0 \mathrm{CO}$ & 1. $S \in S+C 4$ & $2.381+$ & & $1.945+04$ \\
\hline $.00 \mathrm{C}$ & $c 77+c 4$ & $2.016+02$ & 4.7 & $1.057+04$ \\
\hline $6.0 C C$ & $6.640+03$ & $1.71 \varepsilon+c 2$ & $5.5 C 5+00$ & $6.463+03$ \\
\hline 8.000 & & $1.287+$ & $6.8 C 7+00$ & $2.497+04$ \\
\hline & $+c 4$ & $1.0 C 5+C 2$ & co & $1.385+04$ \\
\hline $15 . \mathrm{CCO}$ & $4.5 \varepsilon 2+C 3$ & $6.325+01$ & S. $5 \mathrm{C7}+\mathrm{CO}$ & $4.509+03$ \\
\hline & & $4.343+C 1$ & 1. C $54+C 1$ & $1.980+03$ \\
\hline & & $=395+0$ & $1.1 \in 2+C 1$ & 6.056 \\
\hline & & & & $.577+02$ \\
\hline
\end{tabular}

\begin{tabular}{|c|c|c|c|c|}
\hline & $=2 t$ IRGN & & & \\
\hline ENERGY & TOTAL & COHER - & $1 \mathrm{ACOH}$. & PHCTO \\
\hline & & SCATT. & SCATT & EFFECT \\
\hline . CIC & S. $1 \varepsilon S+C E$ & $4.457+c 2$ & $1.340-c_{4}$ & $5.189+06$ \\
\hline .015 & $9.0 \varsigma 5+C \epsilon$ & $4.457+C 2$ & $3.009-04$ & $9.094+06$ \\
\hline .020 & $e 7+c t$ & $4.457+C 2$ & $5.339-c_{4}$ & $8.337+06$ \\
\hline $.0 \geq 0$ & $E C t+0 t$ & $4.456+C 2$ & $1.157-C 3$ & $7.606+06$ \\
\hline .040 & $15+0 t$ & $4.4 S t+C 2$ & $120-03$ & $6.915+06$ \\
\hline .050 & $4+C E$ & $4.45 t+02$ & $3.300-03$ & $6.254+06$ \\
\hline $.0 \in 0$ & 5. $\epsilon \in 7+C \epsilon$ & $4.455+02$ & $4.735-C 3$ & $5.667+06$ \\
\hline .080 & $27+C 6$ & $4.454+C 2$ & 8.3 & $4 \cdot 726+C 6$ \\
\hline .100 & $4 \cdot C 32+0 t$ & $4.453+C 2$ & $56-c 2$ & $4.032+06$ \\
\hline .150 & $2.561+C \epsilon$ & $4.488+C 2$ & $2.8 \in 1-C 2$ & $2.560+06$ \\
\hline & & $4.481+$ & & \\
\hline .300 & $8.8 C 8+C 5$ & $4.4 \epsilon 3+C 2$ & $1 . c 7 s-c 1$ & $8.804+05$ \\
\hline .400 & $5.206+05$ & $4.438+02$ & $1.840-01$ & $5.202+05$ \\
\hline & & & & $3.364+05$ \\
\hline & & & & $2.314+05$ \\
\hline $.80 \mathrm{C}$ & $1.228+C t$ & $4.291+C 2$ & $\epsilon .117-C_{1}$ & $1.228+06$ \\
\hline 1.000 & $8 . C 50+C 5$ & $4.158+$ & $0-c 1$ & $8.046+05$ \\
\hline & & & & \\
\hline 2.000 & $1.493+C 5$ & $3.657+02$ & $1 . s \varsigma s+00$ & $1.489+05$ \\
\hline 3.000 & 5. $C \in C+04$ & $3.112+$ & $3 . c$ & $5.028+04$ \\
\hline & & & & $2.293+04$ \\
\hline $5 . C C C$ & $1.2 \in 7+$ & $2.241+C_{2}$ & $4.775+C 0$ & $1.244+04$ \\
\hline & $7.738+1$ & $1.914+$ & 5.55 & $7.541+03$ \\
\hline & & & & \\
\hline & 54 & $1.115+C_{2}$ & & $1.584+04$ \\
\hline & 5.2 & $\epsilon \cdot S 1 E+C 1$ & $9.759+\mathrm{CO}$ & $5.212+03$ \\
\hline & & & & \\
\hline & & & & \\
\hline $0=50$ & $3.312+C_{2}$ & $1 . \in 54+01$ & $1.247+C_{1}$ & $3.022+02$ \\
\hline
\end{tabular}

\begin{tabular}{|c|c|c|}
\hline \multicolumn{3}{|c|}{$Z=27$ COBALT } \\
\hline $\begin{array}{l}\text { EAERGY } \\
\text { (KEVV) }\end{array}$ & TOTAL & $\begin{array}{l}\text { COHER. } \\
\text { SCATT }\end{array}$ \\
\hline .010 & $7.956+C E$ & $4.845+c 2$ \\
\hline .015 & $1.150+07$ & $4.84 S+C 2$ \\
\hline .020 & $1.268+07$ & $4.845+02$ \\
\hline .030 & $1.144+07$ & $4.84 S+C 2$ \\
\hline .040 & S. $656+06$ & $4.84 S+C 2$ \\
\hline $.05 C$ & $8.275+06$ & $4.848+C 2$ \\
\hline & $7.175+06$ & $4.848+C 2$ \\
\hline .080 & $5.633+c 6$ & $4.847+02$ \\
\hline .100 & $4.623+06$ & $4.845+c 2$ \\
\hline .150 & $2.584+C 6$ & $4.841+02$ \\
\hline .200 & $2.007+06$ & $4.834+C 2$ \\
\hline .300 & $1.051+06$ & $4.816+C 2$ \\
\hline & & $4.791+C 2$ \\
\hline .500 & $4.215+05$ & $4.762+c 2$ \\
\hline $.6 O C$ & $2.582+0 \leqslant$ & $4.727+c 2$ \\
\hline & & \\
\hline $1 . \mathrm{COC}$ & $9.056+C 5$ & $4 \cdot 55 C+C 2$ \\
\hline 1.500 & $3.641+05$ & $4.284+C 2$ \\
\hline $2 . \mathrm{CC}$ & $1.753+05$ & $3.553+02$ \\
\hline 3.000 & $5.936+C 4$ & $3.41 s+c 2$ \\
\hline $4 . C O C$ & $2.711+04$ & 2. $514+C 2$ \\
\hline & $1.475+04$ & $2.483+02$ \\
\hline & & \\
\hline 8.000 & $3.205+04$ & $1.55 t+C 2$ \\
\hline $1 \mathrm{C} \cdot \mathrm{COO}$ & $1.817+c 4$ & $1.240+C 2$ \\
\hline & $6.085+03$ & $7.658+C 1$ \\
\hline $2 \mathrm{C} . \mathrm{CcO}$ & $2.728+C 3$ & $5.274+C 1$ \\
\hline & $\varepsilon \cdot \in 53+02$ & $2.521+01$ \\
\hline & $=842+c 2$ & $1.833+C 1$ \\
\hline
\end{tabular}

I NCOH. SCATT. 2. $5 \$ 1-C 4$ $5.308-04$ $1.150-C_{3}$ $2.108-C 3$ $3.281-03$ $4.7 C_{7}-C_{3}$ $8.3 \mathrm{C}_{6}-\mathrm{C} 3$ 1.288-02 $2.844-C 2$ $4.559-02$ 1. $828-C 1$ $2.736-\mathrm{Cl}$ $3.765-01$ $6.075-01$ $1.439+00$ $1.981+00$ $2.574+C 0$ $3.9 \mathrm{C} 3+\mathrm{CO}$ $4.776+C O$ $5.587+C C$ $7.003+\mathrm{CO}$ $8.125+00$ $9.552+00$ $1.116+C 1$ $1.238+C 1$
$1.289+01$

$\begin{array}{rrr}\begin{array}{r}\text { ENERGY } \\ \text { (KEV) }\end{array} & \text { TCTAL } & \begin{array}{r}\text { COHER. } \\ \text { SCATT }\end{array} \\ 50 . & 1.543+02 & 1.014+01 \\ 60 . & 9.556+01 & 7.331+00 \\ 80 . & 4.790+C 1 & 4.337+00 \\ 100 . & 3.041+01 & 2.860+00 \\ 150 . & 1.662+01 & 1.322+00 \\ 200 . & 1.255+01 & 7.586-01 \\ 300 . & 9.634+00 & 3.438-01 \\ 400 . & 8.296+00 & 1.953-01 \\ 500 . & 7.445+00 & 1.257-01 \\ 600 . & 6.831+00 & 8.763-02 \\ 800 . & 5.55 C+0 C & 4.953-02 \\ 1000 . & 5.328+00 & 3.179-02 \\ 1500 . & 4.342+00 & 1.418-02 \\ 2000 . & 3.785+00 & 7.994-03 \\ 3000 . & 3.158+00 & 3.560-03 \\ 4000 . & 2.521+00 & 2.004-03 \\ 5000 . & 2.780+0 C & 1.284-03 \\ 6000 . & 2.7 C 6+00 & 8.917-04 \\ 8000 . & 2.645+C C & 5.018-04 \\ 10000 . & 2.627+00 & 3.213-04 \\ 15000 . & 2.643+0 C & 1.428-04 \\ 20000 . & 2.725+C 0 & 8.036-05 \\ 30000 . & 2.578+0 C & 3.572-05 \\ 40000 . & 3.173+00 & 2.010-05 \\ 50000 . & 3.3 C 8+00 & 1.286-05 \\ 60000 . & 3.4 C 5+00 & 8.932-06 \\ 80000 . & 3.582+0 C & 5.025-06 \\ 100000 . & 3.755+00 & 3.216-06\end{array}$
SCATT EFFEC $.219+011.32 \mathrm{C}+02$ $1.218+01 \quad 7.6 C 5+0$ $1.1 \in 4+C 1 \quad 1.551+01$ 1. $C 74+C 1 \quad 4.5 E 2+C C$ 5. $547+O C \quad 1.8 \varepsilon E+C C$ E. $732+O C \quad 5.5 \varepsilon 1-O 1$ $7.856+C C \quad 2.44 t-01$ $\begin{array}{ll}7.18 S+O C & 1.344-C 1 \\ t . E 5 S+C C & 8.513-02\end{array}$ $\begin{array}{ll}\epsilon .65 S+C C & 8.513-02 \\ 5.857+0 C & 4.357-C 2\end{array}$ $5.269+00 \quad 2.7 C \varepsilon-02$ $4.285+0 \mathrm{C} \quad 1.254-02$ $3 . \in 57+0 C 7.753-C$ $2.876+C C \quad 4.24 C-C$ $2.3 \varsigma 5+0 C \quad 2.87 \varsigma-03$ 2. $C 71+C C$ $1.830+C C$ $1.457+0 \mathrm{C}$ $1.274+O C$ 5.425-01 $7.560-C 1$ $5.45 \varepsilon-01$ $4.365-C$ $3 . \in 41-01$ 3. $135-0$

$2.049-01$

$1.7 \equiv 8-03$

$1.241-03$

s.642-04 $6.184-04$ $4.55 \mathrm{C}-04$ 2. $57 €-04$ 2.211-04 $1.755-\mathrm{C}$ $1.4 \in C-04$ $1.050-04$ $1.153+C 1 \quad 3.163+01$ 100000 .

PAI PRCL.

$2.551-\mathrm{C}_{2}$

$1.121-01$

$3.139-\mathrm{Cl}$

$5.176-01$

$8.727-C 1$

$1.146+O C$

$1.351+0 C$

$1.7 \mathrm{CO}+\mathrm{CC}$

$2.428+C C$

$2.737+O C$

$2.543+C C$

3. $\mathrm{C} 96+\mathrm{CC}$

$3.335+C C$

\begin{tabular}{|c|c|c|}
\hline ERGY & CT AL & \\
\hline (KEV) & & \\
\hline 50. & $1.750+02$ & \\
\hline & & $8.168+00$ \\
\hline & $.46 C+01$ & $4.838+00$ \\
\hline 100 & & \\
\hline & $1.8 C 5+01$ & $3+00$ \\
\hline 200 & $1.343+01$ & -01 \\
\hline & $1 . C 13+C 1$ & \\
\hline 400 & $8.678+00$ & -01 \\
\hline & & \\
\hline & & \\
\hline 800 & & \\
\hline 100 & & \\
\hline 15 & $4.515 t$ & \\
\hline & & \\
\hline & & \\
\hline 4000 & 3.056 & 2.24 \\
\hline & & \\
\hline & & \\
\hline & 2.78 & 5.6 \\
\hline & & \\
\hline & & \\
\hline & $2.5 c s t$ & \\
\hline & & \\
\hline & & \\
\hline & & $1.440-05$ \\
\hline & & \\
\hline & & \\
\hline & & \\
\hline
\end{tabular}

INC OH

SCATT

$1.2 \in 1+C$

$1.2 \in 2+C$

$1.237+0$

$1.116+C$

1. $C 33+01$

S. $\mathrm{C} 75+0 \mathrm{C}$

$8.167+0 C$

$7.474+0 C$

t. $524+C$

$6 . C 51+0 C$

$5.4 \varepsilon C+O C$

$4.457+0 C$

2. $C S 1+O C$

$2.454+0 \mathrm{C}$

$2.154+0$

1. $\triangle \mathrm{C} 3+O C$

$1.557+C C$

1. $325+0 C$

5. $\varepsilon[2-01$

7. $\varepsilon \in 2-C 1$

$5.718-0$

$3.78 t-C 1$

$3.78 \mathrm{t}-\mathrm{C1}$

$3.260-01$
$2.56 \mathrm{~s}-\mathrm{Cl}$

$2.131-01$

EFFEC T

1. $551+02$

8. $558+01$

1. $8 \varepsilon t+0$

$5.417+O C$

$2.24 \varepsilon+00$

6. 6t2-01

2. $521-01$

$1 . \in C E-C 1$

1.C17-C

5.211-02

2. $24 \mathrm{C}-02$

$5.27 \epsilon-03$

$5.072-03$

$3.442-C^{3}$

$2.554-0$

2. $C 77-03$

$1.483-03$

$1.152-03$

7. $387-04$

5.434-0

$3.5 \leq 4-04$
$.64 C-04$

$2.1 \mathrm{CC}-04$

$1.743-C_{4}$

1. $3 \mathrm{C} 1-04$

1. $33 \varepsilon-04$

$3.155-C_{2}$

$3.4 \mathrm{CO}-\mathrm{Cl}$

5.562-C1

7.552-C1

. $327-\mathrm{Cl}$

$1.448+C C$

$1.830+C C$

$2.122+O C$

$2.519+C C$

$3.138+C C$

$3.3 \mathrm{Cl}+\mathrm{C}$

$3.559+C$

$3.750+C C$
INCOH. SCA TT

$1.3 \mathrm{C} 6+\mathrm{C}$

1. $3 C^{2} 7+0$

$1.282+0$

$1.252+C$

$1.158+01$

$5.425+C C$

$\varepsilon .4 \varepsilon z+O C$

$7.763+O C$

$7.151+00$

$6.325+C C$

$5 . t S 1+00$

$4.628+0 \mathrm{C}$

$3.550+0 C$

3. $1 C E+C C$

$2.550+C C$

$2.237+O C$

1. $577+0 \mathrm{C}$

1. $617+0 \mathrm{C}$

1. $37 t+O C$

1. $C 18+0 C$

8. $1 \in 5-01$

$5.528-01$

4. 714-C

3. $532-0$

$3.386-01$

2. $\in \in \in 7-01$

2.213-01
PHCTO

EFFEC $1.81 E+C$ $4.39 \varepsilon+01$ $2.223+01$ $6.414+00$ $2.673+0 C$ 7. 577-01 $.51 \mathrm{C}-\mathrm{C}$ . $5 \geq C-01$ 1.220-01 $6.226-C 2$ 1. $7 \mathrm{cl}^{-C} \mathrm{C}$ 1. 1 C -02 $6 . C 35-03$ 4. CSt-03 3. $C \varepsilon E-C 3$ $2.470-03$ $1.7 \in 2-0$ $1.3 \in 8-03$ ع. $77 \mathrm{C}-04$ $6.45 \mathrm{C}-04$ $4.217-\mathrm{C}_{4}$ 3.1 $32-04$ $2.452-04$ 2. $C \in 8-C 4$ 1. $544-\mathrm{CA}_{4}$ 1. $232-04$
PAIR

PRCE.

3.479-02

$1.317-\mathrm{C} 1$ $3.676-01$ $8.203-\mathrm{Cl}$ 1. $\mathrm{C} 11+\mathrm{OC}$ $1.320+C$ $1.550+C C$ 1. $542+0 C$ $2.26 \mathrm{C}+0 \mathrm{C}$ $2.757+C C$ $3.151+O C$ $3.381+0$ $3.549+O C$ $3.816+c C$
$4.067+c$ 


\begin{tabular}{|c|c|c|c|c|}
\hline $\begin{array}{l}\text { ENERGY } \\
\text { (KEV) }\end{array}$ & & $\begin{array}{l}\text { COHER } \\
\text { SCATT: }\end{array}$ & $\begin{array}{l}\text { INCOH. } \\
\text { SCATT. }\end{array}$ & $\begin{array}{r}\text { PHOTC } \\
\text { EFFECT }\end{array}$ \\
\hline .010 & $4 \cdot t 12+06$ & $5.215+02$ & $1.31 c-c 4$ & $4.611+06$ \\
\hline & & & & \\
\hline .02 & $7.418+0 t$ & $5.215+02$ & $.222-04$ & $.419+06$ \\
\hline .030 & $8.244+0 t$ & & & \\
\hline .04 & $62+06$ & & & $.162+06$ \\
\hline .050 & $15+0 t$ & $4+C 2$ & & $.714+06$ \\
\hline .060 & & & & \\
\hline & & & & \\
\hline & 5.3 & & & \\
\hline .150 & & & & \\
\hline & 2.1 & & & \\
\hline .300 & & & & \\
\hline .400 & & & & +05 \\
\hline & & & & \\
\hline .60 & & & & \\
\hline .800 & & & & \\
\hline 000 & & & & \\
\hline & & & & \\
\hline 2.000 & & & & \\
\hline 3 & & & & \\
\hline & & & & \\
\hline & & & & \\
\hline & & & & \\
\hline & & & & \\
\hline C. .000 & & & & \\
\hline & & & & \\
\hline & & & & \\
\hline & S. $944+0$ & & & \\
\hline & $4.417+02$ & $2 . c 25+c 1$ & $1.329+01$ & $4.081+02$ \\
\hline
\end{tabular}

\begin{tabular}{|c|c|c|}
\hline \\
\hline $\begin{array}{l}\text { EERGV } \\
\text { (KEVY) }\end{array}$ & TOTAL & $\begin{array}{l}\text { COHER } \\
\text { SCATT. }\end{array}$ \\
\hline .010 & $5.350+C E$ & \\
\hline & & \\
\hline .020 & $8.50 \hat{z}+c t$ & $5.594+C 2$ \\
\hline $.03 \mathrm{C}$ & $8.894+06$ & $5.594+C 2$ \\
\hline .040 & $8.337+$ & $5.594+C 2$ \\
\hline .050 & $7.6 C 5+$ & $5.553+c 2$ \\
\hline .060 & & \\
\hline & & \\
\hline .10 & 4.90 & $c 2$ \\
\hline & & \\
\hline & & \\
\hline & 1.18 & 5.5 \\
\hline & & \\
\hline .500 & 5.010 & 5.5 \\
\hline .600 & 3.555 & \\
\hline & & \\
\hline $1 . \mathrm{Co}$ & $\operatorname{CSE} t$ & 5.3 \\
\hline 1.50 & & \\
\hline & & \\
\hline 3 & 7.778 & 4.1 \\
\hline & & \\
\hline & & \\
\hline$\epsilon$ & & \\
\hline & & \\
\hline $1 \mathrm{C} .00$ & $2.285+$ & \\
\hline 150 & & \\
\hline & & \\
\hline & & \\
\hline & & \\
\hline
\end{tabular}

I NCOH.

SCATT.

$1.053-04$
$2.364-04$

$4.156-04$

$9.4 \mathrm{C9}-\mathrm{C} 4$

$1.667-C_{3}$
$2.5 C_{6}-C_{3}$

$2.556-C 3$
$3.725-C 3$

6.577-03

$1.020-02$

$2.256-\mathrm{C} 2$

$3.939-\mathrm{C2}$

$8.542-02$
$1.462-01$

$2.155-\mathrm{Cl}$

3. $C 23-C 1$

$4.536-01$

$7.004=01$
$1.221+00$

$1.737+C D$

$2.746+C O$

$3.713+C$

$4.630+C 0$

$5.491+00$

7. $\mathrm{Cl} 12+\mathrm{CC}$

$8.241+C C$

$1.035+01$
$1.168+01$

$1.308+01$

$1.268+01$
FHCTO

EFFECT

$5.349+06$
$7.399+06$

$8.501+06$

$8.893+06$

$8.336+06$

$7.605+06$

$6.908+06$

. $761+06$

$907+06$

$2.067+06$

$1.185+06$

$7.436+05$

$.005+05$

$3.550+05$
$1.991+05$

$1.097+06$

$4.654+05$

$2.245+05$

$7.736+04$

$3.577+04$

$1.954+04$

$5 \cdot 310+03$

$.269+04$

$7.756+c 3$

$3.482+03$

$4.699+02$

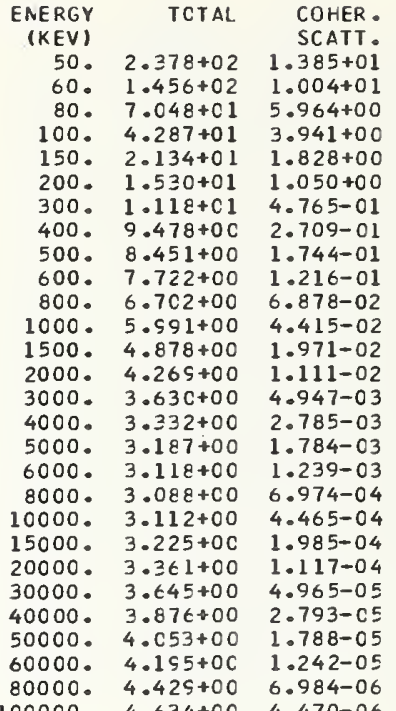

100000 .
$4.634+0 C$

\begin{tabular}{|c|c|c|}
\hline $\begin{array}{l}\text { INC LH. } \\
\text { SCATT. }\end{array}$ & $\begin{array}{r}\text { FHOTO } \\
\text { EFFECT }\end{array}$ & $\begin{array}{l}\text { PAIR } \\
\text { PROD. }\end{array}$ \\
\hline $1.348+01$ & $2.1 C 5+02$ & \\
\hline $1.350+01$ & $1.221+02$ & \\
\hline $1.32 \epsilon+C 1$ & $5.125+01$ & \\
\hline $1.255+01$ & $2.5 s q+01$ & \\
\hline $1.159+C 1$ & $7.521+0 C$ & \\
\hline $1.112+01$ & $3.1 \geqslant 7+00$ & \\
\hline $5.771+0 \mathrm{C}$ & $\varsigma .361-01$ & \\
\hline $8.755+0 \mathrm{C}$ & $4.125-01$ & \\
\hline $8 . C 49+0 C$ & $2.27 t-01$ & \\
\hline $7.456+C C$ & $1.445-01$ & \\
\hline $6.55 s+00$ & $7.4 C 7-02$ & \\
\hline $5 . S C 1+C C$ & $4.6 C z-02$ & \\
\hline $4.75 s+C C$ & $2.124-02$ & $3.735-02$ \\
\hline 4. $\csc 6+00$ & $1.3 C \subseteq-02$ & $1.484-01$ \\
\hline $3.222+O C$ & $7.134-03$ & $3.560-01$ \\
\hline $2.686+0 \mathrm{C}$ & $4.8 \equiv 2-03$ & $6.375-c 1$ \\
\hline $2.319+0 \mathrm{C}$ & $3.6 \geq 7-03$ & $8.616-01$ \\
\hline $2 \cdot c 50+c c$ & $2.51 C-03$ & 1. $C 64+C C$ \\
\hline $1.677+0 \mathrm{C}$ & $2.076-03$ & $1.4 \mathrm{C} 8+0 \mathrm{C}$ \\
\hline $1.427+0 \mathrm{C}$ & $1.612-03$ & $1.682+C C$ \\
\hline $1 . C 56+0 C$ & $1.0 \equiv 3-03$ & $2.168+C C$ \\
\hline$\varepsilon .4 t 8-01$ & $7.5 \subseteq 4-C 4$ & $2.513+c c$ \\
\hline $6.158-01$ & $4.565-04$ & $3.028+0 C$ \\
\hline 4. \& \& S-Cl & $3.6 \varepsilon \varepsilon-04$ & $3.387+C C$ \\
\hline $4 . c 78-C 1$ & $2.533-C 4$ & $3.645+0 \mathrm{C}$ \\
\hline $3.511-\mathrm{Cl}$ & $2.435-04$ & $3.844+C C$ \\
\hline $2.7 \in 6-01$ & $1 . \varepsilon 17-C 4$ & $4.152+0 \mathrm{C}$ \\
\hline & & \\
\hline
\end{tabular}

EAERGY

(KEV)

50
60

80.
100.

100.

200 .

300 .

400 .

500 .

600.
800 .

800.

1500.

2000 .

3000 .

4000 .

6000 .

$80 \mathrm{Co}$.

10000 .

15000 .

20000 .

30000 .

40000 .

50000 .

60000 .

80000 .

100000 .

$\begin{array}{rr}\text { TOTAL } & \begin{array}{c}\text { COHER } \\ \text { SCATT }\end{array} \\ 2.721+C 2 & 1.527+01 \\ 1.6 \epsilon 1+02 & 1.108+01 \\ 7.57 C+C 1 & 6.577+00 \\ 4.794+01 & 4.346+00 \\ 2.321+01 & 2.015+00 \\ 1.634+01 & 1.158+00 \\ 1.174+01 & 5.255-01 \\ 9.888+00 & 2.987-01 \\ 8.753+00 & 1.923-01 \\ 8.023+00 & 1.341-01 \\ 6.554+00 & 7.584-02 \\ 6.214+00 & 4.869-02 \\ 5 . C 57+C C & 2.173-02 \\ 4.425+00 & 1.225-02 \\ 3.77 t+C C & 5.455-03 \\ 3.47 t+0 C & 3.071-03 \\ 3.332+00 & 1.567-03 \\ 3.26 t+00 & 1.367-03 \\ 3.238+00 & 7.691-04 \\ 3.262+00 & 4.524-04 \\ 3.374+0 C & 2.189-04 \\ 3.52 C+C 0 & 1.232-04 \\ 3.84 t+00 & 5.475-05 \\ 4.1 C 8+00 & 3.080-05 \\ 4.3 C 3+00 & 1.971-05 \\ 4.456+00 & 1.369-05 \\ 4.7 C 2+C 0 & 7.701-06 \\ 4.918+00 & 4.929-06\end{array}$

INCOH.

PHOTO

EFFECT

$1.389+01 \quad 2.425+02$

$\begin{array}{ll}1.353+C 1 & 1.411+02 \\ 1.369+01 & 5.544+01\end{array}$

$1.33 s+\mathrm{Cl} \quad 3.02 \mathrm{C}+01$

$1.240+01 \quad 8.753+00$

1. $150+01 \quad 3.67 \varepsilon+0 C$

$1 . \mathrm{C} 11+01 \quad 1.1 \mathrm{C} 1+00$

5.1C4+OC $4.847-\mathrm{Cl}$

$8.334+0 \mathrm{C} \quad 2.666-01$

$7.721+0 C \quad 1.6 \varepsilon 7-01$

$6.752+C C \quad \varepsilon . t \equiv 1-02$

$6.111+0 C \quad 5.3 \in 2-02$

$4.571+C C \quad 2.47 \varepsilon-02$

$4.242+0 C$

$3.337+0 \mathrm{C}$

$2.782+0 \mathrm{C}$

$2.4 \mathrm{C} 2+0 \mathrm{C}$

1. $736+C C$

$1.478+0 \mathrm{C}$

1. $C S_{3}+C C$

$8.77 \mathrm{C}-01$

t. $378-C 1$

5. $0 \in 3-01$

4. $223-01$

3.t $t 3 t-01$

$2 \cdot \varepsilon \in 5-c 1$

. $340-02$

5.657-03

$4.255-03$

$3.4 C \varepsilon-03$

$2.4 \equiv 1-03$

1.887-03

$1.2 \mathrm{CS}-03$
$8.885-\mathrm{C} 4$

5. $811-C 4$

4. $315-04$

3. $432-04$

$2.849-04$

$2.126-04$

4.030-C2

1. $553-\mathrm{C} 1$

4.252-C1

6. 848-0

$5.235-C 1$

$1.138+C C$

$1.782+C C$

$2.275+C C$

$2.642+0 C$

$3.2 \mathrm{C} 7+\mathrm{CC}$

$3.880+C C$

4. $\operatorname{Cs} 2+C C$

$4.415+C C$
$4.680+0 C$

$\begin{array}{rr}\text { INCCH. } & \text { PHCTO } \\ \text { SCATT. } & \begin{array}{r}\text { EFFECT } \\ 1.264-C 4\end{array} \\ 2.507+06 \\ 5.839-C 4 & 3.331+06 \\ 1.129-C 4 & 4.741+06 \\ 2.129-03 & 8.043+06 \\ 2 . C C 0-03 & 9.284+06 \\ 3.114-C 3 & 5.439+06 \\ 4.468-C 3 & 9.156+06 \\ 7.884-C 3 & 8.234+06 \\ 1.223-C 2 & 7.313+06 \\ 2.7 C 0-C 2 & 4.8 C 9+06 \\ 4.708-02 & 3.089+06 \\ 1 . C 18-C 1 & 1.534+06 \\ 1.737-01 & 9.089+05 \\ 2.559-C 1 & 6.013+05 \\ 3.579-01 & 4.279+05 \\ 5.778-C 1 & 2.497+05 \\ 8.117-01 & 1.645+05 \\ 1.374+C C & 5.251+05 \\ 1.858+C C & 2.560+05 \\ 2.876+C 0 & 8.862+04 \\ 3.814+00 & 4.099+04 \\ 4.718+C 0 & 2.238+04 \\ 5.576+C C & 1.359+04 \\ 7.114+C C & 6.113+03 \\ 8.367+00 & 2.540+04 \\ 1 . C 54+01 & 8.752+03 \\ 1.154+01 & 3.948+03 \\ 1.342+C 1 & 1.243+03 \\ 1.4 C 7+C 1 & 5.388+02\end{array}$

ENERG

(KEV)

50 .

60

80 .

150 .

200 .

300 .

400

500 .

600.

800

1000

1500 .

2000 .

3000 .

5000 .

8000 .

8000 .

15000

$2000 \mathrm{C}$.

30000 .

40000 .

50000 .

60000 .

80000 .

100000 .
TCTAL

$3.1 \mathrm{C} 6+\mathrm{C} 2$

$1.894+02$

S. $023+01$

$2.524+01$

$1.745+01$

$1.233+01$

$1 . C 32+01$

$9.146+00$

$8.332+00$

$6.439+00$

$5.235+00$

$4.551+00$

$3.524+00$

$3 . t 2 t+00$

$3.4 \varepsilon \varepsilon+00$

$3.431+00$

$3.41 s+00$
$3.456+0 C$

$3.585+O C$

$3.740+C 0$

$4 . C 84+0 C$

$4.366+c 0$

$4.578+00$

$4.74 t+00$

$5.020+00$
$5.263+00$
COHER

SCATT

$1.670+01$

$1.212+01$
$7.201+00$

$7 \cdot 201+00$
$4.760+00$

$2.208+00$

$1.269+00$

5.760-01

3. 274-01

2.109-01

8.316-C2

5.339-02

2.383-02

$1.343-02$
$5.982-03$

3.368-03

2.157-03

1. $499-03$

8.434-04

5.399-04

2. $401-04$

$1.351-04$
$6.004-05$

3.378-05

2.162-0

1.501-05

8.445-06

5.405-06
INC OH

SCATT.

$1.431+C 1$

$1.436+01$

$1.413+01$

$1.382+C$

$1.150+01$

1. $C 46+C 1$

S. $420+0 \mathrm{C}$

$8.623+0 \mathrm{C}$

$7.588+0 \mathrm{C}$

$7 . \mathrm{C} 27+O \mathrm{C}$

$5.142+00$

$4.389+C C$

$3.452+0 \mathrm{C}$

$2 . \varepsilon 78+0 \mathrm{C}$

$2.485+O \mathrm{C}$

$2.156+C C$

$1.756+\mathrm{CO}$

$1.52 s+c c$

$1.131+00$

5.C72-0

6.598-01

$5.23 \varepsilon-C$

$4.365-0$

2.762-C1

2. $455-01$

FHCTO

EFFEC T

$2.75 t+02$

1. $62 S+02$

t. $\varepsilon \subseteq 0+01$

$3 \cdot 5 \mathrm{C} 5+01$

$4.2 \varepsilon 3+00$

$1.2 \varepsilon \varepsilon+0 C$

$5.6 \varepsilon 4-01$

3.125-01

$1.575=01$

t. $2 \varepsilon z-02$

2.910-02

$1.7 \subseteq \varepsilon-c 2$

5. $80 t-03$

$t \cdot E \geq S-03$

4. $553-03$

$3.553-c 3$

2.844-03

$2.2 \mathrm{CE}-\mathrm{C}$

1.411-03

1. $C ₹ 7-03$

E. $770-c 4$

5. $C 2 t-04$

3. $55 t-04$ 


\begin{tabular}{|c|c|c|c|c|}
\hline \multicolumn{5}{|c|}{$\mathrm{Z}=31 \mathrm{GALLIUM}$} \\
\hline & & & INCOH. & PHOTC \\
\hline (KEV) & & SCATT. & SCATT. & EFFECT \\
\hline .010 & $3.537+C 6$ & $6.393+02$ & $1.355-04$ & \\
\hline & $7.7 \varepsilon 2+C 5$ & $6.392+02$ & $3.133-04$ & $7.775+05$ \\
\hline .020 & $2.083+\mathrm{CE}$ & $6.392+02$ & $5.560-C_{4}$ & $3.083+06$ \\
\hline .030 & $2.475+C 6$ & $6.392+02$ & $246-c 3$ & $2.474+06$ \\
\hline .040 & $4.122+06$ & $6.392+C 2$ & $2.207-03$ & $4.122+06$ \\
\hline .050 & $5.5 t 6+c t$ & $6.351+c 2$ & $3.436-03$ & $5.566+06$ \\
\hline & $t .432+0 t$ & $6.391+02$ & $9-03$ & $6.431+06$ \\
\hline .080 & $7.034+06$ & $6.350+02$ & $8.697-03$ & $7.033+06$ \\
\hline & & & & \\
\hline .150 & $5.375+C t$ & $6.382+02$ & & $5.374+06$ \\
\hline .200 & $3.814+C \epsilon$ & $6.374+02$ & $5.187-C 2$ & $3.814+06$ \\
\hline & $2.051+c$ & & & \\
\hline $.40 \mathrm{C}$ & $234+0 t$ & $t .324+C 2$ & $1.909-01$ & $1.234+06$ \\
\hline .500 & $8.069+05$ & $6.289+02$ & 2.8 & $62+05$ \\
\hline & 5.55 & $\epsilon .24$ & & $5.586+05$ \\
\hline .800 & $3.020+05$ & $6.150+C 2$ & $6.317-01$ & $3.014+05$ \\
\hline 1.000 & $1.803+C 5$ & $6.038+02$ & $1-C 1$ & $1.797+05$ \\
\hline $1.5 \mathrm{CO}$ & 5.53 & & & $5.929+05$ \\
\hline 2.000 & . $8 \varsigma \varsigma+C 5$ & $5.374+02$ & $2.018+$ & $2.893+05$ \\
\hline 3.000 & $1.010+05$ & 4.68 & & $1.005+05$ \\
\hline 4.000 & $4.7 C^{3}$ & 4.07 & co & $4.662+04$ \\
\hline 0 & & & & 2.5 \\
\hline 6.000 & $1.583+c 4$ & $3 . c 73+c 2$ & & $1.552+04$ \\
\hline & $7.236+$ & & $7.267+00$ & $6.994+03$ \\
\hline & & & & \\
\hline $15.00 \mathrm{C}$ & c. $9 \in 9+03$ & $1.08 s+C 2$ & $1.075+01$ & $9.849+03$ \\
\hline & & & $1.218+\mathrm{Cl}$ & $4.477+03$ \\
\hline & & & & \\
\hline 40.000 & $6.582+C 2$ & $2.65 t+C_{1}$ & $1.445+C 1$ & $6.172+02$ \\
\hline
\end{tabular}

\begin{tabular}{|c|c|c|c|c|}
\hline - & $=32$ GERM & $\because I L M$ & & \\
\hline EDERGY & TOTAL & COHER . & I NCOH. & PHOTC \\
\hline (KEV) & & SCATT. & SCATT. & EFFECT \\
\hline .010 & $2.170+c 7$ & $6.812+c 2$ & $1.404-C 4$ & $2.170+07$ \\
\hline & $4.287+C 6$ & $6.812+02$ & $3.153-04$ & $4.286+06$ \\
\hline .020 & $7+06$ & $6.811+C 2$ & $5.5 \subseteq 5-c 4$ & $1.356+06$ \\
\hline .030 & $1.495+C 6$ & $6.811+02$ & $1.254-C 3$ & $1.494+06$ \\
\hline .040 & $2.180+C 6$ & $6.811+0 z$ & $.222-03$ & $2.179+06$ \\
\hline .050 & $3.870+06$ & $6.81 c+02$ & $9-03$ & $3.869+06$ \\
\hline .060 & $5.484+06$ & $6.810+02$ & $4.962-C_{3}$ & $5.483+06$ \\
\hline .080 & $7.4 \epsilon 7+0 t$ & $6.808+02$ & & $7.467+06$ \\
\hline .100 & $8.223+0 t$ & $6.807+C 2$ & & $8.223+06$ \\
\hline .150 & $6.711+\mathrm{CE}$ & $\mathrm{C.} .801+\mathrm{C}_{2}$ & 7-02 & $6.711+06$ \\
\hline & & $6.792+$ & & \\
\hline .300 & & $t .7 \epsilon c+C 2$ & & $2.533+06$ \\
\hline .400 & $1.521+C t$ & $6.738+C 2$ & $1.527-01$ & $1.521+06$ \\
\hline .500 & & $6.700+$ & & \\
\hline .600 & & & & $6.916+05$ \\
\hline .800 & $3.777+C 5$ & $6.550+02$ & $6.4 \mathrm{CO}-\mathrm{Cl}$ & $3.770+05$ \\
\hline 1.000 & 2.28 & $6.428+C 2$ & & \\
\hline & & & & \\
\hline 2.000 & $3.280+C 5$ & $5.6 \varsigma t+C 2$ & $2.083+00$ & $3.274+05$ \\
\hline 3.000 & 1.14 & $4.95 C+$ & & $1.142+05$ \\
\hline & & & & \\
\hline 5.000 & $2.525+$ & $3.760+$ & $5 . \mathrm{C} 27+\mathrm{CC}$ & $2.887+04$ \\
\hline 6.000 & $1.788+$ & $3.287+$ & & $1.754+04$ \\
\hline & & $c 2$ & co $\rightarrow$ & \\
\hline 10.000 & & $1.578+$ & & $4.246+03$ \\
\hline 15.000 & $1.112+C_{4}$ & $1.1 \varsigma \varsigma+C 2$ & $1 . \operatorname{cs} 8+c 1$ & $1.099+04$ \\
\hline & & & & \\
\hline & 1.6 & $4.568+C 1$ & $1.409+01$ & $1.601+c$ \\
\hline 0.000 & $7.425+C 2$ & $2 . \varepsilon q C+C 1$ & $1.484+C 1$ & $6.988+02$ \\
\hline
\end{tabular}

\begin{tabular}{|c|c|c|c|c|}
\hline \multicolumn{5}{|c|}{32} \\
\hline (KEV) & & $\begin{array}{l}\text { COFER } \\
\text { SCATT. }\end{array}$ & $\begin{array}{l}\text { INC OH- } \\
\text { SCATT. }\end{array}$ & $\begin{array}{l}\text { PHCTC } \\
\text { EFFECT }\end{array}$ \\
\hline.$c 10$ & $3.613+07$ & $7.244+02$ & $1.344-04$ & $3.613+07$ \\
\hline.$C 15$ & $291+07$ & $7.244+02$ & $3.018-c 4$ & $1.291+07$ \\
\hline .020 & $80+c 6$ & $7.244+02$ & $5.356-c 4$ & $5.179+06$ \\
\hline .030 & $57+C 6$ & $7.244+02$ & $1.201-C_{3}$ & $1.097+06$ \\
\hline .040 & $57+05$ & $7.243+02$ & $127-03$ & $2.589+05$ \\
\hline .050 & $01+C 6$ & $7.243+02$ & $1-c 3$ & $1.000+06$ \\
\hline .060 & $8.2 \in 5+05$ & $7.242+c 2$ & $4.751=C 3$ & $8.262+05$ \\
\hline .080 & $4.406+06$ & $7.241+c 2$ & $8.386-C 3$ & $4.405+06$ \\
\hline & & & & \\
\hline .150 & $11+c 6$ & $7.233+02$ & $2.873-\mathrm{C2}$ & $7.510+06$ \\
\hline .200 & $4.725+C 6$ & $7.225+02$ & $5.012-C 2$ & $4.724+06$ \\
\hline .300 & & & & \\
\hline .400 & $1.234+06$ & $7.17 \mathrm{C}+\mathrm{C}_{2}$ & $1.852-\mathrm{Cl}$ & $1.233+06$ \\
\hline .500 & $7.547+05$ & $7.131+02$ & $2.775-01$ & $7.940+05$ \\
\hline & & & & \\
\hline & & $t .576+c 2$ & $6.191-01$ & $3.236+05$ \\
\hline 1.000 & $2.163+05$ & $C .847+C 2$ & $4-C 1$ & $2.156+05$ \\
\hline 1.500 & $6.427+$ & $6.471+$ & 1.4 & $6.420+05$ \\
\hline & & $t \cdot C 5$ & $2.076+$ & $3.667+05$ \\
\hline 3.000 & $1.294+C 5$ & $5.236+02$ & $3.172+0 C$ & $1.288+05$ \\
\hline 4. $C 00$ & $6.021+$ & 4.55 & & $5.975+04$ \\
\hline & & 3.58 & 5.1 & $5+04$ \\
\hline 6.000 & $2.021+C_{4}$ & $3.457+C 2$ & t.C3 & $1.985+04$ \\
\hline 8.000 & $9.274+$ & & $7.592+$ & $8.995+03$ \\
\hline & & & $O C$ & \\
\hline 15 & $1.23 t+$ & & 1.1 & 1.222 \\
\hline & $5.725+c$ & $8.807+$ & $1.272+C 1$ & $5.624+03$ \\
\hline & & & & \\
\hline & & & $\mathrm{Cl}$ & \\
\hline
\end{tabular}

\begin{tabular}{|c|c|c|c|c|c|}
\hline $\begin{array}{l}\text { IERGY } \\
\text { (KEV) }\end{array}$ & TOTAL & $\begin{array}{l}\text { COHER . } \\
\text { SCATT. }\end{array}$ & $\begin{array}{l}\text { INCOH. } \\
\text { SCATT. }\end{array}$ & $\begin{array}{r}\text { FHCTO } \\
\text { EFFECT }\end{array}$ & $\begin{array}{l}\text { PAIR } \\
\text { PRCD. }\end{array}$ \\
\hline 50. & $.539+02$ & $1.818+01$ & $1.473+01$ & 3. $21 c+02$ & \\
\hline 60 & & $1.319+01$ & $1.477+01$ & $1.873+02$ & \\
\hline 80 & $8+02$ & $7.841+00$ & $1.45 t+01$ & $7.937+01$ & \\
\hline 100. & & & $1.424+C 1$ & 4. $C 44+01$ & \\
\hline & & $2.406+00$ & $1.324+01$ & $1.1 \varepsilon c+01$ & \\
\hline & & & $1.230+C 1$ & $4.553+0 C$ & \\
\hline & & & & $1.4 \mathrm{CC}+0 \mathrm{C}$ & \\
\hline & & & & $\epsilon . \subseteq \varepsilon 7-C_{1} 1$ & \\
\hline & & & $\varepsilon . \varsigma$ & $3.632-01$ & \\
\hline & & & $\varepsilon .2$ & $2.3 C c-01$ & \\
\hline & 7.4 & & 7.2 & $1.178-01$ & \\
\hline & 6.6 & & $+0 \mathrm{C}$ & $7.325-02$ & \\
\hline & & & 5.3 & $3.3 \varepsilon$ & $4.7 C 3-C 2$ \\
\hline & 4.7 & & 4.5 & $8-02$ & $5-\mathrm{Cl}$ \\
\hline & 4.0 & & & & $899-\mathrm{Cl}$ \\
\hline 4000 & 3.77 & 3.6 & 2.57 & $7.6 \varepsilon 7$ & $c 8-01$ \\
\hline & & & 2.5 & $5.77 s-03$ & $1 . \mathrm{C}^{2} 7+\mathrm{CC}$ \\
\hline & & & & 4.62 & \\
\hline & & & 1.85 & 3.25 & $1.726+C C$ \\
\hline & & & 1.5 & 2.5 & 2. $C 47+C C$ \\
\hline & & & & & $2.6 C 9+C C$ \\
\hline & & 1.4 & & $1.1 c$ & 3. $\mathrm{C} 26+0 \mathrm{C}$ \\
\hline & $4 \cdot 3$ & & $\epsilon . \varepsilon]$ & $7.8 \equiv$ & $.670+c c$ \\
\hline & & & & $5.81 t$ & $4.1 C 5+0 C$ \\
\hline & & & & & $4.4 C 9+0 C$ \\
\hline & & & $3.8 \varepsilon$ & $3.8 \equiv \varepsilon-04$ & $4.635+C C$ \\
\hline & & & & & 5. $C C 4+C C$ \\
\hline & & & & & $5.323+O C$ \\
\hline
\end{tabular}

\begin{tabular}{|c|c|c|c|c|c|}
\hline $\begin{array}{l}\text { NERGY } \\
\text { (KEV) }\end{array}$ & TOTAL & $\begin{array}{l}\text { COHER. } \\
\text { SCATT. }\end{array}$ & $\begin{array}{l}\text { INCCH. } \\
\text { SCATT. }\end{array}$ & $\begin{array}{r}\text { FHCTO } \\
\text { EFFECT }\end{array}$ & $\begin{array}{l}\text { PAIR } \\
\text { PRCL. }\end{array}$ \\
\hline 50. & $3.594+02$ & $1.972+01$ & $1.514+C 1$ & $3 . t 4 t+02$ & \\
\hline 60. & $2.428+02$ & $1.432+01$ & $1.520+01$ & $2.133+02$ & \\
\hline 80. & $1.14 \bar{z}+02$ & $.512+00$ & $1.4 S 5+01$ & S. $c 72+01$ & \\
\hline 100. & $6.663+01$ & $5.630+00$ & $1.467+C 1$ & $4 \cdot 632+01$ & \\
\hline 150 & $2.583+01$ & $2.613+00$ & - $3 t t+C 1$ & $1.256+01$ & \\
\hline & $1.589+01$ & 1.502 & 1. $26 S+01$ & $5.657+0 C$ & \\
\hline 300 . & $1.357+01$ & 6.818 & $1.117+01$ & $1.717+00$ & \\
\hline 400 . & $1.121+01$ & 3.87 & 1. CCE+CI & $7.6 C 7-01$ & \\
\hline & & 2.49 & $5.2 C 4+C C$ & $4.2 C t-C 1$ & \\
\hline & 8. & & $\varepsilon . \Xi$ & & \\
\hline & & & 7.49 & & \\
\hline 1000 . & & 6.32 & $t .74 t+C C$ & 8.51 & \\
\hline & & .8 & 5.4 & $3-02$ & $4.593-02$ \\
\hline & & & & & \\
\hline & & & & & \\
\hline 400 & $3.52 C+00$ & 3.9 & 3. & $-c 3$ & $\varepsilon .375-c_{1}$ \\
\hline & & & & & \\
\hline 600 & & 1.77 & 2.34 & & $386+C c$ \\
\hline & & & & $3.7 \varepsilon$ & $1.826+C C$ \\
\hline & & & & & \\
\hline & & & & & \\
\hline & 4.21 & 1.5 & S.t & & $3.250+00$ \\
\hline & & & & & \\
\hline & & & & $t \cdot t \subseteq 5=04$ & $4.367+C C$ \\
\hline & 5.15 & 2.5 & & $5.323-04$ & $4.689+C 0$ \\
\hline & & 1.7 & & 4.2 & \\
\hline & & $0 s+2$ & 3.16 & $3.2 \subseteq 7-04$ & $+\mathrm{CC}$ \\
\hline & $51+08$ & $6.401-06$ & $2.623-01$ & $2.625-04$ & $5.648+C$ \\
\hline
\end{tabular}

\begin{tabular}{|c|c|c|c|c|c|}
\hline $\begin{array}{l}\text { ENERGY } \\
\text { (KFY }\end{array}$ & TCTAL & COHER . & INCOH. & $\begin{array}{r}\text { PHCTO } \\
\end{array}$ & $\begin{array}{l}\text { PAIR } \\
\text { PRCL. }\end{array}$ \\
\hline $\begin{array}{l}\text { KEVI } \\
50 .\end{array}$ & $455+0$ & $\begin{array}{r}\text { SCATT } \\
2.135+01\end{array}$ & $\begin{array}{r}\text { SCATT } \\
\text { 1. } 554+C 1\end{array}$ & $\begin{array}{r}\text { EFFECT } \\
4.12 t+02\end{array}$ & \\
\hline 60 . & & $1.551+01$ & $1.5 E 1+C 1$ & $2.417+02$ & \\
\hline 80 . & $277+02$ & $9.224+00$ & $1.541+C 1$ & $1.031+02$ & \\
\hline 100 & $.40 c+01$ & $6.102+0 \mathrm{C}$ & 1. $5 C s+01$ & $5.2 \varepsilon C+01$ & \\
\hline 150. & & $2.833+$ & $1.406+C 1$ & $1.552+01$ & \\
\hline & & & $1.3 C 7+01$ & $6.523+0 \mathrm{C}$ & \\
\hline 300. & 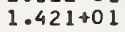 & & $1.151+01$ & 1. $S \in Z+C C$ & \\
\hline 400. & $.165+$ & 4.2 & 1. $c 36+$ & $8.6 \subseteq t-c 1$ & \\
\hline 500. & & & 5.48 & 4.81 & \\
\hline & & & 8.7 & 2.CE & \\
\hline 800. & & & 7.7 & $1.575-01$ & \\
\hline & & & & & \\
\hline & & & & & $5.369-02$ \\
\hline & .08 & 1.7 & $4.828+00$ & $2.77 t-02$ & $2-\mathrm{Cl}$ \\
\hline 30 & 3 & & 3.7 & 1.5 & \\
\hline & & & & & \\
\hline 5000 & 00 & 2.77 & $2.734+0 \mathrm{C}$ & 7.63 & $94+C C$ \\
\hline & & & & & $0+C$ \\
\hline & & & & & $3+c$ \\
\hline & & & & 3.3 & $3 \mathrm{CO}+O C$ \\
\hline & & & 1.2 & & $I+C C$ \\
\hline & & & & $1.5 \varepsilon$ & $3.357+0$ \\
\hline & & & & 1. $C \equiv$ & 4. $C S 2+C C$ \\
\hline & & & 5.7 & & $.583+0 C$ \\
\hline & & & & $t .1 c$ & \\
\hline & & & & & \\
\hline & & 1.085 & $3.260-01$ & $3.77 \varepsilon-c_{4}$ & $5.622+C C$ \\
\hline & & $6.943-06$ & 2. $705-01$ & $3.013-04$ & $5.556+C C$ \\
\hline
\end{tabular}




\begin{tabular}{|c|c|c|c|c|}
\hline & & & & \\
\hline KEV) & & SCATT. & SCATT. & EFFECT \\
\hline .010 & $7.410+C 7$ & $7.690+02$ & $1.3 C 6-C 4$ & $7.410+07$ \\
\hline .015 & $2.492+07$ & $7.69 c+02$ & $2.533-04$ & $2.492+07$ \\
\hline .02 & $9.809+C 6$ & $7.65 C+02$ & $5.205-c 4$ & $9.809+06$ \\
\hline $.0 \geq 0$ & $2.085+0 t$ & $7.689+02$ & $1.1 \in 7-03$ & $2.085+06$ \\
\hline .040 & $5.4 C 2+05$ & $7.68 s+02$ & 2. $c \in 8-C 3$ & $5.394+05$ \\
\hline .050 & $1.494+05$ & $7.688+02$ & 3. & $1.486+05$ \\
\hline .060 & $1.0 t 2+0 t$ & 7.68 & 4.62 & $1.061+06$ \\
\hline .080 & & 7.68 & & $3.211+06$ \\
\hline & 6. & 7.68 & & \\
\hline & 7. & 7.67 & & $b+06$ \\
\hline .200 & $429+c t$ & $7.67 \mathrm{C}$ & 4.8 & $1+06$ \\
\hline .300 & 3. $c 67+0 t$ & $7.647 t$ & & $3.067+06$ \\
\hline .400 & & 7.6 & & +06 \\
\hline .500 & & 7.57 & 2.7 & +06 \\
\hline .600 & $8.813+$ & 7.530 & 3.7 & $6+05$ \\
\hline & & & & \\
\hline 1.000 & 3. $C 14+C 5$ & $7.285+02$ & 8.61 & $3.007+05$ \\
\hline 1.500 & $7.035+05$ & $6.884+02$ & & 7. $c 28+05$ \\
\hline & & 6.42 & & \\
\hline 3.000 & $456+C 5$ & $5.545+02$ & 3.272 & $1.450+05$ \\
\hline c00 & & 4.82 & & \\
\hline & & $4.222+$ & 5.32 & $3.683+04$ \\
\hline 6.000 & $2.276+c 4$ & $3.711+$ & 6.22 & $2.239+04$ \\
\hline & & & & \\
\hline & & 230 & & $5.462+03$ \\
\hline $15 . \mathrm{CCC}$ & 1.25 & $1.384+C$ & $1.143+c 1$ & $1.341+04$ \\
\hline & & & & \\
\hline & & & & \\
\hline & & & & \\
\hline
\end{tabular}

\begin{tabular}{|c|c|c|}
\hline \multicolumn{3}{|c|}{$Z=35$ 8RCMINE } \\
\hline $\begin{array}{r}\text { EAERGY } \\
\text { (KEV) }\end{array}$ & TOTAL & $\begin{array}{l}\text { COHER } \\
\text { SCATT. }\end{array}$ \\
\hline .010 & $1.1 \varepsilon \varepsilon+C 8$ & \\
\hline .015 & $3.719+C 7$ & $\varepsilon .145+02$ \\
\hline .020 & $1.435+C 7$ & $\varepsilon=14 \varsigma+02$ \\
\hline $.03 \mathrm{C}$ & $3.196+C \epsilon$ & $\varepsilon .148+C 2$ \\
\hline .040 & 1. $C 48+06$ & $8.148+02$ \\
\hline .050 & $5.115+0 E$ & $8.147+c 2$ \\
\hline .060 & $3 . t \in 2+c 5$ & $8.147+02$ \\
\hline .080 & $8.906+C E$ & $8.146+02$ \\
\hline .100 & $4.086+06$ & $8.144+C 2$ \\
\hline $.15 \mathrm{C}$ & $6.379+06$ & $8.138+02$ \\
\hline .200 & $5.397+C 6$ & $8.130+02$ \\
\hline .300 & $3.287+c t$ & $8.107+c 2$ \\
\hline .400 & $2.079+C E$ & $8.075+c 2$ \\
\hline .500 & $1.397+06$ & $8.036+02$ \\
\hline .600 & $5.853+C 5$ & 7. $59 \mathrm{C}+\mathrm{C2}$ \\
\hline .800 & $5.441+05$ & $7.876+c 2$ \\
\hline $1 . \mathrm{COC}$ & $3.361+05$ & $7.735+C 2$ \\
\hline 1.500 & $1.333+05$ & $7.317+c 2$ \\
\hline 2.000 & $4.563+05$ & $6.82 s+c 2$ \\
\hline 3.000 & $1.628+C E$ & $5.88 \mathrm{C}+\mathrm{C2}$ \\
\hline 4.000 & $7.557+c 4$ & $5.104+02$ \\
\hline $5.00 \mathrm{C}$ & $4.172+04$ & $4.46 t+02$ \\
\hline $6 . C 00$ & $2.550+04$ & $3.527+02$ \\
\hline 8.000 & $1.171+04$ & $3.078+02$ \\
\hline $1 \mathrm{C.COC}$ & $6.4 C 7+c 3$ & $2.451+c \bar{z}$ \\
\hline 15.000 & $1.457+04$ & $1.488+C 2$ \\
\hline 20.000 & $7.022+03$ & $1 \cdot C 16+c 2$ \\
\hline 30.000 & $2.322+c 3$ & $5.72 s+C 1$ \\
\hline $4 \mathrm{C} \cdot \mathrm{COO}$ & 1. $c 4 t+c 3$ & $3.627+C 1$ \\
\hline
\end{tabular}

I NC OH. \begin{tabular}{l} 
SCATT. \\
$1.229-04$ \\
\hline
\end{tabular} $1.229-04$
$2.7 \in 2-04$ $2.762-04$
$4.502-04$ 1. $C 59-C_{3}$ 1. $548-C 3$ $3 . \mathrm{C} 33-03$ $4.353-03$ 7.687-03 $1.193-C 2$ $2.639-02$ 4. $611-\mathrm{C} 2$ 1. $\mathrm{CCl}-\mathrm{Cl}$ $1.715-C 1$ $2.579-C 1$ $3.5 \epsilon s-C 1$ $8.304-C 1$ $1.465+00$ $2.103+0 C$ $3.342+C C$ $4.473+C C$ $5.452+0 C$ $6.4 \mathrm{Cg}+\mathrm{CO}$ $7.586+C C$ $9.287+C C$ 1. $167+01$ $1.327+01$ $1.508+C 1$ $1.557+C 1$ EFFECT
$.188+08$ $3.719+07$ $1.435+07$ $3.195+06$ $1.047+06$ $5.1 \mathrm{C} 7+05$ $3.654+05$ $8.858+05$ $4.085+06$ $6.378+06$ $5.396+06$ $3.286+06$ $2.079+06$ $1.396+06$ $9.845+05$ $5.433+05$ $3.353+05$ $1.326+05$ $4.556+05$ $1.622+05$ $7.546+04$ $4.127+04$ $2.511+04$ $1.140+04$ $6.152+03$ $1.481+04$ $6.908+03$ $2.249+03$ $9.936+02$
INCOH. PHCTO $\begin{array}{rr}\text { SCATT. } & \text { EFFECT } \\ 1.089-04 & 3.613+08\end{array}$ 2. $448-04 \quad 4.782+07$ $4.345-C_{4} \quad 1.028+07$ $9.746-04 \quad 1.677+06$ $1.727-\mathrm{C} 3 \quad 1.055+06$ $2.691-039.648+05$ $3.8 \in 3-C 3 \quad 8.553+05$ $6.825-c 3 \quad 6.563+05$ 1. C6C-C2 $9.768+05$ 2. $34 \varepsilon-C 2 \quad 4.295+06$ 4.108-02 $4.437+06$ $8.946-02 \quad 3.091+06$ $1.538-\mathrm{Cl} \quad 2.071+06$ $2.321-01 \quad 1.443+06$ 3.224-01 $1.047+06$ $5.313-\mathrm{cl} \quad 6.057+05$ 7.647-01 $3.854+05$ $1.350+C 0 \quad 1.556+C 5$ $\begin{array}{ll}2 . C 48+C C & 4.991+05 \\ 3.365+O C & 1.822+05\end{array}$ $\begin{array}{ll}3.365+0 C & 1.822+05 \\ 4.563+00 & 8.501+04\end{array}$ $5.624+C C \quad 4.640+04$ $6.567+$ CO $2.814+04$ $8.167+C C \quad 1.272+04$ $9.486+00 \quad 6.863+03$ $1.191+01 \quad 1.627+04$ $1.356+01 \quad 7.640+03$ $1.543+\mathrm{Cl} \quad 2.500+03$ 1. $63 t+C 1 \quad 1.106+03$

\begin{tabular}{|c|c|c|c|}
\hline $\begin{array}{l}\text { ENERGY } \\
\text { (KEV) }\end{array}$ & TOTAL & $\begin{array}{l}\text { COHER . } \\
\text { SCATT. }\end{array}$ & $\begin{array}{l}\text { INCOH. } \\
\text { SCA TT. }\end{array}$ \\
\hline 50. & $5.042+02$ & $2.308+01$ & $1.555+01$ \\
\hline 60. & $3.063+02$ & $1.677+01$ & $1.603+01$ \\
\hline 80 & $1.430+02$ & $9.978+00$ & $1.584+01$ \\
\hline 100. & $8.230+01$ & $6.602+00$ & $1.552+01$ \\
\hline $15 \mathrm{C}$ & $3.524+C_{1}$ & $3.066+00$ & $1.447+01$ \\
\hline 200. & $2.267+01$ & $1.763+00$ & $1.345+01$ \\
\hline 300 . & $1.45 C+01$ & $8.006-01$ & $1.185+01$ \\
\hline 400. & $1.212+C_{1}$ & $4.552-01$ & 1. $C 67+C 1$ \\
\hline 500. & $1.062+C 1$ & $2.932-01$ & $5.771+C C$ \\
\hline 600 . & $5.6 C \varsigma+00$ & $2.045-01$ & S. C $52+C C$ \\
\hline 800. & $8.261+00$ & $1.157-01$ & 7. $5 E 4+0 C$ \\
\hline 1000 & $7.352+O C$ & $7.426-02$ & $7.165+c c$ \\
\hline 1500 . & $5.571+00$ & $3.315-02$ & $5.828+0 \mathrm{C}$ \\
\hline 2000 . & $5.244+00$ & $1.868-02$ & $4.574+C C$ \\
\hline 3000 & $4.533+00$ & $8.322-03$ & $3 . \subseteq 12+O C$ \\
\hline 4000 . & $4.243+00$ & $4.686-03$ & $3.2 \in 2+C C$ \\
\hline 5000 . & $4.128+00$ & $3.001-03$ & $2.816+00$ \\
\hline 6000 . & $4.054+00$ & $2.085-03$ & $2.48 \varsigma+0 \mathrm{C}$ \\
\hline & & $1.173-03$ & 2. $c 36+0 c$ \\
\hline 10000 & $4.177+00$ & $7.512-04$ & $1.733+C C$ \\
\hline 15000. & $4.2 \in 2+00$ & $3.340-04$ & $1.282+0 C$ \\
\hline 20000 . & $4.618+00$ & $1.879-04$ & 1. $c>\varepsilon+c c$ \\
\hline 30000 . & $5.16 c+00$ & $8.354-05$ & $7.477-01$ \\
\hline $400 \mathrm{co}$. & $5.538+00$ & $4.700-05$ & $5.536-01$ \\
\hline 50000. & $5.789+00$ & $3.008-05$ & $4.551-01$ \\
\hline 60000. & $5.581+00$ & $2.089-05$ & $4.263-01$ \\
\hline & $6.315+00$ & $1.175-05$ & $3.355-01$ \\
\hline & & & $2.787-$ \\
\hline
\end{tabular}

PHOTO EFFECT $2.735+02$ $1.172+02$ t. $117+01$ $1.771+\mathrm{Cl}$ $7.451+00$ $2.246+C 0$ $5.5 \in 7-01$ $5.52 \varepsilon-01$ 3.522-01 1. $\varepsilon 13-01$ $1.128-01$ 5.1 $57-02 \quad 5.787-\mathrm{C} 2$ $3.1 \subseteq 4-02 \quad 2.1 \subseteq 5-01$ $1.731-C 25.958-01$ $1.1 \in 7-02$ $5.648-01$ $8.7 \in C-C 3 \quad 1.3 \mathrm{CO}+0 \mathrm{C}$ $6.555-03 \quad 1.556+C C$ 4.974-03 2.C76+CC $3.854-03 \quad 2.440+0 C$ $2.4 E 2-C 3 \quad 3 . C 77+C C$ 1. $\varepsilon C \varepsilon-C 3 \quad 3.588+C C$ $1.18 \mathrm{C}-03 \quad 4.411+0 \mathrm{C}$ E. $7 \in C-04 \quad 4.543+C C$ $t .5 \in 4-04 \quad 5.294+C C$ $5.775-C 4 \quad 5.554+C C$ 4.312-04 $5.578+0 C$ $3.4 \geq \varepsilon-04 \quad 6.382+C C$

\begin{tabular}{|c|c|c|c|c|c|}
\hline $\begin{array}{l}\text { ENERGY } \\
\text { (KEV) }\end{array}$ & TCTAL & $\begin{array}{l}\text { COHER } \\
\text { SCATT }\end{array}$ & $\begin{array}{l}\text { INCOH. } \\
\text { SCATT. }\end{array}$ & $\begin{array}{r}\text { PHOTO } \\
\text { EFFEC T }\end{array}$ & $\begin{array}{l}\text { PAIR } \\
\text { PRCE. }\end{array}$ \\
\hline 50 & $5.640+02$ & $2.48 \varsigma+01$ & $1.635+01$ & $5.22 \varepsilon+02$ & \\
\hline 60. & $3.424+02$ & $1.810+01$ & $1.645+01$ & $3 . c 7 \varepsilon+02$ & \\
\hline 80 . & $1.593+02$ & $1.078+01$ & $1.626+01$ & $1.323+c 2$ & \\
\hline 100. & $9.113+01$ & $7.135+00$ & $1.555+01$ & $t . \varepsilon C 5+C 1$ & \\
\hline & $3.827+01$ & $3.316+00$ & $1.487+01$ & $2.0 C \varsigma+01$ & \\
\hline 200 & $2.421+01$ & $1.907+0 \mathrm{C}$ & $1.383+01$ & $\varepsilon .4 \epsilon E+00$ & \\
\hline 300 & $1.561+01$ & $8.663-01$ & $1.21 s+01$ & $2.557+00$ & \\
\hline 400 & $1.261+01$ & $4.92 t-01$ & 1. $c \leqslant 8+01$ & $1.13 t+00$ & \\
\hline 500 . & $1.100+01$ & $3.174-01$ & $1 . C C 5+C 1$ & $6.31 C-01$ & \\
\hline 600 . & $9.940+00$ & $2.214-01$ & $5.316+c c$ & $4 \cdot c 23-01$ & \\
\hline 800 . & $8.52 \varsigma+00$ & $1.252-01$ & $8.197+00$ & $2 \cdot(71-01$ & \\
\hline 1000 & $7.585+00$ & $8.038-02$ & $7.375+c c$ & $1.2 \varepsilon \varepsilon-01$ & \\
\hline 1500 . & $6.155+C C$ & $3.588-02$ & $E_{0} s \varsigma s+c c$ & $5.532-c 2$ & $6.120-02$ \\
\hline 2000 . & $5.425+00$ & $2.023-02$ & $5.120+00$ & $3.643-02$ & $86-01$ \\
\hline 3000 & $4 \cdot t \varepsilon \varepsilon+O C$ & $9.009-03$ & $4 . C 27+O C$ & $1.571-02$ & $\epsilon .321-c 1$ \\
\hline 4000 & $4.370+00$ & & $3.358+0 \mathrm{C}$ & $1.3<s-c 2$ & $9.534-C 1$ \\
\hline 5000 & $4.238+00$ & $3.249-03$ & 2. $\varepsilon S \varsigma+O C$ & S. $\subseteq \in 7-03$ & $1.326+C C$ \\
\hline 6000 & $4.2 \mathrm{C} 2+00$ & $2.257-03$ & $2.5 \in 2+C c$ & $7.556-03$ & $1.630+0 \mathrm{C}$ \\
\hline & $4.261+00$ & $1.270-03$ & $2 \cdot c s t+c c$ & $E .655-03$ & $2.158+C C$ \\
\hline 10000 . & $4 \cdot 384+00$ & $8.133-04$ & $1.784+C C$ & $4.3 \varepsilon 1-03$ & $2.595+C C$ \\
\hline & $4.7 C t+00$ & $3.616-$ & $1.320+0 \mathrm{C}$ & $2.7 \varsigma \varepsilon-03$ & $3.383+C C$ \\
\hline 20000 . & $4 . S E t+00$ & $2.035-04$ & 1. $C 58+O C$ & 2. $C 55-03$ & 3. $S C 5+C C$ \\
\hline 30000 . & $5.4 \mathrm{CC}+0 \mathrm{C}$ & $9.044-05$ & $7.697-01$ & $1.341-c 3$ & $4.629+0 \mathrm{C}$ \\
\hline & $5.765+00$ & $5.088-$ & $6.1111-01$ & S. S52-04 & $5.153+0 \mathrm{C}$ \\
\hline & $6 . c t 6+00$ & $3.257-05$ & $5 . C 57-01$ & $7.511-04$ & $5.555+C c$ \\
\hline & $6.313+00$ & $2.262-05$ & $4.385-01$ & $t .5 t E-04$ & $5.874+C C$ \\
\hline & $6.657+00$ & $1.272-05$ & $3.45 \varepsilon-01$ & $4.858-04$ & $t .350+c c$ \\
\hline & $6.585+C 0$ & $8.142-06$ & $2 . \varepsilon \in 9-01$ & $3.5 C E-04$ & $6.658+0 \mathrm{C}$ \\
\hline
\end{tabular}

INCCH. FHOTO

FHOTO
EFFECT $\begin{array}{ll}1 . E 7 t+C 1 & 5.823+02 \\ 1.687+01 & 3.431+02\end{array}$ $\begin{array}{ll}1.687+01 & 3.471+02 \\ 1.668+01 & 1.477+02\end{array}$ $1.637+017.614+01$ $1.525+C 1 \quad 2.261+01$ $1.423+01 \quad 5.558+0 \mathrm{C}$ $1.254+012.5 C C+O C$ $1.130+01 \quad 1.252+0 C$ 1. $C 34+01 \quad 7.1 \div 2-01$ 5. $584+C C \quad 4.551-01$ $8.432+O C \quad 2.3 E 5-01$ $7.5 \varepsilon 7+C C \quad 1.47 C-01$ $6.171+0 C$ 6.755-02 5. $z t t+C C \quad 4.14 \bar{z}-02$ $4.142+0 C \quad 2.237-02$ $3.454+O C$ 1.ECE-O2 $2.582+0 C \quad 1.125-02$ $2 . t 3 t+0 \mathrm{C} \quad 5 . C 12-03$ $2.156+0 C \quad 6.4 C 3-03$ 1. $83 E+C C \quad 4.55 \subseteq-C 3$ $1.357+C C \quad 3.167-03$ 1. $C B S+C C \quad 2.325-03$ $7.517-011.517-\mathrm{C} 3$ $t .285-C 1$ 1.1 $2 t-C 3$ 5.243-01 8.951-04 4.514-Cl 7.427-04 $3.55 t-C 1 \quad 5.541-04$ $2.551-01 \quad 4.415-04$
PAIF PRCD.

$6.539-c 2$ $2.558-\mathrm{Cl}$ $6.700-C 1$ $1 . C 67+C \mathrm{C}$ $1.428+0 C$ $1.75 C+C C$ $2.715+C C$ $3.473+C C$ $4 \cdot C 31+O C$ $4.875+C C$ $.447+C C$ $5.851+C C$ $6.162+C C$ $6.651+0 \mathrm{C}$
$6.614+0 \mathrm{C}$

$7.007+00$

$1.337-05$

100000 .
$7.364+00 \quad 8.555-06$ 


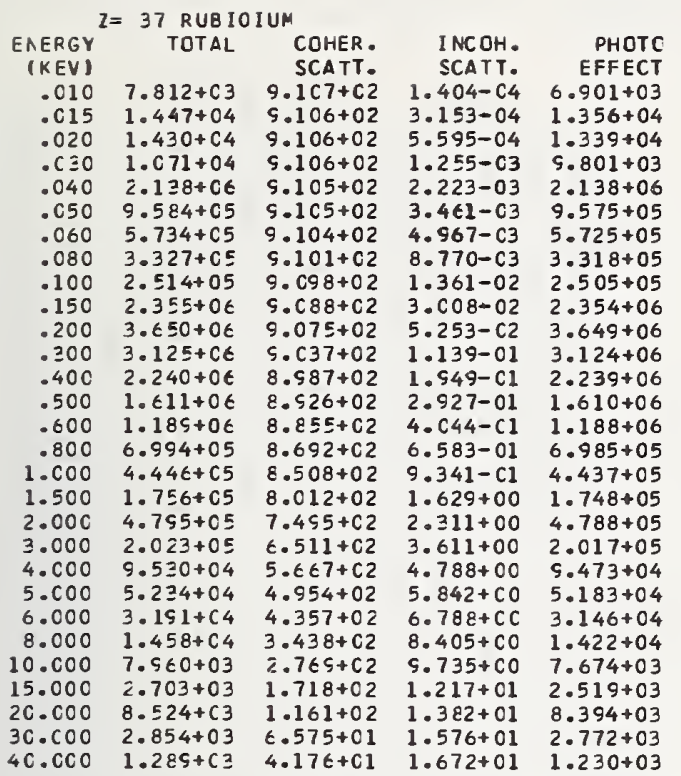

\begin{tabular}{|c|c|c|c|c|}
\hline & & & & \\
\hline (KEV) & & SCATT. & SCATT. & EFFECT \\
\hline .010 & $1.57 s+c 3$ & $9.605+02$ & $1.737-c 4$ & $1.018+03$ \\
\hline .015 & $2.880+04$ & $9.605+02$ & 3. $\mathrm{SCl}-\mathrm{C} 4$ & $2.784+04$ \\
\hline .020 & $5 \in S+04$ & $S .6 C 5+C 2$ & $6.922-04$ & $5.473+04$ \\
\hline .030 & $342+06$ & $5.605+02$ & $1.552-C_{3}$ & $8.341+06$ \\
\hline .040 & $2.626+06$ & $9.604+02$ & $2.749-C 3$ & $2.625+06$ \\
\hline .050 & $\mathrm{CO}+0 \mathrm{t}$ & $5.603+02$ & $4.279-03$ & $1.199+06$ \\
\hline .060 & $7.534+05$ & $5.6 \mathrm{C2}+$ & $6.139-03$ & $7.524+05$ \\
\hline .080 & $5.2 \geq 4+05$ & $c .5 s s+c 2$ & 1.C $83-C 2$ & $5.224+05$ \\
\hline .100 & $4.713+C 5$ & $5.555+C 2$ & $1.680-02$ & $4.703+05$ \\
\hline .150 & $2.297+06$ & $5.583+02$ & $3.710-c 2$ & $2.296+06$ \\
\hline & & & $6.469-C 2$ & $4.565+06$ \\
\hline .200 & $\varepsilon+c \epsilon$ & $c .519+C 2$ & $1.399-01$ & $4.107+06$ \\
\hline $.4 \mathrm{CC}$ & $2.545+C 6$ & $5.457+02$ & $2.387-01$ & $2.944+06$ \\
\hline & & & & \\
\hline .600 & $1.527+\mathrm{C} 6$ & $9.297+02$ & $4.919-01$ & $1.526+06$ \\
\hline $.80 \mathrm{C}$ & $8.6 \in 3+05$ & $5.1 \mathrm{Cl}+$ & 7.94 & $8.653+05$ \\
\hline 1.000 & & $8.887+$ & & $5.214+05$ \\
\hline 1.500 & $1.935+05$ & $8.337+c 2$ & $1.889+C 0$ & $1.930+05$ \\
\hline .000 & $782+0=$ & $7.790+$ & & \\
\hline & & & $3.500+c 0$ & $2.222+05$ \\
\hline 4.000 & $.053+05$ & $5.512+02$ & $5.059+0 C$ & $1.047+05$ \\
\hline $.00 \mathrm{C}$ & $5.815+C 4$ & $5.181+$ & 6.10 & $5.763+04$ \\
\hline & & & $7.043+00$ & $3.520+04$ \\
\hline & & & $8.6 \in 0+C 0$ & $1.607+04$ \\
\hline 000 & S.C10+03 & $2.921+$ & $9.596+\mathrm{CO}$ & $8.708+03$ \\
\hline & & & $1.245+$ & $2.823+03$ \\
\hline & & & & \\
\hline 30.000 & $3.145+C 3$ & 7. CCE +C 1 & $1.609+01$ & $3.063+03$ \\
\hline $4 C .000$ & $1.423+03$ & $4.452+C 1$ & $1.7(9+0)$ & $1.361+03$ \\
\hline
\end{tabular}

\begin{tabular}{|c|c|c|c|c|}
\hline ENERGY & \\
\hline $\begin{array}{l}\text { ENERGY } \\
\text { (KEV) }\end{array}$ & & & INCOH. & PHCTC \\
\hline & & & & \\
\hline & $C 77+06$ & 03 & $.802-c 4$ & \\
\hline .015 & $8+0 t$ & $1.012+03$ & 4. $C 48-04$ & $6.207+06$ \\
\hline .020 & $8 \varepsilon+C 6$ & $1.012+C 3$ & $7.184-04$ & $6.387+06$ \\
\hline .030 & 4. $7 \in 9+C 6$ & $1.012+03$ & $1.610-03$ & $4.768+06$ \\
\hline .040 & $2.2 \epsilon 5+06$ & 1.C12+03 & $2.853-03$ & $2.264+06$ \\
\hline .050 & $1 . t 25+06$ & 1. $C 12+C 3$ & $4.441-03$ & \\
\hline .060 & $1.310+c t$ & $1 . \mathrm{Cl} 1+\mathrm{C} 3$ & $6.371-03$ & $1.309+06$ \\
\hline .080 & $9.310+05$ & $1.011+03$ & $1.12>$ & $9.300+05$ \\
\hline .100 & E. SE0+05 & $1.011+03$ & $1.744-C 2$ & \\
\hline $.15 \mathrm{C}$ & $3.860+C 5$ & 1. $\cos +03$ & $3 . \varepsilon 50-c 2$ & $3.850+05$ \\
\hline .200 & $3.139+06$ & $1.0 C 8+03$ & $6.713-c 2$ & $3.138+06$ \\
\hline & & $1 . \mathrm{CO}_{3}+03$ & & \\
\hline .400 & $2 . t 14+0 \epsilon$ & $9.967+C 2$ & $2.476-01$ & $2.613+06$ \\
\hline .500 & 1. $9 \in 9+C E$ & $5.850+02$ & $3.707-\mathrm{Cl}$ & $1.968+06$ \\
\hline & & & & \\
\hline & & $5.6 \mathrm{CO}+02$ & $8.240-C 1$ & $9.019+05$ \\
\hline 1.000 & $5.693+05$ & $5.37 t+C 2$ & $1.158+C C$ & $5.683+05$ \\
\hline & & & & \\
\hline & $s+c$ & $\mathrm{C} 2$ & $2 . \in 9$ & $1.081+05$ \\
\hline 3.000 & $6+c 5$ & $7.108+C 2$ & $4.033+00$ & $2.438+05$ \\
\hline 4.000 & 1.17 & $6.192+c$ & & $1.165+05$ \\
\hline & +04 & 5.4 & & 6. \\
\hline 6.000 & $5+04$ & $4.785+C 2$ & $7.245+00$ & $3.926+04$ \\
\hline & & $3.801+$ & & \\
\hline & & & & \\
\hline 15.000 & $3.341+03$ & $1.557+C 2$ & $1.273+01$ & $3.132+03$ \\
\hline & $1.019+04$ & $1.315+c 2$ & $1.444+01$ & $1.004+04$ \\
\hline & & & & \\
\hline & & & 1 & \\
\hline
\end{tabular}

\begin{tabular}{|c|c|c|}
\hline $\begin{array}{l}\text { ENERGY } \\
\text { (KEVV) }\end{array}$ & TOTAL & $\begin{array}{l}\text { COHER } \\
\text { SCATT. }\end{array}$ \\
\hline 50. & $6.946+02$ & $2.873+01$ \\
\hline 60. & & \\
\hline 80. & $1.944+02$ & $248+01$ \\
\hline 100. & $1.102+02$ & $275+00$ \\
\hline & $4.492+01$ & $52+00$ \\
\hline 200 . & & $2.217+00$ \\
\hline & $7+01$ & \\
\hline 400. & $1.365+01$ & $3-c_{1}$ \\
\hline 500 . & +01 & $3.695-01$ \\
\hline & & \\
\hline 800 & $9.079+00$ & $1.458-01$ \\
\hline 1000 & $7+0 \mathrm{C}$ & $9.365-02$ \\
\hline & & \\
\hline 20 & t+oc & $2.357-02$ \\
\hline & & \\
\hline 4000 . & & \\
\hline 50 & 4.60 & $3.787-03$ \\
\hline & & $1-03$ \\
\hline & & \\
\hline 100 & $4.76 t+00$ & $9.479-04$ \\
\hline & & \\
\hline & & \\
\hline 300 & $5.916+00$ & $4-04$ \\
\hline & & \\
\hline & & $3.79 t-05$ \\
\hline & $6.932+00$ & $2.636-05$ \\
\hline & & $1.483-05$ \\
\hline & & $.4911-06$ \\
\hline
\end{tabular}

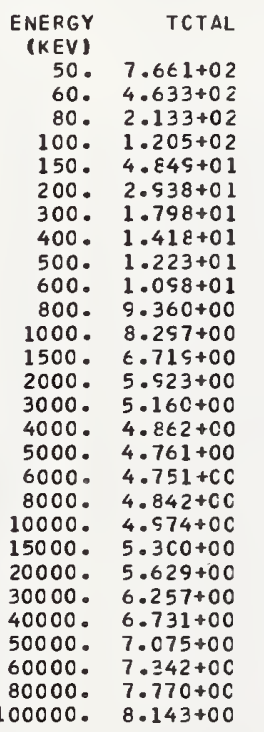

COHER

SCATT

$2.232+01$

$1.332+01$

$8.834+00$

$4.113+0 \mathrm{C}$

$1.077+00$

6.126-01

$3.948-01$

2.754-01

$1.558-01$

$1.000-01$
$4.467-02$

$4.467-02$
$2.518-02$

$1.122-02$

$6.217-03$

4.046-03

2.811-03

$1.582-03$

1. $013-03$

$2.534-04$

$1.126-04$

$6.336-05$

$4.056-05$

2.817-05

$1.584-05$

$1.014-05$
INCOH.

$1.71 E+C 1$

$1.727+C 1$
$1.710+01$

$1.710+01$
$1.675+C 1$

$1.568+C 1$

$1.460+01$

$1.288+01$

$1.160+01$

S. $846+C C$

$8.664+O C$
$7.756+C C$

$7.756+C C$
$6.341+C C$

$5.412+O C$

$4.257+0 C$

$3.550+C C$
$3.065+C C$

$3 . C 65+C 0$
$2.7 C 5+0 C$

$2.215+C C$

1. $886+C C$

$1.395+C C$

$1.115+0 C$

6. $460-01$

5. $388-01$

4.639-01

$3.655-01$
$3.033-01$
PHCTO

PEC

E. $4 \varepsilon 7+C 2$

$3.825+02$
$1.648+02$

$8.511+01$

$2.535+01$

$1 . C 7 \varepsilon+01$

$3.2 \varepsilon \varepsilon+O C$

8.1 $22-01$

5.221-01

2. 6 C C -01

$1.673-01$
$7.6 \varepsilon \varepsilon-02$

4.712-02

$2.542-C 2$

$1.71 \mathrm{C}-02$

$1.281-02$

1.021-02

$7.25 \mathrm{C}-03$

$3.58 \mathrm{C}-03$

$2.627-03$

$1.712-03$

1. $\mathrm{ClC}-03$

$8.3 \varepsilon 1-c 4$

t. $251-04$

4. $5 \varepsilon 5-04$

$1.521+00$

$1.866+C C$

$2.874+O C$

$3.632+C C$

$4.159+C C$

$5.717+C C$

$6.145+C C$

$6.467+C C$

$6.570+C$

$7.4 C 4+C C$

INCCH.
SCATT
$1.754+01$
$1.769+C 1$
$1.752+C 1$
$1.721+01$
$1.6 C 9+01$
$1.455+01$
$1.322+C 1$
$1.152+01$
$1 . C S 1+C 1$
$1 . C 11+01$
$8.85 S+0 C$
$8 . C C 7+0 C$
$6.513+C C$
$5.559+C C$
$4.372+0 C$
$3.646+C C$
$3.148+00$
$2.782+C C$
$2.275+0 C$
$1.527+0 C$
$1.433+00$
$1.149+C C$
$8.357-01$
$6.625-01$
$5.534-01$
$4.765-C 1$
$3.754-01$
$2.115-C 1$

FHCTO

7. $1 \varepsilon C+0$

PAI R

$4.233+c 2$

$1.824+\mathrm{C} 2$

S. $443+01$
$2.82 \varepsilon+01$

$1.2(2+0)$

$3.677+00$

$1.645+00$

S. $22 t-C 1$
$5.51 C-01$

$3 . C 54-C 1$

1. $5(\mathrm{C}-0)$

8.715-02

$5.323 \mathrm{C}-02$
$2.8 \in 8-02$

1. $527-02$

1.442-02

1.145-02

ع.153-03

$6.3 \mathrm{cs}-\mathrm{c} 3$

$2.5 \leq 2-C_{3}$

1. $525-03$

$1.42 \varepsilon-03$

$1.135-03$

7. $117-04$

5.tC1-04

7.406-02 2. $862-C$ $7.482-01$ $1.151+C$

$1.555+C$

$2.557+C$

3. $C 29+0 C$

$3.863+C$

$4.476+C$

t. $C 66+C C$

$6.520+C C$ $6.865+C C$ $7.354+C C$ $7.831+C C$

\begin{tabular}{|c|c|c|c|c|c|}
\hline ENEREY & TCTAL & COHER . & INCOH. & PHCTO & PRCL \\
\hline & & SCATT. & SCATT & EFFEC T & \\
\hline $\begin{array}{l}50 . \\
60 .\end{array}$ & $\begin{array}{l}8.4=0+02 \\
5.108+02\end{array}$ & $\begin{array}{l}3.251+01 \\
2.367+01\end{array}$ & $\begin{array}{l}1.754+01 \\
1.810+C 1\end{array}$ & $\begin{array}{l}7.545+02 \\
4.651+02\end{array}$ & \\
\hline 80. & $2.347+02$ & $1.412+01$ & $1.754+C 1$ & 2. $C \hat{C} E+C 2$ & \\
\hline 100. & $.3 z 1+0 \bar{z}$ & $9.360+00$ & $1.7 \in 3+01$ & 1. $0 \leqslant 1+02$ & \\
\hline 150. & $5.251+01$ & $4.356+00$ & $1.651+01$ & $3.165+01$ & \\
\hline 200. & $3.140+01$ & $2.507+00$ & $1.538+C 1$ & $1.351+01$ & \\
\hline 300 . & $1.886+01$ & $1.140+0$ & $1.357+01$ & $4.153+0 C$ & \\
\hline 400. & $1.474+01$ & $6.485=$ & $1.223+01$ & $1.865+00$ & \\
\hline 500. & $1.26 t+01$ & 4.1 & 1.120 & $1.042+00$ & \\
\hline & & 2.9 & 1.03 & $6 . t E$ & \\
\hline 800 & 9.6 & 1.6 & 5.13 & $3.44 c-01$ & \\
\hline 1000. & 8.5 & & $\varepsilon .21$ & & \\
\hline & $6.5 C$ & 4.72 & $6.684 t$ & 5.82 & $7.841-$ \\
\hline 2000. & $6.1 C 7+00$ & 2.66 & $5.705+00$ & $6.011-02$ & \\
\hline & & 1.18 & $4.487+$ & $3.234-02$ & \\
\hline & & & & $2.171-02$ & \\
\hline 00 . & $4.5 C_{4}+C_{0}$ & $4.281-03$ & $2.231+c c$ & $1.624-02$ & 1 \\
\hline 60 & 4.8 & 2.97 & $2 . \varepsilon 55+C C$ & 1.25 & \\
\hline & & 1.67 & 2.335 & 5.16 & \\
\hline 10000 & $5.2 \mathrm{c3}+$ & 1.072 & 1. $\subseteq 88+0 C$ & 7.05 & $3.2 C 7+$ \\
\hline 15000 . & & 4.7 & 1.4 & 4.5 & \\
\hline & $5.984+00$ & 2.681 & $1.179+O C$ & $3.312-03$ & \\
\hline & $6.544+00$ & 1.192 & $\varepsilon_{.577-C_{1}}$ & $2.15 s-03$ & $5.684+$ \\
\hline & & 6.7 & $\epsilon .80 s$ & 1.6 & \\
\hline & & & $5.67 s-01$ & & \\
\hline & & 2.980 & 4. $890-\mathrm{C1}$ & $1.055-03$ & $7.2 \mathrm{CO}+$ \\
\hline & 8.1 & $1.677-$ & $\equiv .853-$ & $7.87 \mathrm{C}-$ & \\
\hline & $8.510+00$ & $1.073-05$ & $3.157-01$ & $6.275-04$ & $8.190+0$ \\
\hline
\end{tabular}


ENERGY $Z=4 \mathrm{C}$ ZIRCONILM

\begin{tabular}{|c|c|c|c|c|}
\hline KEVI & TOTAL & $\begin{array}{l}\text { COHER. } \\
\text { SCATT. }\end{array}$ & $\begin{array}{l}\text { I NCOH. } \\
\text { SCATT. }\end{array}$ & $\begin{array}{l}\text { PHCTC } \\
\text { EFFECT }\end{array}$ \\
\hline & $7.105 \div 0 t$ & 1. $\mathrm{C}_{64}+\mathrm{C} 3$ & $1.865-c 4$ & \\
\hline & & $1.064+03$ & $4.189-C_{4}$ & \\
\hline & +06 & $64+03$ & $33-04$ & \\
\hline & & . $C 64+03$ & $1.666-03$ & $04+0$ \\
\hline & & $64+03$ & & $8+8>$ \\
\hline - & & +03 & & \\
\hline & & $\mathrm{C} 3$ & 6 & \\
\hline & & $4+03$ & -02 & - \\
\hline & 7. & $-c \in 3+03$ & $+-\mathrm{C} 2$ & \\
\hline & & $C 62+03$ & -02 & 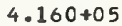 \\
\hline & & +03 & $-0<$ & $8.694+$ \\
\hline & & & & $3+$ \\
\hline & & & & \\
\hline & & & & \\
\hline & & & & \\
\hline & & & & \\
\hline 01 & 6 & 02 & $+\mathrm{CC}$ & \\
\hline $50-25$ & 2. & & & \\
\hline$c 00+8$ & 1.2 & $+\mathrm{C} 2$ & $+\mathrm{CC}$ & $5+0$ \\
\hline 000 & 2.6 & $7.475+c 2$ & $3+0 \mathrm{C}$ & $3+0$ \\
\hline & 1. & $6.503+02$ & $5.310 \div 00$ & $277+$ \\
\hline & & $5.696+02$ & $6.4 \mathrm{C} 2+\mathrm{CC}$ & $7.070+$ \\
\hline & & $.024+02$ & $7 \cdot 389+\mathrm{CO}$ & $4.327 t$ \\
\hline & & $.994+C 2$ & $\mathrm{CO}$ & $.977+$ \\
\hline & & $.248+C 2$ & $1.047+01$ & $1.072+$ \\
\hline & & $-C 7 t+C 2$ & $8+C 1$ & $.495+c$ \\
\hline & & & & \\
\hline & & 7. $5 C C+01$ & $1.676+01$ & $3.679+0$ \\
\hline & $14+C 3$ & $.011+01$ & $1.783+01$ & $1.646+$ \\
\hline
\end{tabular}

\begin{tabular}{|c|c|c|c|c|}
\hline $\begin{array}{r}\text { EAERGY } \\
\text { (KEV) }\end{array}$ & TOTAL & $\begin{array}{l}\text { COHER. } \\
\text { SCATT. }\end{array}$ & $\begin{array}{l}\text { INCOH. } \\
\text { SCATT. }\end{array}$ & $\begin{array}{r}\text { PHOTC } \\
\text { EFFECT }\end{array}$ \\
\hline .010 & 8. $c t \in+c 6$ & $1.118+03$ & $1.626-c 4$ & $8.065+06$ \\
\hline .015 & $1.662+07$ & $1.118+03$ & $3.651-04$ & $1.662+07$ \\
\hline . $\mathrm{C} 2 \mathrm{C}$ & $31+C 7$ & $1.118+C 3$ & $6.480-04$ & $1.031+07$ \\
\hline .030 & $20+C t$ & $1.118+03$ & $1.453-C_{3}$ & $4.519+06$ \\
\hline .040 & $87+06$ & $1.118+03$ & $2.574-03$ & $2.586+06$ \\
\hline . C5C & $67+06$ & $1.118+03$ & $4.007-03$ & $1.766+06$ \\
\hline .060 & $45+C 6$ & $1.118+03$ & $5.750-03$ & $1.344+06$ \\
\hline .080 & $s+c$ & $18+03$ & $1.015-C_{2}$ & $298+05$ \\
\hline .100 & $7.281+c$ & $1.117+03$ & $74-C 2$ & $270+05$ \\
\hline $.15 \mathrm{C}$ & $4.886+$ & $1.116+C_{3}$ & $47 \varepsilon-C 2$ & $.875+05$ \\
\hline .200 & $3.741+C 5$ & $1.115+03$ & $6 . c 70-02$ & +05 \\
\hline .200 & $3.271+c t$ & $1.110+03$ & $1.315-01$ & $3.270+06$ \\
\hline .400 & $3.244+06$ & $1.105+03$ & $2.246-C_{1}$ & $3.243+06$ \\
\hline .500 & 2. $t 4 S+0 t$ & $1.058+03$ & $3.3 \in 8-01$ & $2.648+06$ \\
\hline .600 & $2.065+06$ & $1.050+03$ & $4.646-01$ & $2.064+06$ \\
\hline .800 & $2 S+06$ & $1.071+03$ & $7.535-01$ & $1.228+06$ \\
\hline $1 . \mathrm{COC}$ & $\varepsilon 7+C 5$ & 1. $c 49+03$ & 1. $C E 4+C C$ & $.476+05$ \\
\hline 1.500 & $51+C 5$ & +02 & $1.831+00$ & $741+05$ \\
\hline & 1 & $8+C 2$ & & 105 \\
\hline 3.000 & $2.923+0$ s & 7.945 & 3.94 & $25+05$ \\
\hline 4.000 & $1.414+05$ & $6.887+C 2$ & $5.2 \mathrm{C} 5+0 \mathrm{C}$ & $1.407+05$ \\
\hline $5 . C 00$ & $7.855+C 4$ & $6.010+02$ & $6.352+0 \mathrm{C}$ & $7.798+04$ \\
\hline$t . c C O$ & $4.823+04$ & $5.2 \varepsilon \varepsilon+C 2$ & $7.394+00$ & $4.770+04$ \\
\hline 8.000 & $2.216+04$ & $4.2 \mathrm{C} 2+\mathrm{C} 2$ & $9.187+\mathrm{CO}$ & $2.173+04$ \\
\hline C. $\mathrm{COO}$ & $1.210+C 4$ & $3.428+C 2$ & 1. $C \in 4+01$ & $1.175+04$ \\
\hline 000 & $C 55+03$ & $2.207+C 2$ & $1.322+01$ & $3.821+03$ \\
\hline $2 \mathrm{C}, \mathrm{COO}$ & $02+04$ & $1.484+02$ & $1.457+01$ & $1.185+04$ \\
\hline & $116+c 3$ & +01 & $1.709+C 1$ & $4.015+03$ \\
\hline 40.000 & $1.875+C 3$ & $5 \cdot 314+C 1$ & $1.819+c 1$ & $1.804+03$ \\
\hline
\end{tabular}

\begin{tabular}{|c|c|c|}
\hline \multicolumn{3}{|c|}{$Z=42 \mathrm{MOLY} Y D E N U M$} \\
\hline $\begin{array}{r}\text { ENEREY } \\
\text { (KEV) }\end{array}$ & TOTAL & $\begin{array}{l}\text { COFER } \\
\text { SCATT }\end{array}$ \\
\hline - $C 10$ & $1.232+C 7$ & $1.173+C^{2}$ \\
\hline .015 & $1.605+07$ & $1.173+c 3$ \\
\hline .020 & 1. $C 73+07$ & $1.173+c 3$ \\
\hline .030 & $5.4 t 5+0 t$ & $1.173+03$ \\
\hline .040 & $3.428+C t$ & $1.173+C^{3}$ \\
\hline .050 & $2.445+0 \epsilon$ & $1.173+03$ \\
\hline - $c \in 0$ & 1. $\varepsilon \varepsilon \varepsilon+C \epsilon$ & $1.173+03$ \\
\hline .080 & $1.293+06$ & $1.173+c 3$ \\
\hline .100 & $S .8 \geq S+05$ & $1 \cdot 173+c 3$ \\
\hline .150 & $\epsilon .154+05$ & $1.171+03$ \\
\hline .200 & $4.538+C 5$ & $1.170+c 3$ \\
\hline .300 & $2.910+C \epsilon$ & $1.166+c 3$ \\
\hline .400 & $3.081+0 t$ & $1.16 C+C 3$ \\
\hline $.50 \mathrm{C}$ & $2.584+06$ & $1.154+C 3$ \\
\hline .600 & 2. $C 48+06$ & $1.14 \epsilon+C 3$ \\
\hline .800 & $1.253+0 t$ & $1.127+\mathrm{C} 3$ \\
\hline 1.000 & $7.860+05$ & $1.105+03$ \\
\hline $1.50 \mathrm{C}$ & $3.073+05$ & 1. $C 42+c 3$ \\
\hline 2.000 & $1.527+05$ & $5.729+02$ \\
\hline 3.000 & $3.16 s+C 5$ & $8.390+02$ \\
\hline 4. 000 & $1.546+C 5$ & $7.260+02$ \\
\hline 5.000 & $8.639+C 4$ & $6.324+C 2$ \\
\hline$\epsilon .000$ & $5.319+04$ & $5.55 t+C 2$ \\
\hline 8.000 & $2.452+C 4$ & $4.412+c 2$ \\
\hline 10.000 & $1.341+04$ & $3.603+02$ \\
\hline $15.00 \mathrm{C}$ & $4.455+C 3$ & $2.320+c 2$ \\
\hline 20.000 & $1.305+04$ & $1.575+02$ \\
\hline $3 \mathrm{C} \cdot \mathrm{COO}$ & $4.483+03$ & $\varepsilon .8 \varsigma \varepsilon+C 1$ \\
\hline 40.000 & $2.046+03$ & $5.631+01$ \\
\hline
\end{tabular}

SCATT.

$1.599-C_{4}$

$3.592-c 4$

$6.375-04$

$1.429-C 3$

$3.942-03$

$5.657-03$

$9.586-03$

$1.549-C 2$

3.423-02

5.975-02

$1.254-01$

$2.212-01$

$3.318-01$

$4.579-c 1$

$1.051+00$

$1.051+00$

$2.549+\mathrm{CO}$

$3.541+0 C$

$5.222+C C$

$6.394+00$

$7.464+00$

$5.313+\mathrm{CO}$

$1.081+01$

$1.527+C 1$

$1.527+C 1$
$1.744+C 1$

$1.857+C 1$

$\begin{array}{rr}\begin{array}{r}\text { ENERGY } \\ \text { (KEV) }\end{array} & \text { TCTAL } \\ 50 . & 5.249+02 \\ 60 . & 5.599+02 \\ 80 . & 2.574+02 \\ 100 . & 1.447+02 \\ 150 . & 5.687+01 \\ 200 . & 3.353+C 1 \\ 300 . & 1.577+01 \\ 400 . & 1.531+01 \\ 500 . & 1.305+01 \\ 600 . & 1.170+01 \\ 800 . & 9.925+00 \\ 1000 . & 8.775+00 \\ 1500 . & 7.055+00 \\ 2000 . & 6.263+00 \\ 3000 . & 5.484+00 \\ 4000 . & 5.2 \mathrm{C} 2+00 \\ 5000 . & 5.123+00 \\ 6000 . & 5.135+00 \\ 8000 . & 5.259+0 \mathrm{C} \\ 10000 . & 5.4 \mathrm{C} 8+00 \\ 15000 . & 5.752+0 \mathrm{C} \\ 20000 . & 6.117+00 \\ 30000 . & 6.852+0 \mathrm{C} \\ 40000 . & 7.401+00 \\ 50000 . & 7.789+00 \\ 60000 . & 8 . \mathrm{C} 85+00 \\ 80000 . & 8.560+00 \\ 100000 . & 8.989+00\end{array}$

COHER :
SCATT:
$3.443+01$ $3.443+01$
$2.506+C 1$

$1.494+01$

$9.896+00$

$4.603+00$

$2.648+00$

$1.204+00$

6.846-01

4.411-01

3.077-0

$1.740-01$
$1.117-01$

4.989-02

2.812-02

$1.253-02$

7.054-03

$4.518-03$

$1.766-03$

1.131-03

5.028-04

2.829-04

1.258-04

7.075-05

4.528-0

$3.145-05$

1.132-05

100000 .
COHER -

$3.649+0$

$2.655+01$

$1.581+0$

$4.867+00$

$2.799+00$

$1.272+0 \mathrm{C}$

7.234-01

4.660-01

3.250-01

1.838-01

$1.180-01$

2.970-02

1. $323-02$

7.450-03

$4.771-C^{3}$

3.315-03

$1.86 t-03$

1.194-03

5.311-0

2. $988-04$

1.328-04

7.472-0

4.783-05

3.321-05

$1.868-05$

1.196-05
INCOH.

$\varepsilon 32+C 1$

$1.849+01$

$1.834+01$

1. $805+01$

$1.6 S C+01$

$1.575+C 1$

$1.253+01$

$1.148+01$

1. $C \in 4+C 1$

S. $3 E 4+O C$

$6.855+O C$

c. $\varepsilon 51+C C$

$4.6 C 2+0 C$

3. $\varepsilon 3 \varepsilon+C C$

$3.213+00$

2. $528+O C$

$2.355+O C$

2. $C 35+0 C$

1. $5 c \varepsilon+C C$

8.797-0

6.584-01

5.825-01

5. C $1 \epsilon-C 1$

$3.279-01$
PHOTO

EFFECT

$\varepsilon .722+02$

$2.241+02$

$1.168+02$

$3.5 \geq 7+01$

$1.514+01$

$4.662+00$

$1.172+00$

7.454-01

3. $8 \in S-01$

$2.4 C 7-01$

$8.4 C 2-C 2$

$2.433-02 \quad 1.333+C c$

$1.815-02 \quad 1.787+C C$

$1.44 \varepsilon-02 \quad 2.189+C C$

1. $C 2 E-02 \quad 2.852+C C$

$7.525-03 \quad 3.360+C C$

$3 . C 45-03 \quad 4.239+C C$

$2.411-035.570+C C$

$1.7 \varepsilon \varepsilon-03 \quad 6.7 \mathrm{Cl}+C \mathrm{C}$

$1.420-03 \quad 7.2 \mathrm{C} 5+C \mathrm{C}$

$1.17 \varepsilon-C 3 \quad 7.582+C C$

$\begin{array}{ll}2.787-04 & 8.164+C C\end{array}$

INCOH.

FHOTO

EFFECT

$1.872+01$ S. $5 \varepsilon S+02$

$1.891+01 \quad 5.6 S C+02$

$1.875+01 \quad 2.477+02$

1. $E 4 \epsilon+C 1 \quad 1.2 \div 2+C 2$

$1.730+013.917+01$

$1.613+01 \quad 1.67 \varepsilon+01$

$1.424+015.1 \varepsilon 2+0 \mathrm{C}$

$1.2 \varepsilon 4+01 \quad 2.324+00$

$1.177+01 \quad 1.3 \mathrm{C} 7+O C$

$1 . C 90+C 1 \quad 8.373-01$

5.558+00 4.337-01

E. $627+C \mathrm{C} \quad 2.7 \mathrm{C} 4-01$

7. $C 2 \epsilon+O C$

$5.557+0 \mathrm{C}$

$4.717+C C$

$3.533+0 C$

3. $3 S E+O C$

. $455+C$

2. $\mathrm{CSO}+\mathrm{CC}$

$1.546+C C$

$1.240+00$

$1.245-01$

$7.61 \varepsilon-02$

$4 . C 51-C 2$

$2.74 \mathrm{C}-02$

$2 . C 4 t-C 2$
$1.627-02$

1.151-C2

8. 883-03

$5.64 t-03$

$4.124-03$

$2.6 \$ 1-03$

$7.158-C 1$

5. $571-01$

$1.5 \$ 4-03$

$3.3 \in \mathrm{C}-\mathrm{Cl} 1 \mathrm{~S} \quad \mathrm{7SC}-\mathrm{C} 4$

S. C19-C?

$3.258-C 1$

E. 763-C

$1.894+C C$

$2.321+O C$

$3.016+C C$

$3.536+C C$

$4.412+C C$

. $C 88+C C$

7. $\mathrm{CC}^{2}+\mathrm{CC}$

$7.533+C C$

7. $c 18+C C$

E. $5 \mathrm{C} 2+\mathrm{CC}$

9. $\mathrm{CC}+\mathrm{CC}$

\begin{tabular}{|c|c|}
\hline $\begin{array}{l}\text { EAERGY } \\
\text { (KEVY) }\end{array}$ & TCTAL \\
\hline 50. & $1.107+C 3$ \\
\hline 60. & $6.713+c=$ \\
\hline 80. & $3.084+02$ \\
\hline 100. & $1.724+02$ \\
\hline 150. & $6.632+01$ \\
\hline 200. & $3.817+01$ \\
\hline 300. & $2.173+01$ \\
\hline 400. & $1.652+01$ \\
\hline 500. & $1.400+C 1$ \\
\hline 600 . & $1.245+C 1$ \\
\hline 800 & $1.051+01$ \\
\hline 1000. & $9.274+00$ \\
\hline 1500. & $7.487+0 \mathrm{C}$ \\
\hline 2000 . & $6.624+00$ \\
\hline 3000 . & $5.815+00$ \\
\hline 4000. & $5.516+C 0$ \\
\hline 5000 . & $5.432+00$ \\
\hline 6000 . & $5.452+0 \mathrm{C}$ \\
\hline 8000 . & $5 . \in 18+00$ \\
\hline 10000. & $5.834+00$ \\
\hline 15000. & $6.342+00$ \\
\hline 20000 . & $6.776+0 \mathrm{C}$ \\
\hline 30000 . & $7.5 \mathrm{C} 8+\mathrm{CO}$ \\
\hline 40000 . & $8 . C 71+0 C$ \\
\hline 50000 & $8.5 C 3+00$ \\
\hline 60000 . & $8.850+00$ \\
\hline 80000 . & $9.394+00$ \\
\hline .00000 . & $9.833+00$ \\
\hline
\end{tabular}

EFFECT

$1.232+07$

$1.604+07$

$1.072+07$
$5.464+06$

$3.427+06$

$2.444+06$

$1.887+06$

$1.292+06$

$9.828+05$

$4.527+05$

$2.909+06$

$3.079+06$

.

$1.252+06$

$7.849+05$

$3.063+05$

$3.161+05$

$1.538+05$

$8.575+04$

$5.263+04$

$1.304+04$

$4.24 \mathrm{~s}+03$

$1.288+04$

$4.377+03$
$1.971+03$
COHER SCATT.

INCOH

SCATT

1. $\subseteq 11+C 1$

1. $531+01$

$2.808+C 1$

$1.671+0$

$1.106+01$

$2.953+00$

$1.341+0 C$

7.627-0

4.913-01

1.938-01

1.244-01

5.553-02

$3.130-02$

$1.394-02$

7.851-03

$5.028-03$
$3.493-03$

1.966-03

1.259-03

5.596-04

3.149-04

$1.400-04$

$7.874-05$

3.500-05

1.969-05

1.260-05

$1.917+01$
$1.887+01$

1. $.77 \mathrm{C}+\mathrm{C} 1$

$1.651+01$

$1.459+01$

$1.316+0$

$1.2 \mathrm{C} 5+0$

$.117+0$

$8.848+C C$

$7.1 S 8+C C$

$6.143+C O$

4. $C 29+0$

$3.479+O C$

$3 . \mathrm{C} 75+\mathrm{CC}$

$2.515+00$

$2.141+00$

1. $583+0 \mathrm{C}$

$1.270+0 C$

..237-01

7. 323-C

t.116-0

5. $266-01$

$4.145-01$
$3.442-C 1$

PHCTO

PAIF

EFFEC

PRCE.

$6.235+02$

$2.725+02$

$1.4 Z 5+02$

$4.34 \varepsilon+C 1$

$1.871+01$

5. $\varepsilon C ?+O C$

2. $t 15+O C$ 


\begin{tabular}{|c|c|c|c|c|}
\hline \multicolumn{5}{|c|}{$Z=43$ TECHNETIUM } \\
\hline EAERGY & TOTAL & COrER. & $1 \mathrm{NCOH}$. & $\begin{array}{l}\text { PHOTO } \\
\text { EFFECT }\end{array}$ \\
\hline $\begin{array}{r}\text { (KEV) } \\
. \text { C10 }\end{array}$ & $1.425+07$ & $1.230+C 3$ & $1.876-C 4$ & 1.434007 \\
\hline .015 & $1.185+07$ & $1.230 * 03$ & $4.212-c 4$ & $1.185 \times 07$ \\
\hline .020 & $1.170+C 7$ & $1.230+c 3$ & $7.475-c 4$ & $1.170+07$ \\
\hline $.0 \equiv 0$ & $\varepsilon .5556+C 6$ & $1.230+C 3$ & $1 \cdot \in 76-C 3$ & $8.554 * 06$ \\
\hline .040 & $6.033+06$ & $1.230 \diamond 03$ & $2.968-03$ & $6.032 \$ 06$ \\
\hline .050 & $4.4 C 8+C 6$ & $1.230+c 3$ & $4.620-03$ & $4.406+06$ \\
\hline .060 & $3.34 t+0 t$ & $1.230+C 3$ & $6.628=03$ & $3.345+06$ \\
\hline .080 & $2.11 t+C E$ & $1.229 \times C 3$ & $1.170-c 2$ & $2.115 \bowtie 06$ \\
\hline .100 & $1.4 \epsilon 4+06$ & $1.229+03$ & $1.814-02$ & $1.463+06$ \\
\hline & & & & $7.382+05$ \\
\hline .200 & $4.54 S+C 5$ & $1.226+03$ & $6.980-02$ & 4.537 .05 \\
\hline .300 & $3.5 \varepsilon 1+c \epsilon$ & $1.222+03$ & $1.5 \mathrm{Cs}-\mathrm{Cl}$ & $3.580+06$ \\
\hline & & & & \\
\hline $.5 \mathrm{co}$ & 2. $507+06$ & $1.208+C 3$ & $3.847-\mathrm{Cl}$ & $2.905+06$ \\
\hline .600 & $2.275+C \epsilon$ & $1.1 s s+c 3$ & $5.253-\mathrm{Cl}$ & $2.274+06$ \\
\hline & $1.371+0 t$ & $1.175+C 3$ & $8.533-$ & \\
\hline $1.0 c 0$ & $8.5 \varepsilon 3+C 5$ & $1.155+C 3$ & $1.1 \subseteq 6+C C$ & $8.571 \div 05$ \\
\hline 1.500 & $3.402+05$ & $1 . c 8 s+03$ & $2.012+$ & \\
\hline & $1.710+C 5$ & $1 . C 16+03$ & $2.760+$ & $1.700+C 5$ \\
\hline 3.000 & 3. $c s s+c s$ & $8.77 c+c 2$ & $4.115+00$ & $3.090+05$ \\
\hline $4 . C 00$ & $1.6 E 0+05$ & 7.5 & $5.3 \in 2+$ & 1.672 \\
\hline & $9.418+c 4$ & $6.618+C 2$ & $6.536+C C$ & $9.351+04$ \\
\hline & & $5 . \varepsilon 17+c 2$ & $7.618 * 0 C$ & $5.753+04$ \\
\hline & $2.687 \%$ & 4.621 & 5.5104 & $2.640+04$ \\
\hline & & $3.777+c 2$ & $1.104+$ & $1.433 \approx 04$ \\
\hline & & & & 4.08703 \\
\hline & $2.277+03$ & $1.665+C 2$ & $1.555+C 1$ & $2.095+03$ \\
\hline & & & & \\
\hline & & & & \\
\hline
\end{tabular}

$Z=44$ RUTHENILM

\begin{tabular}{|c|c|c|c|c|}
\hline EAEREY & TOTAL & COtER. & I NCOH. & PHCTC \\
\hline (KEV) & & SCATT. & SCATT. & EFFECT \\
\hline - $C 10$ & $1.524+C 7$ & $1.2 \varepsilon \varepsilon+03$ & $1 . \in 17-c 4$ & $1.924+07$ \\
\hline .015 & $1.1 \in 4+07$ & $1.288+C 3$ & $3.631-c 4$ & $1.164+07$ \\
\hline .020 & $1.28 E+C 7$ & $1.288+c 3$ & $6.444-c 4$ & $1.285+07$ \\
\hline.$<30$ & $1.034+07$ & $1.288+03$ & $1.445-03$ & $1.034+07$ \\
\hline .040 & $7 . t 16+c t$ & $1.28 \varepsilon+03$ & $2.560-C 3$ & $7.614+06$ \\
\hline .050 & $5.723+c 6$ & $1.288+C 2$ & $3.585-03$ & $5.722+06$ \\
\hline.$c \in 0$ & $4.441+C 6$ & $1.2 \varepsilon \varepsilon+03$ & $5.719-C 3$ & $4.439+c 6$ \\
\hline .080 & $2.906+06$ & $1.287+C 3$ & $1.0 \mathrm{C} 9-\mathrm{C}_{2}$ & $2.905+06$ \\
\hline .100 & $2.067+0 t$ & $1.287+03$ & $1.5 \in 6-c 2$ & $2.066+06$ \\
\hline .150 & $1.105+c 6$ & $1.28 t+C 3$ & $3.460-c 2$ & $1.103+06$ \\
\hline .200 & $7.1 C 7+05$ & $1.284+03$ & $\mathrm{C.C} 3 \mathrm{~s}-\mathrm{C} 2$ & $7.094+05$ \\
\hline .300 & $2.2 \varepsilon 4+0 \epsilon$ & $1.281+C 3$ & $1.3 \mathrm{C} 8 \rightarrow \mathrm{Cl}$ & $2.283+06$ \\
\hline .400 & $2.9 t 5+0 t$ & $1.275+C ?$ & $2.235-\mathrm{Cl}$ & $2.964 * 06$ \\
\hline .500 & $2.7 \varepsilon E+C \epsilon$ & $1.268+03$ & $3.352-01$ & $2.786+06$ \\
\hline .600 & $355+C 6$ & $61+C 3$ & $5-01$ & $2.354+06$ \\
\hline & & & & $1.543+C 6$ \\
\hline 1.000 & $9.512+05$ & $1.22 \mathrm{C}+03$ & $1.061+C C$ & $9.899+05$ \\
\hline 1.500 & $3.842+C 5$ & $1.154 \div C 3$ & $1.828+C C$ & $3.831+C 5$ \\
\hline 2.000 & $1.8 \varepsilon 3+C 5$ & $1 . C 81+C 3$ & $2.569+\mathrm{CO}$ & $1.872+05$ \\
\hline 3.000 & $3.251+05$ & $5.344+C 2$ & $3.576+C 0$ & $3.242+05$ \\
\hline 4.000 & $1.822+05$ & & & $1.814 \div 05$ \\
\hline & $1 . C \geqslant 1+C 5$ & $7 . \mathrm{CO}^{2}+\mathrm{C}^{2}$ & $6.513+c c$ & $1.023 * 05$ \\
\hline$t . c 00$ & $6.382+04$ & $6.136+02$ & $7.641+00$ & $6.320+04$ \\
\hline$\varepsilon . \operatorname{ccc}$ & $2.954+$ & $4.85 \varepsilon+C 2$ & $9.6 C 9+00$ & $2.905+04$ \\
\hline & & $3.570+c 2$ & & $1.575+C 4$ \\
\hline & & & & $5.131+c$ \\
\hline 20.000 & $2.459+03$ & $1.764+C 2$ & $1.585+\mathrm{Cl}$ & $2.307+03$ \\
\hline & & $\subseteq . \subseteq 32+c 1$ & $1.811+C 1$ & \\
\hline & & & $1.931+01$ & $2=250$ \\
\hline
\end{tabular}

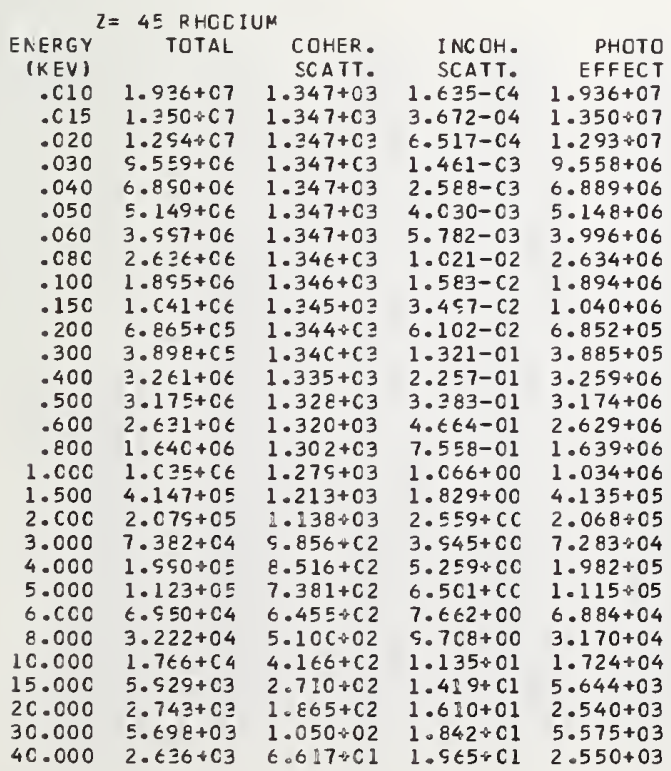

\begin{tabular}{|c|c|c|c|c|c|}
\hline $\begin{array}{l}\text { ENEREY } \\
\text { (KEV): }\end{array}$ & TAL & $\begin{array}{l}\text { COHER. } \\
\text { SCATT. }\end{array}$ & & $\begin{array}{l}\text { PHCTO } \\
\text { EFFECT }\end{array}$ & $\begin{array}{l}\text { PAIF } \\
\text { PRCL. }\end{array}$ \\
\hline 50. & $1.212+03$ & $4.068+01$ & 1. $549+C 1$ & $1.151+03$ & \\
\hline 60. & & $.954+01$ & $1.571 * 01$ & $6.843+c 2$ & \\
\hline 80. & $3.257+02$ & $1.756+01$ & $1 . \subseteq 58+C 1$ & $2 . S E t+02$ & \\
\hline 100. & $1.871+0 \bar{z}$ & $1.1 \in 1+01$ & $1.53 C+01$ & $1.5 \leqslant 2+c 2$ & \\
\hline & $7.125+01$ & $5.390+$ & $1.812+01$ & $4.775+01$ & \\
\hline & $4 \cdot C 5 t+01$ & $3.097 \%$ & $1.65 C+01$ & 2. CEE $+C_{1}$ & \\
\hline & $2.273+01$ & $1.40 t$ & $3+01$ & $6.352+0 \mathrm{C}$ & \\
\hline & & & $1.347+01$ & $2 . \varepsilon \leqslant 2+C C$ & \\
\hline & 1.44 & & $1.234+01$ & $1.622+00$ & \\
\hline & $1.283+01$ & & & 1. $C \equiv S+00$ & \\
\hline & 1.08 & & +01 & 5.3 & \\
\hline 100 & & & & $3.344-01$ & \\
\hline 150 & $7.681+00$ & 5.81 & $7.365+00$ & $1.533-01$ & 1. $c C 6-c 1$ \\
\hline & $\epsilon .78 s+C 0$ & & & $-c 2$ & $c-1$ \\
\hline & & & & & $s c-C$ \\
\hline 400 & 5.78 & 8.2 & $4.125+0 \mathrm{C}$ & $3.35 t-c 2$ & $1.540+C$ \\
\hline & 5.64 & 5.2 & & & $57+c$ \\
\hline & 5.68 & & 3.148 & & \\
\hline 80 & 5.86 & 2.0 & $2.575+C C$ & $1.41 \mathrm{C}$ & $24+C 0$ \\
\hline & 6.06 & & & 1. C\&9-02 & $57+C c$ \\
\hline & $6.5 \mathrm{CO}+0 \mathrm{C}$ & 5.86 & $1 . t$ & & $72+c$ \\
\hline & $6.935+00$ & 3.29 & & & \\
\hline & & & & & $6.840+C D$ \\
\hline & $8.422+00$ & $8.246-$ & & & $7.668+c c$ \\
\hline 50000 & $8.87 c+00$ & $5.277-$ & 6.26 & & \\
\hline & & & 5.3 & & $8.672+0$ \\
\hline & & & & & \\
\hline & & & & $5.5 \varepsilon$ & \\
\hline
\end{tabular}

\begin{tabular}{|c|c|c|c|c|c|}
\hline $\begin{array}{c}\text { ENEREY } \\
\text { (KEVV) }\end{array}$ & TCTAL & $\begin{array}{l}\text { COHER } \\
\text { SCATT }\end{array}$ & $\begin{array}{l}\text { INCOH. } \\
\text { SCATT: }\end{array}$ & $\begin{array}{r}\text { PHCTO } \\
\text { EFFECT }\end{array}$ & $\begin{array}{l}\text { PAIR } \\
\text { PRCL. }\end{array}$ \\
\hline 50. & $1.321+03$ & $4.284+01$ & $1 . \varsigma 8 S+01$ & $1.255+03$ & \\
\hline 60. & $8.006+02$ & $3.108+01$ & 2.c $12+01$ & $7.4 \subseteq 4+02$ & \\
\hline 80. & 3.660402 & $1.845+01$ & 2. $C C C+C 1$ & $\Xi .27 t+02$ & \\
\hline 100. & $.033+02$ & $1.219+01$ & . $\$ 71+01$ & $1.714+02$ & \\
\hline 150. & $7.663+01$ & $5.651+00$ & 1. $852+01$ & $5.24 t+C 1$ & \\
\hline 200 & $4.318+01$ & $3.246+00$ & $1.72 s+01$ & $2 . \overline{\angle E E} E+01$ & \\
\hline 300. & $2.3 \varepsilon 2+01$ & $1.473+0 C$ & $.528+C 1$ & & \\
\hline 400. & $1.782+01$ & $8.369-01$ & $1.378+C 1$ & $3.1 \subseteq 8+0 C$ & \\
\hline 500. & $1.455+01$ & 5.38 & $1.263 * C 1$ & $1.7 E 7+C C$ & \\
\hline 600. & $1.322+01$ & $3.758-$ & $1.170+C 1$ & $1.141+0 C$ & \\
\hline 80 & & 2.1 & & $-C 1$ & \\
\hline 100 & & 1.3 & 5.26 & 3.67 & \\
\hline 1500 & $7.877+4$ & 6.08 & $7.54 \mathrm{C}+\mathrm{CC}$ & 1.65 & 1. $\mathrm{C} 6 \mathrm{~S}-\mathrm{Cl}$ \\
\hline 200 & & & & & \\
\hline & & 1.5 & & & \\
\hline 4000 . & & 8.60 & $4.221+0 C$ & 3.724 & \\
\hline 5000 . & $5.827 \leftrightarrow 00$ & 5.50 & 3.6 & -02 & 2. \\
\hline & & & & & \\
\hline & & & & & \\
\hline 10000 . & 6.251 & 1.37 & 2.2 & $-c 2$ & 4.034 \\
\hline & & & & & \\
\hline & & & & & \\
\hline 00 & 8.0 & 1.5 & 5.6 & 3 . & 7.669 \\
\hline & & 8.62 & & & \\
\hline & $9.187 \div 00$ & & & & \\
\hline & $5.557+0 \mathrm{C}$ & $3.834-05$ & $5.517-$ & & S. $C C 3+c$ \\
\hline & & $2.157-$ & $4.347-$ & 1.325 & \\
\hline & $1.055+01$ & $1.380-05$ & $\exists . \in C t-c]$ & 1. CES- 03 & $1.023+c$ \\
\hline
\end{tabular}

\begin{tabular}{|c|c|c|c|c|c|}
\hline $\begin{array}{l}\text { EAERGY } \\
\text { (KEV) }\end{array}$ & TOTAL & $\begin{array}{l}\text { COHER } \\
\text { SCATT }\end{array}$ & $\begin{array}{l}\text { INCOH. } \\
\text { SCATT. }\end{array}$ & $\begin{array}{r}\text { FHOTO } \\
\text { EFFECT }\end{array}$ & $\begin{array}{l}\text { PAIR } \\
\text { PRCL. }\end{array}$ \\
\hline 50. & $1.435+03$ & $4.527+01$ & 2. $C 26+C 1$ & $1.37 c+03$ & \\
\hline 60. & $8.715+C 2$ & 3.284401 & $2.05 C+C 1$ & $8.185+02$ & \\
\hline 80 & $3.557+02$ & $1.950+01$ & 2. $C 35+01$ & $3.5 \subseteq \varepsilon+C 2$ & \\
\hline 100 . & $2.219+02$ & $1.288+01$ & $2.012+01$ & $1.8 E S+C 2$ & \\
\hline 150. & $8.258+01$ & $5.971+00$ & 1. ESO+CI & $5.771+01$ & \\
\hline 200. & $4.554+C 1$ & $3.429+00$ & 1. $7 \in 5+01$ & $2.44 t+01$ & \\
\hline 300. & $2.451+01$ & $1.556+00$ & $1.5 \in O+C 1$ & $7.752+0 \mathrm{C}$ & \\
\hline 400. & $1.852+C 1$ & $8.841-01$ & 1. $4 C E+C 1$ & $3.555+0 C$ & \\
\hline & $1.548+01$ & $5.693-01$ & $1.2 S 0+01$ & $2 . C C 5+00$ & \\
\hline 600. & 1. $3 \Leftrightarrow 4+01$ & $3.970-\mathrm{cl}$ & $1.15 t+01$ & $1.2 E Z+O C$ & \\
\hline 800. & $1.142+01$ & $2.244-01$ & 1. $C 53+01$ & $\epsilon \in \in 25-01$ & \\
\hline 1000 . & $1.002+01$ & $1.441-01$ & $5.477 .0 \mathrm{C}$ & $4.11 t-01$ & \\
\hline 1500 . & $8.075+00$ & $6.430-02$ & $7.711+O C$ & $1.822-01$ & $1.113-01$ \\
\hline 2000 . & $7.161+00$ & $3.624-02$ & $\epsilon=5 \varepsilon 2+C C$ & $1.147-01$ & $4.280-C 1$ \\
\hline & 6.318 & 1.614 & $5.177+0 \mathrm{C}$ & $6.1 \geq 2-02$ & 1. $C 64+0 \mathrm{C}$ \\
\hline 4000 . & $6.022+00$ & $9.088-03$ & $4.317+00$ & $4.1 C C-C 2$ & $1.655+C C$ \\
\hline 5000 . & $5.557+00$ & $5.820-03$ & $3.728+C C$ & 3.C $E \varepsilon-02$ & $2.153+O C$ \\
\hline & $6.0 C 4+00$ & & $3.254+0 \mathrm{C}$ & $2.431-02$ & $2.6 \mathrm{Q} 1+0 \mathrm{C}$ \\
\hline 8000 & $6.235+00$ & $2.275-03$ & $Z \in C S 4+O C$ & $1.71 \mathrm{~s}-\mathrm{c} 2$ & $3.521+C C$ \\
\hline 10000 . & $6.511+c 0$ & $1.457-03$ & $2.294+C C$ & $1.327-02$ & $4.2 \mathrm{C2}+\mathrm{CC}$ \\
\hline & & $6.477-04$ & $1.657+O C$ & $8.4 \equiv 1-03$ & $5.4 C 7+C C$ \\
\hline & $7.577 * 00$ & $3.644-04$ & $1.361+00$ & $6.174-03$ & $6.210+C C$ \\
\hline 30000 . & $8.378+00$ & $1.620-04$ & S. ESG-C1 & $4.01 \subseteq-03$ & $7.384+C C$ \\
\hline & & $9.113-05$ & $7.857-01$ & $2.57 \varepsilon-03$ & $8.265+C C$ \\
\hline & & & $6.553-c 1$ & $2.365-03$ & 2. $535+C C$ \\
\hline 60000 . & $1 . C 02+01$ & $4.051-05$ & $5.643-01$ & $1.5 \in 2-03$ & S. $454+0 \mathrm{C}$ \\
\hline & 1. $c \in 4+01$ & $2.279-0 E$ & $4.446-\mathrm{Cl}$ & $1.463-03$ & 1. $C 20+C 1$ \\
\hline 100000 & $1.107+01$ & $1.458-05$ & $3.688-01$ & $1.1 \in t-03$ & $1.070+01$ \\
\hline
\end{tabular}




\begin{tabular}{|c|c|c|c|c|}
\hline & & OHER . & I NCOH. & \\
\hline (KEY) & & & SCATT. & EFF \\
\hline .010 & $1.514+07$ & $1.4 C 8+c 3$ & $.470-04$ & \\
\hline & $2.215+07$ & $1.4 C 8+C 3$ & $3.303-04$ & \\
\hline .020 & $99+C 7$ & $1.408+C 3$ & $8 \in 2-c 4$ & $1.799 \div 0$ \\
\hline .030 & $13 \$ 07$ & $1.4 \mathrm{C} 8+\mathrm{C3}$ & & \\
\hline .04 & $0 \epsilon$ & $1.407 * C 3$ & $c_{-}$ & $78+0$ \\
\hline .05 & $46+$ & $1.4 C 7 \$ 03$ & & \\
\hline .060 & $4+$ & & & \\
\hline .080 & $t$ & $1.407-03$ & & \\
\hline .100 & & 1.40 & & \\
\hline .15 & & $1.4 \mathrm{C}$ & & \\
\hline .200 & $C 5$ & $1.4 C 5$ & & \\
\hline .300 & & & & \\
\hline $.4 \mathrm{CO}$ & & 1.3 & & \\
\hline .500 & & & & \\
\hline.$\in C O$ & & & & \\
\hline .800 & $17+$ & 1.36 & & \\
\hline 1.000 & & 1.3 & & \\
\hline & & & & \\
\hline 2.000 & & 1.2 & 2.2 & \\
\hline 3. & & & & \\
\hline & & & & \\
\hline 5.00 & & & & \\
\hline & & & & \\
\hline & $3.544 t$ & & & \\
\hline $0 . C 0 O$ & & 4.383 & 1.1 & \\
\hline & & & & \\
\hline & & & & \\
\hline $3 \mathrm{C} . \mathrm{COO}$ & $\epsilon .126+c$ & 1.1100 & $1.873+$ & \\
\hline & $2.852+C 3$ & $6.594+01$ & $1 . \varsigma \varsigma 9+01$ & $2.762+0$ \\
\hline
\end{tabular}

\begin{tabular}{|c|c|c|c|c|}
\hline \\
\hline $\begin{array}{l}\text { ENEREY } \\
\text { (KEVV) }\end{array}$ & & $\begin{array}{l}\text { COHER. } \\
\text { SCATT. }\end{array}$ & $\begin{array}{l}\text { I NCOH. } \\
\text { SCATT. }\end{array}$ & $\begin{array}{r}\text { PHOTC } \\
\text { EFFECT }\end{array}$ \\
\hline .010 & $4.90 s+14$ & $1.4 \epsilon G+C 3$ & $1.581-\mathrm{C} 4$ & $4.909+14$ \\
\hline & & $1.465+C 3$ & & \\
\hline.$c 2 c$ & $3.077+1$ & $1.4 \in S+03$ & $6.3 c_{4}-c_{4}$ & $3.077+13$ \\
\hline .030 & $6.0 \$ 5+12$ & $1.46 s+c 3$ & & $405+1$ \\
\hline .040 & $1.934+12$ & $1.4 \in G * C 3$ & & \\
\hline .050 & 7. $544+11$ & $1.4 \in G+C 3$ & & $7.944+11$ \\
\hline .060 & $3.842+11$ & 1.46 & & \\
\hline & & & & \\
\hline & $5.034+1$ & 1.4 & & 10 \\
\hline & $1.008+$ & 1.4 & & \\
\hline & 3.2 & 1.4 & & \\
\hline & & 1.4 & & \\
\hline & & & & \\
\hline & & 1.4 & & \\
\hline.$\in 0$ & & 1.4 & & \\
\hline & & & & \\
\hline $1 . C C C$ & -2.416 & 1.4 & & -2.4 \\
\hline & & & & \\
\hline & & & & \\
\hline 3.000 & -9.2 & 1.C & 3.8 & \\
\hline & & & & \\
\hline & & & & \\
\hline & & & & \\
\hline & & & & \\
\hline & & & & \\
\hline & & & & \\
\hline & & & 1.6 & +0 \\
\hline & & & & 6.444 \\
\hline & & & & $76+0$ \\
\hline
\end{tabular}

\begin{tabular}{|c|c|c|c|c|}
\hline \\
\hline $\begin{array}{l}\text { ERGY } \\
\text { (KEY) }\end{array}$ & TOTAL & CCFER. & I NCOH. & PHCTC \\
\hline (KEV) & & $\begin{array}{r}\text { SCA } \\
523\end{array}$ & SCATT, & EFFECT \\
\hline & $\begin{array}{l}1.153+C \\
8.54 \varepsilon+C E\end{array}$ & $\begin{array}{l}1.33 \\
1.53\end{array}$ & $\begin{array}{l}1.8 C s-c 4 \\
4.063-C 4\end{array}$ & $.547+06$ \\
\hline. $\mathrm{C} 2 \mathrm{O}$ & $1.2 \& 7+C 7$ & $1.533+c 3$ & $7.210-C_{4}$ & $.387+07$ \\
\hline & $50+07$ & & .616 & $.290+07$ \\
\hline .040 & S.890+ & & & \\
\hline .050 & $7.570+C E$ & $1.532+c 3$ & $4.456-$ & $7.56 s+06$ \\
\hline & & & & \\
\hline .080 & 3 & & & \\
\hline $.10 \mathrm{C}$ & $2 . E 1 S+C \epsilon$ & $1.532+C 3$ & 1.7 & $8+06$ \\
\hline & & & & \\
\hline & 5.851 & 1.5 & & \\
\hline & $5.424+$ & 1.52 & 1.4 & \\
\hline .400 & $2 . t$ & 1.5 & & \\
\hline & & 1.5 & 3.7 & \\
\hline & 2.8 & $1.504+$ & $E .1$ & +06 \\
\hline .80 & 1.55 & 1.4 & & \\
\hline & & & & \\
\hline 1.500 & 5.9 & 1.3 & 1.5 & \\
\hline & & & & \\
\hline & & & & \\
\hline $4 \cdot C$ & 2.1 & c. 8 & 5.3 & \\
\hline & & & & \\
\hline & & & & \\
\hline & & 5.8 & & \\
\hline & & & & \\
\hline & & & & \\
\hline & & & & \\
\hline & & & & \\
\hline & & & & \\
\hline
\end{tabular}

\begin{tabular}{|c|c|c|c|c|c|}
\hline ENERGY & TCTAL & COHER. & INCOH- & FHOTO & PAIF \\
\hline & & & & & PRCL. \\
\hline 50. & $1.557+C_{3}$ & $4.785+01$ & 2. $c \in 3+01$ & $1.4 \varepsilon \varsigma+03$ & \\
\hline 60. & $5.470+02$ & $3.472+01$ & $2 \cdot \operatorname{cs} 0+01$ & $\varepsilon \cdot \varsigma 14+02$ & \\
\hline 80. & $4.341+02$ & $2.061+01$ & $2.080+01$ & 3. $527+02$ & \\
\hline 100. & $2.4 C 6+02$ & $1.362+01$ & $2 . C 53+01$ & $2.0 \notin 4+02$ & \\
\hline 150. & $8.516+01$ & $6.314+00$ & $1.531+01$ & $6.353+01$ & \\
\hline 200 . & $4.922+01$ & $3.62 t+00$ & $1.803+01$ & $2.756+01$ & \\
\hline 300. & $2 . \in 26+c 1$ & $1.645+00$ & 1. $\subseteq \subseteq 5+C 1$ & $\varepsilon . t \in \varepsilon+00$ & \\
\hline 400. & $1.527+01$ & $9.351-01$ & $1.439+01$ & $3.543+0 \mathrm{C}$ & \\
\hline 500. & $1 . \in C 0+01$ & $6.022-01$ & $1.319+01$ & $2.212+O C$ & \\
\hline 600 . & $1.40 t * 01$ & $4.199-01$ & $1.223+01$ & $1.415+00$ & \\
\hline 800. & $1.173+01$ & $2.374-C 1$ & 1. $C 7 t+01$ & $7.3 c 5-01$ & \\
\hline 1000 . & $1 . c 30+01$ & $1.524-01$ & $9.688+c c$ & $4.547-C 1$ & \\
\hline 1500 . & $8.280+00$ & $6.801-02$ & 7. $\varepsilon 82+O C$ & 2. C $\& 4-01$ & $1.212-01$ \\
\hline & $7.306+00$ & $3.833-02$ & $\epsilon .728+0 C$ & $1.271-01$ & $4.130-C 1$ \\
\hline 3000 . & $6.485+0 \mathrm{C}$ & $1.707-02$ & $5.292+O C$ & $t .75 t-c 2$ & $1.112+c c$ \\
\hline 4000 . & $6.268+00$ & $9.613-03$ & $4.413+0 \mathrm{C}$ & $4.542-C 2$ & $1.8 \mathrm{CO}+\mathrm{CC}$ \\
\hline 5000 . & $6.268+00$ & $6.156-03$ & $3.810+0 \mathrm{C}$ & $3.385-02$ & $2.418+0 \mathrm{C}$ \\
\hline 6000 . & $6.351+0 C$ & $4.277-03$ & 3. $3 \in \varepsilon+c c$ & $2, \epsilon \subseteq C-C 2$ & $2 . \subseteq 52+c c$ \\
\hline 8000 . & $6.562+00$ & $2.407-03$ & $2.754+0 \mathrm{C}$ & $1.8 \varsigma \varsigma-02$ & $3.786+C C$ \\
\hline 10000. & $6.737+C 0$ & $1.541-03$ & $2.345+C C$ & $1.4 \in 5-02$ & $4 \cdot 376+C C$ \\
\hline 15000 . & $7.056+00$ & $6.851-04$ & $1.734+O C$ & $5.295-03$ & $5.351+C c$ \\
\hline 20000 . & $7.6 C 2+00$ & $3.855-04$ & $1 . \equiv S 1+C C$ & $t . \varepsilon \subset 4-c 3$ & $6.204+C C$ \\
\hline 30000 . & $8.759+00$ & $1.713-04$ & 1. $C 12+O C$ & $4.42 t-03$ & $7.743+C C$ \\
\hline 40000 . & $9.554+C C$ & $9.639-05$ & $\varepsilon . C \equiv 1-C 1$ & $3.27 \varepsilon-03$ & E. $748+C C$ \\
\hline & $1 . C 05+01$ & $6.170-C 5$ & $6.659-01$ & $2 \cdot \in C 3-03$ & c. $375+c c$ \\
\hline & 1. $c 4 c+01$ & $4.285-05$ & $E . T \in 8-01$ & $2.155-03$ & $\varsigma .819+C c$ \\
\hline 80000 . & $1.059+01$ & $2.410-05$ & $4.544-01$ & $1 . \in C \varsigma-03$ & 1. $C 53+C 1$ \\
\hline 00000 . & $1.162+01$ & $1.543-05$ & $3.770-01$ & $1.2 \varepsilon 2-03$ & $1.124+C 1$ \\
\hline
\end{tabular}

\begin{tabular}{|c|c|c|c|c|c|}
\hline $\begin{array}{l}\text { ENERGY } \\
\text { (KEV) }\end{array}$ & TCTAL & COHER. & $\begin{array}{l}\text { INCOH. } \\
\text { SCATT. }\end{array}$ & $\begin{array}{r}\text { FHOTO } \\
\text { EFFEC T }\end{array}$ & $\begin{array}{l}\text { PAIF } \\
\text { PRCE. }\end{array}$ \\
\hline 50. & $1.675+03$ & $5.036+01$ & 2. $1 \mathrm{Cl}+0 \mathrm{i}$ & $1 . \in C 7+03$ & \\
\hline 60. & $1.022+03$ & $3.652+01$ & $2.125+01$ & 5. $t 4 t+02$ & \\
\hline 80. & $4.694+02$ & $2.1 \in 7+01$ & $2.121+01$ & $4.2 \epsilon E+02$ & \\
\hline 100. & $2 \cdot \in 02+02$ & $1.431+01$ & 2. CS5+01 & $2.245+02$ & \\
\hline 150. & $9.59 c+01$ & $6.632+00$ & 1. $572+01$ & E. $5 E t+C 1$ & \\
\hline 200. & $5.246+C 1$ & $3.808+00$ & 1. $\varepsilon 42+C 1$ & $3 .(23)+01$ & \\
\hline 300 . & $2.754+C 1$ & $1.728+00$ & $1 . t 29+01$ & $5.51 t+c c$ & \\
\hline 400. & 2. $c C z+C 1$ & $5.817-01$ & $1.471+C 1$ & $4.33 C+0 C$ & \\
\hline 500. & $1.654+01$ & $6.321-01$ & $1.348+01$ & $2.4 \geq 1+0 C$ & \\
\hline 600. & $1.44 S+C 1$ & $4.408-01$ & $1.24 s+C 1$ & $1.5 E t+C C$ & \\
\hline 800 & 1. $2 C_{5}+C_{1}$ & $2.492-01$ & $1.100+01$ & $\varepsilon .042-01$ & \\
\hline 1000 . & 1. $C 56+C 1$ & $1.600-01$ & ૬.८५ +OC & $5 . C C \geqslant-01$ & \\
\hline 1500 . & $8.48 C+00$ & $7.138-02$ & E. C $54+0 C$ & $2.251-01$ & $1.261-C 1$ \\
\hline 2000 & $7 \cdot 5 \mathrm{C} 5+\mathrm{CO}$ & $4.023-02$ & $\epsilon . \varepsilon 74+c c$ & $1.357-01$ & $4.5 C 6-C 1$ \\
\hline 3000 . & $6.666+00$ & $1.792-02$ & $5.4 C 7+C C$ & $7.4 \epsilon E-02$ & $1.166+C C$ \\
\hline 4000. & $6.421+00$ & $1.009-02$ & $4.5 C S+C C$ & $4 . \varsigma \varepsilon 7-c 2$ & $1.852+C C$ \\
\hline $50 \mathrm{co}$. & $6.4 C 5+00$ & $6.461-03$ & $3.853+c c$ & $3.71 \epsilon-02$ & $2.472+C C$ \\
\hline 6000 . & $6.493+00$ & $4.488-03$ & $3.441+00$ & $2.5 \leq 2-02$ & 3. $C 18+O C$ \\
\hline 8000. & $6.742+C 0$ & $2.526-03$ & Z.E14+CC & $2 . c \varepsilon 4-02$ & 3. $5 C 5+C C$ \\
\hline 10000 & $6.984+00$ & $1.617-03$ & 2. $3 \subseteq t+O C$ & $1, \in \subset 7-02$ & $4.571+C C$ \\
\hline $1500 \mathrm{C}$. & $7.484+O C$ & $7.190-04$ & $1.772+C C$ & 1. $C 2 C-02$ & $5.7 \mathrm{Cl}+\mathrm{CC}$ \\
\hline 20000 . & $8.0 C 7+00$ & $4.045-0$ & $1.421+O C$ & $7.4 \in 2-03$ & $6.578+0 C$ \\
\hline 30000 & $9.1<1+00$ & $1.798-04$ & 1. $c 34+c c$ & $4.8 \leq 3-c 3$ & E. $C 62+c c$ \\
\hline 40000 & $9.524+C O$ & $1.012-04$ & E. $2 \mathrm{Cb} 6-01$ & $3.5 \leq 5-03$ & $c .1 C O+C c$ \\
\hline & 1. $C 49+C 1$ & $6.475-0 E$ & t. $845-$ & $2.855-03$ & $5.8 C 4+C C$ \\
\hline & $1.091+01$ & $4.497-05$ & E. \&S ${ }^{-C 1}$ & $2.367-03$ & $1 . C 32+01$ \\
\hline 000. & $1.154+C 1$ & $2.529-05$ & $4.643-01$ & $1.7 \in 4-C 3$ & $1.1 C 7+01$ \\
\hline 00000 & $1.207+01$ & $1.619-05$ & $3.852-\mathrm{Cl}$ & $1.4 c t-03$ & $1.169+01$ \\
\hline
\end{tabular}

\begin{tabular}{|c|c|c|c|c|c|}
\hline $\begin{array}{l}\text { ENEREY } \\
\text { (KEV) }\end{array}$ & TOTAL & $\begin{array}{l}\text { COHER. } \\
\text { SCATT. }\end{array}$ & $\begin{array}{l}\text { INCCH } \\
\text { SCATT }\end{array}$ & $\begin{array}{r}\text { FHCTC } \\
\text { EFFECT }\end{array}$ & $\begin{array}{r}\text { PAIF } \\
\text { PRCE. }\end{array}$ \\
\hline 50. & $1.81 \epsilon+03$ & $5.299+01$ & $2.139+C 1$ & $1.741+C 3$ & \\
\hline 60. & $1.1 C 8+03$ & $3.843+01$ & $2.1 \in 8+C 1$ & 1.C47+03 & \\
\hline 80. & $5.053+02$ & $2.281+01$ & $2.1 \in 1+01$ & $4.64 S+C 2$ & \\
\hline 100 & $2.819+02$ & $1.507+01$ & $2.136+C 1$ & $2.455+02$ & \\
\hline 150. & $1.031+02$ & $6.981+00$ & $2 . C 11+01$ & $7.5 \div 7+C 1$ & \\
\hline 200 . & $5.584+01$ & $4.009 \$ 00$ & $1.879+01$ & $3.3 C 4+01$ & \\
\hline 300. & $2.887+01$ & $1.819+00$ & 1. $t \in 3+C 1$ & 1. $C 42+01$ & \\
\hline 400. & $2.080+01$ & $1.034+00$ & 1. $5 \mathrm{Cl}+\mathrm{Cl}$ & $4.752+C 0$ & \\
\hline $50 \mathrm{C}$. & $1.710+C 1$ & $6.655-c 1$ & $1.276+C 1$ & $2 \cdot G T E+O C$ & \\
\hline 600 . & $1.494+01$ & $4.640-01$ & $1.276+C 1$ & $1.717+c c$ & \\
\hline 800 & $1.238+01$ & $2.623-01$ & $1.123+01$ & $\varepsilon . \varepsilon \varepsilon 7-C l$ & \\
\hline 1000 . & $1.083+01$ & $1.684-01$ & $1 . C 11+01$ & $5.5 \geq c-01$ & \\
\hline 1500 . & $8.685+C 0$ & $7.515-02$ & $8.225+C c$ & $2.53 \mathrm{C}-01$ & $1.320-c 1$ \\
\hline 2000 . & $7.7 C 5+00$ & $4.236-02$ & 7. $\mathrm{C} 2 \mathrm{C}+\mathrm{CC}$ & $1.54 \mathrm{C}-\mathrm{Cl}$ & $4.886-\mathrm{Cl}$ \\
\hline 3000 . & $6.844+00$ & $1.886-02$ & $5.522 * C C$ & $8.215-02$ & $1.221+C C$ \\
\hline 4000 . & $6.573+c c$ & $1.062-02$ & $4 . t C 5 \vee 00$ & $5.481-02$ & $1 . S C 3+c c$ \\
\hline 5000 . & $6.542+00$ & $6.802-03$ & $3 . \leq 7 t+C C$ & 4.CE1-C2 & $2.518+C C$ \\
\hline 6000 . & $6.620+00$ & $4.726-03$ & $\Xi .5144 C C$ & $3.24 \mathrm{C}-02$ & $3.068+C C$ \\
\hline 8000 & $6.8554 c 0$ & $2.660-03$ & 2. $\varepsilon 74+C C$ & $2.2 \varepsilon t-02$ & $3.955+O C$ \\
\hline 10000 . & $7.1 S \varsigma+00$ & $1.703-03$ & $2.447+0 \mathrm{C}$ & $1.7 \in 2-02$ & $4.733+C C$ \\
\hline 15000 . & $7 . \varepsilon \in 7 \ngtr 00$ & $7.570-04$ & 1. $810+C C$ & $1.118-02$ & 6.C4 $C+C C$ \\
\hline 20000 & $8.427+00$ & $4.259-04$ & $1.452+O C$ & $8.175-03$ & $6.577+C C$ \\
\hline 30000 . & $9.425+0 \mathrm{C}$ & $1.893-04$ & $1 . C 56+C C$ & $5.315-03$ & $8.364+0 \mathrm{C}$ \\
\hline 40000 & 1.c18+01 & $1.065-04$ & $8.381-01$ & $3.5 \equiv t-03$ & $5.334+C C$ \\
\hline 50000 . & $1 . C 74+01$ & $6.817-05$ & $\epsilon . \subseteq 50-01$ & $3.125-03$ & 1. $\mathrm{CO}+\mathrm{Cl}$ \\
\hline & $1.117+01$ & $4.734-05$ & $6.019-01$ & $2.551-03$ & 1. C57+01 \\
\hline 80000 . & $1.183 * 01$ & $2.663-05$ & $4.742-C_{1}$ & $1.5 \geq 1-03$ & $1.136+C 1$ \\
\hline 00000 . & $1.235+01$ & $1.704-C 5$ & $3.534-01$ & $1.5 \equiv s-03$ & $1.196+C 1$ \\
\hline
\end{tabular}




\begin{tabular}{|c|c|c|c|c|}
\hline \multicolumn{5}{|c|}{$z=45$ INDIUM } \\
\hline $\begin{array}{l}\text { EAERGY } \\
\text { (KEV) }\end{array}$ & TOTAL & $\begin{array}{l}\text { COFER } \\
\text { SCATT. }\end{array}$ & $\begin{array}{l}\text { INCOH. } \\
\text { SCATT. }\end{array}$ & $\begin{array}{r}\text { PHCTO } \\
\text { EFFECT }\end{array}$ \\
\hline .010 & $2.52 \varepsilon+c \epsilon$ & $1.557+03$ & $.932-04$ & $3.527+06$ \\
\hline & & $1.557+C 3$ & $4.339-c 4$ & $1.045+06$ \\
\hline & $7.6 \epsilon 7+0 t$ & $1.557+C 3$ & $7 . \in S 9-04$ & $7.666+06$ \\
\hline & $7.5 C 3+c t$ & $597+C 3$ & $1.72 t-03$ & $7.501+06$ \\
\hline .040 & $=0+c$ & $7+c 3$ & & $6.058+06$ \\
\hline & $4.5 t$ & $1.557+C 3$ & $3-03$ & $4.966+C 6$ \\
\hline & 4.18 & 1.5 & & $4.180+06$ \\
\hline & & & & \\
\hline & $532+0 t$ & & & \\
\hline & $1 . \in E 9+$ & 1.5 & $1-02$ & $1.687+06$ \\
\hline & & & & \\
\hline & 8.4 & 1.5 & & $8.424+05$ \\
\hline .400 & $6.330+$ & 1.5 & $2 . t$ & $6.314+05$ \\
\hline & & & & \\
\hline $.6 \mathrm{CO}$ & $3.37 t+$ & 1.5 & 5.2 & $74+06$ \\
\hline .800 & $2.224 t$ & 1.5 & & \\
\hline & & & & \\
\hline 1.500 & $6 . C 26+05$ & 1.43 & 2.0 & $6.012+05$ \\
\hline & & & & \\
\hline & & & & \\
\hline & & $1.027+$ & 5.4 & $4+05$ \\
\hline & $1.538+$ & & & \\
\hline & & & & \\
\hline & & & & \\
\hline & 2.475 & & & \\
\hline & & $3.298+$ & & \\
\hline & & & & $3.598+03$ \\
\hline 30.000 & $7.577+03$ & $1.253+02$ & $1.971+01$ & $7.428+03$ \\
\hline & $3.5 \in 5+C 3$ & $8.140+01$ & $2.1 \mathrm{Co}+\mathrm{Cl}$ & $3.462+03$ \\
\hline
\end{tabular}

\begin{tabular}{|c|c|c|c|c|}
\hline \\
\hline $\begin{array}{l}\text { EAERGY } \\
\text { (KEVV) }\end{array}$ & TOTAL & COFER. & $1 \mathrm{NCOH} \cdot$ & $\begin{array}{l}\text { PHCTC } \\
\text { EFFECT }\end{array}$ \\
\hline .010 & $1,435+c 7$ & 1. $t \in 3+C 3$ & 1. $55 c-c 4$ & $1.439+07$ \\
\hline & $=4+06$ & . $t \in 3+03$ & $.380-c_{4}$ & $4.262+06$ \\
\hline & $+c t$ & $1.663+c 3$ & $.772-c 4$ & $1.798+06$ \\
\hline & $8+C 7$ & $1 . \in t \in 3+C 3$ & $1.742-C_{3}$ & $1.348+07$ \\
\hline & $C 2+07$ & $1.6 \in 3+$ & $.0 \varepsilon 6-$ & $201+07$ \\
\hline .050 & $+c \epsilon$ & 3 & & \\
\hline & $7.1 \varepsilon$ & $1.663+03$ & $6.8 \subseteq 2-C 3$ & $7.17 s+06$ \\
\hline & $4.8 t$ & & & +06 \\
\hline & & 1.6 & & +06 \\
\hline & 2.28 & & & $6 \div 06$ \\
\hline & 1.6 & $1 . t 5$ & 7.2 & \\
\hline & & $1 . t E$ & 1.5 & $3+06$ \\
\hline & $6+c 5$ & $1.646 \%$ & $2 . \in 75-C 1$ & $8 \cdot 369+05$ \\
\hline .500 & $4.767+$ & $1 . t 37+$ & 4.00 & $4.766+06$ \\
\hline & & & & \\
\hline .800 & $87+0 \epsilon$ & $1 . \in C 3$ & 8.87 & $2.386+06$ \\
\hline 1.000 & 1.5 & 1.57 & 1.2 & $0+06$ \\
\hline & & & & \\
\hline 2.000 & 3.3 & 1.403 & 2.878 & $1+C 5$ \\
\hline $3 . \mathrm{CC}$ & 1.2 & 1.22 & 4.3 & +05 \\
\hline & & & & \\
\hline & & S.2 $\mathrm{s}$ & 6. & $3+05$ \\
\hline & & & 8.1 & \\
\hline & & & & \\
\hline & & & & \\
\hline 15.000 & $9.013+$ & $3.457 t$ & 1.533 & \\
\hline & & & & $3.888+03$ \\
\hline & & & & \\
\hline & & & & \\
\hline
\end{tabular}

\begin{tabular}{|c|c|c|c|c|}
\hline$=R G Y$ & & COHER . & $1 \mathrm{NCOH}$. & PHOTO \\
\hline (KEV) & & САTT. & SCATT. & EFFECT \\
\hline & $3.327+C 7$ & $1.730+03$ & $1.5 C 9-C_{4}$ & $3 \cdot 327+07$ \\
\hline & $\varepsilon 32+C \epsilon$ & $1.730+c 3$ & $289-c 4$ & $8.630+06$ \\
\hline .02 & $4+0 \varepsilon$ & & & \\
\hline & $12+05$ & $+c 3$ & $-c 3$ & $8.994+05$ \\
\hline & $1.404+C$ & $1.73 c+c 3$ & & $1.403+07$ \\
\hline .050 & $1.120+$ & & & $1.120+07$ \\
\hline & $8.002+$ & & & $01+06$ \\
\hline & $4.44 E+C E$ & $9+c 3$ & $: 2$ & $4.446+06$ \\
\hline & & & & \\
\hline & 1. $7 \epsilon t+c t$ & & & +06 \\
\hline & $1.4 C 4+C 6$ & & & +06 \\
\hline & $1.0 \varepsilon 0+$ & & & \\
\hline & & & & 8.9 \\
\hline & $7 . \in 25+$ & & & 7.6 \\
\hline & & & & \\
\hline & & & & \\
\hline 1.000 & $1.817+0 t$ & $1.638 \%$ & 1.2 & $16+c 6$ \\
\hline 1.5 & $7.264+$ & & & \\
\hline & 3.69 & & & \\
\hline & $1.3 \in 8+$ & 1.26 & 4.3 & 1.3 \\
\hline & & & & \\
\hline & & & & $1.772+05$ \\
\hline & 1.120 & & & $1.112+05$ \\
\hline & $5.2 \epsilon \varepsilon+$ & & & \\
\hline & & & & 2.8 \\
\hline & & & & \\
\hline & $4.52 C+C$ & $2.531+$ & $1.776+01$ & $4.250 * 03$ \\
\hline & $1.538+$ & $1.423+$ & & $1.376+03$ \\
\hline & 4. $C 77+03$ & $\varepsilon .572+\mathrm{Cl}$ & $2.176+C 1$ & $3.965+03$ \\
\hline
\end{tabular}

\begin{tabular}{|c|c|c|c|c|c|}
\hline & TOTAL & COHER . & INCOH. & FHOTO & \\
\hline & & SCATT. & & EFFECT & \\
\hline 50. & $1.556+03$ & $5.566+01$ & $2.17 t+C 1$ & $1.879+03$ & \\
\hline to. & 1.193403 & $4.037+01$ & $2.2 C 7+01$ & $1.13 \mathrm{C}+03$ & \\
\hline 80. & $5.472+02$ & $.395+01$ & $2.1 s s+c 1$ & 5. $C 12+02$ & \\
\hline 100. & $3.025+02$ & $2+01$ & $2.175+01$ & 2. $t \leq c+02$ & \\
\hline & $52+02$ & & - $C 4 S+01$ & $8.240+01$ & \\
\hline & & & - $\subseteq 15+C 1$ & $3.5 \div 7+01$ & \\
\hline 300 & 3 & & $.655+01$ & $1.14 C+01$ & \\
\hline 40 & & & $1+01$ & & \\
\hline & & & $1.4 C 4+01$ & $2.53 \epsilon+00$ & \\
\hline & & & & $1 . \varepsilon \varepsilon 2+C C$ & \\
\hline 800 & $1.271+01$ & $2.756-01$ & & 5.7 & \\
\hline 100 & & 1. & $2+01$ & $-\mathrm{Cl}$ & \\
\hline & & & & $2.773-01$ & $1.355-$ \\
\hline & & 4.44 & 7.16 & 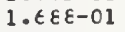 & \\
\hline & & & & & \\
\hline & 6.78 & & & $6.0 C_{3}-$ & \\
\hline 5000 & $6.781+00$ & 7.14 & $4 . c$ & $4.4 \in \varepsilon-C 2$ & $2.671+$ \\
\hline & & & 3.5 & 3.54 & \\
\hline & & & 2.53 & $2.5 \mathrm{CC}-$ & \\
\hline 10000 & $7.430+00$ & 1.7 & 2.45 & $1.527-c 2$ & 4. $\$ 11+$ \\
\hline & & 7.9 & $1 . \varepsilon$ & 1.2 & \\
\hline & 8.64 & 4.47 & $1.482 t$ & $\varepsilon .534-$ & \\
\hline & 9.82 & & 1.078 & $5 . \varepsilon \subset 7-$ & \\
\hline & & & & $4.3 \mathrm{CC}-$ & S. $8 \mathrm{C} 2+$ \\
\hline & $1.123+01$ & & $7.136-01$ & $3.414-$ & 1551 \\
\hline & $1.1 \epsilon \epsilon+01$ & 4.97 & $t .144-01$ & & \\
\hline & & & & $2.11 \mathrm{C}-03$ & $1.185+$ \\
\hline & $1.29 t+01$ & $1.790-05$ & $4.01 t-01$ & $1 . \in 81-03$ & $1.256+0$. \\
\hline
\end{tabular}

\begin{tabular}{|c|c|c|c|c|c|}
\hline $\begin{array}{l}\text { ENERGY } \\
\text { (KEV) }\end{array}$ & TCTAL & $\begin{array}{l}\text { COHER. } \\
\text { SCATT. }\end{array}$ & $\begin{array}{l}\text { INCCH. } \\
\text { SCATT. }\end{array}$ & $\begin{array}{l}\text { FHOTO } \\
\text { EFFECT }\end{array}$ & $\begin{array}{l}\text { PAIF } \\
\text { PRCE. }\end{array}$ \\
\hline 50. & $2 . \operatorname{cs} 7+03$ & $5.845+01$ & $2 . \overline{2} 13+C 1$ & 2. $C 1 t+C 3$ & \\
\hline 60. & $1.282+03$ & 4.239401 & $2.24 E+01$ & $1.217+03$ & \\
\hline 80. & $5.5 C 7+C 2$ & $2.516+01$ & $2.23 s+c 1$ & $5.4 \geq 1+02$ & \\
\hline 100. & $3.271+c 2$ & $1.662+01$ & $2.215+C 1$ & $2.883+02$ & \\
\hline 150. & $1.18 \epsilon+02$ & $7.703+00$ & 2. $c 89+01$ & E. SS $E+01$ & \\
\hline 200. & $6.328+01$ & $4.424 * 00$ & 1. $\$ 52+C 1$ & $3.533+01$ & \\
\hline 300 . & $3.178+01$ & $2.007+00$ & $1.729+01$ & $1.24 \varepsilon+01$ & \\
\hline 400 . & $2.246+C 1$ & $1.140+0 \mathrm{C}$ & 1. $5 \in 2+C 1$ & $5.7 C 5+00$ & \\
\hline 500. & $1.827+01$ & $7.344-C 1$ & $1.432+01$ & $3.213+00$ & \\
\hline 600. & $1.585+01$ & $5.121-01$ & $1.328+01$ & 2. $c \in 1+00$ & \\
\hline 800 . & $1.305+01$ & $2.895-01$ & 1. $1 \in s+C 1$ & 1. $c \in \varepsilon+C C$ & \\
\hline 1000 . & $1.138 * 01$ & $1.859-01$ & 1. $C 53+01$ & $\epsilon \cdot \in 4 \zeta-01$ & \\
\hline 1500 . & $9.1 C 1+C 0$ & $8.294-02$ & 8. $566+c c$ & $3.04 E-01$ & $1.475-C_{1}$ \\
\hline 2000 . & $8.056+0 C$ & $4.675-02$ & $7.212+0 C$ & $1.853-C 1$ & $17-C_{1}$ \\
\hline 3000 . & $7.1 s S+0 C$ & $2.082-02$ & $5.752+C C$ & $5.874-02$ & $1.327+C C$ \\
\hline 4000 . & $6.58 t+00$ & $1.172-02$ & $4.757+C C$ & $\epsilon .580-02$ & $2.112+C C$ \\
\hline 5000. & $7 . \mathrm{Cl} 16+00$ & $7.507-03$ & $4.142+C C$ & $4.85,4-02$ & $2.818+C C$ \\
\hline 6000 . & $7.140+00$ & $5.215-03$ & 3. $t \in C+O C$ & $3.882-02$ & $3.436+C C$ \\
\hline 8000 . & $7.454+0 \mathrm{C}$ & $2.935-03$ & $2 . \subseteq 94+O C$ & $2.735-02$ & $4.430+C C$ \\
\hline 10000 . & $7.742+00$ & $1.879-03$ & $2.549+0 C$ & $2.1<7-02$ & $5.170 .0 \mathrm{C}$ \\
\hline & & $8.354-04$ & 1. $\varepsilon \varepsilon 5+C C$ & $1.334-\mathrm{C}_{2}$ & $6.427+C C$ \\
\hline 000 . & & $4.700-04$ & $1.512+C C$ & $5.753-03$ & $7.417+C C$ \\
\hline 30000 . & 1.C $17+C 1$ & $2.085-04$ & $1.1 C 0+0 C$ & $t .3 \geq 2 t-c 3$ & S. $C 60+C C$ \\
\hline 40000 & $1.104+01$ & $1.175-\mathrm{C}_{4}$ & $\varepsilon .730-01$ & $4 . t \subseteq 1-C 3$ & 1. $C 16+C 1$ \\
\hline 50000 . & $1.1 \in 2+01$ & $7.523-0 E$ & $7.2 \varepsilon 1-01$ & $3.723-03$ & 1. $C 89+C 1$ \\
\hline 60000 . & $1.20 t+01$ & $5.225-05$ & $\epsilon .2 \epsilon S-01$ & $3.087-03$ & $1.143+c 1$ \\
\hline 80000 . & $1.275+01$ & $2.939-05$ & $4.54 \mathrm{C}-\mathrm{Cl}_{1}$ & $2.3 C C-03$ & $1.229+C 1$ \\
\hline 100000 . & $1.349+01$ & $1.881-05$ & 4. CS8-01 & $1.8 \equiv 3-03$ & $1.3 \mathrm{C} 8+\mathrm{Cl}$ \\
\hline
\end{tabular}

\begin{tabular}{|c|c|c|c|c|c|}
\hline $\begin{array}{l}\text { ENERGY } \\
\text { (KEV) }\end{array}$ & TCTAL & $\begin{array}{l}\text { COHER } \\
\text { SCATT }\end{array}$ & $\begin{array}{l}\text { INCOH. } \\
\text { SCA TT }\end{array}$ & $\begin{array}{r}\text { PHCTO } \\
\text { EFFECT }\end{array}$ & $\begin{array}{l}\text { PAIR } \\
\text { PRCLE. }\end{array}$ \\
\hline 50 & $2.247+03$ & $6.138+01$ & $2.250+01$ & $2.163+03$ & \\
\hline 60. & $1.37 t+03$ & $4.454+01$ & $2.284+01$ & $1.3 c \subseteq+C 3$ & \\
\hline 80. & $6.350+02$ & 2.644401 & $2.281+C 1$ & $5.8 E \varepsilon+02$ & \\
\hline 100. & $3.51 \epsilon+C 2$ & $1.747 \varangle 01$ & $2.258+01$ & $3.11 \epsilon+02$ & \\
\hline 150. & $1.270 \$ 02$ & $8.100+00$ & $2.130+c 1$ & $5.75 t+01$ & \\
\hline 200. & $6.731+C 1$ & $4.653+00$ & 1. $5 S 2+01$ & $4.274+01$ & \\
\hline 300 . & $3.335+01$ & $2.111+00$ & $1.764+01$ & $1.3 \in 0+01$ & \\
\hline 400. & $2.33 \epsilon+01$ & $1.200+00$ & $1.5 \varsigma 3+C 1$ & $\epsilon .22 E+00$ & \\
\hline 500 . & $1.889+01$ & $7.726-01$ & $1.4 \in 1+01$ & $3.5 C t+C 0$ & \\
\hline 600 . & $1 . t 332+01$ & $5.387-01$ & $1.355+01$ & $2.24 \varepsilon+C O$ & \\
\hline 800. & $1.340+01$ & $3.046-01$ & $1.193+01$ & $1.1 \in 4+00$ & \\
\hline 1000 . & $1.16 t+01$ & $1.955-01$ & 1. $074+01$ & $7.241-C_{1}$ & \\
\hline 1500. & $9.313+00$ & $8.726-02$ & $\varepsilon .738+0 c$ & $3.313-01$ & $1.564-\mathrm{Cl}$ \\
\hline 2000 & $8.241+00$ & $4.91 s-02$ & $7.45 S+C C$ & $2.016-01$ & $5.313-c 1$ \\
\hline 3000 . & $7.38 \mathrm{C} \$ 00$ & $2.190-02$ & 5. $\varepsilon \in 7+C C$ & $1.074-01$ & $1.383+0 \mathrm{C}$ \\
\hline 4000 & $7.1 \& C \hookleftarrow O C$ & $1.233-02$ & 4. ES $3+C C$ & $7.15 t-02$ & 2. $2 \mathrm{C} 4+C \mathrm{C}$ \\
\hline 5000 & & $7.899-03$ & $4.225+C C$ & $5.323-02$ & 2. $537+C C$ \\
\hline 6000 . & $7.354+00$ & $5.487-03$ & $3.734+C C$ & $4.222-02$ & $3.573+C C$ \\
\hline 8000 & 7.666000 & $3.088-03$ & $3.054+C C$ & $2.575-02$ & $4.57 \mathrm{~s} A C \mathrm{C}$ \\
\hline $100 \mathrm{co}$. & & $1.977-03$ & $2 . \in C C+O C$ & $2.2 \leq 1-c 2$ & $5.312+C C$ \\
\hline 15000 . & $8.5 \mathrm{CO}+\mathrm{CO}$ & $8.791-04$ & $1.92340 \mathrm{C}$ & $1.452-02$ & $6.561 \Vdash O C$ \\
\hline & & $4.946-04$ & $1.542+C C$ & 1. $c \in 1-02$ & $7.616+C C$ \\
\hline 30000 . & $1.056 \div C 1$ & $2.199-04$ & $1.122+0 \mathrm{C}$ & $6.894-03$ & $9.426+0 C$ \\
\hline 40000 & $1.15 \mathrm{C} \bullet 01$ & $1.237-04$ & $\varepsilon . S C 4-c 1$ & $5.1 C 4-03$ & $1.060+C 1$ \\
\hline 50000 . & $1.210+01$ & $7.916-05$ & $7.427-C 1$ & 4. $C 52-03$ & $1.135+C 1$ \\
\hline & & $5.497-05$ & $6.255-01$ & $3.355-03$ & $1.189 * \mathrm{Cl}$ \\
\hline & $1.324+01$ & $3.092-05$ & $5.038-01$ & $2.5 C 3-03$ & $1.273+\mathrm{Cl}$ \\
\hline 100000 & $1.39 t+01$ & $1.979-05$ & $4.180-01$ & $1.5 \$ 4-C 3$ & $1.354+01$ \\
\hline
\end{tabular}




\begin{tabular}{|c|c|c|}
\hline \multicolumn{3}{|c|}{52 TELLURIUM } \\
\hline ENERGY & TOTAL & COHER - \\
\hline (KEV) & & SCATT. \\
\hline .010 & $4.874+C 7$ & $1.759+C 3$ \\
\hline .015 & $1.444+C 7$ & $1.79 s+03$ \\
\hline . $c 20$ & $6 . C 93+0 E$ & $1.795+C 3$ \\
\hline .030 & $1.807+C t$ & $1.7 s s+c 3$ \\
\hline .040 & $7.633+05$ & $1.799+03$ \\
\hline .050 & $1.027+07$ & $1.798+C 3$ \\
\hline .060 & $9.147+06$ & $1.758+C 3$ \\
\hline .080 & $5.574+06$ & $1.798+03$ \\
\hline .100 & $3.545+06$ & $1.798+03$ \\
\hline .150 & $1.711+C t$ & $1.796+c 3$ \\
\hline .200 & $1.180+C t$ & $1.7 \subseteq 4+C \exists$ \\
\hline .300 & $8.180+05$ & $1.789+C 3$ \\
\hline .400 & $\epsilon .5 S \varepsilon+C E$ & $1.781+C 3$ \\
\hline .500 & $5.602+05$ & $1.772+C 3$ \\
\hline .600 & $E .1 C C+C E$ & $1.761+03$ \\
\hline .800 & $2.616+06$ & $1.735+C 3$ \\
\hline 1.000 & $1.771+0 t$ & $1.7 C 4+C 3$ \\
\hline 1.500 & $7.980+05$ & $1.614+03$ \\
\hline $2 . \mathrm{COC}$ & $4 . C 25+C E$ & $1.512+c 3$ \\
\hline 3.000 & $1.4 t 4+C 5$ & $1.313+03$ \\
\hline $4.6 C 0$ & 7. $c \in 3+c 4$ & $1.144+C 3$ \\
\hline $5 . \mathrm{COC}$ & $1.888+C 5$ & $1 . \mathrm{CO2}+03$ \\
\hline$t . C O O$ & $1.203+05$ & $\varepsilon \cdot 83 c+c 2$ \\
\hline 8.000 & $5.715+04$ & $6.587+C 2$ \\
\hline $1 \mathrm{C} . \mathrm{COO}$ & $3.154+C 4$ & $5.671+02$ \\
\hline $15 . \mathrm{COC}$ & $1 . C 55+C 4$ & $3.717+C 2$ \\
\hline $20.0 C 0$ & $4.256+c 3$ & $2.644+02$ \\
\hline $3 \mathrm{c} \cdot \mathrm{ccc}$ & $1 . t 5 \varepsilon+C 3$ & $1.490+C 2$ \\
\hline $4 C .000$ & $4 \cdot 3+2+03$ & $9.40 t+01$ \\
\hline
\end{tabular}

$\begin{array}{rr}\text { INCOH. } & \text { PHCTC } \\ \text { SCATT. } & \text { EFFECT } \\ 1.853-C 4 & 4.873+07 \\ 4.252-04 & 1.444+07 \\ 7.546-C 4 & 6.092+06 \\ 1.652-03 & 1.805+06 \\ 2.557-C 3 & 7.615+05 \\ 4.666-C 3 & 1.026+07 \\ 6.655-03 & 9.145+06 \\ 1.182-02 & 5.572+06 \\ 1.833-C 2 & 3.547+06 \\ 4.048-02 & 1.710+C 6 \\ 7.063-02 & 1.179+06 \\ 1.529-C 1 & 8.162+05 \\ 2.611-01 & 6.580+05 \\ 3.512-C 1 & 5.584+05 \\ 5.353-01 & 5.099+06 \\ 8.733-C 1 & 2.614+06 \\ 1.231+0 C & 1.765+06 \\ 2.1 C 6+00 & 7.964+05 \\ 2.537+C C & 4 . C 10+05 \\ 4.494+00 & 1.451+05 \\ 5.541+0 C & 6.948+04 \\ 7.286+C 0 & 1.878+05 \\ 8.531+C 0 & 1.154+05 \\ 1 . C 72+C 1 & 5.644+04 \\ 1.253+C 1 & 3.096+04 \\ 1.581+01 & 1.016+04 \\ 1.804+C 1 & 4.573+03 \\ 2.670+01 & 1.488+03 \\ 2.214+C 1 & 4.245+03\end{array}$

\begin{tabular}{|c|c|c|}
\hline \multicolumn{3}{|c|}{$Z=53 \quad 10 C \mathrm{INE}$} \\
\hline ENERGY & TOTAL & COHER. \\
\hline (KEV) & & SCATT. \\
\hline .010 & $1.3 \varepsilon 3+C \varepsilon$ & 1. $2 \in C+C 3$ \\
\hline .015 & $2.722+C 7$ & $1.86 s+C 3$ \\
\hline .020 & $8.753+c \epsilon$ & $1.868+03$ \\
\hline .030 & $2.29 t+c t$ & 1. $2 \in 8+c 3$ \\
\hline .040 & $1.259+0 t$ & $1.868+C 3$ \\
\hline .050 & $2.7 t 4+06$ & $1.868+03$ \\
\hline.$c \in 0$ & $4.253+c t$ & $1.868+C 3$ \\
\hline .080 & $4.161+0 t$ & $1.868+03$ \\
\hline $.10 \mathrm{C}$ & $3.475+C t$ & $1.867+C 3$ \\
\hline .150 & $2.279+0 t$ & 1. $2 t t+c 3$ \\
\hline .200 & $1 . t 58+0 t$ & $1.264+C 3$ \\
\hline $.3 \mathrm{CO}$ & 1. $c \in t+0 t$ & 1. $858+C 3$ \\
\hline .400 & $7.781+05$ & $1.851+C 3$ \\
\hline .500 & $6.138+C 5$ & $1.842+C 3$ \\
\hline $.60 \mathrm{C}$ & $5.0 t \varepsilon+05$ & $1.831+C 3$ \\
\hline .800 & $2 . \varepsilon 2 \varepsilon+c t$ & $1.8 C 4+C 3$ \\
\hline 1.000 & $1.9 \geq 1+06$ & $1.773 * C 3$ \\
\hline $1.50 \mathrm{C}$ & $8.104+05$ & $1.678+03$ \\
\hline 2.000 & $4.1 \in 0+0 E$ & $1.571+c 3$ \\
\hline 3.000 & $1.571+C E$ & $1 \cdot 3 \epsilon \hat{z}+c 3$ \\
\hline 4.000 & $7.598+C 4$ & $1.1 \varepsilon 6+C 3$ \\
\hline $5 . c c 0$ & $1.775+05$ & 1. $C 40+C 3$ \\
\hline$t . C O O$ & $1.254+05$ & $S .173+c 2$ \\
\hline$\varepsilon .000$ & $6.117+C 4$ & $7.272+02$ \\
\hline $1 \mathrm{C} .000$ & $3.3 \varepsilon 0+C 4$ & $5.9 C 7+02$ \\
\hline $15.0 C \mathrm{C}$ & $1.139+C 4$ & $3 . \varepsilon t 5+c 2$ \\
\hline 20.000 & $5.267+03$ & $2.7 \notin 4+c 2$ \\
\hline $3 \mathrm{C} \cdot \mathrm{COC}$ & $1.788+03$ & $1.564+C 2$ \\
\hline & & \\
\hline
\end{tabular}

$\begin{array}{lr}\text { INCOH. } & \text { PHCTC } \\ \text { SCATT. } & \text { EFFC T } \\ .833-04 & 1.383+08 \\ .117-C 4 & 2.721+07 \\ .3 C 7-C 4 & 8.791+06 \\ .638-03 & 2.254+06 \\ .5 C 3-C 3 & 1.257+06 \\ .520-C 3 & 2.762+06 \\ .485-C 3 & 4.292+06 \\ .145-C 2 & 4.159+06 \\ .776-C 2 & 3.473+06 \\ .525-C 2 & 2.277+06 \\ .852-02 & 1.656+06 \\ .485-C 1 & 1.059+06 \\ .539-C 1 & 7.762+05 \\ .810-C 1 & 6.120+05 \\ 5260-01 & 5.049+05 \\ .546-C 1 & 2.826+06 \\ .210+C 0 & 1.929+06 \\ .055+C C & 8.087+05 \\ .556+0 C & 4.144+05 \\ .551+C C & 1.558+05 \\ . C 57+00 & 7.479+04 \\ .475+C C & 1.764+05 \\ .735+C C & 1.284+05 \\ . C 93+C 1 & 6.043+C 4 \\ .275+01 & 3.319+C 4 \\ .6 C 7+01 & 1.098+04 \\ .834+C 1 & 4.972+03 \\ .1 C 2+01 & 1.610+03 \\ .248+C 1 & 4.532+C 3\end{array}$

\begin{tabular}{|c|c|c|}
\hline \multicolumn{3}{|c|}{$z=54 \quad$ XENON } \\
\hline $\begin{array}{l}\text { EAERGY } \\
\text { (KEV) }\end{array}$ & TOTAL & $\begin{array}{l}\text { CORER. } \\
\text { SCATT. }\end{array}$ \\
\hline.$C 10$ & $3.220+c t$ & $1.540+03$ \\
\hline .015 & $1.19 t+C 7$ & $1.540+03$ \\
\hline $.02 \mathrm{C}$ & 7. $C 8 C+06$ & $1.940+C 3$ \\
\hline .030 & $2.707+0 t$ & $1.540+C 3$ \\
\hline .040 & $1.36 i+06$ & 1. $540+C 3$ \\
\hline .050 & $8.556+05$ & $1.939+03$ \\
\hline $.0 \in 0$ & $6.191+05$ & $1.539+03$ \\
\hline .080 & $t .45 s+c 6$ & $1.939+03$ \\
\hline .100 & $4.35 C+C E$ & $1.939 * 03$ \\
\hline .150 & $1.6 t 6+06$ & $1.537 * C 3$ \\
\hline .200 & $1 . C 19+C t$ & $1.935+03$ \\
\hline .300 & $8.015+05$ & $1.930+03$ \\
\hline .400 & $7.542+C 5$ & $1.522+03$ \\
\hline $.50 \mathrm{C}$ & $7.09 \varepsilon+05$ & $1 . S 13+C 3$ \\
\hline .600 & $t \cdot 632+0 E$ & $1.5 C_{2}+C_{3}$ \\
\hline .800 & $2.667+06$ & $1.875+C 3$ \\
\hline 1.000 & $2.134+C t$ & $1.843+03$ \\
\hline 1.500 & $8.5 \$ 6+C 5$ & $1.745+C 3$ \\
\hline $2 . C 00$ & $4 \cdot 4 t 4+C 5$ & $1.633+c 3$ \\
\hline 3.000 & $1.698+05$ & $1.412+C_{3}$ \\
\hline 4.000 & $8.223+04$ & $1.22 s+c 3$ \\
\hline 5.000 & 1.404205 & $1.078+03$ \\
\hline $6.00 \mathrm{C}$ & $1.3 \varepsilon 3+C 5$ & $5.51 t+c 2$ \\
\hline 8.000 & $\epsilon .578+04$ & $7.561+C 2$ \\
\hline 10.000 & $3.643 * C 4$ & $c .154+c 2$ \\
\hline $15 . \mathrm{CCC}$ & $1.228+04$ & $4 \cdot C 32+c 2$ \\
\hline $2 C .000$ & $5.677+03$ & $2.881+c 2$ \\
\hline 30.000 & $1.929+03$ & $1.641+02$ \\
\hline 40.000 & $4.565+C 3$ & $1.041+C 2$ \\
\hline
\end{tabular}

\begin{tabular}{|c|c|c|}
\hline EERGY & OTAL & COHER. \\
\hline (KEV) & & \\
\hline 50 & $.408+03$ & $6.440+01$ \\
\hline & & $4.675+01$ \\
\hline & & \\
\hline 100 & & \\
\hline & & $15+00$ \\
\hline & & \\
\hline & & \\
\hline 400 & 36 & \\
\hline & & \\
\hline & & \\
\hline & 1.3 & \\
\hline & & \\
\hline & & \\
\hline & & \\
\hline & & \\
\hline & & \\
\hline & & \\
\hline 60 & & \\
\hline & & \\
\hline & & \\
\hline & & \\
\hline & & \\
\hline & & \\
\hline & & $1.301-04$ \\
\hline & & \\
\hline & & 05 \\
\hline & & \\
\hline & 10 & $2.082-05$ \\
\hline
\end{tabular}

100000 .
INCOH.

EFFECT

$2.28 \mathrm{~s}+01 \quad 2.32 \mathrm{C}+03$

$\begin{array}{ll}2.323+01 & 1.4 C 4+03 \\ 2.015+C 1 & t .2 \varepsilon C+C 2\end{array}$

$2.257+01 \quad 3.343+02$

$2.168+01 \quad 1 . C 51+02$

$2 . C 28+C 1 \quad 4 . t 22+01$

$\begin{array}{ll}1.757+C 1 & 1.475+01 \\ 1.623+01 & 6.8 C C+00\end{array}$

$1.489+01 \quad 3.8 z \epsilon+00$

$1.381+01 \quad z .4 E 2+0 C$

$1.21 t+C 1 \quad 1.274+0 C$

$1 . \operatorname{CS5}+C 1 \quad 7.92 t-01$

$\varepsilon . S C 9+O C \quad 3.621-01$

$7.6 C 5+0 C \quad 2.2 C 1-01$

5. $5 \varepsilon 2+0 C$ 1.171-01

$4.588+C C \quad 7.797-02$

$4.3 C 7+C C \quad 5.79 t-C 2$

$3.8 C 7+C C \quad 4.5 \subseteq t-C 2$

$3.114+C C \quad 3.2 \equiv 7-02$

$2.651+0 C \quad 2.493-02$

$1 . \subseteq \in C+O C \quad 1.575-02$

$1.573+O C \quad 1.154-02$

$1.144+C C \quad 7.454-03$

5.C79-C1 5. $54 \varepsilon-03$

7.573-C1 4.4C4-03

6.520-C1 $3.651-03$

$5.137-01 \quad 2.72 C-03$

$\mathrm{a} .1 \in \varepsilon-03$
PAIF

PRCD.

$1 .+21-01$

$5.659-01$

$1.441+C C$
$2.259+C C$

$2.589+C C$

$3.630+C C$

$4 \cdot 672+C C$

$5.467+C C$

$7.578+C C$

$5.735+C C$

1. $\mathrm{CSO}+\mathrm{Cl}$

$1.169+C 1$

$1.227+C 1$

$1.359+C 1$

\begin{tabular}{|c|c|c|c|c|c|}
\hline $\begin{array}{l}\text { ENERGY } \\
\text { (KEY) }\end{array}$ & TOTAL & $\begin{array}{l}\text { COHER. } \\
\text { SCATT. }\end{array}$ & $\begin{array}{l}\text { INC OH. } \\
\text { SCATT. }\end{array}$ & $\begin{array}{l}\text { PHOTO } \\
\text { EFFECT }\end{array}$ & $\begin{array}{l}\text { PAIR } \\
\text { PREC. }\end{array}$ \\
\hline 50. & $2.576+C 3$ & $6.784+01$ & $2.325+01$ & $2.4 \varepsilon E+03$ & \\
\hline 60. & $1.579+03$ & $4.929+01$ & $2.3 \in 2+C 1$ & $1.5 C t+C 3$ & \\
\hline 80. & $7.277+02$ & $2.931+01$ & $2.35 s+01$ & t. $74 \varepsilon+02$ & \\
\hline 100. & $4.021+C 2$ & $1.939+01$ & $2.33 \varepsilon+01$ & $3.553+02$ & \\
\hline 150. & $1.444+C 2$ & $9.001+00$ & $2.2 C 8+01$ & $1.133+02$ & \\
\hline 200 . & $7.525+c 1$ & $5.173+00$ & 2. $c t t+01$ & $5 . c C 2+c 1$ & \\
\hline 300 . & $3.677+01$ & $2.349+C 0$ & 1. $831+C 1$ & $1 \cdot t 11+01$ & \\
\hline 400. & $2.531+C 1$ & $1.335+00$ & $1 . t 55+01$ & $7.425+0 \mathrm{C}$ & \\
\hline 500. & $2.022+01$ & $8.601-01$ & $1.517+C 1$ & $4.1 \subseteq C+00$ & \\
\hline 600 . & $1.73 t+01$ & $5.998-01$ & $1.4 C 7+C 1$ & $2 \cdot t \varepsilon E+O C$ & \\
\hline 800 . & $1.412+01$ & $3.392-\mathrm{Cl}$ & $1.235+01$ & $1.38 \varepsilon+0 C$ & \\
\hline $100 \mathrm{C}$ & $1.224+01$ & $2.178-01$ & $1.11 t+C 1$ & $\varepsilon, t 33=01$ & \\
\hline 1500 . & $9.741+0 \mathrm{C}$ & $9.719-02$ & $5 . C 80+C c$ & $3.544-01$ & $1.653-\mathrm{Cl}$ \\
\hline 2000 . & $8.652+00$ & $5.478-c 2$ & $7.751+O C$ & $2 . \equiv \subseteq i-C l$ & $6 . c 70-c 1$ \\
\hline 3000 . & $7.754+0 \mathrm{C}$ & $2.440-02$ & $t . c \leqslant 7+O C$ & $1.275-01$ & $1.5 C 5+C C$ \\
\hline 4000 & $7.515+00$ & $1.374-02$ & $5 . C \varepsilon 4+0 C$ & $\varepsilon .484-02$ & $2.336+C C$ \\
\hline 5000. & $7.541+C C$ & $8.799-03$ & $4.350+0 C$ & $t .3 C 5-02$ & 3. $c 79+c c$ \\
\hline 6000 . & $7.673+0 C$ & $6.113-03$ & 3. $\varepsilon 80+C C$ & $4.558-02$ & $3.736+C C$ \\
\hline 8000 & $8 . C 41+00$ & $3.440-03$ & $3.173+O C$ & $3.51 \varepsilon-02$ & $4.829+C C$ \\
\hline 10000 . & $8.417+00$ & $2.202-03$ & $2.7 C 2+c c$ & $2.7 c \varepsilon-c 2$ & $5.686+c c$ \\
\hline 15000 . & $9.2 \geq 2+00$ & $9.793-04$ & $1 . \varsigma s \varepsilon+c c$ & $1.714-02$ & $7.215+C C$ \\
\hline 20000 & $9.561+0 \mathrm{C}$ & $5.510-04$ & $1 . \in C 3+0 C$ & $1.25 \hat{\mathrm{C}}-02$ & $8.346+C C$ \\
\hline 30000 . & & $2.449-04$ & $1.1 t \epsilon+00$ & $8.1 \equiv 3-03$ & 1. $C C 7+C 1$ \\
\hline & $1.218+01$ & $1.378-04$ & $5.254-\mathrm{Cl} 1$ & C.C $\angle C C-C 3$ & $1.125+C 1$ \\
\hline 50000 . & $1.287+01$ & $8.819-05$ & $7.718-C 1$ & & \\
\hline 60000. & $1.340+C 1$ & $6.124-05$ & $t \cdot t 4 t-01$ & 3. $5 \in C-03$ & $1.273+\mathrm{Cl}$ \\
\hline & & $3.445-C 5$ & $5.23 t-c 1$ & $2 . \subseteq 51-c 3$ & $1.373+01$ \\
\hline & & & $4.344-c$ & $2.351-03$ & \\
\hline
\end{tabular}

\begin{tabular}{|c|c|c|c|c|c|}
\hline $\begin{array}{l}\text { EAERGY } \\
\text { (KEV) }\end{array}$ & TCTAL & $\begin{array}{l}\text { COFER } \\
\text { SCATT. }\end{array}$ & $\begin{array}{l}\text { INCCH. } \\
\text { SCATT. }\end{array}$ & $\begin{array}{r}\text { FHCTO } \\
\text { EFFECT }\end{array}$ & $\begin{array}{l}\text { PAIR } \\
\text { PRCL. }\end{array}$ \\
\hline 50 & $2.752+C 3$ & $7.145+01$ & $2.3 t \equiv+C 1$ & $2.657+C 3$ & \\
\hline 60 . & $1.688+03$ & $5.197+01$ & $2.401+01$ & $1 . t 12+03$ & \\
\hline 80 . & $7.791+c 2$ & $3.094+01$ & $2.358+01$ & $7.242+02$ & \\
\hline 100 . & $4.311+02$ & $2.048+01$ & $2.378+01$ & $3.8<s+02$ & \\
\hline 150. & $1.547+02$ & $9.51 t+00$ & $2.247+C 1$ & $1.227+02$ & \\
\hline 200 & $8 \cdot C \varepsilon 7+c 1$ & $5.472 * 00$ & $2.1 C 3+01$ & $5.4 \geq 7+01$ & \\
\hline 300 . & $3.871+01$ & $2.485+0 \mathrm{C}$ & $1 . \varepsilon \in 5+C 1$ & $1.757+\mathrm{Cl}$ & \\
\hline 400. & $2.637+01$ & $1.413+00$ & $1.685+01$ & $8.1 C 3+0 c$ & \\
\hline 500 . & $2 \cdot \operatorname{cs} 3+01$ & $9.104-01$ & $1.545+C 1$ & $4.560+00$ & \\
\hline 600. & $1.788+01$ & $6.350-01$ & $1.433+\mathrm{Cl}$ & $2 . s 1 t+00$ & \\
\hline 800 . & $1.445+C 1$ & $3.591-01$ & $1.263+01$ & $1.5 C E+C C$ & \\
\hline 1000 & $1.253+01$ & $2.306-01$ & $1.137+01$ & $5.3 \in 8-01$ & \\
\hline 1500 . & S.SES+OC & $1.029-01$ & S. $251+0 C$ & $4.287-01$ & $1.770-C 1$ \\
\hline 2000 & $8.869+00$ & $5.801-02$ & 7. $\& 97+O C$ & $2 . t C \varepsilon-01$ & $6.533-c 1$ \\
\hline 3000 . & $7.946+0 C$ & $2.584-02$ & $t .212+O C$ & $1.3 \varepsilon 7-01$ & $1.570+C C$ \\
\hline 4000 & $7.681+0 C$ & $1.455-02$ & $5.180+O C$ & $5.231-02$ & $2.3 \subseteq 4+0 C$ \\
\hline & 7.683400 & $9.318-03$ & $4.473+0 C$ & t. . 557-02 & $3.132+C C$ \\
\hline 6000 . & $7.8 \mathrm{CS}+00$ & $6.474-03$ & $3.953+0 C$ & $5.433-02$ & $3.795+C C$ \\
\hline 2000 . & $\varepsilon .2 C \varepsilon+00$ & $3 . t 43-03$ & $3.233+0 \mathrm{C}$ & $3.822-02$ & $4.533+C C$ \\
\hline 10000 & $8.652+00$ & $2.332-03$ & $2.753+0 \mathrm{C}$ & $2.54 \mathrm{C}-02$ & $5.868+C C$ \\
\hline 15000 . & s.t $31+00$ & $1.037-03$ & $2 . c 36+C C$ & $1.85 \varsigma-02$ & $7.575+C C$ \\
\hline 20000 & $1.035+C 1$ & $5.835-04$ & $1.633 \div 00$ & $1.358-02$ & $8.738+C C$ \\
\hline 30000 . & $1.156+01$ & $2.594-c 4$ & $1.188+C c$ & $8.81 \mathrm{C}-03$ & 1. $C 36+C 1$ \\
\hline 40000 & $1.248+01$ & $1.459-04$ & $9.428-01$ & $6.51 \varepsilon-03$ & $1.153+\mathrm{Cl}$ \\
\hline 50000 . & $1.323+01$ & $9.340-05$ & 7. $E \in 4-C 1$ & $5.172-03$ & $1.244+C 1$ \\
\hline & $1.384+C 1$ & $6.486-05$ & $6.771-01$ & $4.287-03$ & $1.316+01$ \\
\hline 80000 & $1.480+01$ & $3.649-05$ & $5.335-01$ & $3.1 \subseteq 3-03$ & $1.426+C 1$ \\
\hline 00000 . & $1.552+01$ & $2.335-05$ & $4.426-01$ & $2.544-03$ & $1.5 \mathrm{C} 8+\mathrm{Cl}$ \\
\hline
\end{tabular}




\begin{tabular}{|c|c|c|c|c|}
\hline $\begin{array}{l}\text { NERGY } \\
\text { K FV }\end{array}$ & TOTA & COHER - & INCOH. & PHOTO \\
\hline .010 & $7+$ & $\begin{array}{l}\text { SCA } \\
\text { C12 }\end{array}$ & & \\
\hline & 1 & $2.012+03$ & $4.383-04$ & $946+07$ \\
\hline .020 & $53 * c 7$ & 2. $C 12+03$ & $7.779-c_{4}$ & $2.253+07$ \\
\hline .030 & & & $744-C 3$ & \\
\hline .040 & & 2. & 3 & $07+06$ \\
\hline & & & & \\
\hline & & & & \\
\hline .080 & & 2.0 & & \\
\hline & 2.1 & 2.0 & 62 & 6407 \\
\hline & & & & \\
\hline & 1.3 & & & $1.323+06$ \\
\hline $.2 \mathrm{CO}$ & $E t+0$ & l.sc & & .06 \\
\hline & & & & \\
\hline .500 & & 1.5 & 4.0 & $41 * 05$ \\
\hline .600 & & & & \\
\hline & & & & \\
\hline & & $1.887 * C_{3}$ & .00 & -3.14 \\
\hline $1.5 \mathrm{CO}$ & & & & \\
\hline & & & & \\
\hline. $\mathrm{CCO}$ & & 1.4 & 4.8 & \\
\hline 10 & & & & \\
\hline & & & & \\
\hline & & & & \\
\hline & & & & \\
\hline & & & & 2.096 \\
\hline & & & & \\
\hline & & & 1.8 & \\
\hline & & & & \\
\hline & & & & \\
\hline
\end{tabular}

\begin{tabular}{|c|c|c|c|c|}
\hline \multicolumn{5}{|c|}{$=56$ BAR IUM } \\
\hline $\begin{array}{l}\text { EAERGY } \\
\text { (KEV) }\end{array}$ & TOTAL & $\begin{array}{l}\text { CCFER. } \\
\text { SCATT. }\end{array}$ & $\begin{array}{l}\text { INCCH. } \\
\text { SCATT. }\end{array}$ & $\begin{array}{r}\text { PHCTO } \\
\text { EFFECT }\end{array}$ \\
\hline .010 & $9.217+13$ & 2. $C \varepsilon 6+C 3$ & $2.245-c 4$ & $5.217 * 13$ \\
\hline & & 2. $C 86+C 3$ & & \\
\hline 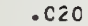 & $57+12$ & $2 . c 2 t+c 3$ & $.547-04$ & $5.757 * 12$ \\
\hline .03 & $36+12$ & . $c 86+03$ & $.0 C 6-C 3$ & $1.136+12$ \\
\hline & $0+1$ & & & \\
\hline & $\varepsilon+11$ & $2.086+$ & & $1.468+11$ \\
\hline $.06 \mathrm{C}$ & $8+1 c$ & & & \\
\hline & & & & \\
\hline & 1 . & $2.084+$ & & \\
\hline & 3.0 & 2.0 & & \\
\hline & & & & \\
\hline & & $2 . c \in S+$ & & \\
\hline .4 & & $2 . c$ & & \\
\hline & & & & \\
\hline.$\in C C$ & & 2. C & & \\
\hline & & 1.5 & & \\
\hline & & & & \\
\hline & & 1.8 & 2. & -2.8 \\
\hline & & & & \\
\hline & & & & \\
\hline & & 1.3 & & \\
\hline & -1 & 1.1 & 8.1 & -1 . \\
\hline & & & & \\
\hline & & & & \\
\hline C & & 6.6 & & \\
\hline & & & & \\
\hline & & & & \\
\hline & $4.135+04$ & $1.757+c$ & $2.2 \mathrm{Cl}+\mathrm{Cl}$ & $4.119+0$ \\
\hline & $\epsilon .51 \varepsilon+04$ & $1.145+c 2$ & $2.354+C 1$ & $6.904 * 04$ \\
\hline
\end{tabular}

\begin{tabular}{|c|c|c|c|c|}
\hline $\begin{array}{l}\text { NERGY } \\
\text { (KEV) }\end{array}$ & TOTAL & $\begin{array}{l}\text { COFER. } \\
\text { SCATT. }\end{array}$ & $\begin{array}{l}\text { INCOH. } \\
\text { SCATT. }\end{array}$ & $\begin{array}{l}\text { PHOTC } \\
\text { EFFECT }\end{array}$ \\
\hline .010 & $5.5 \varepsilon 6 \div C 7$ & $2.1 \in 1+C 3$ & $2.287-C_{4}$ & $5.586 * 07$ \\
\hline .015 & $3.552+C 7$ & $2.161+03$ & $5.137-04$ & $3.551 * 07$ \\
\hline . $C 2 \approx O$ & $1.713+C 7$ & $2.1 \in 1+C_{3}$ & C. $117-04$ & $1.713+c 7$ \\
\hline . $C \geq 0$ & E. $C 73+\mathrm{CE}$ & $2.1 \in 1+C 3$ & $2.044-03$ & $5.071+06$ \\
\hline .040 & $2.115+0 t$ & $2.1 \in 1+C 3$ & $3.621-03$ & $2.112+06$ \\
\hline .050 & $1.185+06$ & $2.1 \in 1 * C_{3}$ & $5.637-03$ & $1.183+06$ \\
\hline - $C \in C$ & $8.358 * 05$ & $2.1 \in 0+c 3$ & 8.CE7-C3 & $8.336 * 05$ \\
\hline .080 & $6.104+C 5$ & $2.160+C 3$ & $1.427-02$ & 6.082405 \\
\hline .100 & $t . c 4 s+C t$ & $2.155+C 3$ & $2.214-02$ & $6.047 * 06$ \\
\hline .150 & $2.524+C E$ & $2.157+C 3$ & 4. $\varepsilon 89-C 2$ & $2.522+06$ \\
\hline .200 & $2.134+06$ & $2.153+C 3$ & $8.529-c 2$ & 2.132406 \\
\hline $.3 \mathrm{CO}$ & $1.54 \subseteq+C \epsilon$ & $2.144+C 3$ & $1.846-\mathrm{Cl}$ & $1.547+06$ \\
\hline .400 & $1.1 \geq 1+C t$ & $2.131 * C 3$ & $3.151-01$ & $1.128 * 06$ \\
\hline .500 & $8.566+c 5$ & & & $8.545 * 05$ \\
\hline .600 & $6.735+05$ & 2. CS\& $C 3$ & $6.503-c 1$ & $6.714 * 05$ \\
\hline $.8 \mathrm{CC}$ & $4.542+05$ & $2 . C 57 \star 03$ & 1. $C 52 \div C C$ & 4.521005 \\
\hline 1.000 & $2.1 \epsilon \varepsilon+C 6$ & $2.012+03$ & $1.482+c C$ & $2.166+06$ \\
\hline 1.500 & $1.040 \div 0 E$ & $1.892+03$ & $2.525 \forall C C$ & $1.038+06$ \\
\hline $2 . \mathrm{COC}$ & $5.501+05$ & $1.77 C+C 3$ & $3.505 * 00$ & $5.484+05$ \\
\hline 3.000 & $2.150+0=$ & $1.541+C 3$ & $5.258+C C$ & 2.135405 \\
\hline & $1.059 * 05$ & $8 \div 03$ & $6.901+00$ & $1.045+05$ \\
\hline $5 . C C C$ & 5. $\varepsilon \in 9+C 4$ & $1.185+c 3$ & $8.338+C C$ & $5.750 * 04$ \\
\hline 6. $\mathrm{CCO}$ & $1.476+C 5$ & $1 . C 4 S+0 ?$ & $5.627 * C C$ & $1.465+05$ \\
\hline 8.000 & & $\varepsilon .371 * 02$ & $184+01$ & $.027 * 04$ \\
\hline C.OCO & $4.527+C 4$ & $t .837+C 2$ & $1.369 * 01$ & $4.457 * 04$ \\
\hline 00 & $1.5 \geq 1+c 4$ & $4.4 \in 8 \& C 2$ & $1.712+C 1$ & $2.485+04$ \\
\hline & $7.070+C 3$ & $3.234+02$ & 1.950401 & $6.727+03$ \\
\hline & & $1.875+C 2$ & $2.236+\mathrm{Cl}$ & $2.193 * 03$ \\
\hline & & & & \\
\hline
\end{tabular}

\begin{tabular}{|c|c|c|c|c|c|}
\hline ENERGY & TCTAL & OHER . & & FHCTO & $\begin{array}{l}\text { PAIF } \\
\text { PRCL. }\end{array}$ \\
\hline $\begin{array}{l}\text { KEV } \\
50 .\end{array}$ & & & SCATT. & $\begin{array}{r}\text { EFFECT } \\
3.63 \epsilon+04\end{array}$ & \\
\hline $\begin{array}{l}50 . \\
60 .\end{array}$ & $\begin{array}{l}3.646004 \\
2.574004\end{array}$ & $\begin{array}{l}7.526+01 \\
5.481+01\end{array}$ & $\begin{array}{l}2.4 C 1+01 \\
2.439\end{array}$ & $\begin{array}{l}3.6 z 6+04 \\
2.567+04\end{array}$ & \\
\hline 80. & $2.175+04$ & $3.270+01$ & $2.4 \equiv 7+01$ & $2.173+c 4$ & \\
\hline 100. & $1.723+04$ & $2.167 * 01$ & $2.418+01$ & $1.71 \varepsilon+04$ & \\
\hline & $1.651 * 02$ & $1.008 * 01$ & $.2 \varepsilon 6+01$ & $1.322+02$ & \\
\hline & $3 * 01$ & $5.800 *$ & $2.140+01$ & $5.863+C 1$ & \\
\hline & & 2.63 & $8+c 1$ & $1.8 \varsigma 5+01$ & \\
\hline & & 1.50 & $1.715 * C 1$ & $8.777 * 0 \mathrm{C}$ & \\
\hline & $2.165+C 1$ & 9.66 & 1.574001 & $4.951+00$ & \\
\hline & 1.844701 & $6.74 \mathrm{C}$ & $1.460 * 01$ & $2.171+c c$ & \\
\hline & 1.428401 & 3.81 & 3.2 & $1 . t$ & \\
\hline 100 & & 2.4 & & $1 . c_{2}$ & \\
\hline & $1 \cdot C 18+01$ & 1.09 & $5.422+C C$ & $4.663-01$ & $1.843-$ \\
\hline & $9 .(883+00$ & 6.16 & E.c & & \\
\hline & & & & & \\
\hline 400 & $7.8 t$ & 1.54 & $5.27 t+c c$ & $1.0 C 1-01$ & \\
\hline & $7.8 t$ & 9.8 & 4.55 & & 3 \\
\hline & & & & & \\
\hline 80 & 8.41 & 3.87 & 3.2 & $4.137-02$ & $5 . c 74 *$ \\
\hline & & & & & \\
\hline & 1.003 & 1.10 & $2 . c$ & $2.011-02$ & \\
\hline 20000 . & $1.085=0$ & 6.19 & $1 . t$ & $1.4 E \varepsilon-02$ & \\
\hline & & 2.7 & 1.21 & & \\
\hline & & $1.550-04$ & s.tC3-01 & $7.045-03$ & \\
\hline & $1.367 * 0$ & $9.920-05$ & E. $(1 C-01$ & $5.553-03$ & \\
\hline & & 6.889 & $6 . \varepsilon \varsigma$ & $4.625-03$ & $361+$ \\
\hline & & 3.875 & 543 & $3.453-$ & \\
\hline & & & & & \\
\hline
\end{tabular}

\begin{tabular}{|c|c|c|c|c|c|}
\hline $\begin{array}{l}\text { ENERGY } \\
\text { (KEV) }\end{array}$ & TCTAL & $\begin{array}{l}\text { COHER. } \\
\text { SCATT. }\end{array}$ & $\begin{array}{l}\text { INCCH. } \\
\text { SCATT. }\end{array}$ & $\begin{array}{r}\text { FHCTC } \\
\text { EFFECT }\end{array}$ & $\begin{array}{r}\text { PAI } \\
\text { PRCC }\end{array}$ \\
\hline 50. & $5.357+04$ & $7.922+01$ & $2.43 t+C 1$ & $E .34 t+C 4$ & \\
\hline 60. & & $5.779+01$ & $2.47 t+01$ & $4.357+04$ & \\
\hline 80 . & $.181+04$ & $.453+01$ & $2.47 t+C 1$ & $3.175+04$ & \\
\hline 100. & $2.5 \mathrm{CO}+04$ & . $291 \div 01$ & $2.459+01$ & $2.4 \subseteq 5 \$ 04$ & \\
\hline & $02 \nleftarrow 03$ & $.067 t$ & $2.326+C 1$ & $4.1 \in \varepsilon+C 3$ & \\
\hline & $2.776+03$ & $6.145+00$ & 2.177001 & $2.748+03$ & \\
\hline & & & 1. $532 * C 1$ & $1.57 \varepsilon+03$ & \\
\hline 400. & $1.107+03$ & $1.591+00$ & $1.746+C 1$ & 1. $C E E+03$ & \\
\hline 500 & $8.4104 \mathrm{C} 2$ & 1.025 & $1 . \in C 2+C 1$ & $\varepsilon .23 \subseteq+C 2$ & \\
\hline & & & & $3.4 \geq 1+0$ & \\
\hline 800 . & $1.527+01$ & 4.045 & $1.3 C s \leftrightarrow 01$ & $1.77 t+c c$ & \\
\hline 1000 & $1.315+01$ & & $1.17 s+C 1$ & $1.1 C t+00$ & \\
\hline & & & & E.CEE- 01 & \\
\hline $2 C$ & $9.278+00$ & $6.540-$ & $E .189+O C$ & 3.c72-01 & \\
\hline 300 & & & $2+c c$ & $1.6 \geqslant 1-01$ & \\
\hline & & & & & \\
\hline 5000 . & $8 . c 85 *$ & 1.0 & $4.635+0 C$ & $8 . C 41-02$ & $0 \%$ \\
\hline & $\varepsilon \cdot 2$ & & 4.10 & $t \cdot z \in \bar{t}-c 2$ & \\
\hline & & & & & \\
\hline 10000. & $9.217+00$ & 2.6 & 2. $E 55+O C$ & $3.441-02$ & 25 \\
\hline & & & & & \\
\hline & & 6.580 & 1.6544 & $1.587-02$ & \\
\hline & & 2.925 & 1.232 & & \\
\hline & $1.342+$ & & & & \\
\hline & & & & & \\
\hline & & & & & \\
\hline & $1.552 * C$ & $4.115-05$ & $5.532-C 1$ & $3.7<5-03$ & \\
\hline & $1 . t 64 \div 01$ & $2.633-05$ & $4.550-C 1$ & $2.571-03$ & $1.618+0$ \\
\hline
\end{tabular}

\begin{tabular}{|c|c|c|c|c|c|}
\hline $\begin{array}{l}\text { EAERGY } \\
\text { (KEV) }\end{array}$ & TCTAL & $\begin{array}{l}\text { COHER } \\
\text { SCATT. }\end{array}$ & $\begin{array}{l}\text { INCCH. } \\
\text { SCATT }\end{array}$ & $\begin{array}{l}\text { PHCTO } \\
\text { EFFECT }\end{array}$ & $\begin{array}{l}\text { PAIR } \\
\text { PRCLE. }\end{array}$ \\
\hline 50 . & $3.325 * C=$ & $8.290+C 1$ & $2.475+0 i$ & 3. $2 \approx 1+C 3$ & \\
\hline 60. & & $051+01$ & $2.515+01$ & $1.968+03$ & \\
\hline 80. & $9.536 * C 2$ & $.619 * 01$ & - $516+C 1$ & $8.523 \geq 02$ & \\
\hline 100. & 5.284002 & $.401+01$ & $.459+01$ & $4.754+02$ & \\
\hline & - $E 8 C+02$ & & $.365+01$ & $1.531+02$ & \\
\hline 200 & 5.664001 & $6.447+00$ & $2.215+01$ & $E \cdot E \subset 4+C 1$ & \\
\hline 300 & $4.4 \epsilon \epsilon+01$ & $2.933 * 00$ & $\subseteq \in 6+01$ & $2.2 C 7+01$ & \\
\hline & & & $1.777+C 1$ & $1.021+01$ & \\
\hline & $2.315+01$ & $1.076+0$ & 1. $E \geq C+O 1$ & $5.771+00$ & \\
\hline 600 & $1.558+01$ & 7.5 & 1.51 & $3.7 C 2+c C$ & \\
\hline & $1.567+$ & & & & \\
\hline 1000 & $1.34 t+C 1$ & & $C_{1}$ & $1.1 \leqq 4+00$ & \\
\hline & $1.063+01$ & & & 5.4 & \\
\hline & & & & & \\
\hline 30 & 8.5 & $3.058-02$ & $6.557+0 C$ & $1.7 \in 1-01$ & $2+6$ \\
\hline 4000 & & & 5.468 & & 2 \\
\hline & & & & & \\
\hline & $8.435+0$ & & 4.1 & $6.871-02$ & 4.186. \\
\hline 800 & 8.90 & & & & $38+$ \\
\hline & $9.434+0 \mathrm{C}$ & & & & \\
\hline & $1 . C 62 * 01$ & $1.228-$ & & $2.344-02$ & $8.445+$ \\
\hline 20 & $1.149+$ & & & & $5.747+8$ \\
\hline & & & 1.254. & & \\
\hline & 1.3 & & & & $1.273+$ \\
\hline 5000 & $1.456+$ & & & & $1.372+$ \\
\hline & & & & $5.3 \subseteq 7-03$ & \\
\hline & & & & $4.015-03$ & $1.569+c$ \\
\hline & 1 & $65-05$ & $4.672-\mathrm{Cl}$ & $3.2 \times 2-03$ & $1.650 * c$ \\
\hline
\end{tabular}




\begin{tabular}{|c|c|c|c|c|}
\hline $\begin{array}{r}\text { ENERGY } \\
(K \varepsilon V)\end{array}$ & TOTAL & $\begin{array}{l}\text { COHER. } \\
\text { SCATT. }\end{array}$ & $\begin{array}{l}\text { I NCOH. } \\
\text { SCATT. }\end{array}$ & $\begin{array}{r}\text { PHOTD } \\
\text { EFF \&CT }\end{array}$ \\
\hline . $C 10$ & $1.194+05$ & $2.238+C 3$ & $2.2 c 8=04$ & $1.172+05$ \\
\hline & $4+C 7$ & & 4.56 & \\
\hline .020 & $2+07$ & 2.2 & 8.80 & $3.232+0$ \\
\hline .030 & $69+0 t$ & & & $5.666+0$ \\
\hline .040 & $5+C 6$ & & & \\
\hline . $C 50$ & & & & \\
\hline & & & & \\
\hline - $\mathrm{C}$ & 1.2 & .2 & 2 & 06 \\
\hline .100 & $64+C 6$ & $2.236+C 3$ & 2.13 & +06 \\
\hline .150 & $69+06$ & 2.23 & $4.724-C 2$ & $1.367+06$ \\
\hline.$\angle \mathrm{CC}$ & $23+C 5$ & $2.23 c+c 3$ & $8.244-C 2$ & $5.701+C 5$ \\
\hline .300 & $5+C t$ & 2.22 & $-C_{1}$ & $.053+06$ \\
\hline $.40 \mathrm{C}$ & $1+C 5$ & 2.2 & 3. C & 05 \\
\hline $.5 \mathrm{co}$ & 9 & & & .05 \\
\hline.$\in C C$ & & & & \\
\hline .8 & & & & \\
\hline & & & & \\
\hline & 1. & & c & \\
\hline & t. & 1.8 & & +05 \\
\hline 3.000 & $18+05$ & 1.60 & 5.2 & 05 \\
\hline $4 . \mathrm{COC}$ & $1.133+05$ & 1.4 & 6.52 & 1.11 \\
\hline 5.000 & $6.406+C 4$ & 1.23 & 8.38 & $6.281+04$ \\
\hline 000 & $\epsilon 4+c$ & $1 . \mathrm{C}$ & 9.67 & $1.153+05$ \\
\hline $8.00 \mathrm{C}$ & $57+C$ & 8.74 & 1.15 & $8.608+04$ \\
\hline. $\mathrm{CCO}$ & $\mathrm{CO}+\mathrm{C}$ & $7.142+C 2$ & 1.3 & $4.827+04$ \\
\hline $\mathrm{CCO}$ & $\in 1+0$ & $4.65 t+02$ & $1.73 c+c$ & $1.613+04$ \\
\hline $\mathrm{COO}$ & $t=26+0$ & $7+C 2$ & 1.9 & $7.280+03$ \\
\hline & $2.588+C$ & 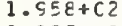 & $2 \cdot 2 \in 7+C l$ & $2.365+0=$ \\
\hline & $1.232+c 3$ & $1.255+C 2$ & $2.426+C 1$ & $1.083+03$ \\
\hline
\end{tabular}

\begin{tabular}{|c|c|c|c|c|}
\hline $\begin{array}{r}E A E R G Y \\
\text { (K\&V) }\end{array}$ & TOTAL & $\begin{array}{l}\text { COFER. } \\
\text { SCATT. }\end{array}$ & $\begin{array}{l}\text { INCCH. } \\
\text { SCATT. }\end{array}$ & $\begin{array}{r}\text { PHCTC } \\
\text { EFFECT }\end{array}$ \\
\hline C 10 & $1.8 \leq 1+0 t$ & $2.316+C 3$ & $2.174-C 4$ & $1.848+06$ \\
\hline & $1.016+08$ & $2.316+C 3$ & 4. $824-C 4$ & $1.016+08$ \\
\hline - $\mathrm{C} 2 \mathrm{C}$ & $737+c 7$ & $5+c 3$ & $8 . t \in 7-c$ & \\
\hline & $83+C 7$ & +03 & - $>4$ & +07 \\
\hline .040 & 8. $\cos 8+c t$ & $+C 3$ & 3.44 & - $c 96+06$ \\
\hline . $C 50$ & $4.455+0 t$ & $+C 3$ & 5.35 & $1+06$ \\
\hline - $c 60$ & $C \varepsilon O+C E$ & $15+C 3$ & 7.65 & $078+06$ \\
\hline .080 & $2.118+C t$ & $.314+C 3$ & $1.357-C_{2}$ & $2.115+06$ \\
\hline $.10 \mathrm{C}$ & $1.81 z+c t$ & $.314+C 3$ & $2.1 C 5-C 2$ & $1.810+06$ \\
\hline .150 & $31+c$ & $.311+C 3$ & 4.65 & $1.725+06$ \\
\hline .200 & $3+06$ & $+\mathrm{C}_{3}$ & -02 & $0+06$ \\
\hline & & & & +06 \\
\hline .400 & & $2 \cdot 2$ & & +05 \\
\hline 500 & e. $3 \varepsilon l+c$ & 2.27 & 4.4 & $\varepsilon$. \\
\hline .600 & $48+C 5$ & $2.254+c 3$ & 6.20 & +05 \\
\hline .800 & $5.152+C 5$ & $2.214+C 3$ & 1. $\mathrm{CO} t+\mathrm{CO}$ & $0+05$ \\
\hline 1.000 & $2.424+C 6$ & $2.17 C+C_{3}$ & 1.415 & $2.422+06$ \\
\hline $1.5 \mathrm{CC}$ & $1.190+0 t$ & 2. $C 51+C 3$ & $2.434+C C$ & $1.188+06$ \\
\hline $2 . \mathrm{cco}$ & $51+05$ & $1.529+C 3$ & $3.4 \mathrm{CO}+\mathrm{CC}$ & $6.372+05$ \\
\hline 000 & $97+05$ & $1.6 \subseteq 3+03$ & $5.154+00$ & $2.370+05$ \\
\hline 200 & $74+05$ & $1.485+C 3$ & $6.809+C O$ & $1.159+05$ \\
\hline & + & $5+C 3$ & $+C c$ & $33+04$ \\
\hline & & & & \\
\hline 0 & $.72+c 4$ & $1+0 \bar{z}$ & 1.18 & $5.079+04$ \\
\hline 10.000 & $197+C 4$ & $7.5 C_{1}+C_{2}$ & 1.37 & $5.121+04$ \\
\hline & $73+c$ & 4. $545+c 2$ & 1.73 & $1.722+c 4$ \\
\hline & $1+($ & $3.515+C 2$ & $1 . s \varepsilon$ & $7.790+03$ \\
\hline & $E 2+C$ & $2 . C 4 \varepsilon+C 2$ & & $2.525+03$ \\
\hline & +03 & $14+C 2$ & $457+C l$ & $41+03$ \\
\hline
\end{tabular}

\begin{tabular}{|c|c|c|c|c|}
\hline $\begin{array}{l}\text { EAERGY } \\
(K \varepsilon V)\end{array}$ & TOTAL & $\begin{array}{l}\text { COFER. } \\
\text { SCATT. }\end{array}$ & $\begin{array}{l}\text { I NC OH. } \\
\text { SCA T T. }\end{array}$ & $\begin{array}{l}\text { PHCTC } \\
F F \& C T\end{array}$ \\
\hline .010 & $1.52 s+C s$ & $2.355+C 3$ & $2.147-C 4$ & $1.529+09$ \\
\hline & $2.225+C E$ & $2.355+C 3$ & $4.822-C_{4}$ & $2.225+08$ \\
\hline C 20 & $64+C 7$ & $2.355+c 3$ & $8.558-c$ & $5.864+07$ \\
\hline & & & & $1.429+07$ \\
\hline .040 & $8.185+$ & .39 & 3 & +06 \\
\hline 50 & $5.851+C t$ & $.394+C 3$ & 5. & 5.849 \\
\hline $.06 \mathrm{C}$ & $4.43 t+0 t$ & $354+C 3$ & $7.5 \subseteq 4-c 3$ & $4.434+06$ \\
\hline . $C \& C$ & $2.724+C 6$ & $2.353+C 3$ & $1.340-C 2$ & $2.721+c 6$ \\
\hline .100 & $1.757+C E$ & $2 \cdot 3 c_{3}+c_{3}$ & $2 . C 75-02$ & $1.754+06$ \\
\hline $.15 \mathrm{C}$ & $E C+C E$ & $2.350+c 3$ & $4.594-$ & $1.947+06$ \\
\hline & $2.4+c t$ & & E. C17- & $1.022+06$ \\
\hline & $+0 t$ & & 1.7 & $1+06$ \\
\hline $.4 \mathrm{CC}$ & 1. $C 44+0 t$ & $.365 t$ & 2.5 & 06 \\
\hline .500 & $8 \cdot 671+C 5$ & $2.35 C+03$ & $4.447-01$ & 8.64 \\
\hline $.6 \mathrm{CO}$ & $7.155+C 5$ & $2.333+c 3$ & $6.134-01$ & $7.132+05$ \\
\hline $.80 \mathrm{C}$ & $5.037+05$ & $2.293+c 3$ & $9.547-C_{1}$ & $5.014+05$ \\
\hline $1 . \mathrm{CCO}$ & $2.5 \varepsilon s+C 6$ & $2.245+c 3$ & $1.4 C 5$ & $2.586+06$ \\
\hline $\mathrm{CO}$ & $244+C 6$ & $2.12 s+c 3$ & $2.415+\mathrm{CO}$ & $1.242+06$ \\
\hline $\mathrm{OCO}$ & $74+C 5$ & $2 \cdot C C 5+C 3$ & 3.381 & $6.754+05$ \\
\hline 00 & & & & $2.530+05$ \\
\hline & & 3 & 6.80 & +05 \\
\hline 0 & $7.243+C 4$ & $1.3 \leftarrow 2+$ & $8 \cdot 2$ & $7.106+04$ \\
\hline$t \cdot C C C$ & $4.552+C 4$ & $1.203+c$ & 9.5 & 4.470 \\
\hline $\mathrm{COC}$ & $9.744+C 4$ & $5.581+C 2$ & $1.190+01$ & $9.647+04$ \\
\hline $\mathrm{IC} \cdot \mathrm{CcO}$ & $5.52 S+C 4$ & $7.8 C 4+C$ & $1.383 t$ & $5.450+04$ \\
\hline $15 . \mathrm{COC}$ & $1.885+04$ & $5 . C 82+02$ & $1.751+C_{1}$ & $1.837+04$ \\
\hline $2 \mathrm{C} \cdot \mathrm{COC}$ & $8.703+C 3$ & $3.658+02$ & $2.0 C 8+C 1$ & $8.317+03$ \\
\hline & $2 \cdot 536+03$ & $2.136+c 2$ & $2.320+C 1$ & $2.699+03$ \\
\hline $4 \mathrm{C} \cdot \mathrm{CCO}$ & $1.282+C 3$ & $372+C 2$ & $50+C 1$ & $.220+0$ \\
\hline
\end{tabular}

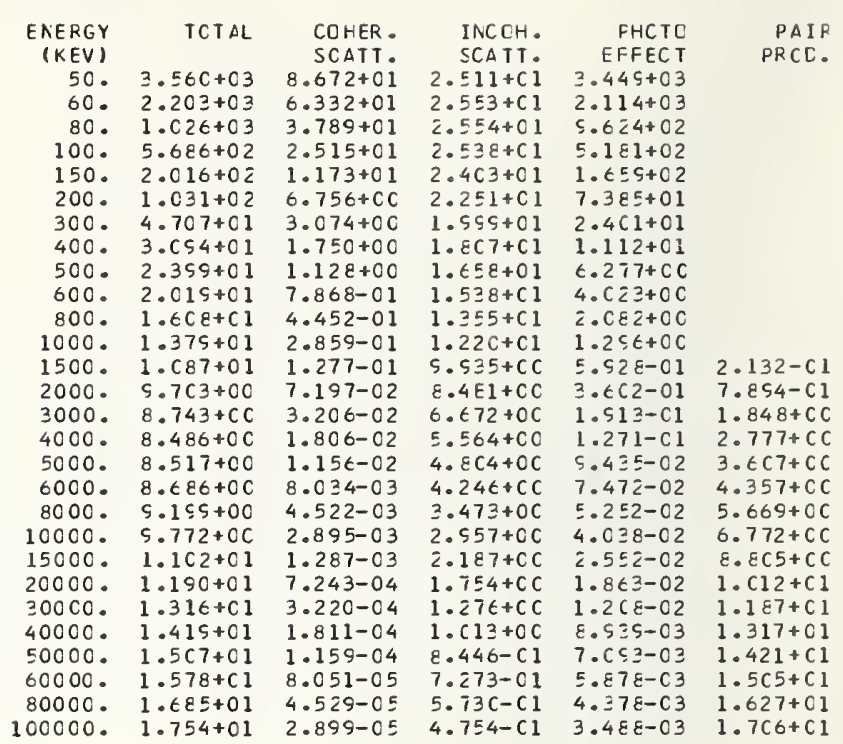

$\begin{array}{rr}\text { COHER. } & \text { INCCH. } \\ \text { SCATT } & \text { SCATT } \\ 9.082+C 1 & 2.54 E+C 1 \\ 6.634+01 & 2.585+C 1 \\ 3.972+01 & 2.551+C 1 \\ 2.637+01 & 2.578+C 1 \\ 1.230+01 & 2.442+C 1 \\ 7.088+00 & 2.288+C 1 \\ 3.226+00 & 2.032+C 1 \\ 1.836+00 & 1.838+01 \\ 1.183+00 & 1.68 C+01 \\ 8.257-01 & 1.565+C 1 \\ 4.672-01 & 1.375+01 \\ 3.001-01 & 1.241+C 1 \\ 1.340-01 & 1 . C 11+01 \\ 7.554-02 & 8.627+0 C \\ 3.365-02 & 6.787+C C \\ 1.895-C 2 & 5.6 E C+C C \\ 1.214-02 & 4.587+C C \\ 8.433-03 & 4.315+C C \\ 4.746-C 3 & 3.553+C C \\ 3.039-03 & 3.0 C 8+C C \\ 1.351-03 & 2.224+0 C \\ 7.602-04 & 1.784+0 C \\ 3.379-04 & 1.258+0 C \\ 1.901-04 & 1 . C 3 C+0 C \\ 1.217-04 & 8.552-C 1 \\ 8.450-C 5 & 7.358-C 1 \\ 4.754-05 & 5.829-01 \\ 3.042-05 & 4.836-C 1\end{array}$

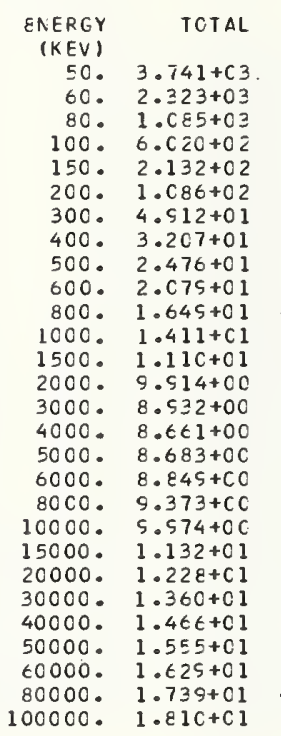

100000 .

\begin{tabular}{|c|c|c|}
\hline $\begin{array}{r}\text { ENERGY } \\
\text { (KEV) }\end{array}$ & TCTAL & SCATT. \\
\hline 50. & $3.551+03$ & 9.491 \\
\hline & & \\
\hline 80 . & $14 \mathrm{ct}$ & $4+0$ \\
\hline 100. & & \\
\hline 150. & & \\
\hline 2 & & \\
\hline 300. & & \\
\hline $40 C$. & & 2 \\
\hline 500 . & & \\
\hline 600 . & & . \\
\hline 800 . & $1 . t 5$ & \\
\hline 1000 & & \\
\hline & $1 \cdot 1$ & \\
\hline 2 & 1. C & \\
\hline 00 & 9.1 & 3 \\
\hline 4000. & 8.52 & $985-$ \\
\hline 5000. & 9. C I & \\
\hline 6000 . & 2 & 8. \\
\hline 8000 . & 9.75 & 4. \\
\hline 10000 & 1.02 & \\
\hline - & 1.13 & \\
\hline & & \\
\hline & & \\
\hline 40000 & & \\
\hline & 1.61 & \\
\hline & & \\
\hline & $.877+C 1$ & .186 \\
\hline
\end{tabular}

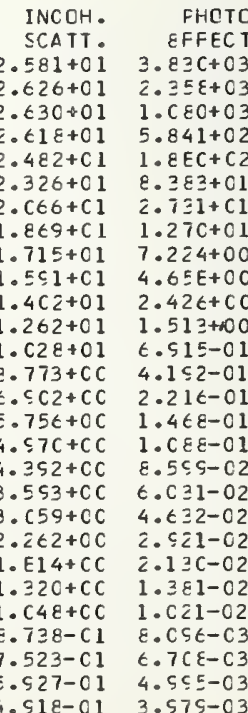

FHCTC
EFFECT 3. $E \geq E+03$ $2.23 \mathrm{C}+03$ $1.01 \varsigma+03$ $5.459+02$ $1.7 \epsilon 5+C 2$ 7. $\varepsilon \in C+01$ $2.557+C$ $1.1 \varepsilon t+C 1$ $t .715+C C$
$4.314+00$ $2.241+0 \mathrm{C}$ $1.3 C 7+C C$ $t .3 \div t-01$ $3 . \varepsilon \varepsilon 5-01$ 2. $C 55-01$ $1.3 t t-01$ 1. $C 12-C 1$ 8.CCE-02 $5 . \in 1 \bar{i}-02$ 4. $314-02$ 2.721-02 $1.5 \varepsilon 5-02$ $1.2 \varepsilon t-02$ $5.51 \mathrm{C}-\mathrm{C} 3$ $7.542-03$ $4 . t 54-C 3$ $3.7 C 7-03$
PAIR PRCL.

$2.220-C 1$ E. $225-C 1$ 1. $5 \mathrm{C} 6+C \mathrm{CC}$ 2. $E 46+C C$ $4.442+C C$ $5.779+C C$ t. $521+C C$ S. $C 71+0 C$ $1.229+01$ $1.362+C 1$ $1.555+C 1$ $1.680+01$ $1.761+C 1$ 


\begin{tabular}{|c|c|c|c|c|}
\hline $\begin{array}{r}\text { ENERGY } \\
\text { (KEV) }\end{array}$ & TOTAL & $\begin{array}{l}\text { COHER. } \\
\text { SCATT. }\end{array}$ & $\begin{array}{l}\text { I NCOH. } \\
\text { SCATT. }\end{array}$ & $\begin{array}{r}\text { PHCTO } \\
\text { EFFECT }\end{array}$ \\
\hline .010 & $3.804+C 7$ & $2.475+03$ & $2.121-c_{4}$ & $3.804+07$ \\
\hline . $C 15$ & $2.536+07$ & $2.475+03$ & $4.7 \in 3-C_{4}$ & $2.536+07$ \\
\hline . $\mathrm{C2O}$ & $2+07$ & $2.475+C 3$ & $8.453-C_{4}$ & $1.902+07$ \\
\hline .030 & $268+07$ & $2.475+C 3$ & $1.895-\mathrm{C3}$ & $1.268+07$ \\
\hline - $\mathrm{C} 4 \mathrm{C}$ & $512+c \epsilon$ & $2.475+C ?$ & $.357-c 3$ & $9.510+06$ \\
\hline .050 & $7 . t 1 c+c t$ & $2.475+03$ & $8-03$ & $7.608+06$ \\
\hline - $c \in 0$ & $t .342+06$ & $2.474+C 3$ & 7.501 & $6.340+06$ \\
\hline . $C \& O$ & $4.757+C \epsilon$ & $2.474+C 3$ & $1.324-02$ & $4.755+06$ \\
\hline .100 & $2.80 t+C t$ & $2.473+C 3$ & 2. C54-C2 & $3.804+06$ \\
\hline .150 & $4 \cdot C 71+C \epsilon$ & $2.471+C 3$ & $4.538-C 2$ & $4 \cdot C 69+06$ \\
\hline .200 & $3.177+06$ & $2.467+03$ & $7.920-c 2$ & $3.174+06$ \\
\hline $.3 \mathrm{CO}$ & 2. $C E 1+C \epsilon$ & $2.458+c 3$ & $716-\mathrm{Cl}$ & $2.079+06$ \\
\hline .400 & & $2.446+C 3$ & $-\mathrm{Cl}$ & $.483+06$ \\
\hline .500 & $1.1 \geq 1+c t$ & $2.431+C 3$ & $C_{1}$ & $9+06$ \\
\hline $.6 \mathrm{CO}$ & $9.023+05$ & $2.414+C 3$ & $6.066-c 1$ & $8.999+05$ \\
\hline $.80 \mathrm{C}$ & $6.315+05$ & $2.374+C 3$ & S. $841-C_{1}$ & $6.292+05$ \\
\hline $1 . \mathrm{COO}$ & $4.8 \mathrm{C} 2+C 5$ & $2.329+C 3$ & $1.391+C 0$ & $4.778+05$ \\
\hline 1.500 & $1 . \equiv 41+C 6$ & $2.205+03$ & $2.395+C C$ & $1.339+06$ \\
\hline 2.000 & $7.235+c 5$ & $2.083+03$ & $3.360+C 0$ & $7.219+05$ \\
\hline 3.000 & $2.730+C 5$ & $1.83 t+C 3$ & $5.166+c c$ & $2.712+05$ \\
\hline $4 . \mathrm{COO}$ & $1.345+C 5$ & $1.615+03$ & $6.799+c 0$ & $1.329+05$ \\
\hline $\mathrm{COC}$ & & $1.421+C 3$ & $274+0 \mathrm{C}$ & $593+04$ \\
\hline $\mathrm{COO}$ & $4.909+04$ & $1.257+C 3$ & $9.6 \mathrm{C} 9+0 \mathrm{C}$ & $4 \cdot 782+04$ \\
\hline $8 . C 00$ & $1.042+05$ & $1 . \mathrm{CO} 1+\mathrm{C}_{3}$ & $1.193+\mathrm{Cl}$ & $1.032+05$ \\
\hline$C . C O O$ & $5 . \& 14+C 4$ & $8.157+c 2$ & $1.389+01$ & $5.731+04$ \\
\hline & $1.986+C_{4}$ & $5.354+02$ & $1.7 \in 3+C 1$ & $1.931+04$ \\
\hline $2 \mathrm{C} .0 \mathrm{CO}$ & $9.252+C 3$ & $3.807+C 2$ & $2.026+C 1$ & $8.851+03$ \\
\hline & $3.165+C 3$ & $2.228+c 2$ & $2.345+01$ & $2.919+03$ \\
\hline & & $1.432+c ?$ & & \\
\hline
\end{tabular}

\begin{tabular}{|c|c|c|c|c|}
\hline $\begin{array}{r}\text { EAERGY } \\
\text { (KEV) }\end{array}$ & TOTAL & $\begin{array}{l}\text { COHER. } \\
\text { SCATT. }\end{array}$ & $\begin{array}{l}\text { I NC CH. } \\
\text { SCATT. }\end{array}$ & $\begin{array}{r}\text { PHCTC } \\
\text { EFFECT }\end{array}$ \\
\hline . C10 & $4.054+07$ & $2.557+C 3$ & 2. $c \in 1-C_{4}$ & $4.094+07$ \\
\hline 15 & $2.730+C 7$ & $2.557+C 3$ & $4 \cdot 63 c-c 4$ & $2.729+c 7$ \\
\hline .020 & $2.047+07$ & $2.557+03$ & $8.217-C_{4}$ & $2.047+07$ \\
\hline - $C \geq 0$ & $1.3 \in 5+C 7$ & $2.557+C 3$ & $1.842-\mathrm{C} 3$ & $1.365+07$ \\
\hline . $\mathrm{C} 4 \mathrm{O}$ & $1.024+07$ & $2.557+C 3$ & $3.264-C 3$ & $1.023+07$ \\
\hline .C50 & $\varepsilon .190+c \epsilon$ & $2.557+C 3$ & 5. C $82-03$ & E.188+06 \\
\hline .060 & $t .826+0 t$ & $2.55 t+C 3$ & $7.253-03$ & $6.823+06$ \\
\hline.$C 80$ & 5. & $2.556+C 3$ & $1.287-C_{2}$ & $5.117+06$ \\
\hline .100 & 4. $C 56+c 6$ & $2.555+C 3$ & $7-c_{2}$ & 4. $C 94+06$ \\
\hline .150 & $7.81 C+c \epsilon$ & $2.553+C 3$ & $4.414-C 2$ & $7.808+06$ \\
\hline .200 & $6.25 \varepsilon+c \epsilon$ & $2.545+C 3$ & $7.7 C 6-C 2$ & $6.256+06$ \\
\hline .300 & $3.97 \bar{c}+\mathrm{C} 6$ & $2.54 C+C 3$ & $1.670-C_{1}$ & $3.969+06$ \\
\hline .400 & $2 . t 26+0 t$ & $2.52 \varepsilon+C 3$ & 2. $856-\mathrm{Cl}$ & $2.624+06$ \\
\hline .500 & $34+06$ & $2.513+c 3$ & $4.286-\mathrm{Cl}$ & $1.831+06$ \\
\hline$\bullet \leftarrow O C$ & $1.341+06$ & $2.49 t+C 3$ & $5.918-C_{1}$ & $338+06$ \\
\hline .800 & $7.93 t+C E$ & $2.456+c 3$ & 9.617 & $2+05$ \\
\hline 1.000 & $5.1 \in 7+05$ & $2.411+C 3$ & $1.362+C c$ & $5.143+05$ \\
\hline 1.500 & $1.342+0 t$ & $2.290+03$ & $2.360+C 0$ & $1.340+06$ \\
\hline $2 . \mathrm{COC}$ & $7.722+C 5$ & $2.1 \in 2+C 3$ & $3.330+C C$ & $7.700+05$ \\
\hline 3.000 & $2.916+05$ & $1.911+03$ & $5.160+\mathrm{CO}$ & $2.897+05$ \\
\hline $4 . C \mathrm{CO}$ & $1.437+05$ & $1 . \in \varepsilon 3+c 3$ & $6.811+C C$ & $1.420+05$ \\
\hline $5.00 \mathrm{C}$ & $58+04$ & $1.482+C 3$ & $8.253+C C$ & $8.109+04$ \\
\hline $\mathrm{CCO}$ & $3 \varepsilon+C 4$ & $1.311+c 3$ & $5.62 s+C C$ & $5.106+04$ \\
\hline$\varepsilon .000$ & $1.087+05$ & 1. $C 45+C 3$ & $1.155+01$ & $1.077+05$ \\
\hline $1 \mathrm{C} \cdot \mathrm{CCC}$ & $6.232+04$ & $\varepsilon .511+C 2$ & $1.393+C_{1}$ & $6.146+04$ \\
\hline $15.00 \mathrm{C}$ & $2.151+C 4$ & $5.562+c 2$ & $1.776+01$ & $2.094+04$ \\
\hline $20.00 \mathrm{C}$ & $5.533+03$ & 3. $\subseteq \in 1+C 2$ & $2.048+01$ & $5.517+03$ \\
\hline & $50+03$ & $2.334+C 2$ & $2.377+C_{1}$ & $3.093+03$ \\
\hline & - $572+C^{2}$ & $1.5 C 7+C 2$ & $2.557+\mathrm{Cl}$ & $1.396+03$ \\
\hline
\end{tabular}

\begin{tabular}{|c|c|c|c|c|}
\hline \\
\hline $\begin{array}{r}\text { EAERGY } \\
\text { (KEV) }\end{array}$ & TOTAL & $\begin{array}{l}\text { COHER. } \\
\text { SCATT. }\end{array}$ & $\begin{array}{l}\text { INCOH. } \\
\text { SCATT. }\end{array}$ & $\begin{array}{r}\text { PHCTC } \\
\text { EFFECT }\end{array}$ \\
\hline .010 & $4.1 E G+C 7$ & $2 . t 4 c+c 3$ & 2. $C 70-C_{4}$ & $4.188+07$ \\
\hline . C 15 & $2.752+c 7$ & $2.64 c+03$ & $4.64 G-C 4$ & $2.792+07$ \\
\hline.$C 2 C$ & $94+C 7$ & $2 . t 4 C+C 3$ & $8.250-C 4$ & $2.054+07$ \\
\hline$. c \geq 0$ & $g \in+C 7$ & $2.64 C+C 3$ & $1.850-\mathrm{C} 3$ & $1.396+07$ \\
\hline .040 & $7+07$ & $2 . t 4 c+c 3$ & $3.277-C_{3}$ & $1.047+07$ \\
\hline .050 & $8 \cdot 379+C \epsilon$ & $2.64 C+C 3$ & $5.102-C_{3}$ & $8.377+06$ \\
\hline - $c \in 0$ & $t .98 z+0 t$ & $2.639+03$ & $7.322-c 3$ & $6.981+06$ \\
\hline .080 & $\varepsilon+C \epsilon$ & $2.639+03$ & 1. $2 \varsigma 2-c 2$ & $5.235+06$ \\
\hline .100 & $4.191+0 t$ & $2 \cdot 63 \varepsilon+c 3$ & $2.0 C 5-C 2$ & $4.188+06$ \\
\hline .150 & $8.341+C t$ & $2 . t 36+c 3$ & $4.431-C 2$ & $8.338+06$ \\
\hline .200 & $\varepsilon+C \epsilon$ & $2.632+C 3$ & $7.734-C 2$ & $6.505+06$ \\
\hline .300 & $C 8+06$ & $2.623+C 3$ & $1.67 t-\mathrm{Cl}$ & $4.105+06$ \\
\hline .400 & $2.724+$ & $2.611+C 3$ & -01 & $2.722+06$ \\
\hline .500 & $1.510+c t$ & $2.596+C 3$ & $4.298-C 1$ & $1.908+06$ \\
\hline .600 & $1.403+06$ & $2.575+c 3$ & $5.932-01$ & $1.400+06$ \\
\hline $.8 C 0$ & $8.380+05$ & $2.539+C 3$ & s.t $635-01$ & $8.355+05$ \\
\hline $1 . \mathrm{COO}$ & $C \varepsilon+C 5$ & $2.494+C 3$ & $1.3 \epsilon 3+c c$ & $5.483+05$ \\
\hline 1.500 & $+C t$ & $2.372+C 3$ & $2.356+C C$ & $1.428+06$ \\
\hline $\mathrm{COO}$ & 05 & $2.243+c 3$ & 3.3 & .05 \\
\hline $3 . \operatorname{CCC}$ & 3. $\mathrm{CS}_{1}+\mathrm{C} 5$ & $1.5 \& 7+C 3$ & $5.129+C C$ & $3.071+05$ \\
\hline $4 . C 00$ & $1.52 \varepsilon+C 5$ & $1.752+C 3$ & $6.773+C C$ & $1.510+05$ \\
\hline $5 . \mathrm{CCC}$ & $8.8 C 2+C 4$ & $1.545+C 3$ & $8.2 \in 2+C C$ & $8.647+04$ \\
\hline t.COO & $5.5 \$ 2+c 4$ & $1.367+c 3$ & $9.612+00$ & $5.455+04$ \\
\hline$\varepsilon . C \mathrm{CO}$ & 1. $\cos +\mathrm{C} 5$ & $1 . c 8 \varepsilon+C 3$ & $1.157+C 1$ & $9.983+C 4$ \\
\hline LC. .000 & $\epsilon .592+04$ & $\varepsilon .845+C 2$ & $1.398+01$ & $6.502+04$ \\
\hline $\mathrm{CCC}$ & $2 \varepsilon 2+c 4$ & $5 \cdot 689+02$ & $1.785+C_{1}$ & $2.223+04$ \\
\hline & $C 58+04$ & $4.120+C 2$ & $2 \cdot c \in 0+C 1$ & $1.015+04$ \\
\hline & $3 \cdot 582+c 3$ & $2.42 C+c 2$ & $2.399+\mathrm{C1}$ & $316+03$ \\
\hline$=\cos$ & $78+C 3$ & $1.559+C 2$ & $2.585+C_{1}$ & $1.497+03$ \\
\hline
\end{tabular}

\begin{tabular}{|c|c|c|c|c|c|}
\hline $\begin{array}{l}\text { ENERGY } \\
\text { (KEV) }\end{array}$ & TCTAL & $\begin{array}{l}\text { COHER. } \\
\text { SCATT. }\end{array}$ & $\begin{array}{l}\text { INC OH. } \\
\text { SCATT. }\end{array}$ & $\begin{array}{r}\text { FHCTO } \\
\text { EFFECT }\end{array}$ & $\begin{array}{l}\text { PAIR } \\
\text { PRCD. }\end{array}$ \\
\hline 50. & $4.191+03$ & $9.917+01$ & $2 \cdot t 15+C 1$ & $4.0 t t+03$ & \\
\hline 60. & 2. $607+03$ & $7.251+01$ & 2. $t \in 2+01$ & $2.5 c \varepsilon+03$ & \\
\hline 80. & $219+03$ & $4.345+01$ & $\bar{z}, \epsilon \in \varepsilon+01$ & $1.145+03$ & \\
\hline 100 & $71+C 2$ & $2.887+01$ & $2.657+01$ & $t .217+C 2$ & \\
\hline 150. & $2.359+0 z$ & $1.348+01$ & $2.520+01$ & $2.012+c 2$ & \\
\hline 200. & $1.21 t+02$ & $767+00$ & $2.362+C 1$ & S. $C 24+01$ & \\
\hline 300 . & 5.417 & $3.536+00$ & 2. $C S G+C 1$ & $2.9 \in 4+01$ & \\
\hline 0. & $3.481+01$ & $2.013+00$ & 1. ESS+C1 & $1.3 \varepsilon 1+01$ & \\
\hline 500 . & 2.65 & $1.298+00$ & $1.743+C 1$ & 7. $817+O C$ & \\
\hline 600. & $2.2 \mathrm{Cs}$ & -01 & $1 . t 17+C 1$ & $5.015+0 C$ & \\
\hline 800 . & $1.73 t$ & -01 & $1.425+01$ & $2.6 c c+00$ & \\
\hline 1000 & 1.478 & & $1.2 \varepsilon 3+01$ & $1.62 \mathrm{C}+00$ & \\
\hline 00. & 1.15 & $1.470-01$ & 1. $C 45+01$ & $7.4 \mathrm{Ct}-01$ & $2.458-0$ \\
\hline & $1 \cdot C 2$ & $8 \cdot 286-02$ & E.S $1 S+O C$ & $4.455-c 1$ & 311 \\
\hline 0. & 9.320 & $3.692-02$ & 7. $\mathrm{C} 17+\mathrm{OC}$ & $2.380-01$ & 28 \\
\hline 4000 & $9.159+00$ & 2.079 & $5 . \varepsilon 52+C C$ & $1.57 \varepsilon-01$ & 129 \\
\hline 00. & +00 & $1.331-02$ & $5 \cdot C 53+C C$ & $1.1 \in G-01$ & $C 5$ \\
\hline 6000. & $9.526+C C$ & $9.251-03$ & $4.4 t \epsilon+0 C$ & $5.24 C-02$ & 4 \\
\hline 8000 & $1.007+01$ & $7-03$ & $3.652+0 C$ & $t .48 c-02$ & \\
\hline 10000. & $1 . C 58$ & $3.333-03$ & $3.11 \mathrm{C}+\mathrm{CC}$ & $4.975-C 2$ & 17 \\
\hline 15000. & & & 2. $3 \mathrm{CO}+\mathrm{OC}$ & $3.1 \geq 7-02$ & \\
\hline 20000 & & 8.340 & 1. $845+C C$ & $2.287-02$ & - $\mathrm{C} 6 \mathrm{~S}+$ \\
\hline & - & $3.707-04$ & $1.342+0 \mathrm{C}$ & $1.4 \varepsilon 2-02$ & $1.297+c$ \\
\hline & $1.562+01$ & $2.086-04$ & 1. $c \in 5+c c$ & $1 . c 55-02$ & $1.454+c$ \\
\hline & & $1.335-04$ & $8.883-c 1$ & $\varepsilon .687-03$ & $1 \cdot 563+c$ \\
\hline & & $9.271-05$ & $7.645-01$ & $7.1 \subseteq 7-c 3$ & $1.645+c$ \\
\hline 000 & $1.827+01$ & $5.215-05$ & t. $C 26-c 1$ & $5.359-03$ & 1. $7 \in 7+c$ \\
\hline 20000 . & $1.515+01$ & $3.338-05$ & 5. $\mathrm{CCC}-\mathrm{Cl}$ & $4.26 \varepsilon-03$ & $1 . \varepsilon \in 5+C$ \\
\hline
\end{tabular}

\begin{tabular}{|c|c|c|c|c|c|}
\hline $\begin{array}{r}\text { ENERGY } \\
(K E V)\end{array}$ & TCTAL & $\begin{array}{l}\text { COHER . } \\
\text { SCATT. }\end{array}$ & $\begin{array}{l}\text { INCOH } \\
\text { SCATT. }\end{array}$ & $\begin{array}{r}\text { PHCTE } \\
\text { EFFECT }\end{array}$ & $\begin{array}{l}\text { PAI } \\
\text { PRCL. }\end{array}$ \\
\hline 50 . & $4.414+03$ & $1.046+02$ & $2.653+01$ & $4.2 \varepsilon z+03$ & \\
\hline 60. & $2.752+03$ & $7.665+01$ & $2.7 C c+C_{1}$ & $2 \cdot 64 \varepsilon+03$ & \\
\hline 80. & $1.294+03$ & $4.604+01$ & $2.706+C 1$ & $1.2<1+03$ & \\
\hline 100. & $7.213+02$ & $3.063+01$ & $2 . \in 96+C 1$ & $\epsilon \cdot \in 3 \varepsilon+02$ & \\
\hline 150. & $2.554+C 2$ & $1.433+01$ & $2.55 c+c 1$ & $2.155+02$ & \\
\hline 200. & $1.2 \varepsilon \varepsilon+02$ & $8.264+00$ & $2.35 c+C_{1}$ & S.t5 $23+01$ & \\
\hline 300. & $5.671+01$ & $3.76 t+00$ & 2. $133+C 1$ & $3.1 \in 1+C_{1}$ & \\
\hline 400 . & $3 . t 17+C 1$ & $2.145+00$ & $1 . \varsigma 30+C 1$ & $1.473+01$ & \\
\hline $5 \mathrm{Co}$. & $2.746+C 1$ & $1.383+0 \mathrm{C}$ & $1.771+\mathrm{C} 1$ & $\varepsilon .3 \in \varepsilon+C C$ & \\
\hline 600 . & 2.27 & $5.652-01$ & $1.644+01$ & $5.2 \subseteq 1+0 \mathrm{C}$ & \\
\hline 800 . & & 5.4 & 1.44 & $\varepsilon(4+0)$ & \\
\hline 1000 & $1.514+01$ & $3.509-01$ & $1.3 C_{4}+01$ & $1.748+O C$ & \\
\hline 1500. & $1.183+01$ & $1.567-\mathrm{Cl}$ & 1. $C 62+01$ & 7. $9 \varepsilon \subseteq-C 1$ & $2.574-C 1$ \\
\hline 2000 . & $1.051+01$ & $8.838-02$ & S. $C \in 5+O C$ & $4.843-C 1$ & $8.6 \subseteq 3-c 1$ \\
\hline 3000 . & $9.530+0 \mathrm{C}$ & $3.938-02$ & $7.132+c c$ & $2.555-01$ & 103 \\
\hline 4000 . & $5.370+00$ & 2.218 & 5. $.48+0 C$ & $1.6 \subseteq 4-01$ & 230 \\
\hline 5000 . & $5.5 C_{4}+0$ & 1.42 & 5.13 & $254-01$ & 225 \\
\hline $600 \mathrm{C}$. & s. & 03 & & $512-02$ & \\
\hline 0 . & $1 . C$ & 5.5 & $+C \mathrm{C}$ & $\epsilon .94 \varepsilon \rightarrow 02$ & $42+c$ \\
\hline 10000 & $1 \cdot(87+01$ & $3.55 t-c 3$ & $3.1 \in 1+0 \mathrm{C}$ & $5.333-c 2$ & $7.653+C$ \\
\hline $50 \mathrm{co}$. & $1.2 \mathrm{C}$ & $1.581-03$ & $2.337+O C$ & $3.3 \in 1-02$ & S. $624+C$ \\
\hline 20000 . & $1.302+C 1$ & $8.897-04$ & $1.875+0 \mathrm{C}$ & $2.45 c-02$ & $1.112+C 1$ \\
\hline 30000 . & $1.48 t+C 1$ & $3.955-04$ & $1.363+0 \mathrm{C}$ & $1.5 \varepsilon 7-c 2$ & 1. $348+C 1$ \\
\hline 40000 . & $1.621+01$ & $2.225-04$ & 1. $C \varepsilon 2+C c$ & $1.173-02$ & $1.511+C$ \\
\hline 50000 . & $1.716+C 1$ & $1.424-04$ & S.C2S-01 & $5.3 C 3-03$ & $1 . t 25+c$ \\
\hline $600 \mathrm{co}$. & $1.788+01$ & $9.891-05$ & $7.774-C_{1}$ & $7.7 c 7-03$ & $1.710+c$ \\
\hline & $.898+01$ & $5.564-05$ & $t .125-C_{1}$ & $5.739-c 3$ & $1.836+0$ \\
\hline & 1. $.9 \varepsilon t+C 1$ & $3.561-05$ & $5 . C \varepsilon z-C 1$ & $4.571-c 3$ & 1. $534+$ \\
\hline
\end{tabular}

\begin{tabular}{|c|c|c|c|c|c|}
\hline \multicolumn{6}{|l|}{ EAERGY } \\
\hline 50. & $4.656+03$ & $1.080+02$ & $2.685+01$ & $4.521+C 3$ & \\
\hline 60. & $2.9 C 5+03$ & $7.904+01$ & $2.735+01$ & $2.755+c 3$ & \\
\hline 80. & $7+C^{2}$ & $4.740+01$ & $2.743+C 1$ & $1.252+03$ & \\
\hline 100. & $18+02$ & $3.151+01$ & $2.735+01$ & 7. $C 2 \mathrm{~S}+02$ & \\
\hline 150. & $7+02$ & $1.472+01$ & $\subseteq \varsigma_{\varepsilon}+C_{1}$ & $2.25 c+02$ & \\
\hline 200 . & $58+02$ & $8.484+0 \mathrm{C}$ & $2.437+C 1$ & 1. $C Z S+02$ & \\
\hline 300 . & $C+01$ & $3.864+0 \mathrm{C}$ & $1 \in 7+01$ & $.3 \varepsilon 7+01$ & \\
\hline 400. & $63+C 1$ & $2.200+0 \mathrm{C}$ & $1.5 \in 1+01$ & $1.5 \varepsilon 2+01$ & \\
\hline 500 . & $2.842+01$ & $1.418+00$ & 1. $\varepsilon C O+C 1$ & S. $\mathrm{CCC}+00$ & \\
\hline 600. & $2.349+C 1$ & $9.897-01$ & $1 . t 7 c+01$ & $5.7 \subseteq 9+0 c$ & \\
\hline 800 . & $1.829+C 1$ & $5.600-01$ & $1.472+C 1$ & 3. $C 17+00$ & \\
\hline 1000 . & $245+C 1$ & $3.597-01$ & $1.325+C 1$ & $1.8 \& 1+0 C$ & \\
\hline 00. & 1.2 & 1.60 & $C 79+C 1$ & $.5 \leq 1-01$ & $2.672-01$ \\
\hline 2000 . & 1.07 & 9.05 & $+C \mathrm{C}$ & $5.2 C 4-01$ & 148 \\
\hline 3000 . & $9.746+C C$ & $4.035-02$ & $7.247+C C$ & $2.747-01$ & 184 \\
\hline 4000 . & $9.576+C C$ & $2.273-02$ & t. $C 43+c c$ & $1.81 \varepsilon-01$ & $328+c$ \\
\hline 5000 . & $9.7 C 7+00$ & $1.456-02$ & $5.21 \varepsilon+0 C$ & $1.245-01$ & $340+c$ \\
\hline 6000 . & $9 . \subseteq \in 1+00$ & $1.011-02$ & $4 \cdot t 12+0 C$ & 1. $c \in 2-01$ & $5.232+c c$ \\
\hline & $7+01$ & $5.692-03$ & $3.772+O C$ & $7.44 t-02$ & $715+c c$ \\
\hline 1000 & $1.115+01$ & $3.644-03$ & $3.212+c c$ & $5.713-02$ & 7. $882+\mathrm{CC}$ \\
\hline 15 & $1.238+01$ & 1.62 & $\overline{6} . \equiv 75+0 \mathrm{C}$ & $3.5 \subseteq 5-c 2$ & $9.963+\mathrm{CC}$ \\
\hline & $1.241+C 1$ & $5.117-c 4$ & $1.5 C 5+0 C$ & $2.623-02$ & $1.148+01$ \\
\hline 30000. & $1.519+C 1$ & $4.053-04$ & $1.285+0 \mathrm{C}$ & $1.655-c 2$ & $1.379+\mathrm{Cl}$ \\
\hline 40000 . & $1.654+01$ & $2.280-04$ & $1.1 \mathrm{CC}+\mathrm{CC}$ & $1.255-02$ & $1.542+C 1$ \\
\hline 50000 . & $1.753+01$ & $1.459-04$ & $5.175-01$ & $5.555-03$ & $1.661+C 1$ \\
\hline & $1.831+C_{1}$ & $1.013-04$ & $7.9 \mathrm{CO}-\mathrm{Cl}$ & E.247-C3 & $1.751+C 1$ \\
\hline & $1.946+01$ & $5.701-05$ & $6.224-01$ & $t .14 C-03$ & $1.883+C 1$ \\
\hline & & .05 & $4-$ & $4 . \varepsilon \subseteq C-C 3$ & 1. $582+C 1$ \\
\hline
\end{tabular}




\begin{tabular}{|c|c|c|c|c|}
\hline $\begin{array}{r}\text { ENERGY } \\
\text { (KEV) }\end{array}$ & TOTAL & $\begin{array}{l}\text { COHER. } \\
\text { SCATT. }\end{array}$ & $\begin{array}{l}\text { I NCOH. } \\
\text { SCAT T. }\end{array}$ & $\begin{array}{r}\text { PHOTC } \\
\text { EFFECT }\end{array}$ \\
\hline .010 & $4.435+07$ & $2.725 * C_{3}$ & 2. $C 78-C 4$ & 4.435 .07 \\
\hline & $2.555 * 07$ & $2.725+c 3$ & $4.6 \in 7-04$ & \\
\hline & 20607 & 3 & & \\
\hline .030 & $42 C+C 7$ & 2.7244 & & \\
\hline - $C 40$ & $10+07$ & $2.724+C 3$ & 3 & 207 \\
\hline . C 50 & $80+06$ & $2.724+C 3$ & 5 & \\
\hline .060 & $01 \div 06$ & $2.724 * C_{3}$ & 7. & $7.398 \div 06$ \\
\hline .080 & $51 * 0 t$ & $2.723+\mathrm{C} 3$ & $1.258-C_{2}$ & $5.548 * 06$ \\
\hline .100 & $41+0 t$ & $2.723 \div 03$ & $3-02$ & $4.439+06$ \\
\hline $.15 \mathrm{C}$ & & $2.720+C_{3}$ & $-c_{2}$ & $\$ 07$ \\
\hline & & & 7.7 & 7.63 \\
\hline .300 & $4.4 \varepsilon$ & & & 4.4 \\
\hline .400 & SO & 2 & & \\
\hline .500 & $2.023+0 t$ & $2 . t$ & & \\
\hline .600 & $1.486+C t$ & $2.662+03$ & $5 . c$ & .48 \\
\hline .800 & $979 * 05$ & $2.621 \div 03$ & 9.6 & 8.953 \\
\hline $1 . \mathrm{COC}$ & $6.0 \mathrm{C} 6+0 \mathrm{E}$ & $2.575 \& C 3$ & $1 \cdot 3$ & $5.981 * 05$ \\
\hline 1.500 & $1.321+06$ & $2.448+c 3$ & 2.370 & $1.319 \diamond 06$ \\
\hline 000 & C. $701+C 5$ & $2.313+03$ & 3.34 & $8.678 \diamond 05$ \\
\hline 3. & $3.255+C E$ & $2 . C_{4} 7+c 3$ & $5.171+\mathrm{CO}$ & $3.274+05$ \\
\hline $4 . \mathrm{CCO}$ & $4 \rightarrow 05$ & $1.2 C 7 \& C_{3}$ & $6 . \varepsilon 35+c 0$ & 1.616 \\
\hline & 4 & $1.556+c$ & 8.32 & 9.270 \\
\hline & + & $1.415+C 3$ & $c .7$ & $5.847+04$ \\
\hline & & & & \\
\hline $\mathrm{C} \cdot \mathrm{COO}$ & $65+$ & $9.2 \mathrm{C} 8+\mathrm{C} 2$ & 1.41 & 6.871 \\
\hline & $21+C 4$ & $6.012+02$ & & $2.359+04$ \\
\hline & & $4.274 \times C 2$ & & $1 . C 80804$ \\
\hline & $3.813+c$ & $2.518+C 2$ & & $3.537+03$ \\
\hline 10.000 & $1.7 \varepsilon 5+C z$ & $1 . t 2 t+C_{2} 2$ & $2.618+01$ & $1.596 \div 03$ \\
\hline
\end{tabular}

\begin{tabular}{|c|c|c|c|c|}
\hline \multicolumn{5}{|c|}{$Z=65$ TER 8 I L } \\
\hline $\begin{array}{r}\text { EAERGY } \\
\text { (KEV) }\end{array}$ & TOTAL & COFER. & I NCOH. & $\begin{array}{l}\text { PHCTC } \\
\text { EFFECT }\end{array}$ \\
\hline . C 10 & $4.539+C 7$ & $2.81 \mathrm{C}+\mathrm{C} 3$ & 2. $\mathrm{C}_{2} \mathrm{O}-\mathrm{C} 4$ & $4.539+07$ \\
\hline - $C 15$ & $3.026+c 7$ & $2.810+c 3$ & $4.537-c 4$ & $3.026+C 7$ \\
\hline$=20$ & $2.270+c 7$ & $810+C 3$ & $8 \cdot c 52-c 4$ & $2.269+07$ \\
\hline .030 & $1.513+C 7$ & & 1. $80 t-C 3$ & $1.513+07$ \\
\hline .040 & $1.135+C 7$ & 2. & & $1.135 \diamond 07$ \\
\hline.$C 50$ & 9. $C \& C \& C t$ & $2.810+C 3$ & 4. $581-C 3$ & $8+06$ \\
\hline . $c \in O$ & $7.5 \epsilon 7+0 t$ & $2.81 C+C 3$ & $7.148-c 3$ & $7.565+06$ \\
\hline .030 & $5.676+c \epsilon$ & $2 \cdot 8 c s+c 3$ & 1. $2 \in 2-\mathrm{C} 2$ & $5.673+06$ \\
\hline .100 & $4.542+06$ & $2.8 C 8 * C 3$ & $58-c 2$ & $4.539+06$ \\
\hline .150 & $1.15 t+C 7$ & $2.806 t$ & $4.328-C 2$ & $1.156+07$ \\
\hline .200 & $\varepsilon .445+C 6$ & 2.8 & $7.557-C 2$ & $8.442+06$ \\
\hline $20 \mathrm{C}$ & $=4 t+C t$ & & $9-01$ & $4.944+06$ \\
\hline .400 & $C t$ & & $-C 1$ & +06 \\
\hline $.5 \mathrm{CC}$ & $2.155+0 t$ & 2.7 & $4 \cdot 2$ & 106 \\
\hline$-€ C O$ & $1.559+0 t$ & $2.74 s+C 3$ & 5.8 & 1.59 \\
\hline $.80 \mathrm{C}$ & $5.4 \subseteq 5+C 5$ & $2.7 C 5+C 3$ & $9.4 \epsilon 2-01$ & 9.47 \\
\hline. $\mathrm{COO}$ & $6.245+05$ & $2 . \in \in 4+03$ & i. $342+c c$ & $6.223 * 05$ \\
\hline 1.500 & $1.405 \times C t$ & $2.54 C+C 3$ & $2.336+c C$ & $1.4 C 3+06$ \\
\hline $2 \cdot \operatorname{coc}$ & $9.236+c 5$ & $2.4 C \varepsilon+c 3$ & $3.311+C C$ & $9.212+05$ \\
\hline 3.000 & $3.5 C 8+C 5$ & $2.143+03$ & $5.167+C C$ & $3.486+05$ \\
\hline 4.600 & $732+C 5$ & $1.897+C 3$ & $6.851+C C$ & $1.713+05$ \\
\hline E. COC & $5+c 4$ & $1 \cdot t 7 t+C 3$ & $8.368+c 0$ & $9.806+04$ \\
\hline & $341+04$ & & $+C C$ & $6.151+04$ \\
\hline $8 . C 00$ & $8.225+c 4$ & $1.185+C$ & 1.21 & 8.10 \\
\hline $1 \mathrm{C} \cdot \mathrm{COO}$ & $7.336+C_{4}$ & $\$ .640+02$ & 1.41 & $7.238 * 04$ \\
\hline & $2.577+C 4$ & $6.257+\mathrm{C} 2$ & $1.818+01$ & $2.512 \checkmark 04$ \\
\hline & $1.200+04$ & $4.455 * C 2$ & $2.103+C 1$ & $1.153 \$ 04$ \\
\hline & $4.070 * \mathrm{C} 3$ & $2.628+C 2$ & $2.454+C 1$ & $3.782+03$ \\
\hline & $4+03$ & $8+02$ & $C+C 1$ & $1.708 \div 03$ \\
\hline
\end{tabular}

\begin{tabular}{|c|c|c|c|c|}
\hline $\begin{array}{r}\text { ENERGY } \\
\text { (KEV) }\end{array}$ & TOTAL & $\begin{array}{l}\text { COFER. } \\
\text { SCATT. }\end{array}$ & $\begin{array}{l}\text { I NC OH. } \\
\text { SCATT. }\end{array}$ & $\begin{array}{r}\text { PHCTC } \\
\text { EFFECT }\end{array}$ \\
\hline . $C 10$ & $4.722+C 7$ & $2 . \varepsilon \varsigma \varepsilon+C 3$ & 1. $\subsetneq \subseteq 1-c 4$ & $4.721+07$ \\
\hline - $C 15$ & $3.14 \varepsilon \star 07$ & $2.858+C 3$ & $4.473-04$ & $3.148+07$ \\
\hline .020 & $2.361+07$ & $2.898+c 3$ & $7 . \subseteq 38-c 4$ & $2.361 \div 07$ \\
\hline - $c \geqslant 0$ & $574 * 67$ & 2. $\varepsilon \subseteq 7+C 3$ & $1.780-C 3$ & 1.574007 \\
\hline .040 & $1.181+07$ & 2. \& $57+C 3$ & $3.154-03$ & $1.180 \div 07$ \\
\hline $.65 C$ & $5.446+0 t$ & 2. ES $7+C 3$ & $4.510-c_{3}$ & 9.443806 \\
\hline . $C 60$ & $7.872 * 06$ & $2.8 \subseteq 7+03$ & $7.047-03$ & 7.869406 \\
\hline.$C 80$ & E. $S C 5+C 6$ & $2.896+C 3$ & $1.244-C 2$ & $5.902+06$ \\
\hline .100 & $4.724 * \mathrm{CE}$ & $2.896+C 3$ & $1.930-02$ & $4.721+C 6$ \\
\hline .150 & $3.15 C+C 6$ & $2.8 \mathrm{~S} 3+\mathrm{C} 3$ & $4.266-c 2$ & $3.148+06$ \\
\hline .200 & $9.303 * \mathrm{Ct}$ & 2.850 & $=-C 2$ & 9.300806 \\
\hline .300 & $5.41 C+C t$ & $2.881+C 3$ & $1 \cdot t 1$ & $7 \& 06$ \\
\hline $.4 \mathrm{CO}$ & $3.4 t 3+C t$ & $2.869 * C ?$ & $2.762-C_{1}$ & $3.460+06$ \\
\hline .500 & $2.383+06$ & $2.854+C 3$ & $4.147-01$ & $2.380+06$ \\
\hline $.60 \mathrm{C}$ & $1.7 \equiv 0+c \epsilon$ & $2.83 T+C 3$ & $5.728-\mathrm{Cl}$ & $1.727+06$ \\
\hline .800 & $1.021 \leqslant C E$ & $2.757+c 3$ & $9.317-01$ & $1.018 \star 06$ \\
\hline $1 . C 00$ & $t \cdot t \in 7 \otimes C 5$ & $2.751+C 3$ & $1 \cdot 321+C C$ & 6.639405 \\
\hline 1.500 & $1.498 \div \mathrm{CE}$ & $2.627+03$ & $6+C C$ & $1.495 * 06$ \\
\hline & & 2.4 & 3. & G \\
\hline 3. $\mathrm{COO}$ & $3.738+C 5$ & $2.224+C 3$ & $5.073+00$ & 3.716405 \\
\hline 4.000 & $1.841 \triangleleft C 5$ & $1.571+C 3$ & $6.743+c 0$ & $1.821+05$ \\
\hline $5.00 \mathrm{C}$ & $1.057 * 05$ & $1.744 * \mathrm{C} 3$ & $8.265 * \mathrm{co}$ & 1.040405 \\
\hline $6 . c 00$ & $6.714 \times C 4$ & $1.548 * C 3$ & $9.654+C C$ & $6.558+04$ \\
\hline 8.000 & $8.882+04$ & $1.235 * 03$ & $1.210+01$ & $8.758 * 04$ \\
\hline $10 \cdot C 00$ & $7.7 \varepsilon 3 \cdot c 4$ & 1. $005+03$ & $1.418+\mathrm{Cl}$ & $7.681 * 04$ \\
\hline $15.00 \mathrm{C}$ & $2.726 * C_{4}$ & $6.510 \leqslant C 2$ & & $2.659+04$ \\
\hline & $273+04$ & $4.632 * C_{2}$ & $2.112+\mathrm{Cl}$ & $.224 \div 04$ \\
\hline 30.000 & $4.3 \geq 5+C 3$ & $2.737+C 2$ & $2.475+C 1$ & $4.040+03$ \\
\hline $4 \mathrm{C} .000$ & $2, C \equiv 3+C 3$ & $1.770 * C_{2}$ & $2.678>01$ & $1.829 \div 03$ \\
\hline
\end{tabular}

\begin{tabular}{|c|c|c|}
\hline $\begin{array}{l}\text { ENERGY } \\
\text { (KEV ) }\end{array}$ & TCTAL & $\begin{array}{l}\text { COHER } \\
\text { SCATT }\end{array}$ \\
\hline 50. & $1.0 C 0 \$ 03$ & $1.128 * 02$ \\
\hline 60 。 & $3 . C_{71} \& 3$ & ع.258^01 \\
\hline 80. & $1.445 \otimes 03$ & 4.957601 \\
\hline 100 。 & $8, C 58+02$ & $3.297 * 01$ \\
\hline 150. & $2.841+C 2$ & $1.541 \vee 01$ \\
\hline 200 。 & $1.425+C 2$ & $8.889+00$ \\
\hline 300 。 & $6.227+01$ & $4.050 \diamond 00$ \\
\hline 400 . & $3.522 * 01$ & $2.306+0 \mathrm{C}$ \\
\hline 500. & 2.545401 & $1.487+00$ \\
\hline 600 。 & $2.424+01$ & $1.038 \leadsto 0 \mathrm{C}$ \\
\hline 800. & 1.879401 & $5.873-01$ \\
\hline 1000. & $1.586 \div 01$ & $3.772-\mathrm{Cl}$ \\
\hline 1500. & 1.233001 & $1 . t 85-01$ \\
\hline 2000 。 & $1.05 t+02$ & $9.500-02$ \\
\hline 3000 . & $5.545 \$ 00$ & $4.233-02$ \\
\hline 4000 . & $5.768 \nleftarrow 00$ & $2.384-02$ \\
\hline 5000 。 & $5.8 \subseteq 7 * 0 C$ & $1.527-02$ \\
\hline 6000 . & $1.015 * 01$ & $1.061-02$ \\
\hline 8000 & $1 . c 78 \otimes 01$ & $5.970-03$ \\
\hline 10000 . & $1.141+01$ & $3.822-03$ \\
\hline 15000. & $2.273+01$ & $1.700-03$ \\
\hline 20000 . & $1.382 \hookleftarrow 01$ & $9.563-04$ \\
\hline 30000 . & $1.5 \epsilon 5 \nLeftarrow 01$ & $4.251-04$ \\
\hline 40000 . & $1.703 \$ 01$ & $2.392-04$ \\
\hline 50000 。 & $1 . \varepsilon \subset 7 \star 01$ & $1.531-04$ \\
\hline 60000 . & $1 . \varepsilon 87+C 1$ & $1.063-04$ \\
\hline 80000 . & $2.0 C 7+C 1$ & $5.980-05$ \\
\hline & & \\
\hline
\end{tabular}

100000 .

\begin{tabular}{|c|c|c|}
\hline ENERGY & TCTAL & COHER . \\
\hline (KEV) & & \\
\hline 50. & $1.067+03$ & $1.179+02$ \\
\hline $6 c$. & $3.235 * 03$ & $8.634+01$ \\
\hline 80 . & $1.53 \in \forall C 3$ & $5.186+01$ \\
\hline 100. & $8.557+C \bar{c}$ & $3.450+01$ \\
\hline 150. & $3 . C 124 C 2$ & $1.614+01$ \\
\hline 200 . & 1.508402 & $9.309+00$ \\
\hline 300 . & $6.512+01$ & $4.242 \$ 00$ \\
\hline 400. & $4.073 \times 01$ & $2.416+00$ \\
\hline 500 . & $3 . C 45+01$ & $1.558+O C$ \\
\hline 600 . & $2.458+C 1$ & $1.087+0 C$ \\
\hline 800 & $1.527+C 1$ & $6.153-01$ \\
\hline 1000 . & $1 \circ t 24+01$ & $3.952-01$ \\
\hline 1500 . & $1.258 \div 01$ & $1.765-01$ \\
\hline 2000 . & $1.121 * C 1$ & $9.954-02$ \\
\hline 3000 . & $1.017+01$ & $4.435-c 2$ \\
\hline 4000 . & $9.57 t+00$ & $2.498-c 2$ \\
\hline 5000 . & $1.00 \subseteq * 01$ & $1.600-02$ \\
\hline 6000 . & $1 . C 35 * C 1$ & $1.111-02$ \\
\hline 8000 & 1.100201 & $6.256-03$ \\
\hline 10000 . & $1.1 \in 7 \vee 01$ & $4.005-03$ \\
\hline 15000 . & $1.312 * 01$ & $1.781-03$ \\
\hline 20000 . & $1.427+01$ & $1.002-03$ \\
\hline 30000 . & $1.611 * 01$ & $4.455-04$ \\
\hline 40000 . & $1.754+C 1$ & $2.506-04$ \\
\hline 50000 . & $1.865 * C 1$ & $1.604-04$ \\
\hline 60000 . & $1.551+01$ & $1.114-04$ \\
\hline 80000 & $2 \cdot C 73+c 1$ & $0.266-05$ \\
\hline & $2.153+01$ & $4.011-05$ \\
\hline
\end{tabular}

100000 . 2.153+01 4.011-0

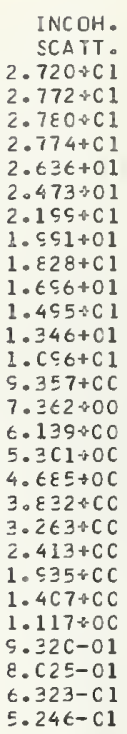

E. $24 t-C 1$

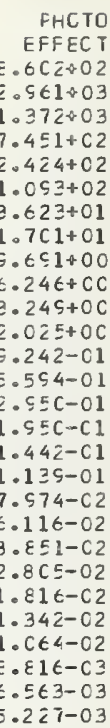

2.782-C $9.515-C 1$ $3.410+C C$ $4 \cdot 436+C$ $5.344+0 C$ $6.865+C C$
$\varepsilon . C 78+0 C$ 1. $C 27+C 1$ $1.186+01$ $1.422+C 1$ $1.550+C 1$ $1.713+C 1$ 1. $8 \mathrm{C} 6+\mathrm{C} 1$ $1 . c 43+C 1$
$2 . C 42+C 1$

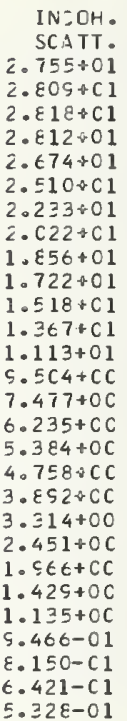

FHCTO EFFEC T C. $22 \mathrm{ZC}+02$ $3.121+c 3$ $1.45 \epsilon+03$ $7.531+02$ $2.5 \varepsilon 4+C 2$ $1.164+02$ 3. $854+C 1$ 1. $\varepsilon C \subseteq+C 1$ 1. $C 33 * 01$ $t .6 t 5+00$ $3.47 t+0 C$ $2.1 \epsilon \varepsilon+C C$ S. $\varepsilon \subseteq \subseteq-01$ ᄃ. $\varepsilon \varepsilon-01$ $3.155-01$ 2.ces $5-01$ $1.54 \bar{z}-01$ $1.217-01$ $\varepsilon .52 \mathrm{C}-\mathrm{C} 2$ $6.534-C 2$ $4.113-02$ Z. $5 \subseteq t-02$ 1. $535,-02$ $1.4 \equiv 3-02$ $1.136-02$ $5.413-03$ 7.CC 7-03

2. $873-01$

1. $C C_{4}+C C$

$2.338+C C$ $3.5 \mathrm{C} 7+\mathrm{CC}$ $4.53 \mathrm{~S}+\mathrm{CC}$ $5.456+C C$
$7 . C 16+O C$ $\varepsilon .288+O C$ 1. $C 62+C 1$

$1.227+01$ $1.466+C 1$ $1.639+C 1$ $1.769+C 1$ $1.8 t 8+C 1$
2. CSS+C 1

\begin{tabular}{|c|c|c|c|c|c|}
\hline $\begin{array}{r}\text { ENERGY } \\
\text { (KEV) }\end{array}$ & TCTAL & $\begin{array}{l}\text { COHER, } \\
\text { SCATT. }\end{array}$ & $\begin{array}{l}\text { INCOH. } \\
\text { SCATT. }\end{array}$ & $\begin{array}{l}\text { PHCTO } \\
\text { EFEC T }\end{array}$ & $\begin{array}{l}\text { PAIF } \\
\text { PRCL. }\end{array}$ \\
\hline 50 & $1.1 \exists \epsilon+C 3$ & $1.230+C 2$ & 2.\rceil \varepsilon 7+C$. & ร. $\varepsilon 4 \subseteq+C 2$ & \\
\hline 60. & $3.413+c 3$ & $9.014+01$ & $2.842+C 1$ & $3.2 \subseteq 4+03$ & \\
\hline 80. & $1.622+03$ & $5.417+01$ & $2.853+c 1$ & $1.53 s+03$ & \\
\hline 100. & 9. $C 33+C 2$ & $3.60 t+01$ & 2. $E \subseteq C+C 1$ & $\varepsilon .3 \varepsilon 7+c 2$ & \\
\hline 150. & $3.17 t+02$ & $1.687+01$ & $2.7111+C 1$ & $2.736 * 02$ & \\
\hline 200 & $1.58 \epsilon \nLeftarrow C 2$ & $9.7 .35+00$ & $2.545+01$ & $1.234+02$ & \\
\hline 300 . & $6.804+01$ & $4.437+00$ & $2.265+01$ & $4.0 \leqq 5+01$ & \\
\hline 400 . & $4.2 \geqslant 0+01$ & $2.528+0 \mathrm{C}$ & 2. $C E 1+01$ & 1. ระz $t+01$ & \\
\hline 500 . & $3.148+C 1$ & $1.630+00$ & 1. $\varepsilon \varepsilon 4+C 1$ & $1.1 \mathrm{Cl}+01$ & \\
\hline 600 . & $2.574+C 1$ & $1.137+O C$ & $1.748+C 1$ & $7.12 \mathrm{C}+0 \mathrm{C}$ & \\
\hline 800 . & $1.577 \diamond 01$ & $6.438-01$ & $1.541+C 1$ & $3.715+00$ & \\
\hline 1000 & $1.6 \in 1 * 01$ & $4.1 \geq 6-01$ & $1.388+01$ & $2 . \overline{31} \varepsilon+O C$ & \\
\hline 1500. & $1.285+01$ & $1.847-01$ & $1.230+C 1$ & 1. $C 57+C C$ & $3.020-\mathrm{C} 1$ \\
\hline 2000 & $1.141+01$ & $1.042-01$ & $5.64 S+O C$ & $6.396-01$ & 1. C1 $8+O C$ \\
\hline $300 \mathrm{C}$. & $1 . c 3 s+01$ & $4.641-02$ & $7.552+C C$ & $3.3 \in \varepsilon-01$ & $2.415+C C$ \\
\hline 4000 & $1.024+01$ & $2.614-02$ & $6 \cdot 331+C C$ & $2.225-01$ & $3.665+O C$ \\
\hline 5000 . & $1 . c 41 \leqslant 01$ & $1.674-02$ & $5.4 E 7+0 C$ & $1.645-01$ & $4.764+C C$ \\
\hline 6000 . & $1.07 C \nLeftarrow 01$ & $1.163-02$ & $4 \cdot \varepsilon \geqq 2+C 0$ & $1.2 \subseteq \varepsilon-01$ & $5.727+C C$ \\
\hline 8000 & $1.13 \in 01$ & $6.547-03$ & $3.5 \leq 2 \otimes 0 C$ & ᄃ.СЕ 7-02 & $7 \cdot 313+c c$ \\
\hline 10000 . & $1.1 \varsigma 5+01$ & $4.192-$ & 3.364. & $6.5 \in 8-02$ & $8.552+c c$ \\
\hline 15000 . & $1 \cdot 330+01$ & $1.864-03$ & $2.48 \varepsilon+C C$ & $4 \cdot 3 \varepsilon t-c 2$ & 1. $C 76+C l$ \\
\hline 20000 & $1.444+01$ & $1.049-03$ & $1.55 t C C$ & $3.1 \subseteq 5-02$ & $1.241+C 1$ \\
\hline 30000 . & $1.644+01$ & $4.662-04$ & $1.451 * 0 C$ & $2 \cdot c \in \varepsilon-02$ & $1.4 \leqq 7+C 1$ \\
\hline & $1.753+01$ & $2.623-04$ & $1.152 \triangleleft C C$ & $1.528-02$ & $1.676+C 1$ \\
\hline 50000 。 & $1.5 C 2 \otimes 01$ & $1.679-04$ & $5.611-01$ & $1.212-02$ & $1.8 C 501$ \\
\hline 60000 . & $1.585 \div 01$ & $1.166-04$ & $\varepsilon .27 t-c 1$ & $1 . C C 4-C 2$ & $1.502+C 1$ \\
\hline & $2.110+01$ & $6.558-05$ & $6.52 C-C 1$ & $7.471-03$ & $2 \cdot C 44 * C 1$ \\
\hline 00000 。 & $2.206+01$ & $4.197-05$ & $5.410-01$ & $5.5 \leq C-03$ & $2.152+C 1$ \\
\hline
\end{tabular}




\begin{tabular}{|c|c|c|c|c|}
\hline \multicolumn{5}{|c|}{$Z=67$ rOLMIUM } \\
\hline EAERGY & TOTAL & COHER. & I NC OH. & PHCTC \\
\hline (KEV) & & SCATY. & SCATT. & EFFECT \\
\hline .010 & $4.848+C 7$ & $2.586+C 3$ & $1.974-\mathrm{C}_{4}$ & $4.848+07$ \\
\hline - $C 15$ & $3.232+C 7$ & $2.586+C 3$ & $4.433-04$ & \\
\hline 20 & $424+C 7$ & $86+C 3$ & $967-04$ & $\leftrightarrow 0$ \\
\hline .030 & $1, t 16+0$ & $6+03$ & $.764-\mathrm{C3}$ & $626+0$ \\
\hline .040 & $1.212 \times 07$ & $86+C 3$ & $.125-c 3$ & $1.212+0$ \\
\hline. $\mathrm{C} 50$ & $9 . t 98 \div 06$ & $6 \div 03$ & $\mathrm{E} 67-\mathrm{C} 3$ & $9.695+0$ \\
\hline $.06 \mathrm{C}$ & \&. CE $2 \div C E$ & $\star C 3$ & $6.984-03$ & 8. \\
\hline .080 & $\epsilon, C \in 3+06$ & $5 * 03$ & $1.233-02$ & $.060+0$ \\
\hline 100 & $4.85160 t$ & $.584+C 3$ & . $513-02$ & .848 \\
\hline 150 & $3.235+c t$ & $2 \div 03$ & $4.225-C 2$ & 3 \\
\hline $2 \mathrm{CC}$ & \&. $s \in 5+c$ & & $3-\mathrm{C} 2$ & $8 \cdot 962+0$ \\
\hline $.30 \mathrm{C}$ & & $\mathrm{S}_{4} \mathrm{C3}$ & $1-01$ & $5.456+0$ \\
\hline .400 & & & & \\
\hline .500 & 2.46 & 2. & $-C_{1}$ & 2. \\
\hline .600 & $1.803+c$ & 2.5 & 5.6 & 1.8 \\
\hline - ECC & $1.073+06$ & $2.882+C 3$ & $9.240-01$ & 1.070 \\
\hline 1.000 & $7.062+C 5$ & $2.836 \times C 3$ & $1.310 \times C C$ & $7.034 \div 0$ \\
\hline 1.500 & $1 . \in 03 * C 6$ & $2.7 C 7+C 3$ & $2.280+0 C$ & $1.6 \mathrm{Cl}+0$ \\
\hline 2.000 & $9.541+05$ & $7+03$ & $3.232+00$ & $9.915+0$ \\
\hline $3 . \mathrm{COC}$ & & $2.28 E+03$ & $5 . C 52+C C$ & $3.940+0$ \\
\hline & 1. & $0+03$ & $6.725+c c$ & $1.936+0$ \\
\hline & 1.12 & 1.79 & 8.25 & $1.105+0$ \\
\hline & $7.117+0$ & $1.5 \varsigma \varsigma+C 3$ & 9.65 & $6.956+0$ \\
\hline $8 . \mathrm{COC}$ & $3.458+c$ & $1.2 \mathrm{EO}+\mathrm{C}_{3}$ & $1.212+\mathrm{C} 1$ & $3.329+C$ \\
\hline & $8.167 \diamond 04$ & 1.04 & 1.42 & $8.061 * 0$ \\
\hline & $2.501+C$ & $6.733+c 2$ & $1.832+\mathrm{Cl}$ & $2.832+0$ \\
\hline & $1.355+c 4$ & $4.802+C 2$ & $2.127+\mathrm{Cl}_{1}$ & $1.3 \mathrm{C} 5+c$ \\
\hline & $4.598 \div C 3$ & $2.843+C 2$ & $2.500+C 1$ & $4 \cdot 289+0$ \\
\hline & $2.14 c+C ?$ & $1 . E 41+C 2$ & $2.709+01$ & $1.938+0$ \\
\hline
\end{tabular}

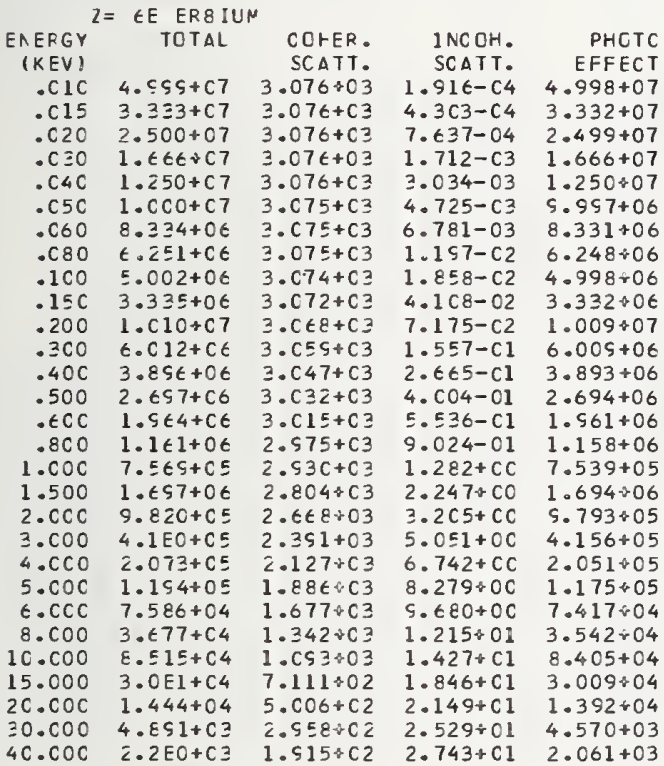

\begin{tabular}{|c|c|c|c|c|}
\hline \multicolumn{5}{|c|}{$8=$} \\
\hline $\begin{array}{r}\text { EAERGY } \\
\text { (KEV) }\end{array}$ & TOTAL & $\begin{array}{l}\text { COFER. } \\
\text { SCATT. }\end{array}$ & $\begin{array}{l}\text { I NCOH. } \\
\text { SCATT. }\end{array}$ & $\begin{array}{r}\text { PHCTC } \\
\text { EFFECT }\end{array}$ \\
\hline .010 & $5.10 \epsilon * C 7$ & $3.1 \in 7 * \mathrm{C} 3$ & $1.53 t-c 4$ & $5.106+07$ \\
\hline 15 & $3.404 \div C 7$ & $3.167+03$ & $4.350-c_{4}$ & $3.404+07$ \\
\hline 20 & $Z+C 7$ & $3.1 \in 7+03$ & $.719-c 4$ & $2.553+07$ \\
\hline $.0 \geq 0$ & $2+c 7$ & $3.167 \div 03$ & $1.731-03$ & $1.702 \nleftarrow 07$ \\
\hline .040 & $77+C 7$ & $3.167+\mathrm{C}_{3}$ & - $C \in 7-C_{3}$ & $1.27 t+07$ \\
\hline .050 & $21+07$ & $3 \cdot 1 \in 7+\mathrm{C}_{3}$ & $4.775-C_{3}$ & $1.021+07$ \\
\hline - $C \in C$ & $\varepsilon$. & $3.1 \in \epsilon * C_{3}$ & $3-c 3$ & +06 \\
\hline .080 & $t .385+0 t$ & $3.1 \in 6+c 3$ & $1.210-C_{2}$ & $6.382+06$ \\
\hline .100 & $E .10 S+C E$ & $165+C 3$ & 1.E $77-C_{2}$ & $5.106+06$ \\
\hline .150 & $3.407+C E$ & $3.163+c 3$ & $4.150-02$ & $3.404+06$ \\
\hline.$\angle O C$ & $1 \overline{2}+\mathrm{C} 7$ & $3.159+\mathrm{C} 3$ & $7.247-02$ & $1.112+07$ \\
\hline$-3 \mathrm{CO}$ & $8+06$ & $3.15 \mathrm{C} \approx \mathrm{C} 3$ & $1.572-\mathrm{Cl}$ & $6.365+06$ \\
\hline $.4 \mathrm{CO}$ & $4.108+C t$ & $3.1 \geq E+C 3$ & $2.68 \varsigma-C 1$ & $4.105+06$ \\
\hline $.5 \mathrm{CO}$ & $2 \cdot 8$ & $-\mathrm{C} 3$ & $-\mathrm{Cl}$ & .06 \\
\hline .600 & $2.076+0 t$ & $2.10 t+C 3$ & $5.579-01$ & $2.073 \$ 06$ \\
\hline $.8 C \mathrm{C}$ & $1.231+0 t$ & $3.0 t \epsilon+C 3$ & $9 . C 82-C 1$ & $1.228+06$ \\
\hline $1 . \mathrm{COO}$ & $8 \cdot C 54+C 5$ & $3.020: 03$ & $1.289+C C$ & $8.023+05$ \\
\hline 1.500 & $1.15 \varsigma+C 6$ & $2.894 * C 3$ & $2.247+C C$ & 1.196 .05 \\
\hline $2 . \mathrm{COC}$ & $1.041+0 t$ & $2.757+C_{3}$ & $3.191+C C$ & $1.039+06$ \\
\hline $\mathrm{COO}$ & $5+05$ & $2.476+c 3$ & 5.00 & 4.35 \\
\hline 4. & 2. & 2.2078 & $6.683+00$ & 3805 \\
\hline $5 . \mathrm{CCO}$ & $1.2 t t+C E$ & $1.5 \in C+03$ & $8.224+C C$ & $1.247+05$ \\
\hline $6 . \mathrm{CCO}$ & $8 . C 48 * 04$ & $1.745+C 3$ & $5.640+00$ & $7.873+04$ \\
\hline 8.000 & $3.8 \varsigma 7+04$ & $1.398 * C 3$ & $1.215+C 1$ & $3.756+04$ \\
\hline $1 \mathrm{C} .000$ & $51+c 4$ & $1.139+03$ & $1.430+01$ & $7.836 \bullet 04$ \\
\hline $15 . \mathrm{COC}$ & $.234+C 4$ & $7.413 \div C 2$ & $1.848+C 1$ & $3.158+04$ \\
\hline $20 \cdot \operatorname{coc}$ & $5 \leq 2+c 4$ & $5.2 C 3+C 2$ & $2.154+01$ & $1.468+04$ \\
\hline & +0 & $C 72+C_{2}$ & $2.546+C 1$ & $4.849+03$ \\
\hline $4 \mathrm{C} .000$ & $17+C 3$ & $1.58 S+C 2$ & $2.7 \epsilon 8+C 1$ & 2.190403 \\
\hline
\end{tabular}

\begin{tabular}{|c|c|c|c|c|c|}
\hline $\begin{array}{l}\text { ENERGY } \\
\text { (KEV) }\end{array}$ & TCTAL & $\begin{array}{l}\text { COHER. } \\
\text { SCATT. }\end{array}$ & $\begin{array}{l}\text { INC OH. } \\
\text { SCATT. }\end{array}$ & $\begin{array}{r}\text { FHCTO } \\
\text { EFFECT }\end{array}$ & $\begin{array}{l}\text { PAIF } \\
\text { PRCD. }\end{array}$ \\
\hline 50 & $1.203+03$ & $1.280+02$ & 2. $E 21 * C I$ & $1.047+03$ & \\
\hline 60. & $3.571+03$ & $9.385+01$ & 2. $E 78+C 1$ & $3.44 E+03$ & \\
\hline 80 & & $5.644 \div 01$ & $2 . E S 2+C 1$ & $1.64 t+C 3$ & \\
\hline 100 & 9. & $3.758+C 1$ & 2. $285+C 1$ & $c . c 4 c+02$ & \\
\hline 150. & $3.42 \mathrm{C}+0$ & $1.759+01$ & $2.751+C 1$ & $2.5 \in S+02$ & \\
\hline 200 & $1 . \in S 7+02$ & $1.015+01$ & $2.584+C 1$ & $1.33 E+C 2$ & \\
\hline 30 & $172+0$ & $4.629 \div 00$ & $2.3 \mathrm{CC}+\mathrm{C} 1$ & $4.4 C 5+01$ & \\
\hline 400 & $4.410 * 01$ & $2.637+00$ & 2. $C 83+01$ & 2. $C E 4+01$ & \\
\hline 500 & & $1.701+00$ & $1.512 \times C 1$ & $1.17 E+01$ & \\
\hline 600 . & $2.655+0$ & $1.187+O C$ & $1.775+C 1$ & $7 . \in 1 C+O C$ & \\
\hline 800 & $2.025+0$ & -01 & 1.565 & $2.574+O C$ & \\
\hline 1000 & $1.701 \div 0$ & $\mathrm{C} 1$ & $1.4 C$ & $2.482+0 \mathrm{C}$ & \\
\hline 1 & 1. & $3-01$ & $1.147+01$ & $1.135 \div 00$ & $3.101-$ \\
\hline & 1 & $87-01$ & S. TSE\&CC & $\epsilon . \varepsilon 71-C_{1}$ & \\
\hline & 1. $C \in C$ & $4.844-02$ & $7 \cdot 7 \mathrm{C} 7+0 \mathrm{C}$ & $3.615-01$ & $85+0$ \\
\hline 40 & 1.044401 & 2.728 & $6.427+0 \mathrm{C}$ & $2.350-01$ & $3.743+C$ \\
\hline & $\therefore C 59$ & $1.747-02$ & $5.550+C C$ & 1. $T \in E-C 1$ & 4. $E 48+C$ \\
\hline & 1.088 & $1.214-\mathrm{C} 2$ & $4.5 \mathrm{C}$ & $1.3 \subseteq 3-01$ & $22+$ \\
\hline 8000 & $1.157+C_{1}$ & $6.833-03$ & 4. $\mathrm{C} 1280 \mathrm{C}$ & $5.73 \epsilon-C 2$ & \\
\hline 10000 & $1.22 t+01$ & $4.375-03$ & $3.415+C C$ & $7.4 \in C-02$ & $76 ?+$ \\
\hline 15000 . & $1.373+01$ & $1.945-03$ & $2.526+0 C$ & $4 . \epsilon \subseteq C-02$ & \\
\hline & $1.496+C 1$ & $1.095-03$ & $2 . C 2 t+O C$ & $3.414-02$ & $250+0$ \\
\hline & $1.657 \div 01$ & $4.866-c 4$ & 1.473 & $2.2 C 8-02$ & 1. $548+c$ \\
\hline & $1.847+C 1$ & $2.737-04$ & $1.17 \mathrm{C}$ & $1 . t \equiv 1-02$ & $1.729+c$ \\
\hline & 1060001 & $1.752-04$ & $5.757-C 1$ & $1.2 \varsigma 3-02$ & $1.862+c$ \\
\hline & & $10217-04$ & $\varepsilon .4 \mathrm{Cl} 1-01$ & $1 . c 71-02$ & $1.564+C$ \\
\hline & & $6.845-05$ & $6.615-01$ & 7. $S \in E-C 3$ & $2.115+C$ \\
\hline & $2 \cdot 280801$ & $4.381-05$ & $\subseteq .4 \subseteq 2-01$ & $6.345-03$ & $2.225+c$ \\
\hline
\end{tabular}

ENERGY

(KEV$$
\begin{aligned}
& 50 \\
& 60 \\
& 80
\end{aligned}
$$

100.

150.

200 .

300.
400.

500 .

600.
800.

800.
1000 .

1500 .

2000 .

$30 \mathrm{CO}$

4000 .

5000.

6000.

8000 .

15000 .

20000 .

30000.

40000

50000 .

60000

80000

\section{TCTAL \\ 1. $275+0$ \\ 3. $756+C$
$1.757+0$ \\ 1. $C \mathrm{C} 5+03$ \\ $3 \cdot 545+C 2$ \\ $1.767+C 2$ \\ 7. $452+01$ \\ 4. $556+C 1$ \\ 2. $742+C$ \\ 2 . $C 84+01$ \\ 1. $741+01$ \\ 1. $328+01$ \\ $1 \cdot C 84+C$ \\ $1.067+01$ \\ 1. $C 82+01$ \\ 1. $111+01$ \\ 1.254001 \\ $1.412+C 1$ \\ $1.541+01$ \\ $1.744 * 01$ \\ 1. $855 \$ 01$ \\ 2. $\mathrm{C} 1 \mathrm{C}+\mathrm{C}$ \\ COHER. \\ $1.331+C 2$ \\ $9.761+01$ \\ $5.870+01$
$3.908+C 1$ \\ $1.829+01$ \\ $1.05 t+01$ \\ $4.814+00$ \\ $2.742+00$ \\ $1.768+00$ \\ $1.234+00$ \\ 6.986-C 1 \\ 4.488-01 \\ 2. $005-01$ \\ $5.037-02$ \\ $2.837-02$ \\ $1.817-02$ \\ 1. 262-02 \\ 7. $105-03$ \\ $4.549-C 3$ \\ $1.138-03$ \\ $5.060-04$ \\ 2.846-04 \\ 1. $822-04$ \\ $1.2 \epsilon 5-\mathrm{C} 4$}

$2.239+01$

$2.339+01$
7.117-0.5

$4.555-C 5$
INC CH.

ATT.

2. $E 5 G+01$
2. $S 1 E+C 1$

2. $533+61$

2. $\mathrm{G} 3 \mathrm{C}+\mathrm{O} 1$

. $753+C I$

2. $t 24+C 1$

i. $115+C 1$

1. $\$ 42+01$

$1.802+C 1$

$1.43 \mathrm{C} \bullet \mathrm{Cl}$

$1.1 \epsilon E+C$

S. $542+0 \mathrm{C}$

$6.523+C C$

5. $t 32+0 C$

4. $578+0 \mathrm{C}$

4. $\mathrm{C} 72+0 \mathrm{C}$

2. $4 E t \$ O C$

. $5 t 4+C C$

2. $C 56+C \mathrm{C}$

$1.455+0 \mathrm{C}$

1. $18740 \mathrm{C}$

8.527-0

t. $718-\mathrm{Cl}$

5. 573-01
FHCTO

EFFEC T
$1.114+03$

3. $t 2 c+c 3$

1. $7 \mathrm{CS}+03$

S. $3 \epsilon \overline{\mathbf{T}}+\mathrm{C} 2$

$3 . c 83+02$

$4.674+01$

$2.2 C \epsilon+C 1$

1. $2 \epsilon \bar{t}+01$

$\mathrm{E} \cdot 1 \in \bar{Z}+O \mathrm{C}$

2. $6 \epsilon 1+C O$

1. $215+\mathrm{CC}$

7. $347-01$

2. 54s-01

1. 8 E2-01

$1.484-01$

1. $C \geq \overline{7}-01$

4. $5 \$ 3-02$

3.634-02

$2.35 \mathrm{C}-02$

1. $7 \equiv \epsilon-02$

1.1 ,

E. $475-03$

t. $751-C 3$
PAIR

PRCL.

$3.222-\mathrm{C} 1$

1. $113+C C$

$3.859+C$

4. $\$ 78+C C$

5. $\subseteq 66+C C$

7. $632+C$

$1.150 \div C 1$

1. $332+01$

$1 \cdot 552+C 1$

1. $\mathrm{C} 1 \mathrm{C}+\mathrm{C}$

2. $C 15+01$

$2.171+C_{1}$

$2.283+C 1$

\begin{tabular}{|c|c|c|c|c|c|}
\hline $\begin{array}{r}\text { ENERCY } \\
\text { (KEV) }\end{array}$ & TCTAL & COHER . & INCCH. & $\begin{array}{r}\text { PHCTC } \\
\text { FFEECT }\end{array}$ & $\begin{array}{l}\text { PAI F } \\
\text { RCC. }\end{array}$ \\
\hline 50 & $1.348+C 3$ & $1.382+02$ & 2. $E E 7 \div C i$ & $1.1 \mathrm{E} 1 * 03$ & \\
\hline 60. & 3.543803 & $1.013+02$ & $2.547 \times 01$ & $3.812+03$ & \\
\hline 80. & $1.8 S_{1+03}$ & $6.093+01$ & $2.5 \in 4+C 1$ & $1.8 \mathrm{CC}+03$ & \\
\hline 100. & $1.058+03$ & $4.056+C 1$ & $2.565 \div 01$ & $S .8 E Z+02$ & \\
\hline 150. & $3.734+C 2$ & $1.899+01$ & E $27 \div C 1$ & $3.262 \div 02$ & \\
\hline 200 . & $1.858+C_{2}$ & $1.09 t+01$ & $2 \cdot t 556+C 1$ & $1.4 E 3+C 2$ & \\
\hline 300 & $7.834 \div C 1$ & $4.996 \div 00$ & $2.3 t t \diamond C 1$ & $4 . S \in \subseteq * 01$ & \\
\hline 400 . & $4.777+01$ & $2.846+C C$ & $2.143+C 1$ & $2.345 \$ 01$ & \\
\hline 500 . & $3.498+C 1$ & $1.835+\mathrm{CO}$ & $1 . \subseteq \in 8+01$ & $1.34 t+\mathrm{Cl}$ & \\
\hline 600 . & $2.826+01$ & $1.281+00$ & 1. $E 27 * C_{1}$ & $E .7 C 3 \$ 00$ & \\
\hline 800 & 2.13 & 7. & $1 . t 11+C 1$ & $4.547 * 00$ & \\
\hline 1000 . & 1.782401 & $4.658-01$ & 1.451 & 2. $E \geqslant C \& C C$ & \\
\hline 1500. & $1.36 t+01$ & $2.080-01$ & $1.182+01$ & $1.257 \times C C$ & $3.366-\mathrm{Cl}$ \\
\hline 2000 . & $1.214+01$ & $1.173-01$ & 1. $C C s+C 1$ & $7.84 C-C 1$ & $.0 \mathrm{C}$ \\
\hline 3000 . & $1.106+01$ & $5.227-02$ & $7.53780 \mathrm{C}$ & $4.122-01$ & $661+00$ \\
\hline $400 \mathrm{C}$. & 1. $C E S+C 1$ & $2.944-02$ & $\epsilon, \epsilon 15 * 0 \mathrm{C}$ & $2.71 E-01$ & $71+C C$ \\
\hline 5000 . & $1.105+c 1$ & $1.886-02$ & $5.715 * 00$ & 2. $c c t-01$ & $5.115 \div 0 \mathrm{C}$ \\
\hline 6000 . & $1.135+01$ & 1.31 & $5 . \mathrm{C} 51.50 \mathrm{C}$ & $1.5 \mathrm{E} 2-\mathrm{C} 1$ & $6.123+C C$ \\
\hline 800 & 1.207 & 7.37 & $4.131+0 \mathrm{C}$ & $1.1 C 5-01$ & 7. $\mathrm{E} 17+\mathrm{CC}$ \\
\hline 000. & $1.275 \nLeftarrow C 1$ & $4.721-03$ & $3.517+O C$ & $E .4 \in 2-02$ & S. $1 E 6 * C C$ \\
\hline 500 & $1.437+01$ & $2.099-03$ & $2 \cdot t C 1+C C$ & $5.316-02$ & $1.171+01$ \\
\hline 0000 . & $1.5 t \in+C_{1}$ & $1.181-03$ & $2 . C 87 * 0 C$ & 3. $E \in E-C 2$ & $1 \cdot 354+C 1$ \\
\hline 0000. & $1.778+0 !$ & $5.251-04$ & $1.517+0 \mathrm{C}$ & $2.5 \mathrm{C} 1-02$ & $1.624+01$ \\
\hline & $1.94 \mathrm{C} \geqslant 01$ & $2.954-04$ & $1.2 \mathrm{C} 5+0 \mathrm{C}$ & $1.84 \overline{\mathrm{T}}-\mathrm{C} 2$ & $1.818+C 1$ \\
\hline & $2.065+01$ & $1.891-04$ & 1. $\mathrm{CC} 5 \div \mathrm{OC}$ & $1.4 \in 4-02$ & 1. $563+C 1$ \\
\hline & $2 \cdot 263+C 1$ & $1.313-04$ & $8 \cdot t \leq 2-01$ & $1.212-02$ & $2 \cdot C 76+C 1$ \\
\hline & $2.308+01$ & $7.386-05$ & 6. E I $7-C 1$ & S.C1S-03 & $2.239+01$ \\
\hline & $2.40 \subseteq \vee 01$ & $4.727-05$ & $5.655-01$ & 7.1 E2-03 & $2 \cdot 352+C 1$ \\
\hline
\end{tabular}

PAIF 


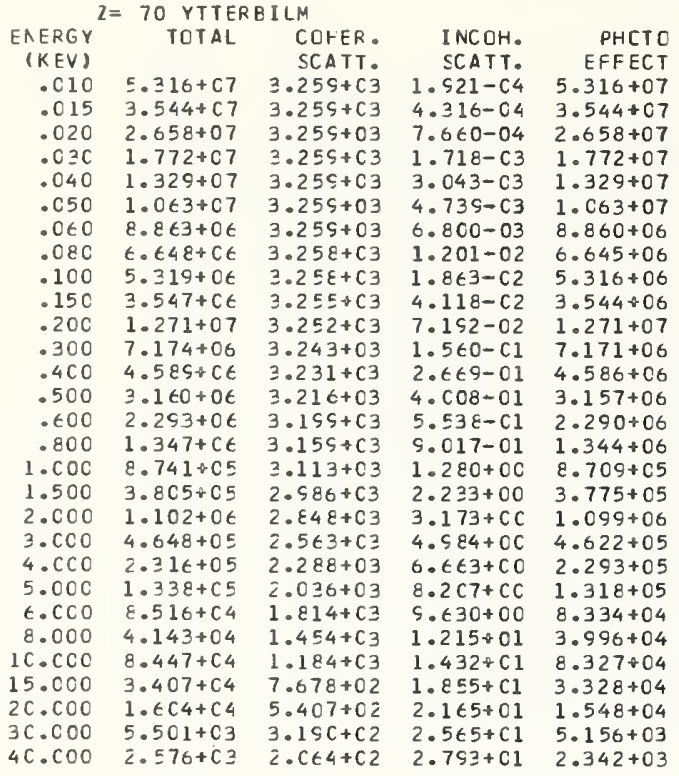

\begin{tabular}{|c|c|c|c|c|}
\hline \\
\hline $\begin{array}{l}E A E R G Y \\
\text { (KEV) }\end{array}$ & TOTAL & CORER. & I $\wedge \mathrm{COH}$. & PHCTC \\
\hline $\begin{array}{l}\text { (KEV) } \\
\text {.C10 }\end{array}$ & $E .4 \in 2+C 7$ & $\begin{array}{r}\text { SCATT } \\
3.352+C 3\end{array}$ & $\begin{array}{r}\text { SCATT. } \\
\text { C8 } 3-C 4\end{array}$ & $\begin{array}{r}\text { EFFECT } \\
5.462+C 7\end{array}$ \\
\hline .015 & $3.641+C 7$ & $3.353+03$ & $4.455-04$ & $3.641+07$ \\
\hline & $2.7 \geq 1+C 7$ & $3.353+C 3$ & $7.5 \mathrm{Cb}_{-} \mathrm{C}_{4}$ & $.731+07$ \\
\hline .030 & $1.221+C 7$ & $.352+03$ & $.773-c 3$ & $.821+07$ \\
\hline .040 & $1.366+c 7$ & $E 2+C$ & 3.141 & +07 \\
\hline $.05 \mathrm{C}$ & $1 . \operatorname{cs} 3+0$ & $3.353+$ & 4.8 & 1. $C 92+07$ \\
\hline.$C 6 C$ & $10 t+C t$ & & & \\
\hline & $R=0+0$ & & & \\
\hline .100 & $5.4 t 5+C t$ & $3.351+C 3$ & $1 . \$ 22-C 2$ & $5.462+06$ \\
\hline & $\in(44+1$ & & & \\
\hline & $1.402+C 7$ & 3.34 & 7.4 & $+c 7$ \\
\hline 200 & $7.854+C 6$ & $3.336+c 3$ & $7-01$ & $7.891+06$ \\
\hline & & & & \\
\hline. & $.425+$ & $3 \cdot 3$ & 4. & $22+06$ \\
\hline .600 & $2.475+06$ & $3.291+03$ & $5.657-01$ & $2.472+06$ \\
\hline & $1.44 t-1$ & & & \\
\hline & $5.3 \leqslant 3-$ & $3.2<>>$ & 1.3 & $9.321+05$ \\
\hline 1.500 & $4.059+C 5$ & $2 . C 7 C+C 3$ & $2.276+C C$ & $4.028+05$ \\
\hline & & & & \\
\hline & $4.503+C 5$ & 2.6 & 5.0 & $4.877+05$ \\
\hline $4 . c 00$ & $2.451+0=$ & $2.351+$ & $1+\infty$ & $2.427+05$ \\
\hline & & & & \\
\hline & & & & \\
\hline & $4.374+1$ & & & \\
\hline & & & & \\
\hline & & & & $+C_{4}$ \\
\hline & & & & \\
\hline & & & & \\
\hline & & & $+C 1$ & $1 / 4+03$ \\
\hline
\end{tabular}

\begin{tabular}{|c|c|c|c|c|}
\hline \\
\hline $\begin{array}{l}\text { ENEREY } \\
\text { (KEV) }\end{array}$ & TOTAL & COHER - & I NC CH. & $\begin{array}{r}\text { PHCTC } \\
\text { EFFECT }\end{array}$ \\
\hline .010 & $t \in 1+07$ & $3.448+C 3$ & 2. $\mathrm{C} 22-\mathrm{C} 4$ & $5.66 \mathrm{C}+07$ \\
\hline & $14+C 7$ & $3.44 \varepsilon+c 3$ & $4.542-04$ & $3.774+07$ \\
\hline .020 & $1+c 7$ & $3.448+C 3$ & \&. $c \in 1-$ & $2.830+07$ \\
\hline .030 & 1. $\varepsilon \varepsilon 7+C 7$ & $3.448+C 3$ & $1.807-\mathrm{C} 3$ & $1.887+07$ \\
\hline .040 & $1.415+07$ & $3.448+C_{3}$ & & $1.415+07$ \\
\hline - $C \leq 0$ & $1.122+c 7$ & $3.44 \varepsilon+C 3$ & & $1.132+07$ \\
\hline $.0 \in \mathrm{C}$ & $9.438+C t$ & $3.44 \varepsilon+C 3$ & $7.154-C_{3}$ & $5.434+06$ \\
\hline - $C \& \mathrm{C}$ & 7. $C 79+06$ & $3.447 t$ & 1.2 & $7.076+06$ \\
\hline .100 & 5. $t \in 4+0 t$ & $3.446+$ & & $1+06$ \\
\hline .150 & $3.777+C t$ & $3.444+C 3$ & $4.330-C_{2}$ & $3.774+C 6$ \\
\hline & $2.824+c t$ & $3.441+$ & & $1+06$ \\
\hline .300 & 8. $t \equiv 3+0 t$ & $3.421+C 3$ & 1.6 & $8.63 c+06$ \\
\hline .400 & $5.598+C 6$ & $3.41 s+c 3$ & $2.8 \mathrm{CO}-\mathrm{Cl}_{1}$ & $5.594+06$ \\
\hline & $22+1<-1$ & & & \\
\hline & $144+0 t$ & 3.38 & $5.7>$ & $2.741+0 \epsilon$ \\
\hline .800 & $1.584+0 t$ & $3.344+c 3$ & $9.415-\mathrm{C1}$ & $1.581+06$ \\
\hline & & & & \\
\hline & & & & +05 \\
\hline 2.000 & $1.0 \epsilon 0+c \epsilon$ & $3.010+03$ & $3.247+C 0$ & $1.057+06$ \\
\hline & & & & \\
\hline & c $=$ & 2.4 & & \\
\hline 5.000 & $1.492+05$ & $2.15 q+C 3$ & 8.3 & $1.470+05$ \\
\hline & & & & \\
\hline & & & & +04 \\
\hline 0.00 & & 1.2 & 1.4 & \\
\hline & & & $1.8 \varepsilon$ & \\
\hline & & & & \\
\hline $\cos \longrightarrow$ & $6.115+c$ & $3.420+02$ & $2 . t 15+01$ & $5.747+03$ \\
\hline O & 2. $\varepsilon \in 4+C\}$ & $2.211+c 2$ & 2. $\varepsilon \subseteq 4+C 1$ & $2.614+03$ \\
\hline
\end{tabular}

\begin{tabular}{|c|c|c|}
\hline $\begin{array}{l}\text { ENEREY } \\
\text { (KEV) }\end{array}$ & OTAL & $\begin{array}{l}\text { COHER } \\
\text { SCATT }\end{array}$ \\
\hline 50. & $1.435+03$ & $1.434+02$ \\
\hline & & \\
\hline & $76+03$ & $6 \cdot 320+01$ \\
\hline & $8+03$ & \\
\hline & 3. & \\
\hline & $. \subseteq 4 t+C 2$ & $1.13 t+01$ \\
\hline & & \\
\hline & $\varepsilon \bar{z}+$ & \\
\hline & & \\
\hline & & \\
\hline & & \\
\hline & & \\
\hline & 1.3 & \\
\hline & & \\
\hline & & \\
\hline & $.115+$ & 3.0 \\
\hline & & \\
\hline & & \\
\hline & 1.2 & 7.6 \\
\hline & & \\
\hline & $1.4 t$ & \\
\hline & $1.6 \mathrm{C}$ & 1.2 \\
\hline & & \\
\hline & & 3.0 \\
\hline & & \\
\hline & & \\
\hline & & \\
\hline & & \\
\hline
\end{tabular}

100000 .

\begin{tabular}{|c|c|c|}
\hline EAERGY & TOTAL & COHER - \\
\hline & & \\
\hline 50. & $1.512+C 3$ & $1.485+c 2$ \\
\hline 60. & $9.469+02$ & $1.08 \varepsilon+02$ \\
\hline 80 & $2.078+03$ & $6.540+01$ \\
\hline 100. & $1.167+03$ & $4 \cdot 353+01$ \\
\hline 150. & $4.120+02$ & $2.037+C 1$ \\
\hline 200 & $2.043+02$ & $1.176+01$ \\
\hline 300. & $8.527+01$ & $5.359+0 \mathrm{C}$ \\
\hline 400. & $5.15 c+01$ & $3.052+0 \mathrm{c}$ \\
\hline 500. & $3.743+C 1$ & $1.968+00$ \\
\hline 600 . & $3.00 \epsilon+01$ & $1.374+0 \mathrm{C}$ \\
\hline 800 & $2.254+C 1$ & $7.77 t-C_{1}$ \\
\hline 1000 . & $1.8 \in 7+C 1$ & $4.995-01$ \\
\hline 1500 . & $1.422+01$ & $2.231-01$ \\
\hline 2000 & $1.2 \epsilon 3+01$ & $1.258-01$ \\
\hline 3000 . & $1.153+01$ & $5.606-02$ \\
\hline 4000 . & $1.138+01$ & $2.157-02$ \\
\hline 5000 . & $1.15 t+C 1$ & $2.022-02$ \\
\hline 6000 . & $1.1 \varepsilon s+c 1$ & $1.405-02$ \\
\hline 8000 & $1.265+01$ & $7.908-03$ \\
\hline 10000 . & $1.342+C 1$ & $5.063-03$ \\
\hline 15000 . & $1.510+C 1$ & $2.251-03$ \\
\hline 20000 . & $1.651+c 1$ & $1.267-03$ \\
\hline 30000 . & $1.877+01$ & $5.631-04$ \\
\hline 40000 . & $2.043+01$ & $3.168-04$ \\
\hline 50000 . & $2.169+01$ & $2.027-04$ \\
\hline 60000 . & $2.268+01$ & $1.408-c 4$ \\
\hline 80000 . & $2.419+01$ & $7.921-05$ \\
\hline & & \\
\hline
\end{tabular}

$100000 \cdot 2.531+01 \quad 5.069-05$

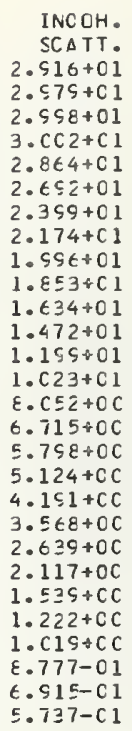
SCA TT. 2. $C 5,1+C 1$ $3 . C 16+01$ $3 . C 35+01$ $3 . \mathrm{C} 4 \mathrm{C}+\mathrm{C} 1$ $2.5 C 2+01$ $2.72 s+C 1$ $2.432+01$ $2 \cdot \overline{2} C 4+C 1$ $2 . C 2 E+01$ $1.820+01$ $1 . E E 7+01$ $1.4 \subsetneq 3+C 1$ $1.21 t+C 1$ 1. $C 3 \varepsilon+c 1$ $8.167+00$ t. $E 11+C C$ 5. $821+C C$ $5.1 c \varepsilon+c c$ $4.251+0 C$ $3.61 S+0 C$ $2 . t 77+0 C$ $2.147+C C$ 1. $561+C \mathrm{C}$ $1.240+C C$ $1.034+00$ E. SC 3-C 1 7. $\mathrm{C} 14-\mathrm{Cl}$ ᄃ. $\varepsilon 15-C 1$
FHOTO EFFECT $1.2 \in 3+03$ 7. $5 \varepsilon \mathrm{C}+\mathrm{C} 2$ 1. $8 \varepsilon\}+03$ 1. $C \equiv \epsilon+C 3$ $3.432+02$ 1. $\leq t 4+C 2$ $5.257+C 1$ $2.45 \Xi+01$ $1 \cdot 433+01$ $4.8 t t+C 0$ 2. $C 4 \mathrm{C}+0 \mathrm{O}$ $1.3 \varepsilon 7+00$ $\varepsilon .37 \varsigma-01$ $4.3 c \varepsilon-C 1$ 2. $\varepsilon \subseteq, 7-01$ 2. $127-01$ 1.684-01 $1.17 \epsilon-01$ $5 . t 53-C 2$ 4.112-02 $2.658-02$ $1.5 \in 3-02$ $1.5 \leq t-C 2$ $1.2 \varepsilon \varepsilon-02$ $\varsigma .5 \varepsilon E-03$ $7 . t \geq 2-03$

$3.523-C$ $1.186+C C$ $2.745+C$ $5.3 C 9+C C$ $0.354+C C$ $\varepsilon \cdot C 8 s+C C$ $1.198+C 1$ 1. $384+C 1$ $1.668+01$ $1.871+01$ $2 . \mathrm{Cl}+\mathrm{C}$ $2.295+C 1$ $2.41 \mathrm{C}+\mathrm{Cl}$
PAIR
PRCD.

\begin{tabular}{|c|c|}
\hline $\begin{array}{l}\text { EN'ERGY } \\
\text { (KEV) }\end{array}$ & TCTAL \\
\hline 50 & \\
\hline 60. & $\begin{array}{l}1.558+03 \\
9.568+02\end{array}$ \\
\hline 80. & $2.174+03$ \\
\hline 100. & $1.2<t+c 3$ \\
\hline 150. & $4.344+02$ \\
\hline 200 . & $2.153+02$ \\
\hline 300 . & $8.538+01$ \\
\hline 400. & $5.3 E s+01$ \\
\hline 500. & $3.870+01$ \\
\hline 600. & 3. $\operatorname{cs} 4+01$ \\
\hline 800 & $2.30 s+01$ \\
\hline 1000 & $1 . \operatorname{sc} 8+01$ \\
\hline 1500. & $1.451+01$ \\
\hline 2000 & $1.283+01$ \\
\hline 3000 & $1.175+01$ \\
\hline 4000 & $1 \cdot 1 \in 8+01$ \\
\hline 5000 & $1.154+C 1$ \\
\hline 6000 & $1.232+01$ \\
\hline 8000. & $1.310+01$ \\
\hline 10000. & $1.380+01$ \\
\hline 15000. & $1.527+01$ \\
\hline 20000 & $1 . \in 71+01$ \\
\hline $3000 \mathrm{C}$ & $1 . \varsigma 28+C 1$ \\
\hline 40000 & $2.106+01$ \\
\hline 50000. & $2.228+01$ \\
\hline 60000 & $2.321+01$ \\
\hline 80000 & $2.4 E 7+C 1$ \\
\hline & $2.595+\mathrm{Cl}$ \\
\hline
\end{tabular}

COHER

$1.536+02$

$1.536+02$
$1.125+02$

$6.763+01$

$4.5 \mathrm{Cl}+\mathrm{Cl}$

$2.106+01$

$1.215+0$

$5.540+0$

$3.155+0 \mathrm{C}$

$2.035+00$

$1.420+00$

8.038-01

$5.164-01$

2.306-0

5.795-0

3.263-02

$2.09 \mathrm{C}-0$

$1.452-02$

8.174-03

5.233-03

$2.327-03$

1.309-03

$5.820-04$

3.274-04

2.096-04

$1.455-04$

$8.188-05$
$5.240-05$

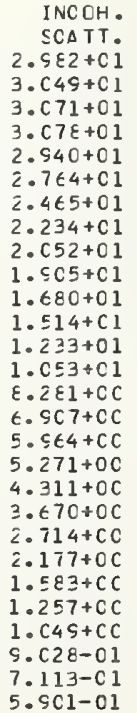

NCOH.

2. SE $2+C 1$

3. $C 49+C 1$

3. $c 7 \varepsilon+01$

2. $940+0$

. $4 \epsilon 5+0$

. $52+0$

1. $\subseteq \mathrm{C} 5+\mathrm{C}$

$1 \cdot 680+0$

. $233+0$

$C \leq 3+C$

t. $9 \mathrm{C} 7+\mathrm{CC}$

5. $\subseteq E 4+C$

$4.311+O C$

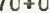

<. $177+0$

$1.257+C$

S. $228-0$

5. 9 C1- 0

\begin{tabular}{|c|c|}
\hline $\mathrm{FHCTC}$ & $P \Delta I F$ \\
\hline EFFEC T & PRCL. \\
\hline$\cdot 3 \equiv 5+c 3$ & \\
\hline $.08 C+02$ & \\
\hline - $s \in 2+C 3$ & \\
\hline $.093+03$ & \\
\hline $.62 t+c 2$ & \\
\hline $.65 \equiv+02$ & \\
\hline $.55 \varsigma+01$ & \\
\hline $.641+C 1$ & \\
\hline $.522+C 1$ & \\
\hline . $\varepsilon \varepsilon \epsilon+C C$ & \\
\hline $.1 \varepsilon 4+O C$ & \\
\hline $.239+00$ & \\
\hline $.477+00$ & $3.63 t-01$ \\
\hline . $512-01$ & $1.233+C C$ \\
\hline $.672-01$ & $2.838+C C$ \\
\hline $.077-01$ & $4.228+C C$ \\
\hline . $2 \in 9-01$ & $5.436+C C$ \\
\hline $.7 \varepsilon \varepsilon-C 1$ & $6.455+C C$ \\
\hline $.24 \varepsilon-C 1$ & $\varepsilon .268+c c$ \\
\hline $.554-C 2$ & $5.657+C C$ \\
\hline . ५५५-02 & $1.236+C 1$ \\
\hline $.3 \in 4-c 2$ & $1.432+C 1$ \\
\hline . $\varepsilon 21-02$ & $1.718+C 1$ \\
\hline $.083-02$ & $1.517+01$ \\
\hline$\epsilon 51-02$ & $2 . c 64+01$ \\
\hline $.2 \in \overline{7}-02$ & $2.178+C 1$ \\
\hline . $017-02$ & $2.348+C 1$ \\
\hline $1 C 1-03$ & $2.472+01$ \\
\hline
\end{tabular}
FFEC T $1.41 E+03$
$\varepsilon .53 \varepsilon+C 2$ 2. $C i t+C 3$ $1.150+03$ $3.84 C+02$ $1.75 E+C 2$ 5. $52 \mathrm{C}+01$ 2. $81 \mathrm{C}+01$ $1 \cdot E 14+01$ $1.04 t+01$
$5.4 \varepsilon 1+0 C$ $3.42 \varepsilon+0 C$ $1.5 \in 7+00$ S. $475-01$ $4.97 t-C 1$ $3.27 \varepsilon-0$. $2.417-01$
$1.5 C 4-01$ $1.325-01$ 1.017-01 $6.375-02$ $4.638-02$ 2. $597-02$ $1.752-02$ 1.451-02 1. $C E C-C 2$ $8.557-03$ 


\begin{tabular}{|c|c|c|c|c|}
\hline \\
\hline $\begin{array}{l}\text { EAERGY } \\
\text { (KEV) }\end{array}$ & TOTAL & $\begin{array}{l}\text { CCHER, } \\
\text { SCATT: }\end{array}$ & $\begin{array}{l}\text { I NCOH. } \\
\text { SCATT. }\end{array}$ & $\begin{array}{r}\text { PHCTC } \\
\text { EFFECT }\end{array}$ \\
\hline .010 & $5.830+C 7$ & $3.545+C 3$ & $1.584-C 4$ & $5.829+07$ \\
\hline .015 & 3. $\varepsilon \varepsilon 7+c 7$ & $3.545+03$ & $4.456-c 4$ & $.886+07$ \\
\hline & $2.915+07$ & $3.545+c 3$ & $7.508-04$ & $2.915+07$ \\
\hline .030 & $1.943+C 7$ & $3.545+c 3$ & $1.773-c^{3}$ & $1.943+07$ \\
\hline .040 & $1.458+C 7$ & $3.54 E+C 3$ & & $.457+07$ \\
\hline & $1.1 \in 6+C 7$ & $3.544+C 3$ & $4.851-$ & $1.166+07$ \\
\hline & $9.719+0 t$ & $3.544 \backsim c 3$ & 7.c15-c3 & $9.715+06$ \\
\hline & & & & \\
\hline & $\Xi .8 \equiv 3+0 t$ & 3.54 & & $.829+06$ \\
\hline & $3.850+0 t$ & $3.54 \mathrm{C}$ & & $.886+06$ \\
\hline & $2.518+$ & & & $5+06$ \\
\hline & $8.391+$ & 3.52 & & +06 \\
\hline & $5.42 \mathrm{~s}+\mathrm{C}$ & $3.5:$ & $-\mathrm{cl}$ & $5.425+06$ \\
\hline & & & & \\
\hline & $2.738+06$ & $3.4 \varepsilon 3$ & & $2.734+06$ \\
\hline & $1 . t \in 17+0 t$ & & -01 & $1.614+06$ \\
\hline & 1. & & & \\
\hline & $4.635 \div C 5$ & 3.253 & 2.2 & $4.607+05$ \\
\hline & $1.12 t+0 t$ & & 3.2 & \\
\hline & 5.46 & & & \\
\hline & & & & +05 \\
\hline 5 & & & 8.3 & +05 \\
\hline & & & & \\
\hline & & & & \\
\hline 10.000 & 7.25 & 1.3 & & \\
\hline & & & $c 1$ & 3. \\
\hline & & & & \\
\hline $3 \mathrm{C} . \mathrm{COC}$ & $6.514+c 3$ & $3.525 \& C 2$ & $2.643+01$ & $6.135+03$ \\
\hline & $3 . C 3 t+C 3$ & $2.275+C 2$ & $2.824+C 1$ & $2.780+03$ \\
\hline
\end{tabular}

\begin{tabular}{|c|c|c|c|c|}
\hline ENEREY & & COHER & & \\
\hline & & & & FECT \\
\hline . $C I C$ & $6.045+07$ & $3.643+c 3$ & $2.035-c_{4}$ & $6.044+07$ \\
\hline & $4 . c 3 c+c 7$ & $3 \cdot t 43+03$ & $4.571-C_{4}$ & $4.030+07$ \\
\hline . $c \approx 0$ & $3.023+C 7$ & $3.643+03$ & $8.112-04$ & $3 . \mathrm{C} 22+07$ \\
\hline.$C 3 C$ & $2 \cdot C 15+07$ & $3.642+C 3$ & & $2.015+07$ \\
\hline $.04 \mathrm{C}$ & $1.511+C 7$ & $3.642+03$ & $3.222-c 3$ & $1.511+07$ \\
\hline $.05 c$ & $1.20 s+c 7$ & $3.642+03$ & $5 . C 17-C 3$ & $1.2 \mathrm{C9}+07$ \\
\hline.$c 6 C$ & $1.008+07$ & $3.642+C 3$ & -03 & $.0 C 7+07$ \\
\hline & $7.555+C 6$ & ‥ $\in 41+$ & & $55+06$ \\
\hline .100 & t. C $48+C \epsilon$ & 3.641 & 1. & $6.044+06$ \\
\hline & & & & $4.030+06$ \\
\hline & $3 . c 26+0 t$ & $3.635+$ & $7 . \in C 2-$ & $3.022 \div 06$ \\
\hline $.30 \mathrm{C}$ & $\varepsilon, \epsilon 58+c \epsilon$ & $3.62 t+$ & 7-01 & $8.654+06$ \\
\hline & & & & \\
\hline .500 & $3.552+c t$ & 3.555 & 4.2 & $8+06$ \\
\hline$\cdot \in C C$ & 2. $\varepsilon 5 C+C 6$ & $3.58 \bar{z}$ & & $7+06$ \\
\hline & & & & \\
\hline $1 . . C C O$ & $1.122+c t$ & $3.451+03$ & $1.335+0 \mathrm{C}$ & $\varepsilon+0 t$ \\
\hline & & 3.348 & & 405 \\
\hline & & & & \\
\hline $3 . c c c$ & $5.7 \in 6+C E$ & 2.857 & 5.0 & $8+05$ \\
\hline $4 .<C 0$ & & & & \\
\hline & & $2.2 \varepsilon$ & & 1.6 \\
\hline & & & & 1.0 \\
\hline & & & & \\
\hline & & & & \\
\hline & & & & \\
\hline & 1. $\subseteq \varepsilon 5+$ & $6.21 \varepsilon+$ & $2.235+$ & $1.920+04$ \\
\hline & & & $2.663+01$ & $6.439+03$ \\
\hline & & & $11+\mathrm{Cl}$ & $40+03$ \\
\hline
\end{tabular}

\begin{tabular}{|c|c|c|c|c|}
\hline \\
\hline $\begin{array}{l}\text { EAERGY } \\
\text { (KEV) }\end{array}$ & TOTAL & COFER. & IACCH. & $\begin{array}{r}\text { PHCTC } \\
\text { EFFEC }\end{array}$ \\
\hline & & & SCATT & EFFECT \\
\hline - C15 & $c .215+c 7$ & $3.742+c 3$ & 2.CE4-C4 & $214+01$ \\
\hline & $3+C 7$ & $42+(-2)$ & 04 & \\
\hline & & & & \\
\hline & & $74 \hat{c}+c \vec{z}$ & $1.836-$ & $1+07$ \\
\hline $.04 c$ & $1.554+c 7$ & $3.741+C 3$ & $3.252-03$ & 1.554007 \\
\hline .050 & $43+07$ & $3.741+c$ & $5.064-03$ & $1.243+07$ \\
\hline.$c \in c$ & $1 . c \geq 6+c 7$ & $2.741+C 3$ & $7.26 \in-C 3$ & $1.036+07$ \\
\hline.$c 8$ & & & & \\
\hline .100 & $+c \epsilon$ & & s-c2 & $4+06$ \\
\hline .150 & $4.147+C E$ & $3.738+C 3$ & $4.355-c 2$ & $4.143+06$ \\
\hline & & & & +06 \\
\hline & & 3.72 & & $5+06$ \\
\hline .400 & $5.757+c 6$ & $3.714+c 3$ & $2.836-\mathrm{Cl}$ & $5.753+06$ \\
\hline & & & & \\
\hline & & & & \\
\hline .800 & $1.78 z+C 6$ & $3.64 C+C 3$ & $9.503-\mathrm{Cl}$ & $1.779+06$ \\
\hline 1. $\mathrm{ccO}$ & $1.174+0 t$ & $3.5 \subseteq 1+c z$ & $1.341+C C$ & $1.171+56$ \\
\hline & & & & \\
\hline $2 . \cos$ & 1.23 & 3.2 & $t+c 0$ & 1.23 \\
\hline $3 . \mathrm{COO}$ & $6 . c \varepsilon$ & 2.538 & $4.985+C C$ & $34+05$ \\
\hline & & & & \\
\hline & & 2.3 & $O C$ & $.729+05$ \\
\hline$\epsilon .000$ & $1.112+05$ & $2.1 C_{2}+03$ & ؟. ع 29+CC & $1.090+05$ \\
\hline & & & & \\
\hline & & & & \\
\hline $15 . \mathrm{CCO}$ & $4.405+C 4$ & & $1.530+C 1$ & 4.31 \\
\hline & & & $2.253 * 01$ & $2.039+04$ \\
\hline & & & & \\
\hline & & & & \\
\hline
\end{tabular}

\begin{tabular}{|c|c|c|c|c|c|}
\hline $\begin{array}{l}\text { ENEREY } \\
\text { (KEV) }\end{array}$ & TCTAL & $\begin{array}{l}\text { COHER } \\
\text { SCATT: }\end{array}$ & $\begin{array}{l}\text { INCGH. } \\
\text { SCATT. }\end{array}$ & $\begin{array}{r}\text { FHCTO } \\
\text { EFFECT }\end{array}$ & $\begin{array}{l}\text { PAI } \\
\text { PRCC }\end{array}$ \\
\hline 50. & $1.685+03$ & $1.577+02$ & 3. $c 1 \epsilon+c 1$ & $1.5 \mathrm{C} 1+03$ & \\
\hline 60. & $1.053+03$ & $1.154+02$ & $3.085+C 1$ & $5.0 \in 5+02$ & \\
\hline 80 . & $2.269+03$ & $6.925+01$ & $.1 \mathrm{C} 7+\mathrm{Cl}$ & $2.1 \in 5+03$ & \\
\hline 100. & $1.283+03$ & $4.604+01$ & $.11 t+01$ & $1.2 \mathrm{C} 5+03$ & \\
\hline & $4.553+C 2$ & $2.152+01$ & $2 . \subseteq 7 \varepsilon+01$ & $4 \cdot C 4 C+C 2$ & \\
\hline & $2.256+02$ & $1.241+C$ & $2.801+01$ & $1 . \varepsilon 51+0$ & \\
\hline & $9.328+C 1$ & 5.65 & $2.458+C 1$ & $t .264+01$ & \\
\hline & 5.5 & 3.2 & $2,2 \in 5+01$ & $2 . \subseteq 82+01$ & \\
\hline & $4.006+c$ & & & $1.71 \varepsilon \nLeftarrow 0$ & \\
\hline & & & 1.5 & $1.11 t+C 1$ & \\
\hline & & & & & \\
\hline 1000. & $1.553+\mathrm{Cl}$ & 5.26 & $1.535+01$ & 3. $E \leqslant \varepsilon+O C$ & \\
\hline & & & & $1 . \in \in C$ & \\
\hline & & 1.3 & & & \\
\hline 000. & $1.198+C 1$ & 5.90 & $\varepsilon \cdot 3 s t+c c$ & 5.282 & \\
\hline & & & & 3.4 & \\
\hline & & & t. C & $2.5 \in 2$ & \\
\hline 6000 . & $1.24 \bar{z}+01$ & 1.4 & 5.3 & $2 . c 1 \varepsilon-c 1$ & \\
\hline & $1.321+01$ & & & & \\
\hline 10000. & $1.357+c$ & 5.3 & 3.7 & $1.077-01$ & \\
\hline 15000. & $1.564+C$ & 2.3 & & $6.75 \varepsilon-02$ & \\
\hline & & & & & \\
\hline & & 5.93 & $1 . \in C 5$ & & \\
\hline 40000 & $2.152+c$ & & $1.275+$ & & \\
\hline & & 2.136 & & $1.857-$ & \\
\hline & & $1.483-$ & & & \\
\hline & $2.547+$ & & $7.212-01$ & $1.144-02$ & \\
\hline & $2 . t 66+c 1$ & $5.340-05$ & E. . ₹3-c1 & $5.1 C \varepsilon-03$ & \\
\hline
\end{tabular}

\begin{tabular}{|c|c|c|c|c|c|}
\hline $\begin{array}{l}\text { AEREY } \\
\text { (KEV) }\end{array}$ & TCTAL & COHER . & INC OH. & FHCTO & \\
\hline (KEV) & & SCATT & SCATT & EFFECT & \\
\hline $\begin{array}{l}50 . \\
60 .\end{array}$ & $1.789+C 3$ & $1.641+02$ & $\begin{array}{l}3 \cdot C 46+C 1 \\
3 \cdot 118+C 1\end{array}$ & $\begin{array}{l}1.5 c 4+c 3 \\
9.63 \varepsilon+02\end{array}$ & \\
\hline 80. & $2.378+03$ & $7.222+01$ & $.143+C 1$ & $2.274+03$ & \\
\hline 100. & $1.347+C 3$ & $4.805+c 1$ & $3.154+C 1$ & $1.267+03$ & \\
\hline 150. & $4.786+02$ & $2.248+01$ & 3. $C 16+01$ & $4.2 E C+C 2$ & \\
\hline 200 . & $2.3 \in 5+02$ & $1.297+01$ & $2 . \varepsilon 37+C 1$ & $1.555+<2$ & \\
\hline 300 . & $5.747+C 1$ & $5.909+00$ & $2.531+c 1$ & $6.625+01$ & \\
\hline 400 . & $5.786+C 1$ & $3.36 t+0 C$ & $2.2 \subseteq 5+c 1$ & & \\
\hline 500 . & $4.143+01$ & $2.170+0 \mathrm{C}$ & $2.1 C \varsigma+C 1$ & 1. $\varepsilon 17+c 1$ & \\
\hline & & & & $1.1 \varepsilon \mathrm{C}+01$ & \\
\hline 800 . & $2.4 \geqslant 2+01$ & 8.572 & $1.727+\mathrm{C}_{1}$ & $t \cdot I \subseteq 2$ & \\
\hline 1000 . & $1 . \operatorname{cs} 8+01$ & $5.507-C_{1}$ & $1.556+C 1$ & 3. $\varepsilon T 4+C 0$ & \\
\hline & & 2.46 & & 1.771 & \\
\hline 00. & $1.33 \varepsilon+01$ & 1.38 & 1. CE2 $+C 1$ & 1.C $C \mathrm{C}+\mathrm{CC}$ & 54 \\
\hline 30 & $1.22 \epsilon+01$ & 6.17 & $\varepsilon .511+c c$ & $5 . t 10$ & \\
\hline & & & 7. CS & $3.692-01$ & \\
\hline 5000 . & $1.238+01$ & 2.22 & $t .12 s+c c$ & $2.721-01$ & $5.556+$ \\
\hline 60 & & 1.5 & & & \\
\hline & 1.35 & 8.71 & 4.43 & $1.4 \div 4-$ & \\
\hline 10000 . & $1.432+01$ & 5.58 & 3.77 & $1.143-01$ & $43+$ \\
\hline & & & & & \\
\hline & $1.765+$ & $1.396=$ & $2.238+$ & $5.2 c s-02$ & \\
\hline & $2 . c 28+$ & $6.207=$ & & $3.3 \in 5-02$ & \\
\hline 40000 & $2.211+$ & $3.492-$ & 1.25 & 2.483 & 075 \\
\hline & $2.343+c$ & $2.235-$ & $700^{\circ}$ & & \\
\hline & & & & & \\
\hline & & $8.731-c$ & $7.31 \mathrm{C}-01$ & $1.212-02$ & $2.527 t$ \\
\hline & $725+0$ & $5.588-05$ & $\in . c \in 5-01$ & $9.64 \varepsilon-03$ & 2.0047 \\
\hline
\end{tabular}

\begin{tabular}{|c|c|c|c|c|c|}
\hline $\begin{array}{l}\text { EAERGY } \\
\text { (KEV) }\end{array}$ & TCTAL & $\begin{array}{l}\text { COHER } \\
\text { SCATT: }\end{array}$ & $\begin{array}{l}\text { INCOH. } \\
\text { SCATT: }\end{array}$ & $\begin{array}{l}\text { FHCTC } \\
\text { EFFEC T }\end{array}$ & $\begin{array}{l}\text { PAIF } \\
\text { PRCC. }\end{array}$ \\
\hline 50. & 1. $\varepsilon \varepsilon \geqq \nleftarrow 03$ & $1 . t 95+02$ & a.c $c \nmid t+c 1$ & 1. $\epsilon \varepsilon \equiv+03$ & \\
\hline 60. & $1.173+03$ & $1.241+02$ & $3.151+C 1$ & $1.018+03$ & \\
\hline 80. & $2.422+03$ & $7.456+01$ & $3.17 \varepsilon+c 1$ & $2.375+03$ & \\
\hline 100. & $1.405+03$ & $4.960+01$ & $3.151+C 1$ & $1.32 \varepsilon+03$ & \\
\hline 150. & $5 . C 25 \div 02$ & $2.320+01$ & 3. $c 53+01$ & $4.4 \varepsilon \varepsilon+02$ & \\
\hline 200 . & $2.487+02$ & $1.338+01$ & $2.873+01$ & 2. $c \in t+02$ & \\
\hline 300 . & 1. $C 2 \mathrm{C}+0 \mathrm{z}$ & $6.099 \div 00$ & $\overline{2} .5 \in 4+C 1$ & 7. CEE+C1 & \\
\hline 400. & $6 . c 20+01$ & $3.473+00$ & $2.325+c 1$ & $3.34 \varepsilon+01$ & \\
\hline 500 . & $4.287+01$ & $2.240 \div 00$ & $2.137+01$ & $1.52 t+C 1$ & \\
\hline tOC. & $3.350+01$ & $1.5 \in 3+00$ & $1.584+01$ & $1.25 \mathrm{C}+\mathrm{Cl}$ & \\
\hline 800 . & $2.494+01$ & $8.846-01$ & 1. $7 E C+C 1$ & $t . E \in 1+00$ & \\
\hline 1000 . & 2. $C 44+01$ & $5.682-01$ & $1.577+\mathrm{Cl}$ & $4.111+00$ & \\
\hline 1500 . & $1.54 C+C 1$ & $2.538-01$ & $1.284+C 1$ & $1.8 \varepsilon 4+C C$ & $4.211-\mathrm{Cl}$ \\
\hline & $1.365+01$ & $1.431-01$ & 1. $C 56+01$ & $1.140+0 \mathrm{C}$ & $1.4 C 3+0 C$ \\
\hline 3000 . & $1.245+C 1$ & $6.37 t-02$ & $\varepsilon .626+c c$ & $5.583-01$ & $3.205+0 \mathrm{C}$ \\
\hline 4000 . & $1.237+01$ & $3.591-02$ & $7.154+0 C$ & $3.937-01$ & $4.746+C C$ \\
\hline 5000. & $1.255+01$ & $2.300-02$ & $\epsilon . \bar{z} 12+c c$ & $2 . \subseteq C C-01$ & t. $\mathrm{CGS}+\mathrm{cc}$ \\
\hline 6000 & $1.255+C 1$ & $1.598-02$ & $=.450+0 \mathrm{C}$ & $2.282-01$ & $7.217+C C$ \\
\hline 8000 & $1.378+01$ & $8.994-03$ & $4.491+C c$ & $1.5 \subseteq c-01$ & $5.118+C c$ \\
\hline 10000 . & $1.4 E C+01$ & $5.758-03$ & 3. $\varepsilon 23+c c$ & $1.215-01$ & $1 . C 65+C 1$ \\
\hline 15000 . & $1.649+01$ & $2.560-03$ & 2. $\varepsilon 28+C C$ & $7.611-02$ & $1.359+C 1$ \\
\hline 20000 . & $1.819+01$ & $1.441-03$ & $2.268+c 0$ & $5.52 q-C 2$ & $1.586+C 1$ \\
\hline 30000 . & 2.CQE+01 & $6.404-04$ & $1.649+0 \mathrm{C}$ & $3.567-c 2$ & $1.516+C 1$ \\
\hline & $2.268+01$ & $3.603-04$ & $1.3 C 5+O C$ & $2.6 \equiv 2-02$ & $2.134+C 1$ \\
\hline 50000 . & $2.4 \mathrm{Cl} 1+01$ & $2.306-c 4$ & $1 . c s_{2}+c c$ & $2.085-02$ & $2.290+C_{1}$ \\
\hline & & $1.601-04$ & $5.4 C 4-C_{1}$ & $1.7 \geq t-C_{2}$ & $2.41 C+C 1$ \\
\hline & & $9.009-05$ & $7.4 \mathrm{CS}-\mathrm{Cl}_{1}$ & $1.283-02$ & $2.553+C 1$ \\
\hline 100000 & $2.757+C 1$ & $5.766-C 5$ & $6.147-C_{1}$ & $1 . C 22-02$ & $2.735+C 1$ \\
\hline
\end{tabular}




\begin{tabular}{|c|c|c|c|c|}
\hline $\begin{array}{r}\text { ENERGY } \\
\text { (KEV) }\end{array}$ & TOTAL & $\begin{array}{l}\text { COHER. } \\
\text { SCATT. }\end{array}$ & $\begin{array}{l}\text { I NCOH. } \\
\text { SCATT. }\end{array}$ & $\begin{array}{r}\text { PHCTC } \\
\text { EFFECT }\end{array}$ \\
\hline & $6.474+C 7$ & $2.842+C 3$ & 2. $c \in 7-C 4$ & $6.474+07$ \\
\hline & $4.316+C 7$ & $3.842+c 3$ & $4.643-C 4$ & $4 \cdot 316+07$ \\
\hline & $27+07$ & $3.842+C 3$ & & \\
\hline & 2 & & & \\
\hline .040 & $619+$ & 3.8 & & +07 \\
\hline - $C 50$ & $S 5+C 7$ & 3. $842+c 3$ & $5 . C S 5$ & $\approx 07$ \\
\hline .060 & $1 . C 79+07$ & $3.842+c$ & 7.3 & 1.07 \\
\hline . $C \& 0$ & $8 \cdot C 56+06$ & $3.841+C 2$ & $1.2 \mathrm{SO}-\mathrm{C} 2$ & $8.052+06$ \\
\hline .100 & $t .478+C t$ & $3.840+C 3$ & $2.001-02$ & $6.474+06$ \\
\hline .150 & 4. & 3.83 & 4.42 & $4.316+06$ \\
\hline & 3 & & 7.71 & 3.23 \\
\hline & 8 & $3 \cdot 8$ & 1. & \\
\hline & 5 & $3 \cdot$ & & \\
\hline & & 3.8 & 4.2 & +06 \\
\hline.$\in C C$ & $3.102+0 t$ & 3.78 & 5.8 & $+c 6$ \\
\hline .800 & $1.872+C 6$ & 3.74 & 9.55. & $1.869+06$ \\
\hline $1 . C C O$ & $1.235+0 t$ & $3.652+03$ & $1.347+C C$ & $1.235+06$ \\
\hline $1.5 \mathrm{CO}$ & $5.593+0$ & $3 \cdot 543+C 3$ & $2.310+C C$ & $5.558+05$ \\
\hline $2.00 \mathrm{C}$ & $8.847+C$ & $3.372+03$ & $3.233+00$ & $8.814+05$ \\
\hline 3.000 & $t .106+c$ & $3 \cdot C_{2} 1+C 3$ & $4.556+C C$ & $6.076+05$ \\
\hline $4 . \mathrm{COO}$ & $199+0$ & $2.65 c+c 3$ & $6.655+00$ & $3.172+05$ \\
\hline $5 . \mathrm{COO}$ & $1.847+0$ & 2.41 & $+\mathrm{CC}$ & $1.823+05$ \\
\hline & & 2 & C & \\
\hline & & & & \\
\hline $1 \mathrm{C} .000$ & & & & $100+04$ \\
\hline 15.000 & $4 . t 2 s+c$ & $9.432+C 2$ & $1.947+\mathrm{Cl}$ & $4.533+04$ \\
\hline & $206+$ & $6 . t 48+C z$ & $2.272+C 1$ & $2.137+04$ \\
\hline & $t 15+c$ & 3. $\subseteq 08+c 2$ & $2.712+\mathrm{Cl}$ & $7.197+03$ \\
\hline c. & $573+C 3$ & $2.522+c 2$ & 2. $s 69+01$ & $3.251+03$ \\
\hline
\end{tabular}

\begin{tabular}{|c|c|c|c|c|}
\hline $\begin{array}{r}E \wedge E R G Y \\
\text { (KEV) }\end{array}$ & TOTAL & $\begin{array}{l}\text { COFER. } \\
\text { SCATT. }\end{array}$ & $\begin{array}{l}\text { I NC CH. } \\
\text { SCATT. }\end{array}$ & $\begin{array}{r}\text { PHCTC } \\
\text { EFFECT }\end{array}$ \\
\hline .010 & $6.7 C_{3}+C 7$ & $3.544+03$ & $2.080-C_{4}$ & $6.702+07$ \\
\hline .015 & $4.4 t 5+07$ & $3.944+C 3$ & $4 . t 71-C 4$ & $4.468+07$ \\
\hline . $C 20$ & $2.3 \leq 2+C 7$ & $3.544+C 3$ & $8.289-04$ & $3.351+c 7$ \\
\hline . $C 30$ & $2.234+07$ & $3.944+C 3$ & $1.858-C 3$ & $4+07$ \\
\hline .040 & 1. $67 \epsilon+07$ & 3.944 & $2-c 3$ & $6+07$ \\
\hline $.05 \mathrm{C}$ & $1.341+07$ & 3.944 & $5-03$ & $1.340+07$ \\
\hline. $\mathrm{C} 60$ & $1.117+07$ & $3.543+c 3$ & $7.354-03$ & $1.117+07$ \\
\hline - $\mathrm{C} 80$ & $8.382+0 t$ & 3. $543+c 3$ & $1.298-02$ & $8.378+06$ \\
\hline .100 & $t .70 t+C t$ & $3.542+c 3$ & $2 . C 13-C 2$ & $6.702+0 t$ \\
\hline .150 & $4.472+C t$ & $3.540+03$ & $4.447-C 2$ & $4.468+06$ \\
\hline .200 & $3.355+06$ & $2.937+C 3$ & $7.759-02$ & $3.351+06$ \\
\hline .300 & $t S+0 t$ & $3.92 \varepsilon+$ & $1 . t 80-01$ & $9.465+06$ \\
\hline .400 & $t+0 t$ & 3.51 & & +06 \\
\hline .500 & $3+c t$ & 3.90 & -01 & +06 \\
\hline .600 & $S S+0 t$ & 3.88 & $-C 1$ & 3 \\
\hline 800 & $1 . S S 5+C t$ & 3.844 & $9.557-\mathrm{Cl}$ & $.991+06$ \\
\hline $1 . \mathrm{COO}$ & $1.321+C E$ & $3.794+C 3$ & $1.353+\mathrm{CC}$ & $1.317+0 t$ \\
\hline 1.500 & $5.955+05$ & $3.644+C 3$ & $2.316+00$ & $5.919+05$ \\
\hline $2 . \mathrm{CcO}$ & $3.2 \in 1+05$ & $3.469+03$ & $3.238+\mathrm{CO}$ & $3.227+05$ \\
\hline 3.000 & $6.434+05$ & $3.1 C \varepsilon+C 3$ & 4. $559+C O$ & $6.403+05$ \\
\hline $4 . C 00$ & $3.377+C 5$ & $2.776+C 3$ & $6.7 C 7+C C$ & $3.349+05$ \\
\hline $5.00 \mathrm{C}$ & $1.951+05$ & $2.48 C+C 3$ & $8 \cdot 360+C 0$ & $1.926+05$ \\
\hline $6 . \mathrm{COO}$ & $6+C 5$ & $2.222+c 3$ & $5 . \$ 40+C 0$ & $1.213+05$ \\
\hline $\mathrm{COO}$ & $+C 4$ & & & +04 \\
\hline C. $\mathrm{COO}$ & $3.412+c 4$ & $1.489+C 3$ & $1.520+C_{1}$ & $3.262+04$ \\
\hline $\mathrm{CCC}$ & 4. $\varepsilon 1 \varepsilon+C 4$ & $5.741+C 2$ & 1. $S \in 4+C 1$ & $4.718+04$ \\
\hline & $2.322+04$ & $6.869+02$ & $2.291+C 1$ & $2.251+04$ \\
\hline & 8. $C \varepsilon_{3}+C 3$ & $4 \cdot c 35+c 2$ & $2.735+C 1$ & $7.652+03$ \\
\hline$C . \mathrm{COO}$ & $04+C 3$ & $2 \cdot \epsilon C 3+C 2$ & $2.595+01$ & $3.513+03$ \\
\hline
\end{tabular}

\begin{tabular}{|c|c|c|c|c|}
\hline $\begin{array}{r}\text { ENERGY } \\
\text { (KEV) }\end{array}$ & TOTAL & $\begin{array}{l}\text { COHER. } \\
\text { SCATT. }\end{array}$ & $\begin{array}{l}\text { I NCOH. } \\
\text { SCATT. }\end{array}$ & $\begin{array}{r}\text { PHCTO } \\
\text { EFFECT }\end{array}$ \\
\hline . C 10 & $t .9 \in 5+C 7$ & 4. $C 47+C 3$ & $1.87 t-04$ & $6.965+07$ \\
\hline .015 & $4.644+07$ & 4. $c 47+c 3$ & $4.214-04$ & $4.643+07$ \\
\hline $.0<0$ & $3.483+C 7$ & $4 \cdot C 47+c 3$ & $7.478-04$ & $3.482+07$ \\
\hline .030 & $2.322+c 7$ & 4. $147+C 3$ & $1.677-03$ & $2.322+07$ \\
\hline .040 & $142+C 7$ & $4 \cdot C 47+03$ & $2.571-c 3$ & $1.741+07$ \\
\hline.$C 50$ & $1.393+C 7$ & $4 \cdot C 47+C 3$ & $4 \cdot \in 25-c 3$ & $1.393+07$ \\
\hline .060 & $1.161+07$ & $4.046+c 3$ & $6.637-03$ & $1.161+07$ \\
\hline & $10+c t$ & $4 \cdot C 4 t+C 3$ & 1.17 & $8.706+06$ \\
\hline .100 & $69+06$ & $4.045+c$ & 1. & $6.965+06$ \\
\hline $.15 \mathrm{C}$ & $4.647+C t$ & 4. $c 43+c 3$ & 4.C 1 & $1+06$ \\
\hline .200 & $3.48 t+c t$ & $4 . c 41+C 3$ & $7.013-02$ & $3.482+06$ \\
\hline .300 & $2.32 t+c t$ & $4 \cdot c 33+c 3$ & $1.520-\mathrm{Cl}$ & $2 \cdot 322+06$ \\
\hline .400 & $6 . t 18+0 t$ & 4. $C 22+C 3$ & $2.55 \mathrm{~s}-\mathrm{Cl}$ & $6.614+06$ \\
\hline .500 & $4.721+c t$ & 4. $C C S+C 3$ & $3.8 \varsigma \varsigma-\mathrm{Cl}$ & $4.717+06$ \\
\hline$\cdot \in C C$ & $3.508+c 6$ & $3.553+03$ & $5.383-01$ & $3.504+06$ \\
\hline .800 & $2 t+0 t$ & $3.554+c 3$ & $8.747-01$ & $2.122+06$ \\
\hline 1.000 & $07+0 t$ & 3.907003 & 1.23 & $1.4 C 3+06$ \\
\hline $1.5 \mathrm{CO}$ & $+C 5$ & $3.75 c+C 3$ & $2.146+C C$ & $6.303+05$ \\
\hline & $3.467+05$ & 3.582 & & +05 \\
\hline 2.000 & $6 \cdot z \in 8+C 5$ & $3.211+c 3$ & $4.788+C C$ & $6.336+C 5$ \\
\hline 4.000 & $3.541+C E$ & $2.865+c 3$ & $6.518+00$ & $3.512+05$ \\
\hline $5 . \mathrm{COC}$ & $2.046+C 5$ & $2.555+C 3$ & $8.211+C C$ & $2.020+05$ \\
\hline $6 . \mathrm{COO}$ & $1.299+05$ & $2.2 \& 7 * C 3$ & $5.841+\mathrm{CO}$ & $1.276+05$ \\
\hline \&.COC & $6.308+04$ & $1.858+C 3$ & $1.281+C 1$ & $6.121+04$ \\
\hline 10.000 & 3. $\mathrm{EC} 1+\mathrm{C}_{4}$ & $1.535+03$ & $1.527+01$ & $3.446+04$ \\
\hline 000 & $c 36+c 4$ & $1 . C 07+C 3$ & 1. $\subseteq 78+c 1$ & $4.933+04$ \\
\hline $\mathrm{COO}$ & $2.4 \epsilon 6+c 4$ & $7 . c 58+c 2$ & $2.3 c 9+01$ & $2.392+04$ \\
\hline & $554+C 3$ & $.16 S+C 2$ & $2.761+C 1$ & $109+03$ \\
\hline
\end{tabular}

\begin{tabular}{|c|c|c|}
\hline $\begin{array}{l}\text { NEREY } \\
\text { (KEV) }\end{array}$ & CTAI $>$ & $\begin{array}{l}\text { COHER. } \\
\text { SCATT. }\end{array}$ \\
\hline 50. & $1.553+03$ & \\
\hline 60. & $1.243+03$ & $1.281+02$ \\
\hline & $5 \mathrm{SC}+02$ & $.694+01$ \\
\hline 100 & $77+$ & \\
\hline & $.276+0$ & $3+01$ \\
\hline & $t C 8+0=$ & \\
\hline & & \\
\hline 40 & $1+$ & +00 \\
\hline & & \\
\hline & & \\
\hline & & 01 \\
\hline 100 & & \\
\hline & 1.5 & 2.6 \\
\hline & $\cdot 3$ & 1.4 \\
\hline & & \\
\hline & $1.263+$ & 3.7 \\
\hline 5 & 1.28 & \\
\hline & & \\
\hline & $.4 \mathrm{C}$ & 5.2 \\
\hline & & \\
\hline & .67 & \\
\hline & & \\
\hline & & \\
\hline & & \\
\hline & & \\
\hline & & \\
\hline & & \\
\hline & $1832+5$ & $5.046-0$ \\
\hline
\end{tabular}

ENERG

(KEV

50
60
80

80.
100.

150 .

200 .

300 .

400 .

500 .

600

800 .

1000 .

1500

3000

4000 .

5000

6000

8000 .

10000 .

15000 .

20000

30000

40000 .

$500 \mathrm{CO}$

60000

80000 .

100000 .

$\begin{array}{rr}\text { TCTAL } & \text { COHER. } \\ 2.124+03 & 1.806+02 \\ 1.324+03 & 1.322+02 \\ 2.655+03 & 7.935+01 \\ 1.542+03 & 5.278+01 \\ 5.518+02 & 2.467+01 \\ 2.726+C 2 & 1.423+01 \\ 1.109+02 & 6.484+00 \\ 6.454+01 & 2.692+00 \\ 4.553+C 1 & 2.381+0 C \\ 3.61 C+01 & 1.661+00 \\ 2.631+01 & 9.403-01 \\ 2.143+01 & 6.040-01 \\ 1.603+01 & 2.697-01 \\ 1.415+01 & 1.521-01 \\ 1.258+01 & 6.777-02 \\ 1.293+01 & 3.817-02 \\ 1.325+01 & 2.444-02 \\ 1.367+01 & 1.698-02 \\ 1.456+C 1 & 9.559-03 \\ 1.535+01 & 6.120-03 \\ 1.7 C 4+C 1 & 2.721-C 3 \\ 1.869+C 1 & 1.531-03 \\ 2.164+C 1 & 6.806-04 \\ 2.368+01 & 3.829-04 \\ 2.510+C 1 & 2.451-04 \\ 2.616+C 1 & 1.702-04 \\ 2.780+C 1 & 9.575-05 \\ 2.516+C 1 & 6.128-05 \\ & \end{array}$

INCOH

$3.14 \mathrm{C}+01$

$3.218+01$

2. $249+C$

3. $129+C$

$2.545+C 1$

2. $630+01$

2. $286+C$

2. $C 36+0$

1. $7 \subseteq 6+C$

1. $\epsilon 18+01$

$1.31 \varepsilon+C$

1. $12 t+C 1$

$\varepsilon \cdot \varepsilon 56+C c$

$7.386+C C$

$t .378+0 C$

$5.637+00$

$4.610+0 C$

3. $525+0 \mathrm{C}$

2. $5 C 3+0 C$

2. $329+0 C$

1. $653+0$

1. $344+C C$

$1.1<1+0 C$

9. $655-01$

$7.6 C 7-01$
t. $311-C$
PHOTO

EFFECT

1. $7 \varepsilon 7+C 3$

1. $C \varepsilon z+03$

2. $4 \varepsilon 1+03$

$1 \cdot 3 \mathrm{C} 3+\mathrm{C} 3$

2. $17 \mathrm{c}+02$

$2.175+02$
$7.41 \epsilon+01$

2. $54 \mathrm{C}+\mathrm{Cl}$

2. $C 42+01$

1. $32 \varepsilon+01$

t. $\triangle E 1+O C$

$4.374+C C$

2. $\mathrm{CC} 2+00$ 4.379-01

$1.21 \mathrm{C}+\mathrm{CC}$

t. $34 C-01$

4. $1 \in 5-01$

3. $C \in S-01$

2. 4 I 4-0 01

$1 . \in 82-01$

1. $2 \varepsilon 5-01$

E. $\mathrm{C} 4 \mathrm{t}-\mathrm{C} 2$

5.844-02

$3.771-\mathrm{C} 2$

2. 782-C2

2. $2 \mathrm{C}_{4}-\mathrm{O} 2$

1. $\varepsilon 24-C 2$

1. $357-02$

1. $\mathrm{C}$ \& $-\mathrm{C} 2$
PAIR PRCC.

1. $446+C C$

3. $3 \mathrm{C} 2+C \mathrm{C}$

$4.851+C C$

$6.252+C C$
$7.428+C C$

5. $361+C C$

1. $\mathrm{CSO}+\mathrm{Cl}$

1. $3 \varepsilon_{1}+\mathrm{C} 1$

$1.540+C 1$

$2.160+C 1$

2. $317+01$

$2.438+01$

$2.623+C 1$

2. $7 \in 9+C 1$
FHCTC

EFFECT

$1.912+C 3$

1. $1 \in \mathrm{C}+\mathrm{C} 3$

2. $5 \varepsilon \overline{7}+03$

$1.457+03$

$2.25 \mathrm{C}+\mathrm{C} 2$

$7.813+C 1$

$3.7 \equiv 5+01$

. $1 \in 2+C 1$

$7.411+00$

$4 \cdot 643+0 C$

$2.123+0 \mathrm{C}$

$1.282+00$

t.710-01

$4.4 \mathrm{C} c-\mathrm{C} 1$

.245-01

$2.553-01$

1. $35 \varepsilon-01$

$\varepsilon .5 C 4-c 2$

$t .177-02$

$3.58 \epsilon-02$

2. $541-02$

$2.325-C 2$

$1.52 \varepsilon-02$

$1.434-02$

$1.14 \bar{z}-c^{2}$
PAIF

PRCE.

$4.587-\mathrm{Cl}$

$1.460+O C$

$5 . C 69+C C$

$t .51 s+C C$

$7.764+C C$

$1.128+01$

$1.4 \mathrm{C} 5+\mathrm{Cl}$

1. $t 3 c+c 1$

$1.550+C 1$

$2.355+C 1$

$2.518+C 1$

$2.702+01$

2. $\varepsilon 52+C 1$

\begin{tabular}{|c|c|c|}
\hline EERGY & ZT AL & \\
\hline & & \\
\hline 50. & $2.210+03$ & $1.864+02$ \\
\hline 60. & $1.375+03$ & $1.365+02$ \\
\hline 80. & $2.827+C 3$ & $8.190+01$ \\
\hline 100. & $1.614+03$ & $5.446+01$ \\
\hline 150. & $5.776+c z$ & $2.546+01$ \\
\hline 200 . & $853+02$ & $1.468+01$ \\
\hline 300 . & $157+C 2$ & $6.689+00$ \\
\hline 400 . & & 3.809 \\
\hline 50 & & OC \\
\hline 600 . & 3.722 & 1.7 \\
\hline 800 . & & \\
\hline 1000 . & $2.153+c 1$ & 6.2 \\
\hline 1500 & $1.634+$ & $2-01$ \\
\hline & 1.44 & \\
\hline 3000 . & $1.325+$ & 6.95 \\
\hline $40 \mathrm{CO}$ & $1.312+0$ & 3.93 \\
\hline & & \\
\hline 6000 . & 1.374401 & 1.7 \\
\hline 8000 & 1.463 & \\
\hline & & \\
\hline 15000 & $1.753+$ & 2.8 \\
\hline & & \\
\hline & & \\
\hline 40000. & & \\
\hline & $2.547+$ & \\
\hline & $2.667+01$ & \\
\hline 80000 & $2 . \varepsilon 45+C 1$ & $9.875-05$ \\
\hline & & \\
\hline
\end{tabular}

INC OH .

SCATT

$3.173+C$

$3.252+01$

3. $28 t+c 1$

3. $3 \mathrm{C} 4+\mathrm{C}$

$3.1 \in 7+C 1$

C. $644+0$

$2.416+C$

$2.221+0$

2. $C \in 3+C$

1. $E \equiv S+C$

$1.235+0$

1. $14 C+C$

$8.571+0 C$

$7.482+0 C$

$6.461+0 C$

$5.71 \mathrm{C}+\mathrm{CC}$

$4.670+0 C$

$3.57 t+C C$

$2.541+C C$

$2.355+C C$

$1.715+C C$

1. $3 \in 2+0$

$1.136+0$

5.780-C

7. $7 \mathrm{Cb}-\mathrm{C}$

$6.393-01$
FHCTC

EFFEC T

$1.551+03$

. $2 \mathrm{C} E+03$

. $712+C_{3}$

1. $52 \overline{7}+C$

. $2 C E+C$

$\varepsilon .23 \varsigma+01$

2. $c 45+01$

$2.2 \varepsilon 4+01$

$1.4 \varepsilon \varepsilon+C$

$4.5 C 5+O C$

$2.24 t+C C$

$1.35 t+C$

7.0 St-0

$4.6 \in 2-01$

$3.42 \mathrm{C}-01$

$2 . t c \varepsilon-01$

$1.875-01$

$1.435-01$

t. $525-02$

$4.21 \mathrm{C}-02$

. $1 \mathrm{C} t-02$

$2.460-02$

$1.514-C_{2}$

$1.2 \mathrm{C5}-\mathrm{C2}$
PAIF

PRCE.

$4.675-C 1$

$1.55 \mathrm{~s}+\mathrm{CC}$

5. $136+C C$

$6.531+C C$

$7.743+C C$

9. $762+C \mathrm{C}$

$1.140+C 1$

$1.450+01$

$1.681+C 1$

. $\mathrm{C} 2 \mathrm{O}+\mathrm{C} 1$

$2.256+C 1$

$2.431+C 1$

$2.767+C 1$

2. $507+01$ 


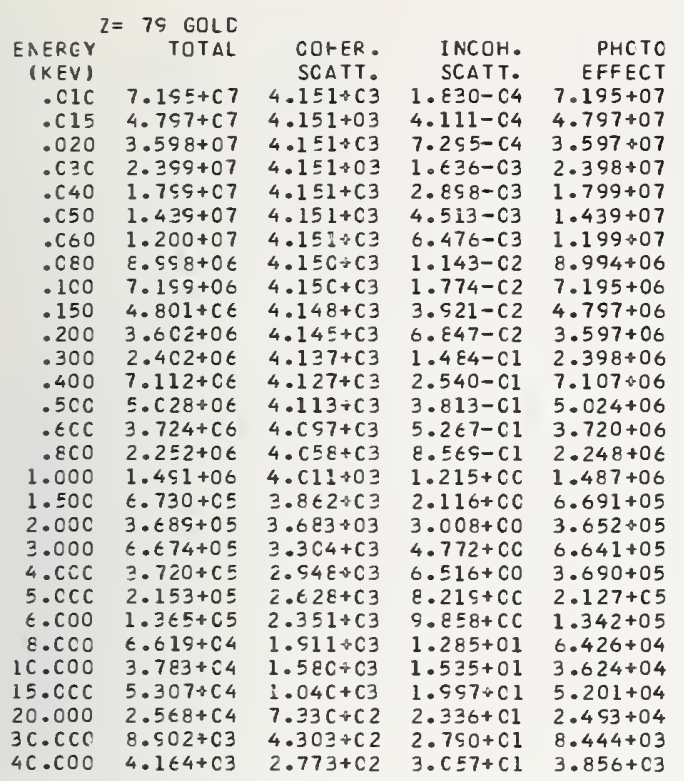

$Z=E C$ MERCURY

\begin{tabular}{|c|c|c|c|c|}
\hline $\begin{array}{r}\text { EA EREY } \\
\text { (KEV) }\end{array}$ & TOTAL & $\begin{array}{l}\text { COHER. } \\
\text { SCATT. }\end{array}$ & $\begin{array}{l}\text { I NCOH. } \\
\text { SCATT. }\end{array}$ & $\begin{array}{r}\text { PHCTC } \\
\text { EFFECT }\end{array}$ \\
\hline .010 & $7.455+C 7$ & $4.257+C 3$ & $2.111-C_{4}$ & $7.494+07$ \\
\hline C1E & $4.557+07$ & $4.257+C 3$ & $4.741-C 4$ & $4.956+07$ \\
\hline .020 & $3.748 * C 7$ & $4.257+C 3$ & $8.413-04$ & $3.747+07$ \\
\hline.$C 3 C$ & $2.459+C 7$ & $4.257+C 3$ & $1.8 \varepsilon \epsilon-C 3$ & $2.498+07$ \\
\hline.$C 4 C$ & $1.874+C 7$ & $4.257+C 3$ & $3.341-C 3$ & $1.874+07$ \\
\hline.$C 5 C$ & $1.455+07$ & $4.257+C 3$ & $5.2 \mathrm{Cl}-\mathrm{C}_{3}$ & $1.455+07$ \\
\hline.$C 60$ & $1.249+07$ & $4.257+03$ & $7 \cdot 4 \in 2-03$ & $1.249+07$ \\
\hline - $C \varepsilon C$ & 5.372 & $4.25 t+C 3$ & $1.317-C_{2}$ & $9.368+c 6$ \\
\hline .100 & $7.455+06$ & $4.25 t+C 3$ & 2. $C 42-c 2$ & $7.494+06$ \\
\hline $.15 \mathrm{C}$ & $E . C C_{1}+C E$ & $4.253+03$ & $4.5 \mathrm{Cc}-\mathrm{C} 2$ & $4.996+06$ \\
\hline $.2 \mathrm{CO}$ & $3.751+C \epsilon$ & $4.251+c 3$ & 7. $\varepsilon \in 5-c 2$ & $3.747+06$ \\
\hline 300 & $2.5 C 2+06$ & $4.242+C ?$ & 1. $7 C 1-C 1$ & $2.498+06$ \\
\hline .400 & $7.437+06$ & $4.231+03$ & 2. $5(3-01$ & $7.432+06$ \\
\hline .500 & 5.30 & $4.217+03$ & $4.345-01$ & $5.302+06$ \\
\hline$\in O C$ & 3.54 & 4.20 & $4-01$ & +06 \\
\hline $.80 \mathrm{C}$ & $2.359+$ & $C 3$ & $-\mathrm{Cl}$ & $.395+06$ \\
\hline $1 . \mathrm{CCO}$ & $1.5 \subseteq 1+06$ & $4.110+03$ & $1.359+C C$ & $1.587+06$ \\
\hline 1.500 & $7.174+C E$ & 3. $.555 * C 3$ & $2.3 C 7+C C$ & $7.134+05$ \\
\hline 2.000 & $3.916+05$ & $3.771+C 3$ & $3.2 \mathrm{CO}+\mathrm{CO}$ & $3.878+05$ \\
\hline 3. $\mathrm{COO}$ & $7.017+05$ & $2.383+C 3$ & $4.9 C 7+C C$ & $6.983+05$ \\
\hline 4.000 & ב.SC $5+05$ & $3 \cdot C 2 C+03$ & $6.608+00$ & $3.874+05$ \\
\hline $5 . \mathrm{COC}$ & $2.266+C E$ & $2 . \in S_{4}+C 3$ & $8 \cdot 3 \mathrm{C} 2+C \mathrm{C}$ & $2.239+C 5$ \\
\hline 6.000 & 1. & $2.412+C 3$ & $9.953+C C$ & $1.413+C 5$ \\
\hline $\mathrm{SCC}$ & 6.55 & $3+c 3$ & $\mathrm{~S}+\mathrm{Cl}$ & +04 \\
\hline 10. & 3. $568+c 4$ & $1 . t 25+c 3$ & $1.552+C 1$ & $3.804+04$ \\
\hline & $5.536+C 4$ & $1.07 \overline{2}+C 3$ & $2.013+C 1$ & $5.427+04$ \\
\hline $\mathrm{CCC}$ & $2.711+C 4$ & $7.563+c 2$ & $2.351 \div \mathrm{Cl}$ & $2.633+04$ \\
\hline & & $4.43 t+C 2$ & $2.812+\mathrm{Cl}$ & $8.972+03$ \\
\hline & 3 & $z \cdot \varepsilon 5 \varepsilon+C 2$ & $C 24+C 1$ & $4.056+03$ \\
\hline
\end{tabular}

\begin{tabular}{|c|c|c|}
\hline \multicolumn{3}{|c|}{$Z=\varepsilon I$ THALLIUN } \\
\hline $\begin{array}{l}\text { ENERGY } \\
\text { (KEV) }\end{array}$ & TOTAL & $\begin{array}{l}\text { CCIER. } \\
\text { SCATT. }\end{array}$ \\
\hline. $\mathrm{ClC}$ & $7.840+C 7$ & $4.364+C 3$ \\
\hline .015 & $5.227+C i$ & $4.364+c 3$ \\
\hline . $C 2 \mathrm{C}$ & $3.520+c 7$ & $4 \cdot 3 \in 4+C 3$ \\
\hline .030 & $2 . \epsilon 14+07$ & $4 \cdot 364+C 3$ \\
\hline .040 & $1.5 \in 0 \div C 7$ & 4.364403 \\
\hline .050 & $1.56 \varepsilon+C 7$ & $4 \cdot 3 \in 4+03$ \\
\hline .060 & $1.307 \div 07$ & $4 \cdot 3 \in 4+C 3$ \\
\hline .080 & $5.804+C t$ & $4.363+c 3$ \\
\hline$\cdot 100$ & $7.244+C t$ & $4 \cdot 3 \in 2+C 3$ \\
\hline .150 & $5 \cdot 231+0 t$ & $4.360+03$ \\
\hline .200 & $3.524 \& C t$ & $4 \cdot 357+C 3$ \\
\hline $.3 \mathrm{CO}$ & $2 . \epsilon 1 \varepsilon+c \epsilon$ & $4 \cdot 347+C 3$ \\
\hline .400 & $7.537+0 t$ & $4 \cdot 335+C 3$ \\
\hline .500 & $5.530 * C \epsilon$ & $4.319+C 3$ \\
\hline .600 & $4 \cdot 1 C 2+C t$ & $4.301+C 3$ \\
\hline .800 & $2.5 C 7+C E$ & $4.256+C 3$ \\
\hline 1. $\mathrm{COO}$ & 1. $674+0 \epsilon$ & $4.202+C 3$ \\
\hline 1.500 & $7 . t 1 \epsilon+05$ & $4.040+C 3$ \\
\hline 2.000 & $4.1 \in C+C 5$ & $3.851+C 3$ \\
\hline $3 . \mathrm{COC}$ & $7 \cdot 3 \in 2+C 5$ & $3.457+C 3$ \\
\hline $4 . C 00$ & $4.07 t+C 5$ & 3. $C E S+C 3$ \\
\hline 5.000 & $2.3 \varepsilon 0+0 \leq$ & $2.759+C 3$ \\
\hline $6 . C 00$ & $1.513+C E$ & $2.472+C 3$ \\
\hline 8.000 & $7.335+04$ & $2.014+03$ \\
\hline 10.000 & $4.185+04$ & $1.669+03$ \\
\hline $15 . \mathrm{COC}$ & $5.0 \varepsilon 4+C_{4}$ & $1.1 C 7+03$ \\
\hline $2 \mathrm{C} .000$ & $2.824 \div C 4$ & 7.802402 \\
\hline $3 \mathrm{C} \cdot \mathrm{COC}$ & $5.859+C 3$ & 4. $5 \in \subseteq+C 2$ \\
\hline 40.000 & $4.625+03$ & $2.94 C+02$ \\
\hline
\end{tabular}

I ACCH.

SCATT.

5. $055-04$

S. $\mathrm{C} 42-\mathrm{C}_{4}$

2. $C 27-03$

$3.5 \mathrm{SO}-\mathrm{C} 3$

$5.589-C 3$

8. C18-C3

1. $415-C 2$

2. $154-C 2$

4. $842-C 2$

1. 825-01

3.111-01

$4.653-\mathrm{Cl}$

6. $4 \mathrm{Cl}-\mathrm{Cl}$

1. $\mathrm{C} 32+\mathrm{CO}$

$1.447+C C$

2. $434+C C$

$3.34 t+C C$
$5.052+O C$

$6.758+C$

$8.453+00$

$1 . \mathrm{Cl1}+\mathrm{OI}$

1. $315+01$

1. 5 ES+ C

$2.032+C 1$

$2.373+01$

3. $113+01$

\begin{tabular}{|c|c|c|}
\hline $\begin{array}{r}\text { ENERGY } \\
\text { (KEV) }\end{array}$ & TCTAL & $\begin{array}{l}\text { COMER. } \\
\text { SCATT. }\end{array}$ \\
\hline 50. & $2.317+03$ & $1.922+02$ \\
\hline 60. & $1.443+03$ & $1.406+02$ \\
\hline 80. & $6.55 t+02$ & $\varepsilon .438 \div 01$ \\
\hline 100. & $1.682+03$ & $5.610+01$ \\
\hline 150. & $6 . C 54+02$ & 2.621601 \\
\hline 200 & $2.986+02$ & $1.512+01$ \\
\hline 300. & $1.2 C 5+02$ & $6.885+00$ \\
\hline 400. & $6.594+C 1$ & $3.920+00$ \\
\hline 500 . & $4.913+01$ & $2.527+00$ \\
\hline 600 . & $3.841+01$ & $1.764+00$ \\
\hline 800 . & $2.774+C_{1}$ & $9.981-01$ \\
\hline 1000 & $2.246+01$ & $6.411-01$ \\
\hline 1500. & $1 . \epsilon 68 * 01$ & $2.863-01$ \\
\hline 2000 . & $1.477+0:$ & $1.615-01$ \\
\hline 3000 . & $1.353 \div 01$ & $7.193-02$ \\
\hline 4000 . & $1.335+01$ & $4.051-02$ \\
\hline 5000 . & $1.362+01$ & $2.594-02$ \\
\hline 6000 . & $1.4 C 1+01$ & $1.8 \mathrm{C} 3-\mathrm{C} 2$ \\
\hline 8000 . & $1.492+01$ & $1.015-02$ \\
\hline 10000 & $1.586+c 1$ & $6.496-03$ \\
\hline 15000 . & $1.798+C 1$ & $2.888-03$ \\
\hline 20000 . & $1.974+C 1$ & $1.625-03$ \\
\hline 30000 . & $2.245+01$ & $7.224-04$ \\
\hline 40000 . & $2.444+C 1$ & $4.064-04$ \\
\hline 50000 . & $2.597 \approx 01$ & $2.601-04$ \\
\hline$\epsilon 0000$. & $2.72 c+c 1$ & $1.807-04$ \\
\hline 80000 . & $2.502+01$ & $1.016-04$ \\
\hline 00000 . & Z. C $2 S+C 1$ & $6.504-05$ \\
\hline
\end{tabular}

INCOH.

3. $2 \mathrm{C} E+\mathrm{C} 1$

3. $288+01$

3. $321+C$

3. $340+C 1$

3. $2 \mathrm{C} 4+01$

3. $\mathrm{Cl} \mathrm{S}+\mathrm{Cl}$

$2.658+01$

2. $447+C 1$

2. $24 S+C 1$

2.

1. $\in \in O+01$

$1.352+01$

1. $155+C 1$

S. $C E 6+C C$

7. $578+C C$

t. $543+00$
$5.783+C C$

5. $723+C \mathrm{C}$
$4.730+0 \mathrm{C}$

4. $C 27+C C$

$2.5 .78+0 C$

2. $389+C C$

1. $727+\mathrm{CC}$

1. $375+C C$

$9.506-C 1$

7. $8 \mathrm{C} 4-\mathrm{Cl}$

t. $475-\mathrm{Cl}$
PHOTO

. $C \subseteq 2+C 3$

1. $2 \in S+03$

5. $7 \varepsilon \mathrm{C}+02$

1. $5 \subseteq 3+C 3$

$5.472+0$
$2.533+C 2$

2. $t \in \hat{Z}+01$

4. $154+01$

$2.411+01$

E. $317+C$ C

$5.21 \epsilon+00$

2. $3 \varepsilon \epsilon+C$

$7.522-01$

4. $53 \mathrm{i}-\mathrm{Cl}$

$3.63 \mathrm{C}-01$

2. $\varepsilon 54-C$

1. 517-C

c. $4 \subseteq 1-C_{2}$

E. $\varepsilon \subseteq 1-C 2$

$4.44 E-02$
$3.27 \varepsilon-02$

2. 5 ऽ $6-C 2$

$2.14 c-02$

$1.5 c \varepsilon-02$

1. $272-02$
PAIR

4. $865-C 1$

1. $623+C C$

$5.274+C C$

$6.688+O C$

7. $515+C C$
5. $584+C C$

$1.167+C 1$

$1.451+01$

1. $728+C 1$

2. $\mathrm{C} 66+\mathrm{C} 1$

2. $480+01$

$2.619+C 1$

2. $823+C 1$

2. $563+C 1$

\begin{tabular}{|c|c|c|}
\hline $\begin{array}{l}\text { ENERGY } \\
\text { (KEV) }\end{array}$ & TCTAL & $\begin{array}{l}\text { COFER } \\
\text { SCATT. }\end{array}$ \\
\hline 50. & 2.445803 & $1.979+02$ \\
\hline$\in 0$. & $1.521+03$ & $1.447+02$ \\
\hline 80. & $7.308+02$ & $2.679+01$ \\
\hline 100. & $1.744+C 3$ & $5.769+01$ \\
\hline 150. & $6.25 C+02$ & $2.695+01$ \\
\hline 200 . & $3.110+C 2$ & $1.553+01$ \\
\hline 300. & $1.256+02$ & $7.074+00$ \\
\hline $4 \mathrm{CO}$ & $7.273+\mathrm{Cl}_{1}$ & $4.028+00$ \\
\hline 500. & $5.089+01$ & $2.597+00$ \\
\hline 600. & $\exists . \subseteq \in 3+01$ & $1.812+00$ \\
\hline 800 . & $2.849+C 1$ & $1.025+0 \mathrm{C}$ \\
\hline 1000 . & $2.3 \mathrm{CC}+01$ & $6.58 t-\mathrm{Cl}$ \\
\hline 1500 . & $1.7 C^{2}+01$ & $2.941-01$ \\
\hline 2000 . & $1.50 t+C 1$ & $1.658-01$ \\
\hline 3000 . & $1.379+01$ & $7.388-02$ \\
\hline 4000. & $1.365+C 1$ & $4.161-02$ \\
\hline 5000 . & $1.390+C 1$ & $2.665-02$ \\
\hline 6000 . & $1.43 c+01$ & $1.851-02$ \\
\hline 8000 . & $1.527+C 1$ & $1.042-02$ \\
\hline 10000 . & $1 \cdot t 25+01$ & $6.671-03$ \\
\hline 15000 & 1. $\varepsilon 4 \epsilon+C 1$ & $2.966-03$ \\
\hline 20000 . & 2. $C 27+01$ & $1.669-03$ \\
\hline 30000 . & 2.303601 & $7.420-04$ \\
\hline 40000 & $2.5 C 7+C 1$ & $4.174-04$ \\
\hline 50000 . & 2. $t \in E 5+01$ & $2.672-c_{4}$ \\
\hline$\epsilon 0000$. & $2.790+01$ & $1.855-04$ \\
\hline 0000 . & $2.573+C 1$ & $1.044-04$ \\
\hline & & $6.680-05$ \\
\hline
\end{tabular}

INCOH.
SCATT
$3.237+C 1$
$3.32 C+C 1$
$3.355+C 1$
$3.378+01$
$3.241+C 1$
$3 . C 54+C 1$
$2.730+C 1$
$2.477+C 1$
$2.277+01$
$2.115+C 1$
$1 . E 66+01$
$1.681+01$
$1.36 C+C 1$
$1.16 S+C 1$
$5.2 C 1+0 C$
$7.674+0 C$
$6.626+C C$
$5 . E 56+C C$
$4.75 C+C C$
$4 . C 78+O C$
$3 . C 16+C C$
$2.415+C C$
$1.759+0 C$
$1.357+O C$
$1.165+0 C$
$1 . C C 3+0 C$
$7.5 C 3-O 1$
$6.557-C 1$

FHOT EFFEC T
$2.21 \mathrm{C}+03$ 1. $344+03$ t. $1 \mathrm{C} 4+02$ 1. $6 \leq 3+03$ $5.65 t+02$ 2. $64 c+0$ 5. $123+01$ 4. $3 \subseteq 3+01$ $2.5 \leq z+01$ 1. $\epsilon \in \bar{T}+0$ ह. $21 \mathrm{C}+00$ 5. $5 \equiv C+00$ 2. $5323+00$ $1.52 \varepsilon+00$ 7. $\subseteq \varepsilon \in-0$ 5. $235-01$ $3.850-C 1$ $3.025-C 1$ 2. 1 C $3-01$ 1. $\in C 5-01$ 1.C $3-0$ 7. $2 \& 2-C$ 4. $t 54-02$ 3. $4 \in 1-02$ 2. $741-C 2$ 2. $2 t \varepsilon-02$ 1. $\in \varepsilon \epsilon-C 2$ 1. 342-02
PAIR

PRCE.

5. $C \in 1-C 1$

1. $t 71+C C$

3. $712+C C$

$5.411+C C$
$6.862+C C$

ع.127+CC

1. $C 25+C 1$

1. $2 \mathrm{CC}+\mathrm{C} 1$

1. $534+C 1$

1. $778+C_{1}$

$2.123+C 1$

$2.364+C 1$

2. $545+C 1$

2. $t 87+C 1$

2. $E \subseteq 2+C 1$

\begin{tabular}{|c|c|c|c|}
\hline $\begin{array}{l}\text { ENERGY } \\
\text { (KEV) }\end{array}$ & TCTAL & $\begin{array}{l}\text { COHER } \\
\text { SCATT. }\end{array}$ & $\begin{array}{l}\text { INCCH. } \\
\text { SCATT. }\end{array}$ \\
\hline 50. & $2.579+C 3$ & $2.03 t+0 z$ & 3. $2 \epsilon \varepsilon+C 1$ \\
\hline 60. & $1.605+03$ & $1.489+02$ & $3.355+01$ \\
\hline 80. & $7.6 \subseteq \subseteq+02$ & $8.923+01$ & $\equiv \varsigma 2+C_{1}$ \\
\hline 100. & $1.818+03$ & $5.930+01$ & $3.41 \epsilon \$ 01$ \\
\hline 150 . & $t .58 c+0 z$ & $2.7 \in G+C 1$ & $\exists .280+C 1$ \\
\hline 200. & $3.258+02$ & $1.596+01$ & 3. $\operatorname{CS} 1+C_{1}$ \\
\hline 300. & $1.310+02$ & $7.267+00$ & 2. $T \in 3+C 1$ \\
\hline 400. & $7.553+C 1$ & $4 \cdot 137+00$ & $2.5 c \varepsilon+c 1$ \\
\hline 500 . & $5.2 \epsilon 4+01$ & $2.667+00$ & $2.3 C 5+C 1$ \\
\hline 600. & $4.087+01$ & $1.861+00$ & $2.141+01$ \\
\hline 800 . & $2.525+01$ & $1.053+00$ & 1. $\varepsilon \varepsilon s+C 1$ \\
\hline 1000 . & $2.353+01$ & $6.764-01$ & 1. $7 \mathrm{C} 2 * \mathrm{C} 1$ \\
\hline 1500. & $1.73 t+\mathrm{Cl}$ & $3.021-01$ & $1.387+01$ \\
\hline 2000. & $1.535 \div 01$ & $1.703-01$ & $1.184: 01$ \\
\hline 3000 . & $1.405+C 1$ & $7.588-02$ & $5.316+C C$ \\
\hline 4000 . & $1.3 s_{1+01}$ & $4.273-02$ & $7.77 C+C C$ \\
\hline $50 \mathrm{co}$. & $1.416+01$ & $2.737-02$ & $6.7 \mathrm{css} C \mathrm{C}$ \\
\hline 6000 . & $1.456+01$ & $1.901-02$ & $5.53 C+0 \mathrm{C}$ \\
\hline 8000 & 1.552601 & $1.070-02$ & $4.25 C+C c$ \\
\hline 10000 . & $1.651+01$ & $6.852-03$ & $4.129+C C$ \\
\hline 15000. & $1 . \varepsilon \varepsilon 0+C 1$ & $3.047-03$ & 3. CE4 4 CC \\
\hline 20000 . & 2. $c 73+01$ & $1.714-03$ & $2.450+00$ \\
\hline 30000 . & $2.3 t t+C 1$ & $7.620-04$ & $1.781+C C$ \\
\hline 40000 . & $2.577+01$ & $4.287-04$ & $1.414 * 00$ \\
\hline 50000 . & $2.739+C 1$ & $2.744-04$ & $1.180+C \mathrm{C}$ \\
\hline 60000 . & $2.868 * \mathrm{Cl}$ & $1.906-04$ & 1. C16*CC \\
\hline 80000 . & $3 . c 62 \div 01$ & $1.072-04$ & $\varepsilon . C C 2-01$ \\
\hline 00000 & $3.15 \subseteq+01$ & $6.861-05$ & $\epsilon, \epsilon \equiv 5-C 1$ \\
\hline
\end{tabular}

FHCT'

EFFEC T

2. $342+03$

$1.423+03$
$6.467+02$

1. $7 \bar{\angle} 5+C 3$

5. $S E E+02$

2. $7 E S+02$

S. $\in 14+01$

4. $t 32+01$

2. $\in \subseteq 2+01$

1. $7 \in \mathrm{C}+\mathrm{Cl}$

$\therefore C 1+0$

$5.837+00$

$2.67 C+C C$

$1.61 \mathrm{C}+0 \mathrm{C}$

E. $41 \mathrm{C}-01$

$5.51 t-C 1$

4. $053-01$

$3.1 \varepsilon 5-01$

2. $215-\mathrm{C}$

1. $6 \mathrm{~S}_{1-01}$

1. C 57-01

7.671-02

4. $54 t-C 2$

3. $647-02$

2. $8 \varepsilon \varepsilon-02$

$2.3 \subseteq C-C 2$

1. $777-02$

1. $414-02$
PAIF

$5.213-C$

1. $725+C C$

5. $547+C C$

7. $\mathrm{C} 15+\mathrm{CC}$

E. $2 \mathrm{SI}+0 \mathrm{C}$

1. $C 43+C 1$

$1.220+C 1$

1. $821 \div \mathrm{C} 1$

$2.183+\mathrm{Cl}$

$2.431+C 1$

$2.61 \mathrm{C}+\mathrm{C} 1$

2. $764+C 1$

2. $521+C 1$ 


\begin{tabular}{|c|c|c|c|c|}
\hline \\
\hline $\begin{array}{l}E \wedge E R G Y \\
\text { (KEV) }\end{array}$ & TOTAL & $\begin{array}{l}\text { COHER. } \\
\text { SCATT. }\end{array}$ & $\begin{array}{l}\text { INCOH. } \\
\text { SCATT. }\end{array}$ & $\begin{array}{r}\text { PHOTC } \\
\text { EFFECT }\end{array}$ \\
\hline .010 & $8.1 \varepsilon \varepsilon+07$ & $4.473+c 3$ & $2.250-04$ & $8.187+07$ \\
\hline & $5.455+c 7$ & $4.473+c 3$ & & $5.458+07$ \\
\hline . $C 20$ & $4.094+07$ & $4.473+C 3$ & $.126-C_{4}$ & $4.094+07$ \\
\hline & $730+07$ & $4.473+03$ & & $2.729+C 7$ \\
\hline .04 & $c 47+c$ & 4.47 & 3.624 & $47+07$ \\
\hline .05 & $1 . t 38+c 7$ & $4.472+C 3$ & 3 & $1.637+07$ \\
\hline & & & & \\
\hline & & 4.47 & & \\
\hline & $8.1 S 2+0 t$ & 4.471 & 2 & 8.1 \\
\hline & & & & \\
\hline & $4.098+06$ & $4.4 t$ & & 4.0 \\
\hline$\cdot 3$ & $2.734+06$ & $4.455 t$ & $\mathrm{Cl}$ & 2.72 \\
\hline & & & & \\
\hline .500 & E. $645+$ & 4.42 & $\mathrm{Cl}$ & $5.640+06$ \\
\hline .60 & $4.301 \%$ & $4.4 \mathrm{C}$ & 01 & \\
\hline & & & & \\
\hline 1.000 & $0+c 6$ & 4.3 & 0 & 1.76 \\
\hline & 8 & & & \\
\hline & & & & \\
\hline 3. & $\epsilon \cdot \in 71+0$ & 3 & & \\
\hline & $4.271+$ & & & \\
\hline & & & & \\
\hline & & & & \\
\hline & & & & \\
\hline & & & & \\
\hline & & & & \\
\hline & & & & \\
\hline & & & & \\
\hline & & & & \\
\hline
\end{tabular}

\begin{tabular}{|c|c|c|}
\hline \multicolumn{3}{|c|}{$z=\varepsilon 38$ ISMUTH } \\
\hline $\begin{array}{l}\text { EAERGY } \\
\text { (KEY) }\end{array}$ & TOTAL & COrER. \\
\hline & & SCATT. \\
\hline .010 & $8.432+C 7$ & $4 \cdot 583+c 3$ \\
\hline .015 & $5.621+07$ & $4.583+C 3$ \\
\hline .020 & $4.21 \epsilon+c 7$ & $4.5 \varepsilon 2+C 3$ \\
\hline .030 & $2.811+c 7$ & $4.582+C 3$ \\
\hline .040 & $2.108+C 7$ & $4 \cdot 5 \varepsilon 2+C 3$ \\
\hline .050 & $1.687+07$ & $4.582+03$ \\
\hline .060 & $1.406+C 7$ & $4.5 \varepsilon 2+c 3$ \\
\hline .080 & $1.054+07$ & $4.581+C 3$ \\
\hline .100 & $\varepsilon \cdot 43 \epsilon+c \epsilon$ & $4.58 C+C 3$ \\
\hline $.15 \mathrm{C}$ & $5 . t 25+c t$ & $4.578+C 3$ \\
\hline .200 & $4.220+C \epsilon$ & $4.574+C 3$ \\
\hline .200 & $2.815+c t$ & $4.564+C 3$ \\
\hline .400 & $2.112+0 t$ & $4.54 \subsetneq+c 3$ \\
\hline .500 & 5. $S C 7+C E$ & $4.532+C 3$ \\
\hline .600 & $4.4 \epsilon^{3}+C \epsilon$ & $4.511+C 3$ \\
\hline $.80 \mathrm{C}$ & $2.7 \epsilon g+c \epsilon$ & $4.4 \in 2+c 3$ \\
\hline 1.000 & 1. $\varepsilon \in 0+c 6$ & $4.403+C 3$ \\
\hline $1.50 \mathrm{C}$ & $8.510+C 5$ & $4.22 t+03$ \\
\hline $2 . \mathrm{CCO}$ & $4 \cdot t \in 2+C E$ & $4 \cdot C 23+C 3$ \\
\hline $3 . \mathrm{COC}$ & $6.572+05$ & $3 \cdot \in C 5+C 3$ \\
\hline 4. $C 00$ & $4.424+C 5$ & $3.225+C 3$ \\
\hline $5.0 \mathrm{CC}$ & $2 . t 01+05$ & $2 . \varepsilon 86+c 3$ \\
\hline $6 . C C O$ & $1 . t \in O+C E$ & $2.552+C 3$ \\
\hline $8 . \mathrm{COC}$ & $8 \cdot C 73+C 4$ & $2.117+C 3$ \\
\hline $1 \mathrm{C} .0 \mathrm{OC}$ & $4.610+c 4$ & $1.757+C 3$ \\
\hline $15.00 \mathrm{C}$ & $4.015 \$ 04$ & $1.168+03$ \\
\hline $2 c \cdot \operatorname{ccc}$ & 3. $c 83+c 4$ & $8.287+C 2$ \\
\hline & 1. $\mathrm{CS} 0+04$ & $4.841+C 2$ \\
\hline & & \\
\hline
\end{tabular}

$\begin{array}{cr}\text { INCOH. } & \text { PHCTC } \\ \text { SCATT. } & \text { EFFECT } \\ .326-C 4 & 8.431+07 \\ 5.225-C 4 & 5.621+07 \\ .272-C 4 & 4.216+07 \\ 2 . C 79-03 & 2.810+07 \\ .682-03 & 2.108+07 \\ .731-C 3 & 1.686+07 \\ 8.223-03 & 1.405+C 7 \\ .451-02 & 1.054+07 \\ .250-C 2 & 8.431+06 \\ .5 E 8-C 2 & 5.621+06 \\ .6 E 2-02 & 4.216+06 \\ .873-C 1 & 2.810+06 \\ .1 S 4-C 1 & 2.108+06 \\ .779-C 1 & 5.903+06 \\ .578-C 1 & 4.458406 \\ . C E 2+C C & 2.765+06 \\ .45 C+C C & 1.856+C 6 \\ .516+00 & 8.468+05 \\ 3.472+0 C & 4.622+05 \\ .266+C C & 6.936+05 \\ . C 27+C C & 4.392+05 \\ .7 \in 0+C 0 & 2.572+05 \\ . C 44+C 1 & 1.634+05 \\ .350+01 & 7.860+04 \\ .6 C 5+C 1 & 4.433+04 \\ .073+C 1 & 3.856+C 4 \\ .418+01 & 2.998+04 \\ .8 S 1+C 1 & 1.035+04 \\ 172+01 & 4.779+03\end{array}$

\begin{tabular}{|c|c|c|c|c|}
\hline \\
\hline $\begin{array}{c}\text { ENEREY } \\
\text { (KEV) }\end{array}$ & TOTAL & $\begin{array}{l}\text { COHER. } \\
\text { SCATT. }\end{array}$ & $\begin{array}{l}\text { I NCOH. } \\
\text { SCATT. }\end{array}$ & $\begin{array}{r}\text { PHOTO } \\
\text { EFFECT }\end{array}$ \\
\hline .010 & $8.683+07$ & $4.654+03$ & $2.255-04$ & $8.682+07$ \\
\hline & & $4.6 \$ 4 \$ 03$ & & \\
\hline . $c 20$ & $4.342+07$ & $4.6 \subseteq 4+C 3$ & $.148-C 4$ & $.341+07$ \\
\hline .030 & $2.895+07$ & $4.653+c 3$ & & \\
\hline .04 & $171+07$ & $4.6 \leq 3+c$ & & \\
\hline & $1.737+C 7$ & $4 \cdot \in S 3+C 3$ & 5.6 & $1.736+07$ \\
\hline & $1.448+c^{7}$ & $4.6 \subseteq 3+03$ & & $.447+07$ \\
\hline & 1.0 & 4.65 & & \\
\hline & & $4.691+$ & & \\
\hline & 5.7 & 4.68 & & \\
\hline & & 4.68 & & \\
\hline & & 4.67 & & 406 \\
\hline & & 4.65 & & \\
\hline & & & & \\
\hline & 4.7 & 4.62 & $6 \cdot 5$ & .06 \\
\hline & & 4.58 & & \\
\hline & & 4.50 & & \\
\hline & & 4.32 & & 05 \\
\hline & & & & \\
\hline & & $3 . t 8$ & & \\
\hline & & 3.25 & & 05 \\
\hline & & & & \\
\hline & & $2.6=$ & & \\
\hline & & & & \\
\hline & & & & \\
\hline & & & & \\
\hline & & 8.53 & & \\
\hline & $1.148+04$ & 4. . $\varepsilon 1+$ & $2.52 \mathrm{C}+$ & \\
\hline 40.600 & $5.3 \subseteq 2+03$ & $3.158+02$ & $3.2 \mathrm{Cl}+\mathrm{Cl}$ & $5.040+03$ \\
\hline
\end{tabular}

\begin{tabular}{|c|c|c|}
\hline $\begin{array}{l}\text { NERGY } \\
\text { GKEY }\end{array}$ & TCTAL & COHER - \\
\hline $\begin{array}{l}\text { (EV) } \\
50 .\end{array}$ & & \\
\hline$\in 0^{\circ}$. & $\in 84+$ & $26+02$ \\
\hline 80. & $.08 C \div 02$ & \\
\hline 100. & & \\
\hline & & $1+01$ \\
\hline & & \\
\hline & & 7.4 \\
\hline & & \\
\hline 50 & & \\
\hline & & \\
\hline & & \\
\hline 1000 & & \\
\hline & & \\
\hline & & \\
\hline 3000 & 1.43 & 7.7 \\
\hline & & \\
\hline & & \\
\hline 6 & .4 & \\
\hline & & \\
\hline & & \\
\hline & 1.89 & \\
\hline & & \\
\hline & & \\
\hline & & \\
\hline & & \\
\hline & & \\
\hline & 3. $c 89+0$ & $1.094-04$ \\
\hline & & $7.003-0$ \\
\hline
\end{tabular}

100000 .

\begin{tabular}{|c|c|c|}
\hline INCOH. & РНСТO & PAIR \\
\hline SCATT & EFFECT & \\
\hline $3.3 \mathrm{C} 2+C 1$ & $2.463+03$ & \\
\hline $3.350+01$ & $1.4 \subsetneq \varepsilon+03$ & \\
\hline $3.426+C 1$ & $t .8<4+C 2$ & \\
\hline $3.451+01$ & 1.7SE+03 & \\
\hline $3.31 \epsilon+01$ & $6.257+02$ & \\
\hline $3.12 t+C 1$ & $2 . c_{2} 23+c 2$ & \\
\hline $2.756+01$ & $1.011+02$ & \\
\hline $2.53 \varepsilon+c 1$ & $4 . \varepsilon \varepsilon z+01$ & \\
\hline $2.333+01$ & $2.841+01$ & \\
\hline $2.1 \in 7+01$ & 1. $\varepsilon \leq \varepsilon+c 1$ & \\
\hline $1 . \varsigma_{12}+01$ & $5.821+0 \mathrm{C}$ & \\
\hline $1.723+C 1$ & $\epsilon .1 \in 2+00$ & \\
\hline $1.404+01$ & $2.817+00$ & $5.472-\mathrm{Cl}$ \\
\hline $1.195+C 1$ & $1 . \in \subseteq \varepsilon+00$ & $1.750+c c$ \\
\hline $5.431+0 \mathrm{C}$ & $2.8 \in 4-01$ & 3. $S 10+C c$ \\
\hline 7. $\varepsilon \in E+C C$ & E. E 12-C1 & $5.728+C C$ \\
\hline $6.752+00$ & $4.27 \mathrm{C}-01$ & $7.27 C+0 C$ \\
\hline$t \cdot c c 3+c c$ & $3.356-01$ & $2.5 \subseteq 5+c c$ \\
\hline $4.510+C C$ & $2.333-01$ & 1. $C 77+C 1$ \\
\hline $4.18 C+0 C$ & $1.781-01$ & $1.245+C 1$ \\
\hline 2. $C s_{1}+c c$ & $1.113-01$ & $1.578+C 1$ \\
\hline $2.480+C C$ & E.C C7 $7-c 2$ & $1.833+\mathrm{Cl}$ \\
\hline 1. $\varepsilon C 3+C C$ & $5.2 C 7-02$ & $2.210+c 1$ \\
\hline $1.4 \cong 2+O C$ & $3.84 C-02$ & $2.464+C 1$ \\
\hline $1.194+O C$ & $3.041-02$ & $2.647+C 1$ \\
\hline 1. $C 28+0 C$ & $2.517-02$ & $2.790+C 1$ \\
\hline$\varepsilon .1 \mathrm{Cl} 1-01$ & $1 . \varepsilon T 1-02$ & 3. $C C E+C 1$ \\
\hline $6.721-01$ & $1.4 \varepsilon \varsigma-02$ & $3.172+01$ \\
\hline
\end{tabular}

\begin{tabular}{|c|c|c|c|c|c|}
\hline $\begin{array}{l}\text { ENERGY } \\
\text { (KEV) }\end{array}$ & TCTAL & $\begin{array}{l}\text { COHER. } \\
\text { SCATT. }\end{array}$ & $\begin{array}{l}\text { INCOH. } \\
\text { SCATT. }\end{array}$ & $\begin{array}{r}\text { FHOTO } \\
\text { EFFECT }\end{array}$ & $\begin{array}{r}\text { PAIF } \\
\text { PRCL. }\end{array}$ \\
\hline 50 . & $2.85 C+C 3$ & $2.152+c 2$ & $3.331+C 1$ & $2 . \in C 1+03$ & \\
\hline 60. & $1.772+C 3$ & $1.572+02$ & $3.421+01$ & $1.5 \varepsilon 1+03$ & \\
\hline 80. & $8.455+c 2$ & $9.417+C 1$ & $3.4 \in 1+C 1$ & $7.2 C 7+c 2$ & \\
\hline 100. & $1.5 \in 8+C 3$ & $6.255+01$ & $3.48 s+C 1$ & $1.871+03$ & \\
\hline 150. & $7.155+C 2$ & $2.919+01$ & $3.253+C 1$ & t. $531+c 2$ & \\
\hline 200. & $3.533+02$ & $1.682+01$ & $3.1 \in 2+C 1$ & $3 .<4 \varepsilon+02$ & \\
\hline 300 . & $1.412+\mathrm{C} 2$ & $7.657+0 \mathrm{C}$ & $2 . \varepsilon 2 \varepsilon+C 1$ & $1 . C 53+02$ & \\
\hline 400. & $8.05 C+01$ & $4.359+00$ & $2.5 \in \mathrm{T}+\mathrm{Cl}$ & 5.CE7+01 & \\
\hline 500 . & $5 . \in 14+01$ & $2.809+00$ & $2.3 \in 1+C 1$ & $2.572+01$ & \\
\hline 600 . & $4.341+C 1$ & $1.960+00$ & $2.153+01$ & $1.952+C 1$ & \\
\hline 800 . & $3.082+01$ & $1.109 \div 00$ & $1.935+01$ & $1.02 t+C 1$ & \\
\hline 1000 . & $2.467+C 1$ & $7.124-01$ & $1.744+C 1$ & $6.51 E+O C$ & \\
\hline 1500. & $1 . \varepsilon \subset 7+01$ & $3.181-01$ & $1.421+C 1$ & 2.SE $4+00$ & $5.64 C-C 1$ \\
\hline 2000 . & $1.594+01$ & $1.794-01$ & $1.213+01$ & $1.7 \subseteq 5+c 0$ & $1.225+c c$ \\
\hline 3000 . & $1.460+01$ & $7.990-02$ & $5.54 t+C C$ & s. $3 \varepsilon 1-c 1$ & $4.040+C C$ \\
\hline 4000 . & $1.450 \div 01$ & $4.500-02$ & $7.5 \in 1+C C$ & $\epsilon .147-01$ & $5.877+C C$ \\
\hline 5000 . & $1.475+01$ & $2.882-02$ & $\epsilon . \varepsilon 75+c c$ & $4 \cdot 513-01$ & $7.433+C C$ \\
\hline 6000 . & $1.523+01$ & $2.002-02$ & t. $C 76+0 \mathrm{C}$ & $3.545-01$ & $8.777+C C$ \\
\hline 8000 . & $1 . \in 24+01$ & $1.127-02$ & $4.57 C+C C$ & $2 \cdot 4 \in \bar{z}-01$ & $1.101+C 1$ \\
\hline 10000 . & $1.725+C 1$ & $7.215-03$ & $4.231+C C$ & $1.875-01$ & $1.282+\mathrm{Cl}$ \\
\hline 15000 . & $1.55 t+c 1$ & $3.208-03$ & $3.125+C C$ & $1.174-01$ & $1.631+C 1$ \\
\hline 20000 . & $2.154+01$ & $1.805-03$ & $2.51 C+C c$ & $8.513-02$ & $1.895+C 1$ \\
\hline & $2.458+01$ & $8.024-04$ & $1.825+C C$ & $5.4 \varepsilon t-02$ & $2.270+C 1$ \\
\hline 40000 . & $2 . t 72+01$ & $4.514-04$ & $1.445+C C$ & $4 \cdot c 45-02$ & $2.523+C 1$ \\
\hline 50000 . & $2.834+C 1$ & $2.889-04$ & $1.2 C S+C C$ & $3.2 C 2-02$ & $2.710+C 1$ \\
\hline & 2. $s \in 4+01$ & $2.007-04$ & $1 . C 41+0 \mathrm{C}$ & $2 . t \leq c-02$ & $2.857+C 1$ \\
\hline & & $1.129-04$ & $\varepsilon . \approx C 0-01$ & $1.57 C-02$ & 3. $C 76+C 1$ \\
\hline & & & & & \\
\hline
\end{tabular}




\begin{tabular}{|c|c|c|c|c|}
\hline $\begin{array}{l}\text { EAEREY } \\
\text { (KEVV) }\end{array}$ & TOTAL & $\begin{array}{l}\text { COHER. } \\
\text { SCATT. }\end{array}$ & $\begin{array}{l}\text { INCOH. } \\
\text { SCATT. }\end{array}$ & $\begin{array}{r}\text { PHCTO } \\
\text { EFFECT }\end{array}$ \\
\hline .010 & $8.85 t+C 7$ & $4.806+C 3$ & $2.337-04$ & $8.856+07$ \\
\hline .015 & $504+07$ & $4.806+C 3$ & $5.250-c 4$ & $5.904+07$ \\
\hline.$<20$ & $4.428+07$ & $4.80 t+C 3$ & $5.316-04$ & $428+07$ \\
\hline .030 & $2.952+07$ & $4.806+C 3$ & 2. CQS-C3 & $2.952+07$ \\
\hline .040 & $.214+07$ & $4.806+c 3$ & $3.700-c 3$ & $2.214+07$ \\
\hline .050 & $1.772+C 7$ & $4.805+C 3$ & $5.760-c 3$ & $1.771+07$ \\
\hline .060 & $1.47 t+07$ & $4.8 C 5 \nrightarrow C 3$ & $8.264-c 3$ & $1.476 \bullet 07$ \\
\hline & $1.107+07$ & $4.8 C 5+C 3$ & $1.459-02$ & $1.107+07$ \\
\hline .100 & $t I+C t$ & $4 . E 04+C 3$ & $2.2 \in 2-C 2$ & $56+06$ \\
\hline .150 & $5.509+06$ & $4.801+C 3$ & $4.556-02$ & $5.904+06$ \\
\hline .200 & $4.4 \equiv 3+0 t$ & $4.757+c 3$ & $8.715-02$ & $4.428+06$ \\
\hline .300 & $2.557+0 \epsilon$ & $4.7 E 6+C 3$ & 1.8 & $2.952 * 06$ \\
\hline $.40 \mathrm{C}$ & $2.215+C E$ & $4.771+C 3$ & $3.215-C 1$ & $2.214+06$ \\
\hline .500 & $1.776+C E$ & $4.753+c 3$ & $4.822-01$ & $1.771+06$ \\
\hline.$\in C O$ & $4.843+06$ & $4.731+C 3$ & $4-01$ & $4.839+0 t$ \\
\hline .800 & $3.017+0 t$ & $4 \cdot 678+03$ & $1.075+00$ & $3.012+06$ \\
\hline. $\mathrm{COC}$ & $2.040+06$ & $4.616+$ & & $2.036+06$ \\
\hline & & & & \\
\hline 2.000 & $5.229+05$ & $4.2 C 5+03$ & $3.551+C C$ & $5.187+05$ \\
\hline 3.000 & $7.745+$ & $3.75 s+$ & & \\
\hline & $4.438+C 5$ & $3.3 \in 2 \triangleleft C 3$ & $7.345+00$ & $4.405+05$ \\
\hline $5.00 \mathrm{C}$ & $2.8 \leq 4+C E$ & 3.C1 $4+C 3$ & S. $128+C C$ & $2.824+05$ \\
\hline & $1.824 t$ & 2.712 & 1. $c 82+$ & $1.797+05$ \\
\hline$\varepsilon \cdot c 00$ & $8.8 \in 1+C 4$ & $2.222+C 3$ & $1.389+01$ & $8.637+04$ \\
\hline c.coc & $5 . C 55+C 4$ & $1.84 \varepsilon+03$ & & $4.869+04$ \\
\hline & $4.417+$ & $1.233+c$ & $2.117+C 1$ & $4.252+04$ \\
\hline & $3.362+04$ & $8.784+C 2$ & $2.4 t 6+C 1$ & \\
\hline $3 \mathrm{C} \cdot \mathrm{COC}$ & $1.1 \leq 3+c 4$ & $5.123+02$ & $2.545+01$ & $1.139+04$ \\
\hline & $5.617+03$ & $3.28 \varepsilon+C 2$ & $3.232+\mathrm{Cl}$ & $5.256+03$ \\
\hline
\end{tabular}

\begin{tabular}{|c|c|c|c|c|}
\hline \multicolumn{5}{|c|}{$z=\varepsilon \epsilon \quad R \triangle D C N$} \\
\hline & & & I NCOH. & PHOTO \\
\hline (KEV) & & SCDTT. & SCATT. & EFFECT \\
\hline .010 & $5.584+C 7$ & $4.52 C+C 3$ & $2.233-c 4$ & $9.584+07$ \\
\hline .015 & $6.350+C 7$ & $4.920+c 3$ & 5.C16-04 & $6.389+07$ \\
\hline .020 & $2+c 7$ & $4.520+c 3$ & $8.5 C 3-04$ & \\
\hline.$C 30$ & $3.155+C 7$ & $4.52 C+C 3$ & $1.596-C 3$ & $3.195+07$ \\
\hline .040 & $2.39 t+07$ & $4.51 \subseteq+03$ & $3.536-c 3$ & $2.396+07$ \\
\hline .050 & $1.517+C 7$ & $4.915+C 3$ & - $5 \mathrm{C} 6-\mathrm{C3}$ & $1.917+07$ \\
\hline $.06 \mathrm{C}$ & $1.598+07$ & $4.515+03$ & $7.501-C 3$ & $1.597+07$ \\
\hline & $1.198 * 07$ & $4.518+C 3$ & $5-c 2$ & $1.198+07$ \\
\hline .100 & $s+c \epsilon$ & $4.518+03$ & $2.164-c 2$ & $5.584 \div 06$ \\
\hline .150 & $\epsilon .3 \subseteq 4+C \epsilon$ & $4.515+C 3$ & $4.781-C 2$ & $6.389+06$ \\
\hline .200 & $4.757+c 6$ & $4.511+03$ & $8.344-C 2$ & $4.792+06$ \\
\hline .200 & $3.1 \subseteq 5+C t$ & & $8-\mathrm{Cl}$ & $3.195+06$ \\
\hline $.4 C \mathrm{C}$ & $2.4 C 1+C \epsilon$ & $4.8 \varepsilon 5+C 3$ & $3.089-01$ & $2.356+06$ \\
\hline .500 & $1.5 \bar{z} z+0 t$ & 4. $\varepsilon t t+C 3$ & $4.634-01$ & $1.917+06$ \\
\hline & & & & \\
\hline $.8 \mathrm{CO}$ & $3.211+0 t$ & $4.751+C 3$ & $1.038+C C$ & $3.207+06$ \\
\hline 1.000 & $2.155+06$ & $4.727+03$ & 1.48 & $2.150+06$ \\
\hline & & & & $5.876+C 5$ \\
\hline 2.000 & $5.453+C 5$ & $4.301+C 3$ & $3.566+\mathrm{CO}$ & $5.450+05$ \\
\hline 3.000 & $5.640+C 5$ & $3 \cdot \varepsilon \equiv S+C 3$ & $5.556+C C$ & $5.602+05$ \\
\hline & & $3.432+C$ & & \\
\hline 5. COC & $4+c \leq$ & $3 . c 7 s+$ & 5.28 & $2.953+05$ \\
\hline $6.0 \mathrm{CO}$ & $1.507+05$ & $2.772+C 3$ & 1.10 & $1.879+05$ \\
\hline & & & & \\
\hline & $+c 4$ & 1.8 & & \\
\hline 15.000 & $4.631+04$ & $1.268+03$ & $2.135+C 1$ & $4.5 \mathrm{C} 2+04$ \\
\hline & & $5.040+c 2$ & & \\
\hline & & & & \\
\hline & & $3.381+$ & $3.2 t 2+c 1$ & $5.520+c$ \\
\hline
\end{tabular}

\begin{tabular}{|c|c|c|c|c|}
\hline \multicolumn{5}{|c|}{$Z=E 7$ FRANCIUN } \\
\hline EAERGY & TCTAL & COHER. & $\mathrm{INCOH}$. & PHCTO \\
\hline (KEV) & & SCATT. & SCATT. & EFFECT \\
\hline $.01 \mathrm{C}$ & S. $5 \in 2+C 7$ & $5.035+c 3$ & $2.5 c 8-c 4$ & $9.961+07$ \\
\hline .015 & $6.641+07$ & $5.035+C 3$ & $5.632-04$ & $6.641+07$ \\
\hline .020 & 4. $5 \varepsilon 1+C 7$ & $5.035+03$ & ร. ऽ૬ร- C४ & $4.981+07$ \\
\hline .030 & $3.321+07$ & $5.035+03$ & $2.241-03$ & $3.320+07$ \\
\hline .040 & $2.4 S_{1}+C 7$ & $5 . c 34+c 3$ & & $2.490+07$ \\
\hline .050 & $1.553+07$ & $5 . c 34+03$ & $6.175-03$ & $1.992+07$ \\
\hline .060 & 1. $\epsilon \in I+C 7$ & 5. $C 34 \div 03$ & $8.8 t t-C 3$ & $1.660+07$ \\
\hline .080 & $1.24 t+C 7$ & $5.033+03$ & & $1.245+c 7$ \\
\hline .100 & $9.5 \in 6+06$ & $5.022+03$ & $2.427-c 2$ & $9.961+06$ \\
\hline .150 & $t . t 46+0 t$ & $5.028+03$ & $5.359-C 2$ & $6.641+06$ \\
\hline .200 & 4. $5 \varepsilon 6+C \epsilon$ & $5.023+03$ & $5.347-02$ & $4.981+06$ \\
\hline .300 & & 5. $\cos +03$ & 2.0 & $3.320+06$ \\
\hline .400 & $2.455+C t$ & $4.585+C 3$ & $3.451-C 1$ & $2.490+06$ \\
\hline .500 & $1.557+0 t$ & 4. $S \in 6+C 3$ & 5.16 & $1.992+06$ \\
\hline & & & & $5.605+06$ \\
\hline .800 & $3.4 \equiv 3+06$ & $4.874+C 3$ & $1.151+C C$ & $3.428+06$ \\
\hline 1.000 & $2.284+C \epsilon$ & $4.800+03$ & & $2.279+06$ \\
\hline & & 4.591 & $2.752+O C$ & $1.035+06$ \\
\hline & & $4.362+C 3$ & $3.815+C C$ & $5.707+05$ \\
\hline & $4+0 E$ & & & \\
\hline 4.000 & $4.875+$ & $3.455+$ & $7.715+$ & $4.844+05$ \\
\hline 000 & $123+05$ & $3.140+C 3$ & $9.526+C O$ & $3.091+05$ \\
\hline $\mathrm{coO}$ & $1.595+05$ & $2.828+$ & $1.124+01$ & \\
\hline & 9.7314 & $2.324+$ & $1.431+01$ & $9.497+04$ \\
\hline & & & & \\
\hline 15.000 & $2.042 \%$ & $1.300+03$ & $2.164+C 1$ & 1.910404 \\
\hline & & $5.290+$ & $2.517+01$ & $3.541 * C_{4}$ \\
\hline & & & & \\
\hline & & & 3.25 & $176+03$ \\
\hline
\end{tabular}

\begin{tabular}{|c|c|c|c|c|c|}
\hline $\begin{array}{l}\text { NERGY } \\
\text { (KEV) }\end{array}$ & TOT $\Delta L$ & $\begin{array}{l}\text { COHER : } \\
\text { SCATT. }\end{array}$ & $\begin{array}{l}\text { INCOH: } \\
\text { SCATT: }\end{array}$ & EFFECT & PRCC. \\
\hline 50. & $3.129+03$ & $2.273+02$ & $3.356+c 1$ & & \\
\hline 60. & $.947+03$ & $.660+02$ & $3.489+01$ & $1.74 t+c 3$ & \\
\hline 80. & $337+02$ & $.939+01$ & $3.5 \geq 1+C 1$ & 7. $c \subseteq c+c 2$ & \\
\hline 100. & $120+03$ & $.599+01$ & $.5 \in 3+c 1$ & $2.01 E+03$ & \\
\hline & $52+02$ & $.078 \div 01$ & $.42 \varepsilon+C 1$ & $7.1 C 2+02$ & \\
\hline & $834+02$ & $1.773+01$ & $0.233+01$ & $3.334+02$ & \\
\hline & $.531+02$ & $.071+00$ & . ES4+01 & $1.1 \in 1+02$ & \\
\hline & & & $628+01$ & $5 . \epsilon 35+01$ & \\
\hline & $6 . C 12+C 1$ & $2.961+00$ & 7.01 & $3.2 s \varsigma+c 1$ & \\
\hline 600. & 4.6 & & C 1 & $2.1 \in 8+01$ & \\
\hline & & & $. \varsigma 82+01$ & $1.153+01$ & \\
\hline 00 . & $2.587+\mathrm{Cl}$ & 01 & $1.7 \varepsilon t+C 1$ & & \\
\hline 1500. & & & 1.4 & $3.22 s+0 C$ & 6.C32-01 \\
\hline & & & & 2. CCE & $\$ 35 * C C$ \\
\hline 3000 . & $1.516+c 1$ & 8.42 & $5.776+C C$ & $1 . C$ & $4.253+C C$ \\
\hline 4000 . & & 4.7 & E.15 & $-\mathrm{Cl}$ & $6.164 \div C C$ \\
\hline & & & & 01 & 7. \\
\hline 6000 . & $1.581 \otimes 0$ & & $t .222+C C$ & 3.545 & \\
\hline 8000 . & $1 . t$ & & $\because C \mathrm{C}$ & $2.738-C 1$ & $1.145+C 1$ \\
\hline & & & & 2.0 & $7+C 1$ \\
\hline & & 3.3 & & $1.3 C_{2}$ & $1.658+c 1$ \\
\hline & & & & $5.44]$ & $1.570+c 1$ \\
\hline & & & & & \\
\hline & & & & & $2 \cdot t 29+c 1$ \\
\hline & & & 1.2 & & $2.828+C 1$ \\
\hline & & & & & $82+C 1$ \\
\hline 000 & $3.255+$ & & & & $3 \cdot 2 c 9+c 1$ \\
\hline & $1427+51$ & $7.612-05$ & $t . \subseteq \in 7-c 1$ & $1.73 t-c_{2}$ & $3 \cdot 3 \in 6+C 1$ \\
\hline
\end{tabular}

\begin{tabular}{|c|c|c|c|c|c|}
\hline $\begin{array}{l}\text { ENERGY } \\
\text { (KEVV) }\end{array}$ & $T C T \Delta L$ & $\begin{array}{l}\text { Corer } \\
\text { SCATt }\end{array}$ & $\begin{array}{l}\text { INCOH. } \\
\text { SCATT. }\end{array}$ & $\begin{array}{r}\text { FHCTO } \\
\text { EFFECT }\end{array}$ & $\begin{array}{l}\text { PAIR } \\
\text { PRCL. }\end{array}$ \\
\hline 50. & $3.282+03$ & $2.337+02$ & $3.428+01$ & 3. $C 14+03$ & \\
\hline 60. & & $.707+02$ & $2.524+C 1$ & $1.8 \equiv t+C 3$ & \\
\hline 80. & $i+c$ & $1.022+02$ & 3. $567+C 1$ & $\varepsilon .3 \subseteq t+02$ & \\
\hline 100. & $2.15 t+03$ & $6.783+01$ & $3 . t C 1+C 1$ & $2.053+03$ & \\
\hline 150. & $8 . c 74+02$ & $3.164+01$ & $3.4 t \epsilon+C 1$ & $7.412+02$ & \\
\hline 200 . & $4 . C C 4+02$ & $1.823+01$ & $3.270+01$ & $2.455+02$ & \\
\hline & & & $2 . \subseteq 27 \& C 1$ & $1.223+02$ & \\
\hline & $5.079+01$ & $4.721+c$ & $2 . t=$ & 5.54 & \\
\hline 500. & $6.225+01$ & $3.043+00$ & $2.445+C 1$ & $3.4 E 0+01$ & \\
\hline & & & & $2.2 \varepsilon \subseteq+01$ & \\
\hline & 3.3 & & & 1.21 & \\
\hline 100 & 2.6 & & 1. & $7 . \epsilon=$ & \\
\hline & & & & & $6.254-c$ \\
\hline & $1 . \in 87+01$ & 1.9 & 1.25 & & $7+c$ \\
\hline & 1.54 & & & & $1+c$ \\
\hline & & & & & \\
\hline & 1.560 & & $7.123+$ & 5.2 & $c 22+c c$ \\
\hline & & & & $4 \cdot 1$ & $27+c c$ \\
\hline & & & & & \\
\hline 10000 & $1.82 \mathrm{C}+\mathrm{Cl}$ & 7.8 & & 2.1 & $359+01$ \\
\hline & & & & & \\
\hline & & & & & \\
\hline & & & & & \\
\hline & & & & & $655+0$ \\
\hline & & & & & \\
\hline & & & & & \\
\hline sove & $\cdot 3 \varepsilon 2+0$ & 1.22 & E.4S6- & $2.252-02$ & $3.255+c$ \\
\hline & $3.535+C 1$ & $7.822-05$ & 7. $C 4 S-C 1$ & 1. $\varepsilon 24-c 2$ & $3 \cdot 4 t 3+C 1$ \\
\hline
\end{tabular}

\begin{tabular}{|c|c|c|c|c|c|}
\hline $\begin{array}{l}\text { EAERGY } \\
\text { (KEV) }\end{array}$ & TCTAL & $\begin{array}{l}\text { COHER : } \\
\text { SCATT. }\end{array}$ & $\begin{array}{l}\text { INCOH. } \\
\text { SCATT. }\end{array}$ & $\begin{array}{r}\text { FHCTO } \\
\text { EFFECT }\end{array}$ & $\begin{array}{l}\text { PAIR } \\
\text { PRCC. }\end{array}$ \\
\hline 50 . & $3.4 \equiv 7+03$ & $2.4 \mathrm{C} 6+02$ & $3.4 \epsilon 0001$ & a. $1 \in 2+03$ & \\
\hline 60. & $2.139+03$ & $1.757+02$ & $3.558+01$ & $1.92 \varepsilon+03$ & \\
\hline 80 . & $23+03$ & 052402 & $\Xi \cdot t C \geqslant \geqslant 01$ & $\varepsilon_{0} \varepsilon 1 s+c 2$ & \\
\hline 100 & $5+c 2$ & $83+01$ & $3 \cdot \epsilon \geq \varepsilon+01$ & $4.8 C 3+02$ & \\
\hline 150 & $.377+02$ & 3.25 & $3.5 \mathrm{C} 3+01$ & 7. $7 \mathrm{Cl} 1+\mathrm{C2}$ & \\
\hline 200. & $4.152+02$ & $1.877+01$ & $3.3 c t+01$ & $3,634+02$ & \\
\hline 300. & & & 2. $\subseteq \notin 1+01$ & $1.272+02$ & \\
\hline 400. & $5.374+C 1$ & 4.8 & $2 . t 8 s+01$ & $t .1 \varsigma s+01$ & \\
\hline 500. & $6.428+01$ & 3.13 & $2.473+01$ & $3.641+01$ & \\
\hline & & & & $2.4 C C+01$ & \\
\hline & & 1.2 & 2. $C 28+c 1$ & $1.28 c+01$ & \\
\hline 1000 & $.713+$ & 7.5 & 1.E & $8.0 \in 2+00$ & \\
\hline & & & & & $.356-c]$ \\
\hline & 1.72 & 2.0 & 1.27 & $2.225+00$ & $79+$ \\
\hline 300 & 1.57 & & $1 . c$ & $1.1 \in 1+C C$ & 4 \\
\hline & & & $\varepsilon .3$ & & \\
\hline 5000 . & 1.58 & 3.2 & $7.2 \mathrm{C}$ & $5.5 \in S-C 1$ & E. $C 6 S+C$ \\
\hline 600 & & & $\epsilon \cdot 3$ & $4.3 \in 5-01$ & $5.479+c$ \\
\hline & & & 5.2 & $3.03 c$ & 1 . \\
\hline & & & 4.4 & $2.3 C s-01$ & $86+C$ \\
\hline 1500 & $2.126+$ & & 3.2 & 1.44 & $\varepsilon 3+c$ \\
\hline & & & $2 . t$ & $1.043-01$ & \\
\hline & & & & $t .71 t-$ & $2.486+C$ \\
\hline & & & 1.5 & 4.545 & $.764+0$ \\
\hline & & 3.2 & 1.2 & 3.517 & \\
\hline & & & & & \\
\hline & & $1.258-04$ & $8.5 \div 5-C 1$ & $2.4 C s-02$ & $3.381+0$ \\
\hline & & $8.053-05$ & $7.131-\mathrm{C} 1$ & $1.51 t-02$ & $3.540+0$ \\
\hline
\end{tabular}




\begin{tabular}{|c|c|c|c|c|}
\hline \multicolumn{5}{|c|}{$Z=88$ RADIUM } \\
\hline $\begin{array}{l}\text { EAERGY } \\
\text { (KEY) }\end{array}$ & TOTAL & $\begin{array}{l}\text { COHER. } \\
\text { SCATT. }\end{array}$ & $\begin{array}{l}\text { I NCOH. } \\
\text { SCATT. }\end{array}$ & $\begin{array}{r}\text { PHCTC } \\
\text { EFFECT }\end{array}$ \\
\hline .010 & $1.044+08$ & $5.151+c 3$ & $2.8 C 9-c 4$ & $1.044+08$ \\
\hline & $\epsilon .5 \in 0+07$ & $5.151+03$ & $t .309-c_{4}$ & $6.960+07$ \\
\hline .020 & $5.220+07$ & $5.151+03$ & $1.119-03$ & $.220+07$ \\
\hline .030 & $3.4 E 0+C 7$ & $5.151+03$ & $2.5 \mathrm{cs}-\mathrm{c3}$ & $.480+07$ \\
\hline .040 & $2.610+07$ & $5.151+c 3$ & $4.445-C 3$ & $2.610+07$ \\
\hline .050 & $2.0 \varepsilon \varepsilon+C 7$ & $5.15 C+C 3$ & $6.519-03$ & $2.088+07$ \\
\hline .060 & $1.740+07$ & $5.15 C+C_{3}$ & s. $926-c 3$ & $1.740+07$ \\
\hline.$C \varepsilon C$ & $1.305+07$ & $5.145+03$ & $1.751-c 2$ & $1.305+07$ \\
\hline & & & & $1.044+07$ \\
\hline $.15 \mathrm{C}$ & $t . s \in 5+c t$ & $5.143+c 3$ & $5.554-C_{2}$ & $6.960+06$ \\
\hline .200 & $5.225+0 t$ & $5.137+C 3$ & 1. $C 45-C 1$ & $5.220+06$ \\
\hline .300 & & & & \\
\hline $.40 \mathrm{C}$ & $2 . t 15+0 t$ & $5.095+03$ & 3. $848-C 1$ & $2.610+06$ \\
\hline .500 & $2.093+0 t$ & $5 . C^{2}+03$ & $5.754-C 1$ & $2.088+06$ \\
\hline & & & & \\
\hline .800 & $3.554+C t$ & $4.968+C 3$ & $1.275+00$ & $3.589+06$ \\
\hline 1.000 & $2.4 C 3+C E$ & $4.88 t+C 3$ & $1.786+$ & $2.398+06$ \\
\hline & $1.1 \mathrm{C} 2+$ & $4 \cdot t t 5+03$ & $2.5 \varsigma 5+c c$ & $1.097+06$ \\
\hline 2.000 & $6.106+C 5$ & $4.432+c 3$ & $4.098+00$ & $6.062+05$ \\
\hline $3 . \mathrm{COO}$ & $2.5 \mathrm{CO}+$ & 3.5 & & $2.460+05$ \\
\hline & 5.13 & & 7. $995+C 0$ & $5.096+05$ \\
\hline $00 \mathrm{C}$ & $3.279+C 5$ & $3.20 \mathrm{C}+\mathrm{C}_{3}$ & 5.7 & $3.247+05$ \\
\hline & $2 . c 96+$ & $2.884+C 3$ & & $2.067+05$ \\
\hline & & & $1.455+$ & $5.988+04$ \\
\hline 10.000 & $5.845+04$ & $1.982+$ & $1.711+$ & $5.645+04$ \\
\hline & $2.128+C 4$ & $1.335+c 3$ & $2.189+01$ & $1.952+04$ \\
\hline & & $9.540+c 2$ & $2.543+C 1$ & $3.717+04$ \\
\hline $3 \mathrm{C} .0 \mathrm{CO}$ & $1 . \geq \in 1+04$ & $=.576+C_{2}$ & $3 . c 29+C 1$ & $1.303+04$ \\
\hline $4 \mathrm{C} .000$ & $6.441+C 3$ & $3.5 \varepsilon 1+C 2$ & $3.322+C_{1}$ & \\
\hline
\end{tabular}

\begin{tabular}{|c|c|c|}
\hline \multicolumn{3}{|c|}{$=\varepsilon S A C T I N I L N$} \\
\hline EAERGY & TOTAL & CORER. \\
\hline (KEV) & & SCATT. \\
\hline .010 & $1 . c 70+c 8$ & $5.2 \epsilon 9+03$ \\
\hline .015 & $7.1 \geq 6+07$ & $5.265+03$ \\
\hline $.02 \mathrm{C}$ & $5.352+c 7$ & $5.2 \in S+0 ?$ \\
\hline .020 & $3.5 \in \varepsilon+07$ & $5.2 \in S+03$ \\
\hline .040 & $2 . t 7 t+C 7$ & $5.2 \epsilon \varepsilon+C \Omega$ \\
\hline .050 & $2.141+07$ & $5.2 \in 8+c 3$ \\
\hline $.0 \in 0$ & $1.784+C 7$ & $5.268+02$ \\
\hline .080 & $1.338+07$ & $5.267+C 3$ \\
\hline .100 & $1.071+07$ & $5.2 \epsilon E+C 3$ \\
\hline .150 & $7.141+0 t$ & $5.2 € 1+C_{3}$ \\
\hline .200 & $5.357+C t$ & $5.254+03$ \\
\hline .300 & $3.573+c t$ & $5.237+C 3$ \\
\hline .400 & $2 . t \varepsilon l+c t$ & $5.214+03$ \\
\hline .500 & $2.14 t+C t$ & $5.18 \epsilon+03$ \\
\hline .600 & $1.789+C t$ & $5.153+C 3$ \\
\hline .800 & $3 . t 15+0 t$ & $5 . c 78+c 3$ \\
\hline 1.000 & $2.474+C 6$ & $4.593+c 3$ \\
\hline 1.500 & $1.1 \in 0+0 t$ & $4.7 \in 2+03$ \\
\hline 2.000 & $t .4 t_{2}+C E$ & $4.515+03$ \\
\hline 3.000 & $2 \cdot t 28+05$ & $4 \cdot 047+C 3$ \\
\hline 4.000 & $5 . \equiv 53+C 5$ & $3 . t 28+c z$ \\
\hline $5 . \mathrm{COC}$ & $3.265+05$ & $3.2 \in 2+C 3$ \\
\hline 6.000 & $2.1 \& 4+C 5$ & $2.543+c 3$ \\
\hline$\varepsilon . \mathrm{COC}$ & $1.0 t t+0 \leq$ & $2.426+c 3$ \\
\hline 10.000 & $\epsilon \cdot c \in 8+04$ & $\angle \cdot C 3 C+C 3$ \\
\hline $15 . \mathrm{COC}$ & $2.211+c 4$ & $1.381+03$ \\
\hline 20.000 & $3.513+c 4$ & $5.795+C 2$ \\
\hline & $1.425+C 4$ & $5.732+c 2$ \\
\hline & & \\
\hline
\end{tabular}
SCATT. 2. $873-\mathrm{C}_{4}$ $6.452-C_{4}$ $1.145-C_{3}$ $2.5 \in 6-\mathrm{C} 3$ $4.545-C 3$
$7.076-C 3$ 7. $C 76-C_{3}$
1. $C 15-C 2$ 1. $751-\mathrm{C}_{2}$ $2.777-02$ t. $130-C 2$ 1. $C \in S-C 1$ 2. $309-01$ $3.936-C 1$ 5. $886-C 1$ $8.057-\mathrm{Cl}$ J. $305+C C$ $1.828+C O$ 3. $C \in 7+C C$ $4.2 C 0+00$ $6.256+C 0$ $8.1 E l+C C$ 1. $\mathrm{COO}+\mathrm{Cl}$ $1.172+01$ $1.478+C 1$ 1. $734+\mathrm{Cl}$ $2.212+C 1$ $2.5 \epsilon 7+C 1$ 3. $C 56+C 1$ $3.351+C 1$
PHOTO

1. $070+08$

$7.136+07$

$3.568+07$

$2.141+07$

$1.784+07$

$1.338+07$

$1.070+07$

$7.136+06$

$5.352+06$

$2.676+06$

$2.141+06$

$1.784+06$

$3.610+0 t$

$2.469+C 6$

$1.155+06$

t. $417+05$

$5.317+05$

$3.233+05$

$2.155+0$

4

2. $071+04$

$3.813+0$

$1.365+0$

$6.343+03$
$5.352+C$

$\begin{array}{rr}\text { ENEREY } & \text { TCTAL } \\ \text { (KEV) } & \\ 50: & 3.555+C 3 \\ 60: & 2.243+03 \\ 80: & 1.074+03 \\ 100: & 6.161+02 \\ 150: & 8.793+02 \\ 200: & 4.355+02 \\ 300: & 1.727+02 \\ 400: & 9.742+C 1 \\ 500: & 6.655+01 \\ 600: & 5.075+01 \\ 800: & 3.527+C 1 \\ 1000: & 2.78 C+C 1 \\ 1500: & 2.0 C 0+01 \\ 2000: & 1.754+C 1 \\ 3000: & 1.605+01 \\ 4000: & 1.554+C 1 \\ 5000: & 1.627+01 \\ 6000: & 1.67 t+01 \\ 8000: & 1.787+01 \\ 10000: & 1.5 C 0+01 \\ 15000: & 2.162+01 \\ 20000: & 2.391+01 \\ 30000: & 2.742+C 1 \\ 40000: & 2.592+C 1 \\ 50000: & 3.181+01 \\ 60000: & 3.332+01 \\ 80000: & 3.559+01 \\ 100000: & 3.724+C 1\end{array}$

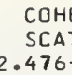
$2.476+02$ $1.809+02$ $7.193+01$ $3.356+01$ $1.934+01$ $8.800+00$ $5 . \cos +00$ $3.229+00$ $2.253+0 \mathrm{C}$ $1.275+00$ $8.186-01$ 3.655-01 $2.0 \in 1-01$ 9.181-02 5.170-0 3.311-02 2.3 $11-02$ 1.295-02 $8.290-03$ 3.686-03 2. $074-03$ $9.220-04$ 5.187-04 3.320-04 2. $305-04$ $1.297-04$ $8.300-05$

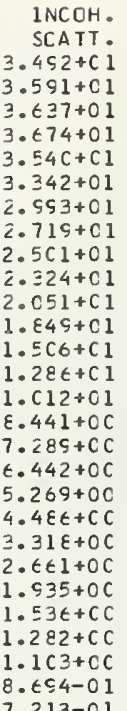

FHCTO EFFECT $3.317+C 3$
$2.02 t+03$ S. $255+02$ E. C $7 E+02$ $\varepsilon .1 C 3+c 2$ $3.831+02$ $1.34 \mathrm{C}+\mathrm{C} 2$ $t .522+C 1$ $3.8 \geq 1+01$ $2.52 t+c 1$ $1.34 \varepsilon+01$ $\varepsilon .45 \varsigma+0 C$ $3.9 \mathrm{C} 2+O C$ $2.353+0 C$ $1.225+00$ E. CC 7-01 $5.8 \in S-C 1$ $3.15 C-01$ $2.43 \mathrm{C}-01$ $1.514-C 1$ 1. $C \subseteq 7-01$

$7.05 t-02$ $5.1 \subseteq \varepsilon-02$ 4.113-02 3. $4 \mathrm{C} 3-02$ $2.52 \varsigma-\mathrm{C}_{2}$ 2. C12-02
PAIF PREC.

6. $724-C 1$ $2.115+C$ $4.612+C C$
$t . t 50+O C$ $8.361+C C$ c. $832+c C$ $1.227+01$ $1.426+C 1$ $1.815+C 1$ $2.542+C 1$ 2. $833+C$ 3. $(49+C 1$ $3.218+C$ $3.470+C 1$ $3.449+C 1$

\begin{tabular}{|c|c|c|c|c|}
\hline NERGY & & $\mathrm{CHE}$ & & \\
\hline (KEV) & & $S C A T$ & TT. & FECT \\
\hline & $1.121+C \varepsilon$ & $E .3 \varepsilon \varepsilon+c 3$ & $2 . \mathrm{sc}_{3}-\mathrm{C}_{4}$ & $1.121+08$ \\
\hline .015 & $474+C 7$ & 5. $3 \varepsilon \varepsilon+03$ & $6.520-04$ & $7.474+07$ \\
\hline & $5.606+07$ & ㄷ. $2 \varepsilon \varepsilon+C 3$ & $1.157-C_{3}$ & $5.605+07$ \\
\hline .030 & $3 \cdot 737+07$ & $5.388+C 3$ & $2.5 \$ 4-C 3$ & $3.737+07$ \\
\hline.$C 4 C$ & $2.803+07$ & $5.388+03$ & $4.594-c$ & $2.8 C 3+07$ \\
\hline .050 & $2.243+C 7$ & $5.3 \varepsilon 7+C 3$ & $7.151-03$ & $2.242+07$ \\
\hline - $c \in 0$ & 1. $8 \in 9+C 7$ & $5.387+c 3$ & $1.026-c$ & $1.868+07$ \\
\hline .080 & $1.402+07$ & $5.386+C 3$ & 1.8 & $1.401+07$ \\
\hline .100 & $1.122+07$ & $5.384+C 3$ & $2.8 \mathrm{c} 7-\mathrm{C}_{2}$ & $1.121+07$ \\
\hline .150 & 7.479 & $5 \cdot 3$ & 6.196 & $7.474+C 6$ \\
\hline .200 & $5.611+0 t$ & $5 \cdot 373+03$ & 1.08 & $5.605+06$ \\
\hline $.3 \mathrm{CO}$ & $3.742+C t$ & $5.35 t+c 3$ & $2.335-01$ & $3.737+C 6$ \\
\hline & $808+0$ & 5.3 & & $03+06$ \\
\hline & 2.24 & 5.3 & 5.5 & $2+06$ \\
\hline $.6 O C$ & $1.874+C E$ & $5.271+03$ & $8.192-01$ & $1.868+06$ \\
\hline . $\varepsilon 00$ & $3 . \varepsilon 86+c 6$ & 5.1 & 1.3 & $3.880+06$ \\
\hline & & 5.1 & & $2.5 \varsigma 5+06$ \\
\hline 1.500 & $1.197+0 t$ & $4.868+C 3$ & $3.114+C C$ & $1.152+06$ \\
\hline 2.000 & $t . t 5 t+$ & $4 \cdot 6$ & & $610+05$ \\
\hline & 05 & 4.1 & & $2.704+05$ \\
\hline 4.000 & $4.79 S+C E$ & $3.695+03$ & $8 \cdot 341+C 0$ & $4.762+05$ \\
\hline $5 . \mathrm{COC}$ & 3.41 & 3.3 & & $3.377+05$ \\
\hline - $\mathrm{COO}$ & +05 & C 3 & 1. & $3+05$ \\
\hline \&.coc & 1.11 & 2.4 & & $1.090+05$ \\
\hline & & $2.075+$ & $1.756+$ & $2+04$ \\
\hline & & & & \\
\hline 20.000 & & $1 . \mathrm{CC}+$ & $2.593+$ & $3.493+04$ \\
\hline & & & & $1.420+04$ \\
\hline & & & & \\
\hline
\end{tabular}

\begin{tabular}{|c|c|}
\hline $\begin{array}{l}\text { ENEFEY } \\
\text { (KEV) }\end{array}$ & TCT $\triangle \mathrm{L}$ \\
\hline 50 & $3.757+C 3$ \\
\hline 60. & $2.334+c 3$ \\
\hline 80. & $1.115+c 3$ \\
\hline 100. & $6.426+c 2$ \\
\hline 150. & $9.071+0 \bar{z}$ \\
\hline 200 . & $4.507+02$ \\
\hline 300 . & $1.792+02$ \\
\hline 400 . & $1.010+02$ \\
\hline 500. & $6.873+01$ \\
\hline 600 . & $5.223+01$ \\
\hline 800 . & $3 \cdot t 12+01$ \\
\hline 1000 & $2.840+c 1$ \\
\hline 1500 . & $2.037+01$ \\
\hline 2000 . & $1.783+01$ \\
\hline 3000 . & $1 . t 31+01$ \\
\hline 4000 . & $1.622+01$ \\
\hline 5000. & $1.655+01$ \\
\hline 6000 . & $1.704+C 1$ \\
\hline 8000 . & $1.811+C 1$ \\
\hline 10000 . & $1.917+01$ \\
\hline 15000 . & $2.170+c 1$ \\
\hline 20000 . & $2.4 C 6+01$ \\
\hline 30000 . & $2.785+C 1$ \\
\hline 40000 . & $3.047+01$ \\
\hline 50000 . & $3.238+01$ \\
\hline 60000 . & $3.289+01$ \\
\hline & $3 . t 1 s+01$ \\
\hline & \\
\hline
\end{tabular}

COHER.
SCATT:
$2.548+C 2$
$1.862+02$
$1.115+C 2$
$7.405+01$
$3.455+C 1$
$1.991+01$
$9.063+00$
$5.158+0 C$
$3.325+0 C$
$2.320+00$
$1.313+0 C$
$8.431-01$
$3.765-C 1$
$2.123-01$
$5.456-02$
$5.325-02$
$3.410-02$
$2.369-02$
$1.334-02$
$8.538-03$
$3.79 t-C 2$
$2.136-03$
$9.455-C 4$
$5.342-04$
$3.419-04$
$2.374-04$
$1.336-C 4$
$8.545-05$

SCATT.

2. $524+C 1$

$3.624+01$

3. $I 1 C+C 1$

$2.577+C 1$

$3.378+01$

2. $C 2 t+C 1$

$2.75 C+C 1$

$2.350+01$

2. $C 74+0$

1. $\varepsilon \in S+C 1$

1. $523+C 1$

1. C $24+C 1$

8. $5.27+00$

$7.371+0 C$

t. $E 15+C C$

$5.325+C$

$4 \cdot 537+\mathrm{CO}$

$3.355+C$

$2 . \in S 1+0 C$

1. $557+C C$

$1.55-4+C$

$1,11 t+C C$

$8.792-c 1$
$7.255-01$

\begin{tabular}{|c|c|}
\hline & \\
\hline $\begin{array}{r}\text { FHCTO } \\
\text { EFFECT }\end{array}$ & PAIR \\
\hline $4 \in 7+C 3$ & \\
\hline $2.111+03$ & \\
\hline $5.672+02$ & \\
\hline $5.315+02$ & \\
\hline$\varepsilon \cdot 3 \in \varepsilon+02$ & \\
\hline $3.97 \mathrm{C}+02$ & \\
\hline $1.3 \subseteq 5+02$ & \\
\hline $6.824+C 1$ & \\
\hline $4 . C 11+C 1$ & \\
\hline 2. $641+01$ & \\
\hline $1.4 C 7+C 1$ & \\
\hline$\varepsilon .8 \epsilon \epsilon+\infty 0$ & \\
\hline $4.072+00$ & $6.8 S \varsigma-C 1$ \\
\hline $2.45 t+0 \mathrm{C}$ & $2.153+C C$ \\
\hline $1.2 \varepsilon C+C C$ & $4.7 \mathrm{CB}+\mathrm{CC}$ \\
\hline$\varepsilon .37 t-01$ & $6.751+C C$ \\
\hline $6.14 \hat{z}-01$ & $8.534+C C$ \\
\hline $4 . E 1 \subseteq-C 1$ & 1. $\mathrm{CC} 2+\mathrm{C} 1$ \\
\hline $3.341-C 1$ & $1.244+C 1$ \\
\hline $2.546-C 1$ & $1.437+01$ \\
\hline $1.5 € \overline{7}-C 1$ & $1 . \varepsilon 18+C 1$ \\
\hline $1.15 c-01$ & $2.125+C 1$ \\
\hline $7.4 C 1-02$ & $2.581+C 1$ \\
\hline $5.453-02$ & $2.886+\mathrm{Cl}$ \\
\hline $4.315-02$ & $3.1 C_{4}+C_{1}$ \\
\hline $2.57 c-02$ & $3.274+C 1$ \\
\hline $2.653-02$ & $3.529+C 1$ \\
\hline $2.111-C_{2}$ & $3.72 \mathrm{C}+\mathrm{Cl}$ \\
\hline
\end{tabular}

\begin{tabular}{|c|c|c|}
\hline $\begin{array}{l}\text { ENERGY } \\
\text { (KEV ) }\end{array}$ & TCT $\Delta \mathrm{L}$ & $\begin{array}{l}\text { COFER } \\
\text { SCATT. }\end{array}$ \\
\hline 50. & $3.55 t+03$ & $2 \cdot t 21+02$ \\
\hline 60. & & \\
\hline & $.184+03$ & $.147+02$ \\
\hline 100 & $6.7 \in 2+02$ & \\
\hline & 9.4 & \\
\hline & $3+02$ & \\
\hline & & \\
\hline & & \\
\hline & $7.105+0$ & \\
\hline & & \\
\hline & & \\
\hline 1000 . & 1 & \\
\hline & & \\
\hline & $1.815+$ & \\
\hline & & \\
\hline & & \\
\hline 5000 & $1.686+$ & \\
\hline & & \\
\hline & & \\
\hline & $1.9 \in 3+$ & \\
\hline & & \\
\hline & & \\
\hline & & \\
\hline & & \\
\hline & & \\
\hline & & \\
\hline & $3.635+c$ & $1.375-0$ \\
\hline & $3.788+01$ & $8.803-05$ \\
\hline
\end{tabular}

INCOH.

SCATT.

FHCTC

EFFECT

3. $E E 5+C 1$ 3.tEE+0?

$3 . t 58+C 1 \quad 2.243+03$

$.7 C t+C 1 \quad 1 . C 32+C 3$

$3.747+01 \quad 5.625+02$

$3 . \in 13+01 \quad 8.7 C 4+C 2$

$3.413+01 \quad 4.137+02$

3. $C 55+C 1 \quad 1.46 \mathrm{C}+02$

$2.77 s+C 1 \quad 7.14 s+01$

$2.557+01 \quad 4.2 c \varsigma+01$

$2.37 t+01 \quad 2.77 \varepsilon+C 1$

2. $C 57+01 \quad 1.4 \varepsilon 2+01$

1. $E \subseteq C+C l \quad \subseteq .344+0 C$

$1.540+C 1 \quad 4.28 j+0 C$

$1.315+01 \quad 2.5 \varepsilon 3+0$

1. $C 35+01$

$2.5 \varepsilon 3+0 C$
$1.344+00$

E. $7 \subseteq C-C$

$7.454+C C \quad 6.443-01$

$7.454+C$

$t .5 \varepsilon \varepsilon+C C$
$5.389+O C$

$5.443-01$

3. 5 C3-01

$4.588+C C$

$3.393+0 \mathrm{C}$

$2.6 \in 5-0$

$1.6 \in 4-0$

$1.2 C t-01$

$2.722+C C$
$1.579+0 C$

$7.75 \varsigma-02$

$1.571+C C$

E. $71 t-C 2$

$1.311+0 \mathrm{C} \quad 4.524-02$

$3 \cdot 743-02$

E.ES1-C1 2.782-02

2.213-02

$7.167-C 1$

$2.232+C C$ $4.837+C C$ $6.558+C C$ $8.731+c c$ $1.274+01$ $1.476+\mathrm{Cl}$ $1.870+\mathrm{Cl}$ $2.175+01$ $2 \cdot-17+01$ $2.915+C 1$ $3.132+\mathrm{Cl}$ $3.300+C 1$ $3.712+\mathrm{Cl}$ 


\begin{tabular}{|c|c|c|c|c|}
\hline & & & & \\
\hline $\begin{array}{l}\text { NERGY } \\
\text { (KEY) }\end{array}$ & TOTAL & COHER. & I NCOH. & $\begin{array}{r}\text { PHCTC } \\
\text { FFEECT }\end{array}$ \\
\hline .010 & $1.147+08$ & $5.502+c 3$ & $2.820-c 4$ & $1.147+08$ \\
\hline & $7.647+07$ & $5.508+03$ & $6.333-04$ & $7.046+07$ \\
\hline .020 & $5.735+07$ & $5.50 \varepsilon+c 3$ & $1.124-03$ & $5.735+07$ \\
\hline .030 & $3.824+07$ & $5.508+03$ & $2.519-03$ & $3.823+07$ \\
\hline .040 & $2.86 \varepsilon+07$ & $5.5 C 8+03$ & $4.463-03$ & $2.867+07$ \\
\hline .050 & $2.254+07$ & $08+c 3$ & $6.947-03$ & $2.294+07$ \\
\hline & $1.512+07$ & $507+03$ & $9.967-03$ & $1.912+07$ \\
\hline & $1.4 \geqslant 4+07$ & $5.506+c 3$ & $1.759-C 2$ & $1.434+07$ \\
\hline & $1.14 \varepsilon+07$ & $5.505+03$ & $-C 2$ & $1.147+07$ \\
\hline .150 & $7.652+0 t$ & $5.50 C+03$ & $1-02$ & $7.646+06$ \\
\hline .200 & $5.740+06$ & $5.454+C 3$ & $1 . C 50-C 1$ & $5.735+06$ \\
\hline & $3.825+0 t$ & $5.477+C 3$ & -01 & $3.823+06$ \\
\hline .400 & $2 . \varepsilon 73+0 \epsilon$ & $5.455+03$ & $3.871-\mathrm{Cl}$ & $2.867+06$ \\
\hline .500 & $2.299+0 t$ & $5.427+C 3$ & $5.7 s 2-01$ & $2.294+06$ \\
\hline & $1.917+0 t$ & $5.3 \subseteq 5+c 3$ & & $1.912+06$ \\
\hline & $3.59 t+C t$ & $5.32 c+c 3$ & $1.286+c 0$ & $3.591+06$ \\
\hline 1.000 & $2.557+06$ & $E .234+C 3$ & $1.8 C 6+C C$ & $2.552+06$ \\
\hline & $1.243+0 t$ & $4 . \varsigma \subseteq 7+C 3$ & & $1.238+06$ \\
\hline $2 . c 00$ & $7.025+05$ & $4.743+C 3$ & $4.155+C C$ & $6.977+05$ \\
\hline 3.000 & $2.895+05$ & $4.244+C 3$ & $6.301+00$ & $2.853+05$ \\
\hline & $5.020+0 E$ & $3.801+03$ & $8.271+C C$ & $4.582+05$ \\
\hline & $3.36 S+C 5$ & $3.413+03$ & $1.013+01$ & $3.335+05$ \\
\hline $6 . C 00$ & $2.380+05$ & $3.077+03$ & $1.187+\mathrm{Cl}$ & $2.349+05$ \\
\hline & $1.1 \in 3+0$ & $2.534+C 3$ & & \\
\hline & & & & \\
\hline 15.000 & $2.404+04$ & $1.431+03$ & $2.249+01$ & $2.259+0$ \\
\hline & $2.681+0$ & $1 . C 34+C 3$ & $2 \cdot t 12+01$ & $2.575+04$ \\
\hline & & & & \\
\hline $40 . C C O$ & $7.372+C 2$ & $3.85 s+c 2$ & $3.4 C 9+C 1$ & $6.948+03$ \\
\hline
\end{tabular}

\begin{tabular}{|c|c|c|c|c|}
\hline $\begin{array}{l}\text { ENERGY } \\
\text { (KEVV) }\end{array}$ & TOT & $\begin{array}{l}\text { COHER } \\
\text { SCATT }\end{array}$ & $\begin{array}{l}\text { I NCOH. } \\
\text { SCATT. }\end{array}$ & $\begin{array}{l}\text { PHCTC } \\
\text { EFFECT }\end{array}$ \\
\hline .010 & $1.20 t+C \varepsilon$ & $5.630+03$ & $2.743-c 4$ & $1.205+08$ \\
\hline & & $5.63 c+03$ & $6.1 \in 0-C 4$ & $8.037+07$ \\
\hline & $\epsilon .028+C \bar{T}$ & $5.63 C+0 \geq$ & $.0 S_{3}-C_{3}$ & $6.027+07$ \\
\hline . $c \geq 0$ & & $5 . t 3 c+03$ & & $4.018+07$ \\
\hline .040 & $3 . \mathrm{C}$ & $5.63 \mathrm{C}+$ & 3 & $14+07$ \\
\hline & $2.412+07$ & $5.629+03$ & & $1+07$ \\
\hline $.0 \in C$ & & $5 . \in 2 S+03$ & & \\
\hline & 1. & 5.62 & & \\
\hline & $6+c 7$ & 5.62 & & +07 \\
\hline & & & & \\
\hline & & & & \\
\hline .30 & & 5.5 & & \\
\hline & & & & \\
\hline .50 & & 5.5 & & +06 \\
\hline & & & & \\
\hline & & & & \\
\hline 1.00 & & 5.3 & & \\
\hline & & & & \\
\hline & & & & \\
\hline & & 4.35 & & \\
\hline & & 3.8 & & \\
\hline & & & & \\
\hline & & & & \\
\hline & & & & \\
\hline & & & & \\
\hline & & & & \\
\hline & & & & \\
\hline & & & & \\
\hline & & $.01 c+c z$ & $3.443+01$ & $7.305+C$ \\
\hline
\end{tabular}

\begin{tabular}{|c|c|c|}
\hline \multicolumn{3}{|c|}{$z=S \geq$ NEPTUN ILM } \\
\hline $\begin{array}{l}\text { ENERGY } \\
\text { (KEY) }\end{array}$ & TOTAL & $\begin{array}{l}\text { COHER. } \\
\text { SCATT. }\end{array}$ \\
\hline .010 & $1.22 \varepsilon+0 \varepsilon$ & $5.753+c 3$ \\
\hline .015 & $8.1 \varepsilon 6+c 7$ & $5.752+c 3$ \\
\hline .020 & $6.140+07$ & $5.753+C 3$ \\
\hline .030 & 4. $\operatorname{cs} 3+C 7$ & $5.753+C 3$ \\
\hline .040 & $3.070+07$ & $5.753+03$ \\
\hline $.05 \mathrm{C}$ & $2.456+C 7$ & $5.752+C 3$ \\
\hline .060 & $2.047+07$ & $5.752+C 3$ \\
\hline $.08 \mathrm{C}$ & $1.535+07$ & $5.751+C 3$ \\
\hline .100 & $1.228+C 7$ & $5.75 c+c 3$ \\
\hline .150 & $8.1 \varsigma_{1+0 t}$ & $5.745+C 3$ \\
\hline .200 & $t .145+0 t$ & $5.739+03$ \\
\hline .300 & $4.098+C 6$ & $5.723+C 3$ \\
\hline .400 & $3.075+C 6$ & $5.7 \mathrm{CO}+03$ \\
\hline .500 & $2.461+06$ & $5.673+03$ \\
\hline$\bullet \in O C$ & $2.052+0 t$ & $5.641+c 3$ \\
\hline .800 & $2.725+C 6$ & $5.567+C 3$ \\
\hline 1.000 & $2.253+c t$ & $5.481+C 3$ \\
\hline 1.500 & $1.315+0 t$ & $5.241+C 3$ \\
\hline 2.000 & $7.847+05$ & $4.580+03$ \\
\hline 3.000 & $3.2 \in 3+C 5$ & $4.459+03$ \\
\hline 4.000 & $5.453+C 5$ & $3.591+\mathrm{C}_{3}$ \\
\hline 5.000 & $3.65 \bar{z}+05$ & $3.58 c+c 3$ \\
\hline t.c00 & $2.564+C 5$ & $3.223+C 3$ \\
\hline$\varepsilon . C 00$ & $1.2 \in 2+C 5$ & $2.652+03$ \\
\hline $1 \mathrm{C} . \mathrm{COC}$ & $7.211+04$ & $2.215+C 3$ \\
\hline $15.00 \mathrm{C}$ & $2.625+04$ & $1.458+C 3$ \\
\hline $2 \mathrm{C} \cdot \mathrm{co0}$ & $2.509+04$ & $1 \cdot \csc c+c 3$ \\
\hline & $1.677+04$ & \\
\hline $\operatorname{coc}$ & C.C $229+c 3$ & $4.12 t+c 2$ \\
\hline
\end{tabular}
SCATT:
2.784-04 $6.252-\mathrm{C}^{2}$ $1.1 \mathrm{C9}-03$ $2.487-C 3$ $4.4 C_{5}-C_{3}$ $6.858-03$ $5.840-C_{3}$ $1.736-c 2$ $2.653-02$ $5.545-c 2$ $1.037 \div 01$ 2.242-01 $3 . \varepsilon 25-\mathrm{Cl}$ $5.725-\mathrm{Cl}$ $7.882-c 1$ $1.273+00$ $1.788+\mathrm{CC}$ 3. $C 26+00$ $4.178+C C$ $6.305+C 0$ $8.299+00$ $1.018+C 1$ $1.155+01$ $1.511+01$ $1.777+\mathrm{C} 1$ $2.279+01$ $2.652+01$ $3.159+01$ $3.465+C 1$
PHCTO EFFECT $1.228+08$ $8.185+07$ $6 \cdot 139+07$ 4. $C 93+07$ $3.070+07$ $2.456+07$ $2.046+07$ $1.535+07$ $1.228+07$ $8.185+06$ $6.139+06$ $4.093+06$ $3.070+06$
$2.456+06$ 2. $046+06$ $2.720+c 6$ $2 .<88+06$ $1.310+06$ $7.757+05$ $3.218+05$ $5.413+05$ $3.616+05$ $1.236+05$ $6.987+C 4$ $2.473+04$ $2.757+04$ $1.610+04$ $7.581+03$

\begin{tabular}{|c|c|c|}
\hline $\begin{array}{l}R G Y \\
E V Y\end{array}$ & & COHER - \\
\hline 50. & $4.113+03$ & $.699+02$ \\
\hline 60. & & $1.973+02$ \\
\hline 80. & & $.182+02$ \\
\hline 100. & $7+0$ & $3+01$ \\
\hline & & $3.66 \epsilon+01$ \\
\hline & 4.8 & \\
\hline 300 . & $1.930+0$ & +00 \\
\hline & & +00 \\
\hline 50 & & \\
\hline 60 & & 2.4 \\
\hline & & 1.3 \\
\hline 1000 & & 8.9 \\
\hline 1 & $2.120+c$ & 3.9 \\
\hline & & 2.2 \\
\hline 00 & $1, \in 9$ & 1.0 \\
\hline 40 & & 02 \\
\hline & & \\
\hline 600 & 1.75 & $2.515-02$ \\
\hline 800 & 1.87 & $1.4]$ \\
\hline & & 9.06 \\
\hline 1500 & 2.27 & 4.03 \\
\hline & $2.520+$ & $2.267-03$ \\
\hline & & $1.008-03$ \\
\hline & $3.164+$ & \\
\hline & & $3.629-04$ \\
\hline & & $2.521-04$ \\
\hline & $3.763+0$ & $1.418-04$ \\
\hline
\end{tabular}

100000 . $3.529+019.075-05$

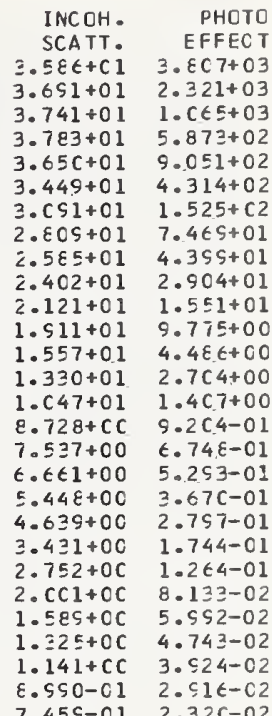

$7.45 s-01$
PAIR

PRCD。

$7.402-\mathrm{Cl}$

$2.302+C$

$7.078+C$

$8.854+0 \mathrm{C}$

1. $\mathrm{C} 38+\mathrm{C} 1$

$1.251+01$

$1.499+01$

$1.512+C 1$

$2.689+\mathrm{Cl}$

2. $599+C 1$

$3.228+01$

$3.4 C 7+01$

$3.852+C 1$

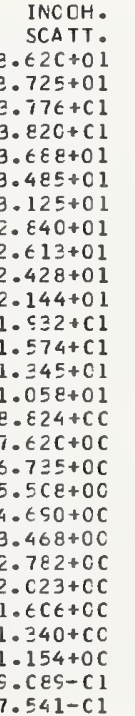

PHCTO EFFEC T $2.45 S+03$ $1.12 c+03$ t. $1 \subseteq C+02$ S. $3 \subseteq 4+C 2$ $4.5 C 5+02$ 1. $t C \underline{5}+02$ $7.8 \subseteq 4+01$ $4 \cdot 656+01$ $3.074+01$ $1.64 \mathrm{C}+01$ $1.0 \equiv 2+01$ $4.72 t+00$ $2 . \varepsilon 44+0 C$ $1.47 \varepsilon+C C$ S. $657-01$ $7.077-01$ $5.545-01$ $2.5 \geqq 1-01$ $1.827-C 1$ $1.324-01$
$8.52 C-02$ t. $27 \varepsilon-02$ 4. $5 \in \varepsilon-02$ $4.111-02$ 3. $C 55-02$ 2. $431-02$

\begin{tabular}{|c|c|c|}
\hline $\begin{array}{c}\text { EAERGY } \\
\text { (KEV) }\end{array}$ & CTAL & $\begin{array}{l}\text { COHER } \\
\text { SCATT. }\end{array}$ \\
\hline 50. & $4.45 S+C 3$ & $2.858+02$ \\
\hline & $2.804+03$ & $2.090+02$ \\
\hline 80 & $3+03$ & \\
\hline 100 & $7.715+C 2$ & 8.32 \\
\hline & & 3.88 \\
\hline & & \\
\hline 300 & $2 . c 87+0$ & $0+01$ \\
\hline & & 5.81 \\
\hline & & \\
\hline & & 2.6 \\
\hline & & \\
\hline & & \\
\hline & & 4.2 \\
\hline & & 2.3 \\
\hline & & \\
\hline & $1.745+01$ & 6.0 \\
\hline & & \\
\hline & & \\
\hline & 1.561 & 1.5 \\
\hline & & \\
\hline & & \\
\hline & $2.583+$ & -03 \\
\hline & & \\
\hline & & \\
\hline & $3.471+$ & 3.85 \\
\hline & $3.624+$ & $2.676-04$ \\
\hline & 1 & $1.505-04$ \\
\hline & & \\
\hline
\end{tabular}
SCATT: 3. $647+01$ 2. $756+C 1$ a. $\varepsilon 11+C 1$ 3. $\varepsilon 5 \varepsilon+01$ $2.726+C 1$ 3. $521+C 1$ $3.158+C 1$ z. $\varepsilon 7 C+C 1$ $2.641+01$ $2.455+01$ $2.167+C 1$ 1. $553+C 1$ $1.551+01$ $1 \cdot 35 s+01$ 1. $\mathrm{C} 7 \mathrm{C}+\mathrm{Cl}$ Q. $\mathrm{C} 2 \mathrm{C}+\mathrm{CC}$ $7.7 \mathrm{C}+\mathrm{CC}$ $5.568+C 0$ $4.741+0 C$ $3.5 C t+C C$ 2. $E 12+C C$ 2. $C 45+C C$ $1 . t 24+0 C$ 1. $354+0 \mathrm{C}$ $1.1 \in 6+O C$ c. 1 \& $7-01$ 7.623-01
PHCTO EFFECT $4.177+03$ $1.17 s+03$ $6.45 i+02$ S. $75 E+02$ $1.66 s+02$ $\varepsilon .21 \mathrm{t}+\mathrm{Cl}$ 4. $\varepsilon 53+C 1$ $3.207+01$ $1.713+01$ 1. $C 7 S+01$ $4.544+0 C$ $1.547+C C$ $1.011+00$ 7.4C7-01 $5.8 C S-C 1$ $4.027-01$ 3. $c \in \varepsilon-C 1$ 1. $513-01$ 1. $3 \varepsilon t-01$ E. S23-02 $6.575-02$ $5.2 C 4-02$ $4.3 \mathrm{C} 5-02$ $3.2 \mathrm{CC}-02$ $2.546-C 2$
PAI

PRCL.

7. $871-01$

$2.387+C$

$5.155+C$

$7.499+0 \mathrm{C}$ $1.103+C 1$ $1.363+C 1$ $1.566+C 1$ $1.561+01$ $2.287+0$ $2.781+C$ $3.331+C 1$ 3. $503+C 1$ 3. $764+C 1$ 3. $566+C$ 


\begin{tabular}{|c|c|c|c|c|}
\hline $\begin{array}{l}\text { ENERG } \\
\text { (KEV) }\end{array}$ & TOTAL & $\begin{array}{l}\text { COHER. } \\
\text { SCATT. }\end{array}$ & $\begin{array}{l}\text { I NCOH. } \\
\text { SCATT. }\end{array}$ & $\begin{array}{l}\text { PHCTC } \\
\text { EFFECT }\end{array}$ \\
\hline .010 & $1.286+08$ & $5.878+C 3$ & $2.733-c 4$ & $1.286+08$ \\
\hline.$C 15$ & $8.574+07$ & $5.878+03$ & $6.138-04$ & $8.573+07$ \\
\hline - $C<0$ & $30+C 7$ & $5.878+C 3$ & $1 . C 8 s-C 3$ & $6.430+07$ \\
\hline 30 & $4.287+07$ & $5.877+C 3$ & $.442-03$ & $4 \cdot 286+07$ \\
\hline .040 & $15+07$ & 5.877 & $5-c_{3}$ & $3.215+07$ \\
\hline 50 & $72+07$ & 5.87 & & $2+07$ \\
\hline - 060 & 2. & 5.87 & & $2.143+07$ \\
\hline - $C 80$ & $1.608+$ & 5.87 & & +07 \\
\hline .100 & 1. $287+c$ & $5 \cdot 874+03$ & $2.644-02$ & $6+07$ \\
\hline .150 & $E .579+C 6$ & E. E $7 C+C 3$ & $5.838-02$ & $8.573+06$ \\
\hline .200 & $t .436+0 t$ & $5.864+03$ & 1.C18-01 & $6.430+06$ \\
\hline .300 & $4.252+C t$ & $5.848+03$ & $2.203-\mathrm{Cl}$ & $4.286+06$ \\
\hline .400 & $3.221+C 6$ & $5.827+C 3$ & $3.758-c 1$ & $3.215+06$ \\
\hline .500 & $2.578+C t$ & $5.8 \mathrm{CO}+\mathrm{C}_{3}$ & $5 \cdot \in 27-01$ & $2.572+06$ \\
\hline .600 & 2 & 5.765 & $7.749-C 1$ & $2.143+06$ \\
\hline .800 & $613+06$ & $5.697+C 3$ & $1.252+c c$ & $1.607+06$ \\
\hline & $74+C 6$ & .612 & $61+00$ & $2.868+06$ \\
\hline 500 & $1.4 \mathrm{C}$ & 3 & $2.588+c c$ & $1.400+06$ \\
\hline OC & & & & +05 \\
\hline 000 & $3.32 t+C E$ & 3 & & 3 \\
\hline 4. $\mathrm{COO}$ & 5. $t \in 7+05$ & $4.111+C 3$ & $8.250+c c$ & 5.6 \\
\hline $5 . \mathrm{CCC}$ & $3.802+05$ & $3 . t 82+C z$ & 1. $\mathrm{C} 12+\mathrm{Cl}$ & $3.765+05$ \\
\hline $6 . c 00$ & $2.671+0$ & $3.312+03$ & $1.188+01$ & $2.638+05$ \\
\hline 8.000 & $1.313+C 5$ & $2.723+C 3$ & $1.503+c 1$ & $1.286+05$ \\
\hline $1 \mathrm{C} . \mathrm{COO}$ & $7.498+04$ & $2.282+c 3$ & $1.772+01$ & $7.268+04$ \\
\hline 15.000 & $2.732+04$ & $1.564+C 3$ & $2.2 s_{1}+01$ & $2.573+04$ \\
\hline $2 \mathrm{C} \cdot \mathrm{COO}$ & $3.044+04$ & $1.11 s+03$ & $2.675+01$ & $2.929+04$ \\
\hline & $1.745+C 4$ & $6.58 s+02$ & $3.1 \mathrm{Eg}+\mathrm{Cl}$ & $1.676+04$ \\
\hline & & $45+C 2$ & $3.458+C 1$ & $7.930+03$ \\
\hline
\end{tabular}

\begin{tabular}{|c|c|c|c|c|}
\hline $\begin{array}{r}\text { EAEREY } \\
\text { (KEV) }\end{array}$ & TOTAL & $\begin{array}{l}\text { COHER. } \\
\text { SCATT. }\end{array}$ & $\begin{array}{l}\text { I KCOH. } \\
\text { SCATT. }\end{array}$ & $\begin{array}{r}\text { PHCTC } \\
\text { EFFECT }\end{array}$ \\
\hline . $C 10$ & $1.316+08$ & 6.CC3+03 & $2.759-04$ & $1.316+08$ \\
\hline - C 15 & $8.7 \overline{7} 1+C 7$ & $6.0 C_{3}+C_{3}$ & $6.157-C_{4}$ & \\
\hline 20 & $7 E+C 7$ & 6. $\mathrm{CO} 3+03$ & $0-03$ & \\
\hline 20 & $86+07$ & $6 . \mathrm{CO}_{3}+\mathrm{C} 3$ & $.465-03$ & 4 \\
\hline .040 & $89+C 7$ & $6.0 \mathrm{C} 3+\mathrm{C} 3$ & $367-03$ & \\
\hline .050 & $2.632+c 7$ & $6 \cdot \mathrm{CO}_{3}+03$ & $6.758-c 3$ & $2.631+$ \\
\hline .060 & $2.153+07$ & $6 . \mathrm{CC}_{2}+\mathrm{C}_{3}$ & $3+c_{3}$ & \\
\hline . $C 80$ & 1. $645+07$ & $6 . \mathrm{Cl}_{1}+\mathrm{C}_{3}$ & $1.721-02$ & $1.644+0$ \\
\hline .100 & $16+07$ & $6 . \mathrm{COC}+03$ & $2.669-02$ & $1 \cdot 316+0$ \\
\hline .150 & $6+C t$ & $5.5 \$ 6+c$ & $5.893-\mathrm{C}_{2}$ & $8.770+0$ \\
\hline .200 & $t .5 E 4+C t$ & E.SSC & 1.0 & 6.57 \\
\hline$=300$ & 4. & 5.97 & 2. & \\
\hline .400 & $3.255+c t$ & $5.553+C 3$ & & 3.2 \\
\hline $.5 \mathrm{CO}$ & $2.637+0 t$ & $5.52 t+C 3$ & $5.673-01$ & $2.631+0$ \\
\hline $.60 \mathrm{C}$ & $2.198+C t$ & $5.8 S t+C 3$ & $7.809-C 1$ & 2.1 \\
\hline .800 & $1 . t 50+06$ & $5.824+03$ & $1.261+C C$ & $1.644+0$ \\
\hline $1 . \mathrm{COO}$ & $3.010+c t$ & $5.74 C+C 3$ & $1.771+C C$ & $3.005+0$ \\
\hline $1.5 \mathrm{CO}$ & $1.470+0 t$ & $5.5 C_{3}+C_{3}$ & $2.994+00$ & $1.464+0$ \\
\hline $2 . C 00$ & $8.282+c 5$ & $5.241+c_{3}$ & $4.131+C C$ & $8.230+$ \\
\hline 3.000 & $3.461+C 5$ & $4 \cdot 7 C 7+C 3$ & $6.231+00$ & $3.414+c$ \\
\hline 4. $\mathrm{COO}$ & $6+C 5$ & $4.213+C 3$ & $8.2 \mathrm{C} 8+00$ & $4.144+$ \\
\hline & & 3.77 & & \\
\hline $6 . \mathrm{COO}$ & $2.677+05$ & $3 \cdot 3 \subseteq 1+C 3$ & 1.18 & $2.643+0$ \\
\hline $8.0 \mathrm{CC}$ & $1 \cdot 365+0=$ & $2.785+C 3$ & $1.505+C 1$ & $1.337+$ \\
\hline $\mathrm{C} . \mathrm{COO}$ & $7.8 C_{2}+C_{4}$ & $2.331+C 3$ & $1.777+01$ & $7.567+0$ \\
\hline 15.000 & $2.840+C 4$ & $1.589+C 3$ & $2.258+C 1$ & $2.679+0$ \\
\hline $2 C \cdot c 00$ & $3.170+04$ & $1.15 \mathrm{C}+\mathrm{C} 3$ & $2.684+01$ & $3.052+0$ \\
\hline $0 . \mathrm{COC}$ & $1.813+04$ & $6.771+c 2$ & $3.2 C 5+01$ & $1.742+0$ \\
\hline & $20+C 3$ & $.365+c 2$ & $20+c 1$ & $.258+0$ \\
\hline
\end{tabular}

\begin{tabular}{|c|c|c|}
\hline \multicolumn{3}{|c|}{$Z=S E$ CUR IUM } \\
\hline ENERGY & TOTAL & COFER. \\
\hline (KEV) & & SCATT. \\
\hline .010 & $1.355+O E$ & $6.130+03$ \\
\hline .015 & $9 \cdot c 33+07$ & $6.13 \mathrm{C}+\mathrm{C} 3$ \\
\hline $.02 \mathrm{C}$ & $6.775+07$ & $6.130+03$ \\
\hline .030 & $4.517+C 7$ & $6.130+03$ \\
\hline . $C_{4} 0$ & $3.38 E+C 7$ & $6.13 C+03$ \\
\hline $.05 \mathrm{C}$ & $2.710+07$ & $6.13 C+C 3$ \\
\hline.$C 60$ & $2.259+07$ & $6.12 c+c 3$ \\
\hline .080 & $1 . \in S 4+C 7$ & $6.128+03$ \\
\hline .100 & $1.355+C 7$ & $6.127+C 3$ \\
\hline $.15 \mathrm{C}$ & S. C $38+06$ & $6.123+03$ \\
\hline .200 & $6.780+c 6$ & $6.117+03$ \\
\hline $.30 \mathrm{C}$ & $4.522+c 6$ & $6.101+03$ \\
\hline .400 & $3.393+06$ & 6.C79+03 \\
\hline .500 & $2.716+C E$ & $6.05 \bar{z}+c 3$ \\
\hline .600 & $2.2 t 4+C t$ & $6.021+C 3$ \\
\hline $.80 \mathrm{C}$ & $1 . t S S+0 \epsilon$ & $5 . \$ 47+C 3$ \\
\hline 1.000 & $3.062+c t$ & $5.862+c 3$ \\
\hline 1.500 & $1.517+06$ & $5.61 \varepsilon+c 3$ \\
\hline 2.000 & $8.564+C 5$ & $5.34 S+C 3$ \\
\hline 3. $\mathrm{COC}$ & $3.587+C 5$ & $4.8 C 2+C 3$ \\
\hline 4.000 & $1.848+05$ & $4.299+03$ \\
\hline E. COC & 4. C SO+C 5 & $3.851+C 3$ \\
\hline 6.100 & $2.765+05$ & $3.464+c 3$ \\
\hline$\varepsilon \cdot \operatorname{coc}$ & $1.404+C 5$ & $2.847+C 3$ \\
\hline $1 \mathrm{C} . \mathrm{COO}$ & 8. $c \geq 8+04$ & $2.383+c 3$ \\
\hline $15.00 \mathrm{C}$ & $2.528+C 4$ & $1.62 E+C 3$ \\
\hline $2 \mathrm{C} .000$ & $3.269+C 4$ & $1.178+03$ \\
\hline $3 \mathrm{C} \cdot \mathrm{COC}$ & $1.872+c 4$ & $t . S 51+C 2$ \\
\hline $4 \mathrm{C} . \mathrm{COO}$ & S. $C 18+C \underline{2}$ & $4.485+02$ \\
\hline
\end{tabular}

$\begin{array}{rr}\text { INCOH. } & \text { PHCTC } \\ \text { SCATT. } & \text { EFFECT } \\ 4.4 C 5-04 & 1.355+08 \\ 5.8 S 1-C 4 & 5.032+07 \\ 1.755-C 3 & 6.774+07 \\ 3.533-C 3 & 4.516+07 \\ 6 . S E 5-C 3 & 3.387+07 \\ 1 . C 84-C 2 & 2.710+07 \\ 1.555-C 2 & 2.258+07 \\ 2.742-C 2 & 1.693+07 \\ 4.251-C 2 & 1.355+07 \\ 9.371-C 2 & 9.032+06 \\ 1.632-C 1 & 6.774+06 \\ 3.517-C 1 & 4.516+06 \\ 5.980-01 & 3.387+06 \\ \text { E. } 116-C 1 & 2.710+06 \\ 1.222+C 0 & 2.258+06 \\ 1.955+0 C & 1.653+06 \\ 2.714+C C & 3.056+06 \\ 4.420+C C & 1.511+06 \\ 5.862+C C & 8.510+05 \\ 8.252+00 & 3.539+05 \\ 1 . C 38+C 1 & 1.805+05 \\ 1.236+01 & 4.051+05 \\ 1.419+C 1 & 2.730+05 \\ 1.741+C 1 & 1.376+05 \\ 2 . C 11+01 & 7.798+04 \\ 2.518+01 & 2.762+04 \\ 2.8 S 1+C 1 & 3.149+04 \\ 3.350+C 1 & 1.799+04 \\ 3.687+01 & 8.533+03\end{array}$

\begin{tabular}{|c|c|c|}
\hline $\begin{array}{r}\text { ENERGY } \\
\text { (KEV) }\end{array}$ & TCTAL & $\begin{array}{l}\text { COHER . } \\
\text { SCATT. }\end{array}$ \\
\hline 50 & $4.722+03$ & $2.941+02$ \\
\hline 60. & $2.951+03$ & $2.152+02$ \\
\hline 80. & $1.414+03$ & $1.290+02$ \\
\hline 100. & $8.084+02$ & $8.574+01$ \\
\hline 150. & $1.085+03$ & $4.004+01$ \\
\hline 200. & $5.483+02$ & $2.308+01$ \\
\hline 300. & $2.171+02$ & $1.051+01$ \\
\hline 400 . & $1.20 s+02$ & $5.985+00$ \\
\hline 500. & $8.148+01$ & $3.858+00$ \\
\hline 600. & $6.128+01$ & $2.692+0 \mathrm{C}$ \\
\hline 800 . & $4.151+01$ & $1.523+00$ \\
\hline $10 \mathrm{Co}$ & $3.211+c 1$ & $9.784-01$ \\
\hline 1500. & $2.255+01$ & $4.369-01$ \\
\hline 2000 . & $1.55 t+01$ & $2.464-01$ \\
\hline 3000. & $1.784+C 1$ & $1.098-01$ \\
\hline 4000. & $1.77 t+C 1$ & $6.181-02$ \\
\hline 5000. & $1.816+01$ & $3.959-02$ \\
\hline 6000. & $1.87 C+C 1$ & $2.751-02$ \\
\hline 8000 & $1.987+01$ & $1.548-02$ \\
\hline 10000 & 2. $c s s+01$ & $9.911-03$ \\
\hline 15000. & $2.369+c 1$ & $4.407-03$ \\
\hline 20000 . & $2.632+01$ & $2.480-03$ \\
\hline 30000 . & $3.063+01$ & $1.102-03$ \\
\hline 40000 . & $3.353+01$ & $6.201-04$ \\
\hline 50000. & $3.55 \varepsilon+C 1$ & $3.969-04$ \\
\hline 60000 & $3.71 t+C 1$ & $2.756-\mathrm{C} 4$ \\
\hline 80000 & 3. $557+C 1$ & $1.551-04$ \\
\hline & $4.146+01$ & $9.924-05$ \\
\hline
\end{tabular}

INCOH.
SCATT:

3. $682+01$

3. $751+01$

3. $E 44+C 1$

3. $E \subseteq 3+C 1$

3. $556+C$

3. $19 \mathrm{C}+\mathrm{Cl}$

2. $900+C$

2. $669+01$

2. $4 E 1+C$

2. $190+01$

1. $574+01$
$1.6 C 5+01$

1. $374+01$

1. $C 81+0$

S. $\mathrm{C} 16+\mathrm{CC}$

7. $785+C C$

6. $E 81+0 C$

5. $628+0 c$

4. $7 \subseteq 2+C C$

3. $544+0 C$
2. $E 43+C C$

2. $E 43+C C$

1. $641+0 C$

1. $369+C C$

$1.179+C \mathrm{C}$

S. $286-01$
$7.7 C 5-01$
PHOTO

$4.3 \subseteq 1+03$

2. $658+03$

1. $247+C 3$

$6.837+02$

1. $012+03$

1. $747+02$

E. $5 \subsetneq 6+01$

5. $053+01$

$3.37 E+01$

$1.135+01$

5. $21 \mathrm{C}+0 \mathrm{C}$

$1.623+00$

I. $C \in C+O C$

7. $7 \in 1-01$

6. $0 \varepsilon 5-01$

$3.212-\mathrm{C} 1$

$2.002-01$

1. 451-01

S. 341-02

E. 447-02

4. 5C7-02

$3.35 \mathrm{C}-02$

$2 . t \in t-02$
PAIR
PRCL.

ع. $178-C 1$

2. $448+C C$

$7.623+C C$

S. $554+0 \mathrm{C}$

$1.118+C 1$

$1.3 E C+C$

1. $\subseteq 94+C 1$

$2.333+C 1$

2. $847+C 1$

$3.182+01$

$3.416+01$

$3.554+C 1$

3. $861+01$
4. $C 66+C 1$

\begin{tabular}{|c|c|c|c|c|c|}
\hline $\begin{array}{l}\text { ENERGY } \\
\text { (KEV) }\end{array}$ & TCTAL & $\begin{array}{l}\text { COHER - } \\
\text { SCATT }\end{array}$ & $\begin{array}{l}\text { INCCH. } \\
\text { SCATT. }\end{array}$ & $\begin{array}{r}\text { FHOTC } \\
\text { EFFEC T }\end{array}$ & $\begin{array}{l}\text { PAIF } \\
\text { PRCL. }\end{array}$ \\
\hline 50 & $4.511+03$ & $3.026+02$ & 3. $7 C_{6} 6+C 1$ & $4.572+C 3$ & \\
\hline 60. & 3. $c 68+03$ & $2.214+02$ & 3. E $1 S+01$ & 2. $\varepsilon C \varepsilon+C 3$ & \\
\hline 80 . & $1.47 c+03$ & $1.328+02$ & $3.876+C I$ & $1.2 S E+03$ & \\
\hline 100 & $8.415+02$ & $8.827+01$ & $3.528+c 1$ & $7.144+C 2$ & \\
\hline & $1 \cdot 1$ & $124+01$ & 3. $7 S_{E}+C 1$ & 1. $C \equiv C+03$ & \\
\hline 0 & $5.702+02$ & $2 \cdot 377+01$ & $3.589+01$ & $5.1 C 5+02$ & \\
\hline 300. & $2.255+02$ & $1.083+01$ & $\cdot \angle 21+C 1$ & $1.825+02$ & \\
\hline 400 & $1 \cdot 253+C 2$ & $=00$ & 2.52 & \&.SE4+01 & \\
\hline 500 & 8.42 & 3.97 & $2 \cdot 6$ & 5.32 & \\
\hline 600 . & 6.32 & 2.773 & $2 \cdot 5$ & $+C 1$ & \\
\hline 800 & $4.265+01$ & $1.569+00$ & $2.213+c 1$ & $1.8 \subsetneq 5+C .1$ & \\
\hline 1000 。 & $3.289+01$ & $1.008+00$ & 1. $s \varsigma 5+01$ & $1.154+01$ & \\
\hline 00 . & $2 \cdot 301+C 1$ & $4.502-01$ & $1 \cdot t 2 t+C 1$ & $5.457+0 C$ & $E .43$ \\
\hline 2000 & 1. Sऽs+0 1 & 2.538 & $1.388+C 1$ & $3.277+0 \mathrm{C}$ & \\
\hline & 1.8 & 1 & & $1 . t$ & \\
\hline 4000 & 1.807 & -02 & S. $112+00$ & 1.10 & 7 \\
\hline 5000 & 1.84( & $4.079-02$ & T. EE & $8.121-01$ & $\varsigma$ \\
\hline & 1.89 & 2.83 & 6.55 & $6.3 t t-01$ & \\
\hline & $2 . \mathrm{CO}$ & 1.59 & $5.6 E$ & $4.4 I 1-C 1$ & \\
\hline 10000 & $2.121+01$ & 1.021 & 4. E & $3.361-C 1$ & $1.602+C 1$ \\
\hline 150 & $2.4 \mathrm{I} 3+\mathrm{Cl}$ & $4.541-C_{3}$ & $3.582+c c$ & $2 \cdot c \leqq 5-01$ & - C $34+c$ \\
\hline & $2 \cdot 684+0$ & $2.555-03$ & $2.873+0 \mathrm{C}$ & $1.51 \varepsilon-01$ & $382+c$ \\
\hline 3000 & 3.10 & $1.136-\mathrm{C} 3$ & 2. $C E S+O C$ & $5.773-02$ & $882+c$ \\
\hline 4000 & $3 \cdot 385+0$ & $6.390-04$ & $1.655+0 C$ & $7.2 \mathrm{Cl} 1-\mathrm{C} 2$ & $3.212+C$ \\
\hline & $3.554+0$ & $4.090-04$ & $1.2 E 3+$ & $5.7 \mathrm{CC}-02$ & $3.450+C$ \\
\hline & $3.7 \in C+C 1$ & $2.840-04$ & $1.1 \varsigma 1+00$ & $4.716-c 2$ & $3.636+C 1$ \\
\hline & $4.010+01$ & $1.598-04$ & S. $385-01$ & $3 \cdot 5 C 5=02$ & 3. $\subsetneq 13+C 1$ \\
\hline & $4 \cdot 1 \subseteq 2+C 1$ & $1.023-04$ & $7.786-C 1$ & $2.7 \varepsilon \varsigma-\mathrm{C} 2$ & $4.111+C$ \\
\hline
\end{tabular}

\begin{tabular}{|c|c|c|c|}
\hline ENERGY & TCTAL & COHER . & INCOH. \\
\hline 50 & 5. $c 84+03$ & $3.110+02$ & 3. $\varepsilon 58+C 1$ \\
\hline 60. & $3.183+03$ & $2.277+02$ & 3. $c 58+C 1$ \\
\hline 80. & $25+03$ & $1.36 t+02$ & $592+01$ \\
\hline 100. & $755+02$ & $9.082+01$ & 4. $C \equiv 1+01$ \\
\hline 150. & 1. $1 \in C+C 3$ & $4.244+01$ & 3. $E \in 1+C 1$ \\
\hline 200. & 5. EG4+C 2 & $2.447+01$ & 3. $635+C 1$ \\
\hline 300 . & $2.319+02$ & $1.115+01$ & $55+C 1$ \\
\hline 400 . & 1. $2 E \&+C 2$ & $6 \cdot 34 t+00$ & $5 s+C_{1}$ \\
\hline 500. & $8.661+01$ & $4.091+00$ & $2.723+01$ \\
\hline 600 . & $6.45 t+01$ & $2.855+0 \mathrm{C}$ & $.531+C 1$ \\
\hline 800 . & $4.371+C_{1}$ & $1.616+0 \mathrm{C}$ & $23 t+c 1$ \\
\hline 1000 & $3.363+C_{1}$ & $1.038+00$ & $2 . C 15+01$ \\
\hline 1500 . & 01 & 4.63 & $=642+01$ \\
\hline 2000 . & 2. $C 35+01$ & $2.613-01$ & $1.4 C 3+01$ \\
\hline 3000 . & $1.850+C_{1}$ & $1.164-01$ & 1. $1 C_{4}+C_{1}$ \\
\hline 4000 . & 1. $828+01$ & $6.557-02$ & $5.2 C 8+C C$ \\
\hline 5000 & $1.85 t+01$ & $4.200-02$ & $7.551+C C$ \\
\hline 6000 . & $1.5 C 2+01$ & $2.918-02$ & T. C $227+C C$ \\
\hline 8000 . & $2.015+01$ & $1.642-02$ & E. $748+C C$ \\
\hline 10000 . & $2.138+01$ & $1.051-02$ & $4.8 \varsigma 4+0 \mathrm{C}$ \\
\hline 15000. & $2.44 S+01$ & $.675-03$ & $19+0 \mathrm{C}$ \\
\hline 20000 . & $727+C_{1}$ & $2.630-03$ & $\mathrm{SC} 3+0 \mathrm{C}$ \\
\hline 30000. & $3.135+C 1$ & $1.169-03$ & $2.111+0 \mathrm{C}$ \\
\hline 40000 . & $3.413+C 1$ & $6.578-04$ & $1.676+0 \mathrm{C}$ \\
\hline 50000 & $3.625+01$ & $4.210-04$ & $1.358+0 C$ \\
\hline 60000 & $3.793+C 1$ & $2.924-04$ & $1.2 C 4+C C$ \\
\hline 80000 . & 4. $c 45+01$ & $1.645-04$ & $5.484-01$ \\
\hline & $4.217+01$ & $1.053-04$ & 7. $E \in \&-C I$ \\
\hline
\end{tabular}

FHCTO

EFFECT

$4.734+03$

2. $\subseteq 15+C\}$

$1.3 \leq 2+03$

$7.444+C 2$

1. $C 75+03$

$5.25 t+02$

1. $8 \varepsilon 2+C 2$

5. $2 E S+C 1$

$5.52 \mathrm{E}+\mathrm{Cl}$

$3.675+01$

I. $574+\mathrm{Cl}$

$5.67 t+0 C$

$8.617-01$

$\begin{array}{ll}3.4 C E+O C & 2.652+O C \\ 1.7 t 5+00 & 5.577+C C\end{array}$

$1.152+00 \quad 7.857+C C$

$E .442-01$ S. $720+C C$

t. $t 21-011.130+C 1$

$4.5 \div 2-01 \quad 1.393+C 1$

$3.5 \mathrm{CC}-01 \quad 1.612+\mathrm{Cl}$

$2.1 E 5-012.065+C 1$

$1.5 \varepsilon 4-C 1 \quad 2.421+C 1$

$1.021-012.513+01$

$7.523-02 \quad 3.238+C 1$

$5.956-02 \quad 3.479+C 1$

4. $525-02 \quad 3.668+01$

$3.664-02 \quad 3.547+C 1$

2. $\$ 16-02 \quad 4.136+C 1$ 


\begin{tabular}{|c|c|c|}
\hline \multicolumn{3}{|c|}{$Z=97$ 8ERKELILM } \\
\hline ENERGY & TOTAL & COHER - \\
\hline $\begin{array}{l}\text { (KEV) } \\
.01 \mathrm{C}\end{array}$ & $1.393+08$ & $\begin{array}{l}\text { SCATT. } \\
.25 s+C 3\end{array}$ \\
\hline & S. $2 \varepsilon \varepsilon+C 7$ & $.259+03$ \\
\hline.$<20$ & $6.566+c 7$ & $6.259+c 3$ \\
\hline & $4 \cdot 645+07$ & $G .25 S+C 3$ \\
\hline .040 & $3.4 \varepsilon 4+07$ & $6.258+03$ \\
\hline .050 & $2.787+C 7$ & $.258+03$ \\
\hline .060 & $2.323+07$ & $.258+c 3$ \\
\hline.$c \varepsilon 0$ & $1.742+C 7$ & $6.257+03$ \\
\hline & $1.394+C 7$ & $.255+03$ \\
\hline .150 & $9.254+06$ & $251+c 3$ \\
\hline .200 & $6 . \$ 72+0 €$ & $6.245+03$ \\
\hline & $4.650+06$ & $6.229+03$ \\
\hline .400 & $3.489+$ & $+c_{3}$ \\
\hline .500 & $2.793+C \epsilon$ & $6.181+C 3$ \\
\hline & $2.32 \varepsilon+C \varepsilon$ & $6.145+C 3$ \\
\hline & $1.748+C \epsilon$ & $6.07 \epsilon+C 3$ \\
\hline $1 . \mathrm{COC}$ & $3.190+06$ & $5.55 C+C 3$ \\
\hline 1.50 & $1.5 \varepsilon 0+C 6$ & $5.746+c 3$ \\
\hline 2.000 & & $5.475+C 3$ \\
\hline 3.000 & $3.810+C 5$ & $4.520+c 3$ \\
\hline $4 . C$ & $1.553+$ & $4.4 C E+C 3$ \\
\hline 5.000 & 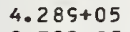 & $3.546+c 3$ \\
\hline 6.00 & $2.733+C 5$ & $3.54 \varepsilon+C 3$ \\
\hline 8.00 & $1.47 t+C$ & $2.514+C 3$ \\
\hline 12.00 & & \\
\hline $15 . \mathrm{COC}$ & $3.067+c$ & $1.6 E 7+C 3$ \\
\hline & & $1.209+C 3$ \\
\hline & & \\
\hline $4 \mathrm{C} . \mathrm{COO}$ & S. $461+C 3$ & $4.61 c+c 2$ \\
\hline
\end{tabular}

\begin{tabular}{|c|c|c|c|c|}
\hline \\
\hline $\begin{array}{l}\text { EAERGY } \\
\text { (KEVV) }\end{array}$ & TOTA & $\begin{array}{l}\text { COHER, } \\
\text { SCATT. }\end{array}$ & $\begin{array}{l}\text { INCOH. } \\
\text { SCATT. }\end{array}$ & $\begin{array}{l}\text { PHCTC } \\
\text { EFFEC }\end{array}$ \\
\hline & $1.41 \varepsilon+c \varepsilon$ & $6.389+02$ & $4.448-04$ & $1.418+08$ \\
\hline .015 & & $6.385+03$ & $9.588-04$ & \\
\hline & $7.051+07$ & $6.388+03$ & $1.772-C_{3}$ & $7.090+07$ \\
\hline .030 & & $\epsilon .3 \varepsilon \varepsilon+C 3$ & $3.572-03$ & $4.727+C 7$ \\
\hline .040 & $3.546+C 7$ & $\epsilon .38 \varepsilon+C 3$ & $7 . C \geq 3-c 3$ & \\
\hline & $7+c 7$ & $6.388+03$ & & $2.836+07$ \\
\hline & & $6.387+03$ & & $2.363+07$ \\
\hline .08 & & & & \\
\hline & $1.419+07$ & $6.385+c 3$ & 4.25 & $1.418+07$ \\
\hline & 5.4 & & & 9.45 \\
\hline .200 & & & & \\
\hline & 4.73 & & & +06 \\
\hline .400 & & & $-c 1$ & 3.5 \\
\hline & & & & \\
\hline $.60 \mathrm{C}$ & 2.3 & & 1.2 & +06 \\
\hline & 1.7 & & & \\
\hline & & & & \\
\hline 1.500 & 1.64 & $5.877+c 3$ & 4.4 & 1.6 \\
\hline 2.000 & & & & \\
\hline & & & & \\
\hline $4 . C 00$ & $2.028+$ & 4.515 & 1.0 & +05 \\
\hline & & & & \\
\hline & $2.820+$ & $3.635+C 3$ & & \\
\hline & & & & \\
\hline & & & & \\
\hline & & & & \\
\hline & & & & \\
\hline $3 \mathrm{C} .00 \mathrm{C}$ & $2.016+C 4$ & $7.331+02$ & $3.441+C 1$ & $1.939+0$ \\
\hline & $9.645+03$ & $4.737+c 2$ & $3.745+C 1$ & $9.134+03$ \\
\hline
\end{tabular}

\begin{tabular}{|c|c|c|}
\hline $\begin{array}{l}\text { IERGY } \\
\text { (KEV) }\end{array}$ & ICTAL & $\begin{array}{l}\text { COHER. } \\
\text { SCATT. }\end{array}$ \\
\hline & $5.344+03$ & $198+02$ \\
\hline & & \\
\hline & $2+03$ & $.405+02$ \\
\hline 100 & & \\
\hline & & \\
\hline & & \\
\hline & & \\
\hline & & \\
\hline & & \\
\hline & & \\
\hline & & \\
\hline & & \\
\hline & & \\
\hline & & \\
\hline & & \\
\hline 400 & 1.87 & \\
\hline & & \\
\hline & & \\
\hline & 2.07 & 1.6 \\
\hline & & \\
\hline & & \\
\hline & & 2.7 \\
\hline & & \\
\hline & & \\
\hline & & \\
\hline & & \\
\hline & & \\
\hline & $4.341+C 1$ & $1.084-$ \\
\hline
\end{tabular}

\begin{tabular}{|c|c|}
\hline $\begin{array}{l}\text { ENERGY } \\
\text { (KEV) }\end{array}$ & TOTAL \\
\hline 50. & $5 \cdot 477+C_{3}$ \\
\hline 60. & $3.4 \epsilon z+02$ \\
\hline 80. & $1.68 t+03$ \\
\hline 100. & $9.680+0 \bar{z}$ \\
\hline 150. & $1.237+03$ \\
\hline 200. & $6.305+02$ \\
\hline 300 . & $2.491+02$ \\
\hline 400 . & $1.377+c 2$ \\
\hline 500. & $9.224+01$ \\
\hline 600 . & $6.897+01$ \\
\hline 800 . & $4.6 C 5+01$ \\
\hline 1000 . & $3.52 \epsilon+01$ \\
\hline 1500. & $2.438+01$ \\
\hline 2000 . & $2.10 \epsilon+01$ \\
\hline 3000 . & $1.513+c 1$ \\
\hline 4000 . & $1.857+C 1$ \\
\hline 5000 . & $1.533+01$ \\
\hline 6000 . & $1.585+01$ \\
\hline 8000 & $2.103+01$ \\
\hline 10000 . & $2.222+01$ \\
\hline 15000 . & $2.512+01$ \\
\hline 20000 . & $2.788+01$ \\
\hline 30000 . & $3.230+01$ \\
\hline 40000 . & $3.535+C 1$ \\
\hline 50000 . & $3.75 \varepsilon+c 1$ \\
\hline 60000 . & $3.931+01$ \\
\hline 80000 . & $4.18 s+01$ \\
\hline & \\
\hline
\end{tabular}

COHER $3.288+02$ $2.408+02$ $2.408+02$
$1.446+02$ $9.615+01$ $4.455+01$ $2.592+C 1$ $1.181+01$ $6.726+00$ $4 \cdot 336+00$ $3.026+00$ $1.100+00$ $4.913-01$ $4.913-01$
$2.770-C 1$ 1.234-01 6.951-02 4. $452-02$ 3.093-02 $1.741-02$ $1.115-02$ $2.789-03$ $1.240-03$ 6.974-04 4.464-04 3.100-04 $1.744-04$ $1.116-04$
INCOH

SCATT.

$3.524+01 \quad 4.985+03$

4. $C 24+01 \quad 3.07 \epsilon+03$

4. $C 71+01 \quad 1.431+03$

4. $\operatorname{cst} t+C 1 \quad 7.8 \subseteq t+02$

$3.534+01 \quad 1.124+03$

3. $7 C \varepsilon+C 15.5 C \varepsilon+02$

$3.314+01 \quad 1.57 \varepsilon+02$

$\begin{array}{ll}3 . C C E+C 1 & 9.773+01\end{array}$

$2.763+01 \quad 5 . \varepsilon 21+C 1$

$2.5 \epsilon 6+C 1 \quad 3.87 \varepsilon+01$

z. $\bar{\varepsilon} \epsilon z+c 1 \quad 2 .(\varepsilon \varepsilon 4+01$

$2 . C 39+01 \quad 1.315+01$

$1.6 E 1+C 1 \quad \epsilon . C 1 C+0 C$

$1.418+01 \quad 3.6 C 7+00$

$1.11 \epsilon+C 1 \quad 1 . \varepsilon \epsilon 7+C 0$

S. $3 C 4+C C \quad 1.217+0 C$

8.C34+Co $\varepsilon .51 C-01$

$7.1 C 1+0 C$ 6.581-01

5. $\varepsilon C 8+0 C$

$4.545+00$

$3.657+C C$

$2.533+0 \mathrm{C}$

$2.133+c c$

$4 \cdot 23=-01$

$2.2 \div 4-01$

$1.662-01$

1. CES-01

$7.87 \varepsilon-02$

$1.413+0 C \quad \epsilon .235-02$

$1.413+0$

$1.216+0 C 5.155-02$

$\begin{array}{ll}5.583-C 1 & 3 . \varepsilon 24-02 \\ 7.55 C-01 & 3.051-02\end{array}$

\&. $525-c 1$

$2.712+C C$

$.735+C C$

$8.122+0 \mathrm{C}$

1. $C C 8+C 1$

$1.446+C 1$

$1.671+01$

$2.127+01$

$2.452+01$

$3 . \mathrm{CC} 7+\mathrm{C} 1$

$3.589+C 1$

$3.780+C 1$

4. $C 61+C 1$

$4.258+C 1$

\begin{tabular}{|c|c|c|c|c|}
\hline \\
\hline $\begin{array}{l}\text { ENERGY } \\
\text { (KEV) }\end{array}$ & 1016 & $\begin{array}{l}\text { COFER } \\
\text { SCATT. }\end{array}$ & $\begin{array}{l}\text { I NC OH. } \\
\text { SCATT. }\end{array}$ & $\begin{array}{r}\text { PHCJC } \\
\text { EFFECT }\end{array}$ \\
\hline .010 & $1.463+C \varepsilon$ & $6.52 \mathrm{c}+\mathrm{C} 3$ & $4.5 C 4-C 4$ & $1.463+08$ \\
\hline .015 & $9.756+C 7$ & $6.520+03$ & . $\mathrm{C} 11-03$ & $9.756+07$ \\
\hline & $7.317+c 7$ & $+c^{3}$ & $1.7 \subseteq 5-C 3$ & $7.317+07$ \\
\hline & $4.878+C 7$ & $6.519+03$ & $4.022-c 3$ & $4.878+07$ \\
\hline $.04 C$ & & $6.515+C 3$ & $7.122-\mathrm{C3}$ & $3.658+07$ \\
\hline .050 & $2.927 t$ & & 1.1 & $27+07$ \\
\hline. $\mathrm{C} 60$ & $2.440+07$ & & 1.5 & $2.439+07$ \\
\hline & & & & \\
\hline & 1.46 & & & \\
\hline & 5.762 & & 9.9 & +06 \\
\hline .200 & 7.32 & & & \\
\hline .30 & $4.8 \varepsilon$ & & & 4. \\
\hline .40 & 3.665 & 6.4 & 6.1 & +06 \\
\hline & & & & \\
\hline .600 & $2.445+$ & & 1.2 & \\
\hline .800 & 1.836 & & & 1.8 \\
\hline 1.00 & 3.36 & & & \\
\hline & & & 4.5 & \\
\hline 2.000 & S. $849+05$ & $5.769+03$ & 5.55 & $9.791+05$ \\
\hline & & & 8.4 & \\
\hline & & & & \\
\hline $5 . \mathrm{COC}$ & $3.594+0$ & 4.19 & 1.2 & 3.95 \\
\hline & & & 1.45 & \\
\hline & & & & \\
\hline $1 \mathrm{C} . \mathrm{COO}$ & $9.201+$ & & 2.0 & \\
\hline & & & & \\
\hline & & & & \\
\hline & $112+$ & $7.632+02$ & $3.495+C 1$ & $2.032+0$ \\
\hline $4 \mathrm{C} \cdot \mathrm{COO}$ & $1 . C 2 S+C 4$ & $4.936+02$ & 3. $8 \mathrm{C} 5+\mathrm{Cl}$ & $5.761+03$ \\
\hline
\end{tabular}

\begin{tabular}{|c|c|c|}
\hline $\begin{array}{l}\text { NERGY } \\
\text { (KEVV) }\end{array}$ & TCTAL & $\begin{array}{l}\text { COHER } \\
\text { SCATT }\end{array}$ \\
\hline 50. & $5.819+03$ & $3.428+02$ \\
\hline 60. & $3.643+03$ & $2.511+02$ \\
\hline & $.74 \varepsilon+C 3$ & \\
\hline 100 & & \\
\hline & $.288+03$ & $4.694+01$ \\
\hline & $.607+02$ & $2.708+01$ \\
\hline & & \\
\hline & & \\
\hline & .5 & 4.5 \\
\hline & & \\
\hline & & \\
\hline & & 00 \\
\hline & & \\
\hline & $2.154 t$ & 2.8 \\
\hline & & 1.2 \\
\hline & & \\
\hline & 1.57 & 4.65 \\
\hline & & \\
\hline & & \\
\hline & & \\
\hline & & \\
\hline & & \\
\hline & & \\
\hline & $3.633+$ & 7.28 \\
\hline & & \\
\hline & & \\
\hline & $4.301+$ & $1.822-04$ \\
\hline & & $1.166-04$ \\
\hline
\end{tabular}

INCOH

SCATT.

2. $\subseteq \varepsilon 7+C$

4. $\operatorname{cs} 0+01$

$4.138+01$

$4.169+01$

4. $C(6+C)$

$3.777+\mathrm{C}$

2. $277+01$

$2.818+c 1$

$2.617+0$

$2.617+0$
$2.3 \mathrm{CS}+\mathrm{C}$

$2 \cdot 3 C g+C 1$
$2 \cdot C 20+C 1$

1. $\epsilon 95+01$

$1.447+01$

$1.139+01$

$5.456+C$

$\varepsilon . \angle C C+C C$

7. $247+00$

5. $\subseteq 2 \varepsilon+00$

$5 . C 47+0 C$

3. $732+C C$

$2.554+0 C$

$2.177+0 \mathrm{C}$

$1.728+0 \mathrm{C}$

$1.442+C C$

$1.241+0 C$

S. $780-C 1$
E. $114-01$

FHOTO

EFFEC T

$5.1 \mathrm{CS}+03$

$3.1 \varepsilon 1+03$
$1.5 C 1+03$

$1.5 C 1+03$
$\varepsilon \cdot 3 C \varepsilon+02$

$1.152+03$

$5.675+02$

2. $C 41+02$

1. $C \subset \varepsilon+02$

e. $C 11+01$

.

$1.35 s+01$

$6.155+00$

$3.717+C C$
$1.525+00$

1. $2 \epsilon \epsilon+\mathrm{CO}$

$5.2 \mathrm{C} t-01$

7.221-01

$5.0 \mathrm{Cs}-0$

$3 . \varepsilon 1 \subseteq-C$

. $3 \varepsilon 4-0$

1.725-01

1.114-01

E. 214-0

6. $5 \mathrm{C} 4-02$

$5.3 \varepsilon 3-0$

$4 . C C z-0$

$3.1 \varepsilon 5-02$

PAIF

PRCD.

5. $24 t-01$

2. $746+C C$

$\varepsilon .248+C C$

1. $C 25+C 1$

$1.153+\mathrm{Cl}$

$1.683+01$

$2.118+C 1$

$2.474+01$

3. $\mathrm{CC}+\mathrm{C} 1$

$3.608+01$

$3.803+C 1$

4. $C 88+C 1$

$4.291+C 1$

PHCTO

EFFECT

$5.4 \equiv \epsilon+03$

$3 \cdot 351+03$

1. $5 \leq t+03$

1. $2 \mathrm{Cl}+\mathrm{O}_{3}$

$5.95 \varepsilon+02$

$2.141+C 2$

$6.321+01$

$4.233+01$

$2.2 \varepsilon z+01$

$1.44 \mathrm{C}+01$

$3.53 \varepsilon+0 C$

2. $C \equiv E+00$

$1.32 t+00$

S. $7 \mathrm{CC}^{-\mathrm{C} 1}$

7.6C3-01

5. $2 t t-01$

4. C 11-01

$2.5 C C-C 1$

$1.812-01$

$1.167-01$

$8.59 t-02$

$6.8 \mathrm{C} 4-0$

$5.63 \mathrm{C}-02$

9. 568-C1

$2.846+C C$

$8.518+0 C$

1. $C 56+C 1$

$1.228+01$

$1.729+C 1$

$2.181+C 1$

$2.553+C 1$

$3.451+C 1$

$3.7 \mathrm{C} 5+\mathrm{Cl}$ $3.33 \mathrm{C}-02 \quad 4.419+\mathrm{Cl}$ 
BIGGS ANC LIGHTHILL IA BARASIATOM

\begin{tabular}{|c|c|c|c|c|}
\hline \multicolumn{5}{|c|}{1 CO FERM } \\
\hline $\begin{array}{l}\text { ENERGY } \\
\text { (KEV) }\end{array}$ & TOTAL & $\begin{array}{l}\text { COHER. } \\
\text { SCATT. }\end{array}$ & $\begin{array}{l}\text { INCQH. } \\
\text { SCATT. }\end{array}$ & $\begin{array}{r}\text { PHCTC } \\
\text { EFFECT }\end{array}$ \\
\hline .010 & $1.469+C 8$ & $6.652+03$ & $4.5 \times 7-04$ & $1.469+08$ \\
\hline & & 6 & 1. $C_{1} 12-C_{3}$ & S. $792+07$ \\
\hline 20 & $45+$ & 6.6 & 1 & +07 \\
\hline 30 & $4 . \varepsilon 57+$ & $6 . t$ & 4.0 & $.896+07$ \\
\hline 40 & 3.6 & 6.6 & 7.1 & +07 \\
\hline 50 & $2^{\prime \prime}$. & 6.6 & 1.1 & +07 \\
\hline .060 & $445+07$ & 6.6 & -02 & $2.448+07$ \\
\hline & 1. & 6.6 & 22 & $1.836+07$ \\
\hline & & & 2 & $469+07$ \\
\hline & & & & $792+06$ \\
\hline & & & & \\
\hline & & 6.6 & & \\
\hline & & $t .6$ & & \\
\hline .500 & & $\epsilon .5$ & & 06 \\
\hline .600 & $2.455+$ & 6.5 & 1.2 & 2.4 \\
\hline .80 & $1.842+0 t$ & 6.4 & & +06 \\
\hline $\mathrm{CcO}$ & $3.455+C t$ & $t .4$ & 2.7 & 3.44 \\
\hline 1 & 1. $789+c t$ & 6.1 & 4.52 & $1.78=$ \\
\hline 2 & $.02 t+C$ & 5.5 & 6.0 & $1.020+06$ \\
\hline c & $33 \varepsilon+$ & 5.3 & $C$ & $4.284+05$ \\
\hline $\mathrm{c}$ & & 4.7 & 1.0 & $3+05$ \\
\hline & $4.156+c$ & & & +05 \\
\hline & $3.048+C$ & & 1.45 & $3.009+05$ \\
\hline & & & 1.78 & $1.616+05$ \\
\hline & S. $532+c$ & & & $5+04$ \\
\hline & $=.466+$ & $1.811+$ & & $3.282+04$ \\
\hline & $1.712+$ & 1.32 & 2.5 & $1.577+04$ \\
\hline & $2.1 \varepsilon 0+C$ & 7.8 & 3.50 & $2.098+04$ \\
\hline & $1.068+04$ & $5 \cdot c 71+c 2$ & $3.819+01$ & $1.013+04$ \\
\hline
\end{tabular}

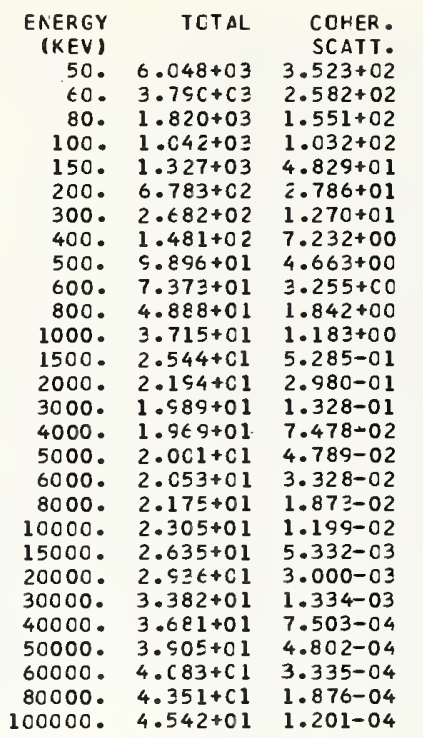

INCOH.
SCATT $4.0 \mathrm{CO}+01$

$4.105+01$

4. $153+01$

4. $1 \subseteq 2+C 1$ 4. $C 28+C 1$ 3. $7 \varsigma \varepsilon+C 1$ 3. $4 \mathrm{CO}+01$ 3. $C 88+01$ 2. $\varepsilon 41+C 1$ 2. $\epsilon 4 \mathrm{C}+01$ 2. $330+01$

2. $1 \mathrm{CC}+01$

1. $711+\mathrm{Cl}$

1. $4 \in 1+C 1$ 1. $150+C 1$ S. $592+C C$ e. $282+00$ 7. $22 \mathrm{C}+\mathrm{CC}$ 5. $\$ 87+0 C$ E. $C \subseteq E+C C$ 3. $770+C C$ 3. $C 24+C C$ 2. $159+0 C$ 1. $74 t+C C$ 1. $45 t+O C$ 1. $254+C C$ S. $875-C 1$ E. $15 t-01$
FHOTO EFFEC T 5. $t E E+03$ 3. $451+03$ $1.62 z+03$ E. $s \in 4+02$ 1. $2 \geq \varepsilon+03$ $\epsilon .125+02$ $2.21 \epsilon+02$ 1. $1 \mathrm{C} \mathrm{C}+02$ t. $5 \varepsilon c+01$ 4. $4 C \varepsilon+01$ $2.374+01$ 1. $4 c t+01$ $6.8<C+00$ 4. $085+00$ z. $112+0 \mathrm{C}$ 1. $27 i+00$ 1. $C C E+O O$ 7. $S C 2-C$ $5.47 \varepsilon-01$ $4.175-01$ $2.6 C 5-01$ 1. EES 01 1.2 17-0 E. $S \in S-02$ 7.1 C 1-02 5. $87 t-02$ $4.3 \in c-C 2$ $3.477-02$
PAIF PRCC.

5. $771-C 1$ $2.539+C C$ $6.148+C C$ $\varepsilon \cdot-46+C C$ 1. $C 68+C 1$ 1. $239+C 1$ 1. $520+C 1$ 1. $752+C 1$ $2.231+01$ $2 . t 14+C 1$ $3.149+C 1$ $3.45 \varepsilon+C 1$ 3. $752+01$ 3. $551+C 1$ $4.248+C 1$ $4.456+C 1$ 
ENDF/ 8 IN BARNS/ATCM

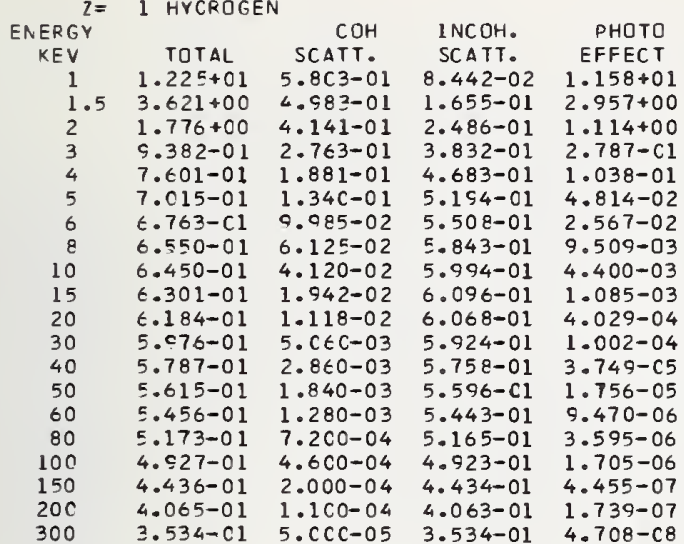

\begin{tabular}{|c|c|c|c|c|}
\hline$Z=$ & $\approx$ HEL IUM & & & \\
\hline ERGY & & $\mathrm{COH}$ & I NCOH. & PHOTC \\
\hline KEV & TOTAL & SCATT. & SCATT. & EFFECT \\
\hline 1 & $4.331+02$ & $2.512+00$ & $7.039-02$ & $4.3 \mathrm{C} 5+02$ \\
\hline 1.5 & $1.140+02$ & $47+0 C$ & $1.492-01$ & $1.115+02$ \\
\hline 2 & $4.438+01$ & $2.148+00$ & $2.447-01$ & $39+01$ \\
\hline 3 & $1.255+01$ & $1.726+00$ & $4.447-01$ & $1.038+01$ \\
\hline 4 & $5.780+00$ & $1.353+00$ & $6.251-01$ & $3.802+00$ \\
\hline 5 & $2+00$ & $t 0+00$ & $s-01$ & $36+00$ \\
\hline 6 & 2.62 & $8-01$ & 8. & $9-01$ \\
\hline 8 & 1.89 & $5.542-\mathrm{Cl}$ & 1. C & -01 \\
\hline 10 & $1.621+00$ & $3.891-C 1$ & 1.0 & 1.48 \\
\hline 15 & $1.38 B+00$ & $1.948-01$ & $1.158+00$ & $3.523-02$ \\
\hline 20 & $1.305+00$ & $1.159-01$ & $1.177+00$ & $1.269-02$ \\
\hline 30 & $1.224+00$ & $5.410-02$ & $1.167+00$ & $3.030-03$ \\
\hline 40 & $1.174+O C$ & $3.1 C C-C 2$ & $1.141+00$ & $1.105-03$ \\
\hline 50 & $1.133+00$ & $2.000-02$ & $1.113+00$ & $5.083-04$ \\
\hline 60 & $1.098+0 \mathrm{C}$ & $1.4 \mathrm{CO}-02$ & $1 . \mathrm{C} 84+0 \mathrm{C}$ & $2.708-c 4$ \\
\hline 80 & $03 \varepsilon+00$ & $7.900-03$ & $1.030+00$ & $1.012-04$ \\
\hline $10 \mathrm{C}$ & $7 s-01$ & $5 . c \in C-03$ & S. $828-01$ & 4.76 \\
\hline 150 & $8.883-01$ & $2.25 c-03$ & 8.86 & $1.239-05$ \\
\hline $20 \mathrm{C}$ & $35-01$ & $1.26 C-03$ & $8.123-01$ & $4.874-06$ \\
\hline 300 & $7.071-01$ & $5.60 \mathrm{C}-04$ & $7.066-01$ & $1.354-06$ \\
\hline
\end{tabular}

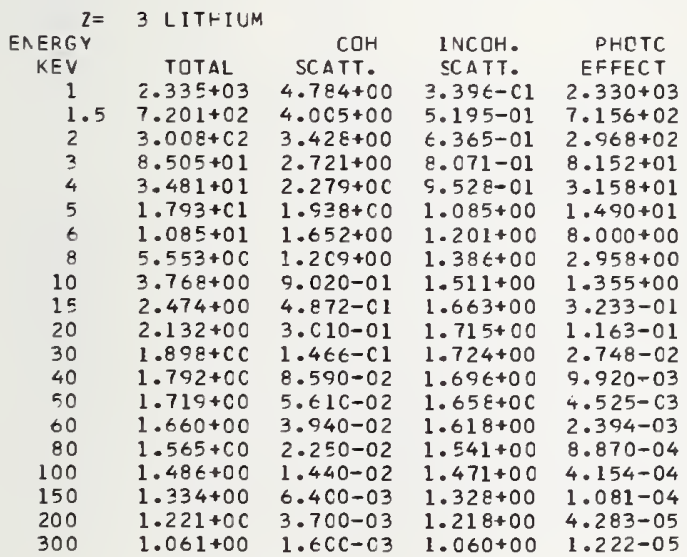

\begin{tabular}{|c|c|c|}
\hline \multirow{2}{*}{$\begin{array}{r}Z= \\
\text { ENERGY }\end{array}$} & \multirow{2}{*}{\multicolumn{2}{|c|}{ 8ER YLL IUM }} \\
\hline & & \\
\hline KEV & TOTAL & SCATT. \\
\hline 1 & $8.486+c 3$ & $8.886+00$ \\
\hline 1.5 & $2.607+03$ & $7.4 \in 1+00$ \\
\hline 2 & $1.089+03$ & $6.215+00$ \\
\hline 3 & 3. $065+02$ & $4.517+0 \mathrm{C}$ \\
\hline 4 & $1.231+02$ & $3.548+c 0$ \\
\hline 5 & $6.093+01$ & $2.945+0 \mathrm{C}$ \\
\hline 6 & $3.479+01$ & $2.521+00$ \\
\hline 8 & $1 \cdot 527+C 1$ & $1.923+00$ \\
\hline 10 & $8.815+00$ & $1.501+00$ \\
\hline 15 & $4.331+00$ & $8.65 c-01$ \\
\hline 20 & $3.267+00$ & $5.5 c 3-01$ \\
\hline 30 & $2.661+00$ & $2.759-01$ \\
\hline 40 & $2.448+00$ & $1.643-01$ \\
\hline 50 & $\overline{2} .325+00$ & 1. $C 85-01$ \\
\hline 60 & $2.235+00$ & $7.68 C-02$ \\
\hline 80 & $2.057+00$ & $4.410-02$ \\
\hline 100 & $1.988+00$ & $2.840-02$ \\
\hline 150 & $1.782+00$ & $1.280-02$ \\
\hline 200 & $1.630+00$ & $7.200-03$ \\
\hline 300 & $1.415+C 0$ & $3.2 \mathrm{CO}-03$ \\
\hline
\end{tabular}

$1 \mathrm{NCOH}$.

PHOTO

SCATTECT

4.011-01 $8.477+03$

t. $975-012.598+03$

9.247-01 $1.082+03$

$1.197+00 \quad 3.012+02$

$1.360+00 \quad 1.182+02$

$1.487+00 \quad 5.650+01$

$1.597+00 \quad 3.068+01$

$1.781+C 0 \quad 1.157+01$

$1.92 t+00 \quad 5.389+00$

$2.137+0 \mathrm{C} \quad 1.328+00$

$\begin{array}{ll}2.227+00 & 4.891-01 \\ 2.265+00 & 1.196-01\end{array}$

$2.240+00 \quad 4.424-02$

$2.196+00 \quad 2.055-02$

$2.045+00 \quad 4.174-03$

$1.958+00 \quad 1.985-03$

1. $768+C 0 \quad 5.288-04$

$1.622+00 \quad 2.125-04$

$1.412+00 \quad 6.142-05$

$\begin{array}{rcc}\text { ENERGY } & \text { TOTAL } & \text { COH } \\ \text { KEV } & \text { SCATT } \\ 400 & 3.167-01 & 3.000-05 \\ 500 & 2.891-01 & 1.900-05 \\ 600 & 2.675-01 & 1.300-05 \\ 800 & 2.349-01 & 7.300-06 \\ 1000 & 2.112-01 & 4.700-06 \\ 1500 & 1.716-01 & 2.096-06 \\ 2000 & 1.465-01 & 1.181-06 \\ 3000 & 1.157-01 & 5.256-07 \\ 4000 & 9.700-02 & 2.959-07 \\ 5000 & 8.430-02 & 1.894-07 \\ 6000 & 7.501-02 & 1.316-07 \\ 8000 & 6.239-02 & 7.410-08 \\ 10000 & 5.431-02 & 4.747-08 \\ 15000 & 4.222-02 & 2.091-08 \\ 20000 & 3.588-02 & 1.159-08 \\ 30000 & 2.910-02 & 4.967-09 \\ 40000 & 2.558-02 & 2.681-09 \\ 50000 & 2.357-02 & 1.630-09 \\ 60000 & 2.217-02 & 1.066-09 \\ 80000 & 2.058-02 & 5.250-10 \\ 100000 & 1.982-02 & 3.035-10\end{array}$

INCOH.
SCATT.
$3.16 t-01$
$2.891-01$
$2.675-01$
$2.345-C 1$
$2.112-01$
$1.716-C 1$
$1.464-01$
$1.151-01$
$9.600-02$
$8.287-02$
$7.323-C 2$
$5.988-02$
$5.099-02$
$3.771-02$
$3.025-C 2$
$2.200-02$
$1.74 t-C 2$
$1.457-02$
$1.254-C 2$
$9.882-C 3$
$8.199-C 3$

PHOTO

EFFECT

$1.890-C 8$
$9.397-09$

5.343-0s

$2.219-\mathrm{CS}$

$1.134-05$

$5.56 \mathrm{~S}-1 \mathrm{C} \quad 4.35 \mathrm{C}-\mathrm{C} 5$

$3.362-1 \mathrm{C} \quad 1.750-\mathrm{C}_{4}$

$1.890-1 \mathrm{C} \quad 5.522-\mathrm{C4}_{4}$

$1.318-10 \quad 9.950-\mathrm{C}_{4}$

9.972-11 1.434-03

8.090-11 1.775-03

5.815-11 2.511-C 3

4.502-11 3.317-03

2.949-11 4.513-C3

2.184-11 5.633-03

$1.431-117.098-03$

$1.060-118.114-03$

$8.397-12$ S. 0 C5-C 3

$6.943-12 \quad 9.627-\mathrm{C} 3$

$5.142-12 \quad 1.070-02$

$\begin{array}{rc}\begin{array}{c}\text { ENERGY } \\ \text { KEV }\end{array} & \text { TCTAL } \\ 400 & 6.335-01 \\ 500 & 5.784-01 \\ 6 C 0 & 5.350-01 \\ 800 & 4.699-01 \\ 1000 & 4.224-01 \\ 1500 & 3.433-01 \\ 2000 & 2.935-01 \\ 3000 & 2.323-01 \\ 4000 & 1.957-01 \\ 5000 & 1.708-01 \\ 6000 & 1.527-01 \\ 8000 & 1.283-01 \\ 10000 & 1.129-01 \\ 15000 & 8.997-02 \\ 20000 & 7.835-02 \\ 30000 & 6.614-02 \\ 40000 & 6.003-02 \\ 50000 & 5.680-02 \\ 60000 & 5.453-02 \\ 80000 & 5.226-02 \\ 100000 & 5.148-02\end{array}$

$\mathrm{COH}$
$\mathrm{CATT}$.

PHOTC

EFFECT

3.200-04 6.332-C1 5.611-07

$\begin{array}{lll}1.900-04 & 5.782-01 & 2.882-C 7\end{array}$

$1.300-04 \quad 5.349-01 \quad 1.653-07$

$7.000-05$

$4.000-05$

$1.785-05$

$1.006-05$

$4.481-06$

$1.616-06$

$1.122-06$

$6.316-07$

$4.046-07$

$1.001-07$

$4.338-08$

$2.368-08$

$1.466-08$

$9.778-09$

$4.999-09$

4.698-01 7.478-08

4.224-01 4.052-CE

3.432-01

$2.927-01$

$1.551-08$

$1.203-C 8 \quad 7.160-04$

$1.920-01$

4.695-09

$3.544-C S$

$2.875-05$

$1.465=0$

$1.198-01$

$2.066-\mathrm{CS}$

$1.599-C 9$

$1.047-09$

$6.050-C 2$
$4.400-02$

$4.400-02$
$3.493-C 2$

$2.914-02$

$7.748-1$

$5.072-10$

$3.754-1 \mathrm{C}$

$2.973-1 \mathrm{C}$

$2.457-1 \mathrm{C}$

$1.640-02$

$1.819-10$

1. $441-1 \mathrm{C}$

$3.649-03$

$6.150-03$

$8.492-\mathrm{C}_{3}$

1. $053-02$

$1.454-02$

$1.785-\mathrm{C} 2$

$2.509-\mathrm{C}^{2}$

$2.7 \in 6-C 2$

$2.944-C 2$

$3.249-C 2$
$3.5 C 8-C 2$

$\begin{array}{cc}\begin{array}{c}\text { ENERGY } \\ \text { KEV }\end{array} & \text { TCTAL } \\ 400 & 9.504-01 \\ 500 & 8.677-01 \\ 600 & 8.026-01 \\ 800 & 7.049-01 \\ 1000 & 6.336-01 \\ 1500 & 5.151-01 \\ 2000 & 4.407-01 \\ 3000 & 3.500-01 \\ 4000 & 2.959-01 \\ 5000 & 2.596-01 \\ 6000 & 2.329-01 \\ 8000 & 1.976-01 \\ 10000 & 1.758-01 \\ 1500 C & 1.432-01 \\ 20000 & 1.274-01 \\ 30000 & 1.111-01 \\ 40000 & 1.032-01 \\ 50000 & 9.953-02 \\ 60000 & 9.688-02 \\ 80000 & 9.474-02 \\ 100000 & 9.463-02\end{array}$

SCATT.

$1 \mathrm{NCOH}$.

PHOTC

EFFECT

$\begin{array}{lll}5.700-04 & 9.495-01 & 5.228-C E \\ & 8.672-01 & 2.777-0 t\end{array}$

$\begin{array}{lll}3.800-04 & 8.022-C 1 & 1.687-0 t\end{array}$

2.200-04 7.047-01 7.962-C7

$1.300-04 \quad 6.335-01 \quad 4.593-c 7$

5.804-05

$3.272-05$

$1.458-05$

$8.209-06$

$5.258-06$
$3.653-06$

$3.653-06$

$2.056-06$
$1.316-06$

5.859-07

$3.277-07$

$1.427-07$

$7.828-08$

$4.883-08$

$3.283-08$

$1.714-08$

$5.147-01$

4.390-01

$2.88 \mathrm{C}-01$

2.258-07

$1.365-C 7$

$7.645-08$

$5.311-c 8$

$2.486-01 \quad 4.003-C \varepsilon$

$2.197-01$

$1.796-\mathrm{Cl}$

$3.247-0 \varepsilon$

$2.333-c 8$

1.806-08

$1.1 \varepsilon 1-0 \varepsilon$

$1.131-\mathrm{Cl}$

9.074-02

$6.595-c_{2}$

5.239-02

$4.370-\mathrm{C} 2$

3. $762-\mathrm{C} 2$

$8.741-C 5$
$5.718-C 5$

$5.718-c c$
$4.231-C 9$

3.350-09

2.768-05

$2.048-C 5$

2. $459-02$
PAIR

PRCO.

3. $922-\mathrm{C}_{4}$

1. $C 11-C_{3}$

7. $9 \in \mathrm{C}-\mathrm{C} 3$

1. $\mathrm{CSt}-\mathrm{C2}$

$1.324-C 2$
$1.754-02$

2. $282-\mathrm{C} 2$

$3.0 \mathrm{CE}-\mathrm{C2}$

$3.663-02$

$5.085-C_{2}$

5. 584-02

$5.926-c 2$

$6.510-C 2$

7. $004-02$

$\begin{array}{rr}\begin{array}{r}\text { ENERGV } \\ \text { KEV }\end{array} & \text { TCTAL } \\ 400 & 1.268+00 \\ 500 & 1.157+00 \\ 600 & 1.070+00 \\ 800 & 9.400-01 \\ 1000 & 8.449-01 \\ 1500 & 6.870-01 \\ 2000 & 5.883-01 \\ 3000 & 4.687-01 \\ 4000 & 3.979-01 \\ 500 C & 3.505-01 \\ 6000 & 3.158-01 \\ 8000 & 2.704-01 \\ 10000 & 2.429-01 \\ 15000 & 2.019-01 \\ 20000 & 1.829-01 \\ 30000 & 1.639-01 \\ 40000 & 1.551-01 \\ 50000 & 1.517-01 \\ 60000 & 1.491-01 \\ 80000 & 1.478-01 \\ 100000 & 1.489-01\end{array}$
SCATT.

$1.800-03$

$1.200-03$
$8.000-04$

$8.000-04$
$4.200-04$

2.600-04

$1.161-04$

6.549-0

2.918-05

$1.644-05$

$7.315-06$

4.116-06

2.636-06

$1.173-06$

6.583-07

2.874-07

$1.583-07$

$9.891-08$

3.535-08

2.093-08
INCOH -

SCATT.

$1.266+00$

1. $156+0 \mathrm{C}$

$1.070+0 \mathrm{C}$

$8.396-C 1$

6.862-Cl

5.853-C1

4.603-01

3. $840-\mathrm{Cl}$

3.314-01

2.929-01

2.395-01

2.039-0

$1.210-01$

$8.798-\mathrm{C} 2$

6.985-02

5.826-C2

$5.016-C_{2}$

$3.952-C$

$3.279-02$
EFFEC T

$2.639-05$

$1.402-05$

$8.501-C t$
$3.982-0 t$

$2.275-C E$

$1.11 \mathrm{c}-\mathrm{CE}$

6.765-C 7

3.785-C7

2.626-07

$1.978-C 7$

$1.604-07$

$1.152-07$

$8.910-0 \varepsilon$

$5.832-C \varepsilon$

4.315-C $\varepsilon$

$2.821-0 \varepsilon$

$2.087-08$

$1.652-0 \varepsilon$

$1.365-0 \varepsilon$

$7.953-c$
PAIR

PRED.

$6.975-04$

$2.864-C 3$ $8.343-03$
$1.393-02$ 1. 9 C $6-02$ 2.2ऽ3-02 3. $C 84-02$ $3.897-\mathrm{C2}^{2}$ $5.1 \mathrm{C5}-02$ $7.589-C 2$ $8.529-\mathrm{C} 2$ $9.340-C 2$ $9.850-02$ 1.083-01 1. 162-01 


$\begin{array}{ccccc}\begin{array}{c}Z= \\ \text { ENEREY } \\ \text { KEV }\end{array} & \begin{array}{c}\text { BORON } \\ \text { TOTAL }\end{array} & \begin{array}{c}\text { COH } \\ \text { SCAT. }\end{array} & \begin{array}{c}\text { INCOH. } \\ \text { SCATT. }\end{array} & \begin{array}{c}\text { PHOTO } \\ \text { EFFECT }\end{array} \\ 1 & 2.098+04 & 1.449+01 & 3.561-01 & 2.096+04 \\ 1.5 & t .681+03 & 1.256+01 & 6.698-01 & 6.668+03 \\ 2 & 2.860+03 & 1.067+01 & 9.562-01 & 2.848+03 \\ 3 & 8.280+02 & 7.740+00 & 1.365+00 & 8.198+02 \\ 4 & 3.368+02 & 5.868+00 & 1.625+00 & 3.293+C 2 \\ 5 & 1.667+02 & 4.673+00 & 1.920+00 & 1.601+02 \\ 6 & 9.402+01 & 3.879+00 & 2.048+00 & 8.810+01 \\ 8 & 3.903+C 1 & 2.890+00 & 2.226+00 & 3.392+01 \\ 10 & 2.065+01 & 2.271+00 & 2.336+00 & 1.604+01 \\ 15 & 8.018+00 & 1.363+00 & 2.597+00 & 4.658+00 \\ 20 & 5.126+00 & 8.88 C-01 & 2.718+00 & 1.520+00 \\ 30 & 3.622+00 & 4.543-C 1 & 2.787+00 & 3.803-01 \\ 40 & 3.188+0 C & 2.737-01 & 2.772+00 & 1.427-01 \\ 50 & 2.975+00 & 1.823-01 & 2.726+00 & 6.697-02 \\ 60 & 2.835+00 & 1.298-01 & 2.669+00 & 3.624-02 \\ 80 & 2.642+00 & 7.51 C-02 & 2.553+00 & 1.387-02 \\ 100 & 2.407+00 & 4.87 C-02 & 2.442+00 & 6.652-03 \\ 150 & 2.232+00 & 2.190-02 & 2.208+00 & 1.796-03 \\ 200 & 2.040+0 C & 1.240-02 & 2.027+00 & 7.271-04 \\ 300 & 1.771+00 & 5.500-C 3 & 1.765+00 & 2.120-04\end{array}$

\begin{tabular}{|c|c|c|c|c|}
\hline EA EREY & $\epsilon C \Delta$ & $\mathrm{COH}$ & INCCH. & PHOTC \\
\hline KEV & TOTAL & SCATT. & SCATT. & EFFECT \\
\hline 1 & $4.385+04$ & 2. $1 \in C+01$ & $3.025-01$ & $4 \cdot 382+04$ \\
\hline 1.5 & $1.415+04$ & 1. $\subsetneq 25+01$ & $5.989-01$ & $1.413+04$ \\
\hline 2 & $6.125+03$ & $1 . t 85+01$ & $5.035-01$ & $6.108+03$ \\
\hline 3 & $1.807+03$ & $1.255+01$ & $1.413+00$ & $1.793+03$ \\
\hline 4 & $7.43 c+02$ & $9.54 \varepsilon+00$ & $1.785+00$ & $7.317+02$ \\
\hline 5 & $3.695+02$ & $7.45 \mathrm{C}+00$ & $2.086+00$ & $3.603+02$ \\
\hline 6 & $2.08 \varepsilon+02$ & 6. $C 84+00$ & $2.291+00$ & $2.004+02$ \\
\hline 8 & $8.54 t+C 1$ & $4.361+00$ & 2.56 & $7.854+01$ \\
\hline 10 & $4.372+01$ & $3.3 t$ & 2.6 & $6+0$ \\
\hline$: 5$ & $1 \cdot 483+01$ & $2 . c 30+00$ & & +00 \\
\hline 20 & $8.250+00$ & $1.343+00$ & 3.1 & +00 \\
\hline 30 & $4.749+00$ & 7.c C $3-01$ & 3.29 & 9.53 \\
\hline 40 & $4.083+0 \mathrm{C}$ & $4.262-01$ & $3.253+00$ & $3.634-01$ \\
\hline 50 & $3.706+c C$ & $2.856-C_{1}$ & $3.248+00$ & $1.725-01$ \\
\hline 60 & $3.484+0 \mathrm{C}$ & $2.043-01$ & $3.18 t+0 C$ & $9.411-02$ \\
\hline 80 & $3.208+00$ & $1.190=01$ & $3.053+00$ & $3.046-02$ \\
\hline 100 & $3 . C 18+0 C$ & $7.75 c-c 2$ & $2.923+00$ & $1.76 !-C 2$ \\
\hline 150 & $2.68 t+00$ & $3.51 c-02$ & $2.646+00$ & $4.804-03$ \\
\hline 200 & $2.452+00$ & $1.550-02$ & $2.430+00$ & $1.953-03$ \\
\hline 300 & $2.12 t+00$ & $8.800-03$ & $2.117+00$ & $5.698-0$ \\
\hline
\end{tabular}

$\begin{array}{ccccc}\begin{array}{c}\text { ENERGY } \\ \text { KEV }\end{array} & \begin{array}{c}\text { NITROGEN } \\ \text { TOTAL }\end{array} & \begin{array}{c}\mathrm{COH} \\ \text { SCATT. }\end{array} & \begin{array}{c}\text { INCOH. } \\ \text { SCATT. }\end{array} & \begin{array}{c}\text { PHCTC } \\ \text { EFFECT }\end{array} \\ 1 & 7.898+C 4 & 3 . C 11+01 & 2.524-01 & 7.895+04 \\ 1.5 & 2.610+04 & 2.755+01 & 5.157-01 & 2.608+04 \\ 2 & 1.149+04 & 2.465+01 & 8.128-01 & 1.147+04 \\ 3 & 3.468+03 & 1.9 C 5+01 & 1.381+00 & 3.448+C 3 \\ 4 & 1.447+03 & 1.473+01 & 1.854+00 & 1.430+C 3 \\ 5 & 7.269+02 & 1.155+01 & 2.215+00 & 7.131+02 \\ 6 & 4.125+02 & 5.352+00 & 2.487+0 C & 4.007+02 \\ 8 & 1.688+02 & 6.544+0 C & 2.856+C 0 & 1.594+C 2 \\ 10 & 8.535+01 & 4.542+00 & 3 . C 95+00 & 7.731+01 \\ 15 & 2.683+01 & 2.925+00 & 3.454+00 & 2.045+01 \\ 20 & 1.350+01 & 1.545+0 C & 3.651+00 & 7.899+00 \\ 30 & 6.880+00 & 1.021+00 & 3.802+00 & 2.056+00 \\ 40 & 5.223+00 & t .2 C 6-01 & 3.811+00 & 7.917-01 \\ 50 & 4.560+00 & 4.162-01 & 3.765+C 0 & 3.785-01 \\ 60 & 4.205+00 & 2.982-01 & 3.659+00 & 2.076-01 \\ 90 & 3.804+C 0 & 1.740-01 & 3.545+C C & 8.102-02 \\ 100 & 3.555+0 C & 1.136-01 & 3.402+00 & 3.933-02 \\ 150 & 3.145+00 & 5.15 C-02 & 3.083+00 & 1.080-02 \\ 200 & 2.866+C 0 & 2.920-02 & 2.833+00 & 4.400-03 \\ 300 & 2.483+C C & 1.3 C 0-02 & 2.468+00 & 1.285-03\end{array}$

$\begin{array}{rc}\begin{array}{c}\text { ENERGY } \\ \text { KEV }\end{array} & \text { TCTAL } \\ 400 & 1.585+00 \\ 500 & 1.447+00 \\ 600 & 1.338+00 \\ 800 & 1.175+00 \\ 1000 & 1.057+00 \\ 1500 & 8.592-01 \\ 2000 & 7.365-01 \\ 3000 & 5.886-01 \\ 4000 & 5.017-01 \\ 5000 & 4.438-01 \\ 6000 & 4.015-01 \\ 8000 & 3.467-01 \\ 10000 & 3.144-01 \\ 15000 & 2.661-01 \\ 20000 & 2.450-01 \\ 30000 & 2.245-01 \\ 40000 & 2.156-01 \\ 50000 & 2.131-01 \\ 60000 & 2.109-C 1 \\ 80000 & 2.111-01 \\ 100000 & 2.141-01\end{array}$

$\begin{array}{rc}\text { ENFRGY } & \\ \text { KEV } & \text { TCTAL } \\ 400 & 1.903+00 \\ 500 & 1.736+00 \\ 600 & 1.6 C 6+00 \\ 800 & 1.410+00 \\ 1000 & 1.268+00 \\ 1500 & 1.031+00 \\ 2000 & 8.848-01 \\ 3000 & 7.093-01 \\ 4000 & 6.071-01 \\ 5000 & 5.351-01 \\ 6000 & 4.896-01 \\ 8000 & 4.263-01 \\ 10000 & 3.900-01 \\ 15000 & 3.357-01 \\ 20000 & 3.136-01 \\ 3000 C & 2.928-01 \\ 40000 & 2.846-01 \\ 50000 & 2.836-01 \\ 60000 & 2.822-01 \\ 80000 & 2.844-C 1 \\ 100000 & 2.896-01\end{array}$

ENERGY

KEV

$$
\begin{aligned}
& 400 \\
& 500
\end{aligned}
$$

600
800

1000

1500

2000

4000

5000

6000

8000
10000

10000
15000

20000

30000

40000

50000

60000

80000

100000 2. $896-01$

COH
SCATT.
$3.100=03$
$2.000-03$
$1.300-03$
$7.000-04$
$5.000-04$
$2.234-04$
$1.260-04$
$5.616-05$
$3.164-05$
$2.026-05$
$1.408-05$
$7.923-06$
$5.074-06$
$2.258-06$
$1.270-06$
$5.557-07$
$3.068-07$
$1.920-07$
$1.305-07$
$6.934-08$
$4.150-08$

INCOH.
SCATT.
$1.582+00$
$1.445+00$
$1.337+00$
$1.174+00$
$1 . C 56+0 C$
$8.575-01$
$7.319-01$
$5.755-C 1$
$4.801-01$
$4.144-C 1$
$3.6 \in 2-01$
$2.995-C 1$
$2.550-01$
$1.886-01$
$1.513-01$
$1.100-01$
$8.733-02$
$7.285-02$
$6.272-C 2$
$4.942-02$
$4.100-02$

INCOH.
SCATT.

PHOTO EFFECT

$9.153-C 5$ $4.877-C 5$ $1.389-0=$ 7.935-0E $3.904-C 6$ 2. $360-0$ $1.320-C E$

๑. 150-07 $6.886-C 7$ 5. 584-0 $4 . C 12-C 7$

$3.104-07$

$2.029-C^{7}$ $1.501-07$ c. $813-C$ $7.258-08$

$5.744-08$ $4.745-C 8$ $3.50 \varsigma-C$ $2.777-c 8$

$\begin{array}{cc}\text { COH } & \text { INCOH. } \\ \text { SCATT. } & \text { SCATT. } \\ 5.000-03 & 1.898+O C \\ 3.100-03 & 1.733+C O \\ 2.200-03 & 1.6 C 4+O C \\ 1.200-03 & 1.4 C 9+00 \\ 7.000-04 & 1.267+00 \\ 3.128-04 & 1.029+00 \\ 1.765-04 & 8.781-C 1 \\ 7.867-05 & 6.906-C 1 \\ 4.432-05 & 5.760-C 1 \\ 2.839-05 & 4.972-01 \\ 1.972-05 & 4.394-01 \\ 1.110-05 & 3.593-C 1 \\ 7.108-06 & 3.060-C 1 \\ 3.164-06 & 2.263-C 1 \\ 1.781-06 & 1.815-C 1 \\ 7.811-07 & 1.320-01 \\ 4.320-07 & 1.048-01 \\ 2.709-07 & 8.741-C 2 \\ 1.844-07 & 7.52 t-C 2 \\ 9.856-08 & 5.92 C-02 \\ 5.941-08 & 4.519-C 2\end{array}$

PHOTO PHOTO
EFFECT $2.451-04$ $1.295-C_{4}$ $7.837-\mathrm{C}=$ $3.62 \varepsilon-C$ $2.04 t-0$ $1.007-05$

$\epsilon .085-0 t$ $3.402-C E$ 2.357-0E $1.773-0 t$ $1.438-C E$

$1.033-C E$ $7.552-C 7$ $5.223-C^{-}$ $3.863-\mathrm{Cl}$ $2.525-C^{2}$

$1.867-C 7$ 1.477-07 $1.220-C$ $9.024-0 \varepsilon$

$7.141-C \varepsilon$

1.092-C3 $4.475-c 3$ $1.258-c 2$ 2.937-02 $3.52 t-\mathrm{C} 2$ 4. $720-\mathrm{C} 2$ 5. $538-02$ 7. $75 \mathrm{C}-\mathrm{C2}$ $9.37 t-\mathrm{C}^{2}$ $1.145-01$ $1.2 \varepsilon 3-\mathrm{Cl}$ $1.4 \mathrm{C} 2-\mathrm{Cl}$ $1.482-\mathrm{Cl}$ $1.617-\mathrm{Cl}$ 1. $730-\mathrm{Cl}$ $.044-03$ $1.864-c 2$
$3 . c 83-c 2$ $4.150-c 2$ $5.021-\mathrm{c} 2$ $6.7 \mathrm{CC}-02$ $8.4 \mathrm{C} 4-02$ $1.054-\mathrm{Cl}$ $1.321-\mathrm{Cl}$ $1.603-01$ $.7 \div 0-\mathrm{Cl}$ $1.961-01$ $2.0 \in 5-C 1$ $2.251-C 1$ $2.4 \mathrm{C} 4-\mathrm{C}$

$\begin{array}{cc}\text { PHOTO } & \text { PAIR } \\ \text { EFFECT } & \text { PROO. } \\ 5.518-C 4 & \\ 2.917-0 C & \\ 1.755-C 4 & \\ 8.073-C 5 & \\ 4.523-C E & \\ 2.224-0 E & 2.147-C 3 \\ 1.344-0 E & 9.785-C 3 \\ 7.498-C \epsilon & 2.523-C 2 \\ 5.152-C \epsilon & 4.177-02 \\ 3.505-0 \epsilon & 5.666-02 \\ 3.164-C \epsilon & 6.780-C 2 \\ 2.271-0 \epsilon & 5 . C 22-C 2 \\ 1.75 t-C \epsilon & 1.125-C 1 \\ 1.147-0 \epsilon & 1.466-C 1 \\ 8.482-07 & 1.767-01 \\ 5.542-C 7 & 2.148-C 1 \\ 4.098-C 7 & 2.40 C-01 \\ 3.242-C 7 & 2.615-01 \\ 2.677-07 & 2.757-C 1 \\ 1.580-C 7 & 2.556-C 1 \\ 1.56-C 7 & 3.196-01\end{array}$

$\begin{array}{ccc}\begin{array}{c}\text { ENERGY } \\ \text { KEV }\end{array} & \text { TCTAL } & \text { COH } \\ 400 & 2.541+C 0 & 1.080-02 \\ 500 & 2.317+00 & 0.900-03 \\ 600 & 2.143+00 & 4.700-03 \\ 800 & 1.881+0 C & 2.600-03 \\ 1000 & 1.691+00 & 1.600-03 \\ 1500 & 1.376+0 C & 7.155-04 \\ 2000 & 1.183+00 & 4.038-04 \\ 3000 & 9.539-01 & 1.800-04 \\ 4000 & 8.225-01 & 1.014-04 \\ 5000 & 7.366-01 & 6.498-05 \\ 6000 & \epsilon .739-01 & 4.515-05 \\ 8000 & 5.959-C 1 & 2.542-05 \\ 10000 & 5.539-01 & 1.627-05 \\ 25000 & 4.909-01 & 7.240-06 \\ 20000 & 4.697-01 & 4.078-06 \\ 30000 & 4.524-01 & 1.796-06 \\ 40000 & 4.482-01 & 5.959-07 \\ 50000 & 4.526-01 & 6.264-07 \\ 60000 & 4.543-01 & 4.267-07 \\ 80000 & 4.634-01 & 2.303-07 \\ 100000 & 4.753-01 & 1.401-07\end{array}$
1.573-01-1.221+05 3.617-01 $4.167+04$ €. 304-01 $1.873+04$ $1.220+00 \quad 5.796+C_{3}$ $1.770+00 \quad 2.451+\mathrm{Cl}^{3}$ $2.171+00 \quad 1.240+03$ $2.54 \mathrm{C}+00 \quad 7.046+02$ $3.063+00 \quad 2.851+02$ $3.354+0 \mathrm{C} \quad 1.400+02$ $3.844+00 \quad 3.782+01$ $4.086+00 \quad 1.481+01$ $4.278+00 \quad 3.021+00$ $4.305+00 \quad 1.526+00$ $4.271+0 \mathrm{C} \quad 7.350-01$ $4.203+00 \quad 4.054-01$ $4.042+00 \quad 1.595-01$ $3.878+0 \mathrm{C} \quad 7.782-02$ $3.515+00 \quad 2.153-02$ $3.235+00 \quad 8.807-03$ $2.820+00 \quad 2.580-03$
INCCH:
SCATT
$2.213+00$
$2.022+C C$
$1.871+C C$
$1.644+C C$
$1.478+00$
$1.201+00$
$1.024+0 C$
$8.057-01$
$6.720-C 1$
$5.801-C 1$
$5.126-C 1$
$4.192-01$
$3.565-C 1$
$2.64 C-01$
$2.118-01$
$1.54 C-C 1$
$1.223-01$
$1.020-C 1$
$8.780-C 2$
$6.518-C 2$
$5.739-C 2$

$1.5 t t-C$
$3.196-01$

\begin{tabular}{|c|c|c|}
\hline INCOH. & PHOTC & $P \Delta I R$ \\
\hline SCATT. & EFFECT & PROO. \\
\hline $2.525+C C$ & $1.105-03$ & \\
\hline $2.310+00$ & $5.859-04$ & \\
\hline $2.138+c 0$ & $3.522-c_{4}$ & \\
\hline $1.878+C C$ & $1 . t i \in-04$ & \\
\hline $1.689+0 C$ & $9.02 t-C E$ & \\
\hline $1.372+C C$ & $4.434-C 5$ & $2.811-c 3$ \\
\hline $1.171+00$ & $2.678-0=$ & $1.14 \mathrm{c}-\mathrm{C} 2$ \\
\hline $9.207-01$ & $1.492-05$ & $3.3 c t-c 2$ \\
\hline $7.68 \mathrm{C}-01$ & 1.c $c 33-c 5$ & $5.435-c 2$ \\
\hline $6.625-01$ & $7.765-\mathrm{c} 6$ & $7.363-02$ \\
\hline $5.858-C l^{2}$ & $t .28 \mathrm{C}-\mathrm{CE}$ & $8.8 \mathrm{CO}-\mathrm{C2}$ \\
\hline $4.791-C 1$ & $4.5 \mathrm{CS}-\mathrm{CE}$ & $1.168-C 1$ \\
\hline $4.075-01$ & $3.483-0 t$ & $1.455-\mathrm{Cl}$ \\
\hline $3.017-C 1$ & $2.275-0 \epsilon$ & $1.8 \subseteq 2-\mathrm{Cl}$ \\
\hline $2.420-C 1$ & $1.682-0 t$ & $2.277-01$ \\
\hline $1.760-\mathrm{Cl}$ & 1. CSS-CE & $2.764-\mathrm{Cl}$ \\
\hline $1.397-\mathrm{Cl}$ & $8.123-c 7$ & $3.085-01$ \\
\hline $1.165-\mathrm{Cl}$ & $6.42 t-07$ & $3.36 \mathrm{C}-\mathrm{Cl}$ \\
\hline $1.003-\mathrm{Cl}_{1}$ & $5.306-c 7$ & $3.540-\mathrm{Cl}$ \\
\hline $7.905-c 2$ & $3.523-c 7$ & $3.844-\mathrm{Cl}$ \\
\hline & & \\
\hline
\end{tabular}


$Z=$ G FLUCRINE

$\begin{array}{ccccc}\begin{array}{c}\text { ENERGY } \\ \text { KEV }\end{array} & \text { TOTAL } & \begin{array}{c}\text { COH } \\ \text { SCATT. }\end{array} & \begin{array}{c}\text { INCOH. } \\ \text { \$CATT. }\end{array} & \begin{array}{c}\text { PHOTO } \\ \text { EFFECT }\end{array} \\ 1 & 1.793+C 5 & 5.122+01 & 1.469-01 & 1.792+C 5 \\ 1.5 & 6.422+C 4 & 4.826+01 & 3.388-01 & 6.417+04 \\ 2 & 6.974+04 & 4.467+01 & 5.950-01 & 2.969+04 \\ 3 & 5.545+03 & 3.694+01 & 1.180+00 & 9.511+03 \\ 4 & 4.135+03 & 2.588+01 & 1.757+00 & 4.103+03 \\ 5 & 2.129+03 & 2.414+01 & 2.247+00 & 2.103+03 \\ 5 & 1.229+03 & 1.970+01 & 2.660+00 & 1.206+03 \\ 8 & 5.112+02 & 1.372+01 & 3.276+0 C & 4.942+02 \\ 10 & 2.584+02 & 1.015+01 & 3.652+00 & 2.446+02 \\ 15 & 7.674+01 & 5.745+C 0 & 4.230+00 & 6.677+01 \\ 20 & 3.457+01 & 3.785+00 & 4.516+00 & 2.627+01 \\ 30 & 1.375+01 & 2 . C 13+00 & 4.754+00 & 6.985+00 \\ 40 & 8.769+00 & 1.243+00 & 4.804+00 & 2.722+00 \\ 50 & 6.924+00 & 8.405-01 & 4.772+0 C & 1.312+00 \\ 60 & 6 . C 33+00 & 6 . C 52-01 & 4.704+00 & 7.236-01 \\ 80 & 5.170+00 & 3.545-01 & 4.531+0 C & 2.844-01 \\ 100 & 4.723+00 & 2.329-01 & 4.351+0 C & 1.387-01 \\ 150 & 4.098+C 0 & 1.066-01 & 3.953+00 & 3.832-02 \\ 200 & 3.712+00 & 6 . C 60-02 & 3.636+00 & 1.567-02 \\ 300 & 3.203+00 & 2.710-02 & 3.171+00 & 4.592-03\end{array}$

$Z=10$ NEON

$\begin{array}{ccccc}\begin{array}{c}\text { ENERGY } \\ \text { KEV }\end{array} & \begin{array}{c}\text { TOTAL } \\ 1\end{array} & \begin{array}{c}\text { SCATT } \\ \text { SCA }\end{array} & \begin{array}{c}\text { INCDH. } \\ \text { SCATT. }\end{array} & \begin{array}{c}\text { PHOTC } \\ \text { EFFECT }\end{array} \\ 1.5 & 9.101+04 & 6.379+01 & 1.824-C 1 & 2.549+05 \\ 2 & 4.221+04 & 5.685+01 & 3.890-01 & 9.095+04 \\ 3 & 1.364+04 & 4.821+01 & 1.448-01 & 4.215+04 \\ 4 & 5.552+03 & 3.988+01 & 1.793+00 & 1.359+04 \\ 5 & 3.087+03 & 3.276+01 & 2.318+C 0 & 5.910+03 \\ 6 & 1.792+03 & 2.7 C 2+C 1 & 2.774+00 & 1.761+03 \\ 8 & 7.533+02 & 1.895+01 & 3.478+00 & 7.308+02 \\ 10 & 3.834+02 & 1.4 C 4+C 1 & 3.956+00 & 3.654+02 \\ 15 & 1.142+02 & 7.848+C 0 & 4.612+00 & 1.018+02 \\ 20 & 5 . C 7 C+01 & 5.123+00 & 4.935+00 & 4.064+01 \\ 30 & 1.898+01 & 2.721+00 & 5.224+00 & 1.104+01 \\ 40 & 1.135+01 & 1.687+00 & 5.294+00 & 4.366+00 \\ 50 & 8.542+00 & 1.146+00 & 5.269+00 & 2.127+00 \\ 60 & 7.211+00 & 8.272-01 & 5.2 C 0+00 & 1.184+00 \\ 80 & 5.972+00 & 4.839-01 & 5.017+00 & 4.713-01 \\ 100 & 5.373+0 C & 3.184-01 & 4.822+00 & 2.319-01 \\ 150 & 4.598+00 & 1.463-01 & 4.386+00 & 6.494-02 \\ 200 & 4.14 t+0 C & 8.340-02 & 4.036+00 & 2.675-02 \\ 300 & 3.566+00 & 3.730-02 & 3.521+00 & 7.886-03\end{array}$

\begin{tabular}{|c|c|c|c|c|}
\hline \multicolumn{5}{|c|}{$Z=11$ SOCIUM } \\
\hline ENERGY & & $\mathrm{COH}$ & I NCOH. & PHOTC \\
\hline KEV & TOTAL & SCATT. & SCATT. & EFFECT \\
\hline I & $2.795+04$ & $7.376+01$ & $4.636-01$ & $2.787+C_{4}$ \\
\hline 1.5 & $1.235+05$ & $6.840+01$ & $7.506-\mathrm{Cl}$ & $1.235+05$ \\
\hline 2 & $5.980+04$ & $6.341+01$ & $1.003+00$ & $5.974+04$ \\
\hline 3 & $2.024+04$ & $5.457+01$ & I. $50 s+00$ & $2.018+04$ \\
\hline 4 & $9.036+03$ & $4 . t 4 t+C 1$ & $2.022+00$ & $8.988+03$ \\
\hline 5 & $4.747+03$ & $3.918+01$ & $2.51 c+00$ & $4.706+03$ \\
\hline 6 & $2.778+C 3$ & $3.298+01$ & $2.955+0 \mathrm{C}$ & $2.742+03$ \\
\hline 8 & $1.175+03$ & $2.373+01$ & $3.687+00$ & $1.148+03$ \\
\hline 10 & $5.979+02$ & $1.770+01$ & $4.218+00$ & $5.760+02$ \\
\hline 15 & $1.755+02$ & $9 . \varsigma 28+00$ & $4.973+00$ & $1.606+02$ \\
\hline 20 & $7.574+01$ & $6.472+0 C$ & $5.348+00$ & $6.392+01$ \\
\hline 30 & $2.636+01$ & $3.457+0 \mathrm{C}$ & $5.679+00$ & $1.722+01$ \\
\hline 40 & $1.470+01$ & $2.170+00$ & $5.772+00$ & $6.762+00$ \\
\hline 50 & $1.052+01$ & $1.490+00$ & $5.756+00$ & $3.274+00$ \\
\hline 60 & $8.588+0 c$ & 1. $c \in \epsilon+00$ & $5.689+00$ & $1.813+00$ \\
\hline 80 & $6.849+00$ & $6.346-01$ & $=.4 \varsigma \varepsilon+00$ & $7.167-01$ \\
\hline 100 & $.060+00$ & $4-1 \div 3-01$ & $5.290+00$ & $3.510-01$ \\
\hline 150 & $5.110+00$ & $1.941-01$ & $4.818+00$ & $9.779-02$ \\
\hline 200 & $4.587+0 \mathrm{C}$ & $1.110-01$ & $4.435+00$ & $4.028-02$ \\
\hline 300 & $2.933+00$ & $4.9 \varepsilon C-C 2$ & $3.871+00$ & $1.195-02$ \\
\hline
\end{tabular}

\begin{tabular}{|c|c|c|c|c|}
\hline ENERGY & & $\mathrm{COH}$ & $1 \mathrm{NCOH}$. & PHETC \\
\hline KEV & TOTAL & SCATT. & SCATT. & EFFECT \\
\hline 1 & $4.011+C 4$ & $8.719+01$ & $5.939-01$ & $4.002+04$ \\
\hline 1.5 & $1.580+05$ & $7.585+01$ & $1.001+C 0$ & $1.579+05$ \\
\hline 2 & $7.858+c 4$ & $7.287+01$ & $1.321+00$ & $7.850+04$ \\
\hline 3 & $2.746+C 4$ & 6. I $t \in+01$ & $1.842+00$ & $2.739+C 4$ \\
\hline 4 & $1.249+04$ & $5.282+01$ & $2.315+00$ & $1.244+04$ \\
\hline 5 & $6.643+03$ & $4.525+01$ & $2.772+00$ & $6.595+03$ \\
\hline 6 & $3.921+03$ & $3.873+01$ & $3.195+00$ & $3.879+03$ \\
\hline 8 & 1.6 & $2.8 \subseteq 7+01$ & $3.924+00$ & $1.644+03$ \\
\hline 10 & $\varepsilon .582+02$ & $2.157+01$ & $7+00$ & $8.321+02$ \\
\hline 15 & $2.523+02$ & $1.218+C 1$ & $5.330+00$ & $2.348+02$ \\
\hline 20 & $1.077+02$ & $7.54 C+00$ & $5.753+00$ & $9.405+01$ \\
\hline 30 & $3.590+01$ & $4.263+0 c$ & $6.125+50$ & $2.550+01$ \\
\hline 40 & $1.900+01$ & $2.7 C 8+00$ & $6.243+00$ & $1.005+01$ \\
\hline 50 & $1.300+C 1$ & $1.884+0 \mathrm{C}$ & c. $237+C C$ & $4.874+00$ \\
\hline 60 & $1.026+01$ & $1.390+00$ & $6.174+00$ & $2.702+00$ \\
\hline 80 & & $C E-C)$ & +00 & \\
\hline 100 & $6.817+00$ & $5.375-01$ & $5.756+00$ & $5.244-01$ \\
\hline 150 & $5.645+00$ & $2.507-01$ & $5.24 \varepsilon+00$ & $1.463-01$ \\
\hline 200 & $5.038+00$ & $1.438-01$ & $4.834+C 0$ & $6.036-02$ \\
\hline 300 & $.304+00$ & $6.480-02$ & $4.221+00$ & $1.797-\mathrm{C2}$ \\
\hline
\end{tabular}

$\begin{array}{rcc}\text { ENERGY } & \text { TCTAL } & \begin{array}{c}\mathrm{COH} \\ \text { KEV }\end{array} \\ 400 & 2.861+00 & 1.520-02 \\ 500 & 2.610+00 & 9.700-03 \\ 600 & 2.412+00 & 6.700-03 \\ 800 & 2.117+00 & 3.700-03 \\ 1000 & 1.902+00 & 2.300-03 \\ 1500 & 1.549+00 & 1.029-03 \\ 2000 & 1.332+00 & 5.806-04 \\ 3000 & 1.078+00 & 2.589-04 \\ 4000 & 9.328-01 & 1.459-04 \\ 5000 & 8.387-01 & 9.347-05 \\ 6000 & 7.700-01 & 6.495-05 \\ 8000 & 6.859-01 & 3.657-05 \\ 10000 & 6.421-01 & 2.341-05 \\ 15000 & 5.765-01 & 1.041-05 \\ 20000 & 5.572-01 & 5.866-06 \\ 30000 & 5.435-01 & 2.588-06 \\ 40000 & 5.426-01 & 1.436-06 \\ 50000 & 5.507-01 & 9.042-07 \\ 60000 & 5.547-01 & 6.165-07 \\ 80000 & 5.682-01 & 3.338-07 \\ 100000 & 5.843-01 & 2.035-07\end{array}$

INCOH.
SCATT
$2.844+00$
$2.5 S 5+00$
$2.405+00$
$2.113+00$
$1.9 C 0+00$
$1.544+00$
$1.317+C 0$
$1.036+00$
$8.640-01$
$7.458-01$
$6.591-C 1$
$5.39 C-C 1$
$4.585-01$
$3.394-01$
$2.723-C 1$
$1.980-01$
$1.572-C 1$
$1.311-01$
$1.129-01$
$8.894-C 2$
$7.379-02$

$\mathrm{COH}$
SCATT
$2.100-0$

ENERG
KEV

KEV

400

600

800
1000

1000
1500

1500

3000

4000

5000

6000

8000

1000

15000

20000

30000
40000

50000

60000

80000

80000
100000

$T C T A L$
$3.184+00$
$2.902+00$
$2.682+0 C$
$2.353+00$
$2.114+00$
$1.721+00$
$1.482+00$
$1.203+00$
$1.045+00$
$9.431-01$
$8.687-01$
$7.792-01$
$7.344-01$
$6.673-01$
$6.510-01$
$6.421-01$
$6.453-01$
$6.578-01$
$6.644-01$
$6.830-01$
$7.039-01$

INCOH.

SCAT T
$3.159+0 C$

$2.887+0 \mathrm{C}$

$2.671+0 \mathrm{C}$

$2.348+C 0$

$2.111+00$

1. $715+00$

1. $463+00$

9. $60 \mathrm{C}-01$

$8.286-01$

7. $323-C$

5.988-01

5. $099-01$

$3.771-\mathrm{Cl}$

3.025-01

2. 200-01

1. $746-C 1$

1. 457-01

$1.254-\mathrm{C} 1$

$5.882-C_{2}$
$8.159-02$

$8.886-07$

2. $947-07$
ENERG
KEV

KEV

400
500
600

600

800
1000

1500

2000

3000

4000
5000

5000
6000

8000

10000

15000

20000

30000

40000

50000

60000

80000

100000

$T C T A L$
$3.507+00$
$3.195+0 C$
$2.952+00$
$2.590+00$
$2.325+00$
$1.854+00$
$1.633+00$
$1.329+00$
$1.158+00$
$1.050+0 C$
$9.701-C 1$
$8.760-01$
$8.310-01$
$7.635-01$
$7.510-01$
$7.481-01$
$7.561-01$
$7.738-C 1$
$7.834-01$
$8.078-01$
$8.339-01$

$\mathrm{COH}$
SCATT
$2.800-02$
$1.790-02$
$1.240-02$
$0.900-03$
$4.400-03$
$1.969-03$
$1.111-03$
$4.957-04$
$2.793-04$
$1.790-04$
$1.244-04$
$7.003-05$
$4.484-05$
$1.995-05$
$1.123-05$
$4.969-06$
$2.760-06$
$1.742-06$
$1.190-06$
$6.466-07$
$3.961-07$
I NCOH

SCATT .

$3.175+00$

2. $938+0 \mathrm{C}$

2. $582+\mathrm{CO}$

2. $322+00$

1. $887+00$

1. $610+0 \mathrm{C}$

$1.266+00$

i. $05 t+C C$

9. $116-C$

$8.056-C 1$

6. 587-01

5.609-01

4.149-01

3. $328-C$

$2.420-C$

1. $921-01$

1. $6 \mathrm{C} 3-\mathrm{C} 1$

1. 380-01

$1.087-C 1$
$9: 019-C 2$
PHOTO EFFEC T

1.976-0

$1.04 \mathrm{C}-\mathrm{C} 3$

6.304-04

2. $907-04$

$1.632-C_{4}$

8. $012-C 5$

$4.837<05$

$2.690-C 5$

1. $862-C 5$

$1.40 \mathrm{C}-05$

.133-05

$8.115-C$

$6.265-C$

$.092-06$

$3.024-C 6$

1. 460-OC

$155-C$

$.534-C 7$

7.047-07

$5.574-C 7$

PHOTO

EFFECT

$3.396-C 3$

$.795-03$

$.935-C_{4}$

$2.747-C_{4}$

1. $348-C 4$

8. $131-C 5$

$.517-C 5$

.126-CE

$1.900-C E$

$1.360-05$

1.C49-C5

$6.852-C$

$5.0 E 4-C E$

$3.306-C$

$2.443-06$

$1.932-06$

$1.595-\mathrm{C}$

1.179-Ot

$9.325-C 7$
1. $575-06$

PAIR
PROD.

$3.568-C 3$

1. 457-02

. $182-C 2$

9. $2 \varepsilon 3-C 2$

1. $108-C 1$

$.831-C 1$

2. $371-C 1$

. $849-\mathrm{Cl}$

$3.455-01$

$4.196-01$

4. $418-C 1$

5. $752-\mathrm{Cl}$

PAIR

PROO.

$4.41 t-C$

. $8 \mathrm{Cl}-\mathrm{C2}$

$8.447-C 2$

1. $142-01$

1. $363-\mathrm{Cl}$

-8C3-01

2. $902-01$

$3.485-C_{1}$

$4.221-C 1$

. 7 CE-C1

$5.389-01$

$5.842-C 1$

t. $215-C 1$

PHOTO

EFFEC T

2.782-C 3

.693-0

7. $545-04$

$4.535-C 4$

2.223-0

$1.341-C 4$

$7.441-05$

$5.147-0=$

$3.868-C 5$

2.127-0

$2.237-C 5$

$1.725-C 5$

$1.126-C 5$

$8.319-C E$

$5.421-C E$

4. $013-06$

$3.174-C 6$

$2.620>06$

$1.53 E-C t$

$1.531-0 t$
PAIR

PROO.

$5.357-C 3$

2. $183-02$

$1.020-C 1$

$1.37 \mathrm{~s}-\mathrm{Cl}$

$1.643-C 1$

$2.172-C 1$

$3.48 t-01$

4. 1 \&2-01

5. C61-C1

$.64 c-0$

$6.454-01$

$6.990-C$

$7.437-01$ $\begin{array}{rccc}\begin{array}{c}\text { ENERGV } \\ \text { KEV }\end{array} & \text { TCTAL } & \text { COH } & \text { INCOH. } \\ 400 & 3.833+00 & 2.650-02 & 3.78 E+C C \\ 500 & 3.490+0 C & 2.330-02 & 3.462+C 0 \\ 600 & 3.223+00 & 1.610-02 & 3.2 C 5+C 0 \\ 800 & 2.827+00 & 9.000-03 & 2.816+00 \\ 1000 & 2.539+C 0 & 5.600-03 & 2.533+C 0 \\ 1500 & 2.068+00 & 2.507-03 & 2.058+0 C \\ 2000 & 1.784+00 & 1.415-03 & 1.756+C C \\ 3000 & 1.456+0 C & 6.312-04 & 1.381+00 \\ 4000 & 1.274+00 & 3.557-04 & 1.152+0 C \\ 5000 & 1.159+00 & 2.279-04 & 9.545-01 \\ 6000 & 1.074+0 C & 1.584-04 & 8.78 E-C 1 \\ 9000 & 9.762-01 & 8.918-05 & 7.187-C 1 \\ 10000 & 9.317-01 & 5.710-05 & 6.119-01 \\ 15000 & 8.649-01 & 2.540-05 & 4.526-C 1 \\ 20000 & 8.572-01 & 1.430-05 & 3.630-01 \\ 30000 & 8.615-C 1 & 6.335-06 & 2.64 C-01 \\ 40000 & 8.752-01 & 3.520-06 & 2.096-01 \\ 50000 & 8.986-C 1 & 2.223-06 & 1.748-C 1 \\ 60000 & 9.116-01 & 1.520-06 & 1.505-01 \\ 80000 & 9.423-C 1 & 8.271-07 & 1.186-01 \\ 100000 & 9.743-01 & 5.078-07 & 9.839-C 2\end{array}$
PHOTO

EFFECT

$7.844-C$

$4.209-C 3$

$2.569-C 3$

1.214-03

t. SES-C 4

$3.415-C$

2. $059-04$

1. 141-04

$7.892-C 5$

$5.929-C$

$4.792-C$

$3.425-C E$

$2.639-05$

1. $722-0$ 5

1.273-0

8.307-CE

e. $137-C$

$4.853-C 6$

$4.00 t-C t$

$2.959-C t$

2. 340-0t
PAIR

PROO.

6. 352-03

2. 6 C2-02

7. 431-02

$1.212-\mathrm{Cl}$

$1.637-01$

1. 950-01

2. $574-\mathrm{Cl}$

$3.157-01$

4.122-C1

$5.975-01$

$6.656-01$

$7.238-\mathrm{Cl}$

7.610-01

$8.237-01$
$8.755-01$ 
ECT I $C A$

$C O M P A R I S O N$

ENOF/E IN BARNS/ATCM

$=13$ ALUMINUM

$\begin{array}{ccccc}\begin{array}{c}\text { ENERCY } \\ \text { KEV }\end{array} & \text { TOTAL } & \begin{array}{c}\text { COH } \\ \text { SCATT. }\end{array} & \begin{array}{c}\text { INCOH. } \\ \text { SCATT. }\end{array} & \begin{array}{c}\text { PHOTC } \\ \text { EFFECT }\end{array} \\ 1 & 5.274+04 & 1 . C 16+02 & 6.214-01 & 5.263+04 \\ 1.5 & 1.765+04 & 5.243+01 & 1.093+00 & 1.756+04 \\ 2 & 1.033+05 & 8.372+01 & 1.483+00 & 1.032+05 \\ 3 & 3.615+C 4 & 6.574+01 & 2.088+00 & 3.608+04 \\ 4 & 1.655+04 & 5.940+01 & 2.591+00 & 1.649+04 \\ 5 & 8.868+C 3 & 5.123+01 & 2.998+00 & 9.814+03 \\ 6 & 5.271+C 3 & 4.440+01 & 3.424+00 & 5.224+03 \\ 8 & 2.284+03 & 3.352+01 & 4.163+00 & 2.246+03 \\ 10 & 1.182+03 & 2.567+01 & 4.785+00 & 1.151+03 \\ 15 & 3.534+02 & 1.466+01 & 5.676+0 C & 3.330+02 \\ 20 & 1.516+02 & 5.557+00 & 6.152+00 & 1.359+02 \\ 30 & 4.057+01 & 5.142+C C & 6.588+00 & 3.784+01 \\ 40 & 2.517+01 & 3.279+00 & 6.717+00 & 1.517+C 1 \\ 50 & 1.646+01 & 2.292+0 C & 6.719+00 & 7.453+00 \\ 60 & 1.253+01 & 1.659+00 & 6.657+00 & 4.171+00 \\ 80 & 9.115+C C & 5.857-01 & 6.452+00 & 1.673+00 \\ 100 & 7.7 C 3+C C & 6.565-01 & 6.219+00 & 8.266-01 \\ 150 & 6.217+00 & 3 . C 71-C 1 & 5.677+00 & 2.327-01 \\ 200 & 5.5 C 4+00 & 1.763-01 & 5.232+00 & 9.609-02 \\ 300 & 4.679+0 C & 7.950-02 & 4.571+C 0 & 2.839-02\end{array}$

$\begin{array}{rc}\begin{array}{r}\text { ENERGY } \\ \text { KEV }\end{array} & \text { TCT AL } \\ 400 & 4.160+00 \\ 500 & 3.785+00 \\ 600 & 3.495+00 \\ 800 & 3.044+00 \\ 1000 & 2.751+00 \\ 1500 & 2.240+00 \\ 2000 & 1.934+00 \\ 3000 & 1.584+0 \mathrm{C} \\ 4000 & 1.390+00 \\ 5000 & 1.269+00 \\ 6000 & 1.180+00 \\ 8000 & 1.079+0 \mathrm{C} \\ 10000 & 1.036+00 \\ 15000 & 9.713-01 \\ 20000 & 9.693-01 \\ 30000 & 9.821-01 \\ 40000 & 1.002+00 \\ 50000 & 1.032+00 \\ 60000 & 1.049+00 \\ 80000 & 1.087+00 \\ 100000 & 1.125+00\end{array}$

100000

ENEFCY
KEV

500

600
800

1000

1500

2000

3000

4000

5000

6000

8000

10000

15000

20000

3000

40000

50000

60000

80000

100000

TCTAL
$4.484+00$
$4.079+00$
$3.7 \in 4+C 0$
$3.3 C 0+00$
$2.963+00$
$2.414+00$
$2.086+00$
$1.713+00$
$1.509+00$
$1.382+00$
$1.250+00$
$1.186+00$
$1.145+00$
$1.083+00$
$1.088+00$
$1.111+0 C$
$1.138+0 C$
$1.175+00$
$1.196+0 C$
$1.241+00$
$1.287+00$

\begin{tabular}{|c|c|c|c|c|}
\hline ENERGY & & $\mathrm{COH}$ & I NCOH. & PHOTC \\
\hline KEV & TOTAL & SCATT. & SCATT. & EFFECT \\
\hline 1 & $7.50 C+04$ & $1.187+02$ & $6.053-01$ & $7.488+04$ \\
\hline 1.5 & $2.532+04$ & 1. $c 80+02$ & $1.107+00$ & $2.521+04$ \\
\hline 2 & $1.270+05$ & $9.738+01$ & $1.555+C 0$ & $1.269+05$ \\
\hline 3 & $4.647+04$ & $7.584+01$ & $2.265+00$ & $4.639+04$ \\
\hline 4 & $2.176+04$ & $6.720+01$ & $2.824+00$ & $2.16 s+04$ \\
\hline 5 & $1.181+04$ & $5.782+01$ & $3.238+00$ & $75+0$ \\
\hline 5 & $7.080+03$ & $5.034+01$ & $3.672+0 \mathrm{C}$ & $C 26+03$ \\
\hline 8 & $3.094+03$ & $2.867+01$ & $4.421+00$ & $050+03$ \\
\hline 10 & $1 \cdot t 05+03$ & $3.00 t+01$ & 5. $C 73+0 C$ & $1.570+c 3$ \\
\hline 15 & $4.788+c 2$ & $1.739+01$ & $6.023+00$ & $4.554+02$ \\
\hline 20 & 2. C $35+02$ & $1.13 t+01$ & $6.548+00$ & $1.856+02$ \\
\hline 30 & $6.449+01$ & 6. $c \varepsilon \varepsilon+00$ & $7.032+00$ & $5.137+01$ \\
\hline 40 & $3.150+\mathrm{Cl}$ & $3.83 \epsilon+00$ & $7.1 \& 1+00$ & $2 . C 4^{\circ}+01$ \\
\hline 50 & $1.986+C 1$ & $2.643+00$ & $7.192+00$ & $1.002+01$ \\
\hline$\epsilon 0$ & $1.46 t+01$ & $1 . s_{3} 1+c 0$ & $7.134+00$ & $5.591+00$ \\
\hline 80 & l. $028+01$ & $1.12 t+00$ & $6.924+00$ & $2.232+00$ \\
\hline 100 & $8.52^{5}+00$ & $7.434-01$ & $6.681+0 \mathrm{C}$ & $1.100+00$ \\
\hline 150 & $6.755+C C$ & $3.442-C 1$ & $6.105+00$ & $3.054-01$ \\
\hline 200 & $5.954+0 \mathrm{C}$ & 1. $s \in 8-01$ & $5.625+00$ & $1-281-0$ \\
\hline 300 & $5.04 \epsilon+00$ & $8.84 \mathrm{C}-02$ & $4.91 s+00$ & $3.825-02$ \\
\hline
\end{tabular}

$\begin{array}{ll}1.032+00 & 2.746-06 \\ 1.049+00 & 1.878-06 \\ 1.087+00 & 1.024-06 \\ 1.125+00 & 6.297-07\end{array}$

$\mathrm{COH}$
SCATT.

$4.980-02$

3. 180-02

1. $220-02$

7. 700-03

$3.448-03$

1. $947-03$

$8.685-04$

4. $895-04$

$3.13 t-04$

2.180-04

1. $227-04$

3. $496-05$

1. $969-05$

8.735-06

$4.859-06$

3. $072-06$

2.102-06

$1.147-06$
$7.068-07$

ENERGY

$\begin{array}{ccc}\text { ENERCY } & \text { TOTAL } & \text { SCATT } \\ \text { KEV } & \text { TOH } \\ 1 & 1.040+C 5 & 1.375+02 \\ 1.5 & 3.325+04 & 1.257+02 \\ 2 & 1.560+C 4 & 1.134+02 \\ 3 & 5.733+C 4 & 5.214+01 \\ 4 & 2.739+C 4 & 7.655+01 \\ 5 & 1.507+04 & 6.536+C 1 \\ 6 & 5.116+03 & 5.682+01 \\ 8 & 4.035+03 & 4.4 C 8+01 \\ 10 & 2.111+03 & 3.472+01 \\ 15 & 6.356+C 2 & 2 . C 41+01 \\ 20 & 2.703+C 2 & 1.336+01 \\ 30 & 8.438+C 1 & 7.128+00 \\ 40 & 4.004+01 & 4.453+C C \\ 50 & 2.440+C 1 & 3.034+00 \\ 60 & 1.746+C 1 & 2.195+00 \\ 80 & 1.174+01 & 1.2 E C+0 C \\ 100 & 5.493+00 & 8.417-01 \\ 150 & 7.344+00 & 3.867-01 \\ 200 & 6.422+C 0 & 2.2 C 3-01 \\ 300 & 5.420+C C & 5.87 C-C 2\end{array}$

I NCEH.

SCATT.
$5.569-C_{1}$

PHCTC

$1.055+0 C \quad 3.312+C 4$

$1.544+00 \quad 1.549+C 4$

$2.371+00 \quad 5.723+C 4$

3. $C 18+00 \quad 2.731+04$

$3.535+00 \quad 1.5 \mathrm{CO}+04$

$3.975+00 \quad 9.055+03$

$4.713+00 \quad 3.986+03$

$5.321+00 \quad 2.071+03$

$6.356+00 \quad 6.089+02$

$6.935+00 \quad 2.500+C 2$

$7.455+C 0 \quad 5.979+01$

$7.63 \mathrm{C}+00 \quad 2.795+01$

$7.655+00 \quad 1.371+C 1$

$7.602+00 \quad 7.663+00$

$7.391+00 \quad 3.066+00$

$7.138+00 \quad 1.514+00$

$t .531+00 \quad 4.267-C$

$6.024+00 \quad 1.769-01$

$5.268+00 \quad 5.297+02$

\begin{tabular}{|c|c|c|}
\hline NERGY & & $\mathrm{COH}$ \\
\hline KEV & TCTAL & SCAT T. \\
\hline 400 & $4.80 s+00$ & $5.550 \div 02$ \\
\hline 500 & $4.373+0 C$ & $3.550-02$ \\
\hline 600 & $4.03 t+00$ & $2.450-02$ \\
\hline 800 & $3.537+00$ & $1.360-c 2$ \\
\hline 1000 & $3.175+0 C$ & $8.500-03$ \\
\hline 1500 & $2.587+00$ & $3.807-03$ \\
\hline 2000 & $2.238+00$ & $2.150-03$ \\
\hline 3000 & $1.844+00$ & $9.591-04$ \\
\hline 4000 & $1.630+O C$ & $5.406-04$ \\
\hline 5000 & $1.498+00$ & $3.464-04$ \\
\hline 6000 & $1.401+00$ & $2.407-04$ \\
\hline 8000 & $1.297+00$ & $1.356-04$ \\
\hline 10000 & $1.258+00$ & $8.681-05$ \\
\hline 15000 & $1.200+00$ & $3.861-05$ \\
\hline 20000 & $1.213+0 C$ & $2.174-05$ \\
\hline 30000 & $1.247+00$ & $8.654-06$ \\
\hline 40000 & $1.282+0 C$ & $5.372-06$ \\
\hline 50000 & $1.326+00$ & $3.398-06$ \\
\hline 60000 & $1.352+00$ & $2.326-06$ \\
\hline 80000 & $1.4 C 6+00$ & $1.270-06$ \\
\hline & $1.459+00$ & $843-07$ \\
\hline
\end{tabular}

\begin{tabular}{|c|c|c|}
\hline $\begin{array}{l}Z= \\
\text { ENFRCY }\end{array}$ & 16 SULFLR & $\mathrm{COH}$ \\
\hline KEV & TOTAL & SCATT. \\
\hline$?$ & $1.358+05$ & $2.578+02$ \\
\hline 1.5 & $4.582+C 4$ & $1.452+c 2$ \\
\hline 2 & $2.082+04$ & $1.315+02$ \\
\hline 3 & $7.254+04$ & $1.0 \in 6+02$ \\
\hline 4 & $3.41 \varepsilon+04$ & $\varepsilon .778+c 1$ \\
\hline 5 & $1.869+04$ & $7.419+01$ \\
\hline 6 & $1.125+04$ & $6.414+01$ \\
\hline 8 & $5.000+C 3$ & $4.98 t+01$ \\
\hline 10 & $2.627+C_{3}$ & $3.5, t \in+01$ \\
\hline 15 & C. $005+C 2$ & $2.371+01$ \\
\hline 20 & $3.436+02$ & $1.557+C 1$ \\
\hline 30 & i. $c 7 s+c 2$ & $8.3 C 4+00$ \\
\hline 40 & $5.062+01$ & $5.14 E+0 C$ \\
\hline 50 & $3.020+C 1$ & $3.482+00$ \\
\hline 60 & $2.109+01$ & $2.5 C 6+00$ \\
\hline 80 & $1.360+C 1$ & $1 \cdot 4 \in 2+00$ \\
\hline 100 & $1.069+01$ & $5.580-01$ \\
\hline 150 & $8.002+0 \mathrm{C}$ & $4.375-01$ \\
\hline 200 & $6.925+00$ & $2.489-01$ \\
\hline $30 \mathrm{C}$ & $5.804+C 0$ & $1.113-01$ \\
\hline
\end{tabular}

$\begin{array}{lc}\text { INCOH. } & \text { PHCTC } \\ \text { SCATT. } & \text { EFEECT } \\ 4.442-01 & 1.357+05 \\ 5.218-01 & 4.567+04 \\ 1.437+00 & 2.069+04 \\ 2.361+00 & 7.243+04 \\ 3.107+00 & 3.4 C 9+04 \\ 3.701+00 & 1.861+04 \\ 4.188+00 & 1.122+C 4 \\ 4.97 C+00 & 4.946+03 \\ 5.648+00 & 2.581+03 \\ 6.708+00 & 7.701+C 2 \\ 7.325+00 & 3.207+02 \\ 7.9 C C+00 & 9.16 t+01 \\ 8.052+00 & 3.738+01 \\ 8.124+00 & 1.860+01 \\ 8.074+00 & 1 . C 51+01 \\ 7.859+00 & 4.279+C C \\ 7.596+C 0 & 2.137+00 \\ 6.957+0 C & 6.132-01 \\ 6.420+00 & 2.564-C 1 \\ 5.616+00 & 7.7 C 3-C 2\end{array}$

$\begin{array}{rr}\text { ENERGY } & \\ \text { KEV } & \text { TCTAL } \\ 400 & 5.140+00 \\ 500 & 4.670+00 \\ 6 C 0 & 4.3 C 8+00 \\ 800 & 3.773+00 \\ 1000 & 3.387+0 C \\ 1500 & 2.760+00 \\ 2000 & 2.350+00 \\ 3000 & 1.975+0 C \\ 4000 & 1.751+C C \\ 5000 & 1.616+00 \\ 6000 & 1.516+C 0 \\ 8000 & 1.410+00 \\ 10000 & 1.375+C C \\ 15000 & 1.323+00 \\ 20000 & 1.345+0 C \\ 30000 & 1.351+00 \\ 40000 & 1.434+0 C \\ 50000 & 1.487+0 C \\ 60000 & 1.517+00 \\ 80000 & 1.580+00 \\ 100000 & 1.641+0 C\end{array}$
$\mathrm{COH}$
$\mathrm{SCATT}$.
$.260-02$
$.990-02$
$2.760-02$
$1.530-02$
$.600-03$
$.300-03$
$2.428-03$
$1.084-03$
$.107-04$
$.914-04$
$2.720-04$
$1.532-04$
$5.809-05$
$4.363-05$
$2.456-05$
$1.091-05$
$6.076-06$
$3.845-06$
$2.634-06$
$1.438-06$
$8.900-07$ SCATT.

$4.1 \mathrm{C} 3+00$

$3.750+C C$

$3.471+C 0$

$3.0 \leq 1+C C$
$2.743+C 0$

$2.229+C O$

$1.9 \mathrm{C} 2+0 \mathrm{C}$

$1.49 t+0 C$

$1.248+00$

$1.077+00$

$9.518-C 1$

$7.782-C 1$

$6.627-01$

4. $901-01$

$3.932-01$

$2.859-\mathrm{Cl}$

$2.270-C 1$

$1.893-C$

$1.630-01$

$1.0 \in 6-C$

SCATT.

$4.417+00$

$4.038+C 0$

$3.737+C C$

$2.954+C \mathrm{C}$

$2.4 \mathrm{Cl}+00$

$2.048+00$

$1.611+00$

$1.344+00$

$1.160+C$

8.382-01

$7.137-C$

5.279-01

4.234-01

3.079-CI

$2.445-C 1$

2. $039-\mathrm{Cl}$

$1.75 t-C 1$

1.383-01

i. 14 e-C1
PHOTC EFFECT

1.224-02

$6.477=03$

$3.895-C 3$

$1.787-C^{3}$

$9.97 C-C 4$
$4.883-c 4$

$2.542-04$

$1.62 s-c 4$

$1.127-04$

c. $4 \in 2-05$

$6.837-0$ 5

$4.883-C 5$

$3.7 \in 1-C E$

$2.454-05$

$1.813-C E$

8.740-06

$6.911-C E$

$5.7 C 4-C E$

$4.214-C E$

$3.331-0 t$

7. $520-03$

3. $059-02$

8.722-C2

$1.420-\mathrm{Cl}$

1. $518=01$

3.010-01

$3.734-\mathrm{Cl}$

4. $811-C 1$

$5.7 \notin 1-C 1$

7.752-01

$8.428-01$

$8.858-01$

$5.521-C 1$

PHOTC

EFFECT

$1.671-02$

$8.97 c-C^{3}$

5.472-03

$2.581-C^{2} 3$

$7.24 \mathrm{C}-04$

$4.359-c 4$

$2.415-04$

$1.660-C_{4}$

1.251-04

$1.01 \mathrm{C}-04$

7.213-05

$5.553-C 5$

$3 . \in 23-C 5$

$2.67 t-C E$

$1.74 t-05$

$1.289-05$

$1.01 \subseteq-05$

8.412-0t

$6.213-0 t$

4. $911-C E$

PHCTO

SCATT EFFECT

$4.731+C 0 \quad 2.320-c 2$

$4.325+0 C \quad 1.248-02$

$4.0 C 4+C 0 ? .631-C 3$

$3.51 s+C 0 \quad 3.616-C \equiv$

$3.165+C 0 \quad 2 . C 82-03$

$2.572+0 C \quad 1 . C 17-02$

$2.155+c 0 \quad 6.120=c 4$

$1.72 t+00 \quad 3.392-04$

$1.440+C C$

$1.243+C 0$

$2.3 .35-04$

$1.754-C_{4}$

$1.41 t-c 4$

$1.010-c 4$

$7.772-C 5$

5.069-05

7.647-0

$5.656-\mathrm{Cl}$

$4.537-C 1$

$3.743-0$

$2.441-C 5$

$1.803-C$

$1.425-C$

$2.62 \mathrm{C}-01$

$2.185-01$

$1.881-C 1$

$1.482-C 1$

$.176-0$

$8.683-i \epsilon$

$6.863-06$
PAIR

PROO.

$8.77 c-c 3$

$3.556-C 2$

1. $\mathrm{C} 12-\mathrm{Cl}$

$2.64 t-C 1$

$2.642-\mathrm{Cl}$

$3.479-01$

$4.313-\mathrm{Cl}$

$5.553-\mathrm{Cl}$

$6.648-01$

$8.936-C 1$

c.710-C1

1. $C 20+00$

$1.1 \mathrm{C}^{2}+00$

$1.172+c c$

$1.012-c 2$

$4.0 \varsigma_{2}-C_{2}$

$1.1 \in 2-01$

$1.828-C 1$

3. $C 27-\mathrm{Cl}$

3. $c 22-\mathrm{Cl}$

4. $.32-C 1$

$6.348-01$

$5.171-\mathrm{Cl}$

$1.020+00$

$1.1 C 8+O C$

$1.164+00$

1. $258+0 \mathrm{C}$

$1.336+C 0$
PHOTO

$\begin{array}{ll}\text { SCATT. EFFECT } \\ 5.044+C O & 3.360-C 2\end{array}$

$\begin{array}{ll}5.044+C O & 3.360-C 2 \\ 4.612+C O & 1.794-C 2\end{array}$

$4.270+00 \quad 1 . C 8 t-C 2$

$3.753+C C \quad 5.038-C$

$3.375+C C \quad 2.836-C 3$

$2.743+00$

$2.340+0 C$

$1.841+C O$

1. $52 t+C 0$

$1.325+00$

$1.171+0 C$

c. $575-C$

$8.156-C 1$

$6.032-C 1$

4. $23 s-C 1$

$3.51 \mathrm{~s}-\mathrm{Cl}$

$2.794-01$

$2.33 \mathrm{C}-\mathrm{C} 1$

2.006-01

$1.581-C 1$

1.311-01

$1.384-03$

$4.614-C^{2}$

$3.17 \varepsilon-04$

$2.380-04$

1. $520-c$

$1.36 \mathrm{C}-\mathrm{C}_{4}$ 
$Z=17$ CHLCRINE

$\begin{array}{ccccc}\begin{array}{c}\text { ENEREY } \\ \text { KEV }\end{array} & \text { TOTAL } & \begin{array}{c}\text { COH } \\ \text { SCATT. }\end{array} & \begin{array}{c}\text { INCCH. } \\ \text { SCATT. }\end{array} & \begin{array}{c}\text { PHOTC } \\ \text { EFFECT }\end{array} \\ 1 & 1.634+C 5 & 1.7 S 5+02 & 4.213-01 & 1.633+05 \\ 1.5 & 5.906+C 4 & 1.663+02 & 8.899-01 & 5.890+04 \\ 2 & 2.738+C 4 & 1.514+C 2 & 1.413+00 & 2.723+04 \\ 2 & 8.432+04 & 1.231+C 2 & 2.39 C+00 & 8.420+04 \\ 4 & 4.155+04 & 1 . C C 9+02 & 3.205+00 & 4.144+04 \\ 5 & 2.337+04 & 8.451+01 & 3.869+0 C & 2.328+04 \\ 6 & 1.438+04 & 7.253+01 & 4.404+00 & 1.431+04 \\ 8 & 6.523+C 3 & 5.615+01 & 5.236+0 C & 6.461+03 \\ 10 & 3.470+03 & 4.491+01 & 5.936+00 & 3.419+03 \\ 15 & 1.07 C+03 & 2.731+01 & 7.047+0 C & 1.036+03 \\ 20 & 4.591+C 2 & 1.801+01 & 7.707+00 & 4.334+02 \\ 30 & 1.417+02 & 5.518+00 & 8.326+00 & 1.238+02 \\ 40 & 6.475+01 & 5.966+00 & 8.541+00 & 5.025+01 \\ 50 & 3.750+01 & 4 . C 38+00 & 8.584+00 & 2.488+C 1 \\ 60 & 2.543+01 & 2.5 C 5+00 & 8.538+00 & 1.399+01 \\ 80 & 1.567+01 & 1.696+0 C & 8.322+00 & 5.647+00 \\ 100 & 1.196+01 & 1.111+00 & 8.050+00 & 2.803+00 \\ 150 & 8.683+0 C & 5 . C 77-C 1 & 7.380+00 & 7.952-01 \\ 200 & 7.434+00 & 2.884-C 1 & 6.815+00 & 3.305-01 \\ 300 & 6.192+00 & 1.290-01 & 5.964+0 C & 9.897-02\end{array}$

\begin{tabular}{|c|c|c|c|c|}
\hline ENERGY & $\Delta R$ & $\mathrm{COH}$ & $1 \mathrm{NCOH}$. & PHCTC \\
\hline KEV & TOTAL & SCATT. & SCATT. & EFFECT \\
\hline 1 & $2.190+05$ & $2.027+02$ & $4.617-01$ & $2.188+05$ \\
\hline 1.5 & $7.453+04$ & $1.889+02$ & $9.335-01$ & $7.434+04$ \\
\hline 2 & $2.423+04$ & $1.7 \geq 1+C 2$ & $1.455+00$ & $3.405+C 4$ \\
\hline 3 & $1.124+04$ & $1.417+02$ & $2.453+00$ & $1.110+04$ \\
\hline 4 & $5.235+04$ & $1.158+02$ & $3.322+00$ & $5.223+04$ \\
\hline 5 & $2.919+04$ & $9.647+01$ & 4. $C 35+00$ & $2.909+C 4$ \\
\hline 6 & $1.786+04$ & $8.219+01$ & $4.623+00$ & $1.777+04$ \\
\hline 8 & $8.040+C 3$ & $6.311+01$ & $5.511+00$ & $7.972+03$ \\
\hline 10 & $4.260+c 3$ & $5 . C 56+01$ & $6.182+c c$ & $4.203+03$ \\
\hline 15 & 1. $3 C 8+C 3$ & $3.120+C 1$ & $7.352+00$ & $1.270+03$ \\
\hline 20 & $5.60 C+02$ & $2.065+01$ & $8.07 c+00$ & $5.313+02$ \\
\hline 30 & $1.720+02$ & $1.1 C 8+01$ & $8.748+00$ & $1.522+02$ \\
\hline 40 & $7.792+01$ & $6 . \varepsilon \varepsilon 7+C C$ & $8.986+00$ & $6.205+01$ \\
\hline 50 & $4.455+01$ & $4.667+00$ & $5.040+00$ & $3.084+01$ \\
\hline 60 & $2.977+01$ & $3.360+00$ & $8.97 s+00$ & $1.741+C 1$ \\
\hline 80 & $1.782+01$ & 1. $s \in 4+C C$ & $\varepsilon .780+00$ & $7.073+00$ \\
\hline 100 & $1.332+\mathrm{C} 1$ & $1.288+00$ & $8.50 c+00$ & $3.529+00$ \\
\hline 150 & $5.403+0 \mathrm{C}$ & $5.884-01$ & $7.803+00$ & 1. $C 12+00$ \\
\hline $2 \mathrm{CO}$ & $7.566+00$ & $3.344-01$ & $7.208+00$ & $4.239-01$ \\
\hline 300 & $6.589+00$ & $1.455-01$ & $6.311+00$ & $1.283-01$ \\
\hline
\end{tabular}

\begin{tabular}{|c|c|c|c|c|}
\hline NEREY & & $\mathrm{COH}$ & I NCOH. & PHOTC \\
\hline KEV & TOTAL & SCATT. & SCATT. & EFFECT \\
\hline 1 & $2.653+05$ & $2.189+02$ & $7.655-01$ & $2.651+05$ \\
\hline 1.5 & c. $336+C 4$ & $2.022+02$ & $1.261+00$ & $9.316+04$ \\
\hline 2 & $63+04$ & $1.858+02$ & $1.751+00$ & $4.344+04$ \\
\hline 3 & $1.455+04$ & $48+C 2$ & $2.696+00$ & $1.439+04$ \\
\hline 4 & $.944+C 4$ & $.28 z+02$ & $44+00$ & $5.931+04$ \\
\hline 5 & $3.403+04$ & $1 . C 71+02$ & 4.2 & $22+04$ \\
\hline 6 & $2.121+04$ & $9.115+01$ & $4.878+00$ & $2.111+04$ \\
\hline 8 & $9.776+c 3$ & $6.5 \in 4+01$ & $5.812+00$ & $9.700+03$ \\
\hline 10 & $5.253+03$ & $5.585+01$ & $6.504+00$ & $5.150+03$ \\
\hline 15 & $1.640+C 3$ & $3.498+01$ & $7.701+00$ & $1.597+03$ \\
\hline 20 & $7 . C 57+02$ & $2.341+01$ & $8.451+0 \mathrm{C}$ & $6.738+02$ \\
\hline 30 & $2.158+02$ & $1.251+01$ & $5.177+00$ & $1.941+02$ \\
\hline 40 & 01 & $735+\mathrm{CO}$ & 5.43 & $8+0$ \\
\hline 50 & 5.40 & $5.221+00$ & 9.4 & 3.9 \\
\hline 60 & $2.53 \varepsilon+C 1$ & $3.750+0 c$ & 9.46 & $2.217+01$ \\
\hline 80 & $2.042+01$ & $2.153+00$ & $5.239+00$ & $8.984+00$ \\
\hline 100 & $1.48 t+01$ & $1.4 \geq 4+00$ & $\varepsilon .951+00$ & $4.473+00$ \\
\hline 150 & $1.01 \epsilon+01$ & $6.5 \geq 2-01$ & $\varepsilon .225+C C$ & $1.278+00$ \\
\hline $20 \mathrm{C}$ & $8.507+00$ & 3. $7 \mathrm{CE}-\mathrm{Cl}$ & $7.601+00$ & $5.351-01$ \\
\hline & $.986+00$ & $1.655-01$ & $6.658+00$ & $1.623=0$ \\
\hline
\end{tabular}

$\begin{array}{rc}\begin{array}{c}\text { ENERGY } \\ \text { KEV }\end{array} & \text { TCTAL } \\ 400 & 5.474+00 \\ 500 & 4.968+00 \\ 600 & 4.582+00 \\ 800 & 4.012+00 \\ 1000 & 3.6 C 1+00 \\ 1500 & 2.535+00 \\ 2000 & 2.544+00 \\ 3000 & 2.107+00 \\ 4000 & 1.875+00 \\ 5000 & 1.735+00 \\ 6000 & 1.633+00 \\ 8000 & 1.527+00 \\ 10000 & 1.496+00 \\ 15000 & 1.450+0 C \\ 20000 & 1.482+00 \\ 30000 & 1.542+0 C \\ 40000 & 1.594+C 0 \\ 50000 & 1.655+00 \\ 60000 & 1.691+00 \\ 80000 & 1.764+C 0 \\ 100000 & 1.833+00\end{array}$

ENERG

KEV

500

800

1000

1500

2000

3000

4000
5000

5000
6000

8000

10000

15000

20000

30000

40000

$6 \mathrm{C000}$

86000

80000
100000

DORY

KEV

KEV

500

800

1000

$\begin{array}{ll}2000 & 2.851+C C \\ 3000 & 2.375+O C\end{array}$

$40002.127+00$

$5000 \quad 1.982+00$

$\begin{array}{ll}5000 & 1.982+00 \\ 6000 & 1.875+0 C \\ 8000 & 1.771+00\end{array}$

10000

$15000 \quad 1.721+00$

$20000 \quad 1.776+00$

$30000 \quad 1.865+00$

$40000 \quad 1.938+C 0$

$50000 \quad 2.019+00$

$60000 \quad 2.066+c 0$

$80000 \quad 2.160+C 0$

100000

COH
SCATT.
$7.250-02$
$4.630-02$
$3.200-02$
$1.780-02$
$1.110-02$
$4.973-03$
$2.808-03$
$1.253-03$
$7.064-04$
$4.527-04$
$3.146-04$
$1.772-04$
$1.135-04$
$5.046-05$
$2.841+05$
$1.263-05$
$7.036-06$
$4.452-06$
$3.052-06$
$1.667-06$
$1.033-06$

COH

INCOH.

$5.358+O C$

$4.8 \subseteq 5+C 0$

$4.536+C O$

$3.98 \varepsilon+C 0$

$3.586+C C$
$2.915+C 0$

$2.915+C 0$
$2.487+00$

$1.956+C C$

$1.632+00$

$1.4 C E+C O$

$1.245+c 0$

$1.018+00$

$8.666-\mathrm{Cl}$

$6.410-C 1$

$5.142-01$
$3.739-01$

$3.735-01$
$2.968-01$

2.476-01

$2.132-01$

$1.132-01$
$1.680-01$
$1.394-01$
PHOTO EFFECT

$4.325-C 2$
$2.32 C-C 2$

$1.414-02$

$t .65 t-C$

$1.115-0$

6.187-04

$4.25 t-C^{-C 4}$

$3.184-04$

$2.568-c 4$

$1.830-C 4$
$1.4 C 7-04$

$9.175-C 5$

$6.773-C 5$

4.415-0

$3.25 s-C 5$

2.124-0

$1.568-C$

$1.239-05$
$3.808-03$
$1.857-03$

PAIR

PRCD.

1.314-02

$5.280-02$
$1.454-C 1$

2.421-CI

$3.263-\mathrm{Cl}$

$3.875-01$
$5 . c 88-C 1$

6.252-01

8. $\mathrm{Cs} 3-\mathrm{Cl}$

9.681-C1

$1.168+C 0$

$1.297+0 C$

$1.478+0 C$

$1.5 \xi t+C C$

$1.6 \subseteq 4+C C$

PHOTC

$\begin{array}{ll}\text { SCATT. } & \text { EFFECT } \\ 5.671+00 & 5.644-02\end{array}$

PAIR

$5.18 \epsilon+O C \quad 3 . C 42-C 2$

$4.801+C 0 \quad 1.860-C 2$

$\begin{array}{ll}4.222+C C & 8.80 C-C \\ 3.797+00 & 5.04 c-C\end{array}$

$3.797+C 0$
$3.08 t+C C$

$2.633+00$

$2.071+C C$

$1.728+C C$

$1.451+C 0$

$1.318+C C$

$1.078+0 C$

$6.788-C 1$

$5.485-01$

$3.055-\mathrm{Cl}$

$3.143-C 1$

2.257-01

$2.257-01$
$1.779-C 1$

$5.04 G-C 3$
$2.45 S-C^{2}$

$1.47 \epsilon-03$

$8.192-C_{4}$

$5.628-C_{4}$

$4.20 t-C_{4}$

$3.393-C 4$
$2.416-C 4$

$1.857-C_{4}$

$1.21 \mathrm{C}-04$

$8.933-c$

$5.822-05$

$3.395-c$

$2.800-C 5$

$2.067-C 5$

$3.5 \leq 4-06$

1.206-06

$1.779-C 1$
$\therefore .476-C 1$

$1.633-05$

PROD.

$1.4 \varepsilon !-c 2$

$5.534-C 2$

2.713-CI

3.655-01

$4.338-C 1$

$5.651-0$

9.043-01

1. $C \& 1+C C$

$1.3 C 4+C$

$1.44 \varepsilon+C C$

$1.648+0 C$

$1.779+C C$
$1.888+C C$

PHCTC

PAIR

$7.182-\mathrm{Cz}$

3.897-C2

$2.40 \mathrm{i}-\mathrm{C} 2$

$1.154-02$

$6.725-C 3$

$3.272-C 3$

$1.0 \in 2-C^{3}$

1. $\csc -\mathrm{C}^{2}$

$7.478=04$

$5.584-C_{4}$

4.502-C4

$3.205-04$

2.462-04

$1.6 \mathrm{C} 4-04$

$1.184-C_{4}$

$7.713-C_{5}$

$5.692-C 5$

$4.496-05$

$3.708-C 5$

5.751-06 2.767-C

3.947-06

$2.158-06$

$2.383-01$

$2.737-C 5$
$2.162-C 5$

PROD.

$1.65 \mathrm{~s}-\mathrm{c2}$

$6.627-\mathrm{C2}$

3. $520-c 1$

4. CES-CI

4. $827-C$

$4.327-01$
$7.813-C 1$

$1.0 \mathrm{C} 5+0 \mathrm{C}$

$1.2 \mathrm{Cl}+0 \mathrm{O}$

$1.448+00$
$1.6 C 6+C C$

$1.742+C C$

$1.828+\mathrm{CO}$

1. $572+00$

$2 . c \leq 2+00$

$\begin{array}{rr}\text { ENERGY } & \\ \text { KEV } & \text { TCTAL } \\ 400 & 6.498+00 \\ 500 & 5.878+00 \\ 600 & 5.410+0 C \\ 800 & 4.729+C 0 \\ 1000 & 4.242+00 \\ 1500 & 3.458+00 \\ 2000 & 3.0 C 6+00 \\ 3000 & 2.512+00 \\ 4000 & 2.256+0 C \\ 5000 & 2.109+00 \\ 6000 & 2.000+00 \\ 8000 & 1.898+00 \\ 10000 & 1.883+00 \\ 15000 & 1.864+C 0 \\ 20000 & 1.932+00 \\ 30000 & 2.028+00 \\ 40000 & 2.122+00 \\ 50000 & 2.213+00 \\ 60000 & 2.267+00 \\ 80000 & 2.372+0 C \\ 100000 & 2.470+00\end{array}$

$\begin{array}{cc}\text { INCOH. } & \text { PHOTO } \\ \text { SCATT. } & \text { EFFECT } \\ 9.496-01 & 3.292+05 \\ 1.543+00 & 1.153+05 \\ 2.051+0 C & 5.372+04 \\ 2.976+0 C & 1.784+04 \\ 3.804+00 & 8.006+03 \\ 4.525+C C & 4.088+C 4 \\ 5.145+00 & 2.520+C 4 \\ 6.120+00 & 1.149+04 \\ 6.837+00 & 6.147+03 \\ 8.055+0 C & 1.911+03 \\ 8.835+00 & 8.177+02 \\ 5.605+00 & 2.417+02 \\ 5.884+00 & 1.0 C 7+02 \\ 9.954+0 C & 5.085+01 \\ 5.92 C+00 & 2.906+01 \\ 5.696+00 & 1.201+01 \\ 5.40 C+00 & 6.061+00 \\ 8.64 t+C C & 1.764+00 \\ 7.954+00 & 7.429-01 \\ 7.005+00 & 2.244-01\end{array}$

$\begin{array}{cc}\text { COH } & \text { INCOH. } \\ \text { SCATT. } & \text { SCATT. } \\ 1.038-01 & 6.296+C O \\ 6.630-02 & 5.759+C C \\ 4.580-02 & 5.333+00 \\ 2.550-02 & 4.685+0 C \\ 1.610-02 & 4.218+C 0 \\ 7.216-03 & 3.425+C C \\ 4.076-03 & 2.52 \epsilon+C C \\ 1.819-03 & 2.3 C 1+00 \\ 1.026-03 & 1.920+C C \\ 6.572-04 & 1.657+0 C \\ 4.568-04 & 1.464+C C \\ 2.573-04 & 1.198+00 \\ 1.648-04 & 1.020+00 \\ 7.328-05 & 7.541-C 1 \\ 4.125-05 & 6.045-01 \\ 1.835-05 & 4.395-C 1 \\ 1.024-05 & 3.492-C 1 \\ 6.484-06 & 2.913-C 1 \\ 4.451-06 & 2.508-C 1 \\ 2.436-06 & 1.57 t-01 \\ 1.515-06 & 1.639-01\end{array}$

PHOTC

EFFECT

S.795-02

$5.22 c-c^{2}$

$3.154-C_{2}$

$1.453-C^{2}$

$8.125-C 3$

$3.549-C^{3}$

2.367-03

$1.315-03$

c. $013-\mathrm{C}_{4}$

6.724-C4

5.419-C4

$3.85 t-C_{4}$

2. $S \in 1-C_{4}$

$1.423=C 4$

c.271-05

$6.840-C 5$

$5.403-C s$

$4.456-C 5$

$3.287-C 5$

$3.287-C$
$2.597-C E$
PAIR

PROD.

$1.848-C 2$

$7.360-c 2$

2.072-01

3. $34 t-C$

5.342-01

$6.996-C 1$

$8.633-01$

1. $327+C C$

$1.598+c 0$

$1.773+C O$

1. $522+C$

$2.016+00$

$2.175+00$
$2.306+00$ 


\begin{tabular}{|c|c|c|c|c|}
\hline & & $\mathrm{COH}$ & I NCOH. & PHOTC \\
\hline & TOTAL & SCATT. & SCATT. & EFFECT \\
\hline & $3.985+05$ & $2.674+02$ & $8.902-01$ & $3.983+05$ \\
\hline 1.5 & $1.461+05$ & $2.4 \leqq 4+02$ & $1.483+0 \mathrm{C}$ & $1.458+05$ \\
\hline & $6.922+04$ & $2.243+02$ & $2.002+00$ & $6.899+04$ \\
\hline & $2.305+04$ & $1.87 t+02$ & & $2.286+C 4$ \\
\hline & $1.023+04$ & $1.571+02$ & 3.8 & $1.007+04$ \\
\hline & $4.748+C 4$ & & & \\
\hline & $2.998+04$ & $27+02$ & $5.232+00$ & $2.586+04$ \\
\hline & $1.40 \varepsilon+04$ & $\varepsilon .523+C 1$ & $6.292+$ & $1.399+04$ \\
\hline & $7.661+03$ & $6.790+01$ & $7.023+00$ & $7.586+03$ \\
\hline & $43 e+03$ & $4 \cdot 3$ & 8.3 & 2.3 \\
\hline & & $2.939+$ & 9.1 & 1.0 \\
\hline & $3.238+02$ & $1.578+01$ & 9.962 & $2.980+02$ \\
\hline & $.427+02$ & 9.7 & 1.0 & \\
\hline & $7.825+01$ & $6.567+00$ & $1.032+01$ & $6.130+01$ \\
\hline & $4.980+01$ & 4.7 & & \\
\hline & $2.706+01$ & $2.7 t \leq t$ & 1.01 & $1.416+01$ \\
\hline & & $1.8 \mathrm{C} 8$ & 9.83 & $7.077+0.0$ \\
\hline & & $8.225-$ & $5.061+$ & \\
\hline & & $4.664-$ & $8.383+00$ & $8.551-$ \\
\hline & & & $7.35 \mathrm{C}+00$ & $2.606-0$ \\
\hline
\end{tabular}

$\begin{array}{ccc}\text { ENERGY } & 22 \text { TITANIUM } \\ \text { KEV } & \text { TOTAL } & \begin{array}{c}C O H \\ \text { SCATT }\end{array} \\ 1 & 4.929+05 & 2.558+02 \\ 1.5 & 1.737+C 5 & 2.729+C 2 \\ 2 & 2.138+04 & 2.5 C 1+02 \\ 3 & 2.728+04 & 2 . C \subseteq 8+02 \\ 4 & 1.237+04 & 1.762+02 \\ 5 & 5.566+04 & 1.486+02 \\ 6 & 3.491+04 & 1.265+02 \\ 8 & 1.632+04 & 5.520+01 \\ 10 & 8.887+03 & 7.540+01 \\ 15 & 2.852+C 3 & 4.785+01 \\ 20 & 1.251+03 & 3.281+01 \\ 30 & 2.892+02 & 1.768+01 \\ 40 & 1.729+02 & 1 . C \subseteq 1+01 \\ 50 & 5.484+01 & 7.373+0 C \\ 60 & 6 . C 00+01 & 5.3 C 5+C C \\ 80 & 3.185+01 & 3.111+0 C \\ 100 & 2.152+01 & 2 . C 36+00 \\ 150 & 1.310+01 & 9.270-01 \\ 200 & 1 . C 44+01 & 5.257-01 \\ 300 & 8.277+00 & 2.347-01\end{array}$

$\begin{array}{cc}\text { INCOH. } & \text { PHCTC } \\ \text { SCATT. } & \text { EFECT } \\ 8.304-01 & 4.926+05 \\ 1.417+0 C & 1.734+05 \\ 1.944+00 & 8.113+04 \\ 2.923+00 & 2.707+C 4 \\ 3.810+00 & 1.219+04 \\ 4.555+00 & 5.550+04 \\ 5.256+00 & 3.478+04 \\ 6.434+00 & 1.622+04 \\ 7.245+00 & 8.805+03 \\ 8.657+0 C & 2.795+03 \\ 9.527+00 & 1.2 C 9+03 \\ 1.035+01 & 3.612+02 \\ 1.071+01 & 1.513+C 2 \\ 1.082+01 & 7.664+01 \\ 1 . C 8 C+01 & 4.389+01 \\ 1.058+01 & 1.820+01 \\ 1.028+01 & 9.203+00 \\ 9.478+00 & 2.691+00 \\ 8.773+00 & 1.138+00 \\ 7.696+00 & 3.468-01\end{array}$

$z=23$ VANADIUM

\begin{tabular}{|c|c|c|c|c|}
\hline NEREY & & $\mathrm{COH}$ & I NCOH. & PHOTC \\
\hline$K \varepsilon V$ & TOTAL & SCATT. & SCATT. & EFFECT \\
\hline 1 & $5.692+05$ & $3.257+02$ & $7.712-01$ & $5.689+05$ \\
\hline 1.5 & $\bar{z} .034+05$ & 3. $C 1 \varepsilon+C 2$ & $1.345+00$ & $2.031+C 5$ \\
\hline 2 & $c .5 c c+c 4$ & $2.777+02$ & $1.87 \mathrm{~s}+0 \mathrm{C}$ & $9.571+04$ \\
\hline 3 & $3.242+04$ & $2.340+02$ & $2.879+00$ & $3.218+04$ \\
\hline 4 & $1.474+04$ & $1.971+02$ & $3.793+00$ & $1.454+04$ \\
\hline 5 & $7.32 t+03$ & $1.6 \in 5+02$ & $4.611+00$ & $7.755+c 3$ \\
\hline 6 & $4.140+14$ & $1.418+02$ & $5.34 c+00$ & $4.125+04$ \\
\hline 8 & 1. SCS+O4 & 1. $C \in 4+02$ & $6.545+00$ & $1.898+04$ \\
\hline 10 & $1.032+04$ & 8. $381+01$ & $7.447+00$ & 1. $\mathrm{C} 23+04$ \\
\hline 15 & $3.292+03$ & $5.2 \div 7+01$ & $8.943+00$ & $3.230+03$ \\
\hline 20 & $1.444+03$ & 3. $645+01$ & c. $863+00$ & $1.398+03$ \\
\hline 30 & $4.50 t+02$ & $1.977+01$ & $1.074+01$ & $4.201+02$ \\
\hline 40 & $2.005+02$ & $1.222+01$ & $1.114+01$ & $1.771+02$ \\
\hline 50 & $1.098+02$ & $\varepsilon .26 t+00$ & $1.12 t+01$ & $9.028+01$ \\
\hline to & $\epsilon, c 1 s+01$ & $5 . \subseteq 53+00$ & $1.125+01$ & $5.198+01$ \\
\hline 80 & $3.627+01$ & $3.4 C E+0 C$ & $1.1<3+01$ & $2.174+01$ \\
\hline 100 & $2.408+01$ & $2.290+00$ & $1.072+01$ & $1.1 \mathrm{C7}+01$ \\
\hline 150 & $1.421+01$ & $1.044+00$ & $9.895+00$ & $3.273+00$ \\
\hline 200 & $1.115+01$ & $5.9<2-01$ & $9.163+00$ & $1.393+00$ \\
\hline $30 c$ & $8.732+\mathrm{Co}$ & $2.645=01$ & $8.041+00$ & $4.263-C_{1}$ \\
\hline
\end{tabular}

$Z=24$ CHRCMIUM

\begin{tabular}{|c|c|c|c|c|}
\hline EY & & $\mathrm{COH}$ & I NCCH. & PHOTC \\
\hline & TOTAL & SCATT. & SCATT. & EFFECT \\
\hline 1 & $6.761+c 5$ & $3.5 \subseteq 4+C 2$ & $7.136-01$ & $6.757+C 5$ \\
\hline 1.5 & $2.4 \mathrm{CC}+0=$ & $3.368+02$ & $1.271+00$ & $2.396+05$ \\
\hline 2 & $.129+05$ & 3. $12 t+C 2$ & $1.80 s+00$ & $1.125+05$ \\
\hline$?$ & $96+c 4$ & $2.6 \in 1+c$ & $2.827+C C$ & $.769+04$ \\
\hline 4 & $1.722+04$ & $2.246+02$ & $2.764+00$ & $1.699+04$ \\
\hline 5 & .24 & $1.8 \subseteq t+c$ & $4.608+00$ & $9.055+03$ \\
\hline$\epsilon$ & .7 & 1.6 & 5.36 & $4.720+04$ \\
\hline 8 & +04 & 1.2 & $\epsilon .6$ & $2.196+04$ \\
\hline 10 & 404 & & & $3+04$ \\
\hline 15 & $3.882+03$ & $5.859+01$ & $\$ .219+00$ & $3.814+03$ \\
\hline 20 & 1.7 & 4.0 & & $1 . t$ \\
\hline 30 & & & & \\
\hline 40 & $2.390+02$ & $1.371+$ & $1.147+C$ & $2.138+02$ \\
\hline 50 & & 5.2 & & \\
\hline 60 & & & 1. & \\
\hline 80 & & & & +01 \\
\hline 100 & $2.723+$ & $2.5 \varepsilon$ & $1.114+01$ & $1.352+C 1$ \\
\hline 150 & $1.548+01$ & $1.177+C C$ & $1.030+01$ & $4.004+00$ \\
\hline 200 & $1.1 \subseteq 2+01$ & $6.682-01$ & $9.547+00$ & $1.704+C 0$ \\
\hline & $5.204+00$ & $2.9 \varepsilon 7-C l$ & $8,384+C C$ & \\
\hline
\end{tabular}

$\begin{array}{rr}\begin{array}{c}\text { ENERGY } \\ \text { KEV }\end{array} & \text { TCTAL } \\ 400 & 6.841+00 \\ 500 & 6.183+00 \\ 600 & 5.688+0 C \\ 800 & 4.970+00 \\ 1000 & 4.457+0 C \\ 1500 & 3.634+0 C \\ 2000 & 3.161+00 \\ 3000 & 2.648+0 C \\ 4000 & 2.387+0 C \\ 5000 & 2.237+0 C \\ 6000 & 2.126+00 \\ 8000 & 2.028+0 C \\ 10000 & 2.021+00 \\ 15000 & 2.012+00 \\ 20000 & 2.093+00 \\ 30000 & 2.217+00 \\ 40000 & 2.313+00 \\ 50000 & 2.416+00 \\ 60000 & 2.477+00 \\ 80000 & 2.554+00 \\ 100000 & 2.703+00\end{array}$

$\mathrm{COH}$
$\mathrm{SCATT}$
$1.170-01$
$7.470-02$
$5.160-02$
$2.870-02$
$1.810-02$
$8.114-03$
$4.583-03$
$2.046-03$
$1.153-03$
$7.391-04$
$5.137-04$
$2.893-04$
$1.853-04$
$8.240-05$
$4.639-05$
$2.064-05$
$1.153-05$
$7.299-06$
$5.013-06$
$2.745-06$
$1.709-06$

INCOH.
SCATT.
$6.6 C 8+C O$
$6.045+C O$
$5.558+C C$
$4.923+00$
$4.428+C C$
$3.6 C C+C 0$
$3.072+00$
$2.416+C C$
$2.016+00$
$1.740+00$
$1.537+C 0$
$1.257+C C$
$1.071+C C$
$7.918-C 1$
$6.351-01$
$4.615-01$
$3.667-01$
$3.058-C 1$
$2.633-C 1$
$2.075-C 1$
$1.721-C 1$

ENERGY

KEV$$
\begin{aligned}
& 400 \\
& 500 \\
& 600
\end{aligned}
$$

$7.2 C 5+00$
$500 \quad 6.497+00$

$600 \quad 5.972+00$

$\begin{array}{rr}800 & 5.213+0 C \\ 1000 & 4.673+00\end{array}$

$\begin{array}{ll}1000 & 4.673+00 \\ 1500 & 3.810+00\end{array}$

$2000 \quad 3.317+00$

$3000 \quad 2.787+00$

$4000 \quad 2.519+00$

$\begin{array}{ll}5000 & 2.367+00 \\ 6000 & 2.256+00\end{array}$

$\begin{array}{ll}6000 & 2.256+00 \\ 8000 & 2.161+00\end{array}$

$10000 \quad 2.163+0 \mathrm{C}$

$15000 \quad 2.166+00$

$20000 \quad 2.261+00$

$30000 \quad 2.4 \mathrm{C} 3+00$

$40000 \quad 2.512+C C$

$50000 \quad 2.627+00$

$60000 \quad 2.695+00$

$80000 \quad 2.826+C C$

$100000 \quad 2.946+C C$

$.709-06$

$\mathrm{COH}$

SCATT.

$1.320-01$
$8.420-02$

$5.820-02$

$3.240-02$

$2.040-02$

$9.145-03$

$5.167-03$

$1.300-03$

8.333-04

$5.792-04$

$3.262-04$

$2.089-04$

$5.230-05$

$2.328-05$

$1.301-05$

$5.659-06$

$3.100-06$

$1.931-06$
INCOH.
SCATT
$6.920+00$
$6.331+C C$
$5.864+C O$
$5.157+0 C$
$4.635+00$
$3.771+C O$
$3.218+00$
$2.531+C C$
$2.112+C 0$
$1.823+C C$
$1.611+O C$
$1.317+C C$
$1.122+C O$
$8.296-O 1$
$6.654-C 1$
$4.835-01$
$3.842-C 1$
$3.204-C 1$
$2.75 C-C 1$
$2.174-01$
$1.804-C 1$ EFFECT

$1.15 t-C 1$

$6.288-C z$

$3.882-C 2$

$1.871-C_{2}$

$1.093-02$

$5.306-C 3 \quad 2.051-C 2$

3.177-03 8.144-02

$1.7 \in 2-C \geq 2.286-C 1$

$1.207-C^{3} 3.688-C 1$

2. $598-C 4 \quad 4.556-C 1$

$7.248-04 \quad 5 . \varepsilon 77-\mathrm{Cl}$

$5.152-C_{4} \quad 7.6 \subseteq 7-C 1$

3.954-04 $9.499-01$

$\begin{array}{ll}2.575-C 4 & 1.220+C C\end{array}$

$\begin{array}{ll}1.900-04 & 1.45 e+C C \\ 1.237-04 & 1.7 .55+C C\end{array}$

$\begin{array}{ll}1.237-04 & 1.0755+C C \\ 5.125-C E & 1.947+00\end{array}$

$7.21 \mathrm{C}-C=2.110+00$

$5.946-C=2.213+C 0$

$4.387-05 \quad 2.387+C 0$

$3.465-C 5 \quad 2.531+C O$

\begin{tabular}{|c|c|c|c|c|c|}
\hline$\varepsilon \wedge \varepsilon R \in Y$ & & $\mathrm{COH}$ & INCOH. & PHOTE & PAIR \\
\hline$k \varepsilon V$ & TCT AL & SCATT. & SCATT. & $\varepsilon F F E C T$ & PROC. \\
\hline 400 & $7.568+00$ & $1.488-01$ & $7.232+00$ & $1.875-C 1$ & \\
\hline 500 & $6.813+c 0$ & $9.500-02$ & $6.617+00$ & $1.005-01$ & \\
\hline 600 & $6.256+0 \mathrm{C}$ & $6.570-02$ & $t .129+c 0$ & $6.095-02$ & \\
\hline 800 & $5.456+0 c$ & $3.650-02$ & $5.391+C 0$ & $2.823-\mathrm{Cz}$ & \\
\hline 1000 & $4.888+0 C$ & $2.310-02$ & $4.849+C 0$ & $1.583-c 2$ & \\
\hline 1500 & $3.985+00$ & $1.036-02$ & $3.942+C 0$ & $7.6 \in 5-03$ & $2.454-02$ \\
\hline 2000 & $3.473+0 \mathrm{C}$ & $5.852-03$ & $3.364+00$ & $4.582-c 3$ & $\subsetneq .839-c 2$ \\
\hline 3000 & $2.926+00$ & $2.612-03$ & $2.646+00$ & $2.534-C 3$ & $2.7 \angle 7-C 1$ \\
\hline 4000 & $2.653+0 \mathrm{C}$ & $1.473-03$ & $2.208+C 0$ & $1.732-C^{2}$ & $4.423-\mathrm{Cl}$ \\
\hline 5000 & $2.500+00$ & c.439-04 & $1.5 C 5+C 0$ & $1.290-c_{3}$ & $5.92 \mathrm{C}-\mathrm{Cl}$ \\
\hline 6000 & $2.388+C C$ & $\epsilon .561-04$ & $1.684+00$ & $1.03 \varepsilon-C 3$ & 7.C21-Cl \\
\hline 8000 & $2.298+00$ & $3.695-04$ & $1.377+00$ & $7.362-04$ & $9.158-01$ \\
\hline 10000 & $2 \cdot 3 \mathrm{C} 8+\mathrm{CO}$ & $2.366-04$ & $1.172+c 0$ & $5.641-04$ & $1.135+C C$ \\
\hline & $2.324+00$ & $1.053-04$ & $8.672-\mathrm{Cl}$ & $3.673-04$ & $1.45 t+C C$ \\
\hline 20000 & $2.435+00$ & $5.924-05$ & $6.95 t-c 1$ & $2.71 \mathrm{C}-\mathrm{C} 4$ & $1.73 s+c c$ \\
\hline 30000 & $2.5 \subseteq 6+$ & $2.637-05$ & 5.05 & $1.765-c 4$ & $50+0 C$ \\
\hline 40000 & $2.719+00$ & $1.474-05$ & $4.016-01$ & $1.2 c 2-04$ & $17+C C$ \\
\hline & & & & 1.028 & $11+C C$ \\
\hline 60000 & $2.922+C O$ & $6.417-0 t$ & $2.884-C 1$ & $8.477-05$ & $634+c c$ \\
\hline & $3.067+00$ & $3.517-$ & $2.272-$ & 6.253 & $2.839+00$ \\
\hline & $8+00$ & $2.193-06$ & $1.885-\mathrm{Cl}$ & $4.938-c 5$ & $10+00$ \\
\hline
\end{tabular}

\begin{tabular}{|c|c|c|c|c|c|}
\hline & & & INCOH. & РНОTO & PAIR \\
\hline KEV & TCT AL & SCATT & SCATT & $\varepsilon F F E C T$ & PRCD. \\
\hline 400 & $7.940+00$ & $1.680-01$ & $7.543+C 0$ & $2.291-\mathrm{Cl}$ & \\
\hline $50 \mathrm{C}$ & $7.132+00$ & $1.073-01$ & $6.902+C C$ & $1.22 t-C 1$ & \\
\hline 600 & $6.542+0 c$ & $7.420-02$ & $6.354+00$ & $7.421-c 2$ & \\
\hline 800 & $5.699+00$ & $4.130-02$ & $5.624+00$ & $3.427-02$ & \\
\hline 1000 & $5.1<4+00$ & $2.600-02$ & $5.059+C C$ & $1.16-02$ & \\
\hline 1500 & $4.161+00$ & $1.166-02$ & $4.113+C C$ & $9.266-c 3$ & $2.734-c 2$ \\
\hline 2000 & $3.629+00$ & $6.587-03$ & $3.510+C C$ & $5.534-03$ & $1.075-\mathrm{Cl}$ \\
\hline 3000 & $3.066+00$ & $2.941-03$ & $2.761+C C$ & $3.057-03$ & S53-C1 \\
\hline 4000 & $2.789+00$ & $1.658-03$ & $2.3 \mathrm{C} 3+\mathrm{CO}$ & $2.088-03$ & 4. $E 15-C 1$ \\
\hline 5000 & $2.634+00$ & $1.063-03$ & $1.988+00$ & $1.553-03$ & $6.433-01$ \\
\hline 6000 & $2.522+0 C$ & $7.387-04$ & 1.75 & $1.245-C 3$ & $7.630-01$ \\
\hline 8000 & $2.438+00$ & $4.160-04$ & $1.437+O C$ & $8.851-C_{4}$ & $9.957-01$ \\
\hline 10000 & $2.458+0 C$ & & $1.223+\mathrm{CO}$ & $6.777-c_{4}$ & $34+O C$ \\
\hline 15000 & 2.48 & 1.18 & 9.04 & 4.41 & $22+O C$ \\
\hline 000 & $2.614+O C$ & $6.670-05$ & 7.25 & $3.255-04$ & $32 \varepsilon+c c$ \\
\hline 30000 & $2.795+\mathrm{CO}$ & $2.969-05$ & $5.27 \varepsilon-c 1$ & $2.120-c_{4}$ & $2.2 \epsilon 7+00$ \\
\hline 40000 & $2.933+00$ & & $4.191-$ & & $14+0 \mathrm{C}$ \\
\hline & & 35 & 3.49 & & $24+0 C$ \\
\hline 60000 & 3.158 & $7.232-06$ & 3.0 & $1.018-04$ & $=6+C C$ \\
\hline & $3.316+00$ & $3.967-06$ & $2.371-\mathrm{Cl}$ & $7.51 \mathrm{C}-\mathrm{CE}$ & $3 . c 7 s+c c$ \\
\hline & $3.461+00$ & $2.474-06$ & $1.9 \in 7-\mathrm{Cl}$ & $5.931-C=$ & $3.264+00$ \\
\hline
\end{tabular}




\begin{tabular}{|c|c|c|c|c|}
\hline \multicolumn{5}{|c|}{$z=25$ MANGANESE } \\
\hline ENEREY & & $\mathrm{COH}$ & I NCOH. & PHOTC. \\
\hline KEV & TOTAL & SCATT. & SCATT. & EFFECT \\
\hline 1 & $7.875+05$ & $3.894+02$ & $8.108-01$ & $7.871+05$ \\
\hline 1.5 & $2.813+c 5$ & $3.642+02$ & $1.42 \varepsilon+C C$ & $2.810+C 5$ \\
\hline 2 & $1.328+05$ & $3.37 t+C 2$ & 1.984400 & $1.325+05$ \\
\hline 3 & $4.484+04$ & $2.877+02$ & $2.986+00$ & $4.455 \div 04$ \\
\hline 4 & $2.038+04$ & $2.443+02$ & $3.90 \varepsilon+00$ & $2.013+04$ \\
\hline 5 & $1.095+04$ & $2 . C 75+02$ & $4.746+C C$ & $1.074+04$ \\
\hline 6 & $6.562+C 3$ & $1.772+02$ & $5.502+00$ & $6.380+d_{3}$ \\
\hline$\varepsilon$ & $2.520+04$ & 1. $\equiv 27+02$ & $6.793+00$ & $2.507+C 4$ \\
\hline 10 & $1.277+04$ & 1. $c 3 \epsilon+C 2$ & $7.812+00$ & $1.366+04$ \\
\hline 15 & $4.460+03$ & $t .4 t 1+01$ & $5.497+00$ & $4.386+03$ \\
\hline 20 & $1.974+03$ & $4 \cdot 473+01$ & $1.052+0 i$ & $1.919+03$ \\
\hline 30 & $\epsilon .20 \varepsilon+02$ & $2.451+01$ & $1.160+01$ & $5.847+02$ \\
\hline 40 & $2.759+02$ & $1 \cdot 523+c 1$ & $1.204+01$ & $2.486+C 2$ \\
\hline 50 & $1.500+c 2$ & 1. $c 33+01$ & $1.218+01$ & $1.275+02$ \\
\hline 60 & $9.340+01$ & $7.456+C C$ & $1.217+01$ & $7.377+01$ \\
\hline 80 & $4.740 \div 01$ & $4.35 C+0 C$ & $1.154+01$ & $3.107+01$ \\
\hline 100 & $3.040+01$ & $2.883+0 C$ & $1.161+01$ & $1.590+\mathrm{Cl}$ \\
\hline 150 & $1.679+01$ & $1.318+0 \mathrm{C}$ & $1.074+01$ & $4.73 c+c 0$ \\
\hline 200 & $1.272+01$ & $7.482-01$ & $5.548+00$ & $2.027+00$ \\
\hline 300 & $9.654+00$ & $3.348-01$ & 2. $734+00$ & $6.248-01$ \\
\hline
\end{tabular}

\begin{tabular}{|c|c|c|c|c|}
\hline $\begin{aligned} z= \\
\text { ENERGY }\end{aligned}$ & IFON & $\mathrm{COH}$ & $1 \mathrm{NCOH}$. & PHOTC \\
\hline KEV & TOTAL & SCATT. & SCATT. & EFFECT \\
\hline 1 & $8.647+05$ & $4 \cdot 232+c 2$ & $7.43 C-C 1$ & $8.643+05$ \\
\hline 1.5 & $3.2 C 7+05$ & $3 .<7 t+02$ & $1.332+0 \mathrm{C}$ & $3.203+c 5$ \\
\hline 2 & $1.545+05$ & $3.655+02$ & $1.883+0 \mathrm{C}$ & $1.541+05$ \\
\hline 3 & $5.219+04$ & $3.170+c 2$ & 2. $508+00$ & $5.287+04$ \\
\hline 4 & $2.435+04$ & $2.7 C 5+02$ & $3.860+00$ & $2.408+c 4$ \\
\hline 5 & $1.311+C 4$ & $2.3 c \varepsilon+02$ & $4.722+00$ & $1.288+04$ \\
\hline 6 & $7.849+03$ & $1.573+02$ & $5.503+c 0$ & $7.646+03$ \\
\hline 8 & $2.861+04$ & $1.478+02$ & $6.845+C \mathrm{C}$ & $2.845+c 4$ \\
\hline 10 & $1.603+04$ & $1.151+02$ & $7.886+00$ & $1.591+c 4$ \\
\hline 15 & $5.3<7+03$ & $7.125+01$ & $5.6 S 9+00$ & $5.266+03$ \\
\hline 20 & $2.38 \varepsilon+03$ & $4.033+C 1$ & $1.080+01$ & $2.327+03$ \\
\hline 30 & $7.495+02$ & $2.718+C 1$ & $1.191+01$ & $7.1 \mathrm{C} 8+02$ \\
\hline 40 & $3.3 \mathrm{CO}+02$ & $1.6 \subseteq 5+01$ & $1.241+01$ & $3.007+02$ \\
\hline 50 & $1.772+C 2$ & $1.152+01$ & $1.25 \varepsilon+C 1$ & $1.531+C 2$ \\
\hline 60 & $1.085+02$ & $8.322+0 C$ & $1.25 \varepsilon+01$ & $8.795+01$ \\
\hline 80 & $5.384+01$ & $4.9 \mathrm{C} 5+\mathrm{CC}$ & $1.237+01$ & $3.657+01$ \\
\hline $10 \mathrm{C}$ & $3.372+01$ & $3.22 t+O C$ & $1.204+01$ & $1.852+01$ \\
\hline 150 & $1.805+01$ & $1.477+0 \mathrm{C}$ & $1.115+01$ & $5.420+00$ \\
\hline $2 \mathrm{CC}$ & $1.346+C 1$ & $8.4 \mathrm{CO}-\mathrm{Cl}$ & $1.033+01$ & $2.205+C C$ \\
\hline 300 & 1. $C 15+01$ & $3.758-01$ & $5.077+00$ & $7.021-\mathrm{Cl}$ \\
\hline
\end{tabular}

$\begin{array}{rc}\text { ENERGY } & \\ \text { KFV } & \text { TCTAL } \\ 400 & 8.322+00 \\ 500 & 7.459+0 C \\ 600 & 6.834+00 \\ 800 & 5.547+00 \\ 1000 & 5.323+00 \\ 1500 & 4.339+00 \\ 2000 & 3.788+00 \\ 3000 & 3.2 C 8+00 \\ 4000 & 2.926+00 \\ 5000 & 2.771+0 C \\ 6000 & 2.659+0 C \\ 8000 & 2.581+C 0 \\ 10000 & 2.612+00 \\ 15000 & 2.656+0 C \\ 20000 & 2.799+C 0 \\ 30000 & 3.0 C 2+00 \\ 40000 & 3.155+0 C \\ 50000 & 3.309+00 \\ 60000 & 3.402+00 \\ 80000 & 3.575+0 C \\ 100000 & 3.732+06\end{array}$

ENERGY

KEV

$$
\begin{aligned}
& 40 \\
& 50 \\
& 60
\end{aligned}
$$

600
800

800
1000
150

$1500 \quad 5.541+00$

$2000 \quad 3.946+00$

$3000 \quad 3.351+C C$

$5000 \quad 2.510 .00$

$6000 \quad 2.790+0 \mathrm{C}$

$8000 \quad 2.728+C C$

$100 C 0 \quad 2.770+C C$

$15000 \quad 2.829+0 \mathrm{C}$

$20000 \quad 2.951+C C$

$30000 \quad 3.215+00$

$40000 \quad 3.384+0 \mathrm{C}$

$50000 \quad 3.553+C 0$

$80000 \quad 3.843+C C$

100000
$4000 \quad 3.066+0 C$

$6 \mathrm{COOC} \quad 3.654+0 \mathrm{C}$

$\mathrm{COH}$
$\mathrm{SCATT}$
$1.884-01$
$1.203-01$
$8.320-02$
$4.640-02$
$2.930-02$
$1.314-02$
$7.425-03$
$3.315-03$
$1.869-03$
$1.198-03$
$8.327-04$
$4.689-04$
$3.003-04$
$1.336-04$
$7.519-05$
$3.347-05$
$1.873-05$
$1.187-05$
$8.161-06$
$4.478-06$
$2.795-06$

7. $258+C 0$

$7.150+C \mathrm{C}$

6. $660+00$

$5.85 \varepsilon+C C$

$5.270+C C$

$4.285+0 C$

$3.65 t+C C$

2. $\varepsilon 76+C C$

$2.4 \mathrm{CC}+0 \mathrm{C}$
$2.071+00$

$1.830+00$

1. $457+C C$

$1.274+C C$

$9.427-C 1$

7. $561-C 1$

$5.499-01$

3. $641-C 1$

$3.135-01$

2. $470-01$

$2.045-C 1$
PHOTO

EFFEC T

$2.762-01$

$6-C$

$.20 t-C^{2}$

$2.367-\mathrm{C} 2$

1. $143-\mathrm{C} 2$

$6.823-03$

$3.7 \in 3-C^{3}$

$2.56 \varepsilon-C 3$

$1.909-0$

$1.534-C$
$1.086-C$

$8.312-04$

5. $412-04$

$3.592+\mathrm{C} 4$

$2.595-\mathrm{C} 4$

$1.917-\mathrm{C} 4$

$1.514-C^{4}$

1. $248-C 4$

S. $2 C t-C 5$

$7.27 \mathrm{C}-\mathrm{C} 5$
PAIP
PRGO.

2. SEt-CZ

$1.171-\mathrm{Cl}$

$3.251-C 1$
$5.223-C 1$

t. $5 \in 6-c 1$

8. $264+C 1$

1. $C 83+C C$

$1.337+C 0$

$1.713+C C$

$2.042+C C$

$2.718+00$

2. $545+C C$

3. $088+C C$

$3.527+C$

$\begin{array}{cc}\text { PHCTO } & \text { PAIR } \\ E F F E C T & \text { PROD. } \\ 3.1 C E-C 1 & \\ 1.682-01 & \\ 1.032-C 1 & \\ 4.5 C 7-C 2 & \\ 2.828-C 2 & \\ 1.364-C 2 & 3.257-C 2 \\ 8.135-03 & 1.271-C 1 \\ 4.481-03 & 3.51 C+C 1 \\ 3 . C 55-C 2 & 5.05 C-01 \\ 2.27 C-C 3 & 7.520-C 1 \\ 1.823-C 2 & 8.521-C 1 \\ 1.250-C 3 & 1.169+C C \\ 5.859-C 4 & 1.443+C C \\ 6.419-C 4 & 1.849+C C \\ 4.734-C 4 & 2.2 C 4+C C \\ 3 . C 82-04 & 2.643+0 C \\ 2.273-C 4 & 2.53 C+C C \\ 1.795-C 4 & 3.174+C C \\ 1.490-04 & 3.328+C O \\ 1 . C 52-C 4 & 3.586+O C \\ 8.62 C-C 5 & 3.8 C C+C C\end{array}$

$\begin{array}{rc}\begin{array}{c}\text { EAERGY } \\ \text { KEV }\end{array} & \text { TCTAL } \\ 400 & 9.094+00 \\ 5 C 0 & 8.119+0 C \\ 600 & 7.422+00 \\ 800 & 6.444+C C \\ 1000 & 5.763+00 \\ 150 C & 4.656+00 \\ 2000 & 4.1 C 6+00 \\ 3000 & 3.496+0 C \\ 4000 & 3.207+0 C \\ 5000 & 3.052+0 C \\ 6000 & 2.942+0 C \\ 8000 & 2.878+C C \\ 10000 & 2.931+0 C \\ 15000 & 3.007+00 \\ 20000 & 3.187+0 C \\ 30000 & 3.439+00 \\ 4 C 000 & 3.624+00 \\ 50000 & 3.807+0 C \\ 60000 & 3.918+00 \\ 80000 & 4.123+00 \\ 100000 & 4.307+C 0\end{array}$

COH
SCATT
$2.372-01$
$1.516-01$
$1.049-01$
$5.850-02$
$3.700-02$
$1.660-02$
$5.379-03$
$4.188-C 3$
$2.362-03$
$1.514-03$
$1.052-03$
$5.925-04$
$3.795-04$
$1.688-04$
$5.502-05$
$4.230-05$
$2.368-05$
$1.502-05$
$1.033-05$
$5.674-06$
$3.544-06$

INCOH

SCATT .

PHOT

EFFECT

$3.77 \mathrm{C}-\mathrm{CI}$

$7.7 \in 2+C C$

$7.15 \mathrm{C}+00$

$6.325+0 C$

$5.691+00$

$4.627+C 0$

$3.945+C 0$

2. $1 \mathrm{C} t+C \mathrm{C}$

2. $552+\mathrm{CO}$

$2.237+C O$

1. $977+C C$

1. $617+C D$

$1.377+C C$

1. $01 \varepsilon+00$

$8.1 \in 7-C 1$

$5.935-C 1$

$4.715-C 1$

3. $933-01$

$3.386-C 1$

$2.668-C 1$

2. $214-\mathrm{C} 1$

26

t. $083-02$

3. $527-02$

1. $7 \mathrm{CC}-\mathrm{C}_{2}$

1. $\mathrm{C}_{13}-\mathrm{C} 2$

E. $5 t t-C^{2}$

$3.757-C 3$

$2.2<2-C$

$2.265-03$

1.602-03

$1.224-03$

$7.97 \mathrm{C}-\mathrm{C} 4$

$5.87 t-04$

$2.82 \mathrm{C}-\mathrm{C}$

2. $227-04$

1. $83 t-C 4$

$1.353-C_{4}$
$1.068-C_{4}$

2. $533-\mathrm{C2}$

$3.759-C 1$

t. $c 53-c 1$

$\varepsilon .1 \mathrm{Cb}-\mathrm{Cl}$

$1.259+C C$

$1.553+C C$

1. $588+0 C$

2. $3 \in 5+00$

$2.844+C O$

$3.414+O C$

$3.57 s+C C$

$3.85 t+C C$

4. $C 85+0 \mathrm{C}$
$\mathrm{COH}$
SCATT.
$2.657-01$
$1.698-01$
$1.175-01$
$6.550-02$
$4.150-02$
$1.862-02$
$1.052-02$
$4.699-03$
$2.649-03$
$1.658-03$
$1.180-03$
$6.648-04$
$4.258-04$
$1.894-04$
$1.066-04$
$4.746-05$
$2.658-05$
$1.686-05$
$1.160-05$
$6.375-06$
$3.981-0$.

PHOTO

EFFECT

$\begin{array}{ll}8.792+C O & 4.291-01 \\ 8 . C 48+C C & 2.343-01\end{array}$

$7.455+0 C \quad 1.451-C 1$

$6.55 s+C C \quad 7.024-02$

$5.901+C O \quad 4.11 c-02$

$4.758+C O \quad 1.984-C 2$

$4.095+C C$

i. $182-C^{2}$

$t .475-c 3$

$2.688+O C$

$2.320+00$

$4.41 \varepsilon-C 3$

$3.284-C 3$

$2.050+C C$

$1.427+C \mathrm{CO}$

$1.8 \varepsilon 4-C$

$1.424-C$ ?

$1.056+0 \mathrm{C}$

$2.46 \mathrm{C}-\mathrm{Cl}$

5.265-04

$6.830-C_{4}$

$6.159-C 1$
$4.890-C 1$

4.444-C

$3.27 t-C_{4}$

4.078-01

$2.586-C_{4}$

$3.512-C 1$

$2.132-C_{4}$

$\begin{array}{ll}2.767-C 1 & 1.571-C 4 \\ 2.295-C 1 & 1.24 C-C 4\end{array}$

PAIR

PRCD.

3. $22 \varepsilon-c 2$

$1.484=01$

4. $C 91-0$

8. $715-C 1$

1. $C 33+C$

$1.351+O C$

$1.6 t t+C C$

$2.132+00$

$2.54 \mathrm{C}+\mathrm{CC}$

$3 . C 53+C C$

$3.382+0 C$

$3.662+00$

$3.83 \varepsilon+C C$

$4.135+00$ 
S E C T I G A

C C M P AR I SC N

ENDF/E IN EARNS/ATCM

$Z=25$ CCPPER

\begin{tabular}{|c|c|c|c|c|}
\hline VEREY & & $\mathrm{COH}$ & I NCCH. & PHOTC \\
\hline KEV & TOTAL & SCATT. & SCATT. & EFFECT \\
\hline 1 & $1.324+06$ & $5 . \equiv 54+02$ & t. C72-01 & $1.323+C 6$ \\
\hline 1.5 & $5.324+05$ & $5.106+02$ & $1.134+00$ & $5.319+05$ \\
\hline 2 & $2.47 c+05$ & $4.821+02$ & $1.672+0 \mathrm{C}$ & $2.474+05$ \\
\hline 2 & $\varepsilon .30 I+C 4$ & $4.227+02$ & $2.726+c 0$ & $8 \cdot 258+04$ \\
\hline 4 & $3.778+c 4$ & 3. $t \in 1+02$ & $3.725+C C$ & $3.741+04$ \\
\hline 5 & 2. $041+04$ & $3.151+02$ & $4.643+0 \mathrm{C}$ & $2.009+04$ \\
\hline$t$ & 1.230 & $2.713+02$ & $=.48 t+0 C$ & $1.203+0$ \\
\hline 8 & $.515+c 3$ & 2. $C 40+02$ & t. $96 c+00$ & $5.3 C 5+0$ \\
\hline $1 C$ & $2+314+04$ & $1.582+C 2$ & $8.203+00$ & $2.2 \div 7+0$ \\
\hline 15 & $7.8 \div 1+C 3$ & $5.585+01$ & $1.034+01$ & $7.785+C$ \\
\hline 20 & $? .574+C 3$ & $6.589+01$ & $1.165+01$ & $3.497+0$ \\
\hline 30 & $1.141+03$ & $3.670+01$ & $1.305+C 1$ & $1.091+0$ \\
\hline 40 & $=.05:+02$ & $2.316+01$ & $1.36 t+01$ & $4.683+0$ \\
\hline 50 & $2.707+02$ & 1. $5.85+C 1$ & $1.398+01$ & $2.410+c$ \\
\hline $6 C$ & $1.650+02$ & $1.0150+01$ & $1.391+01$ & $1.396+0$ \\
\hline$A C$ & $7.521+01$ & $6.751+C C$ & $1.37 c+01$ & $5.872+0$ \\
\hline In & 4. $782+01$ & 4. $484+00$ & $1.335+01$ & $2.998+0$ \\
\hline 150 & $2.335+c 1$ & 2. $C E G+O C$ & $1.239+01$ & $8.892+$ \\
\hline 200 & 1.64 & 1. $178+O C$ & $1.150+01$ & $3.793+0$ \\
\hline $30 \mathrm{C}$ & $1.181+01$ & $=.25 C-01$ & $1.011+01$ & $1.169+0$ \\
\hline
\end{tabular}

\begin{tabular}{|c|c|c|c|c|}
\hline ENERGY & & $\mathrm{COH}$ & INCCH. & PHCTC \\
\hline KFV & TOTAL & SCATT. & SCATT. & EFFECT \\
\hline 1 & $1.733+05$ & $5.720+C 2$ & $6.515-01$ & $1.728+C 5$ \\
\hline 1.5 & $5.70 P+C 5$ & $5.44 E+C 2$. & $1.27 t+c c$ & $5.703+05$ \\
\hline 2 & $2 \cdot+t 4+C 5$ & $5.137+02$ & $1.934+00$ & $2.658+c 5$ \\
\hline 3 & C. $045+C 4$ & $4.5 C 4+02$ & $2 \cdot 8+3+0 C$ & c. $0 \mathrm{Cr}+\mathrm{C}^{4}$ \\
\hline 4 & $4.1<1+C 4$ & $3.918+02$ & 2. $834+00$ & $4.151+04$ \\
\hline 5 & C. ${ }^{2} \mathrm{C} 5+\mathrm{C} 4$ & 3. $\geqslant 92+02$ & $4.743+00$ & $2.271+04$ \\
\hline$t$ & $1.415+04$ & $2.936+02$ & $5.585+00$ & $1.385+04$ \\
\hline a & $\epsilon .54 c+0 x$ & $2.2 i<+C 2$ & $7.072+c c$ & $0.32 c+03$ \\
\hline 10 & L. $A 4 x+C 4$ & $1.725+C 2$ & $8.323+00$ & $2.625+04$ \\
\hline 15 & $C .114+C 3$ & 1. $c 44+02$ & $1.054+01$ & $8.500+C 3$ \\
\hline 20 & $4.140 \div 03$ & $7.1 \in c+C 1$ & $1.1 \leqslant 1+01$ & $4.056+c 3$ \\
\hline 30 & $1.220+0^{2}$ & 4.C $11 * 01$ & $1.33 c+c 1$ & $1.266+c 3$ \\
\hline 40 & 5. $815+C 2$ & $2.545+C 1$ & $1.404+01$ & $5.420+12$ \\
\hline $5 \mathrm{C}$ & $2.100+c 2$ & $1.748+01$ & $1.42 c+01$ & $\angle .7 \& 2+c i 2$ \\
\hline$t C$ & $1 . A 7 \varepsilon+C 2$ & $1.271+01$ & $1 \cdot 4.32+01$ & $=.607+C 2$ \\
\hline 80 & $8.8 \zeta 8+C 1$ & $7.51 \mathrm{C}+\mathrm{CC}$ & $1.413+01$ & $6.734+C 1$ \\
\hline 100 & $=.3 \mathrm{C} 4+C 1$ & 4. $5 \in 7+00$ & $1.37 \varepsilon+C 1$ & $3.42^{\circ}+c 1$ \\
\hline 150 & $2.524+01$ & $2.2 c 5+C C$ & $1.281+01$ & $1 . c 14+C 1$ \\
\hline 200 & $1.752+\mathrm{C} 1$ & $1.31 c+00$ & $1.189+01$ & $4.22<4+C 0$ \\
\hline 300 & 1. 23 $8+C 1$ & $5 . \varepsilon \varepsilon c-01$ & $1.04 t+01$ & $1.340+00$ \\
\hline
\end{tabular}

$\begin{array}{rcc}\begin{array}{c}\text { ENEREY } \\ \text { KEV }\end{array} & \text { TCTAL } & \text { COH } \\ 400 & 9.920+00 & 2.97 T-01 \\ 500 & 8.805+00 & 1.903-01 \\ 600 & 8.025+00 & 1.318-01 \\ 800 & 6.948+00 & 7.350-02 \\ 1000 & 6.2 C 5+00 & 4.650-02 \\ 1500 & 5.054+00 & 2.086-02 \\ 2000 & 4.426+00 & 1.179-02 \\ 3000 & 3.788+C C & 5.266-03 \\ 4000 & 3.495+00 & 2.969-03 \\ 5000 & 3.343+C 0 & 1.903-03 \\ 6000 & 3.235+00 & 1.323-03 \\ 8000 & 3.186+00 & 7.451-04 \\ 10000 & 3.264+00 & 4.772-04 \\ 15000 & 3.376+C C & 2.123-04 \\ 20000 & 3.595+0 C & 1.195-04 \\ 30000 & 3.907+0 C & 5.319-05 \\ 40000 & 4.127+00 & 2.981-05 \\ 50000 & 4.341+0 C & 1.991-05 \\ 60000 & 4.470+0 C & 1.301-05 \\ 80000 & 4.709+C 0 & 7.154-06 \\ 100000 & 4.922+00 & 4.468-06\end{array}$

ENERG

400

400

600

800

1000

1500

2000

3000

4000

5000
6000

8000

10000

15000

30000

40000

50000

50000
60000

8000

100000
TCTAL SCATT.

$\begin{array}{ll}1.035+01 & 3.313-01 \\ 9.159+0 C & 2.118-01\end{array}$

$\begin{array}{ll}\$ .159+0 C & 2.118-01 \\ 8.335+00 & 1.467-01\end{array}$

$7.207+00 \quad 8.190-02$

$6.431+c 0$

$5.236+C C$

$4.588+00$

$3.937+C 0$

$3.642+00$
$3.452+00$

$3.385+00$

$3.346+C C$

$3.435+c 0$

$3.567+00$
$3.807+00$

$4.148+C C$

$4.386+C 0$

$4.616+C 0$

$4.756+C C$

$5.012+00$

$5.24 \mathrm{C}+0 \mathrm{C}$

5. 190-02

2. 329-02

1. 316-02

5.879-03

2. 315-03

$2.125-03$
$1.477-03$

8. 318-04

5. $328-04$

2. $370-04$

1. 334-04

$5.939-05$

$2.112-05$

$1.453-05$

$7.996-06$

$4.954-06$

$\begin{array}{cc}\text { ENERGY } & \\ \text { KEV } & \text { TCTAL } \\ 40 C & 1.078+C 1 \\ 500 & 9.518+00 \\ 600 & 8.650+C C \\ 800 & 7.46 C+00 \\ 1000 & 6.661+00 \\ 150 C & 5.42 C+0 C \\ 2000 & 4.753+0 C \\ 3000 & 4.088+00 \\ 4000 & 3.791+00 \\ 5000 & 3.644+0 C \\ 6000 & 3.539+00 \\ 8000 & 3.5 C 9+C C \\ 1000 C & 3.611+0 C \\ 15000 & 3.763+00 \\ 20000 & 4.025+C C \\ 30000 & 4.355+00 \\ 4000 C & 4.652+0 C \\ 50000 & 4.900+00 \\ 60000 & 5.050+0 C \\ 80000 & 5.324+0 C \\ 100000 & 5.567+0 C\end{array}$

SCATT

$3.710-01$

$2.374-01$

$1.643-01$

$9.180-02$

5.820-02

i. $612-02$

$1.476-02$

$6.594-03$

3.718-03

2. $383-03$

$1.657-03$

$9.331-04$

$5.976-04$

z. $658-04$

$1.49 t-04$

$6.661-05$

$3.736-05$

2.371-05

$1.631-05$

$8.979-06$
$5.609-06$

\section{0}

ENEREY

$$
\begin{aligned}
& \text { KEV } \\
& 400
\end{aligned}
$$

$\begin{array}{ll}400 & 1.124+C I\end{array}$

SCC $8.971+0 C$

$800 \quad 7.728+00$

$\begin{array}{r}8000 \quad \\ 1000\end{array} \quad .888+00$

$\begin{array}{ll}1000 & 6.888+00 \\ 1500 & 5.6 C 3+00\end{array}$

$2000 \quad 4 . \subseteq 17+00$

$30004.239+C 0$

$4000 \quad 3.941+00$

$5000 \quad 3.757+00$

$6000 \quad 3.655+0 \mathrm{C}$

$8000 \quad 3.674+00$

$10000 \quad 3.790+c 0$

$15000 \quad 3.963+00$

$\begin{aligned} & 15000 \\ & 20000\end{aligned} \quad 4.248+C 0$

30000

$40000 \quad 4.925+0 \mathrm{C}$

$50000 \quad 5.150+00$

$60000 \quad 5.351+0 \mathrm{C}$

$\begin{array}{ll}60000 & 5.351 .00 \\ 80000 & 5.644 .00\end{array}$

$100000 \quad 5.9 \mathrm{C} 3+0 \mathrm{C}$

INCGH.

SCATT.

2.628-01

$1.810-01$

1.017-01

6.440-02

$2.890-02$

$1.634-02$

$7.297-03$

$4.115-03$

2.638-03

$1.834-03$

$1.033-03$

$2.942-04$

1. $t 5 t-04$

$7.373-05$

$4.137-05$

$2.625-05$

$1.806-05$
$5.949-06$

c. $949-06$

$2.343+\mathrm{CO}$

$1.916+C 0$

$1.632+c 0$

$1.207+00$

C. $68 \mathrm{C}-\mathrm{CI}$

$7.040-C 1$

$5.589-C 1$

4. $t \in 2-01$

$4.014-C$
PHOTO
EFFECT

1. $044 \mathrm{Cl} 7.9 \mathrm{CS}-01$

$9.150+C C \quad 4.37 t-C 1$

$8.515+C C \quad 2.744-C 1$

$7.4 S C+C C \quad 1.3 \in 1-C 1$

$6.742+C 0 \quad 8.163-02$

$5.483+O C \quad 3.924-C 2$

$4.68 \mathrm{C}+\mathrm{CO} \quad 2.333-\mathrm{C2}$

$3.681+C C \quad 1.271-C 2$

$3 . C 72+0 C \quad 8.657-03$

$2.651+C 0$

$3.162-C 1$
$2.624-01$

$8.657-03$
$6.427-03$

$5.152-0$

$3.635-c^{3}$

$2.772-C 3$

$1.803-03$

$1.328-03$

$8 \cdot t 3 c-04$

E. $357-c 4$

$4.131-C_{4}$

$3.043-c 4$

$2.4 \mathrm{Cl} 1-\mathrm{C}$
PAIR

PROD.

$4.138-C 2$

$1.598-\mathrm{Cl}$

$4.393-C 1$
$7.031-C 1$

c. $345-C 1$

$1.1 C 7+00$

$1.447+C C$

$1.783+C C$

$2.281+C C$

$2.717+C C$

$3.2 t G+C C$
$3 . t 2 C+C C$

$3.918+00$

$4.1 \mathrm{C} 7+\mathrm{CO}$

$4.423+C C$

$4.684+0 C$

PAIR

PRCE.

$4.472-C_{2}$

$1.718-C 1$

$4.7 \mathrm{CS}-\mathrm{Cl}$

$7.526-C 1$
$5.597-C I$

$1.184+C C$

$1.546+C C$

1. $5 \mathrm{C} 4+\mathrm{CC}$

$2.434+0 C$

$2.895+C C$
$3.487+00$

3. $8 \in 1+00$

$4 \cdot 179+0 C$

$4.379+C C$

4. $716+C O$

4. $c \subseteq 4+C C$

PAIR

$4.821-C 2$

1. $844-\mathrm{Cl}$

5.037-C1

$8.037-\mathrm{Cl}$

1. $067+C 0$

1. $2 \in 3+C C$

1. $64 \varepsilon+00$

$2.028+C C$

3. $C \& \epsilon+00$

$3.712+00$

$4.11 C+C C$

$4.447+C 0$

$4.6 \in C+C C$

5. $C 17+O C$

$5.313+0 \mathrm{C}$

PAIR

PFCO.

$5.1 \& 7-C 2$

$1.974-C 1$

$5.37 t-01$

$1.137+C C$

$1.344+O C$

$1.753+C C$

$2.755+C C$

$3.27 c+C C$

3. $544+C C$

$4.3 \in 5+C C$

$.724+C 0$

$4 . c 4 \mathrm{~s}+\mathrm{CC}$

$5.327+00$
$5.641+00$ 


\begin{tabular}{|c|c|c|c|c|}
\hline NERGY & & $\mathrm{COH}$ & I NCOH. & PHOTC \\
\hline KEV & TOTAL & SCATT. & SCATT. & EFFECT \\
\hline 1 & $2.798+C 5$ & $6.882+02$ & $7.057-01$ & $2.791+05$ \\
\hline 1.5 & $7.117+05$ & $6.5] 6+c 2$ & $1.341+00$ & $7.110+05$ \\
\hline 2 & $3 . \subseteq 42+05$ & $6.105+02$ & $1.985+00$ & $3.936+05$ \\
\hline 3 & $1.362+C 5$ & $5.322+02$ & $3.177+00$ & $1.357+05$ \\
\hline 4 & $t .320+c 4$ & $4 \cdot 645+02$ & $4.233+00$ & $6.273+04$ \\
\hline 5 & $3.458+04$ & $4.067+02$ & $5.174+0 \mathrm{C}$ & $3.417+04$ \\
\hline 6 & $2.104+04$ & $3.572+02$ & t. $C 28+00$ & $2.068+04$ \\
\hline 8 & $9.54 t+03$ & $2.775+C 2$ & $7.532+00$ & $9.261+03$ \\
\hline 10 & $5.149+03$ & $2.18 t \div 02$ & $8.810+00$ & $4.922+03$ \\
\hline 15 & $1.219+04$ & $1.323+02$ & $1.117+01$ & $1.205+04$ \\
\hline 20 & $5.737+03$ & $9.034+01$ & $1.271+01$ & $5.634+03$ \\
\hline 30 & $1.883+03$ & $5.111+01$ & $1.440+01$ & $1.818+03$ \\
\hline 40 & $8 \cdot 3 \in 7+02$ & $3.301+01$ & $1.519+01$ & $7.885+02$ \\
\hline 50 & $4.455+C 2$ & $2.302+01$ & $1.552+01$ & $4.069+02$ \\
\hline 60 & $2.681+02$ & $1 . \in \$_{2}+01$ & $1.560+01$ & $2.356+02$ \\
\hline 80 & $1.241+02$ & S. SE $7+00$ & $1.542+01$ & $9.874+01$ \\
\hline 100 & $7.191+01$ & $6.640+00$ & $1.5 \mathrm{Cb}+\mathrm{Cl}$ & $5.021+01$ \\
\hline 150 & $3.193+\mathrm{C} 1$ & $3.055+00$ & $1.404+01$ & $1.480+01$ \\
\hline 200 & $2.113+01$ & $1.774+00$ & $1.304+01$ & $6.311+00$ \\
\hline 300 & $1.425+01$ & $7.990-01$ & $1.149+01$ & $1.968+00$ \\
\hline
\end{tabular}

ENERGY
KEV
400
500
600
800
1000
1500
2000
3000
4000
5000
6000
8000
10000
15000
20000
30000
40000
50000
60000
80000
100000

$Z=34$ SELENIUN

\begin{tabular}{|c|c|c|c|c|}
\hline AERGY & & $\mathrm{COH}$ & INCCH. & PHCTC \\
\hline KEV & TOTAL & SCATT & SCATT. & EFFEC $T$ \\
\hline 1 & $3.211+05$ & $7.31 t+c 2$ & t. $14 s-01$ & $3.204+05$ \\
\hline 1.5 & $7.834+05$ & $6.531+02$ & $1.245+00$ & $7.827+05$ \\
\hline 2 & $4.423+05$ & $6.455+02$ & $1.517+00$ & $4.417+05$ \\
\hline$\underline{2}$ & $1.5 \leqq 8+c 5$ & $5.642+02$ & $3.183+00$ & $1.553+C 5$ \\
\hline 4 & & $4.511+02$ & $4.310+00$ & $7.241+C 4$ \\
\hline 5 & $4.003+04$ & $4.3 \mathrm{Cl} 1+02$ & $5.29 E+00$ & $3.559+C 4$ \\
\hline t & $2.437+C 4$ & $3.785+C 2$ & $6.17 s+00$ & $2.399+04$ \\
\hline 8 & & $2.562+02$ & & $1.072+04$ \\
\hline 10 & $918+03$ & $2.346+C 2$ & $9.075+00$ & $5.674+03$ \\
\hline 15 & $.322+04$ & $1.424+C 2$ & $1.145+01$ & $1.3 \mathrm{C} 7+04$ \\
\hline 20 & & $5.7 C 8+C 1$ & & $6.275+03$ \\
\hline 30 & $.157+03$ & $5.4 \subseteq s+01$ & $1.47 t+01$ & $2.088+03$ \\
\hline $4 \mathrm{C}$ & & $3.560+01$ & $1.558+01$ & $9.210+02$ \\
\hline 50 & & 2.4884 & $1.553+\mathrm{Cl}$ & $4.8 \mathrm{C} 4+\mathrm{C} 2$ \\
\hline$\in 0$ & $2.144+02$ & $1.832+$ & $1.602+01$ & $2.8 \mathrm{CO}+\mathrm{C} 2$ \\
\hline $8 \mathrm{C}$ & & 1. $c \varepsilon$ & $1.585+01$ & $1.183+02$ \\
\hline 100 & $8.31 c+01$ & $7.153+$ & $1.549+01$ & $6.042+01$ \\
\hline 150 & & 3.35 & $1.445+01$ & $1.785+01$ \\
\hline 200 & $2.255+C 1$ & $1.92 t+00$ & $1.343+01$ & $7.596+c 0$ \\
\hline 300 & $1.500+01$ & $8.680-01$ & $1.178+01$ & $2.350+00$ \\
\hline
\end{tabular}

$Z=35 \quad B R C M I N E$

\begin{tabular}{|c|c|c|c|c|}
\hline & & & I NC CH. & PHOTC \\
\hline KEV & TOTAL & SCATT. & SCATT. & EFFEC $T$ \\
\hline 1 & $3.492+05$ & $7.767+C 2$ & $6.000=01$ & $3.484+05$ \\
\hline 1.5 & $1.33 t+05$ & $7.3 t t+C 2$ & $1.232+00$ & $1.329+05$ \\
\hline 2 & $4.847+C 5$ & $6 . C C 5+C 2$ & $1.91 S+C C$ & $4.840+05$ \\
\hline 3 & $1.701+05$ & $5 . c 8 \epsilon+02$ & $3.232+C 0$ & $1.695+05$ \\
\hline 4 & $.5 \in 1+04$ & $5.1 \subseteq t+02$ & $4.414+00$ & $7.908+04$ \\
\hline s & $4.378+c 4$ & $4.545+c 2$ & $5.444+00$ & $4.332+04$ \\
\hline 6 & $2.672+04$ & $4 . C C 5+02$ & $6.351+0 c$ & $2.631+04$ \\
\hline 8 & .216 & $\Xi .1 \leq 1+02$ & +00 & $1.183+04$ \\
\hline 10 & $\epsilon .561$ & $2.510+C 2$ & $5.28 \mathrm{C}+0 \mathrm{C}$ & $6.301+C 3$ \\
\hline 15 & $1.453+04$ & $1.525+02$ & $1.168+01$ & $1.436+C 4$ \\
\hline 20 & . C134 & $1 . c 41+02$ & $1.325+01$ & $6.856+C 3$ \\
\hline 30 & $2.372+03$ & $5.8 \subseteq 9+01$ & $1.51 \mathrm{C}+\mathrm{Cl}$ & $2.257+03$ \\
\hline 40 & 1. $c 7 c+03$ & $3.823+C 1$ & $1.596+C 1$ & $1.016+03$ \\
\hline 50 & 2 & $2.674 t$ & 1.6 & $5.307+c 2$ \\
\hline 60 & .46 & $1.671+$ & $4+01$ & $3.059+02$ \\
\hline 80 & $1.5 a 3+c 2$ & $1.163+01$ & $1.627+01$ & $1.314+C 2$ \\
\hline $1 \mathrm{CO}$ & 9.088 & 7.735 & $1.591+01$ & $6.724+C 1$ \\
\hline 150 & $.843+01$ & $3.612+00$ & 1.48 & \\
\hline $2 \mathrm{CO}$ & $2.441+$ & $2.072+\mathrm{CO}$ & $1.381+01$ & $8.530+00$ \\
\hline 300 & $1.572+01$ & $5.240-01$ & $1.213+01$ & $2.654+00$ \\
\hline
\end{tabular}

$z=3 \in$ KRYPTON

\begin{tabular}{|c|c|c|c|c|}
\hline ENERGY & & & INCCH. & PHOTC \\
\hline KEV & TOTAL & SCATT. & SCATT. & EFFECT \\
\hline & $2.571+C 5$ & $8.234+02$ & $6.454-01$ & $3.963+05$ \\
\hline 1.5 & $1.593+C 5$ & $7.820+02$ & $1.252+0 \mathrm{C}$ & $1.585+C 5$ \\
\hline 2 & $5.3 S 2+C 5$ & $7.337+02$ & $1.985+O C$ & $5.385+05$ \\
\hline 3 & $1.897+05$ & $6.355+02$ & $3.324+0 \mathrm{C}$ & $1.8 \div 1+05$ \\
\hline 4 & E.898+04 & $5.501+02$ & $4.544+C O$ & $8.842+c 4$ \\
\hline 5 & $4.904+C 4$ & $4.8 \mathrm{C} 3+02$ & $5.613+0 \mathrm{C}$ & $4.855+C 4$ \\
\hline$\epsilon$ & $2.009+04$ & $4.232+02$ & $6.545+00$ & $2.956+C 4$ \\
\hline 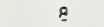 & $1.369+04$ & $3.343+02$ & $8.114+00$ & $1.335+04$ \\
\hline 10 & & $2 \cdot 677+c 2$ & $5.41 c+00$ & $7.132+03$ \\
\hline 15 & $1 . \in 20+04$ & $1.639+02$ & $1.188+01$ & $1.603+04$ \\
\hline 20 & $7.756+03$ & $1.115+02$ & $1.354+01$ & $7.631+03$ \\
\hline 30 & & $6.312+01$ & $1.542+01$ & $2.523+03$ \\
\hline 40 & $1.170+C 3$ & $4.05 C+01$ & $1.632+01$ & $1.112+03$ \\
\hline 50 & $6.263+02$ & 2. $8 \in 1+C 1$ & $1.672+01$ & $5.810+02$ \\
\hline$\in 0$ & $.773+c 2$ & $2.1 C 8+01$ & $1.684+01$ & $3.394+02$ \\
\hline 80 & $1.733+02$ & $1.243+01$ & $1.665+01$ & $1.441+02$ \\
\hline $10 \mathrm{C}$ & $5.855+01$ & $\varepsilon .2 \in \varsigma+00$ & $1.633+01$ & $7.395+01$ \\
\hline & & & $1.526+01$ & $2.209+01$ \\
\hline bo & & $2.214+0 \mathrm{C}$ & $1.420+01$ & 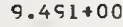 \\
\hline 300 & $1.649+01$ & $5.580-01$ & $1.251+01$ & $. \subsetneq 78+00$ \\
\hline
\end{tabular}

$\begin{array}{ccc}\begin{array}{c}\text { ENEFGY } \\ \text { KEV }\end{array} & \text { TCTAL } & \text { COH } \\ 400 & 1.220+01 & 4.89 T \text { T } \\ 500 & 1.065+01 & 3.130-01 \\ 600 & 5.624+00 & 2.167-01 \\ 800 & 8.261+00 & 1.213-01 \\ 1000 & 7.346+00 & 7.680-02 \\ 1500 & 5.971+00 & 3.447-02 \\ 2000 & 5.247+00 & 1.949-02 \\ 3000 & 4.546+0 C & 8.706-03 \\ 4000 & 4.248+00 & 4.910-03 \\ 50 C 0 & 4.1111+00 & 3.147-03 \\ 6000 & 4.014+00 & 2.188-03 \\ 8000 & 4.015+C C & 1.232-03 \\ 10000 & 4.160+00 & 7.893-04 \\ 15000 & 4.378+C 0 & 3.511-04 \\ 20000 & 4.711+00 & 1.976-04 \\ 30000 & 5.175+0 C & 8.798-05 \\ 40000 & 5.453+00 & 4.940-05 \\ 50000 & 5.755+00 & 3.136-05 \\ 60000 & 5.978+00 & 2.158-05 \\ 80000 & 6.309+00 & 1.189-05 \\ 100000 & 6.6 C 1+0 C & 7.434-06\end{array}$

TCTAL
$1.169+01$

$\mathrm{COH}$
$\mathrm{SCATT}$
$4.510-01$

PHOTO $\begin{array}{llll}1.026+01 & 4.510-01 & 1.035+01 & 8.920-01\end{array}$ $9.251+00$ $7.954+00$ $7.118+00$ $1.995-01$ $1.117-01$ $7.070-02$ $5.787+0 \mathrm{C} \quad 3.173-02$ $5.082+0$ $4.054+00$ $3.953+C C$ $3.843+00$ $8.013-03$ $4.519-03$ $2.856-03$ 2.013-03 $3.973+00$ $1.134-03$ $4.169+00$ $4.477+00$ $7.264-04$ $3.231-04$ $1.819-04$ $.905+00 \quad 8.097-05$ $5.205+00$ $4.544-05$ $.489+00$ $5.660+00$ $5.973+00$ $2.885-05$ $1.985-05$ $1.094-05$ $9.475+00$

$7.726+C C \quad 1.564-C 1$

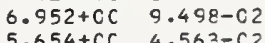
$5.654+C C$

$4.826+C O$

3. $756+C 0$

$3.16 \varepsilon+c 0$

$2.734+C O$

$2.417+C 0$
$1.97 t+C C$

$1.683+00$

$1.244+C C$

9.983-01

7. $260-C 1$

$4.807-C 1$

$4.139-\mathrm{Cl}$

$3.261-C 1$

$2.713=02$

$1.47 t-02$

1. $C 05-\mathrm{C}^{2}$

$7.455-C 3$

$5.974-03$

$4.213-C 3$

$3.213-03$
$2.088-03$

$1.537-C 3$

$5.590-04$

$7.357-04$

$4.781-C_{4}$

$3.521-c 4$
$2.777-04$

$2 \cdot 706-C 1$

SCATT:

PHCTO

$1.0 \mathrm{C} 6+C 1$ 1.CEt+C

$9.760+C O \quad 5.807-C 1$

$9.045+O C \quad 2 . t 2 E-C$

$7.96 C+C C \quad 1.792-C 1$

$7.1 \in 2+C O \quad 1 . C 74-C$ ?

$5.825+C 0$

$5.158-c 2$

$4.972+C O$

3. $512+00$

$3.264+C 0$

$2.817+C C$

$1 . t \in t-C 2$

$1.132-02$

$8.404-c^{3}$

$2.03 t+C O \quad 4.732-C$

$1.734+C 0$

$1.282+C C$

$1.029+00$

$3.617-03$

$2.345-c ?$

$1.73 c-c 3$

$7.48 \mathrm{C}-\mathrm{C} 1$

$4.553-c 1$

$1.124-C$
$8.276-C$

$6.527-C_{4}$

$4.265-C 1 \quad 5.37 t-04$

$3.360-C 1 \quad 3.555-04$

$2.78 \mathrm{E}-\mathrm{Cl}$

$3.122-c_{4}$

ENEREY
KEV

400 TCTAL

$5001.104+01$

$6009.955+0 \mathrm{C}$

$800 \quad 8.525+C C$

$100 \mathrm{C} \quad 7.578+0 \mathrm{C}$

$1500 \quad 6.156+C \mathrm{C}$

$2000 \quad 5.414+00$

$3000 \quad 4.701+00$

$4000 \quad 4.404+00$

5000

6000

8000

10000

15000

20000

30000

$4 \mathrm{CCOO}$

50000

60000

80000

100000

$4.272+0 \mathrm{C}$

$4.189+0 \mathrm{C}$

$4.350+0 C$

$4.5 \subseteq 2+\mathrm{CO}$

$4.951+0 C$

$5.447+0 C$
$5.787+C 0$

$0.108+00$

$6.3 \mathrm{C} 3+0 \mathrm{C}$

$6.654+0$

$t .964+00$

$\mathrm{COH}$
$\mathrm{SCATT}$.
$5.270-01$
$3.370-01$
$2.333-01$
$1.305-01$
$8.270-02$
$3.712-02$
$2.099-02$
$9.376-03$
$5.288-03$
$3.389-03$
$2.356-03$
$1.327-03$
$8.501-04$
$3.782-04$
$2.129-04$
$5.476-05$
$5.322-05$
$3.379-05$
$2.325-05$
$1.282-05$
$8.015-06$

INCOH。

SCATT:

PHOTC

$1.057+C 1 \quad 1.196+C C$

$1.004+C 1 \quad 6.595-C 1$

5. $3 C 9+C C \quad 4.131-C$

$8.150+C C \quad 2.047-01$

$7.372+C C \quad 1.230-C I$

$5.557+C \mathrm{CC} \quad 5.5 \mathrm{C} 4-\mathrm{C2}$

$5.118+00$

$3.507-C^{2}$

$4.02 t+C O 1.505-C 2$

$3.360+c 0$

2. $\mathrm{SCC}+\mathrm{CC}$

$1.255-02$

$2.563+\mathrm{CO} \quad 7.684-03$

$2 . C 9 t+C C \quad 5.412-C 3$

$1.785+C C$

$1.320+C O$

$1.055+C C$

7. $70 \mathrm{C}-\mathrm{Cl}$

$4.124-c^{3}$

$2.678-03$

$1.572-03$

$1.281-C^{3}$

$6.113-C_{1}$

$5.05 S-C 1$
$4.350-C 1$

$4.35 C-C 1$
$3.45 S-C 1$

$5.428-C$

$7.434-c 4$
$6.123-04$

$6.123-04$
$4.508-c 4$

$2.87 \mathrm{C}-\mathrm{Cl}$

$\begin{array}{rrr}\begin{array}{c}\text { EAEFEY } \\ \text { KEV }\end{array} & \text { TCTAL } & \begin{array}{c}\text { COH } \\ \text { SCATT }\end{array} \\ 400 & 1.319+C 1 & 5.630-01 \\ 500 & 1.144+01 & 3.600-01 \\ 600 & 1.029+C 1 & 2.493-01 \\ 800 & 8.800+C C & 1.395-01 \\ 1000 & 7.812+00 & 8.840-02 \\ 1500 & 6.343+0 C & 3.969-02 \\ 2000 & 5.581+00 & 2.244-02 \\ 3000 & 4.859+0 C & 1.002-02 \\ 4000 & 4.562+C 0 & 5.654-03 \\ 5000 & 4.435+C 0 & 3.624-03 \\ 6000 & 4.344+00 & 2.519-03 \\ 8000 & 4.368+00 & 1.419-03 \\ 10000 & 4.544+C 0 & 9.090-04 \\ 15000 & 4.811+C 0 & 4.043-04 \\ 20000 & 5.195+00 & 2.276-04 \\ 30000 & 5.726+C C & 1.013-04 \\ 40000 & 6.088+0 C & 5.691-05 \\ 50000 & 6.429+00 & 3.614-05 \\ 60000 & 6.635+00 & 2.488-05 \\ 80000 & 7.0 C 7+00 & 1.372-05 \\ 100000 & 7.334+00 & 8.579-06\end{array}$$$
3.555-c_{4}
$$

PHOTC

SCATT- EFFECT

$1.128+01 \quad 1.251+C C$

$9.574+C C \quad 4.7 C B C C$

$8.42 t+C O \quad 2.347-C 1$

$7.582+C C \quad 1.416-01$

E. $16 E+C C \quad 6.794-C Z$

$5.264+C 0 \quad 4.034-C \overline{2}$

$4.141+C C \quad 2.185-C 2$

$3.45 t+C C \quad 1.487-02$

$2.983+C C \quad 1.101-C 2$

$2.63 t+C C \quad 8.81 t-03$

$2.15 t+C C \quad 6.20 t-03$

$1.83 t+C C \quad 4.727-C 3$

$1.35 \varepsilon+C C \quad 3 . C E S-C 3$

$1.089+C \mathrm{C} 2.255-03$

$7.520-C 1 \quad 1.467-C 3$

$6.288-C 1 \quad 1.080-C 3$

$5.244-C 1 \quad 8.512-C 4$

$4.51 t-C 1$

8. $512-C 4$
$7.01 C-C 4$

$\begin{array}{ll}3.55 E-C 1 & 5.16 C-C 4 \\ 2.552-C 1 & 4.0 \leq 8-C 4\end{array}$
PAIR

PROO.

$5.569-C_{2}$

$.1 \mathrm{CS}-\mathrm{Cl}$

$0.112-c 1$

$1.2 \mathrm{Cs}+\mathrm{CC}$

$1.429+0 C$

. $\varepsilon \in 1+C C$

$.287+O C$

$3.477+00$

$4.1 \mathrm{El}+\mathrm{CC}$

$.628+C 0$

C $7+\mathrm{CC}$

$5.646+00$

$5.577+00$

$2.25 \mathrm{C}-\mathrm{Cl}$

$.05 \mathrm{C}-\mathrm{Cl}$

$9.675-01$

$1.283+0 C$

1. $c 73+C C$

$2.422+C C$

. $C \subseteq 3+C C$

$3 . t 81+0 C$

$.426+C 0$

5. $2 c s+C c$

$5.551+C C$

$5.573+0 C$

$t .322+C C$

$6.383-\mathrm{C} 2$

$2 \cdot 3 \div 5-c$

1. $026+\mathrm{CO}$

1. $355+0$

$.6 C 4+C C$

2. $5 \in 1+C$

$3.26 \mathrm{~S}+\mathrm{CC}$

3. $88 \mathrm{~S}+\mathrm{C}$

5. $174+C$

5. $598+C$

5. $863+C C$

$\epsilon \cdot 3 C \varepsilon+C$

t. $676+C C$

$F \triangle 1 R$

. $816-C 2$

$.546-C 1$

$6.850-C 1$

$1.437+0 C$

1. $t \subseteq 6+C$

2. $7 \mathrm{C}^{3}+0 \mathrm{CO}$

$3.450+C C$

$4.1 \mathrm{C} 4+\mathrm{CC}$

$.45 E+O C$

$5.5 C 4+C C$

$6.183+C$

7. $C 35+0 C$ 


\begin{tabular}{|c|c|c|c|c|}
\hline ENERGY & & $\mathrm{COH}$ & I NCOH. & PHCTC \\
\hline KEV & TOTAL & SCATT. & SCATT. & EFFECT \\
\hline 1 & $4.561+C 5$ & $8.558+02$ & $\subseteq .64 \subseteq-01$ & $4.552+05$ \\
\hline 1.5 & $1.738+c 5$ & e.c86+02 & $1.620+00$ & $1.730+05$ \\
\hline 2 & $5.13 C+C 5$ & $7.596+02$ & $2.287+00$ & $5.123+05$ \\
\hline 3 & $2.105+05$ & $6.634+02$ & $3.578+C C$ & $2.102+05$ \\
\hline 4 & $9.923+04$ & $5.764+C 2$ & $4.773+C 0$ & $5.865+04$ \\
\hline 5 & $5.478+04$ & $5 . c 35+02$ & $5.844+00$ & $5.427+C 4$ \\
\hline 6 & $3.352+04$ & $4.439+02$ & $6.787+00$ & $3.306+04$ \\
\hline a & $1.530+c 4$ & $3.5=2+02$ & e. $368+00$ & $1.494+04$ \\
\hline 10 & $8.269+03$ & 2. $836+02$ & $9.672+00$ & $7.575+03$ \\
\hline 15 & $2.676+03$ & $1.749+C 2$ & $1.214+01$ & $2.489+03$ \\
\hline 20 & $2.387+03$ & $1.1 \varepsilon S+C 2$ & $1.383+01$ & $8.254+03$ \\
\hline 30 & $2.874+03$ & $6.740+C 1$ & $1.576+01$ & $2.7 s_{1}+\mathrm{C} 3$ \\
\hline 40 & $1.304+03$ & $4.384+01$ & $1.670+01$ & $1.243+03$ \\
\hline 50 & $7.005+c 2$ & 3. $c 78+01$ & $1.712+\mathrm{Cl}$ & $6.526+c 2$ \\
\hline 60 & $4.223+02$ & $2.276+c_{1}$ & $1.72 t+01$ & $3.823+02$ \\
\hline $8 \mathrm{C}$ & $1.932+02$ & $1.341+01$ & $1.711+01$ & $1.627+02$ \\
\hline 100 & $1.052+02$ & $8.5 \geqq 1+00$ & $1.675+01$ & $8.351+01$ \\
\hline $15 \mathrm{C}$ & $4.47 t+01$ & $4.178+0 \mathrm{C}$ & $1.567+\mathrm{Cl}$ & $2.491+01$ \\
\hline 200 & $2.766+C 1$ & $2.3 \div 5+00$ & $1.458+01$ & $1.068+C 1$ \\
\hline 300 & $1.72 \varepsilon+C 1$ & 1. $c e 2+00$ & $1.28 t+01$ & $3 \cdot 346+00$ \\
\hline
\end{tabular}

\begin{tabular}{|c|c|c|c|c|}
\hline ENEREY & & $\mathrm{COH}$ & INCGH. & PHCTC \\
\hline KEV & TOTAL & SCATT. & SCATT. & EFFECT \\
\hline 1 & $5.182+C 5$ & $\varepsilon . \uparrow t 4+02$ & $1.174+0 \mathrm{C}$ & $5.173+05$ \\
\hline 1.5 & $1.550+05$ & $8.419+02$ & $1.917+c 0$ & $1.982 \div 05$ \\
\hline 2 & 3. $520+05$ & $7 . \varepsilon \varepsilon 1+02$ & $2.5 \$ 3+00$ & $3.912+05$ \\
\hline 3 & $2.321+05$ & $6 . E S 3+02$ & $3.864+C 0$ & $2 \cdot 314+C 5$ \\
\hline 4 & $1.103+05$ & $6 . C 16+02$ & $5.032+00$ & $1 . C 97+C 5$ \\
\hline 5 & $6.110+04$ & $5.268+02$ & $6 . \operatorname{cs} 7+00$ & $6.057+04$ \\
\hline$t$ & $3.741+04$ & $4.64 E+02$ & $7.04 t+00$ & $3.694+04$ \\
\hline ? & $1.702+C 4$ & $3.6<7+02$ & $8.64 c+00$ & $1.664+04$ \\
\hline 10 & S. $145+03$ & $2.9 \div 2+02$ & $5.544+00$ & $8.840+03$ \\
\hline 15 & $2.505+c 3$ & $1 . e 61+02$ & $1.241+01$ & $2.711+c 3$ \\
\hline $2 \mathrm{C}$ & $9.080+03$ & $1.26 t+c 2$ & $1.412+01$ & $8.939+03$ \\
\hline 30 & $3.114+C 3$ & $7.161+C 1$ & $1.61 c+01$ & $3.026+03$ \\
\hline 40 & $1.419+03$ & $4 \cdot 645+01$ & $1.707+01$ & $1.355+C 3$ \\
\hline 50 & $7.659+02$ & $3.252+c 1$ & $1.752+\mathrm{C} 1$ & $7.159+02$ \\
\hline $6 \mathrm{C}$ & $4.635+02$ & $2.3 \varsigma \varepsilon+01$ & $1.767+01$ & $4.218+c 2$ \\
\hline 80 & $2.131+c 2$ & $1.413+01$ & $1.753+01$ & $1.814+c 2$ \\
\hline 100 & $1.204+02$ & $5.4 \mathrm{CO}+0 \mathrm{C}$ & $1.717+\mathrm{Cl}$ & $9.386+01$ \\
\hline $15 \mathrm{C}$ & $4.885+01$ & $4.3 E S+O C$ & $1.607+01$ & $2.839+01$ \\
\hline 200 & $2.975+01$ & $2.517+00$ & $1.496+01$ & $1.227+01$ \\
\hline 300 & $1.820+c 1$ & $1.135+00$ & $1.320+01$ & $3.864+00$ \\
\hline
\end{tabular}

$\begin{array}{rrr}\begin{array}{c}\text { ENERGY } \\ \text { KEV }\end{array} & \text { TCTAL } & \begin{array}{c}\text { COH } \\ \text { SCATT. }\end{array} \\ 400 & 1.372+01 & 6.110-01 \\ 500 & 1.185+01 & 3.9 C 0-01 \\ 600 & 1.064+01 & 2.699-01 \\ 800 & 9.078+0 C & 1.515-01 \\ 1000 & 8.051+0 \mathrm{C} & 9.600-02 \\ 150 \mathrm{C} & 6.533+C 0 & 4.311-02 \\ 2000 & 5.752+00 & 2.437-02 \\ 3000 & 5.018+00 & 1.089-02 \\ 4000 & 4.723+C 0 & 6.141-03 \\ 5000 & 4.6 \mathrm{C1}+00 & 3.937-03 \\ 6000 & 4.513+00 & 2.737-03 \\ 8000 & 4.549+C \mathrm{C} & 1.542-03 \\ 10000 & 4.742+00 & 9.874-04 \\ 15000 & 5.035+00 & 4.392-04 \\ 20000 & 5.445+0 \mathrm{C} & 2.472-04 \\ 30000 & 6.012+00 & 1.101-04 \\ 40000 & 6.356+00 & 6.184-05 \\ 50000 & 6.757+0 \mathrm{C} & 3.928-05 \\ 60000 & 6.976+C \mathrm{C} & 2.703-05 \\ 80000 & 7.369+00 & 1.491-05 \\ 100000 & 7.713+C 0 & 9.330-06\end{array}$

ENEREY
KEV
400
500
600
800
1000
1500
2000
3000
4000
5000
6000
8000
10000
15000
20000
30000
40000
50000
60000
80000
$10000 \mathrm{C}$

$\begin{array}{cc}\text { COH } \\ \text { TCTAL } & \begin{array}{c}\text { SCATT } \\ 1.429+01\end{array} \\ 6.400-01 \\ 1.228+01 & 4.090-01 \\ 1.059+01 & 2.830-01 \\ 9.351+0 C & 1.588-01 \\ 8.283+0 C & 1.007-01 \\ 6.719+00 & 4.522-02 \\ 5.520+00 & 2.557-02 \\ 5.177+0 C & 1.142-02 \\ 4.884+C 0 & 6.443-03 \\ 4.768+C 0 & 4.130-03 \\ 4.684+0 C & 2.871-03 \\ 4.734+C 0 & 1.617-03 \\ 4.943+0 C & 1.036-03 \\ 5.262+00 & 4.608-04 \\ 5.700+00 & 2.594-04 \\ 6.3 C 3+00 & 1.155-04 \\ 5.711+00 & 6.489-05 \\ 7.0 \subseteq 3+00 & 4.123-05 \\ 7.324+0 C & 2.838-05 \\ 7.738+00 & 1.566-05 \\ 8.1 C 1+C C & 9.799-06\end{array}$

EA ERCY

KEV

400
500
800

800
1000

1500

2000

3000
4000

5000

6000

800

10000

15000

30000

40000

50000

60000

80000

100000

INCOH.
SCATT.
$1.15 S+C 1$
$1.062+C 1$
$5.838+C C$
$8.655+C C$
$7.753+C C$
$6.340+C O$
$5.411+00$
$4.257+C C$
$3.553+C C$
$3.066+C C$
$2.710+C C$
$2.216+C C$
$1.887+C C$
$1.356+C C$
$1.119+C C$
$8.141-C 1$
$6.463-C 1$
$5.351-C 1$
$4.642-C 1$
$3.657-01$
$3.034-C 1$

PHOTC

EFFEC 7

$1.518+C C$

$8.433-C 1$
$5.314-C 1$

$2.6 \in 6-\mathrm{C} 1$

$1.621-C 1$

$7.773-c 2$

$4.515-02$

2.501-C2

$1.658-02$

$1.257-C 2$

1. CCE-C2

$7.07 t-C 3$

$5.387-C 3$

$3.457-03$

$2.574-C 3$

$1.671-c^{3}$
$1.230-c^{3}$

5.692-0\%

7. $981-\mathrm{C}_{4}$

$5.873-C_{4}$

$4.63 c-c 4$
PAIR

PRCC.

$7.266-\mathrm{C} 2$

2. $7 \mathrm{C} 2-\mathrm{C} 1$

$1.147+00$

$1.518+c 0$

$1.750+C C$

$2.325+C C$

$2.849+00$

$3.635+C C$

$4.323+C 0$

$5.1 \mathrm{C} 6+\mathrm{CC}$

$5.74 \varepsilon+C O$

列

7. $\mathrm{CC} 2+\mathrm{CO}$

$7.41 \mathrm{C}+\mathrm{CC}$
PAIR

SCATT. EFFECT PROD.

$1.190+C 1 \quad 1.752+C C$

$1.090+C 1 \$ .687-01$

$1.010+c 1 \quad 6.06 t-01$

$8.893+C C \quad 2.997-C 1$

$8.0 C 3+C C \quad 1.791-C 1$

$6.511+C C \quad 8.585-02$

$5.557+C C$ 5. C $55-C 2$

$4.372+00 \quad 2.758-c 2$

$3.645+C 0 \quad 1.871-02$

$3.145+C C \quad 1.385-02$

$2.783+C C \quad 1.1 C^{2}-C_{2}$

$2.276+C C$

$1.538+C 0$

$1.433+C C$

$7.788-C 3$

$5.92 t-C 3$

$3.846-C=$

$2.830-C^{3}$

$1.836-C^{2}$

8. $361-C$

$6.638-C 1$

$5.537-C 1$
$4.767-C 1$

$3.75 t-C 1$

1. $C 65-C^{2}$

$\varepsilon .765-c 4$

$6.452-04$

5. Cet-c4
7. $734-\mathrm{C} 2$

$2.864-\mathrm{C} 1$

$1.21 \mathrm{C}+\mathrm{CC}$

1. $E C 1+C C$

$1 . \varepsilon \varepsilon 7+C C$

$2.44 \varepsilon+C C$

$3.825+C C$

$4.547+00$

$5.465+0 \mathrm{C}$

$.045+00$

$6.846+C C$

$7.262+C C$

$7.7 E^{c}+O C$
PHCTO

EFFECT

$1.077+0 C$

$6.782-\mathrm{Cl}$

3.394-01

2.054-01

$5.84 \mathrm{C}-\mathrm{C2}$

$5.83 \varepsilon-C 2$

$3.15 \varepsilon-c$

$2.140-02$

$1.583-\mathrm{C}$

$1.266-C$

$8.896-C_{3}$

$6.767-C^{2}$

$4 \cdot 390-03$

$3.230-\mathrm{C}$

$2 . \operatorname{cs} 5-C^{3}$

$1.542-C$

$1.215-c$

$1.000-C^{2}$

$5.7 \subseteq 5-c_{4}$
PAIR

PROO.

8.220-c2

3. $\mathrm{C} 30-\mathrm{C} 1$

e. $C \& 0-C 1$

$1.274+C O$

$1.6 \varepsilon t+C C$

$2.575+\mathrm{CO}$

$3.151+C C$

4. $018+C C$

$4.777+C C$

$6.866+C C$

$7.1 \varepsilon S+C C$

$7.72 s+C C$

$8.17 t+C C$

4

ENEEGY
KEV
400
500
600
800
1000
1500
2000
3000
400
5000
6000
8000
10000
15000
20000
30000
40000
50000
60000
80000
100000

$400 \quad 1.533+C 1$

$6001.168+01$

$8009.9 C 8+00$

$1000 \quad 8.762+00$

1500 7.CSS+CC

$2000 \quad 6.263+00$

$2000 \quad 5.5 \mathrm{C} 1+00$

$4000 \quad 5.213+C C$

$5.110+C C$

$000 \quad 5.035+00$

$8000 \quad 5.112+0 \mathrm{C}$

$1000 C \quad 5.356+C C$

$15000 \quad 5.731+C 0$

$400007.360+C 0$

$500007.785+00$

$60000 \quad \varepsilon . C 42+C C$

10000
$30000 \quad 6.504+00$

उ. $19 \varepsilon-C$

\section{$\mathrm{COH}$}

SCATT.

4. $570-01$

$3.160-01$
$1.772-01$

$1.124-01$

5.048-02

2.854-02

$1.276-02$

$7.194=03$

$3.206-03$

$1.806-03$

$1.157-03$

$5.146-04$

$1.289-04$

$7.249-05$

4.608-05

3.172-05

1.751-05

$1.056-05$
INCOH.

$1.252+C 1$

$1.147+C$

1. $C 63+C 1$

e. $423+C C$

$6 \cdot \varepsilon 53+c c$

$5.850+C O$

$4.6 C^{2}+C C$

$3.841+00$

$3.315+O C$

$2.930+C C$

$2.35 t+c c$

$2.040+C 0$

$1.505+C$

e. $8 \mathrm{C} 2-\mathrm{Cl}$

t. SeE-Cl

5. $829-C 1$

5.C19-C

$3.954-\mathrm{Cl}$

$3.28 \mathrm{C}-\mathrm{Cl}$
PHOTC

EFFECT

2. $C 96+C C$

$1.16 t+c C$

$7.3 \in 1-C 1$

$3.71 \mathrm{C}-\mathrm{Cl}$

$1.086-C 1$

$6.438-02$

$3.475-C_{2}$

$2.35 t-C 2$

$1.74 \hat{z}-\mathrm{C}$

$1.393-0$

$5.790-C 3$
$7.43 t-C$

$4.823-C$

$3.548-c$

2. $3 \mathrm{C}_{1-03}$

$1 . \in 52-C$

$1.334-03$

1. $C S \varepsilon-c 3$

$8.075-04$

t. $363-04$
PAIR

PROO.

8. 725-C2

$3.2 \mathrm{Cz}-\mathrm{Cl}$

$8.515-01$

$1.341+C C$

2. $0 \varepsilon 8+O C$

$2.7 \mathrm{C}_{4}+\mathrm{CC}$

$3.3 C^{2}+O C$

$4.217+C C$

$6.022+C C$

$7.2 \mathrm{C} 1+\mathrm{OC}$

$7.535+C C$

$8.1 \mathrm{C} 4+\mathrm{CC}$

$\varepsilon .572+C C$
. $C 11+C C$ 


\begin{tabular}{|c|c|c|c|c|}
\hline & & & INC CH. & PHCTC \\
\hline KEV & TOTAL & SCATT. & SCATT. & EFFECT \\
\hline 1 & $7.628+05$ & 1. C $57+03$ & $1.054+00$ & $7.617+05$ \\
\hline 1.5 & $2.773+05$ & 5. $57 t+02$ & $1.825+0 \mathrm{C}$ & $2.763+05$ \\
\hline $\bar{z}$ & $1.355+C 5$ & $9.331+02$ & $2.55 t+c C$ & $1.345+05$ \\
\hline 2 & $3.045+05$ & 8. $\operatorname{cs~} 8+02$ & $3.916+00$ & $3.037+0$ \\
\hline 4 & $.468+05$ & 7. $C 2 t+02$ & $5.166+00$ & $1.461+05$ \\
\hline 5 & $8.220+04$ & 6.1 & 6.3 & \\
\hline t & $5.070+04$ & $5.390+02$ & $7.365+00$ & $5.015+04$ \\
\hline 8 & .32 & & & $+c<$ \\
\hline 10 & . $255+04$ & 3.5 & $1.054 \div 01$ & $223 * 04$ \\
\hline 15 & 4. $C 28+03$ & $2.228+$ & $7+01$ & $2+c_{3}$ \\
\hline 20 & $.193+0$ & $1.521+$ & $1.498+01$ & $1.176+04$ \\
\hline${ }^{2} \mathrm{C}$ & $4.175+03$ & $\varepsilon .582+01$ & $1.710+01$ & $4.072+03$ \\
\hline 40 & $1.919+03$ & 5.5 & 1.8 & $1.845+C 3$ \\
\hline 50 & $1.039+03$ & $3.88 \mathrm{E}$ & $1.869+01$ & $9.816+c 2$ \\
\hline 60 & $6.285+02$ & 2. $E \in 2+C 1$ & $1.888+01$ & $5.810+C$ \\
\hline & 2.8 & 1.6 & 1.8 & $+C^{2} 2$ \\
\hline $10 \mathrm{C}$ & 1.5594 & $1.121+$ & 1. $842+01$ & $1.303+02$ \\
\hline & & & $1.72 c+01$ & $3.547+01$ \\
\hline 200 & $3.616+$ & 2. $596+00$ & $1.611+01$ & $1.7 C 5+01$ \\
\hline 300 & $2.09 \varepsilon+01$ & $1.350+O C$ & 1.427801 & $5.362+00$ \\
\hline
\end{tabular}

$Z=4 \bar{Z}$ MOLYROENUM

\begin{tabular}{|c|c|c|c|c|}
\hline NERGY & & $\mathrm{COH}$ & INCEH. & PHOTO \\
\hline KEV & TOTAL & SCATT. & SCATT. & EFFECT \\
\hline 1 & $8.544+C 5$ & $1.111+03$ & $9.916-01$ & $8.533+c 5$ \\
\hline 1.5 & $3.12 t+05$ & $1.051+03$ & $1.76 t+00$ & $3.116+05$ \\
\hline 2 & $.534+C 5$ & $9.843+02$ & $2.511+00$ & $1.524+05$ \\
\hline 3 & $3.347+05$ & $8.54 t+C_{2}$ & $3.904+00$ & $3.338+05$ \\
\hline 4 & $1.607+C 5$ & $7.400+02$ & $5.183+00$ & $1.600+05$ \\
\hline 5 & 4 & $6.445+02$ & $1+\infty$ & $8.523+04$ \\
\hline 6 & $5.547+04$ & $5 . t \in 7+02$ & $7.43 s+00$ & $5.490+04$ \\
\hline & $5=5$ & $4.5 C t+C$ & & $2.511+04$ \\
\hline 10 & $.389+04$ & $3.693+02$ & $1.104+01$ & $1.351+04$ \\
\hline 15 & $.4 c 9+03$ & $2.359+02$ & $1.358+01$ & $4.250+C 3$ \\
\hline 20 & 2 & 1.6 & & $1.274+C 4$ \\
\hline 30 & $.521+C 3$ & c. $113+c 1$ & $1.74 E+C 1$ & $4.412+03$ \\
\hline 40 & $088+$ & 5.92 & 1. 8 & $10+03$ \\
\hline 50 & 1 & 4.1 & & $1.077+03$ \\
\hline$\in 0$ & +02 & 3.CE & 1.9 & $6.414+02$ \\
\hline 80 & 2 & 1.803 & 1.9 & $2.8 c 5+c 2$ \\
\hline 100 & $7+02$ & 1 & $1.884+C 1$ & \\
\hline $1 \leq 0$ & & & $1.7>>$ & $4.529+C 1$ \\
\hline 200 & 54 & 3.20 & 1.64 & $1.978+01$ \\
\hline & $220+01$ & $5+0 C$ & $1.457+01$ & $6.290+00$ \\
\hline
\end{tabular}

\begin{tabular}{|c|c|c|c|c|}
\hline ENEREY & & & & \\
\hline KEV & TOTAL & SCATT. & SCATT. & EFFECT \\
\hline 1 & c. $37 t+05$ & $1.1 \in 3+03$ & $1.101+00$ & S. $364+05$ \\
\hline 1.5 & $3.482+05$ & 1. $c \subseteq 8+03$ & $1.937+00$ & $3.471+05$ \\
\hline 2 & $1.727+05$ & 1. $C 28+03$ & $2.703+00$ & $1.717+C 5$ \\
\hline 3 & $3.200+05$ & \&. ५ $25+02$ & $4.097+00$ & $3.191+05$ \\
\hline 4 & $1.76 E+C 5$ & $7.74 C+C 2$ & $5.367+00$ & $1.761+05$ \\
\hline 5 & c. $951+C 4$ & $6.745+02$ & $t .533+00$ & $9.783+04$ \\
\hline 6 & $E . C E C+C 4$ & $5.935+02$ & $7.607+00$ & $6.009+04$ \\
\hline$\varepsilon$ & $2.79 t+c 4$ & $4.71 t+c 2$ & c. $48 C+00$ & $2.748+04$ \\
\hline 10 & $1.521+04$ & $3.85 \varepsilon+C 2$ & 1. CSS+01 & $1.481+04$ \\
\hline 15 & $4.559+03$ & $2.487+02$ & $1.365+01$ & $4.697+03$ \\
\hline 20 & $2.225+03$ & $1.7 c t$ & 1.5 & $2.039+c$ \\
\hline 30 & $4.8 \subseteq 8+C 3$ & $5.6 C 5+01$ & $1.777+\mathrm{Cl}$ & $4.784+03$ \\
\hline $4 C$ & 2.26 & $6.1 \div 2+c 1$ & $0+01$ & $2.187+c$ \\
\hline 50 & $1+231+03$ & $4.3 C 3+C 1$ & $4 t+01$ & $1.169+0$ \\
\hline 60 & 7.44 & $\equiv .1 \leq 2+$ & $1.565+01$ & $6.935+02$ \\
\hline 80 & 3.38 & $1.859+$ & $1.961+01$ & $3.0 C 5+c$ \\
\hline 100 & $1.87 t+C 2$ & $1.233+01$ & $1.924+01$ & $1.560+02$ \\
\hline 150 & & $5.725+00$ & & \\
\hline 200 & $.068+0$ & $3.27 t+O C$ & $1.687+01$ & $2.053+0$ \\
\hline 300 & $2.289+01$ & $1.474+00$ & $1.49 \mathrm{C}+01$ & $6.512+00$ \\
\hline
\end{tabular}

\begin{tabular}{|c|c|c|}
\hline \multicolumn{3}{|c|}{$Z=\angle 4$ RUTHENIUM } \\
\hline EAEREY & & $\mathrm{COH}$ \\
\hline KEV & TOTAL & SCATT. \\
\hline 1 & 1. $C \in G+C 6$ & $1.2 \bar{z} t+0 z$ \\
\hline 1.5 & $3.906+05$ & $1.163+02$ \\
\hline 2 & $1.918+05$ & 1. $c 53+c 3$ \\
\hline 3 & $2.387+05$ & S. $5 c \subseteq+02$ \\
\hline 4 & $1.90 \hat{z}+05$ & $\varepsilon .227+02$ \\
\hline 5 & $1.070+05$ & $7.145+02$ \\
\hline 6 & $6.633+04$ & $6.268+02$ \\
\hline 8 & $3.078+04$ & 4. $964+02$ \\
\hline 10 & $1.679+04$ & $4 . C 5 c+C 2$ \\
\hline 15 & $5.47 t+C 3$ & $z \cdot t 2 c+c 2$ \\
\hline 20 & $2.44 t+02$ & $1.808+02$ \\
\hline 30 & $5.28 t+03$ & $1.015+c 2$ \\
\hline 40 & $2.459+03$ & $6.459+01$ \\
\hline 50 & $1.338+C 3$ & $4.4 \subseteq 1+01$ \\
\hline 60 & $8.101+02$ & $3.27 t+C 1$ \\
\hline$\therefore C$ & $3 .+84+02$ & $1.533+01$ \\
\hline 100 & $2.036+02$ & $1.27 c+c 1$ \\
\hline 150 & $7.551+01$ & $5.917+00$ \\
\hline 200 & $4.324+C 1$ & $3.3 \varepsilon C+0 C$ \\
\hline 300 & $2.397+01$ & $1.518+C C$ \\
\hline
\end{tabular}

INCCH. PHCTC

SCATT EFFECT

c. $44 \varepsilon-01 \quad 1.065+06$

$1.748+00 \quad 3.894+05$

$2.560+001.508+05$

$4.010+00 \quad 3.377+05$

$5.305+C C \quad 1.893+C 5$

$6.507+00 \quad 1.063+05$

$7.611+0 \mathrm{C} \quad 6.570+\mathrm{C}^{4}$

$5.449+00 \quad 3.027+04$

$1.103+01 \quad 1.637+04$

$1.387+C 1 \quad 5.199+03$

$\begin{array}{ll}1.578+C 1 & 2.250+03 \\ 1.807+C 1 & 5.167+03\end{array}$

$\begin{array}{ll}1.807+01 & 5.167+03 \\ 1.925+01 & 2.375+03\end{array}$

$1.584+01 \quad 1.273+03$

$2.008+01 \quad 7.573+02$

$2.0 C 1+01 \quad 3.290+02$

$1.965+01 \quad 1.712+02$

$1.845+01 \quad 5.210+01$

$1.725+01 \quad 2.261+01$

$1.527+01 \quad 7.181+00$

$\begin{array}{rc}\begin{array}{c}\text { ENERGY } \\ \text { KEV }\end{array} & \text { TCTAL } \\ 400 & 1.602+01 \\ 500 & 1.358+01 \\ 600 & 1.208+01 \\ 800 & 1.020+01 \\ 1000 & 9.0 C 0+C 0 \\ 1500 & 7.289+00 \\ 2000 & 6.434+00 \\ 3000 & 5.665+00 \\ 4000 & 5.380+0 C \\ 5000 & 5.284+0 C \\ 6000 & 5.214+00 \\ 8000 & 5.3 C 5+00 \\ 10000 & 5.567+00 \\ 15000 & 5.972+00 \\ 20000 & 6.456+00 \\ 30000 & 7.214+C 0 \\ 40000 & 7.655+00 \\ 50000 & 8.142+00 \\ 60000 & 8.412+00 \\ 80000 & 8.853+00 \\ 100000 & 9.313+00\end{array}$

COH
SCATT.
$7.610-01$
$4.870-01$
$3.370-01$
$1.887-01$
$1.197-01$
$5.377-02$
$3.040-02$
$1.358-02$
$7.662-03$
$4.912-03$
$3.415-03$
$1.924-03$
$1.232-03$
$5.482-04$
$3.085-04$
$1.373-04$
$7.722-05$
$4.910-05$
$3.380-05$
$1.867-05$
$1.169-05$

$1.169-05$
INCOH.
SCATT:
$1.283+C 1$
$1.175+C 1$
$1 . C S C+C 1$
$9.55 C+C 0$
$8.633+C C$
$7.024+C 0$
$5.996+C O$
$4.718+C 0$
$3.937+C C$
$3.398+0 C$
$3.003+C 0$
$2.456+C C$
$2 . C 91+0 C$
$1.547+C C$
$1.241+0 C$
$9 . C 22-C 1$
$7.163-01$
$5.975-C 1$
$5.144-C 1$
$4.053-C 1$
$3.363-C 1$

PHCTO

$2.42 t+C C$

$1.340+O C$

$8.380-C 1$

$4.13 t-C 1$

$2.471-\mathrm{Cl}$

$1.183-01$

$7.012-02$
$3.785-C 2$

$2.562-\mathrm{C} 2$

$1.853-c 2$

$1.513-C_{2}$

$1.062-02$

$8.07 c-02$

$5.233-0 \equiv$

$3.848-C 3$

$2.495-C^{3}$
$1.835-C^{3}$

$1.44 t-03$

$1.150-02$

$8.751-\mathrm{C}_{4}$

$6.855-C 4$

(5)

$\begin{array}{cc}\begin{array}{c}\text { EAERGY } \\ \text { KEV }\end{array} & \text { TCTAL } \\ 400 & 1.681+01 \\ 500 & 1.414+01 \\ 600 & 1.251+01 \\ 800 & 1 . C 51+01 \\ 1000 & 9.255+0 C \\ 1500 & 7.487+0 C \\ 2000 & 6.611+00 \\ 3000 & 5.833+00 \\ 4000 & 5.551+C C \\ 5000 & 5.462+00 \\ 6000 & 5.356+00 \\ 8000 & 5.502+C 0 \\ 10000 & 5.783+00 \\ 15000 & 6.217+C C \\ 20000 & 6.771+00 \\ 30000 & 7.530+0 C \\ 40000 & 8.036+C 0 \\ 50000 & 8.5 C 6+C 0 \\ 60000 & 8.789+C 0 \\ 80000 & 9.294+0 C \\ 100000 & 5.733+00\end{array}$

COH
SCATT.

PHOTC

$\begin{array}{lll}8.150-01 & 1.314+C 1 & 2.857+C C\end{array}$

$5.220-01$

$3.610-01$

$2.019-01$

$1.282-01$

$5.758-02$
$3.257-02$

$1.455-02$

$8.208-03$

$5.262-03$

$3.658-03$
$2.061-03$
$1.320-03$

$2.061-03$
$1.320-03$

5. $873-04$

$3.305-04$

$1.471-04$

$8.274-05$

$5.261-05$

$2.001-05$

$1.253-05$

$1.116+C 1$

$9.825+C C$

$8.843+C C$

$7.155+00$

$0.142+00$

$4.033+00$

$3.481+00$

$3.076+00$

$2.516+C O$

$2.142+\mathrm{CO}$

$1.271+00$

9.243-01

$7.3 \geq 8-01$

$6.121-C 1$

$5.27 \mathrm{C}-01$

$4.152-C 1$
$3.445-C 1$

$1.577+C C$
$C .841-C 1$

$4.813-\mathrm{Cl}$

$2.842-\mathrm{C} 1$

$1.360-01$

\&. $C 5 \varepsilon-c 2$

$4.34 t-02$
$2.940-c 2$

$2.171-02$

$1.734-c 2$

$1.217-02$

c. $241-03$

$5.991-C 3$

$4.405-03$
$2.85 t-C^{3}$

$2.85 t-C 3$
$2.10 \mathrm{C}-\mathrm{C} 3$

$1.654-03$

$1.361-03$

$1.0 \mathrm{Cl} 1-\mathrm{C} 3$

$7.884-C 4$

$\begin{array}{cc}\begin{array}{c}\text { ENERGY } \\ \text { KEV }\end{array} & \text { TCTAL } \\ 400 & 1.725+C 1 \\ 500 & 1.452+01 \\ 600 & 1.284+01 \\ 800 & 1.080+01 \\ 1000 & 9.509+00 \\ 1500 & 7.684+00 \\ 2000 & 6.789+00 \\ 3000 & 6.0 C 2+00 \\ 4000 & 5.723+00 \\ 5000 & 5.642+0 C \\ 6000 & 5.581+0 C \\ 8000 & 5.701+00 \\ 10000 & 5.999+00 \\ 15000 & 6.465+00 \\ 20000 & 7.051+0 C \\ 30000 & 7.849+00 \\ 40000 & 8.382+0 C \\ 50000 & 8.876+C C \\ 60000 & 9.173+00 \\ 80000 & 5.7 C 1+C 0 \\ 100000 & 1.016+01\end{array}$

$\mathrm{COH}$

SCATT.

INCOH.

PHOTC

SCATT. EFFECT

$\begin{array}{lll}5.345+01 & 2.977+C C\end{array}$

$3.680-01$

$2.060-01$

$1.306-01$

5.866-02

$3.318-02$

$1.483-02$

$5.362-03$

$5.362-03$
$3.728-03$

$3.728-03$
$2.100-03$

$1.345-03$

$5.084-04$

$3.368-04$
$1.499-04$

$1.499-04$
$8.432-05$

$5.363-05$

3.693-05

$2.040-05$

$1.232+C 1 \quad 1.663+C C$

$1.142+C 1 \quad 1 . C E 2+C C$

$1.00 t+C 1 \quad 5.319-C 1$

$9.053+C C \quad 3.253-C 1$

$7.36 t+C 0 \quad 1.555-C 1$
$6.28 \theta+c 0$

$6.2 \varepsilon 8+C 0 \quad 5.2 C C-C 2$

$4.948+C C \quad 4.57 C-02$

$\begin{array}{ll}4.129+C C & 3.3 t C-C 2 \\ 3.5 t 4+00 & 2.48 C-C\end{array}$

$\begin{array}{ll}3.564+00 & 2.48 C-C 2 \\ 3.150+0 C & 1.980-C 2\end{array}$

$2.57 t+C C \quad 1.38 \varepsilon-C 2$

$2.193+00 \quad 1.054-C 2$

$1.622+00 \quad 6.831-c 3$

$1.301+00 \quad 5.022-03$

$9.464-C_{1} 3.255-C^{3}$

7.513-C1 2.393-C3

$6.267-C 11.885-C 3$

$5.35 t-C 1$ 1.551-CZ

$4.251-C 1 \quad 1.140-C^{3}$

$3.527-C 1 \quad \& .58 C-C 4$

1. $037-C_{1}$

3. $753-c 1$

$.893-C 1$
$1.552+C C$

$2.048+C C$

$2.4 \mathrm{C} 8+00$

$3.11 C+C C$

3. $754+C O$

$5.744+C C$

t. $8 \subseteq S+C C$

$7.628+00$

$8.248+C 0$

. $275+0 C$
c. $8 C 6+C O$

$\begin{array}{cc}\begin{array}{c}\text { ENEFGY } \\ \text { KEV }\end{array} & \text { TCTAL } \\ 400 & 1.7 C 1+C 1 \\ 500 & 1.500+01 \\ 600 & 1.324+01 \\ 800 & 1.110+01 \\ 1000 & 9.761+00 \\ 1500 & 7.882+00 \\ 2000 & 6.957+00 \\ 3000 & 6.172+00 \\ 4000 & 5.898+00 \\ 5000 & 5.824+C C \\ 6000 & 5.768+C 0 \\ 8000 & 5.5 C 3+C C \\ 10000 & 6.220+00 \\ 15000 & 6.717+C C \\ 20000 & 7.335+00 \\ 30000 & 8.173+0 C \\ 40000 & 8.735+00 \\ 50000 & 9.253+C 0 \\ 60000 & 9.564+0 C \\ 80000 & 1.012+01 \\ 100000 & 1.060+C 1\end{array}$

PHOTC

SCATT.

$8.560-01$
$5.480-01$

$3.790-01$

$2.120-01$

$1.344-01$

$6.037-02$
$3.415-02$

$3.415-02$
$1.526-02$

$8.608-03$

$5.518-03$

3.837-03

$2.161-03$
$1.384-03$

$1.384-03$
$6.159-04$

3.466-04

$1.543-04$

$8.680-05$

$5.522-05$

$3.803-05$

$1.317-05$

$1.376+C$

EFFECT

$1261+01-289+0 C$

$1.169+C 1 \quad 1.16 t+O C$

$1.03 C+C 1 \quad 5.513-c 1$

$9.2 E 4+C C \quad 3 . t 2 E-C 1$

$7.538+00 \quad 1.732-C$

$6.435+O C \quad 1.025-C 1$

5. $C E 4+C O \quad 5.538-C 2$

$4.22 t+C O \quad 3.741-C 2$

$3.647+0 C \quad 2.76 C-C 2$

$3.224+00$

2.203-02

$2.636+00 \quad 1.543-C \bar{c}$

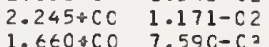

$1.332+C O \quad 5.575-C 3$

$9.685-C 13.615-0 \equiv$

7.685-01 2.657-C3

$6.413-C 1 \quad 2.093-C \equiv$

$5.522-C 1 \quad 1.722-C 3$

4. 351-01 $1.266-C 3$

3. $610-01$ $9.967-c 4$
PAIR

$1+C \subseteq 7-C 1$

1. $C 38+C 0$

$1.626+00$

$2.144+C C$

$3.25 C+C C$

$3.9 \in 2+00$

. $C 4 a+c C$

$7.2 \mathrm{Cl}+\mathrm{OC}$

$7.563+C C$

$\varepsilon \cdot 61 C+C C$

$9.01 C+0 C$
$9.680+00$ 


\begin{tabular}{|c|c|c|c|c|}
\hline \multicolumn{5}{|c|}{$Z=45$ RHOCIUM } \\
\hline EAERGY & & $\mathrm{COH}$ & $1 \mathrm{NCOH}$. & PHCTC \\
\hline KEV & TOTAL & SCATT. & SCATT. & EFFECT \\
\hline 1 & $1.242+06$ & $1.285+03$ & $8.293-01$ & $1.241+06$ \\
\hline 1.5 & $4.313+05$ & $1.22 z+03$ & $1.5 \varsigma \varsigma+00$ & $4.301+05$ \\
\hline 2 & $2 . C 40+05$ & $1.150+03$ & $2.42 C+00$ & $2.028+05$ \\
\hline 3 & $7.130+04$ & 1. $C C_{2}+03$ & $3.912+0 \mathrm{C}$ & $7.030+04$ \\
\hline 4 & $2.07 \epsilon+05$ & $8.67 t+C 2$ & $5.235+0 \mathrm{C}$ & $2.067+05$ \\
\hline 5 & $1.167+05$ & $7.533+C 2$ & $6.467+00$ & $1.160+05$ \\
\hline 6 & $7.240+04$ & $t .55 t+C 2$ & $7.602 * 0 C$ & $7.173+04$ \\
\hline 8 & $3.363+04$ & $5.210+c 2$ & S. $511+00$ & $3.310+04$ \\
\hline 10 & $1.837+04$ & $4.258+C 2$ & $1.124+01$ & $1.793+C 4$ \\
\hline 15 & $6.012+03$ & $2.771+C 2$ & $1.414+01$ & $5.720+03$ \\
\hline $2 \mathrm{C}$ & $2.694+03$ & $1.512+02$ & $1.605+01$ & $2.486+C 3$ \\
\hline 30 & $5.604+03$ & $1.07 C+C 2$ & $1.841+C 1$ & $5.478+03$ \\
\hline 40 & $2.637+03$ & $6.819+01$ & 1. $562+01$ & $2.550+c 3$ \\
\hline 50 & $1.444+03$ & 4. $E S I+C 1$ & $2.022+01$ & $1.377+03$ \\
\hline 60 & $8.776+C 2$ & $3.412+01$ & 2. $c 48+01$ & $8.230+02$ \\
\hline 80 & $4.002 \div 02$ & $2 \cdot C 13+01$ & $2.042+01$ & $3.596+C_{2}$ \\
\hline 100 & $2.210+02$ & $1.330+01$ & $2.006+01$ & $1.876+02$ \\
\hline 150 & $8.233+01$ & $6.125+00$ & 1. $\varepsilon \subseteq C+01$ & $5.73 C+C 1$ \\
\hline 200 & $4.602+01$ & $3.5 \mathrm{CO}+00$ & $1.763+01$ & $2.489+C 1$ \\
\hline $30 \mathrm{C}$ & $2.505+C 1$ & $1.571+C 0$ & $1.557+01$ & $7.912+00$ \\
\hline
\end{tabular}

\begin{tabular}{|c|c|c|c|c|}
\hline \multicolumn{5}{|c|}{$Z=46$ PALLACIUM } \\
\hline ENERGY & & $\mathrm{COH}$ & I NCOH. & PHOTC \\
\hline KEV & TOTAL & SCATT. & SCATT. & EFFEC T \\
\hline 1 & $1.3 \varepsilon<+C 6$ & $1.352+03$ & $7.408-01$ & $1.382+06$ \\
\hline 1.5 & $4.8 \varepsilon 5+05$ & $1.252+03$ & $1.481+0 \mathrm{C}$ & $4.872+C 5$ \\
\hline 2 & $2.337+C 5$ & $1.220+03$ & $2.282+0 \mathrm{C}$ & $2.324+05$ \\
\hline 3 & $\varepsilon .2 \varsigma \varsigma+04$ & 1. $c 6 t+03$ & $3.797+00$ & $8.152+04$ \\
\hline 4 & $2.224+05$ & S. $213+C 2$ & $5.159+00$ & $2.215+05$ \\
\hline 5 & $1.261+05$ & $7.591+02$ & $6.413+00$ & $252+C 5$ \\
\hline 6 & $7.858+C 4$ & $6.970+02$ & $7.581+C O$ & $787+04$ \\
\hline$\varepsilon$ & $3.672+04$ & $5.48 E+C 2$ & $5.663+00$ & $3.616+0$ \\
\hline 10 & $2.012+04$ & $4.47 t+02$ & $1.13 \bar{i}+01$ & $1.966+04$ \\
\hline 15 & $6.592+03$ & $2 . \$ 2 !+02$ & $1.437+01$ & $6.286+03$ \\
\hline 20 & $2 . \subseteq 4 \varepsilon+03$ & $2 . c 22+02$ & $1.636+01$ & $2.729+03$ \\
\hline 30 & $6.205+C 3$ & $1.1 \geq 0+02$ & $1.873+01$ & $6.077+03$ \\
\hline 40 & $2.860+C 3$ & $7.1 \epsilon S+01$ & $1.997+C 1$ & $2.768+C 3$ \\
\hline 50 & $1.550+C 3$ & 4. $\subseteq 17+0 !$ & $2.06 C+01$ & $1.481+03$ \\
\hline 60 & c. $37 t+02$ & $3.569+01$ & $2.087+01$ & $8.811+02$ \\
\hline $8 \mathrm{C}$ & $4.26 t+02$ & $2.1 C t+C 1$ & $2 . c 83+01$ & $3.847+0$ \\
\hline 100 & $2.357+02$ & $1-390+C 1$ & $2.047+01$ & $2.014+C$ \\
\hline 150 & $\varepsilon .788+C 1$ & $6.3 \subseteq 7+O C$ & $1.929+01$ & $6.219+0$ \\
\hline 200 & $4.894+01$ & $3 . t 4 \epsilon+0 C$ & $1.801+01$ & $2.728+01$ \\
\hline 300 & $2.634+01$ & $1 \cdot \in 35+O C$ & $1.593+C 1$ & $8.784 * C$ \\
\hline
\end{tabular}

$Z=47$ SILVER

\begin{tabular}{|c|c|c|c|c|}
\hline$R \in Y$ & & $\mathrm{COH}$ & I NCOH. & PFOTC \\
\hline KEV & TOTAL & SCATT. & SCATT. & EFFECT \\
\hline 1 & $1.423+06$ & $1.4(8+03$ & $8.694-01$ & $1.422+c 6$ \\
\hline 1.5 & $=.213+05$ & $1.344+03$ & $1.625+00$ & $5.199+05$ \\
\hline 2 & $2.560+C 5$ & $1.2 \epsilon \subseteq+C 3$ & $2.359+C C$ & $2.547+05$ \\
\hline 3 & c. $.426+04$ & $1.111+03$ & $3.884+0 \mathrm{C}$ & $9.315+04$ \\
\hline 4 & $2.401+05$ & $9.6 \geq 7+02$ & $5.25 \mathrm{C}+0 \mathrm{C}$ & $2+391+05$ \\
\hline 5 & $1.358+05$ & $8.363+02$ & $6.5 C 5+C C$ & $1.350+05$ \\
\hline 6 & 8.46 & $7.3 C 8+02$ & $7.673+00$ & $1+04$ \\
\hline 8 & $3.561+04$ & $5.743+02$ & $9.768+00$ & 3. $903+04$ \\
\hline 10 & $2.17 t+04$ & 4. $t .81+C 2$ & $1.152+01$ & $2.128 \mapsto 04$ \\
\hline 15 & $7.190+03$ & 2. $C E E+C 2$ & $1.460+01$ & $6.869+03$ \\
\hline 20 & $3.241+03$ & $2.1 \equiv 1+02$ & $1.663+01$ & 3. $C 11+03$ \\
\hline 30 & $t .545+c 3$ & $1.150+02$ & $1.505+01$ & $6.411+03$ \\
\hline 40 & $3.079+03$ & $7.525+C 1$ & $2.032+01$ & $2.984+C 3$ \\
\hline 50 & $1.691+03$ & $5.151+01$ & 2. $\csc 8+01$ & $1.618+C 3$ \\
\hline 60 & $1.031+03$ & $3.725+01$ & $2.126+01$ & $9.722+02$ \\
\hline 80 & 4.72 & $2 \cdot 2 \mathrm{C} 4+01$ & $2.124+C 1$ & $4.294+C 2$ \\
\hline 100 & $2.61 \epsilon+02$ & $1.453+C_{1}$ & $2.088+01$ & $2.262+02$ \\
\hline 150 & $5.663+01$ & $6.675+0 C$ & $1.969+01$ & $7.026+C 1$ \\
\hline 200 & $5.300+01$ & $3.8 C 4+C C$ & $1.839+01$ & $3.080+01$ \\
\hline $30 \mathrm{C}$ & $2.782+C 1$ & $1.7 C 5+C O$ & $1.627 \leftrightarrow 01$ & $9.848+00$ \\
\hline
\end{tabular}

\begin{tabular}{|c|c|c|c|c|}
\hline \multirow{2}{*}{\multicolumn{2}{|c|}{ ENERGY $Z=48 C A C M I L M$}} & \multirow[b]{2}{*}{$\mathrm{COH}$} & \multirow[b]{2}{*}{ INCCH. } & \multirow[b]{2}{*}{ PHCTC } \\
\hline & & & & \\
\hline KEV & TOTAL & SCATT. & SCATT. & EFFECT \\
\hline 1 & $1.707+C 6$ & $1.467+03$ & S. $726-C 1$ & $1.706 \div 06$ \\
\hline 1.5 & $6.13 t+C 5$ & 1. $358+03$ & $1.784+0 \mathrm{C}$ & $6.122+05$ \\
\hline $\bar{z}$ & $2.572+05$ & $1.219+03$ & $2.570+00$ & $2.959+05$ \\
\hline 3 & 1. $C 73+05$ & $1.156+C 3$ & $4.035+00$ & $1.062+05$ \\
\hline 4 & $2.343+05$ & 1. $C C 5+03$ & $5.401+00$ & $2.333+05$ \\
\hline 5 & $1.516+05$ & $\varepsilon .741+C 2$ & $6.653+0 \mathrm{C}$ & $1.507+C 5$ \\
\hline 6 & $9.362+04$ & $7.647+02$ & $7.815+00$ & $5.285 * 04$ \\
\hline 8 & $4.339+C 4$ & $6.007+02$ & S. SC7 $7+00$ & $4.278+04$ \\
\hline 10 & $2.374+04$ & $4.89 C+02$ & $1.168+01$ & $2.324+04$ \\
\hline 15 & $7.84 \varepsilon+03$ & $3.2 \mathrm{Cs}+\mathrm{C} 2$ & $1.424+01$ & $7.512+C 3$ \\
\hline 20 & $3.55 c+C 3$ & $2.241+02$ & $1.691 * 01$ & $3.318+03$ \\
\hline 30 & $7.115+03$ & $1.251+02$ & 1. $\$ 3 \varepsilon+C 1$ & $6.971+03$ \\
\hline 40 & $3.368 \div 03$ & 7. $5 C_{1}+01$ & $2.062+c 1$ & $3.268+C 3$ \\
\hline 50 & $1.852+C 3$ & $5.4 C 2+01$ & $2.135+01$ & $1.776+C 3$ \\
\hline 60 & $1.128 \div 03$ & $3.914+01$ & $2.165+01$ & $1.067+c 3$ \\
\hline $8 \mathrm{C}$ & $5.150+02$ & $2.311+01$ & $2.164 \times 01$ & $4.702+02$ \\
\hline 100 & $2.835+02$ & $1.523+01$ & $2.12 \varepsilon+C 1$ & $2.470+02$ \\
\hline 150 & $1.033+02$ & $6.952+0 C$ & $2 \cdot C C S+01$ & $7.622+01$ \\
\hline 200 & $5.607+01$ & $3.980+0 \mathrm{C}$ & $1.877+01$ & $3.332+01$ \\
\hline 300 & $=.905+C 1$ & $1.7 \varepsilon 4+C C$ & $1.660 \div 01$ & $1.0 \epsilon t+01$ \\
\hline
\end{tabular}

$\begin{array}{rc}\text { ENERGY } & \\ \text { KEV } & \text { TCTAL } \\ 400 & 1.857+C 1 \\ 500 & 1.540+C 1 \\ 600 & 1.363+01 \\ 800 & 1.144+01 \\ 1000 & 1.002+01 \\ 1500 & 8.080+0 C \\ 2000 & 7.145+00 \\ 3000 & 6.344+0 C \\ 4000 & 6.073+00 \\ 5000 & 6.008+00 \\ 6000 & 5.957+0 C \\ 8000 & 6.108+00 \\ 10000 & 6.443+00 \\ 15000 & 6.973+00 \\ 20000 & 7.624+00 \\ 3000 C & 8.5 C 3+C C \\ 40000 & 9.053+00 \\ 50000 & 9.637+00 \\ 60000 & 9.962+00 \\ 80000 & 1.054+C 1 \\ 100000 & 1.104+C 1\end{array}$

$\mathrm{COH}$
SCATT
$8.850-01$
$5.660-01$
$3.920-01$
$2 ! 190-01$
$1.390-01$
$6.245-02$
$3.532-02$
$1.579-02$
$8.904-03$
$5.708-03$
$3.969-03$
$2.236-03$
$1.432-03$
$6.372-04$
$3.586-04$
$1.596-04$
$8.980-05$
$5.713-05$
$3.936-05$
$2.175-05$
$1.363-05$

ENERGY
KEV

400

500

$\begin{array}{ll}500 & 1.603+01 \\ 600 & 1.406+C 1 \\ 800 & 1.171 * 01\end{array}$

$1000 \quad 1.027+01$

$1500 \quad 8.276+00$

$2000 \quad 7.323+00$

$3000 \quad 6.516+C 0$

$4000 \quad 6.251+0 C$

$\begin{array}{ll}5000 & 6.155+C 0 \\ 6000 & 6.149+00\end{array}$

$8000 \quad 6.315+00$

10000

$15000 \quad 7.233+00$

$200007.918+00$

$30000 \quad 8.838+C \mathrm{C}$

40000

50000

$60000 \quad 1.037+01$

80000

100000

$1.037+01$
$1.057+01$

$1.057+01$
$1.149+01$
$\mathrm{COH}$

5. $900-01$

4. $080-01$

$2.280-01$

1. $446-01$

6. 497-02

$3.675-02$

1.643-02

9.265-03

5. $940-03$

$4.130-03$

2. $326-03$

$1.490-03$

$6.631-04$

3. $731-04$

1. $661-04$

5. $946-05$

$4.097-05$

z. 265-0

1. $420-05$
S.210-01
$5 \quad 4.44$ c -01

INCOH.

SCATT.

$1.406+C 1$
$1.285+C 1$

1. $195+01$

1. $057+C 1$

$9.473+C$

$7.7 C 5+C O$
$6.581+C C$

5. $17 \varepsilon+C C$

4. $322+C C$

$3.730+C 0$

$3.257+C 0$

2. $696+00$

2. $296+\mathrm{CC}$

1. $\in S \varepsilon+O C$

$1 \cdot 362+C C$

9. $905-01$

$7.863-01$

6. $555-C 1$

$5.647-01$
PHOTO

EFFECT

$3.626+C C$

$2.030+0 C$
$1.289+C C$

t. $554-C 1$

4. $C 3 t-C 1$

$1.525-C 1 \quad 1.155-01$

$1.139-C 1 \quad 4.151-C 1$

$6.156-C=1.088+C C$

$4.15 t-02 \quad 1.7 C 1+C C$

3. $064-02 \quad 2.242+00$

$2.445-C \bar{L} \quad 2.632+0 C$

$1.712-C \bar{C} \quad 3.3 \subseteq 3+O C$

$1.295-024.133+C C$

$0.412-03 \quad 5.26 \epsilon+C C$

$6.182-03 \quad 6.256+C C$

$4 . C 05-C 3 \quad 7.5 C E+C C$

$2.943-03 \quad 8.3 C 4+0 C$

1. $906-0=0.378+C$

$1.4 \mathrm{Cl} 1-03 \quad 1.0 \mathrm{C} 9+\mathrm{C} 1$

1.103-03 1. C $A 7+C 1$
ENERGY
KEV

400
500

600

800
1000

1500

300

4000

4000
5000

6000

8000

10000

15000

20000

40000

50000

60000

80000

100000
TCTAL 2. $014+C 1$

$1.447+\mathrm{C} 1$

1. $200+01$

1. C51*0

$8.468+00$

$6.688+0 \mathrm{C}$

$6.429+0 \mathrm{C}$

$6.322+00$

$6.342 \triangleleft C C$

$6.525+C C$

6. $898+00$

$7.497+\mathrm{CO}$

$8.216+0$

$9.179+0 C$

9.828400

1. $078+0$

$1.140+0$

$1.155+01$
INCOH.

1. $437+C 1$

1. $317+C 1$

1. $222+\mathrm{Cl}$

1. $076+01$

9. $682+0 C$
7. $87 \mathrm{~s}+\mathrm{CC}$

$6.72 t+C C$

5. $2 \leqq 3+00$

$4.41 \varepsilon+C C$

$3.813+\mathrm{CO}$

$3.37 C+C C$

2. $755+C C$

$2.346+C C$

1. $735+\mathrm{CO}$

$1.392+0 \mathrm{C}$

$1.013+C C$

8. $03 \varepsilon-C$

$6.704-C 1$

$5.772-C 1$

$4.548-C 1$
$3.773-01$
PHOTO

EFFECT

4. $048+C C$

2. $265+O C$

$1.437+00$

4. $355-C 1$

2.095-01

1. $23 \varepsilon-C 1$

$6.6 \subseteq 8-02$

$4.515-C 2$

$3.325-C 2$

$2.655-C 2$

$1.4 \mathrm{CS}-\mathrm{C}$

$5.125-C \equiv$

$6.705-03$

4. 343-0

$3.191-C_{3}$

$2.512-C 3$

2. $066-0$

$1.518-C$

$1.19 \epsilon-C ?$
PAIR

1. 223-01

$4.355-01$

$.13 c+C 0$

$1.779+C C$
$2.343+O C$

2. $748+C 0$

3. $53 \varepsilon+C 0$

$4.3 C 7+C C$

$5.48 \varepsilon+C$

t. $518+O C$

7. $821+C C$

$8.650+C C$

S. $7 \varepsilon 7+C C$

1. $C 51+C$

$1.111+C 1$
INCOH.

SCATT .

1. $34 t+C$

1. $248 \mathrm{C} 5+\mathrm{C}$

9. $852+C C$

8. $051+C C$

6. $873+C C$

$5.4 C 8+C O$

$5.4 \mathrm{C} 8+\mathrm{CO}$
$4.514+\mathrm{CC}$

3. $896+C C$

$3.443+C C$

2. $\varepsilon 16+C O$

2. $398+C 0$

1. $773+0 \mathrm{C}$

1. $422+C C$

1. $035+C C$

8. $213-01$

6. $850-C 1$

$5.898-C 1$

$4.647-01$

$3.856-C 1$
PHCTC

EFFEC T

$4.496+C C$

$2.495+00$

$1.566+C C$
$7.745-C$

4. $631-01$

z. $205-C 1$

$7.052=02$

4. $754-C 2$

3. $501-\mathrm{C} 2$

2. $791-\mathrm{C} 2$

$1.952-\mathrm{C} 2$

$1.475-02$

$5.575-C$

7. $037-03$

$4.55 t-C 3$

$3.347-C ?$

$2.635-C 3$

$2.16 \overline{1}-\mathrm{C} 3$

1. $592-C$

$1.253-C 3$
PAIR

PRCO.

1. $2 \varepsilon c-C$

4. 573-C1

$1.1 \subseteq 2+C C$

$1.858+C C$
$2.445+O C$

2. $\varepsilon 67+C O$

3. $6 \varepsilon 7+O C$

$4.4 \varepsilon 4+0 C$

5. $713+C C$

ह. $140+C$

9. $0 \mathrm{C} 3+0 \mathrm{C}$

5. $736+C C$

1. $018+C$

1. $C 54+C 1$
$1.15 t+C 1$

ENERGY
KEV

400

600

800

1000
1500

1500
2000

3000

4000

5000

6000

8000

10000

15000 
$2=49$ IN CIUM

\begin{tabular}{|c|c|c|c|c|}
\hline RGY & & $\mathrm{COH}$ & I NCOH. & $\begin{array}{l}\text { PHOTC } \\
\text { FFFECT }\end{array}$ \\
\hline EV & TOTAL & SCATT. & SCATT. & EFFECT \\
\hline 1 & $1.816+c \epsilon$ & $1.5 z 2+03$ & 1. $C 07+00$ & $1.815+0$ \\
\hline 1.5 & $17+05$ & $447+C 3$ & $1.850+00$ & \\
\hline 2 & 405 & $1.365+03$ & $2.6 \epsilon 4+00$ & $.222+0$ \\
\hline 3 & +05 & $1.158+03$ & $5+00$ & $<$ \\
\hline 4 & +05 & 1. $C 44+03$ & $5 \epsilon+00$ & \\
\hline 5 & & $07+02$ & $14+00$ & \\
\hline$\epsilon$ & & $1+02$ & & \\
\hline 8 & 04 & $6.273+02$ & $\epsilon+01$ & 4.6 \\
\hline 10 & $.548+C 4$ & $5.101+02$ & $1.202+01$ & $2.456+C$ \\
\hline 15 & $9.40 t+03$ & $.354+02$ & $1.519+01$ & 8. $C 56+0$ \\
\hline 20 & 3.82 & $2.353+02$ & $1.727+01$ & $3.573+0$ \\
\hline 30 & 03 & $1.315+02$ & $1.575+01$ & $.210+0$ \\
\hline 40 & & $25 t+01$ & $106+01$ & $3.456+$ \\
\hline 50 & & $6 \in 9+01$ & $74+01$ & $1.905+$ \\
\hline 60 & & $7+01$ & & $5+0$ \\
\hline 80 & $5 . \in 1$ & $2.426+01$ & $205+01$ & $153+$ \\
\hline $10 \mathrm{C}$ & 3. & $1.5 \subseteq \varepsilon+C 1$ & $2.169+01$ & $724+0$ \\
\hline & 1. & $7.334+00$ & $04 S+01$ & $8.470+0$ \\
\hline 200 & $41+01$ & $4.174+0 C$ & $915+01$ & \\
\hline & $66+01$ & $70+$ & 01 & \\
\hline
\end{tabular}

ENERGY $Z=50 \quad \mathrm{TIN}$

\begin{tabular}{|c|c|c|c|c|}
\hline NERGY & & $\mathrm{COH}$ & I NCCH. & PHCTC \\
\hline KEV & TOTAL & SCATT. & SCATT. & $E F F \subseteq C T$ \\
\hline 1 & $2.101+C 6$ & $1.583+C_{3}$ & $1.013+0 \mathrm{C}$ & $2.100+c 6$ \\
\hline 1.5 & $7.427+05$ & 1. $5 C 3+c 3$ & $1.87 t+00$ & $7.412+05$ \\
\hline$\vec{z}$ & $3.555+05$ & $1.415+03$ & $2.724+00$ & $3.541+C 5$ \\
\hline 3 & $1.262+05$ & $1.241+C 3$ & $4.295+00$ & $1.250+0.5$ \\
\hline 4 & $1.841+05$ & 1. $c 83+03$ & $5.70 \varepsilon+00$ & $1.830+C 5$ \\
\hline 5 & $1.712+05$ & $5.472+02$ & $6.981+00$ & $1.702+05$ \\
\hline 6 & $1.073+05$ & $8.318+02$ & $8.145+00$ & $1.064+05$ \\
\hline ? & $5.061+04$ & $6.545+02$ & $1.023+01$ & $4.995+04$ \\
\hline 10 & $2.796+C 4$ & $5.318+02$ & $1.215+01$ & $2.742+04$ \\
\hline 15 & $5.323+03$ & $3.5 \mathrm{CO}+02$ & $1.542+01$ & $8.958+03$ \\
\hline $2 C$ & $4.224+C 3$ & $2.466+02$ & $1.755+01$ & $3.960+c 3$ \\
\hline 30 & $8.100+03$ & $1.381+02$ & $2.008+01$ & $7.942+03$ \\
\hline 40 & $3.843+03$ & E. $7 \mathrm{C} 8+\mathrm{Cl}$ & $2.142+01$ & $3.734+C 3$ \\
\hline 50 & $2.115+03$ & $5 . c 5 C+01$ & $2.212+01$ & $2 . c 37+c 3$ \\
\hline 60 & $1.254+C 3$ & $4.311+01$ & $2.244+01$ & $1.228+03$ \\
\hline 80 & $2 \varepsilon+C 2$ & $2.54 E+C 1$ & $\bar{c} .245+01$ & $5.449+C 2$ \\
\hline 100 & $2.26 \epsilon+02$ & $1.678+\mathrm{C} 1$ & $2.210+01$ & $2.877+C 2$ \\
\hline $15 \mathrm{C}$ & $1.182+02$ & $7.7 \mathrm{C} 2+\mathrm{CO}$ & $2 . C 89+0 I$ & $8.960+01$ \\
\hline 200 & $6.327+01$ & $4.383+00$ & 1. $953+01$ & $3.936+01$ \\
\hline 300 & $3.18 \varepsilon+C 1$ & $1.5 \in 3+00$ & $1.72 \varepsilon+C 1$ & $1.263+01$ \\
\hline
\end{tabular}

$Z=51$ ANTIMONY

\begin{tabular}{|c|c|c|c|c|}
\hline ERGY & & $\mathrm{COH}$ & I NCOH. & PHCTC \\
\hline KEV & TOTAL & SCATT. & SCATT. & EFFECT \\
\hline 1 & $2.252+0 t$ & $1 . \in 47+03$ & $5.894-01$ & $2.251+06$ \\
\hline 1.5 & $\varepsilon . C_{01}+C_{5}$ & $1.562+03$ & $1.863+00$ & $7.986+05$ \\
\hline 2 & $3.843+C 5$ & $1.4 \in 5+03$ & $2.745+00$ & $3.828+C 5$ \\
\hline 3 & $1.371+C 5$ & $1.295+c 3$ & $4.397+00$ & $1.358+05$ \\
\hline 4 & $6 . \in 25+C 4$ & $1.123+C 3$ & $5.857+0 \mathrm{C}$ & $6.512+04$ \\
\hline 5 & $1.810+05$ & $5.83 \epsilon+02$ & $7.152+0 C$ & $1.800+C 5$ \\
\hline 6 & $1.145+05$ & $\varepsilon .655+02$ & $8.325+00$ & $1.136+05$ \\
\hline ? & $5.463+C 4$ & $6.823+02$ & $1.041+01$ & $5.393+04$ \\
\hline 10 & $3.025+04$ & $5.54 C+C 2$ & $1.222+01$ & $2.578+04$ \\
\hline 15 & $1.015+04$ & $3 . t 48+02$ & $1.55 t+01$ & $9.766+C 3$ \\
\hline 20 & $4.581+C 3$ & $\hat{z} .580+C \hat{z}$ & $1.775+01$ & $4.305+03$ \\
\hline 30 & 1.47 & $1.449+02$ & $2.03 t+01$ & $1.304+03$ \\
\hline 40 & $4.17 \pm+C 3$ & ؟. $139+01$ & $2.174+01$ & $4.062+03$ \\
\hline 50 & $2.35 \epsilon+03$ & $6.246+01$ & $2.247+01$ & $2.271+03$ \\
\hline 60 & $1.453+03$ & $4.527+C 1$ & $2.282+01$ & 1. $385+03$ \\
\hline 80 & $\epsilon .67 \mathrm{C}+02$ & $2.677+01$ & $2.284+01$ & $6.174+02$ \\
\hline 100 & $3.64 t+02$ & $1.764+01$ & $2.24 \xi+01$ & $3.245+02$ \\
\hline$\$ 50$ & $1.285+02$ & 8. $\operatorname{cs} 4+00$ & $2.12 c+01$ & $5.909+01$ \\
\hline 200 & $6.731+01$ & $4.6 C 7+0 C$ & $1.990+01$ & $4.280+01$ \\
\hline 300 & $3.319+01$ & 2. $C \in 3+O C$ & $1.7 \in 2+01$ & $1.350 * 01$ \\
\hline
\end{tabular}

\begin{tabular}{|c|c|c|c|c|}
\hline GY & & $\mathrm{COH}$ & I NCOH. & PHOTC \\
\hline KEV & TOTAL & SCATT. & SCATT. & EFFECT \\
\hline 1 & $\overline{2} .337+0 t$ & $1.712+03$ & c. $055-01$ & $2.335+06$ \\
\hline 1.5 & $8.760+05$ & $1.624+03^{\circ}$ & $1.785+C C$ & $8.744+C 5$ \\
\hline 2 & $4.222+05$ & $1.525+03$ & $2.701+00$ & $4.207+05$ \\
\hline 3 & $1.513+C 5$ & $1.3 \bar{z} 2+C 3$ & $4.44 \epsilon+00$ & $1.5 \mathrm{CO}+\mathrm{CE}$ \\
\hline 4 & $7.334+04$ & $1.1 \epsilon^{2}+03$ & $5.968+00$ & $7.217+0$ \\
\hline 5 & $1.995+C 5$ & 1. $C 2 C C+03$ & $7.3 C 5+0 C$ & $1.589+C$ \\
\hline$\epsilon$ & $1.248+05$ & $93+02$ & $\varepsilon .496+0 C$ & $1.239+0$ \\
\hline$\varepsilon$ & $5.872+04$ & $7.1 \mathrm{C} \epsilon+02$ & $1.060+01$ & $300+C$ \\
\hline 10 & $3.244+04$ & $5.77 C+C 2$ & $1.25 \epsilon+01$ & $3.185+C 4$ \\
\hline 15 & 1. $c .8 \epsilon+c 4$ & $3.798+c 2$ & $1.553+C 1$ & $1.047+04$ \\
\hline $2 C$ & 4. $554+c 3$ & 2. $\epsilon^{t} t+c 2$ & $1.814+01$ & $4.666+03$ \\
\hline 30 & $1 . \in 2 \varepsilon+c 3$ & $1.520+C 2$ & $2.074+01$ & $1.455+03$ \\
\hline 40 & $4.350+c 3$ & $C .588+01$ & $2.213+01$ & $4.232+03$ \\
\hline 50 & $.423+03$ & $\epsilon .55 t+C 1$ & $\epsilon+C 1$ & $2.334+C$ \\
\hline 60 & $1.487+03$ & $4.754+C 1$ & $2.321+01$ & $1.416+C 3$ \\
\hline 80 & $6.832+02$ & $2.813+01$ & $2.325+01$ & $6.318+02$ \\
\hline 100 & $3.792+02$ & 1. $854+01$ & $2.25 c+01$ & $3.342+02$ \\
\hline 150 & $1.343+C 2$ & $8.511+C \mathrm{C}$ & $2.169+C 1$ & $1.041+C 2$ \\
\hline 200 & $7.09 \mathrm{C}+\mathrm{Cl}_{1}$ & $4.845+00$ & $2.028+01$ & $4.578+C 1$ \\
\hline 300 & $.4 \div 0+01$ & $2.17 C+C C$ & 1. $75 t+01$ & $1.477+01$ \\
\hline
\end{tabular}

$\begin{array}{rc}\begin{array}{c}\text { ENERGY } \\ \text { KEV }\end{array} & \text { TCTAL } \\ 400 & 2.178+01 \\ 500 & 1.773+01 \\ 600 & 1.539+01 \\ 800 & 1.268+01 \\ 1000 & 1.106+01 \\ 1500 & 8.887+00 \\ 2000 & 1.872+00 \\ 3000 & 7.046+00 \\ 4000 & 6.797+00 \\ 5000 & 6.769+00 \\ 6000 & 6.740+00 \\ 8000 & 6.955+00 \\ 10000 & 7.367+00 \\ 15000 & 8.037+00 \\ 20000 & 8.827+00 \\ 30000 & 9.877+00 \\ 40000 & 1.059+01 \\ 50000 & 1.124+01 \\ 60000 & 1.162+01 \\ 80000 & 1.230+01 \\ 100000 & 1.288+01\end{array}$

$\mathrm{COH}$
$\mathrm{SCATT}$
$1.053+00$
$6.740-01$
$4.670-01$
$2.610-01$
$1.650-01$
$7.416-02$
$4.195-02$
$1.875-02$
$1.058-02$
$6.782-03$
$4.715-03$
$2.656-03$
$1.701-03$
$7.571-04$
$4.250-04$
$1.896-04$
$1.067-04$
$6.794-05$
$4.683-05$
$2.591-05$
$1.625-05$

INCOH.
SCATT
$1.530+C 1$
$1.4 C 3+01$
$1.3 C 1+01$
$1.14 E+C 1$
$1.031+C 1$
$8.352+C C$
$7.164+C O$
$5.638+00$
$4.7 C E+C O$
$4.061+C C$
$3.589+C C$
$2.535+00$
$2.455+0 C$
$1.845+00$
$1.483+C C$
$1.075+0 C$
$8.562-C 1$
$7.142-01$
$6.145-C 1$
$4.845-C 1$
$4.015-C 1$

PHOTO

EFFECT

$5.424+C C$

$1.911+O C$

$5.619-01$

$2.784-01$

$1.642-01$

$8.908-02$

$5.596-02$

4. $411-02$

$3.514-C 2$

$2.454-02$

$1.858-C 2$

$1.203-02$

$8.832-03$

$5.716-C ?$

$4.158-C 3$

3. 304-03

$2.717-C_{3}$

$1.595-C 3$

$1.625-05 \quad 4.015-C 1$
$3.02 \epsilon+C C$

PAIR

PROC.

$1.429-01$

5. $C 18-C 1$

$1.3 \mathrm{C} 1+\mathrm{CC}$

2. $657+0 C$

$3.111+00$

3. $593+\mathrm{CO}$

$6.175+0 C$

$7.3=5+00$

8. $7 C_{2}+C \mathrm{CC}$

S. $727+\mathrm{CC}$

1. $052+C 1$

$1.1 \mathrm{CO}+\mathrm{Cl}$

$1.248+C 1$

$\begin{array}{rr}\text { ENERGY } & \\ \text { KEV } & \text { TCTAL } \\ 400 & 2.251+01 \\ 500 & 1.825+01 \\ 600 & 1.580+01 \\ 800 & 1.259+01 \\ 1000 & 1.131+01 \\ 1500 & 9.085+00 \\ 2000 & 8.053+00 \\ 3000 & 7.224+0 C \\ 4000 & 6.582+00 \\ 5000 & 6.944+00 \\ 6000 & 6.942+00 \\ 8000 & 7.173+00 \\ 10000 & 7.6 C 5+00 \\ 15000 & 8.312+C 0 \\ 20000 & 9.139+00 \\ 30000 & 1.023+01 \\ 40000 & 1.098+01 \\ 50000 & 1.165+01 \\ 60000 & 1.205+C 1 \\ 80000 & 1.276+C 1 \\ 100000 & 1.336+01\end{array}$

$\mathrm{COH}$
$\mathrm{CATT}$.

INCOH

SCATT:

PHOTC

$1.106+00 \quad 1.561$

$\begin{array}{lll}7.070-01 & 1.431+01 & 3.234+00\end{array}$

$4.900-01$

$2.740-01$

1.740-01

$7.820-02$

4. $425-02$

1. $978-02$

1.116-02

$7.153-03$

4. $973-03$

$2.801 \div 03$

$1.794-03$

7. $986-04$

$4.493-04$

$2.000-04$

1. $126-04$

$7.1 \in 8-05$

4. $941-05$

$2.734-05$

$1.327+0$

$1.169+01 \quad 1.021+C C$

$8.563+C 0 \quad 2.530-01$

$7.311+C 0 \quad 1.727-C 1$

$\begin{array}{ll}5.753+C C & 9.377-02 \\ 4.852+C 0 & 6.307-02\end{array}$

$4.145+C C$ 4.637-02

$3 . t t^{2}+C O \quad 3.652-02$

$2.955+00 \quad 2.578-C_{2}$

$2.551+C C \quad 1.55 C-C 2$

$1.887+\mathrm{CO} \quad 1.262-\mathrm{C} 2$

$1.513+\mathrm{CO}$

1. $1 \mathrm{C} 1+0 \mathrm{O}$

$9.267-03$

$8.738-C$

$5.596-0$

$4.4 \mathrm{C} 2-03$

$3.4 \in 4-C ?$

$6.275-\mathrm{Cl}$

$4.944-C$

4. $102-C 1$
PAIR

PROD.

$1.503-\mathrm{Cl}$

$5.250-01$

$2.1 C 5+00$

2. $7 \in 6+C C$

$3.237+0 C$

$4.149+00$

$6.033+00$

$7 . \in 16+C C$

9. $127+C C$

1. $C 10+01$

$1.142+C 1$

1. $22 t+C 1$

1. $2 c 5+01$
SCATT.

$1.591+C 1 \quad 6.184+0 C$

$1.45 s+C 1 \quad 3.478+0 C$

$\begin{array}{ll}1.353+01 & 2.227+C C\end{array}$

$1.192+C 1 \quad 1.1 \in 0+O C$

$\begin{array}{ll}1 . C 73+C 1 & 7.3 \in 1=C \\ 8.734+C C & 3.493-01\end{array}$

$\begin{array}{ll}8.734+C C & 3.493-01 \\ 7.457+C C & 2.058-C 1\end{array}$

$5 . \varepsilon \epsilon \varepsilon+C C \quad 1.11 \epsilon-01$

$4.8 s 8+C C \quad 7.5 C 2-c 2$

$4.227+00 \quad 5.513-02$

$3.73 t+C C \quad 4.389-02$

$3.055+0 \mathrm{C} \quad 3.0 \in 2-C 2$

$2.6 C 2+O C \quad 2.316-0 \bar{Z}$

$1.524+C C \quad 1.459-C 2$

$1.544+001.100-02$

$1.123+C 07.120-C \equiv$

8.912-01 5.228-03

$7.434-C$

6.401-01

$5.043-01$

$5.228-03$

$3.382-C 3$

$2.483-03$

$1.954-03$
PAIR

1. $5 E 1-\mathrm{Cl}$

$5.457-01$

$0.415+0 C$
$2.191+0 C$

$2.877+C C$

$3.3 E 4+O C$

$4 \cdot 3 C 8+C C$

$4.223+C C$
$6.651+0 C$

$7.899+C \mathrm{C}$

$4 \epsilon \epsilon+C C$

$1.047+C 1$

$1.185+C 1$

$1.272+C 1$

$1 \cdot 343+C 1$

\begin{tabular}{|c|c|c|}
\hline ENERGY & & $\mathrm{COH}$ \\
\hline KEV & TCTAL & SCATT. \\
\hline 400 & $2.428+C 1$ & $1.223+00$ \\
\hline 500 & $1.952+01$ & $7.820-01$ \\
\hline 600 & $1.681+01$ & $5.420-01$ \\
\hline $80 \mathrm{C}$ & $1.372+01$ & $2.030-01$ \\
\hline 1000 & $1.192+01$ & $920-01$ \\
\hline 1500 & $9.5 \geq 2+C C$ & $8.631-02$ \\
\hline 2000 & $8.448+C 0$ & $4.884-02$ \\
\hline 3000 & $7.6 \mathrm{CO}+0 \mathrm{C}$ & $2.183-02$ \\
\hline 4000 & $7.367+00$ & $1.232-02$ \\
\hline 5000 & $7.356+C 0$ & $7.895-03$ \\
\hline 6000 & $7.355+0 C$ & $5.489-03$ \\
\hline 8000 & $7 . \epsilon 21+C C$ & $3.092-03$ \\
\hline 10000 & $8.054+00$ & $1.921-03$ \\
\hline 15000 & $8.873 \cdot \mathrm{CC}$ & $8.816-04$ \\
\hline 20000 & $9.772+C c$ & $4.961-04$ \\
\hline 30000 & 1. $\csc \theta+\cos$ & $2.208-04$ \\
\hline 40000 & $1.177+01$ & $1.243-04$ \\
\hline 50000 & $1.25 C+C 1$ & $7.917-05$ \\
\hline 60000 & $1.29 \overline{3}+01$ & $5.458-05$ \\
\hline 80000 & $1.369+C 1$ & $3.021-05$ \\
\hline 100000 & $1.435+01$ & $1.896-05$ \\
\hline
\end{tabular}

INCOH.

PHOTO

SCATT

$1.622+C$

$1.4 E \varepsilon+C$

$1.380+01$
$1.215+C 1$

$1.054+C 1$

$8.9 C 5+C C$

$7.6 C 3+C 0$

$5.584+C \mathrm{CO}$

$4 . \exists 55+C C$

$4 \cdot 311+C C$

$3.81 C+C C$

2. $11 c+c 0$

2. $\epsilon E 3+C C$

$1.5 \in 2+C C$

$1.574+C C$

$1.145+C 0$

c. $.088-C 1$

$7.58 \mathrm{C}-\mathrm{Cl}$

$\epsilon .527-C 1$

$5.742-C 1$
PHOTO
EFFECT

$t .837+C C$

$3.85 \varepsilon+c C$

$2.465+0 C$

$1.2 t c+c c$

$3.744-C 1$

$2.206-C 1$

$\begin{array}{ll}.022-02 & 2.280+O C\end{array}$

$.9 S_{2}-C 2 \quad 2.5 \varepsilon 9+C C$

$4.585-C 2 \quad 3.453+0 C$

$2.47 \equiv-C 2 \quad 5.415+C O$

$1 .+C C-02 \quad 6.854+C C$

$1.175-C 2 \quad 8.185+C C$

$7 . \in C 1-C_{3}$

$5.581-C \equiv$

$4.392-03$

$3.611-c$

C. $811+C C$

1. $C E 5+C 1$

$1.174+C 1$

$1.228+C 1$

$1.318+c$ 


\begin{tabular}{|c|c|c|c|c|}
\hline AEREY & & $\mathrm{CCH}$ & $1 \mathrm{NCOH}$. & PHOTC \\
\hline KEV & TOTAL & SCATT & SCATT. & EFFECT \\
\hline 1 & $2.426+0 t$ & $1.78 \mathrm{C}+\mathrm{C} 3$ & $8.94 t-C 1$ & $2.424 \div 06$ \\
\hline 1.5 & $9.321+05$ & $1.6 \varepsilon S+03$ & $1.780+00$ & $9.304+05$ \\
\hline 2 & $4.572+c 5$ & $1.585+03$ & $2.715 * C 0$ & $4.556+05$ \\
\hline 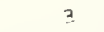 & $1.679+05$ & $1.381+03$ & $4.521 * 00$ & $1.666+05$ \\
\hline 4 & $8.277+04$ & $1.2 \mathrm{Cs}+03$ & $6.1 \mathrm{CO}+00$ & $8.156+C 4$ \\
\hline 5 & $1.872+C 5$ & 1. $C 57+C 3$ & $7.47 t+00$ & $1.861 \div 05$ \\
\hline 6 & $1.34 C+05$ & 9.335402 & $E .686+0 \mathrm{C}$ & $1.330+05$ \\
\hline 8 & $6.232+04$ & $7.3 \subseteq 5+02$ & $1.080 \nleftarrow 01$ & $6.157+64$ \\
\hline IC & $3.42 \sec 4$ & $6 . C C 5+02$ & 1.267401 & $3.367+C 4$ \\
\hline $1 \subseteq$ & $1.151+04$ & $3.551+02$ & $1.608 \$ 01$ & $1.110+04$ \\
\hline $2 \mathrm{C}$ & $5.3 \mathrm{C} 2+\mathrm{C}^{3}$ & $2.814+02$ & $1.835-01$ & $5.002+C 3$ \\
\hline 30 & $1.783+03$ & $1.5<2+c 2$ & $2.104+01$ & 1.603403 \\
\hline 40 & $4.72 C+03$ & 1. $C C t+C 2$ & $2.247+C 1$ & $4.597+03$ \\
\hline 50 & $2 . t 3 \epsilon+03$ & $6.882+01$ & $2.323+01$ & $2.544 * 03$ \\
\hline 60 & 1. $\in 22+C 3$ & $4.553+01$ & $2.359+01$ & $1.548+C 3$ \\
\hline 80 & $7.47 E+02$ & $2.5 \subseteq 6+C l$ & $2.364+01$ & $6.946+02$ \\
\hline 100 & $4.115 \div 02$ & $1.55 C+01$ & $2.33 \mathrm{C}+01$ & $3.691+02$ \\
\hline 150 & $1.46 s+02$ & C. $\subseteq 54+C C$ & $2.208+01$ & $1.159 \div 02$ \\
\hline $2 \mathrm{CO}$ & $7.688+01$ & 5. $C S E+C C$ & $2.0 t t+C 1$ & $5.113+01$ \\
\hline$\equiv \mathrm{co}$ & $3.711+01$ & 2.284400 & $1.830+01$ & 1.652401 \\
\hline
\end{tabular}

\begin{tabular}{|c|c|}
\hline \multirow{2}{*}{\multicolumn{2}{|c|}{$\begin{aligned} & Z== \\
& \text { ENERCY }\end{aligned}$}} \\
\hline & \\
\hline & TOTAL \\
\hline 1 & $2.452+C E$ \\
\hline 1.5 & $5.540+C 5$ \\
\hline 2 & $4.7 \overline{Z 2+C} 5$ \\
\hline 2 & $1.756+05$ \\
\hline 4 & $E .732+C 4$ \\
\hline 5 & $1.437+05$ \\
\hline 6 & $1.4 E C+C E$ \\
\hline 8 & $6.847+C 4$ \\
\hline 10 & $3.777+C 4$ \\
\hline 15 & $1.263+c 4$ \\
\hline 20 & $5.757+C 3$ \\
\hline 30 & $1.853+c 3$ \\
\hline 40 & $4.872+C 3$ \\
\hline 50 & $2.741+C 3$ \\
\hline$\in c$ & $1.693+03$ \\
\hline 80 & $7.83 c+02$ \\
\hline $10 \mathrm{C}$ & $4.313+02$ \\
\hline 150 & 1.534102 \\
\hline 200 & $7.596+01$ \\
\hline 300 & $3.83 \varepsilon+c 1$ \\
\hline
\end{tabular}

$C C H$
$S C \Delta T T$.
$1 . E 5 O+C 3$
$1.755+C 3$
$1.648+C 3$
$1.433+03$
$1.24 \varepsilon+0 ?$
$1 . C C 5+03$
$C .67 C+C 2$
$7.687+02$
$6.248+02$
$4.1 C t+C 2$
$2.533+02$
$1.667+02$
$1 . C 54+C 2$
$7.222+01$
$5.243+C 1$
$3.1 C 7+01$
$2 . C 50+C 1$
$C .423+0 C$
$5.36 t+00$
$2.4 C 5+C C$

$\begin{array}{lc}\text { INCCH. } & \text { PHOTC } \\ \text { SCATT: } & \text { EFFECY } \\ 9.421-01 & 2.450+C 6 \\ 1.83 C+0 C & 9.523+05 \\ 2.78 E+C C & 4.705+C 5 \\ 4.610+00 & 1.742+05 \\ 6.254+0 C & 8.6 C 7+04 \\ 7.664+C C & 1.426+05 \\ 8.895+00 & 1.450+05 \\ 1.101+01 & 6.769+C 4 \\ 1.283+01 & 3.713+C 4 \\ 2.630+01 & 1.220+04 \\ 1.561+01 & 5.445+C 3 \\ 2.135+01 & 1.705+C 3 \\ 2.281+01 & 4.744+C 3 \\ 2.359+01 & 2.645+03 \\ 2.3 C 7+01 & 1.617+03 \\ 2.404+01 & 7.279+C 2 \\ 2.37 C+01 & 3.871+C 2 \\ 2.247+C 1 & 1.215+C 2 \\ 2.1 C 3+01 & 5.356+01 \\ 1.864+01 & 1.734+C 1\end{array}$

\begin{tabular}{|c|c|}
\hline \multirow{2}{*}{\multicolumn{2}{|c|}{ EAERTYY $=$}} \\
\hline & \\
\hline$K \in V$ & TOTAL \\
\hline 1 & $2.1 \epsilon^{2}+c t$ \\
\hline i.5 & $1.14 C+C \theta$ \\
\hline 2 & $5.511+05$ \\
\hline ? & $1.583+c 5$ \\
\hline 4 & c. $632+04$ \\
\hline 5 & $5.515+C 4$ \\
\hline$t$ & $\therefore 5 \epsilon z+C 5$ \\
\hline 8 & $7.27 \varepsilon+C 4$ \\
\hline 10 & $4.005+0<$ \\
\hline 15 & $1.343+04$ \\
\hline 20 & $6.174+c 3$ \\
\hline 30 & C. $C, 68+C 3$ \\
\hline $4 C$ & $5.206+C 3$ \\
\hline 50 & $2.527+C 3$ \\
\hline $6 C$ & $1.80 E+C 3$ \\
\hline 80 & $8.362+02$ \\
\hline 100 & $4.606+C 2$ \\
\hline $15 \mathrm{C}$ & 1.634402 \\
\hline 200 & $8.482+01$ \\
\hline 300 & 4. $(28+0)$ \\
\hline
\end{tabular}

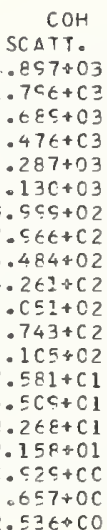

$\begin{array}{cc}\text { INCCH. } & \text { PHOTC } \\ \text { SCATT. } & \text { EFFECT } \\ 1.274+00 & 2.161+06 \\ 2.165+C 0 & 1.138+06 \\ 3.082+00 & 5.4 C 4+05 \\ 4.856+C 0 & 1.968+05 \\ 6.485+0 C & 9.5 C 3+C 4 \\ 7.912+00 & 5.402+04 \\ 5.153+0 C & 1.553+C 5 \\ 1.127+01 & 7.198+04 \\ 1.308+01 & 3.939+C 4 \\ 1.65 t+01 & 1.299+04 \\ 1.851+01 & 5.850+03 \\ 2.169+01 & 1.872+C 3 \\ 2.317+C 1 & 5.073+03 \\ 2.357+C 1 & 2.827+C 3 \\ 2.436+C 1 & 1.728+C 3 \\ 2.443+01 & 7.790+02 \\ 2.410+01 & 4.145+02 \\ 2.287+01 & 1.306+C 2 \\ 2.141+01 & 5.776+C 1 \\ 1.858+C 1 & 1.877+01\end{array}$

$\begin{array}{cc}Z= & 5 E \text { EAR IUM } \\ \text { FNERGY } & \text { TOTAL } \\ \text { KEY } & \text { TOT } \\ 1 & 2.405+0 \epsilon \\ 1.5 & 1.26 C+C 6 \\ 2 & \epsilon . C 34+05 \\ 3 & 2.143+05 \\ 4 & 1.031+05 \\ 5 & 5.8 \epsilon 3+C 4 \\ \epsilon & 1.665+05 \\ E & 7.772+04 \\ 10 & 4.290+C 4 \\ 15 & 1.45 C+04 \\ 20 & \epsilon .708+C 3 \\ 30 & 2.270+03 \\ 40 & 5.556+C 3 \\ 50 & 3.173+C 3 \\ 60 & 1.568+03 \\ 80 & 8.11 \epsilon+02 \\ 1 C C & 5.011+02 \\ 150 & 1.760+C 2 \\ 200 & 5.032+01 \\ 300 & 4.219+C 1\end{array}$
$\mathrm{COH}$ $S C A T T$.
$1 . C 55+C$. $1.843+03$
$1.730+03$ $1 \cdot 730+03$ 1. $51 t+C 3$ i. $1 \in 5+03$ $1.032+03$ $8.241+02$ $6.721+02$ $3.1 \in 9+C 2$ $1.8 \overline{\angle 0}+02$ $1.157+02$ 7.947401 $3.43 ?+C i$ $2.268+01$ $1 . C 45+C 1$ $5 . \subseteq 55+C C$ $1 \mathrm{NCOH}$. SCATT. $2.4 C 3+C 6$ $2.47 C+C C \quad 1.258+05$

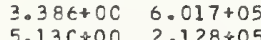
$5.13 \mathrm{C}+00 \quad 2.128+05$ $6.744+00 \quad 1.018 \div 05$ $8.17540 \mathrm{C} \quad 5.745+\mathrm{C} 4$ $5.426+00 \quad 1.654+05$ $1.154+C 1 \quad 7.689+04$ $1.334+01 \quad 4.222+04$ $1.683+01 \quad 1.404+04$ $1.921 * 01 \quad 6.372 * 03$ $2.203+012.066+03$ $2.353+01 \quad 5.456+C 3$ $2.434+01 \quad 3.069+03$ $2.483+\mathrm{Cl} \quad 8.525+02$ $2.45 C+01 \quad 4.539+C 2$ $2.326+01 \quad 1.422+02$ $2.178+01 \quad 6.258401$ i. $531+01$

$\begin{array}{cc}\text { ENERGV } & \\ \text { KEV } & \text { TCTAL } \\ 400 & 2.545+C 1 \\ 500 & 2.027+01 \\ 600 & 1.735+C 1 \\ 800 & 1.409+01 \\ 1000 & 1.220+01 \\ 1500 & 9.745+0 C \\ 2000 & 8.639+00 \\ 3000 & 7.786+C C \\ 4000 & 7.559+0 C \\ 5000 & 7.569+C 0 \\ 6000 & 7.564+C C \\ 8000 & 7.848+00 \\ 10000 & 8.342+0 C \\ 15000 & 9.159+C 0 \\ 20000 & 1.009+01 \\ 30000 & 1.134+01 \\ 40000 & 1.217+01 \\ 50000 & 1.293+01 \\ 60000 & 1.338+01 \\ 80000 & 1.417+01 \\ 100000 & 1.485+01\end{array}$

COH
SCATT.
$1.287+00$
$8.230-01$
$5.700-01$
$3.190-01$
$2.020-01$
$9.081-02$
$5.138-02$
$2.297-02$
$1.296-02$
$8.308-03$
$5.776-03$
$3.254-03$
$2.085-03$
$5.277-04$
$5.220-04$
$2.323-04$
$1.308-04$
$8.332-05$
$5.745-05$
$3.180-05$
$1.997-05$

ENEREY

$400 \quad 2.624+01$

$5002.0 E 6+C 1$

$600 \quad 1.784+C 1$

$800 \quad 1.44 \epsilon+01$

1000

1500

$9.973+00$

$20008.841+C C$

$30007.975+00$

$4000 \quad 7.757+C C$

5000

$6000 \quad 7.777+C C$

$8000 \quad 8.075+00$

$100008.594+0 \mathrm{C}$

$15000 \quad 9.449+0 \mathrm{C}$

$200001.042+C 1$

$30000 \quad 1.171+01$

$40000 \quad 1.258+C 1$

$500001.337+01$

$600001.384+01$

80000

100000

$1.466+01$

ENERG

KEV

$400 \quad 2.730401$

$500 \quad 2.158+01$

$6001.839+01$

$800 \quad 1.484+01$

$1000 \quad 1.282+01$

$1500 \quad 1.020+01$

$2000 \$ .040+00$

$3000 \quad 8.171+00$

$400 \mathrm{C} \quad 7.955+0 \mathrm{C}$

$5000 \quad 7 .<84+00$

60007.552800

$800 C \quad 8.312+C C$

$10000 \quad 8.84 c+c 0$

$15000 \quad 0.741 . C C$

$20000 \quad 1.0 ? 5+C 1$

$300001.210+01$

$40000 \quad 1.300+C 1$

$60000 \quad 1.430 \cdot C$

80000

100000

$1.420+00$

$6.140-01$
$6.330-01$

3.550-01

2.250-01

$1.012-01$

$5.724-02$

2.559-02

$5.257-03$

$6.436-03$

$3.62 t-03$

$2.323-03$

$1.034-03$

$5.817-04$

$5.289=05$

6.405-05

$3.547-05$

$2.228-05$
$50000 \quad 1.382+\mathrm{C}$

$2.588-04$
$1.457-04$

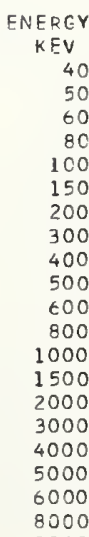

$2.020+01 \quad 8000$

TCTAL

$600 \quad 1.893 .01$

$1.523 * 01$

$1.314+01$

$1.043+0$

e. $368+00$

8. $157+O C$

$8.156+C 0$

$8.211+0$

$8.548+C$

$9.1 C 7+00$

$\mathrm{COH}$
SCATT
$1.355+00$
$.660-01$
$.010-01$
$3.350-01$
$2.130-01$
$.576-02$
$.418-02$
$2.422-02$
$.366-02$
$.762-03$
$.092-03$
$3.432-03$
$.198-03$
$.784-04$
$.505-04$
$2.450-04$
$.379-04$
$.790-05$
$6.061-05$
$2.356-05$
$2.107-05$

\author{
$\mathrm{COH}$
} CCH
SCATT.

INCOH.
SCATT.
$1.653+01$
$1.51 t+C 1$
$1.4 C 6+01$
$1.239+C 1$
$1.115+C 1$
$5.071+C C$
$7.75 C+C C$
$6.055+C O$
$5.051+C C$
$4.354+C C$
$3.883+O C$
$3.17 t+C C$
$2.7 C 4+C C$
$2.0 C O+C C$
$1.6 C 4+0 C$
$1.167+C C$
$9.263-01$
$7.72 t-C 1$
$6.653-C 1$
$5.241-01$
$4.34 S-C 1$

Incoho INCOH.

$1.684+01$

$1.544+C I$

$1.432+C$

$1.262+01$

$1.136+C 1$

$9.248+C C$

7. ESt+CC

$6.214+C C$

5. $1 E i+C C$

$4.477+C C$

$3.557+C C$

$3.23 t+C O$

$2.755+c c$

2. $C 38+C 0$

$1.635+C C$

$1.18 S+C C$

$9.439-C 1$

$7.8 .73-C 1$

$6.77 \mathrm{~s}-\mathrm{Cl}$

4.431-01

INCOH.

INCOH.

$\therefore 715+C 1$

1. $573+C 1$

$1.459+01$

$1.2 \mathrm{E} 5+\mathrm{Cl}$

$.157+C$

$5.415+C C$
$8.043+C C$

$6.330+C C$

$5.284+C O$

$4.5 E C+C O$

$4 . C 30+C C$

$3.25 t=c c$

2. $8 C E+C C$

$2.07 t+C C$

1. $t \epsilon E+00$

$1.211+C 0$

$9.614-C 1$

$8.015-C 1$

6.305-C1

$5.44 C-C]$

4. $513-C 1$

$\mathrm{COH}$
SCAT
$.504+00$
$.630-01$
$.670-01$
$.730-01$
$.370-01$
$.066-01$
$.030-02$
$.696-02$
$.521-02$
$.753-03$
$.781-03$
$.820-03$
$.447-03$
$.089-03$
$.128-04$
$.727-04$
$.535-04$
$.788-05$
$.751-05$
$.739-05$
$.348-05$

$\begin{array}{ll} & \\ 0 & 1 \\ 1 & 1.74 \\ 1 & 1.48 \\ 1 & 1.30 \\ 1 & 1.17 \\ 1 & 5.59 \\ 2 & 8.19 \\ 2 & 6.44 \\ 2 & 5.38 \\ 3 & 4.64 \\ 3 & 4.16 \\ 3 & 3.35 \\ 3 & 2.8 \\ 3 & 2.11 \\ 4 & 1.69 \\ 4 & 1.23 \\ 5 & 8.16 \\ 5 & 7 . \\ 5 & 5.54 \\ 5 & 4.59\end{array}$

INCOH.

INCOH.

$74 t+C 1$

$t C 1+01$

PHO TO

EFFEC.T

$7.625+C C$

$4.285+C C$

$1.383+C C$

$\varepsilon .48 \varepsilon-C 1$

4.024-C1 $1.747-\mathrm{Cl}$

$2.37 C-01$

$1.28 z-C 1$

$8.604-02$

$6 . \equiv 1 \epsilon-c 2$

5.C25-C2

$3.503-C^{2}$

$2.648-02$

$1.713-C^{2}$

$1.258-02$

e.138-0

$4.702-C^{2}$

$3.86 t-c$

$2.838-c 3$

6. $C 11-C$

$1.53 t+C C$

$2.3 E S+C C$
$3.1 C 4+0 O$

$3.625+0 \mathrm{C}$

$4.634+0 \mathrm{C}$

$5.61 C+C C$

$7.141+C C$
$8.476+C C$

1. $\mathrm{Cl} t+\mathrm{Cl}$

$1.124+C 1$

:. $21 t+C$ !

$1.271+C 1$

$\therefore .365+C 1$

PHOTC

PAIR

EFFEC T

PROC.

$.547+C C$

$1.505+C C$

S. $4 \mathrm{C} z-\mathrm{C}$ ?

$4.45 t-C 1$

$2.624-01$

$1.417-C$

c. $5 C_{4} 4-C_{2}$

$6.574-C 2$
$5.547-C^{2}$

$3.865-\mathrm{C} 2$

2. $521-C 2$

$1.88^{4}-C 2$

$1.387-C 2$

$6.58 \mathrm{~s}-\mathrm{C}^{3}$

$5.185-C$

$4.263-C^{3}$

$3.13 \mathrm{C}-0$

1.E33-C?

$6.27 c-C 1$
$1.55 \varepsilon+C C$

$2.4 t i+C C$

$3.220+C C$

$2.759+O C$

4. $8 \mathrm{Cl}+\mathrm{CC}$

5. $E C E+C C$
$7.3 C 1+O C$

$E .771+C C$

$1.051+C 1$

$1.163+01$

$1.258+C 1$

1. $31 t+C$ ?

$1.452+i 1$

PHOTC

EFFEC T

$8.724+C C$
$4.539+C C$

$3 . i \in 4+C C$

$1.635+C C$

$4.837-C 1$

2.847-C

$1.535-C 1$

$7.548-C 2$

$6.002-02$

$4.180-02$

$3.158-02$

$2 . C 43-02$
$1.50 C-C 2$

$\mathrm{S} .7 \mathrm{C} 3-\mathrm{C} 3$

$7.123-03$

$5.6 C 5-C 3$

$4.6 \mathrm{C} 8-\mathrm{C}^{3}$

$3.383-C^{3}$

$2.662-03$

$1.523-C$

t. $554-C 1$
$1 . t \in E 2+C C$

2. $554+C C$

3. $\varepsilon S_{t}+C C$

4. $571+00$

6.008 .00

$7.644+C C$

S.C $7 \mathrm{C}+\mathrm{CC}$

$1.203+01$

1. $3 \mathrm{Cl}+\mathrm{Cl}$

$1.361+01$

$1.543+\mathrm{Cl}$

EFEECT

PAIR

5. $371+C C$

$.485+C 1 \quad 3.4 C 7+C C$ 


\begin{tabular}{|c|c|c|c|c|}
\hline ENERGY & & $\mathrm{COH}$ & INCCH. & РНОTO \\
\hline KEV & TOTAL & SCATT & SCATT. & EFFECT \\
\hline 1 & $2.62 \varepsilon+06$ & $2.028+03$ & $1.603+00$ & $2.626+06$ \\
\hline 1.5 & $1.394+06$ & 1. $S C S+03$ & $2.57 s+C C$ & $1.382 \div 06$ \\
\hline 2 & $6.592+C 5$ & $1.789+03$ & $3.491+00$ & $6.574+05$ \\
\hline 3 & $2.32 \hat{z}+C 5$ & $1.5 \epsilon 4+C 3$ & $5.237+c 0$ & $2.307+C 5$ \\
\hline 4 & $1.111+05$ & $1.3 \leqslant 8+03$ & $6.845+00$ & $1.057+05$ \\
\hline 5 & $6.287+04$ & $1.202+03$ & $8.287+00$ & $6.166+04$ \\
\hline 6 & $1.492+05$ & 1. $C<5+C 3$ & $5.550+00$ & $1.481+05$ \\
\hline 8 & $8.261+$ & $\varepsilon_{0}=28+$ & & $5+04$ \\
\hline 10 & $4, t 15+0<$ & $6.5 \in 7+02$ & $1.308+01$ & $4.544+04$ \\
\hline 15 & $1.573+C 4$ & $4.57 c+02$ & 01 & $1.525+C 4$ \\
\hline 20 & $7.240+c$ & $3.2 c c+c 2$ & & $6.892+c 3$ \\
\hline $3 c$ & $2.401+03$ & $1.5 \mathrm{CO}+02$ & $2.221+01$ & $2.189+03$ \\
\hline $4 C$ & $5.965+$ & $1.212+C 2$ & & $5.820+03$ \\
\hline 50 & 3.37 & $8.338+$ & 2. & $7+03$ \\
\hline 60 & $2.052+c 3$ & $6 . C 71+01$ & $2.5 C 9+01$ & $2.006+03$ \\
\hline 80 & $c .68$ & $3.6 C 7+01$ & 2.521 & 9.08 \\
\hline 100 & 5.33 & $2.3 \varepsilon .7+C 1$ & 2.4 & $4.850+C 2$ \\
\hline 150 & 1.87 & $1.101+01$ & $2.365+01$ & $1.530+02$ \\
\hline 200 & $9 . \notin 09$ & $6.27 c+0 C$ & & $6.765+01$ \\
\hline bo & $.442+01$ & $.817+00$ & $1.965+01$ & $2.195+01$ \\
\hline
\end{tabular}

\begin{tabular}{|c|c|c|c|c|}
\hline & & & & \\
\hline EN ERGY & & $\mathrm{CCH}$ & INCCH. & PHOTO \\
\hline$K \bar{E} V$ & TOTAL & SCATT. & SCATT. & EFFECT \\
\hline 1 & $2.83 t+c t$ & $2.1 C 7+0 \Omega$ & $1.657+00$ & $2.834+06$ \\
\hline 1.5 & $1.504+C 6$ & $1.5 \$ 2+03$ & $2.647+00$ & $1.502+06$ \\
\hline 2 & $7.167+05$ & $1.874+C 3$ & $3.557+C C$ & $7.14 R+05$ \\
\hline 3 & $2.526+05$ & $1 . t 48+03$ & $5.3 \mathrm{C} 2+00$ & $2.500+05$ \\
\hline 4 & $1.208+05$ & $1.444+03$ & $6.9 C s+00$ & $1.194+05$ \\
\hline 5 & $E .839+04$ & $1.2 \epsilon s+C 3$ & $8.363+00$ & $6.711+04$ \\
\hline 6 & $1.159+05$ & $1.123+03$ & c. $535+00$ & $1.147+05$ \\
\hline 3 & $8.530+c 4$ & $8.57 c+02$ & $1.179+01$ & $8.840+C 4$ \\
\hline 10 & 4. $568+04$ & $7 . \equiv 12+02$ & $1.31 a+01$ & $4.894+104$ \\
\hline 15 & $1 . \mathrm{C}_{\mathrm{C} 3}+\mathrm{C}_{4}$ & $4.788+02$ & $1.677+01$ & $1.644+04$ \\
\hline 20 & $7.843+03$ & $3.437+02$ & $1.934+01$ & $7.48 C+C 3$ \\
\hline 30 & $2.643+03$ & $1 . c c_{1}+c_{2}$ & $2.249+01$ & $2.422+C 3$ \\
\hline$\therefore 0$ & $? .223+03$ & $1.273+c_{2}$ & $2.413+01$ & $1.074+03$ \\
\hline 50 & $3 . \leqslant 65+03$ & $8.77 c+01$ & $2.5 \mathrm{C} 2+01$ & $3.552+c 3$ \\
\hline$\in c$ & $2.271+C 3$ & $E .3 C S+01$ & $2.546+C 1$ & $2.182+03$ \\
\hline 90 & 1. $C 5 C+03$ & $3.8 C 4+C 1$ & $2.559+01$ & $9.866+02$ \\
\hline 100 & $5.757+C 2$ & $2.51 c+C 1$ & $2.528+01$ & $5.253+02$ \\
\hline 150 & $2.0<3+02$ & $1.1 \in 4+C 1$ & $2.4 C 5+01$ & $1.64 t+02$ \\
\hline 200 & $1 . \mathrm{C} 16+\mathrm{C} 2$ & $6.641+00$ & $2.253+01$ & $7.247+01$ \\
\hline 300 & $4.635+01$ & $2.581+C C$ & 1. $\$ \subseteq 8+01$ & $2.343+01$ \\
\hline
\end{tabular}

$Z=$ EC PRASECCYMILN

\begin{tabular}{|c|c|c|}
\hline$R C E_{Y}$ & & $\mathrm{COH}$ \\
\hline Ev & TOTAL & SCATT. \\
\hline & $3.01 \varepsilon+c 6$ & $2.184+03$ \\
\hline 1.5 & $\therefore .527+C t$ & 2. $c \in \varepsilon+03$ \\
\hline 2 & $7.733+05$ & $1.949+C 3$ \\
\hline & $2.737+C 5$ & $1.717+C_{3}$ \\
\hline & $1.313+C 5$ & $1.5 \mathrm{C} t+\mathrm{C} 3$ \\
\hline & $7.446+04$ & $1.3=5+c 3$ \\
\hline & $1.24 t+C 5$ & $1.173+03$ \\
\hline & $9.538+04$ & $9.3 \leq 2+02$ \\
\hline 10 & $5.29 c+04$ & $7 . t^{3} c+c z$ \\
\hline 15 & $1.785+04$ & $4.9 \varepsilon t+C 2$ \\
\hline 20 & $\varepsilon .23 c+03$ & $3.579+C 2$ \\
\hline 30 & $2.75 \mathrm{c} \cdot \mathrm{C} 3$ & 2. $C 8:+02$ \\
\hline 40 & $1.27 t+C 3$ & $1.3 \div t+C 2$ \\
\hline 50 & $2.734+c 3$ & c. $225+01$ \\
\hline 60 & $2.227+03$ & $6.735+C 1$ \\
\hline 80 & $1.007+03$ & $4.00 t+01$ \\
\hline 100 & t.CCS+C2 & $2.656+01$ \\
\hline 150 & $2.11 \varepsilon+C 2$ & $1.2 \overline{Z s}+01$ \\
\hline 200 & -. $C 79+02$ & 7. $C 17+0 \mathrm{C}$ \\
\hline & $4.8 \subset 5+01$ & $3.151+O C$ \\
\hline
\end{tabular}

INCDH.

SCAIT.

$1.673+00$

$2 \cdot 671+0 C$

$3.581+00$

$6.535+00$

8. $4 \mathrm{C} 4+\mathrm{CO}$

$5.695+00$

$1.187+01$

$1.327+C 1$

$1.692+01$

$1.554+01$
$2.277+01$

$2.277+C 1$
$2.445+01$

$2.537+01$

$2.583+01$

$2.59 \varepsilon+c 1$

$2.567+01$

$2.251+01$

PHCTC

EFFECT

$3.015+06$
$1.525+06$

$7.714+05$

$2.720+C 5$

$1.258+05$

$7.315+04$

$1.234+05$

$9.443+04$

$1.734+C 4$

$7.853+03$

$2.528+03$

$1.118+c^{2}$

$2.233+03$

$1.021+03$

$5.487+02$

$1.751+02$

$7.796+01$

$2.032+012.552+\mathrm{Cl}$

$\begin{array}{rr}\begin{array}{c}\text { ENERGY } \\ \text { KEV }\end{array} \\ 400 & 2.954 A+01 \\ 500 & 2.308+C 1 \\ 600 & 1.951+01 \\ 800 & 1.563+01 \\ 1000 & 1.344+01 \\ 1500 & 1.065+01 \\ 2000 & 9.445+0 C \\ 3000 & 8.562+C 0 \\ 4000 & 8.355+0 C \\ 5000 & 8.4 C 8+0 C \\ 6000 & 8.430+00 \\ 8000 & 8.786+00 \\ 10000 & 9.367+00 \\ 15000 & 1.034+01 \\ 20000 & 1.142+01 \\ 30000 & 1.287+01 \\ 40000 & 1.385+C 1 \\ 5000 C & 1.472+01 \\ 60000 & 1.524+C 1 \\ 80000 & 1.616+01 \\ 100000 & 1.694+01\end{array}$

$C O H$
$S C A T T$.
$1.588+00$
$1.016+0 Q$
$7.040-01$
$3.940-01$
$2.500 \rightarrow 01$
$1.124-01$
$6.361-02$
$2.844-02$
$1.605-02$
$1.029-02$
$7.154-03$
$4.030-03$
$2.582-03$
$1.149-03$
$6.465-04$
$2.877-04$
$1.620-04$
$1.033-04$
$7.124-05$
$3.946-05$
$2.479-05$

INCOH.
SCATT.

PHOTO

$1.776+C 1$

$1 .-62 s+C 1$

$1.332+C 1$

$1.199+01$

$9.763+c 0$

$8.33 t+00$
$t .5 t 1+C 0$

$5.477+00$

$4.727+C 0$

$4.178+00$

$3.416+C 0$

$2.152+0 \mathrm{C}$

$1.72 t+C O$

$1.255+C C$

$9.9 \in \varepsilon-C 1$

$7.157-C 1$

5.639401

$4.675-C 1$
EFFECT

$1.020+C 1$

$3.77+c c$

1.912100

$1.155+0 C$

$5.657-01$

$3.327-0 ?$
$1.789-01$

$1.198-01$

$8.777-02$

$6.575-C 2$

$4.853-c 2$

$2.370-02$

$1.74 C-C^{2}$

i. $125-02$

$8 \cdot 2 \in 2-C^{3}$

$5.345-C \equiv$

$3.924-C 3$

3. $C 27-03$
PAIR

$2.110-\mathrm{Cl}$

$7.126-C 1$

$2.746+C 0$

$3.5 E 3+C C$

$4.175+C C$
$5.318+00$

$6.41 \varepsilon+C C$

$8.1 \in 1+00$

$1.161+01$

$1.284+01$

$1.388+01$

$1.452+C 1$

$1.559+C 1$
$1.647+C 1$
INCOH.

PHOTO

EFFECT

$\begin{array}{cc}\text { KEV } & \text { TCTAL } \\ 400 & 3.064+01\end{array}$

$\begin{array}{ll}400 & 3.064+01 \\ 500 & 2.38 ?+01\end{array}$

$600 \quad 2.010+C 1$

$800 \quad 1.604+01$

$1000 \quad 1.378+01$

$\begin{array}{ll}1500 & 1.090+01 \\ 2000 & 5.655+0 \mathrm{C}\end{array}$

$3000 \quad 8.765 \cdot 00$

$4000 \quad 8.568+00$

$5000 \quad 8.625+00$

t000 $\quad 8.5 .54+0 \mathrm{C}$

10000

20000
30000

40000

$5000 \mathrm{C}$

80000
100000

ENEFOY

KEV

KEV
40
50

500

600
800

800
1000
1500

1500
2000

3000

$8.964+00$

$4000 \quad 3.774+C C$

$5000 \quad 8.842+C 0$

$\begin{array}{ll}6000 & 8.878+C C \\ 8000 & 9.272+0 C\end{array}$

10000

15000

20000

30000

40000
50000

60000

80000

100000

$1.055+01$

$1.211+01$

$1.367+01$

$1.471+01$

$1.565+01$

$1.621+01$

SCATT.

$1.080+00$

$1.807+01$

1. $\mathrm{C} 8 \mathrm{~S}+\mathrm{C}$

$\begin{array}{lll}1.075+00 & 1.658+C 1 & 6.180+0 C \\ 7.450-01 & 1.538+C 1 & 3.97 t+C C\end{array}$

$4.170-01 \quad 1.354+C 1$

2.650-01

$1.192-01$
$6.743-02$

3.015-02

1.701-02

$1.091-02$

$7.58,4-03$
$4.273-03$

2.737-03

1.219-03

6.854-04

$3.050-04$
$1.717-04$

$1.005-04$

$7.555-05$

$4.185-05$
$2.630-05$

$1.220+C 1$

S. $934+C C$

$6.617+C C$

$5.573+C 0$

$4.81 \mathrm{C}+\mathrm{CO}$

$4.251+C C$

$3.47 t+C C$

2.9 totco

$1.757+C C$

$1.277+C C$

$1 . C 14+C C$

$8.455-C 1$

$5.73 \varepsilon-61$

4. $761-C 1$

$2.080+0 \mathrm{C}$

1. $318+C C$

$t .23 t-C 1$

$3.6 \in 7-c 1$
$1.065-c$

$1.318-31$

$5.048-02$

$7.665-02$

$5.331-C_{2}$

$2.602-02$

$1.910-02$

$1.23 t-c^{2}$

$5.071-\mathrm{C}=$

$7.137-C^{3}$

$5.867-C^{2}$

$4.3 C 7-C^{3}$
$3.389-C^{3}$

INCOH.

PHOTC

SCATT.

SCATT.

EFFEC

$1.777+00$

$1.836+C 1$

$1.191+C 1$

7.880-01

i. $564+C 1 \quad 4.336+C C$

$1.377+012.245+C C$

$4.410-01$

2.800-01

$1.259-01$
$7.126-02$

$3.186-02$

$1.798-02$

$1.153-02$

$8.015-03$

$4.515-03$

2.893-03

$1.287-03$

$7.244-04$

$3.223-04$
$1.815-34$

$1.158-04$

$7.586-05$

$1.718+01 \quad 4.425-05$

$1.241+C 1$

$1.011+01 \quad t . t 40-C 1$

$\varepsilon .629+C C$

$5.72+0 C$

$4.893+C 0$

$4.325+00$

$3.537+C C$

$2.228+C C$

$1.737+C C$

$1.300+c 0$

$1.032+c c$

$\varepsilon \cdot \in C 5-C 1$

$5.839 \rightarrow C 1$

4.843-C1

$3.504-C 1$

$2.053-C 1$

$1.4 \mathrm{CO}-\mathrm{Cl}$

1. $024-C 1$

8. $135-c 2$

$4.256-c$

$2.759-C_{2}$

$2.026-02$

$1.310-02$

S. $618-03$

$7.567-C^{3}$

$6.221-C 3$

$4.567-C \equiv$
$3.593-C \equiv$

PAIR

PRCD.

$2.2 c 8-c$

$7.423-c 1$

$1.8 \mathrm{El+C}$

$3.7 C E+C O$

$4.318+C C$

$5.455+0$
$t .627+0 C$

$8.425+C C$

5. $\mathrm{C} \subseteq \mathrm{C}+\mathrm{CC}$

$1.1 c 8+c 1$

$1.4 \geq 3+01$

$1.4 c 5+C 1$

$1 . t C s+C 1$

$1.700+21$ PAIR

$1.531+C C$

$2.54 E+C C$

$4.4 E 4+C C$

$5.675+0 \mathrm{C}$

$6.835+00$

$8.652+00$

$1.030+C 1$

$1.236+C 1$

$1.3 \in 7+C 1$

$1.478+C 1$

$1.660+$

$1.753+C 1$

\begin{tabular}{|c|c|c|}
\hline \multicolumn{3}{|c|}{$\mathrm{Z}=E C$ NEDCYMIL'M } \\
\hline EAERGY & & $\mathrm{COH}$ \\
\hline KEV & TOTAL & SCATT. \\
\hline 1 & $2.21 \varepsilon+c t$ & $2.2 \in 4+C 3$ \\
\hline 1.5 & $1 . t 7 C+C E$ & $2.14 i+03$ \\
\hline 2 & $8.41 C+05$ & $2.025+03$ \\
\hline 3 & $2.553+05$ & $1.7 .88+03$ \\
\hline 4 & $1.40 \varsigma+05$ & $1.571+C 3$ \\
\hline 5 & 7.957404 & $1.382+03$ \\
\hline$t$ & $4.059+04$ & $1.224+03$ \\
\hline 8 & $1.015+C 5$ & $9.772+C 2$ \\
\hline 10 & $5.654+04$ & $7.5 \in 2+02$ \\
\hline 15 & $1.520+C 4$ & $5.1 \subseteq 3+c 2$ \\
\hline 20 & $8.8 \geq 3+0 \geq$ & $3.724+C 2$ \\
\hline 30 & $2 . \div 32+C 3$ & $2.172+02$ \\
\hline 40 & $1.341+C 3$ & $1.400+02$ \\
\hline 50 & $3.564+03$ & $9.6 c 4+01$ \\
\hline 60 & $2.467+C 3$ & 7. $C 84+C 1$ \\
\hline 80 & $1.152+03$ & $4.21 t+C 1$ \\
\hline 100 & $6.377+02$ & $2.798+C 1$ \\
\hline $15 \mathrm{C}$ & $2.251+02$ & $1.297+01$ \\
\hline $20 c$ & $1.145+02$ & $7.41 C+00$ \\
\hline$\equiv 00$ & $\equiv .15 t+C 1$ & $3.325+0 \mathrm{C}$ \\
\hline
\end{tabular}

INCEH.

PHCTC

SCATT.

$1.654+00 \quad 2.216+C 6$

$2.652+0 \mathrm{C} \quad 1.667+06$

$3.564+C 0 \quad 8.390+105$

$5.317+0 \mathrm{C} \quad 2.535+05$

$6.535+001.353+05$ 
$Z=E I$ PRONETHIUM

\begin{tabular}{|c|c|c|c|c|}
\hline NFRGY & & $\mathrm{COH}$ & INCCH. & РHOTC \\
\hline KEV & TOTAL & SCATT. & SCATT. & EFFECT \\
\hline 1 & t. $274+05$ & $2.344+03$ & $1.602 * 0 \mathrm{C}$ & $6.250+05$ \\
\hline 1.5 & 1. $\varepsilon C C+06$ & $2.226+0 \geq$ & $2.593+c 0$ & $1.798+C 6$ \\
\hline 2 & S.051+05 & $2.103+03$ & $3.50 \varepsilon+00$ & 9. $C 30+C 5$ \\
\hline 3 & $\geq .170+C 5$ & $1.8 \in 1+C ?$ & $5.27 \mathrm{C} * \mathrm{CC}$ & $3.152+05$ \\
\hline 4 & $1.510+05$ & $1.638+03$ & $6.899+00$ & $1.493+05$ \\
\hline 5 & $8.512+c 4$ & $1.442+03$ & $\varepsilon .388+00$ & $8 \cdot 367+C 4$ \\
\hline 6 & $5.340+04$ & $1.277+C 3$ & $5.711+00$ & $5.212+04$ \\
\hline 8 & $1.091+C 5$ & 1. $C 2 C+03$ & $1.195+01$ & $1.081+05$ \\
\hline 10 & $6.038+04$ & $8.308+02$ & $1.348+01$ & $5.954+04$ \\
\hline 15 & $2 . c 38+04$ & $5.4 C G+C 2$ & $1.724+C 1$ & $1.983+04$ \\
\hline 20 & $9.37 \varepsilon+03$ & $3.875+02$ & $1.55 t+01$ & $8 . c 71+03$ \\
\hline 30 & $3.132+03$ & $2.265+C 2$ & $2.333+C 1$ & $2.981+03$ \\
\hline 40 & $1.442 \div 03$ & $1.467+02$ & $2.51 c+01$ & $1.270+03$ \\
\hline 50 & $4.201+03$ & $1 . C 18+02$ & $2.607+01$ & $4.073+03$ \\
\hline 60 & $2.619+02$ & $7.454+C 1$ & $2.556+01$ & $2.518+C 3$ \\
\hline $8 \mathrm{C}$ & $1.225+03$ & $4.437+01$ & $2.675+01$ & $1.153+03$ \\
\hline 100 & $\epsilon .775+C 2$ & 2. $\varsigma 48+C 1$ & $2.645+01$ & $6.215+C 2$ \\
\hline 150 & $2.383+02$ & $1.369+01$ & $2.522+c 1$ & $1.954+02$ \\
\hline 200 & $1.207+02$ & $7.8<6+00$ & $2.365+C 1$ & $9.921+01$ \\
\hline 300 & $5.399+01$ & 2. $518+0 \mathrm{C}$ & 2. $c s s+0 l$ & $2.538+01$ \\
\hline
\end{tabular}

\begin{tabular}{|c|c|c|}
\hline \multicolumn{3}{|c|}{$Z=E 2$ SAMARIUM } \\
\hline ENEREY & & $\mathrm{CGH}$ \\
\hline KEV & TOTAL & SCATT. \\
\hline 1 & $t \cdot t ? t+c 5$ & $2.4 \overline{26}+03$ \\
\hline 1.5 & $1.7 E \varepsilon+C 6$ & $2.3 C 7+C 3$ \\
\hline 2 & $5.762+C 5$ & $2.182+03$ \\
\hline 3 & $3.355+05$ & 1. $53 t+03$ \\
\hline 4 & $1.60 c+05$ & $1.70 t+03$ \\
\hline 5 & C. $C \geqq \epsilon+C 4$ & $1.5 \mathrm{C} 4+\mathrm{C} 3$ \\
\hline$\leqslant$ & $5.652+04$ & $1,333+03$ \\
\hline 8 & $1.148+C 5$ & $\therefore C 64+03$ \\
\hline $1 \mathrm{C}$ & $\epsilon .379+04$ & $\varepsilon \cdot t \in \subseteq+C 2$ \\
\hline 15 & $2.170+04$ & $5.534+02$ \\
\hline $2 \mathrm{C}$ & $1 . C 05+04$ & 4. $c 32+c 2$ \\
\hline 30 & $3.384+03$ & $2.3 \in 7+02$ \\
\hline $4 \mathrm{C}$ & $1.56 \varepsilon+C ?$ & $1.527+C 2$ \\
\hline 50 & $4.461+C 3$ & 1. $C 7 C+02$ \\
\hline$\in \mathrm{C}$ & $2.772+c ?$ & 7. $\varepsilon<1+01$ \\
\hline 80 & $1.29 z+C z$ & $4.66 c+C 1$ \\
\hline 100 & $7 \cdot 135+02$ & 3. $1 C 5+C 1$ \\
\hline 150 & $2.5 C \epsilon+C_{2}$ & $1.444+01$ \\
\hline 200 & $1.2 \in 7+02$ & $\varepsilon .2 t 4+C C$ \\
\hline 300 & $5.627+01$ & $3.718+00$ \\
\hline
\end{tabular}

INCCH.

$1.521+C \mathrm{C}$

$2.49 \varepsilon+00$

$3.418+00$

$5.1 \subseteq 1+C 0$

\&. $334+C C$

$9.674+00$

$1.19 t+01$

$1.359+01$

$2.017+01$

$2.361+01$

$2.543+01$

$2.641+01$

$2.693+01$

$2.713+\mathrm{Cl}$

$2.684+01$

$2.561+01$

$2.402+01$

$2.132+01$

$\mathrm{PHCTC}$

EFFECT

$6.672+C 5$

$1.76 t+C t$

$9.740+C 5$

$3.376+C 5$

$1.592+05$

$8.885+04$

$5.517+C 4$
$1.137+C 5$

$6.291+04$

$2.112+C 4$

$9.622+c 3$

$3.123+03$

$1.289+c 3$

$4.328+03$

$2.667+03$

$6.556+C 2$

$2.106+02$

$9.439+C 1$

\begin{tabular}{|c|c|c|}
\hline$\triangle \triangle E R G Y=$ & EUR & $\mathrm{COH}$ \\
\hline$E V$ & TOTAL & $S C \triangle T T$ \\
\hline & $7.154+C 5$ & $2.5 C c+C 3$ \\
\hline 1.5 & $1.92 !+0 \epsilon$ & $2.3 \subseteq 0+C 3$ \\
\hline 2 & 1. $c t c+c t$ & $2 \cdot 2+3+03$ \\
\hline 3 & $3.685+05$ & $2.012+03$ \\
\hline 4 & $1.735+05$ & $1.777+C 3$ \\
\hline 5 & $5.69 t+C 4$ & $1.5+8+C z$ \\
\hline$t$ & E. $C 4 C+04$ & $1.39 c+03$ \\
\hline$\varepsilon$ & $1.06 \%+C 5$ & $1.116+03$ \\
\hline 10 & $\epsilon .84 i+04$ & C. $c 43+c 2$ \\
\hline 15 & $2.317+04$ & $5.8 \in 8+02$ \\
\hline 20 & $1 . C 71+04$ & $4.1 \subseteq 4+C 2$ \\
\hline 30 & $z . \leqslant 17+03$ & $2.4 E \mathrm{E}+02$ \\
\hline $4 C$ & $1.693+03$ & $1.6 C ?+02$ \\
\hline 50 & $4.712+03$ & $1.1<2+02$ \\
\hline$\epsilon 0$ & $2.040+92$ & $9.242+C 1$ \\
\hline 80 & $1.37 t+03$ & $4.5 C P+C ?$ \\
\hline 100 & $7.619+02$ & Z. $2 \in \varepsilon+01$ \\
\hline$=0$ & $2 \cdot 675+02$ & $1.522+C 1$ \\
\hline $2 \mathrm{CC}$ & $1.347+02$ & $\varepsilon .71 c+00$ \\
\hline 300 & $5.924+01$ & $3.525+C C$ \\
\hline
\end{tabular}

INCCH

$1.418+00$

$2.374+0 C$

$3.29 \varepsilon+00$

$5.082+00$

$6.741+00$

9. $605+00$

I. $154+01$

1. $390+01$

1. $777+01$

$2.06 C+C 1$
$2.401+01$

$2.401+01$
$2.582+01$

$2.680+01$

$2.732+01$

$2.752+01$

2. $724+C 1$

$2.500+0$ ?

$2.44 \mathrm{C}+01$
PHCTC

EFFFCT

$1.920+C b$

$1.0 \in 7+06$

$3.665+C 5$

$1.717+C 5$

$5.900+04$

$1.058+05$

$6.755+C 4$

$1.027+C 4$

$3.346+C 3$

$1.496+C 3$

$4.573+C$

$1.300+03$

$7.020+02$

$2.263+c^{2}$

1. $C 16+C 2$

$\begin{array}{cc}\text { ENEFGY } & \\ \text { KEV } & \text { TCTAL } \\ 400 & 3.474+01 \\ 500 & 2.653+01 \\ 600 & 2.208+01 \\ 800 & 1.733+01 \\ 1000 & 1.478+01 \\ 1500 & 1.1 \in 1+01 \\ 2000 & 1.029+01 \\ 3000 & 9.375+0 C \\ 4000 & 5.198+0 C \\ 5000 & 9.287+0 C \\ 6000 & 9.337+00 \\ 8000 & 9.769+C C \\ 10000 & 1.044+01 \\ 15000 & 1.157+C 1 \\ 20000 & 1.282+C 1 \\ 30000 & 1.449+01 \\ 40000 & 1.560+C 1 \\ 50000 & 1.660+01 \\ 60000 & 1.720+C 1 \\ 80000 & 1.824+C 1 \\ 100000 & 1.913+01\end{array}$

EAEFGY$$
40
$$

600

1000

1500

$4000 \quad 9.414+00$

5000

6000

8000

$1 \mathrm{CONO}$

15000

20000

30000

40000

50000

60000

80000

10000 r

ENERGY

KEV

400
500
500

500
800

1000

1500

2000
3000

3000
4000

5000

6000

9000

10000

15000

20000

30000

40000

50000

60000

80000

100000

$0.5 \in G+C 0$

$1.002+C 1$

$1.189+C$

$1.317+01$

$1.491+01$

$1.6 \mathrm{C} 5+\mathrm{Cl}$

$1.709+01$

$1.770+C 1$

$1.970 . \mathrm{Cl}$

TCTAL

$3.762+C I$

$2.843+01$

$2.34 \varepsilon+C 1$

$1.328+C 1$

$1.212+01$

1. $C 73+\mathrm{C}$

$9.79 t+00$

$9.633+00$

$9.742+0 \mathrm{C}$

c. $8 \mathrm{C} 5+\mathrm{CC}$

$1.028+0$ :

$1.059+01$

$1.221+c 1$

$1.354+C$

$1.532+C 1$

1.759 .0

$1.821+01$

$1.932+0$

$2.027+01$

COH
SCATT.
$1.985+00$
$1.27 C+00$
$8.800-01$
$4.930-01$
$3.130-01$
$1.408-01$
$7.967-02$
$3.563-02$
$2.010-02$
$1.289-02$
$8.962-03$
$5.049-03$
$3.2 \equiv 5-03$
$1.440-03$
$8.101-04$
$3.605-04$
$2.030-04$
$1.295-04$
$8.935-05$
$4.952-05$
$3.113-05$

3. $113-05$

INCOH.
SCATT:
$1.95 C+C 1$
$1.743+C 1$
$1.617+01$
$1.423+C 1$
$1.283+C 1$
$1.045+C 1$
$8.923+C O$
$7.023+C C$
$5.863+C D$
$5 . C 6 C+C C$
$4.472+C C$
$3.657+C C$
$3.114+C C$
$2.3 C 3+C C$
$1.848+C C$
$1.344+C C$
$1.067+C O$
$8.859-C 1$
$7.662-C 1$
$6.037-C 1$
$5 . C C 8-C 1$

$\mathrm{COH}$

$S C A T T$.
$2.097+0.0$

1

SCATT.

1. $C \geq 0+C 1$

$1.771+C$

$\begin{array}{ll}5.300-01 & 1.643+C 1 \\ 5.210-01 & 1.447+C 1\end{array}$

$3.310-01$

1. $489-01$

$8.426-02$

$3.7 \in 8-02$

$2.12 t-02$

$1.363-02$

$1.071+C 1$

C.479-0

$3.421-03$

$1.523-03$

ع. $56 ?-04$

$3.812-04$

2.147-04

$1.370-04$

$0.454-05$

$3.245-05$

1. $C \in \bar{Z}+C$

S. $C 65+C 0$

$7.13 \varepsilon+C 0$

$5.9 \in 0+C O$

$5.143+O C$

$4 \cdot 545+C C$

$3.717+C 0$
$3.105+C C$

$2.341+C C$

$1.878+\mathrm{CC}$

$1.3 t t+C 0$

$1.084+C C$

9. $C 45-C 1$

$7.788-C 1$

6. $13<-0$

ENERGY

PHCTC

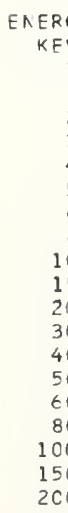

ERGY
$K E V$
1
1.5
2
3
4
5
$\epsilon$
8
10
15
20
30
40
$5 C$
60
80
100
150
$2 C 0$
$3 C 0$

\begin{tabular}{|c|c|}
\hline & \\
\hline & \\
\hline $.65 \varepsilon+05$ & $2.5^{c} 1+C 3$ \\
\hline $.4 G L+D E$ & $2.4 t \epsilon+03$ \\
\hline $0 \leq c+c 6$ & $2.323+03$ \\
\hline $82 c+0=$ & $2.073+03$ \\
\hline$\varepsilon 17+C 5$ & 1. $832+C 3$ \\
\hline & $1.619+03$ \\
\hline & $1.437+C 3$ \\
\hline 5 & $1.150+C 3$ \\
\hline $245=+$ & $=381+02$ \\
\hline $45 \varepsilon+04$ & $6 . C 8 G+C 2$ \\
\hline $13 \theta+C 4$ & $4.348+02$ \\
\hline & $2.5 \in 7+02$ \\
\hline +03 & $1.67 c+02$ \\
\hline $5+c$ & $1.175+C 3$ \\
\hline & $\varepsilon .645+01$ \\
\hline & $5.14 c+$ \\
\hline+ & $3.431+$ \\
\hline 78. & 1 \\
\hline $20+0$ & \\
\hline & \\
\hline
\end{tabular}

SCATT. EFFECT

$1.4 \mathrm{C} 4+0 \mathrm{C} \quad 7.632+05$

$2.351+C 0 \quad 1.489+06$

$3.271+0 \mathrm{C} \quad 1.097+06$

5.C5t+CC $3.809+05$

$6.721+001.798+C 5$

$\varepsilon .235+001.005+05$

c. $6 \mathrm{C} t+00 \quad 6.245+04$

$\begin{array}{ll}1.195+01 & 1.118+05\end{array}$

$1.377+01 \quad 7.15 \mathrm{C}+04$

$1.771+\mathrm{Cl} \quad 2.355+\mathrm{C} 4$

$2.414+01 \quad 3.572+c 3$

$\begin{array}{ll}2.414+01 & 3.572+C 3 \\ 2.605+01 & 1.601+03\end{array}$

$2.71 \mathrm{C}+\mathrm{Cl} \quad 8.553+02$

$2.765+C 1 \quad 3.070+C 3$

$2.788+01 \quad 1.416+03$

$2.7 \epsilon 1+01 \quad 7.643+02$

$2.639+01 \quad 2.446+02$

$2.477+01 \quad 1.089+C 2$

$2.159+01 \quad 3.568+C 1$
$\mathrm{COH}$

$\begin{array}{ccc}\text { COH } & \\ T C T \Delta L & S C A T T . \\ 3.893+01 & 2.333+00 & 1 \\ 2.928+01 & 1.494+00 & 1 \\ 2.413+C 1 & 1.035+00 & 1 \\ 1.873+C 1 & 5.800-01 & 1 \\ 1.587+01 & 3.690-01 & 1 \\ 1.239+01 & 1.650-01 & 1 \\ 1.096+C 1 & 9.395-02 & 9 \\ 1.001+C 1 & 4.202-02 & 7 \\ 9.855+00 & 2.371-02 & 6 \\ 9.974+0 C & 1.520-02 & 5 \\ 1.004+01 & 1.057-02 & 4 \\ 1.053+01 & 5.956-03 & 3 \\ 1.127+01 & 3.815-03 & 3 \\ 1.253+C 1 & 1.698-03 & 2 \\ 1.390+01 & 9.555-04 & 1 \\ 1.575+C 1 & 4.251-04 & 1 \\ 1.697+01 & 2.394-04 & 1 \\ 1.807+01 & 1.529-04 & 9 \\ 1.872+01 & 1.055-04 & 8 \\ 1.987+01 & 5.848-05 & 6 \\ 2.094+01 & 3.678-05 & 5\end{array}$

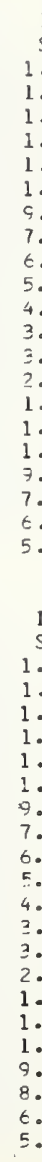

SCATT:

$.759+C 1$

.$\leq E s+C 1$

$.471+C 1$

$.325+C 1$

$.079+01$

$.21 \leqslant+c c$

$.254+C \mathrm{C}$

. $0 E E+C C$

$227+00$

$t \div c+c 0$

$.778+C 0$

$.217+C C$

$.390+C C$

$1.9 \mathrm{CS}+\mathrm{CC}$

$.1 C 2+00$
$1 C 2$

$\exists .1 \subseteq 2-C 1$

$7.915-c i$

5. $174-\mathrm{C} 1$

INCOH.

SCATT:

$0 c_{1+C}$

$.82 \varepsilon+C 1$

$\epsilon^{4} 6+c 1$

$.453+C 1$
$.346+C 1$

$.05 t+C ?$

$3 \in 2+C C$

$.36 \subseteq+C C$

$.153+C C$

$.31 \mathrm{C}+\mathrm{CC}$

$\varepsilon \subseteq 2+C C$

8 a $\varepsilon+C 0$

$2 \epsilon \varepsilon+C C$

$.417+C C$

$.41 C+C C$

$.120+C C$

$.33 \varepsilon-C 1$

$8.041-01$

$5.25 t-c 1$

PHOTS

EFFECT

$1.377+C 1$

$1.83+c c$
$5.03 z+c C$

$2.61 C+C C$

$1.633+O C$

$7.7: 7-C_{1} \quad 2.515-C 1$

$4.534-C 1 \quad 8.36 \mathrm{C}-01$

$0.425-C 1 \quad 2 . C 74+C C$

$1.61 C-01 \quad 3.153+C C$

$1.183-01 \quad 4 . C C t+C C$

$9.393-C 2 \quad 4.7 t 2+C C$

$6.523-C_{2} \quad t . C 42+C C$

4. ${ }^{\circ} 1 \in-C 2 \quad 7.271+C C$ 


\begin{tabular}{|c|c|c|c|c|}
\hline \multicolumn{5}{|c|}{$Z=E 5 T E R B I U M$} \\
\hline ENEREY & & $\mathrm{COH}$ & I NCOH. & PHOTO \\
\hline$K E V$ & TOTAL & SCATT. & SCATT. & EFFECT \\
\hline 1 & $8.214+C 5$ & $2.69 \mathrm{C}+03$ & $1.350+00$ & $8.187+05$ \\
\hline 1.5 & $1.5 t S+C t$ & $2.558+C 3$ & $2.330+00$ & $1.5 t 6+c t$ \\
\hline 2 & $1 . \geqslant 19+C t$ & $2.428+c 3$ & $3.24 t+00$ & $1.216+0 t$ \\
\hline 3 & $4.191+C 5$ & $2.1 \in c+03$ & $5.032+00$ & $4.169+05$ \\
\hline 4 & $1.970+25$ & 1. $c 23+03$ & $6.703+00$ & $1.551+05$ \\
\hline 5 & 1. Cबs+05 & $1.7 C 1+03$ & $8.225+00$ & $1.082+05$ \\
\hline t & $t .83 c+04$ & $1.5 C s+03$ & $c 4+00$ & $6.687+04$ \\
\hline q & $8 \cdot 6 C 9+04$ & $1 \cdot 2 C 7+C_{3}$ & $7+C 1$ & $7+04$ \\
\hline 10 & $5+0$ & $9.8 \geq 5+02$ & +01 & $5+04$ \\
\hline $1=$ & $2 \cdot t ? c+c 4$ & $6.3 t 4+C 2$ & $\mathrm{~S}+\mathrm{Cl}$ & $2.574+C 4$ \\
\hline $2 \mathrm{C}$ & $1.223+04$ & $4.535+C 2$ & $2.079+01$ & $1.176+04$ \\
\hline 30 & $4.137+C 3$ & $2 \cdot t 80+02$ & $2.442+01$ & $3.845+03$ \\
\hline 45 & $3.926+03$ & $1.75 \varepsilon+C 2$ & $2.637+01$ & $1.723+03$ \\
\hline 50 & $1 . C>1+0]$ & $1.234+02$ & $2.744+\mathrm{Cl}$ & $9.190+02$ \\
\hline$\triangle C$ & $3.247+03$ & $C . C \subseteq 7+C 1$ & $2.801+01$ & $3.128+c 3$ \\
\hline $9 \mathrm{C}$ & $1.528+C 3$ & $5.416+01$ & $6+01$ & $1.446+03$ \\
\hline $10 \mathrm{C}$ & $E .4 \& C+C 2$ & $3.613+01$ & $2.8 \mathrm{CC}+01$ & $7.839+02$ \\
\hline 150 & $2.980+02$ & $1.68 \varsigma+C_{1}$ & $2.678+01$ & $2.543+02$ \\
\hline $2 \mathrm{CC}$ & $1.455+C 2$ & $5.689+C C$ & $2.514+C 1$ & $1.146+02$ \\
\hline 390 & $6.485+01$ & $4 \cdot 367+00$ & $2.233+01$ & 3. $815+C 1$ \\
\hline
\end{tabular}

\begin{tabular}{|c|c|c|c|c|}
\hline EA FRGY & & $\mathrm{COH}$ & INCCH. & PHOTC \\
\hline KEV & TOTAL & SCATT. & SCATT. & EFFECT \\
\hline 1 & $E .736+C 5$ & $2.7 \in 7+03$ & $1.37 t+C 0$ & $8.708+05$ \\
\hline 1.5 & $1.7 \varepsilon c+c t$ & $2 \cdot t 45+C 3$ & $2.311+00$ & $1.787+0 t$ \\
\hline $\bar{z}$ & $1.225+c t$ & $2.513+C 3$ & $3.223+00$ & $1.222+c 6$ \\
\hline 3 & $\angle . \angle 82+0 E$ & $2.250+03$ & $5.011+00$ & $4.460+C 5$ \\
\hline 4 & 2. $C S S+C 5$ & $1.958+03$ & $6.686+0 \mathrm{C}$ & $2.079+C 5$ \\
\hline 5 & $1.168+05$ & $1.770+03$ & $E .22 \mathrm{C}+0 \mathrm{O}$ & $1.150+05$ \\
\hline 6 & $7.24 c+04$ & $1.572+03$ & $9.605+0 \mathrm{C}$ & 7. $C S ?+C 4$ \\
\hline a & c. $207+04$ & $1.258+03$ & $1.1 s s+01$ & $9.080+C_{4}$ \\
\hline 10 & F. $257+04$ & 2. $C 25+03$ & $1.4 C_{3}+01$ & $8.153+0<$ \\
\hline 15 & $2.758+C 4$ & E. $\in 2 \mathbf{2 7 + 0 2}$ & $1.8 C 8+C 1$ & $2.720+04$ \\
\hline 20 & $1.2 c c+c 4$ & $4.715+02$ & $2.102+01$ & $1.240+C 4$ \\
\hline $3 \mathrm{C}$ & $4.369+03$ & $2.79 c+02$ & $2.469+01$ & $4.065+03$ \\
\hline 40 & $2.041+03$ & $1 . E 37+C 2$ & $2.669+01$ & $1.831+c 3$ \\
\hline 50 & $1.135+03$ & $1.2 \div 2+02$ & $2.778+C 1$ & c. $823+02$ \\
\hline$\in \mathrm{C}$ & $3.424+03$ & $5.547+C 1$ & $2.836+C 1$ & $3.3 \mathrm{CO}+03$ \\
\hline $8 \mathrm{C}$ & $1.613+C 3$ & $5 . t 83+01$ & $2.864+c 1$ & $1.527+03$ \\
\hline 100 & ค. $550+02$ & $3.755+C 1$ & $2.838+C 1$ & $8.255+c 2$ \\
\hline $15 \mathrm{C}$ & $3.14 \varepsilon+02$ & $1.777+01$ & $2.71 t+01$ & $2.699+02$ \\
\hline $2 \mathrm{CC}$ & $1.57 t+C 2$ & $1 . c 2 c+c 1$ & $2.551+01$ & $1.210+C 2$ \\
\hline $3 \mathrm{C}(1$ & $6.7 c 5+01$ & $4 \cdot C C C+O C$ & $2.267+01$ & $4.058+01$ \\
\hline
\end{tabular}

\begin{tabular}{|c|c|c|c|c|}
\hline $7=$ & $67+5 L M I L M$ & & & \\
\hline EAER\&Y & & $\mathrm{CDH}$ & INCOH. & PHCTC \\
\hline KEV & TITAL & SCATT & SCATT. & EFFECT \\
\hline 1 & c. $33=8+C 5$ & $2.85 t+C 3$ & $1.362+00$ & $9.310+05$ \\
\hline 1.5 & 1. $c 2 \varepsilon+C 6$ & $2.733+C^{2}$ & $2.253+0 \mathrm{C}$ & 1. $525+06$ \\
\hline 2 & $1.31 \varepsilon+c t$ & $2 \cdot t C C+C 3$ & $3.2 C 3+00$ & $1.315+C 8$ \\
\hline 3 & $4.772+C 5$ & $2.333+03$ & $4.59 \mathrm{C}+00$ & $4.749+C 5$ \\
\hline 4 & $-2.218+05$ & $2 . C 75+C 3$ & $6.671+00$ & $2.197+05$ \\
\hline 5 & $1.227+C=$ & $1.841+C 3$ & $8.212+c 0$ & $1.208+05$ \\
\hline t & $7.58 c+04$ & $1.6 \equiv t+03$ & $5.6 C z+00$ & $7.415+04$ \\
\hline 8 & $2.5 c^{2}+c^{2}$ & $1.311+03$ & $1.2 C 2+C 1$ & $3.431+C 4$ \\
\hline 10 & $8.854+C 4$ & i. $c \in S+C 3$ & $1.417+C 1$ & $3.74 t+04$ \\
\hline 15 & $2.5 \in 3+04$ & $6.5(0+02$ & $1.827+C 1$ & $2.852+04$ \\
\hline 20 & $1.370+C 4$ & $4.902+02$ & $2.125+01$ & $1.319+c 4$ \\
\hline 30 & $4.673+03$ & $2.5 \mathrm{C} 3+02$ & $2.497+01$ & $4.358+03$ \\
\hline 40 & $2.205+C 3$ & $1 . c 17+02$ & $2.701+C 1$ & 1. $\mathrm{set}+\mathrm{C}_{3}$ \\
\hline 50 & $1.243+03$ & $1.352+C 2$ & $2.312+0 ?$ & 1. $c 80+03$ \\
\hline 60 & $3.674+03$ & 1. $\mathrm{CCl}+\mathrm{C}_{2}$ & $2.872+01$ & $3.545+03$ \\
\hline$\therefore C$ & $1.752+C 3$ & $5.957+C 1$ & $2.501+01$ & $1.6 \in 4+03$ \\
\hline 100 & $c .754+02$ & $3 . \subseteq 81+01$ & $2.877+02$ & $9.068+02$ \\
\hline 150 & $3.400+02$ & 1. $\varepsilon 67+C 1$ & $2.755+C 1$ & $2.938+02$ \\
\hline 200 & $1 . t \otimes 2+c 2$ & $1 . c 72+c 1$ & $2.538+01$ & $1.316+02$ \\
\hline 300 & $7.12 C+01$ & $4.53 c+00$ & $2.301+C 1$ & $4 \cdot 3=5+01$ \\
\hline
\end{tabular}

\begin{tabular}{|c|c|c|c|c|}
\hline $7=$ & ER 8 IUN & & & \\
\hline AEFGY & & $\mathrm{CCH}$ & I NCGH. & РНСТС \\
\hline KEV & TOTAL & SCATT. & SCATT. & EFFECT \\
\hline 1 & $1.021+0 t$ & 2. $C_{4} 5+C 3$ & $1.348+00$ & $1.018+C 6$ \\
\hline I. 5 & $2.023+c t$ & $2.822+C 3$ & $2.277+0 C$ & $2.020+06$ \\
\hline 2 & $1.252+0 t$ & $2 . t 88+03$ & $3.184+O C$ & $.250+06$ \\
\hline 3 & 5. C $C 5 C+05$ & $2.417+03$ & $4.972+O C$ & $025+C 5$ \\
\hline 4 & $2.349+05$ & $2.154+03$ & $t .65 t+0 c$ & $2.327+05$ \\
\hline 5 & $1.3 C O+C 5$ & 1. $)_{14+03}$ & $E .206+0 \mathrm{C}$ & $1.281+05$ \\
\hline 6 & $\varepsilon .03 t+04$ & $1.7 C 3+03$ & c. $513+00$ & $7.864+04$ \\
\hline ?ִ & $2.780+04$ & $1.365+03$ & $1.2 \mathrm{C} 5+\mathrm{Cl}$ & $3.642+04$ \\
\hline 10 & 5.58 & $1.113+03$ & +01 & +04 \\
\hline 15 & $\equiv .1 \in 7+C 4$ & $7.184+02$ & 1.8 & $3.093+04$ \\
\hline 20 & $1.450+04$ & 5. $C \subseteq 5+C 2$ & $8+01$ & $1.397+C 4$ \\
\hline 30 & $4.879+c 3$ & 3. $C 1 S+C_{2}$ & $2.525+C ?$ & $4.552+c 3$ \\
\hline 40 & $2.281+03$ & $1.95 c+02$ & $2.732+c 1$ & 2. $C 54+03$ \\
\hline $5 C$ & $1.277+03$ & $1.414+C 2$ & $2.84 t+C 1$ & $1.1 C ?+C 3$ \\
\hline so & $3.831+C 3$ & 1. $c 49+02$ & $2.908+01$ & $3.6 \div 7+03$ \\
\hline 80 & $1.804+C 3$ & $6.23 t+01$ & $2.939+0 !$ & $1.7 ! 2+C 3$ \\
\hline $10 \mathrm{C}$ & $1.002+C 3$ & $4.171+C 1$ & $2.915+01$ & $5.310+02$ \\
\hline $15 \mathrm{C}$ & $3.517+C 2$ & 1. $c 58+01$ & $2.794+01$ & $3.042+c 2$ \\
\hline $2 \cap C$ & $1.754+C 2$ & $1.12 t+01$ & $2.625+01$ & $1.379+02$ \\
\hline 300 & $7.468+01$ & 5. $C E 2+O C$ & $2.334+0 i$ & $4.626+c 1$ \\
\hline
\end{tabular}

$\begin{array}{rc}\text { ENERGY } & \\ \text { KEV } & \text { TCTAL } \\ 400 & 4.069+01 \\ 500 & 3.044+01 \\ 600 & 2.497+C 1 \\ 800 & 1.925+01 \\ 1000 & 1.625+01 \\ 1500 & 1.264+01 \\ 2000 & 1.119+01 \\ 3000 & 1 . C 23+01 \\ 4000 & 1.0 C 8+C 1 \\ 5000 & 1.021+01 \\ 6000 & 1.028+01 \\ 8000 & 1.080+01 \\ 10000 & 1.155+C 1 \\ 15000 & 1.286+01 \\ 20000 & 1.427+C 1 \\ 30000 & 1.618+01 \\ 40000 & 1.744+C 1 \\ 50000 & 1.857+C 1 \\ 60000 & 1.524+C 1 \\ 80000 & 2 . C 42+01 \\ 100000 & 2.143+01\end{array}$

COH
SCATT
$2.465+00$
$1.578+00$
$1.074+00$
$6.130-01$
$3.900-01$
$1.755-01$
$9.930-02$
$4.441-02$
$2.506-02$
$1.507-02$
$1.117-02$
$6.295-03$
$4.033-03$
$1.795-03$
$1.010-03$
$4.494-04$
$2.531-04$
$1.616-04$
$1.115-04$
$6.184-05$
$3.890-05$

$\begin{array}{rc}\text { ENEREY } & \\ \text { KEV } & \text { TCTAL } \\ 400 & 4.235+01 \\ 500 & 3.152+C 1 \\ 600 & 2.575+C 1 \\ 800 & 1.977+C 1 \\ 1000 & 1.664+01 \\ 1500 & 1.291+01 \\ 2000 & 1.142+01 \\ 3000 & 1.045+01 \\ 4000 & 1.031+01 \\ 500 C & 1 . C 45+C 1 \\ 6000 & 1.053+C 1 \\ 8000 & 1.1 C 6+01 \\ 10000 & 1.184+C 1 \\ 15000 & 1.319+C 1 \\ 20000 & 1.464+01 \\ 30000 & 1.662+01 \\ 40000 & 1.791+C 1 \\ 50000 & 1.9 C 8+01 \\ 60000 & 1.077+01 \\ 80900 & 2.098+01 \\ 100000 & 2.202+C 1\end{array}$

COH
SCATT.
$2.597+00$
$1.663+00$
$1.152+00$
$6.460-01$
$4.110-01$
$1.840-01$
$1.047-01$
$4.681-02$
$2.641-02$
$1.694-02$
$1.178-02$
$6.636-03$
$4.251-03$
$1.802-03$
$1.065-03$
$4.737-04$
$2.668-04$
$1.704-04$
$1.176-04$
$6.520-05$
$4.102-05$

$\begin{array}{cc}\text { ENERGY } & \\ \text { KFV } & \text { TCTAL } \\ 400 & 4.392+01 \\ 500 & 2.251+01 \\ 600 & 2.650+C 1 \\ 800 & 2.029+C 1 \\ 1000 & 1.707+01 \\ 2500 & 1.320+01 \\ 2000 & 1.166+C 1 \\ 3000 & 1.068+C 1 \\ 4000 & 1.054+01 \\ 5000 & 1.065+C 1 \\ 6000 & 1 . C 78+C 1 \\ 80 C 0 & 1.133+C 1 \\ 10000 & 1.213+01 \\ 1500 C & 1.352+01 \\ 20000 & 1.502+C 1 \\ 30000 & 1.705+01 \\ 40000 & 1.339+01 \\ 50000 & 1.959+01 \\ 60000 & 2.030+01 \\ 90000 & 2.155+01 \\ 10000 C & 2.261+C 1\end{array}$

$1.103-0$

$4.932-02$

2.783-02

1. $785-02$

$1.241-02$

$6.992-03$

$4.470-03$
$1.994-03$

$1.122-0$

$4.991-04$

2. $311-04$

$1.796-04$

$1.237-04$

$4.324-0$

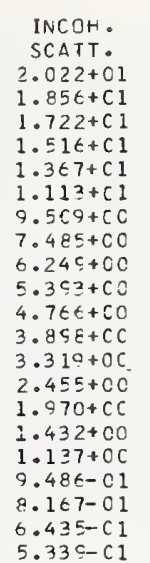

INCOH.

SCATT

1

$9.65 . t+C C$
$7.6 C 1+C C$

$6.34 t+C$

$5.477+C O$

$4.84 C+C C$

$3.95 C+C C$

$3.371+C C$
$2.453+C C$

$2.453+C C$

2. $0 C C+\hat{C}$

?. $155+C$

$9.032-C 1$

8. $254-C$

$6.534-C .1$
$5.422-01$

$\mathrm{INCOH}$

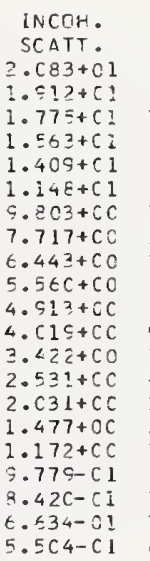

5. 5 C $4-C$
2. C $52+C$ ?

EFFECT

$1.801+C 1$

$1.030+C 1$

$6.653+C C$

$3.478+0 \mathrm{C}$

$2.18 C+0 \mathrm{C}$

$1.033+0 \mathrm{C}$

. $c \in 0-C 1$

3. $222-C 1$

$2.145-01$
$1.565-01$

$1.240-C$

\&. 5 st-C 2

$t .4 t \varepsilon-C 2$

$4.1 \& 3-C 2$

2. $07 \mathrm{C}-\mathrm{C}$

$1.9 \& t-02$

$1.457-c$

$1.14 t-02$

$5.424-C$

t.917-c

$2.978-\mathrm{C} 1$

$2.277+C C$

3. $5 \subseteq 0+C C$

$5 \cdot 3 \varepsilon 4+C$

6. $8 \mathrm{CO}+\mathrm{CC}$

8. $167+\mathrm{CC}$

1. $227+C 1$

$1.473+01$

$1.625+c 1$

1. $7 \in 1+C 1$

1. $577+C 1$

2. $0 E S+C$ :

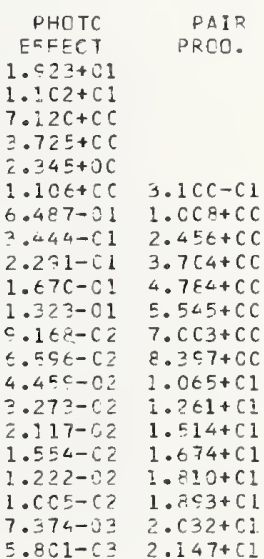

PHOTC

FAI:

$2.732+0 C \quad 2 . C 83+C 1 \quad 2 . C 3 t+C 1$

$1.750+00 \quad 1.1612+C 1 \quad 1.01$

$1.212+100 \quad 1.775+C 1 \quad 7.534+C C$

4.330-01 $1.409+C 1 \quad 2.54 t+C C$

7. $c 38-01$

3. $731-C I$

$2.48 C-C 1$

1. $8 C 7-61$

$1.431-01$

9. $\$ 10-C 2$

$7.451-C 2$

$4.818-C 2$

$3.53 t-c 2$

$1.678-02$

1. $32 \mathrm{C}-\mathrm{C}=2$

1. $085-\mathrm{C} 2$

$7.5 \leq 6-6$

$3.22 t-c 1$

2. $537+C C$

3. $819+C C$

., $28+C C$

$7.2 C 2+C C$

E. $E=C+C C$

1. $\mathrm{CC}_{4}+\mathrm{Cl}$

1. $2 \subseteq 5+\mathrm{C} 1$

$1.555+C !$

1. $85 \mathrm{C}+\mathrm{C} 1$

1. $C 45+C 1$

2. $C E 7+C 1$

$\begin{array}{rcc}\text { ENEREY } & \text { TCTAL } & \text { SCATT } \\ \text { KEV } & \text { SCAT } \\ 400 & 4.555+C 1 & 2.870+00 \\ 500 & 3.383+01 & 1.839+00 \\ 600 & 2.743+C 1 & 1.274+0 C \\ 800 & 2.084+C 1 & 7.150-01 \\ 1000 & 1.743+01 & 4.540-01 \\ 1500 & 1.345+C 1 & 2.043-01 \\ 2000 & 1.189+01 & 1.15 t-C 1 \\ 3000 & 1.050+01 & 5.172-02 \\ 4000 & 1.077+01 & 2.918-02 \\ 5000 & 1.092+C 1 & 1.872-02 \\ 6000 & 1.102+01 & 1.301-02 \\ 8000 & 1.150+01 & 7.322-03 \\ 10000 & 1.242+C 1 & 4.597-03 \\ 15000 & 1.38 t+C 1 & 2.091-03 \\ 20000 & 1.540+C 1 & 1.176-03 \\ 30000 & 1.749+C 1 & 5.234-04 \\ 40000 & 1.887+01 & 2.948-04 \\ 50000 & 2.010+C 1 & 1.883-04 \\ 60000 & 2.084+C 1 & 1.300-04 \\ 80 C 00 & 2.212+C 1 & 7.207-05 \\ 100000 & 2.321+01 & 4.537-05\end{array}$

\begin{tabular}{|c|c|c|}
\hline InCOH. & PHOTC & PAIR \\
\hline SCATT. & EFFECT & PRCC. \\
\hline $.114+C 1$ & $2.194+C_{1}$ & \\
\hline $940+01$ & $1.255+01$ & \\
\hline $80 \mathrm{i}+\mathrm{Cl}$ & $9.143+06$ & \\
\hline$=E T+C 1$ & $4.2 \in O+C C$ & \\
\hline $430+c 1$ & $2.678+0 c$ & \\
\hline $155+C 1$ & i. $2 \epsilon 2+C C$ & R. 355-C1 \\
\hline$C 49+C C$ & $7.378-01$ & 1. $C E 2+C C$ \\
\hline $832+00$ & $3.915-01$ & 2. $t 2 C+C C$ \\
\hline $40+C C$ & $2.601-01$ & $3.537+C C$ \\
\hline $643+c c$ & $1.954-C_{1}$ & $5.074+C C$ \\
\hline $987+C C$ & $1.5 \mathrm{CC}-\mathrm{Cl}$ & $5.874+C C$ \\
\hline$C 79+C C$ & 1. $[3 \varepsilon-c]$ & $7.4 C 4+C C$ \\
\hline $474+C C$ & $7.800-02$ & 8. $8 \in 5+C 0$ \\
\hline $569+C C$ & $5 . C_{4} 4-02$ & $1.124+C 1$ \\
\hline $0 \in 1+C c$ & $3.7 \mathrm{C} 1-\mathrm{C} 2$ & $1 \cdot 330+C 1$ \\
\hline$\angle S S+C C$ & $2.3 \div 3-c 2$ & $1.5 c 7+c 1$ \\
\hline $19 C+C C$ & $1.757-C_{2}$ & 1. $7 t t+c 1$ \\
\hline - $32 t-C_{1}$ & $1.392-02$ & $2 . S C S+C 1$ \\
\hline $.54 t-c 1$ & $1.13 t-c 2$ & $1.5 C_{7}+\mathrm{Cl}_{1}$ \\
\hline $.734-C_{1}$ & $8.33 t-C^{3}$ & $2.143+C 1$ \\
\hline $.587-<1$ & $6.55 E-c 3$ & $2.265+C 1$ \\
\hline
\end{tabular}


$C R C S S$ SECTIOA

C C MPARISON

ENDF/ I IN EARNS/ATCM

\begin{tabular}{|c|c|c|c|c|}
\hline ENEKGY & & $\mathrm{CCH}$ & I NCCH. & PHCTO \\
\hline KEV & TOTAL & SCATT. & SC ATT. & EFFECT \\
\hline 1 & $1.082+0 t$ & $3 \cdot C 37+03$ & $1.334+0 \mathrm{C}$ & $1.075+C$ \\
\hline 1.5 & $1.4^{c} C+C 6$ & $2.913+03$ & $2.2 \in 2+00$ & $1.487+C 6$ \\
\hline 2 & $i .327+0 t$ & $2.777+C 3$ & $3.168+00$ & $1.324+c 6$ \\
\hline 3 & $.343+05$ & $2.5 C 2+c 3$ & $4.954+00$ & $5.317+05$ \\
\hline 4 & $2.483+c 5$ & $: 5+03$ & $t .6$ & $2.460+0$ \\
\hline 5 & 1.37 & 1. SE $8+03$ & & $+c .5$ \\
\hline 6 & $8.482+04$ & $1.771+03$ & 5.6 & $9.304+04$ \\
\hline 8 & $3.98 t+C 4$ & $1.4 \overline{2} 1+03$ & $1.2 \mathrm{Cs}+01$ & $3.842+C 4$ \\
\hline 10 & $8.26 \geq+04$ & $1.159+03$ & $1.44 t+C 1$ & R. $246+04$ \\
\hline 15 & $3.3 C G+C 4$ & $7.477+02$ & $1.8 \in 4+C ?$ & $3.232+04$ \\
\hline 20 & $1.542+C 4$ & $5.255+02$ & $2.171+01$ & $1.487+04$ \\
\hline 30 & $5.271+C 3$ & $3.12 \varepsilon+02$ & $2.552+01$ & $4.531+03$ \\
\hline 40 & $2.475+03$ & $2 \cdot C E Z+C 2$ & $2.764+01$ & $2.239+03$ \\
\hline 50 & $1.385+03$ & $1.47 t+02$ & $2.88 C+01$ & $1.2 C 9+03$ \\
\hline $6 C$ & $4.039+03$ & $1 . c \subseteq 7+02$ & $2.943+01$ & $3.900+03$ \\
\hline 80 & $1.896+03$ & $6.522+C 1$ & $2.97 t+01$ & $1.801+C 3$ \\
\hline $10 n$ & $1.052+03$ & $4 \cdot 3 \in 5+C 1$ & $2.953+01$ & $9.787+C 2$ \\
\hline 150 & 3.60 & $2.052+01$ & $2.832+01$ & $3.201+02$ \\
\hline 200 & $1.838+c 2$ & 1. $1 \mathrm{EO}+01$ & $2.6 t 2+01$ & $454+c$ \\
\hline 300 & $7.800 \div 01$ & $5.331+00$ & $2.368+01$ & $4.899 \div 01$ \\
\hline
\end{tabular}

\begin{tabular}{|c|c|c|}
\hline ENEEEY & & $\mathrm{COH}$ \\
\hline KEV & TOTAL & SCATT. \\
\hline 1 & $1.103+06$ & $2.129+03$ \\
\hline 1.5 & $4.182+C 5$ & 3. $C C 5+03$ \\
\hline 2 & $1.3 \varepsilon \&+0 \epsilon$ & $2 . \varepsilon \in \theta+03$ \\
\hline 2 & $5.64 C+05$ & $2.58 \subseteq+c 3$ \\
\hline 4 & $2.622+05$ & $2.317+03$ \\
\hline 5 & $1.451+05$ & 2. $C \in 4+03$ \\
\hline$\epsilon$ & $8.943+04$ & $1.8<\mathrm{C}+\mathrm{C} 3$ \\
\hline 8 & $4.213+c 4$ & $1.478+03$ \\
\hline 10 & $8.3 \angle 8+04$ & $1 . ? \mathrm{C}^{2}+03$ \\
\hline 15 & $3.414+C 4$ & $7.782+\mathrm{C} 2$ \\
\hline 20 & $1.61 \epsilon+04$ & $5 \cdot 5 C_{2}+C_{2}$ \\
\hline 30 & $5.58 C+03$ & $3 \cdot 2 \in 1+02$ \\
\hline 40 & $2.61 \varepsilon+03$ & $2.1 \epsilon 8+02$ \\
\hline 50 & $1.455+03$ & $1.54 \mathrm{C}+02$ \\
\hline$\epsilon 0$ & a.c22 02 & $1.14 t+02$ \\
\hline RO & $? .014+03$ & $6.811+01$ \\
\hline 100 & ].1 $14 \epsilon+03$ & $4.5 \in 1+C 1$ \\
\hline $1=0$ & $4.085+02$ & $2.147+01$ \\
\hline 200 & $2.023+C 2$ & $1.235+c 1$ \\
\hline 300 & $8.381+01$ & $5.582+00$ \\
\hline
\end{tabular}

$\begin{array}{rc}\text { ENERGY } & \\ \text { KEV } & \text { TCTAL } \\ 400 & 4.776+01 \\ 500 & 3.502+C 1 \\ 600 & 2.829+C 1 \\ 800 & 2.140+01 \\ 1000 & 1.784+01 \\ 1500 & 1.372+01 \\ 2000 & 1.212+01 \\ 3000 & 1.112+01 \\ 4000 & 1.100+01 \\ 5000 & 1.117+01 \\ 6000 & 1.127+01 \\ 8000 & 1.186+01 \\ 10000 & 1.271+01 \\ 15000 & 1.420+01 \\ 20000 & 1.578+C 1 \\ 30000 & 1.794+C .1 \\ 40000 & 1.535+01 \\ 50000 & 2.0 \pm 2+01 \\ 60000 & 2.137+01 \\ 800 C 0 & 2.259+01 \\ 100000 & 2.382+01\end{array}$

ENERGY

KEV

400

500
600

200

1000

2000

3000
4000

5000

6000

8000

10000

15000

20000

20000

40000

50000

60000

80000

100000
TCTAL
$5.032+01$
$.0644+01$

$3.644+01$

$2.922+c$

$2.195+C$

$1.825+C$

$1.235+01$

$1.135+C 1$

$1.123+c)$

$1.141+01$

$1.153+01$

$1.301+C 1$

$1.454+C 1$

$.617+\mathrm{Cl}$

$1.329+01$
$1.594+0.1$

$2.114+01$

$2.192+c 1$

$2.328+c 1$

$443+01$

$\begin{array}{cc}C O H & \text { INCOH. } \\ \text { SCATT. } & \text { SCATT } \\ 2.011+00 & 2.144+C 1 \\ 1.929+00 & 1 . S 6 C+C 1 \\ 1.336+00 & 1.827+C 1 \\ 7.500-01 & 1 . t 10+01 \\ 4.770-01 & 1.45 C+C 1 \\ 2.146-01 & 1.181+C 1 \\ 1.215-01 & 1 . C 09+C 1 \\ 5.434-02 & 7.943+C C \\ 3.067-02 & 6.532+0 C \\ 1.967-02 & 5.723+C C \\ 1.367-02 & 5 . C 5 E+C C \\ 7.704-03 & 4.137+C C \\ 4.93 t-03 & 3.523+C C \\ 2.197-03 & 2 . E C t+C C \\ 1.235-03 & 2.050+C C \\ 5.500-04 & 1.52 C+C 0 \\ 3.098-04 & 1.2 C 7+C C \\ 1.979-04 & 1.0 C 7+0 C \\ 1.3 t t-04 & 8 . t t 7-C 1 \\ 7.576-05 & t .82 S-C 1 \\ 4.769-05 & 5.06 t-C 1\end{array}$

4. $769-05$ $\begin{array}{rcc}\text { ENERGY } & \text { TCTAL } & \begin{array}{c}\text { COH } \\ \text { SCATT }\end{array} \\ 400 & 5.174+01 & 3.286+00 \\ 500 & 3.756+C 1 & 2.106+00 \\ 60 C & 3.011+01 & 1.458+00 \\ 80 C & 2.256+01 & 8.190-01 \\ 1000 & 1.850+01 & 5.210-01 \\ 1500 & 1.430+01 & 2.345-01 \\ 2000 & 1.261+01 & 1.327-01 \\ 3000 & 1.253+C 1 & 5.937-02 \\ 4000 & 1.147+C 1 & 3.351-02 \\ 5000 & 1.166+C 1 & 2.148-02 \\ 6000 & 1.178+C 1 & 1.494-02 \\ 8000 & 1.241+C 1 & 8.418-03 \\ 10000 & 1.331+01 & 5.393-03 \\ 15000 & 1.488+01 & 2.400-03 \\ 20000 & 1.656+01 & 1.351-03 \\ 30000 & 1.884+01 & 6.009-04 \\ 40000 & 2.034+C 1 & 3.385-04 \\ 50000 & 2.167+01 & 2.163-04 \\ 60000 & 2.247+01 & 1.453-C 4 \\ 80000 & 2.386+01 & 8.281-05 \\ 10 C 000 & 2.5 C 5+C 1 & 5.215-05\end{array}$
SCATT

$3.153+00$

$2.020+00$

$7.350-01$

$5.000-01$

$2.250-01$

$1.274-01$

$5.607-02$

$0.215-02$

$1.034-02$

$8.077-03$

$5.175-03$

$2.303-03$
$1.206-03$

$5.766-04$

$3.248-04$

2.075-04

$1.433-34$

$5.002-05$

INCOH.

SCATT.

$2.175+C 1$

$1.5 C 7+C 1$

$1.853+C 1$

$1 . t 24+C 1$

$1.471+C 1$

$1.024+C 1$

$8.055+C C$

$\varepsilon \cdot 725+C C$

$5.807+00$

$4.157+c 0$

$3.574+C C$

$2.544+C C$

$2.121+c 0$

$1.542+00$

$1.225+\mathrm{CO}$

$1.021+.00$

$8.794-61$

$6.92 E-C 1$

$5.748-c 1$

\begin{tabular}{|c|c|c|}
\hline$\wedge E R G Y$ & & $\mathrm{COH}$ \\
\hline KEV & TחTAL & SCATT. \\
\hline 3 & $1.184+06$ & $3.2<0+03$ \\
\hline 1.5 & $4.47 t+C 5$ & 3. $C S C+C 3$ \\
\hline 2 & $1.059+06$ & $2 . \subseteq 47+03$ \\
\hline 3 & $5.964+05$ & $\bar{z}, \epsilon \leq 8+03$ \\
\hline 4 & $2.777+05$ & $2.381+03$ \\
\hline 5 & $1.528+c 5$ & $2.126+c 3$ \\
\hline 6 & $0.512+04$ & $1.89 \varepsilon+03$ \\
\hline 8 & $4.47 t+04$ & $1.5<8+c 3$ \\
\hline 10 & $6.4 C_{4}+04$ & $1.250+c 3$ \\
\hline 15 & $3 . t ? ?+04$ & e. $C 7 C+02$ \\
\hline 20 & $1.713+C^{4}$ & $5.7 \mathrm{Cl}+\mathrm{C} 2$ \\
\hline 30 & $5.921+03$ & $3.379+02$ \\
\hline $4 C$ & $2.792+03$ & $2.25 \mathrm{C}+02$ \\
\hline $5 \mathrm{C}$ & $1.564+c 3$ & $1.6 \mathrm{CC}+02$ \\
\hline$\in 0$ & $9.79 C+C 2$ & $1.1 \div 2+02$ \\
\hline 80 & $? \cdot 097+03$ & 7. $C E t+C 1$ \\
\hline 100 & $1.1 t t+c z$ & $4.74 t+C 1$ \\
\hline 150 & $4.099+0 ?$ & 2. $\geqslant \geqslant 6+01$ \\
\hline 200 & $2.037+c 2$ & $1.287+01$ \\
\hline & $0.552+01$ & $5.818+0 \mathrm{C}$ \\
\hline
\end{tabular}

INCCH. PHCTC

EFFECT

$1.32 C+C O \quad 1.180+06$

$2.262+00 \quad 4.445+05$

$3.172+0 C \quad 1.096+c 6$

$6.64 t+00=2.753+05$

E. $222+00 \quad 1.517+C 5$

$5.671+00 \cdot 9.321+04$

$1.221+C ? \quad 4.322+04$

$1.467+C 1 \quad 6.278+04$

$1.500+013.549+C 4$

$2.212+01 \quad 1.653+04$

$2.602+01 \quad 5.557+\mathrm{Cl}^{2}$

2. $824+C 1 \quad 2.538+C 3$

$3.94 t+01 \quad 1.375+03$

$3 . \mathrm{C} 5 \mathrm{C}+01-1.995+03$

$3.025+01 \quad 1.088+03$

$2.90 c+C 1 \quad 3.583+02$

$2.436+01 \quad 5.534+C 1$

$Z=72$ HAFNTUM

ENEREY
KEV
1
1.5
2
3
4
5
6
8
10
15
20
30
40
50
50
80
100
150
200
300

$\begin{array}{cc}\text { TOTAL } & \text { SCATT. } \\ 1.274+C t & 3.313+03 \\ 4.778+C 5 & 3.180+03 \\ 1.145+C t & 3 . C 31+C 3 \\ t .285+05 & 2.731+03 \\ 2.930+C 5 & 2.448+C 3 \\ 1.625+05 & 2.188+C 3 \\ 1.005+05 & 1.55 t+C 3 \\ 4.737+C 4 & 1.579+03 \\ t .29 t+C 4 & 1.253+C 3 \\ 2.758+C 4 & 8.3 t 3+02 \\ 1.808+C 4 & 5.5 C 3+02 \\ 5.292+03 & 3.45 C+02 \\ 2.527+03 & 2.333+C 2 \\ 1.612+03 & 1.6 t 2+02 \\ 5.508+C 2 & 1.24 C+C 2 \\ 2.205+03 & 7.36 t+C 1 \\ 1.237+03 & 4.536+01 \\ 4.327+02 & 2.327+C 1 \\ 2.126+C 2 & 1.340+01 \\ 8.785+C 1 & 6 . C 58+00\end{array}$

INCCH.

PHOTC

EFFECT

$1.313+00 \quad 1.270+06$

$2.2 \epsilon \subseteq+00 \quad 4.74 t+C 5$

$3.184+0 \mathrm{OC} \quad 1.146+C 6$

$4.962+C 0 \quad 6.257+05$

$6.657+0 \mathrm{C} \quad 2.5 \mathrm{C} 6+\mathrm{C5}$

$8.244+00 \quad 1.6 C 3+C 5$

$5.713+00 \quad 9.858+04$

$1.230+01 \quad 4.578+C 4$

$1.47 t+01 \quad 6.166+04$

$1.915+01 \quad 3.673+04$

$2.23 \mathrm{C}+01 \quad 1.747+04$

$2.627+01 \quad 5.006+03$

$2.853+01 \quad 2.665+03$

$2.578+01 \quad 1.415+03$

$3.047+\mathrm{Cl} \quad 8.364+02$

$3.086+01 \quad 2.101+C 3$

$3.067+01 \quad 1.157+03$

$2.542+01 \quad 3.739+C 2$

$2.773+c 1 \quad 1.715+02$

$2.47 \mathrm{C}+01 \quad 5.710+C 1$

$\begin{array}{rrr}\text { ENERGY } & & \text { COH } \\ \text { KEV } & \text { TCTAL } & \text { SCATT } \\ 400 & 5.283+C 1 & 3.422+00 \\ 500 & 3.832+C 1 & 2.193+00 \\ E C 0 & 3.077+C 1 & 1.518+00 \\ 800 & 2.313+C 1 & 8.530-01 \\ 1000 & 1.923+01 & 5.420-01 \\ 1500 & 1.464+01 & 2.439-01 \\ 2000 & 1.285+C 1 & 1.381-01 \\ 3000 & 1.183+C 1 & t .177-02 \\ 4000 & 1.172+01 & 3.486-02 \\ 5000 & 1.152+C 1 & 2.235-02 \\ 6000 & 1.2 C 5+01 & 1.554-02 \\ 8000 & 1.270+C 1 & 8.758-03 \\ 10000 & 1.361+C 1 & 5.011-03 \\ 15000 & 1.524+C 1 & 2.457-03 \\ 20000 & 1.695+01 & 1.4 C 5-03 \\ 30000 & 1.930+01 & t .252-04 \\ 40000 & 2.084+C 1 & 3.522-04 \\ 50000 & 2.221+C 1 & 2.251-04 \\ 60000 & 2.3 C 3+C 1 & 1.554-04 \\ 80000 & 2.445+C 1 & 8.619-05 \\ 100000 & 2.567+C 1 & 5.428-05\end{array}$

SCATT

2. $23 t+C 1$

$2.053+C 1$

1. $50 t+C 1$

$1.513+\mathrm{Cl}$

$1.233+c 1$

$1.053+C 1$

$8.29 C+C 0$

$t .522+c 0$

$5.574+C C$

$5.279+C C$

$4.219+C C$
$3.577+C C$

$2.720+C C$

$2.182+C C$

$1.587+C C$

1. $260+c 0$

$1.051+C \mathrm{C}$

$9.047-C 1$

$7.12 \mathrm{E}-\mathrm{Cl}$
$5.514-\mathrm{Cl}$
PHCTO

EFFECT

$1.34 \mathrm{C}+\mathrm{C} 1$

$E \cdot t 8 z+C \cdot C$

$4.551+C C$

2. $8 * 2+C C$

$1.34 E+C C \quad 3.497-C 1$

$7.50 c-01 \quad 1.12 c+0 c$

$4.175-C .1 \quad 2.7 C 4+C C$

$2.77 z-01 \quad 4 . C 56+C C$

2.C17-Cl $5.222+c c$

$1.597-016.042+C C$

$1.104-C 17.5 C \varepsilon+C C$

$8.255-C^{2} \quad 5.1 C 3+C C$

$5.3 t z-c 2 \quad 1.154+c 1$

$3.03 t-02 \quad 1.3 t 5+C 1$

$2.545-c 2 \quad 1 . E 3 c+c 1$

1. $\varepsilon \leqslant 8-C 2 \quad$ 1. $E 13+C 1$

$1.4 t c-C 2 \quad 1 . c t C+C 1$

$1.2 C E-C 2 \quad 2 . C 5 C+C 2$

$\left.8.864-C_{2} 2.2 C c+c\right]$

Protc

PHCTC
EFFEC

$z \cdot 54 z+C$ :

$1.44 t+01$

$5.292+0 \mathrm{C}$

$4.83 c+c c$

$3.03 t+0 C$

$1.429+C C$

$8.374-C !$

$4.415-C$

$2.5 \geq 1-c 1$

$2.132-C 1$

$1 . \in 87-0$

$1.1 \in t-01$

$8.75 t-0$

$5 . t 6 ́ 1-02$

$4.155-0$

$1.571-.22$

$1.551-i 2$

$1.275-6$

c. $355-03$

$7.35 c-63$

2. $623-C 1$

$2.1 \mathrm{CO}+\mathrm{CC}$

2. $7 E C+C C$

$4.17 E+c C$
$5.373+c c$

$6.212+C C$

7. $\varepsilon 15+c c$

c. $343+c c$

$1.1 E 4+C$ ?

$3.4 C C+c$

I. $E \leq C+C 1$

$2.011+c$

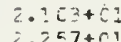

$2 .=\varepsilon c+C$

PHCTC PAI

EFFEC T

$2 . \leqslant 4 C+C 1$
$1.521+C 1$

c. $8 \in 3+50$

$5.173+3 c$

$3.253+0 \mathrm{C}$

$1.531+C$

$8.9 t t-c$

$4.724-61$

$3.13 i-c 1$

$2.27 t-c !$

$1.000-6$

$1.244-c$ :

$t . c 3 \leq-c$

$4.425-C 2$

$2.5 \leqslant 4-c 2$

z. $102-c 2$

$1.653-c$

$1.355-C_{2}$

$5.972-C 3$

$7.944-63$

2. $7+2-C 1$

$1.2 r .0+00$

$2.87 t+C C$

$=3 \mathrm{Cl}+\mathrm{CC}$

$t .584+c c$

a. $C \geq \angle+C C$

c. $5 E 5+C C$

i. $214+01$

$1.43 t+C 1$

$1.725+C 1$

1. $C \mathrm{C} 7+\mathrm{Cl}$

2. $C \in 2+C 1$

$2.315+C$

$2.446+01$

PHETC

PAIR

EFFECT

$1.5 t C+C]$

1. $C 15+C 1$

$5.477+0 C$

$3.5 t^{2}+c c$

$1.67 t+C C$

c. $813-C$

$5.162-C 1$

$3.415-C 1$

$2.484-0$

1. $5 \in 4-C 1$

$1.35 t-01$

$\epsilon .578-02$

$4.827-C^{2}$

$3.121-C 2$

$2.290-c 2$

$1.8 \mathrm{C} 1-\mathrm{C}^{2}$

1. $481-02$

1. $C 8 t-C 2$

$8.547-63$

PROD.

$3.904-C 1$

$1.241+C 0$

2. $\subseteq 65+C C$

$4.426+C C$

$6.558+C C$

$8.235+\mathrm{CC}$

5. $830+C C$

$1.245+C 1$ 
ENEREY $=7$ IANTALUM

\begin{tabular}{|c|c|c|c|c|}
\hline EREY & & $\mathrm{COH}$ & $1 \mathrm{NCCH}$. & $\begin{array}{l}\text { PHOTC } \\
\text { EFFCCT }\end{array}$ \\
\hline EV & TOTAL & SCATT & & \\
\hline 1 & $1.37 E+0 t$ & $3.4 C 5+03$ & $1.255+00$ & $1.371+0$ \\
\hline 1.5 & $5.065+C 5$ & $3.273+03$ & $2.267+00$ & กคด \\
\hline 2 & $+0 \epsilon$ & $119+C^{3}$ & $89+00$ & $82+0$ \\
\hline 3 & $1+05$ & $07+03$ & $6 t+C 0$ & $3.469+C$ \\
\hline 4 & +05 & +0 & +0 & $8+0$ \\
\hline 5 & +05 & $c+c 3$ & +00 & 1.6 \\
\hline 6 & $1 \cdot c_{4}$ & 2. C14+0 3 & $+\mathrm{CO}$ & 1.0 \\
\hline$\varepsilon$ & $4.515+c 4$ & $1.629+03$ & $8+01$ & $4+0$ \\
\hline 10 & $6.970+04$ & $1.32 t+03$ & $1.486+01$ & $0.835+0$ \\
\hline 15 & Z. $c 8 t+04$ & $8.5 \in 1+02$ & $1.93 c+01$ & $3.857+0$ \\
\hline 20 & 1.88 & $6.110+02$ & $2.248+01$ & $1.823+c$ \\
\hline $3 \mathrm{C}$ & 6.53 & $21+02$ & $1+01$ & $6.148+0$ \\
\hline 40 & & $17+c 2$ & & $9+C$ \\
\hline 50 & 1.72 & 1.72 & & 1.5 \\
\hline$\epsilon 0$ & 1.07 & $1.288+02$ & $1+\mathrm{Cl}$ & 9.1 \\
\hline 80 & & $7 . \in 47+C 1$ & $3.123+01$ & $2.1<0+0$ \\
\hline 100 & 03 & $5.12 t+01$ & $3.104+01$ & $1.189+0$ \\
\hline & $.511+C 2$ & $2.418+01$ & $2.586+01$ & $3.970+0$ \\
\hline 200 & $2 \geq 1+C z$ & $203+01$ & $8 C \div+C 1$ & $1.811+0$ \\
\hline 300 & $13+01$ & $298+00$ & $3+01$ & \\
\hline
\end{tabular}

$Z=74$ TUNESTEA

FREY
KEV
1
1.5
2
3
4
5
6
8
10
15
20
30
40
50
60
20
120
150
$2 C 0$
300

TOTAL

TOTAL $\mathrm{COH}$

$\begin{array}{ll}1.4 L E+C E & 3.5 C 7+C 3 \\ 5.4 S 5+C 5 & 3.3 t C+C 3\end{array}$

$1.173+C t \quad 3.21 C+C 3$

$t .613+05 \quad 2 . E 87+C 3$

$0.12 t+05 \quad 2.58 t+C 3$

$1.762+05 \quad 2.314+C ?$

$5.275+c 4 \quad 1 . t 7 c+c^{2}$

$4.342+54$ E. $5,64+C 2$

$0.046+C 4 \quad 6.322+02$

$3.27 \epsilon+03 \quad 2 . E C 2+C 2$

1. $82 t+c 3 \quad 1.7 \varepsilon ?+C 2$

$1.12 t+03 \quad 1.325+c$

$2.37 \varepsilon+C 3 \quad 7 . C \angle E+C$

$1.353+03 \quad 5.315+C 1$

4. $18+02 \quad 2.5 C E+C 1$

c. $t \Omega 2+01$ t. $53 t+C O$

INCCH.

SCATT.

PHCTC

$1.2 \varepsilon 1+C C \quad 1.402+06$

$2.25 \varepsilon+O C \quad 5.461+05$

$3.1 \& 7+O C \quad 1.1+0+C .6$

4. $c^{c} t t+C 0 \quad 6.5 .8 \angle+C 5$

$6.66 E+00 \quad 3.11 C+C 5$

$8.27 c+00 \quad 1.738+C 5$

$5.789+00 \quad 1.081+05$

$1.247+015.10 t+04$

1. $C 43+C 1 \quad 4.25 C+C 4$

$2.265+C 1 \quad 1.981+04$

$z .675+01 \quad 6.618+03$

$2.911+C 1 \quad 2.59 t+C 3$

1. $6 C 7+03$

$\begin{array}{ll}1.5+C 1 & 9.6 \mathrm{CO}+02\end{array}$

$3.141+C 1 \quad 1.269+03$

$3.024+014.265+02$

$2.84 t+01 \quad 1.944+02$

$2.537+C 1 \quad 6.452+01$
$1.102+05 \quad 2 . C 73+C 3$

$7.015+03 \quad 3.745+C 2$

$.454+01 \quad 2.854+04$

$\begin{array}{rc}\text { EAERGY } & \\ \text { KEV } & \text { TCTAL } \\ 400 & 5.502+01 \\ 500 & 3.5 \epsilon 2+C 1 \\ 600 & 3.1 \epsilon 1+C 1 \\ 800 & 2.357+C 1 \\ 1000 & 1.545+C 1 \\ 1500 & 1.484+C 1 \\ 2000 & 1.309+01 \\ 3000 & 1.2 C 4+C 1 \\ 4000 & 1.195+C 1 \\ 5000 & 1.216+C 1 \\ 6000 & 1.230+01 \\ 9000 & 1.257+01 \\ 10000 & 1.391+C 1 \\ 15000 & 1.558+C 1 \\ 20000 & 1.734+C 1 \\ 30000 & 1.976+01 \\ 40000 & 2.134+01 \\ 50 C 00 & 2.274+01 \\ 60000 & 2.355+C 1 \\ 80000 & 2.505+C 1 \\ 10000 C & 2.63 C+C 1\end{array}$

EAERGY

KEV

40
500
60
800

1000

1500

$200 \mathrm{C}$

4000

5000

6000

8000

15000

20000

$300 C 0$

40000

50000

60000

100000

\begin{tabular}{|c|c|c|}
\hline \multicolumn{3}{|c|}{$z=75$ FHEN ILM } \\
\hline$\triangle E E G Y$ & & $\mathrm{CDH}$ \\
\hline$K E V$ & TOTAL & SCATT. \\
\hline 1 & $1.5 \geq 1+c t$ & $3 . \in C 5+03$ \\
\hline 1.5 & $5 . \subseteq 18+05$ & $3.4 t 5+C 3$ \\
\hline$?$ & $1.274+C 6$ & $2 \cdot 3 C 3+c 3$ \\
\hline$\Xi$ & $7.13 c+c 5$ & 2. $c t c+c z$ \\
\hline 4 & $3.3 \times 4+$ & $2 \cdot t \leq \varepsilon+c 3$ \\
\hline 5 & $1.882+05$ & $2.27 E+C 3$ \\
\hline$t$ & $1.173+25$ & $2.1 \equiv 1+03$ \\
\hline 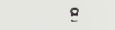 & $5.58 t+54$ & $1.7 z c+c 3$ \\
\hline 10 & $3.157+04$ & $1.4 z 4+03$ \\
\hline 15 & $4.55 t+1$ & $0.272+02$ \\
\hline 20 & $2 \cdot 12 t+04$ & $t \cdot 537+02$ \\
\hline $3 c$ & $7.31 \mathrm{C}+$ & $3.871+C 2$ \\
\hline 40 & $3.453+c 3$ & $2.588+02$ \\
\hline 5. & $1.94 ?+03$ & $1.848+C 2$ \\
\hline 60 & 1.222 & 1. $382+02$ \\
\hline $8 \mathrm{C}$ & $2.497+03$ & $8.2 C 3+01$ \\
\hline $1 \mathrm{CC}$ & $1.44 t+$ & $5.4 c 9+C 1$ \\
\hline 150 & $5.185+$ & $2.596+01$ \\
\hline $2 \mathrm{CC}$ & $2.537+c 2$ & $1.4 c t+C 1$ \\
\hline $30 \mathrm{C}$ & $1 . c 2 C+C 2$ & $6.7 \in 5+00$ \\
\hline
\end{tabular}

INCCH.

SCATT.

$2.242+0 \mathrm{C} \quad 5.883+05$

$3.17 \mathrm{E}+00 \quad 1.27 \mathrm{C}+06$

4. $5 \in 1+C C \quad 7.100+05$

$6.668+00 \quad 3.337+05$

E. $283+\mathrm{CO} \quad 1.858+\mathrm{C}$

c. $824+00 \quad 1.151+05$

$1.25 t+C 1 \quad 5.411+C 4$

$1.484+C 1 \quad 3.013+C 4$

$1.524+C 1 \quad 4.461+04$

$2.253+01 \quad 2.05 c+C 4$

$2.68 \mathrm{E}+01 \quad 6.896+\mathrm{C} 3$

$2.933+01 \quad 3.165+03$

3. $C 71+01 \quad 1.727+03$

$3.147+01 \quad 1.052+C 3$

$3.193+01 \quad 2.383+03$

$2.177+C 1 \quad 1.35 c+C^{3}$

3. $C E 2+C 2 \quad 4 . t 1 s+C 2$

$2.883+01 \quad 2.095+C_{2}$

$2.569+01$

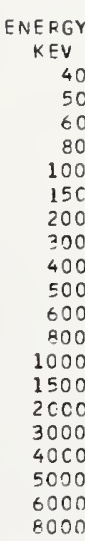

KEV
400
500
600
800
1000
1500
2000
3000
4000
5000
6000
8000
10000
15000
20000
30000
40000
50000
60000
80000
100000

100000
$\mathrm{COH}$

SCATT.

TCTAL

$4.085+01$

$2.242+C 1$

$1.581+01$

$1.508+01$

$1.331+01$

$1.219+C 1$

$1.241+01$

$1.256+\mathrm{Cl}$

$1.325+01$
$1.422+01$

$1.553+C 1$

$1.774+\mathrm{Cl}$

$2.023+01$
$2.184+C 1$

$2.329+01$

$2.415+c 1$

$2.565+C 1$

$.6 \subsetneq 4+01$

TCTAL
$5.977+C 1$

$4.257+C 1$
$3.27 t+C 1$

$2.5 C 5+C 1$

$2.071+01$

?. $555+C 1$

$1.36 \%+n 1$

$1 \cdot 257+C 1$

$1.248+C$

$1.285+01$

$1.355+C 1$

$1.454+01$

$1.815+C 1$

$2.070+\mathrm{Cl}$

$2.236+c 1$

$2.3 \varepsilon 3+c 1$

$2.472+01$

$2.626+01$

$2.75 a+c 1$

SCATT.

$3.822+00$

$2.450+00$
$1.65 t+0$

s. $530-01$

t.050-01

2.723-C1

$1.542-01$
$6.897-02$

$2.496-02$

$1.736-02$

$5.781-03$

$1.570-03$

$6.982-04$

3. $933-04$

$2.514-04$
$1.737-04$

c. $633-05$

$6.070-05$
$3.525+00$

$2.3 t t+0$
$1.63 z+0$

5. $200-0$

$5.850-0$

$2.633-01$
$1.451-01$

$t . t \in G-0$

$2.763-0$

$1.678-02$

$9.456-0$

$2.656-0$

6.751-04

$3.802-C_{4}$

$2.431-04$
$1.679-04$

$1.679-04$
$0.310-05$

$5.866-05$ t. $.558-03$

$5.266-03$
$2.789-03$
INCCH. PHCTC SCATT. EFFECT

$1.16 C+C C \quad 1.590+C t$

$2.133+00 \quad 6.46 t+c 5$

2. $C 81+00 \quad 9.562+05$

$4.884+00 \quad 6.975+C 5$

$6.614+0 C \quad 3.533+C 5$

$8.242+00 \quad 1.564+05$

c. $813+00 \quad 1.21 \epsilon+c 5$

$1.26 \mathrm{C}+\mathrm{Cl} \quad 5.7 \mathrm{Cl}+\mathrm{CC}_{4}$

$1.517+\mathrm{Cl} \quad 3.169004$

$1.57 \mathrm{E}+01 \quad 4.580+04$

$2.3 C 5+C 1 \quad 2.155+C 4$

$2.724+C 1 \quad 7.323+03$

2. $c t c+C 1 \quad 3.36 t+C 3$

$3.107+01 \quad 1.829+03$

$3.184+01 \quad 1.1 \mathrm{C} 7+\mathrm{Cl}^{2}$

$3.231+01 \quad 2.54 C+C 3$

$3.21 \varepsilon+01 \quad 1.351+03$

$\begin{array}{ll}3.10 C+01 & 4.63 C+02 \\ 2.91 c+C 1 & 2.133+02\end{array}$

$2.605+01 \quad 7.327+01$

$\begin{array}{cc}\begin{array}{c}\text { EAEREY } \\ \text { KEV }\end{array} & \text { TCTAL } \\ \angle 00 & 6.282+01 \\ 500 & 4.464+01 \\ 600 & 3.518+C 1 \\ 800 & 2.574+C 1 \\ 1000 & 2.101+01 \\ 15 C 0 & 1.583+01 \\ 2000 & 1.391+01 \\ 3000 & 1.280+C 1 \\ 4000 & 1.272+01 \\ 5000 & 1.254+C 1 \\ 6000 & 1.310+C 1 \\ 8000 & 1.393+01 \\ 10000 & 1.485+01 \\ 15000 & 1.666+C 1 \\ 20000 & 1.855+C 1 \\ 30000 & 2.117+01 \\ 40000 & 2.287+01 \\ 5 C 000 & 2.438+C 1 \\ 600 C 0 & 2.525+C 1 \\ 80000 & 2.688+01 \\ 100000 & 2.823+C 1\end{array}$

COH
SCATT
$.558+00$

$3.558+00$
$2.280+00$

$1.579+00$

$8: 860-01$

$5.540-01$

2. $539-01$

$1.437-01$
$6.429-0$

$3.628-02$

2. $326-02$

1.618-02

9.115-03

5. $840-03$

2. $599-03$

$1.463-03$

t. 507-04

$3.665-04$
$2.343-04$

$1 . t 18-0$

8.973-05

$5.652-05$

INCOH.

2. $2 t \epsilon+C$

$2.081+C 1$

$1.932+01$
$1.704+C 1$

$1.534+C 1$

$1.25 c+c 1$

$1.06 E+01$

$8.4 C E+C C$
$7.019+C 0$

$6.057+C C$

$5.353+c c$

$4 \cdot 37 \varepsilon+c c$

$2.72 \varepsilon+C O$

$2.758+C \mathrm{C}$

l. $\in C G+C C$

$1.277+C C$

1. $C E F+C C$

9.174-C

$7.22 \mathrm{E}-\mathrm{Cl}$

5. $\subseteq \subsetneq 7-C]$

INCOH

SCATT.

$2.1 C S+C 1$
$1.958+C 1$

$1.558+C 1$
$1.727+C 1$

$1.555+C$

1. $267+C$

$1.082+C 1$

$8.5 z 2+C C$

$7.11 t+c c$

$6.14 ?+C C$

$5.427+00$

$4.43 c+c 0$

3. $78 \mathrm{C}+\mathrm{CC}$

$2 \cdot 79 t+C$

$1.631+C C$

$1.2 \subseteq 5+c c$

1. $C 30+C 0$

5. $20 \mathrm{C}-\mathrm{Cl}$

$7.327-c 1$

$t . c 7 c-c 1$

$3.873-02$
INCOH. SCATT.

$2.327+C$

$2.137+01$
$1.9 E 4+C 1$

$1.749+C 1$

$1.57 t+C 1$

$1.284+C 1$

1. $C 57+01$

$\varepsilon \cdot t 38+c c$

$7.213+c$

$6.224+C C$

$5.5 C 1+C C$
$4.45 C+O$

$3 \cdot E 31+C C$

$2.834+C C$

$2.273+C C$

$1.653+C C$

$1.313+C c$

$1.055+C 0$

C. $427-C l$

$7.427-C 1$
$t .1 \in 3-C 1$

\section{PHCTC}

EFFECT

$2 \cdot 8 \varepsilon 1+C 1$

$1 . t 5 z+01$
$1 . C 71+C 1$

$5.644+C C$

$3.58 t+C C$

$1.6 B t+C C \quad 4 . C 5 C-C 1$ 


\begin{tabular}{|c|c|c|c|c|}
\hline ENERGY & 7 IR & $\mathrm{COH}$ & I NC CH. & PHCTC \\
\hline KEV & TOTAL & SCATT. & SCATT. & EFFECT \\
\hline 1 & $1.697+0 t$ & $3.8 C ?+03$ & $1.110+00$ & $1.693+06$ \\
\hline 1.5 & $6.431+C E$ & $3.6 t 4+03$ & $2.058+00$ & $6.394+05$ \\
\hline 2 & $3.23 c+05$ & $=4 c t+03$ & $3.013+00$ & $3.204+C 5$ \\
\hline 3 & $7.532+05$ & $3.141+03$ & $4.832+\mathrm{CO}$ & $7.501+05$ \\
\hline 4 & $3.81 C+05$ & $2.8 C s+C^{2} 3$ & $t .57 \subseteq+0 \mathrm{C}$ & $3.781+C 5$ \\
\hline 5 & $2.11 s+05$ & $2.512+c 3$ & $8.214+0 \mathrm{C}$ & $2.054+05$ \\
\hline 6 & $1.315+05$ & $2.252+C 3$ & c. $813+00$ & $1.2 \mathrm{~s} 2+\mathrm{cs}$ \\
\hline 8 & $t .21 t+04$ & $1.832+03$ & $1.2 t 5+01$ & $6.032+C 4$ \\
\hline 10 & $2.493+04$ & $1.513+c 3$ & $1.524 * 01$ & $3.340+C 4$ \\
\hline 15 & $4.814+C 4$ & S. SCE+02 & 1. $58 \varepsilon+C 1$ & $4.713+c 4$ \\
\hline 20 & $2.345+04$ & $6.583+02$ & $2.31 E+01$ & $2.273+04$ \\
\hline 30 & $\varepsilon .271+c 3$ & $4.128+02$ & $2.74 t+01$ & $7.831+03$ \\
\hline 40 & $3.885+03$ & $2.75 \varepsilon+c 2$ & $2.95 t+01$ & $3.57 c+c 3$ \\
\hline $5 \mathrm{C}$ & $2.148+C 3$ & $1.57 c+c 2$ & $3.13 \varepsilon+01$ & $1 . \leqslant 20+c 3$ \\
\hline 60 & $1.322+03$ & $1.472+02$ & $3.217+\mathrm{Cl}$ & $1.143+03$ \\
\hline 80 & $2.72 z+c 3$ & $8.737+C 1$ & $3.267+01$ & $2.6 \mathrm{Cl}+03$ \\
\hline 100 & $1.531+c 3$ & $5.85 t+C 1$ & $3.252+01$ & $1.440+C 3$ \\
\hline 150 & $5.443+02$ & $2.7 t 3+C 1$ & $3.138+C 1$ & $4.853+c 2$ \\
\hline 200 & $2.702+02$ & 1. $5 \div 2+C 1$ & $2.956+01$ & $2.247+C 2$ \\
\hline 300 & $1.111+02$ & $7.2 \mathrm{Cl}+\mathrm{CC}$ & $2.63 \varepsilon+01$ & $7.747+01$ \\
\hline
\end{tabular}

\begin{tabular}{|c|c|c|c|c|}
\hline $\begin{array}{r}Z= \\
E \wedge E Q G Y\end{array}$ & $E P L A T I$ & $\mathrm{COH}$ & I NCCH. & PHCTE \\
\hline KEV & TOTAL & SCATT. & SCATT. & EFFECT \\
\hline 1 & $1.7 t 0+c t$ & $3.91 t+03$ & $1 . c 72+c c$ & $1.75 t+c 6$ \\
\hline 1.5 & t. $814+C 5$ & $3.77 t+03$ & 2. $C O S+C O$ & $6.776+05$ \\
\hline 2 & $3.484+05$ & $3.6 c 8 \div 03$ & $2.967+c 0$ & $3.44 \varepsilon+C 5$ \\
\hline 2 & $7.46 S+0 E$ & $3.245+C 3$ & $4.801+C 0$ & 7.437505 \\
\hline 4 & $4.18 z+05$ & 2. ESC+C3 & $6.557+c 0$ & $4.153+C 5$ \\
\hline 5 & $2.2^{c} 1+C 5$ & $2.589+c 3$ & $\varepsilon .2 C t+c c$ & $2.265+05$ \\
\hline 6 & $1.404+C 5$ & $2.315+C 3$ & $5.82 \mathrm{C}+\mathrm{CO}$ & $1.381+05$ \\
\hline$\stackrel{8}{2}$ & $t .50 s+c 4$ & $1.8 \varepsilon t+c z$ & $1.27 C+C 1$ & $6.31 c+c 4$ \\
\hline 10 & $2.604+04$ & $1.555+c 3$ & $1.53 C+01$ & $3.447+C 4$ \\
\hline 15 & $5.2 C 5+04$ & 1. $C 2<+C 3$ & 1. $S S E+C 1$ & $5.1 \mathrm{Cl}+\mathrm{C4}$ \\
\hline 20 & $2.470+c 4$ & $7.217+c 2$ & $2.331+01$ & $2.395+04$ \\
\hline 30 & $8.53 \varepsilon+c 3$ & $4 \cdot 2 t 1+c 2$ & $2.7 \in \varepsilon+C 1$ & $8.084+03$ \\
\hline $4 \mathrm{C}$ & $4.001+03$ & $2.844+02$ & $3.024+01$ & $3.687+03$ \\
\hline$\leq 0$ & $2.223+c 3$ & 2. $c z \varepsilon+c 2$ & $3.1 t c+01$ & $1.088+03$ \\
\hline 60 & $1.37 \varepsilon+03$ & $1 \cdot 514+C 2$ & $2.25 \mathrm{c}+\mathrm{Cl}$ & $1.1<4+03$ \\
\hline 80 & $2.843+C 3$ & $\varepsilon . \varsigma \varepsilon \varepsilon+c 1$ & $3.3 C 2+c 1$ & $2.720+c 3$ \\
\hline 100 & $1.585+C 3$ & $0 . C 22+01$ & $3.28 s+C 1$ & $1.402+03$ \\
\hline$i \leq 0$ & $5.571+c 2$ & $2.835+01$ & $\Xi .17 t+C 1$ & $4.569+02$ \\
\hline 200 & $2.751+02$ & $1 \cdot t \equiv E+C 1$ & $2.953+01$ & $2.2 \varepsilon q+c 2$ \\
\hline 300 & $1.128+02$ & $7 \cdot 3 c 4+C C$ & $2.672+C 1$ & $7.873+01$ \\
\hline
\end{tabular}

\begin{tabular}{|c|c|c|c|c|}
\hline ENEREY & OLL & $\mathrm{CCH}$ & INCCH. & PFCTC \\
\hline KEV & TOTAL & SCATT. & SCATT. & EFFECT \\
\hline 1 & $1.871+c t$ & 4. $C 21+C 3$ & $1.042+00$ & $1.8 \in 7+c 6$ \\
\hline 1.5 & $7.302+05$ & $2.88 \mathrm{C}+03$ & 1. $c \in t+00$ & $7.263+C 5$ \\
\hline $\bar{z}$ & $3.754+C 5$ & $3.710+C 3$ & $2 . \$ 1 t+c 0$ & $3.717+05$ \\
\hline 3 & $t .51 t+05$ & $3.2 \geq \varepsilon+03$ & $4.7 t 8+C 0$ & $6.883+05$ \\
\hline 4 & $\angle . C 2 C+C E$ & 2. $\subseteq E I+C Z$ & $t .525+00$ & $3 . \subseteq 0 \subseteq+C 5$ \\
\hline 5 & $2.27 t+05$ & $2 \cdot t \in 2+c 3$ & $8.207+00$ & $2.250+05$ \\
\hline$\leqslant$ & $1.43 c+05$ & $c .38 \equiv+c 3$ & $C . E 17+C C$ & $1.40 t+05$ \\
\hline$\stackrel{9}{9}$ & $t .8 \$ 1+c 4$ & $1.535+03$ & $1.27 t+C 1$ & $0.556+04$ \\
\hline $1 \mathrm{c}$ & $3 . \subseteq 2 c+c 4$ & $1.6 C 5+03$ & $1.525+01$ & $3.767+C 4$ \\
\hline 15 & $\varepsilon .384+C 4$ & 1. $C \leqslant 7+03$ & 1. $5 \subseteq 1+01$ & $5.277+04$ \\
\hline $2 \mathrm{C}$ & $2.5 t 2+04$ & $7.453+c 2$ & $2.330+C 1$ & $2.485+04$ \\
\hline 30 & $8.906+03$ & 4. $3 c_{t}+c 2$ & $2.784+01$ & $8.438+03$ \\
\hline 40 & $4.193+03$ & $2.530+02$ & $3.04 \mathrm{E}+\mathrm{Cl}$ & $3.870+03$ \\
\hline 50 & $2.32 c+c 3$ & $2 \cdot c 87+c 2$ & $3.1 \varsigma \varepsilon+C 1$ & $2 . c 58+c 3$ \\
\hline 60 & $1.455+03$ & $1.557+c 2$ & $3.282+01$ & $1.266+03$ \\
\hline $8 \mathrm{C}$ & $\epsilon .912+02$ & $s .23 c+01$ & $3.337+\mathrm{Cl}$ & $5.655+02$ \\
\hline $1 C C$ & $1 \cdot t 75+03$ & $6.187+01$ & $3.325+C 1$ & $1.580+03$ \\
\hline 150 & $\leq .05<+02$ & $2.91 E+01$ & $3.214+01$ & $5.441+02$ \\
\hline 200 & $2.982+02$ & $1 . t 7 \xi+01$ & $3 . \mathrm{C2}, \mathrm{Cl} 1$ & $2.511+C 2$ \\
\hline 300 & $1.19 \varepsilon+c 2$ & $7.5 E Q+C O$ & $2.703+01$ & $8.517+01$ \\
\hline
\end{tabular}

\begin{tabular}{|c|c|c|}
\hline ENERGY $\mathrm{Z}=$ & EC NERCURY & $\mathrm{COH}$ \\
\hline KEV & TOTAL & SCATT \\
\hline 1 & $2 \cdot c 2 t+c 6$ & $4.121+C 3$ \\
\hline 1.5 & 7. $.0 \mathrm{CO}+\mathrm{C5}$ & $3.575+03$ \\
\hline 2 & $4 . C E C+C 5$ & $3.75 \mathrm{c}+\mathrm{C} 3$ \\
\hline 2 & 7. $534+05$ & $3.41 \varepsilon+c 3$ \\
\hline 4 & $4.353+0.5$ & 3. CE $4+C 3$ \\
\hline 5 & $2.443+05$ & $2.729+C 3$ \\
\hline 5 & $1.527+C 5$ & $2.445+C 3$ \\
\hline 8 & $7.29 t+04$ & 1. $c c c+03$ \\
\hline 10 & $4.133+04$ & $1.650+03$ \\
\hline 15 & $5 . t 07+04$ & 1. $C \varepsilon s+C z$ \\
\hline 20 & $2.7 C_{2}+C_{4}$ & $7.689+02$ \\
\hline 30 & $c .5 c c+03$ & $4.532+c 2$ \\
\hline 40 & $4.457+03$ & 3.c1 $\mathrm{C}+\mathrm{C} 2$ \\
\hline 50 & $2.51 C+C 3$ & $2.14 \varepsilon+C 2$ \\
\hline $6 C$ & $1.550+03$ & $1 . \in \mathrm{C} 1+02$ \\
\hline 80 & $7.378+02$ & $5.5 C 3+C 1$ \\
\hline 100 & $1.722+c^{3}$ & t. $: \quad \in 2+C 1$ \\
\hline 150 & $5.894+02$ & 2. $5 \subseteq 6+01$ \\
\hline $2 \mathrm{CO}$ & $2.508+C 2$ & $1.725+C 1$ \\
\hline 300 & $1.203+02$ & $7.7 C 4+C C$ \\
\hline
\end{tabular}

$\begin{array}{cc}\begin{array}{c}\text { ENERGV } \\ \text { KEV }\end{array} & \text { TCTAL } \\ 400 & 6.530+C 1 \\ 500 & 4.621+01 \\ 600 & 3.629+01 \\ 800 & 2.642+01 \\ 1000 & 2.152+01 \\ 1500 & 1.615+01 \\ 2000 & 1.418+01 \\ 3000 & 1.305+01 \\ 4000 & 1.297+C 1 \\ 5000 & 1.321+C 1 \\ 6000 & 1.337+01 \\ 8000 & 1.412+C 1 \\ 10000 & 1.516+C 1 \\ 15000 & 1.702+01 \\ 20000 & 1.896+C 1 \\ 30000 & 2.165+01 \\ 40000 & 2.339+01 \\ 50000 & 2.493+01 \\ 60000 & 2.587+C 1 \\ 80000 & 2.749+C 1 \\ 10000 C & 2.888+C 1\end{array}$

ENERG

\begin{tabular}{|c|c|c|}
\hline FGY & & $\mathrm{CEH}$ \\
\hline KEV & TCTAL & SCATT. \\
\hline $40 C$ & $6.644+C 1$ & $4.177+100$ \\
\hline 500 & $4.709+C 1$ & $2.677+00$ \\
\hline 600 & $3.703+\mathrm{Cl}$ & $1.854+00$ \\
\hline $8 \mathrm{CO}$ & $2.702+C 1$ & $1.041+00$ \\
\hline 1000 & $2.202+01$ & $6.620-01$ \\
\hline 1500 & $1.648+01$ & $2.780-01$ \\
\hline 2000 & $1.44 t+01$ & $1.687-01$ \\
\hline 3000 & $1.331+01$ & $7.550-c_{2}$ \\
\hline 4000 & $1.323+C 1$ & $4 . ? \in 1-02$ \\
\hline 5000 & $1.347+C 1$ & $2.732-02$ \\
\hline toco & $1.3 \in 4+C 1$ & $1.500-02$ \\
\hline 8000 & $1.442+01$ & $1.071-02$ \\
\hline 10000 & $1.548+01$ & $t .860=03$ \\
\hline 15000 & $1.738+01$ & $3.053-03$ \\
\hline 23000 & $1 . c 37+C 1$ & $1.718-03$ \\
\hline 30000 & $2.213+01$ & $7.644=04$ \\
\hline $4000 \mathrm{C}$ & $2.391+01$ & $4.206-04$ \\
\hline 50000 & $2.549+c 1$ & $2.753-04$ \\
\hline$E 0000$ & $2.645+C 1$ & $1.902-04$ \\
\hline 80000 & $2.812+01$ & $1.055-04$ \\
\hline & & \\
\hline
\end{tabular}

100000

$C O H$
SCATT.
$4.068+00$
$2.507+00$
$1.805+00$
$1.014+00$
$6.450-01$
$2.904-01$
$1.644-01$
$7.355-02$
$4.151-02$
$2.662-02$
$1.851-02$
$1.042-02$
$6.683-03$
$2.574-03$
$1.674-03$
$7.446-04$
$4.194-04$
$2.682-04$
$1.852-04$
$1.028-04$
$6.478-C 5$
SCATT

$2.38 \varepsilon+01$

$2.154+C 1$

$2.02 i+C 1$

$1.797+C 1$

$1.317+01$

$1.12 t+01$

$8.2 t 5+C 0$

$7.40 t+00$

$6.351+C C$

5. $64 \mathrm{C}+\mathrm{C}$

$4 \cdot+2 C+C C$

$3 . \subseteq 34+C 0$

$2.510+C C$

$2.334+C O$

$1.658+C C$

$1.34 E+O C$

$1.124+C O$

$9.68 \mathrm{C}-01$

$7.627-C 1$

$6.32 \varepsilon-c 1$

$\begin{array}{cc}\text { PHCTC } & \text { PAIR } \\ E F F E C T & \text { PRCO. } \\ 3.735+C 1 & \\ 2.166+C 1 & \\ 1.411+C 1 & \\ 7.440+C C & \\ 4.690+C C & \\ 2.2 C 7+C C & 4.6 E 1-C 1 \\ 1.252+C C & 1.459+00 \\ 6.745-C 1 & 3.433+C C \\ 4.45 C-C 1 & 5 . C 8 C+C C \\ 3.223-C 1 & 6.465+C C \\ 2.548-C 1 & 7.44 C+C C \\ 1.75 \varepsilon-C 1 & C .317+C C \\ 1.315-C 1 & 1.1 C C+C 1 \\ 8.492-02 & 1.4 C 2+C 1 \\ 6.213-02 & 1.656+C 1 \\ 4 . C C 1-C 2 & 1.5 C 1+C 1 \\ 2 . C 28-C 2 & 2.2 C 1+C 1 \\ 2.258-C 2 & 2.375+01 \\ 1.885-C 2 & 2.488+C 1 \\ 1.38 C-C 2 & 2.672+01 \\ 1.082-C 2 & 2.823+C 1\end{array}$

1NCOH. PHOTO

SCATT . EFFECT

$2.41 c+C 1 \quad 3.8 C 7+C 1$

$2.222+c 1 \quad \overline{2} .215+01$

$2.0 t 3+C 1 \quad 1.455+C 1$

$1.821+C 1 \quad 7.773+C C$

$1 . t 25+C 1 \quad 4.9 t c+C C$

$1.33 c+01 \quad \therefore .34 c+c C$

$1.141+C 1 \quad 1.371+C C$

$8.585+C C \quad 7.14 C-C 1$

$7.5 \mathrm{C} 3+\mathrm{CC}$

$6.475+C C$

$5.722+C C$

$4 . t E C+C C$

$3.98 t+C C$

$2.94 \varepsilon+C C$

$2.365+c c$

$1.720+c 0$

$1.3 \in 6+C C$

$1.13 .9+\mathrm{CO}$

c. $8 \mathrm{C} 7-\mathrm{Cl}$

$7.727-C$

$t .411-c 1$

$3.407-c 1$

$2 \cdot+54-01$

$1.855-C 1$

$1.395-C 1$

$9 . C 7 C-C^{2}$

$t .557-c 2$

$4.21 t-C z$

$3.08 ?-02$

$2.418-c 2$

$1.582-c 2$

$1.44 \mathrm{c}-\mathrm{C}$

$1.137-c$

$2.44 G+C 1 \quad C . C 5 C+C 1$

$2.250+C 1 \quad 2.343+01$

$2.085+c 1 \quad 1.52 s+C 1$

$1.842+C 1 \quad 8.192+O C$

$1.659+C 1 \quad 5.3 C 5+C C$

$1.352+01 \quad 2.5 C 1+C C$

$1.155+C 1 \quad 1.4 t t+0 C$

5. $c 5 t+c 0 \quad 7 . t 2 c-c 1$

$7.55 t+C C \quad 5 . C 19-01$

$6.555+C C \quad 3.531-C 1$

$5.793+C C$ 2. $871-C 1$

$4.7 \equiv s+c 0 \quad 1 . c \varepsilon z-c 1$

$4.035+C 0 \quad 1.487-01$

$2.7 B E+C C \quad 9.553-C 2$

$2.394+C 0 \quad 6.57 \pi-02$

$1.74 I+C C \quad 4.481-02$

$1.392+O C$

$4.421=02$
$3.272-0$

$2.5 \in E-c 2$

$9.928-C 1$

$7.822-c 1$

$2.1 C 2-C$

$1.535-C_{2}$

$1.2 c 3-c 2$

5.c $c 21-c 1$

$1.553+C C$

$3 .+32+C C$

$t .7 \varepsilon c+c c$

7. $81 t+c c$

c. $7 \in 3+c c$

$1.1 E 1+C 1$

1. $732+c 1$

2. $c \varepsilon z+c 1$

$2.3 c 2+c$ :

$2.4 E 7+C 1$

$2 \cdot E C 2+C 1$

$2.755+C 1$
$2.554+C 1$

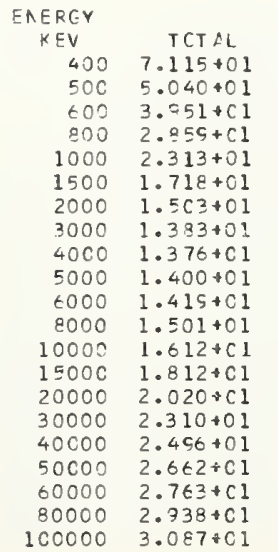

CCH
ATT.

INCOH.

PHCTC

PAIR

SCATT. EFFECT

$4.1 \subseteq 5+C 1$

$2.480+C 1$

$1 . t 41+C 1$

$8.834+C C$

$5.63 c+C$

$2.654+C C \quad 5.1 \subseteq 7-C l$

$1.55 t+C C \quad 1.6 C 2+O C$

8.C74-Cl $3.734+C C$

$5.315-C 1 \quad 5.4 C 5+C C$

3.843-C1 $\quad . .554+C C$

$3 . C 3 C-C 1 \quad 8.0 C 2+O C$

$2 . C S S-C 15.985+C C$

$1.575-C 1 \quad 1.187+C 1$

$1.01 C-C 1 \quad 1.5 C C+C 1$

$1.371-C^{2}$
$4.77 C+C 1$

$4.728-C 2 \quad 2.129+C 1$

$3.45 C-c 2 \quad 2.353+c 1$

$2.702-C 2 \quad 2.542+C 1$

$2.213-c^{2}$

2. $t \in C+C 1$

$1.615-02 \quad 2.857+C$

$1.265-C^{2} \quad 3 . C 2 C+C 1$ 


\begin{tabular}{|c|c|c|c|c|}
\hline ERGY & & $\mathrm{COH}$ & INCCH. & PHOTC \\
\hline KEV & TOTAL & SCATT. & SCATT. & EFFECT \\
\hline 1 & $2.0 \varepsilon 3+0 t$ & $4.216+03$ & $1.17 C+00$ & $2.079+c 6$ \\
\hline 1.5 & $8.230+05$ & $4 . C E 1+C 3$ & $2.17 c+00$ & $8.289+05$ \\
\hline 2 & $4.256+05$ & $3.878+03$ & $3.1 \in \varepsilon+00$ & $4.317+C 5$ \\
\hline 3 & $c \in 6+05$ & $3.452+03$ & 5. $C 64+00$ & $7 . \subseteq 31+05$ \\
\hline 4 & $4.590+C 5$ & $3.1<4+03$ & $3 \varepsilon+c 0$ & \\
\hline 5 & $.571+C 5$ & $2.753+C 3$ & $8.511+00$ & $2.543+05$ \\
\hline 6 & $.604+05$ & $2.5 C 5+C 3$ & $1.010+C 1$ & $1.578+C 5$ \\
\hline 8 & .6423 & $2 . C 42+c$ & +01 & 7.43 \\
\hline 10 & $.31 \subseteq+04$ & $1 . t \subseteq 4+C 3$ & $57+01$ & $4.148+C 4$ \\
\hline 15 & .18 & $1.122+03$ & $2.023+01$ & $5.0 t$ \\
\hline 20 & $.845+0$ & 7. $528+02$ & $2.36 \varepsilon+01$ & $2.764+c 4$ \\
\hline ?0 & $.515+C 2$ & $4.674+C 2$ & $s+01$ & $9.419+03$ \\
\hline 40 & 60 & $3.11 t+02$ & $7+01$ & $4.351+03$ \\
\hline 50 & 2.63 & $2.2 \angle 0+C 2$ & $3.2 \in 3+C l$ & $2.378+03$ \\
\hline 60 &.$\in 4$ & $1.65 t+$ & 3.35 & 1.4 \\
\hline${ }^{2} \mathrm{C}$ & .85 & $9.830+C 1$ & $2.4 C 5+01$ & $6.572+C 2$ \\
\hline 150 & .84 & $6.583+C 1$ & 3.30 & $1.749+C 3$ \\
\hline 150 & .50 & $3.1 \mathrm{Cl}+\mathrm{Cl}$ & $3.28 s+01$ & $5.865+c 2$ \\
\hline 200 & 2 & $1.785+C 1$ & $\Xi 102+01$ & $2.724+C 2$ \\
\hline 10 & $307+c z$ & $8 . C 71+O C$ & $2.772+\mathrm{Cl}$ & $9.493+01$ \\
\hline
\end{tabular}

$z=\varepsilon \varepsilon L E \Delta C$

\begin{tabular}{|c|c|c|c|c|}
\hline ERGY & & $\mathrm{COH}$ & I NCCH. & PHOTC \\
\hline$x \in V$ & TOTAL & SCATT & SC $\triangle T T$ & EFFECT \\
\hline 1 & $2.14 t+C t$ & $4.317+03$ & $1.185+00$ & $2.142+06$ \\
\hline 1.5 & $\varepsilon .633+05$ & $4.154+C 3$ & $2.210+00$ & $8.591+c 5$ \\
\hline 2 & $4.533+C 5$ & $3.5 \in 4+C 2$ & $3.242+0 \mathrm{C}$ & $4.453+C 5$ \\
\hline 3 & $6.283+05$ & $3.567+c 3$ & $5.156+0 c$ & $6.248+C 5$ \\
\hline 4 & $4.86 C+05$ & $2.1 c 3+C 3$ & $6.5 \varsigma 7+00$ & $4.828+C 5$ \\
\hline 5 & $2.722+05$ & 2. $.5 \varepsilon+C 3$ & $8.675+00$ & $2.654+C 5$ \\
\hline$t$ & $. \in S E+C 5$ & $2 . \equiv t 5+03$ & $1.026+C 1$ & $1.672+05$ \\
\hline g & $8.000+04$ & 2. $c \leqslant 3+c 3$ & $1.31 c+01$ & $7.879+04$ \\
\hline 10 & $4.571+C 4$ & $1.738+C 3$ & $1.55 t+01$ & $4.356+C 4$ \\
\hline 15 & & & & $3.754+C 4$ \\
\hline 20 & $2.555+04$ & $8.170+02$ & $2.367+01$ & $2.875+04$ \\
\hline$=0$ & 1.0 & $4.212+C 2$ & $=4+C 1$ & $9.745+c 3$ \\
\hline+0 & $\therefore 833+03$ & $3.2 \mathrm{Cl}+\mathrm{O} 2$ & $3.132+01$ & $4.482+03$ \\
\hline 50 & $2.701+03$ & $2.275+02$ & $=.252+\mathrm{Cl}$ & $2.441+C 3$ \\
\hline$\in 0$ & & $1 . t \varsigma 3+c 2$ & 3.3 & $1.480+03$ \\
\hline 80 & $.035+$ & $1 . C C 5+C 2$ & $3.443+C 1$ & $6.686+02$ \\
\hline 0 & & & & $1.777+03$ \\
\hline 150 & $6.8522^{\circ}$ & $3.164+01$ & $3.327+C_{1}$ & $6.2 C 3+C 2$ \\
\hline $20 \mathrm{C}$ & $.401+$ & $1.521+C 1$ & $3.139+01$ & $2.505+02$ \\
\hline & & & & \\
\hline
\end{tabular}

\begin{tabular}{|c|c|c|c|c|}
\hline$\Lambda E R G Y$ & s & $\mathrm{COH}$ & INCCH. & PHCTL \\
\hline KEV & TOTAL & SE $\triangle T T$ & SCATT. & EFFECT \\
\hline 1 & $2 \cdot 3 \varepsilon t+0 t$ & $4.417+C 3$ & $1.192+00$ & $2.381+C 6$ \\
\hline 1.5 & $5.37 c+05$ & $4.24 t+C 3$ & $2.228+00$ & c. $336+05$ \\
\hline 2 & $\angle .845+C 5$ & 4. $C<8+C 3$ & $3.257+02$ & $4.804+C 5$ \\
\hline 3 & $\leqslant .<13+05$ & $3 . \leqslant 41+03$ & $22+00$ & $6.376+05$ \\
\hline 4 & $.03 \hat{z}+$ & & & +05 \\
\hline 5 & $\overline{2} .83 t+05$ & $2.522+03$ & $2.844+00$ & $2.807+05$ \\
\hline$\leq$ & & $2 \cdot t$ & & \\
\hline$\varepsilon$ & $\varepsilon \cdot 525+0$ & $2.145+03$ & 1.3 & $8.323+04$ \\
\hline 10 & $4.853+$ & $1.782+C 3$ & 1. & $4 \cdot 673+04$ \\
\hline 15 & 2.58 & $1.1 \varepsilon \varepsilon+$ & & 3.8 \\
\hline $2 \mathrm{C}$ & $3.0 p t+C$ & $8.41 t+C 2$ & $2.421+01$ & $2.990+04$ \\
\hline 30 & $1.091+c 4$ & 4. $S \leq 1+C 2$ & 2.8 & $1.03^{\circ}+C_{4}$ \\
\hline 40 & 3 & 3.2844 & & $4.838+c 3$ \\
\hline 50 & .9 & $2.3 z \varepsilon+c z$ & 3.3 & $2.657+03$ \\
\hline$\leqslant 0$ & 82 & 2.7 & 3.4 & $1 . t-21+c 3$ \\
\hline 80 & $?$ & 1.02 & 3.4 & $7.370+02$ \\
\hline 0. & & $\epsilon$. & 3. & 1.5 \\
\hline $15 \mathrm{C}$ & 7.21 & 3.2 & & $5.551+C 2$ \\
\hline 200 & & & & $3.045+C 2$ \\
\hline & $\varepsilon+02$ & $.371+00$ & $2.936+01$ & $1.051+02$ \\
\hline
\end{tabular}

\begin{tabular}{|c|c|c|c|c|}
\hline$E R G Y=$ & & $\mathrm{CCH}$ & INCEH. & PHGTC \\
\hline KEV & TOTAL & SCATT. & SC $\triangle I T$ & EFFECT \\
\hline 1 & $2.42 \varepsilon+c \varepsilon$ & $4.740+C 3$ & $1.15 C+00$ & $2.423+C 6$ \\
\hline 1.5 & $1.051+0 t$ & $4.54 C+C 3$ & $2.232+00$ & 1. $C 47+C 6$ \\
\hline 2 & $5.71 t+C 5$ & $4 \cdot 3 c^{c} c+c 3$ & $3.374+C C$ & $5.672+05$ \\
\hline 3 & $5.515+25$ & $3.87 \varepsilon+C 3$ & $5.591+C 0$ & $5.477+05$ \\
\hline 4 & $4.845+05$ & $3.4 \in 9+0 ?$ & $7.607+00$ & $4.210+05$ \\
\hline 5 & $3.309+05$ & $3.114+C 3$ & c. $375+0 C$ & $3.278+05$ \\
\hline$\varepsilon$ & $2.0 t$ & 2. $a C 5+C 3$ & $1.095+01$ & $2.037+C 5$ \\
\hline & $3.848+04$ & $2.3<2+$ & $1.390+01$ & $9.616+04$ \\
\hline 10 & $=.565+C 4$ & $1 . \subseteq 18+0 ?$ & 1. $64 \varepsilon+C 1$ & $5.372+04$ \\
\hline 15 & 4.51 & $1.288+0 ?$ & 2.1 & $4.381+04$ \\
\hline 20 & .5 & 9.1 & & 3.438 \\
\hline $3 C$ & $1.254+34$ & $5.375+02$ & $2.965+01$ & $1.157+04$ \\
\hline 40 & $\Xi .5$ & $3.535+C 2$ & $3.253+01$ & $5.591+C 3$ \\
\hline 50 & 3.34 & $2.4 \varepsilon 4+C 2$ & $3.422+01$ & $\therefore .062+03$ \\
\hline$\in 0$ & $2 . C^{8}$ & $1.8 \equiv z+02$ & $3.51 \varepsilon+C !$ & i. $865+03$ \\
\hline 90 & 2 & 1.685 & & $8.445+C 2$ \\
\hline $10 c$ & $c 3$ & $7.2 \in 1$ & $3.582+01$ & 2.14 \\
\hline & & & & \\
\hline 200 & $3.5 E 5+02$ & $1.54 t+c 1$ & $3.285+01$ & $3.441+C 2$ \\
\hline & $1.557+C 2$ & $8.772+c 0$ & $2.935+01$ & a.216+02 \\
\hline
\end{tabular}

$\begin{array}{cc}\begin{array}{c}\text { ENEAGY } \\ \text { KEV }\end{array} & \text { TCTAL } \\ 400 & 7.585+C 1 \\ 500 & 5.254+01 \\ 600 & 4.108+01 \\ 800 & 2.938+01 \\ 1000 & 2.362+01 \\ 1500 & 1.750+01 \\ 2000 & 1.531+01 \\ 3000 & 1.409+01 \\ 4000 & 1.403+01 \\ 5000 & 1.427+01 \\ 6000 & 1.447+01 \\ 8000 & 1.531+01 \\ 10000 & 1.644+01 \\ 15000 & 1.845+01 \\ 20000 & 2.062+01 \\ 30000 & 2.360+01 \\ 40000 & 2.550+01 \\ 50000 & 2.719+C 1 \\ 60000 & 2.822+C 1 \\ 80000 & 3.002+01 \\ 100000 & 3.154+01\end{array}$

$C O H$
SCAT
$4.559+00$
$2.922+00$
$2.023+00$
$1.136+00$
$7.230-01$
$3.255-01$
$1.843-01$
$8.248-02$
$4.655-02$
$2.586-02$
$2.076-02$
$1.17 C-02$
$7.495-03$
$3.336-03$
$1.877-03$
$8.352-04$
$4.704-04$
$3.009-C 4$
$2.079-04$
$1.154-04$
$7.276-04$
76

INCOH.
SCATT
$2.510+C 1$
$2.306+C 1$
$2.141+C 1$
$1.890+C 1$
$1.7 C 1+C 1$
$1.387+C 1$
$1.185+C 1$
$5.321+C C$
$7.785+C C$
$6.72 C+C 0$
$5 . C 4 C+C 0$
$4.85 S+C C$
$4.138+C 0$
$3.061+C C$
$2.455+C C$
$1.78 E+C C$
$1.418+C 0$
$1.182+C C$
$1.01 E+C C$
$8.022-C 1$
$6.655-01$

PHOTC

$4.615+C 1$

$2.696+c 1$

$1.7 E 4+C 1$

$5.335+0 C$

$5.824+0 C$
$-.775+C C$
$1.628+0 C$

$1.628+0 C$

\&.431-C1

$5.54 t-C 1$

4. $C 07-C 1$

$2.170-c 2$

$2.1 s c-c 1$

$1.0 \leq 3-C 1$

7.674-02

4. $515-C 2$

$3.58 z-c z$

2. $304-02$

$1,672-C 2$

$1.305-02$

$5.377-C 1$

$1.651+O C$

$5.637+0$

$7.12 \mathrm{C}+\mathrm{CC}$

$\varepsilon .1 c \mathrm{C}+\mathrm{CC}$

$1.0 ? 2+C 1$

$1.213+61$

1. $8 \mathrm{C} c+\mathrm{Cl}$

$2.176+01$

$2.4 C 4+C 1$

$2.718+C 1$

$2 . c 2 C+C !$
$3.0 \varepsilon t+c !$

$\begin{array}{ccc}\text { ENERGY } & & \text { CGH } \\ \text { KEV } & \text { TCTAL } & \text { SCATT. } \\ 400 & 7.837+01 & 4.647+00 \\ .500 & 5.425+01 & 2.977+00 \\ 600 & 4.188+01 & 2.062+00 \\ 800 & 2.983+01 & 1.158+00 \\ 1000 & 2.398+C 1 & 7.360-01 \\ 1500 & 1.777+01 & 3.314-01 \\ 2000 & 1.555+C 1 & 1.877-01 \\ 3000 & 1.433+C 1 & 8.357-02 \\ 4000 & 1.429+C 1 & 4.739-02 \\ 500 C & 1.453+C 1 & 3.940-02 \\ 6000 & 1.474+01 & 2.114-02 \\ 8000 & 1.560+C 1 & 1.191-02 \\ 10000 & 1.677+01 & 7.621-03 \\ 15 C 00 & 1.987+C 1 & 3.395-03 \\ 20000 & 2.104+01 & 1.911-03 \\ 30000 & 2.4 C 5+C 1 & 8.503-04 \\ 40000 & 2.603+01 & 4.750-04 \\ 50000 & 2.776+C 1 & 3.064-04 \\ 60000 & 2.882+01 & 2.117-04 \\ 80000 & 3.066+C 1 & 1.175-04 \\ 100000 & 3.222+01 & 7.410-05\end{array}$

INCOH
SCATT
$2.54 C+C 1$
$2.334+C 1$
$2.1 \epsilon T+C 1$
$1.512+C 1$
$1.722+C 1$
$1.404+C 1$
$1.155+C 1$
$9.444+C C$
$7.887+C C$
$6.8 C E+C D$
$6.015+C O$
$4.515+C O$
$4.125+C C$
$3.055+C C$
$2.48 t+C C$
$1.8 C 5+O C$
$1.425+C O$
$1.157+C C$
$1 . C 31+C O$
$8.122-C 1$
$6.738-C 1$

PHOTC

$E=F E C T$

PAIR

$4 \cdot 823+C 1$
$2.793+C 1$

$1.815+C 1$

$\varsigma .54 c+c c$

$\leftarrow \cdot C 2 \varepsilon+c c$

$2.844+C C$

$1 . \hat{c} \in S+C C$

$5.57>-01$

4. $C$ C $t-C 1$

3.241-01

2. $235-01$

7.581-0

1. $075-C 1$

$7.831-c 2$
$5.005-C 3$

$5.0 C 5-C 2$
$2.64 E-C 2$

$2.253-c 2$

$2.334-02$

$1.7 \mathrm{CO}-\mathrm{C}$
$1.3 \mathrm{C} \mathrm{c}-\mathrm{c}$

5. $5 \in 1-C 1$

$3.54+C$

$5.782+C C$

$7.2 \varepsilon \varepsilon+C C$

$8.3 \varepsilon C+0 C$

$1 . C 45+C 1$

$1.3 E t+c 1$

1. $848+C 1$

$2.223+C 1$

$2.4=6+01$

$2.777+\hat{01}$

2. $\{\varepsilon 3+C 1$

$3.153+C 1$

$\begin{array}{rcc}\text { ENEFGY } & & \text { CCH } \\ \text { KEV } & T C T A L & \text { SCATT } \\ 400 & 8.11 \equiv+C 1 & 4.728+00 \\ 500 & 5.615+01 & 2.329+00 \\ 600 & 4.335+C 1 & 2.058+00 \\ 800 & 3.086+C 1 & 1.179+00 \\ 1000 & 2.481+C 1 & 7.490-01 \\ 1500 & 1.825+01 & 3.373-01 \\ 2000 & 1.592+C 1 & 1.510-01 \\ 3000 & 1.464+C 1 & 8.545-02 \\ 4000 & 1.459+01 & 4.824-02 \\ 5000 & 1.484+C 1 & 3.094-02 \\ 6000 & 1.504+01 & 2.151-02 \\ 8000 & 1.552+C 1 & 1.212-02 \\ 10000 & 1.710+C 1 & 7.767-03 \\ 15000 & 1.524+C 1 & 3.457-03 \\ 20000 & 2.146+C 1 & 1.946-33 \\ 30000 & 2.458+C 1 & 8.655-114 \\ 40000 & 2.557+01 & 4.975-04 \\ 50000 & 2.834+01 & 3.110-04 \\ 60000 & 2.942+01 & 2.155-04 \\ 80000 & 3.130+01 & 1.195-04 \\ 100000 & 3.259+01 & 7.545-05\end{array}$

INCOR.
SCATT

PHOTC

PAIR

$2.571+C 1 \quad \equiv .071+01$

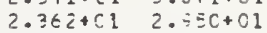

$2.1 \subsetneq 4+C 1 \quad 1 . \subsetneq 31+C 1$

$1.535+C 1 \quad 1 . C 33+C 1$

$1.742+C 1=0.27+0 C$

$1.421+C 1$

$1.214+C 1$

$5.555+C C$

$7.993+C C$

$t . \varepsilon \varepsilon c+C O$

$6.08 \varepsilon+C C$

$4.98 C+C C$

$4.241+C 0$

$3.127+C 0$

$2.51 t+C C$

$1.83 C+C D$

$1.453+00$

$1.212+c 0$

1. $044+00$

$3.125+0 \mathrm{C} \quad 5.75 \mathrm{C}-\mathrm{Cl}$

$1.833+C C \quad 1.753+C C$

$\begin{array}{ll}5.453-C 1 & +.651+C C \\ 6.23 t-01 & 5.5 ? 8+0 C\end{array}$

$4.502-C 1 \quad 7.4 \leq 7+C 0$

$3 . E \leq 1-C 1 \quad 8.577+C C$

$2.4 \in 1-21 \quad 1.0 \leqslant 8+C 1$

$1.847-51$

$1.192-c 1$
$0.621-c 2$

$1.55 \varepsilon+C 1$

$\begin{array}{ll}4 . C 23-C 2 & 2.5 C 8+C 1 \\ 3.14 \varepsilon-C 2 & 2.710+C 1\end{array}$

$2.576-02$

$1.878-0 ?$

$6.821-C 1$

$1.465-C^{2} 2$

$3.04 t+01$
$3.22 C+C l$

\begin{tabular}{|c|c|c|}
\hline & & $\mathrm{COH}$ \\
\hline KEV & $T S T \Delta L$ & SCATT \\
\hline 400 & $9.119+01$ & $4.752+00$ \\
\hline 500 & $6.261+01$ & $3.272+00$ \\
\hline 600 & $4.728+C 1$ & $2.159+0$ \\
\hline 800 & $3.348+i 1$ & $1.233+0$ \\
\hline 1000 & $2.554+01$ & $7.840-0$ \\
\hline 1500 & $1.934+01$ & \\
\hline 2000 & $1.632+01$ & 2.000 \\
\hline 3000 & $1.548+0 ?$ & $8.749-$ \\
\hline 4000 & $1.543+C 1$ & $5.051-0$ \\
\hline 5000 & 1.571 .01 & $3.239-0$ \\
\hline 6000 & $1.553+c 1$ & $2.253-$ \\
\hline 8000 & $1 . \in 85+01$ & $1.269-0$ \\
\hline 10000 & $1.808+01$ & $8.133-0$ \\
\hline 15000 & $2.037+01$ & $3.620-0$ \\
\hline 20000 & 2.2734 & $2.037-0$ \\
\hline 30000 & $2.607+01$ & $9.063-0$ \\
\hline 40000 & $2.821+\mathrm{Cl}$ & $5.105-0$ \\
\hline 50000 & $3.011+02$ & 3.266 \\
\hline 60000 & $3.12 \leqslant+C 1$ & $2.258-0$ \\
\hline & 3.325 & \\
\hline & $494+01$ & $9-0$ \\
\hline
\end{tabular}

\begin{tabular}{|c|c|c|}
\hline & Fut & $P \Delta[R$ \\
\hline$S C \Delta T T$. & EFFECT & PROD. \\
\hline $2 . t \in 2+c 1$ & $5 . \subseteq \notin 1+C 1$ & \\
\hline $2.447+C 1$ & $3.457+C ?$ & \\
\hline $2.272+C 1$ & $2.2 s t+C 1$ & \\
\hline $2.0 C 4+C 1$ & $1.220+c 1$ & \\
\hline $1.905 * 01$ & $7.655+0 C$ & \\
\hline $1.473+01$ & $3.523+0 \mathrm{C}$ & $6.341-C 1$ \\
\hline $2.25 \varepsilon+C 1$ & $2.122+c k$ & $1 . S_{16}+0 \mathrm{C}$ \\
\hline $3.709+50$ & $1.205+0 C$ & $4.382+C C$ \\
\hline $8.275+C C$ & $7.248-0 i$ & $6.378+0 c$ \\
\hline $7.141+00$ & $5.22 t-01$ & $8 . c 15+c c$ \\
\hline $6.310+C 0$ & $4.134-C 1$ & $5.183+c c$ \\
\hline$=.1 \in 2+C C$ & $2.855-C 1$ & $1.13 c+c !$ \\
\hline $4.35 t+2 C$ & $2.143-01$ & $1.34 t+C 1$ \\
\hline $3.252+c c$ & $1.376-01$ & $1.6=7+01$ \\
\hline $2 \cdot \in C \varepsilon+O C$ & $1.005-01$ & $2 . c c 2+c 1$ \\
\hline $1.937+C C$ & $6.455-02$ & $2.411+C 1$ \\
\hline $1.5 c t+c c$ & $4.718-C 2$ & $2.6 t t+C 1$ \\
\hline $1 \cdot 25 \epsilon+C C$ & $3.658-02$ & 2. $\& E 2+C 1$ \\
\hline $1 . C 32+C 0$ & $3.031-c 2$ & $3.015+01$ \\
\hline $8.522-c 1$ & $2.214-02$ & $3.237+01$ \\
\hline$c>c-c)$ & $1.735-02$ & $3.422+i 1$ \\
\hline
\end{tabular}


ENDF/E IN EARNS/ATCM

\begin{tabular}{|c|c|c|c|c|}
\hline ENERGY & 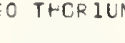 & $\mathrm{COH}$ & INCOH. & PHOTC \\
\hline KEV & TOTAL & SCATT. & SCATT. & EFFECT \\
\hline 1 & $2.54^{c}+C_{6}$ & $5.127+03$ & $1.035+00$ & $2.544+06$ \\
\hline 1.5 & $1.2 n E+C t$ & $4.855+C 3$ & $2.13 t+0 C$ & $1.200+06$ \\
\hline$?$ & t. Stc +05 & 4. $t<t+C 3$ & $3.31 \epsilon+00$ & $6.923+05$ \\
\hline 3 & $3.230+05$ & $4 \cdot 165+03$ & $5.798+00$ & $3.189+05$ \\
\hline 4 & $4.275+05$ & 3.734703 & $\varepsilon .181+C 0$ & $4.238+05$ \\
\hline 5 & $3.691+05$ & $3 \cdot 358+c 3$ & $1.015+01$ & $3.658+05$ \\
\hline t & $2.406+05$ & 3. C34+C3 & $1.184+01$ & $2.466+C 5$ \\
\hline 8 & $1.197+C 5$ & $2.5 \mathrm{CO}_{0}+\mathrm{C}_{3}$ & $1.474+01$ & $1.172+05$ \\
\hline 10 & $6.792+04$ & 2. $C 57+C 3$ & $1.593+01$ & $0.581+04$ \\
\hline 15 & $\check{2} \times 451+04$ & $1.421+03$ & $2.118+01$ & $2.307+04$ \\
\hline 20 & 3.741004 & 1.021403 & $2.475+01$ & $3.637+04$ \\
\hline 30 & $1.49 t+04$ & $5 .<\varepsilon t+C 2$ & $2.593+01$ & $1.433+04$ \\
\hline 40 & 7. $C 73+03$ & 3. $5 \mathrm{C} 7+02$ & 3.333401 & $6.649+C 3$ \\
\hline 50 & $3.070+03$ & $2.728+C 2$ & $3.526+C_{1}$ & $3 \cdot t \leqslant 2$ \\
\hline 60 & $2.485+C 3$ & $2.0 C 4+02$ & $3.634+01$ & $2.248+03$ \\
\hline 80 & $1.15 t+C 3$ & $1.1 \subset 2+c 2$ & $3.719+01$ & $1.040+C 3$ \\
\hline 100 & $6.879+02$ & $7.931+01$ & $3.722+01$ & $5.714+C 2$ \\
\hline 150 & $c .35 t+02$ & $3.651+01$ & $3.629+01$ & $8.624 * 02$ \\
\hline 200 & $4 \cdot t \geq 0+02$ & $\bar{c} .114+C 1$ & $3.432+01$ & 4. $c 75+02$ \\
\hline 300 & $1.945+02$ & $9.514+C C$ & $3.027+01$ & $1.447+02$ \\
\hline
\end{tabular}

$Z=$
EAERGY
KEV
1
1.5
2
3
4
5
5
8
10
15
20
30
40
50
60
20
100
150
200
1500

C 2 URANILM TOTAL

2. $904+06$

$1.2 \epsilon 1+C t$
$t .871+C 5$

$\therefore .930+05$

$4.320+05$

$3.292+05$

$2.54 C+C 5$

$1.224+05$

$2.534+04$

$\overline{2}, \overline{1} 3 t+04$

?. $603+04$

$7.748+C 3$

$4.391+C 3$

$2.75 t+C^{3}$

$1.322+03$

7. $522+C 2$

1. $C 18+03$

$1.997+C 2$

$\mathrm{COH}$

SC, ATT.

$5.385+03$
$5.15 C+03$

$5.15 C+03$
$4 . E C t+C^{3}$

$4.357+C$

3. $539+C 3$

$2.535+C 3$

3. $187+03$

$2 . t 28+C 3$

$2.155+C$

$1.455+C 3$
$1.077+C ?$

t. $32 t+C 2$

4. $124+C 2$

$+\mathrm{ta}$

$2.11 C+C 2$
$1.25 t+C 2$

E. $255+01$

2. E E $t+C I$

$2.224+0$

1. $-C \mathrm{Cl}+\mathrm{O}$
ENERGY
KEV

400

500

600
800

800
1000

1500

2000

3000

4000
5000

5000

8000

8000
10000

$150 \mathrm{CO}$

20000

30000

40000

50000

60000

80000

100000

$\begin{array}{cc}\text { 1NCCH. } & \text { PHCTC } \\ \text { SCATT. } & \text { EFFECT } \\ 5.545-C 1 & 2.899+06 \\ 2.047+00 & 1.256+06 \\ 3.22 C+0 C & 6.922+C 5 \\ 5.827+00 & 2.886+C 5 \\ 8.452+C 0 & 4.281+05 \\ 1.056+01 & 3.257+05 \\ 1.233+C 1 & 2.508+05 \\ 1.521+01 & 1.198+05 \\ 1.710+01 & 6.750+C 4 \\ 2.117+01 & 2.382+04 \\ 2.483+01 & 2.026+04 \\ 3 . C 14+01 & 1.536+C 4 \\ 3.375+01 & 7.302+03 \\ 3.580+C 1 & 4 . C 67+03 \\ 3.593+02 & 2.5 C 8+C 3 \\ 3.784+C 1 & 1.159+03 \\ 3.793+01 & 6.307+02 \\ 3.704+C 1 & 9.417+02 \\ 3.506+01 & 4.445+C 2\end{array}$

$3.073+03 \quad 2.58 \mathrm{C}+\mathrm{C2}$

ENERGY
KEV

EV

400
500

600

800

1000
1500

1500
2000

3000

4000

5000

$\leq 000$

$\$ 000$

10000

15000

20000

30000

40000

50000

60000

80000
100000

$z=\$ 4$ PLUTOAILM

ENERGY
KEV
$?$
1.5
$?$
3
4
5
6
8
10
15
20
30
40
50
60
80
100
150
200
300

\begin{tabular}{|c|c|c|c|}
\hline & & & \\
\hline TOTAL & SCATT & & \\
\hline $7 E z+C E$ & $5.64 C+03$ & $\varepsilon, t \rightarrow 6-C$ ? & \\
\hline $3+$ & $5.4 \mathrm{CS}+\mathrm{C3}$ & & \\
\hline$+\mathrm{C}$ & $15 t+03$ & & \\
\hline & $4 . t 42+03$ & 0 & \\
\hline & $15 t+C 3$ & 0 & \\
\hline$c$ & $724+C 3$ & $1.0 \div 8+0 !$ & $1+6=$ \\
\hline 0 & $352+C 3$ & $1.285+01$ & $2.060+05$ \\
\hline & $757+03$ & $1+4$ & $392+0$ \\
\hline & & 1 . & $5+0$ \\
\hline & & & $3+0$ \\
\hline & & & +0 \\
\hline & 2 & & $\mathrm{C}$ \\
\hline & $4.354+C 2$ & $3.415+$ & $0+0$ \\
\hline .7 & $3 \cdot C 35+C 2$ & $3.631+$ & $76+0$ \\
\hline & $2.227+C 2$ & $3.750+01$ & $2.694+0$ \\
\hline & $1.326+c 2$ & $3.852+01$ & $1.228+0$ \\
\hline & $E .81 s+C 1$ & $3.962+01$ & $5.630+0$ \\
\hline & $\cdot 1 \mathrm{CO}+\mathrm{C} 1$ & $3.77 E+01$ & $1.000+c$ \\
\hline & $347+01$ & $3.580+C$ & \\
\hline & & & \\
\hline
\end{tabular}

ENERGY
KEV

400
500

600

$8 \mathrm{CO}$

1000

1000
1500

2000

3000

4000

5000

6000

8000

10000

15000

20000

30000

40000

50000

60000

80000

100000

TETEL SCATT.

SCATT.

PHOTC

EFFEC T

$\begin{array}{llll}1.045+02 & 5.369+00 & 2.787+C 1 & 7.128+01 \\ 7.110+01 & 3.438+00 & 2.565+C 1 & 4.2 C 1+01\end{array}$

$\begin{array}{llll}5.392+01 & 2.383+00 & 2.381+01 & 2.772+C 1\end{array}$

$\begin{array}{llll}3.695+01 & 1.337+00 & 2.072+01 & 1.49 C+01\end{array}$

$2.931+01 \quad 8.510-01 \quad 1.894+C 1 \quad 9.524+C C$

$2.102+01 \quad 3.833-01 \quad 1.545+C 1 \quad 4.469+0 C$

$\begin{array}{llll}1.817+01 & 2.171-01 & 1.320+C 1 & 2.613+0 C\end{array}$

$1.671+01 \quad 9.716-02$

$1.664+01 \quad 5.485-02$

$1.695+01 \quad 3.518-02$

$1.718+01 \quad 2.446-02$

$1.814+01 \quad 1.379-02$

$1.942+01 \quad 8.832-03$

$2.191+01 \quad 3.931-03$

$2.445+012.213-03$

$2.811+01$

$3.044+01$

$3.252+01$

c. $843-04$

$5.544-04$

3. $549-04$

2. $453-04$

$.590+C 1$

$1.363-04$

1. $C 39+C 1$

$8.681+0 \mathrm{C}$

$2.613+0 \mathrm{C}$
$1.370+\mathrm{CC}$

E. $P>C-O 1$

$6.45 C+00 \quad 6.457-01$

$6.621+00 \quad 5.107-01$

$5.415+C C$

4. $612+C C$

$3.412+00$

$2.737+\mathrm{CC}$

1. $S C C+C C$

$1.5 \mathrm{aO}+\mathrm{CC}$

1. $318+C C$

1. $135+0 \mathrm{C}$

$8.941-01$

$3.773+01$

$8.600-05$

$7.418-C 1$

$.527-C_{1}$

$2.045-21$

$1.7 C 7-C 1$

$1.251-01$

$8.060-C^{2}$

$5.910-02$

$4.64^{3}-C 2$

3. $812-\mathrm{C} 2$

$2.753-C_{2}$

$2.194-02$

7. $191-\mathrm{Cl}$

$2.146+0 C$

4. $847+C C$

7. $C C 8+C C$

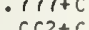

$1.235+C 1$

i. $454+C 1$

1. $832+C$

$2.159+C 1$

$2 \cdot \mathrm{CC}_{4}+\mathrm{Cl}$

$2.880+C 1$

$3.115+C$

$3.259+C$

$3.656+C 1$

TCTAL SCATT.

$1.121+02 \quad 5.647+00$

$7.555+01 \quad 3.616+00$

$5.735+C 1 \quad 2.507+00$

$.855+01 \quad 1.406 \$ 00$

$8.950-01$

$1.890+01 \quad 2.284-01$

$1.736+01 \quad 1.022-01$

$1.727+015.770-02$

$1.757+\mathrm{Cl} 3.701-02$

$1.792+C 1 \quad 2.574-02$

$1.880+01 \quad 1.450-02$

$2.011+013.292-03$

$2.269+C 1 \quad 4.136-03$

$2.533+012.328-03$

$2.914+01 \quad 1.035-03$

$3.152+015.832-04$

$2.374+C 1 \quad 3.733-04$

$3.503+C 12.581-04$

$3.725+C 1 \quad 1.434-0$

3. $014+C 1$

$1.434-04$
$0.053-05$

INCOH.

PHOTO

PAIR

EFFECT

$2.624+C 1 \quad 4.6 C 5+C 1$

2. $435+C 1 \quad 3 . C 50+C 1$

$2.10 t+C 1 \quad 1.648+01$

$1.538+C 1 \quad 1 . C 5 S+C 1$

$1.581+C 1 \quad 4.563+C$

$1.351+C 1 \quad 2.808+0 C$

1. $C E 4+C 1 \quad 1.52 t+C C$

$8.885+C \mathrm{C} \quad 9.077-01$

$7.6 t+t+00$

$0.77 t+C C$

$5.543+\mathrm{CO}$

$\begin{array}{ll}4.72 C+C O & 2.940-01 \\ 3.452+C C & 1 . C C C-C\end{array}$

$7.17 \in-C$

$5.675-C 1$

$2.037+00$

1. $517+0 \mathrm{C}$

1. $349+60$

$1+1 \leqslant 2+00$

$9.151-\mathrm{Cl}$

$7,5 \div 3-C 1$

$1.394-01$

5. $112-02$

$6.012-02$

$5.2 \mathrm{C} 1-\div 2$

$4.274-02$

$3.13 t-52$

$2.46=-62$

$7.543-C$

$2.2 s s+C$

5. $C \subseteq 1+C C$

$7.333+C$

C.: $71+0$

$1.285+C$

1. $5.3 \mathrm{c}+\mathrm{Cl}$

1. $\mathrm{C} C \mathrm{C}+\mathrm{C}$

$2.238+01$

2. $7 \mathrm{C} 2+\mathrm{C}$

2. $C \varepsilon G+C$

3. $234+r$

$3.382+C ?$

$\equiv . t 3 ?+c 1$

3. $83 t+C 1$

$\mathrm{COH}$

INCOH.

PHOTO

$.206+C^{2} \quad 5.957+00$

SCATT.

EFFEC

$8.149+01 \quad 3.815+00$

$6.125+012.645+00$

$4.057+01 \quad 1.484+00$

$3.22 t+01$

$2.283+01$

c. $440-0$ =

$4.253-01$

2. $409-01$

$1.759+C 1 \quad 1.078-01$

$1.791+01 \quad 6.087-02$

$1.824+C 1 \quad 3.904-02$

$1.848+\mathrm{Cl} 2.715-02$

$1.948+C 1 \quad 1.530-02$

c. $803-03$

$2.348+C$

$2.620+01$

$3.020+01$

$4.363-03$

2. $456-03$

1. $092-03$

$3.501+C 1$

3. $939-04$ 


\section{FIGURE CAPTION}

Fig. 1. Elements and grid energies (see Table I) for which the Biggs-Lighthill total cross section is at least $3 \%$ less $(-)$ or $3 \%$ greater $(+)$ than the corresponding ENDF/B total cross section entry. 



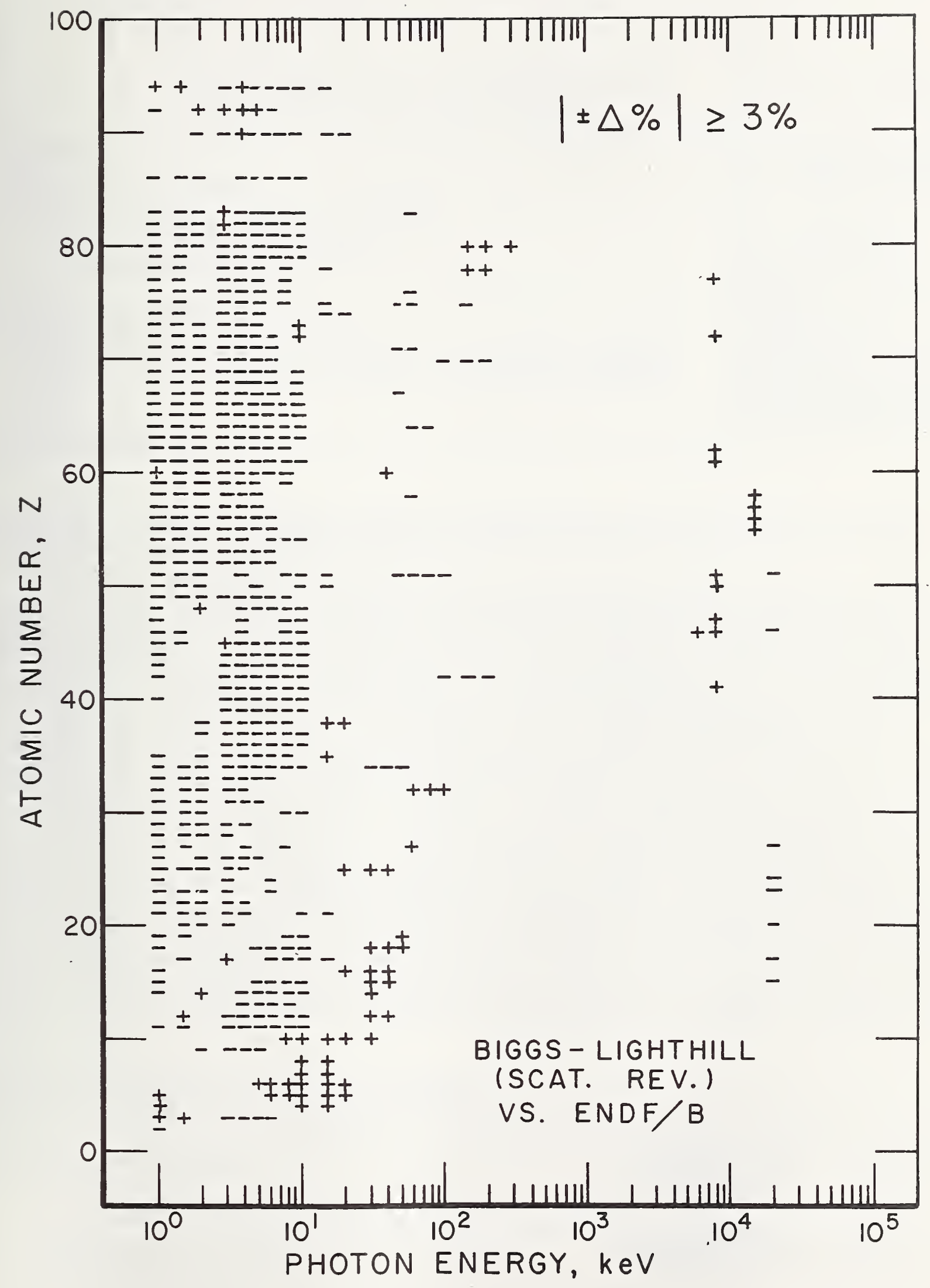



ORM NBS.114A (1.71)

\begin{tabular}{|c|c|c|}
\hline $\begin{array}{l}\text { U.S. DEPT. OF COMM. } \\
\text { BIBLIOGRAPHIC DATA } \\
\text { SHEET }\end{array}$ & $\begin{array}{l}\text { 1. PUBLICATION OR REPORT NO. } \\
\text { NBSIR } 73-241\end{array}$ & 3. Recipient's Accession No. \\
\hline \multicolumn{2}{|l|}{ 4. TITLE AND SUBTITLE } & 5. Publication Date \\
\hline \multicolumn{3}{|c|}{$\begin{array}{c}\text { Comparison of Photon Interaction Cross Section Data Sets. } \\
\text { VII. Biggs-Lighthill (Rev.) and ENDF/B.* }\end{array}$} \\
\hline 7. AUTHOR(S) & - & $\begin{array}{l}\text { 8. Performing Organization } \\
\text { NBSIR 73-241 }\end{array}$ \\
\hline \multicolumn{2}{|c|}{ 9. PERFORMING ORGANIZATION NAME AND ADDRESS } & $\begin{array}{l}\text { 10. Project/Task/Work Unir No. } \\
2400432\end{array}$ \\
\hline \multicolumn{2}{|c|}{$\begin{array}{l}\text { NATIONAL BUREAU OF STANDARDS } \\
\text { DEPARTMENT OF COMMERCE } \\
\text { WASHLNGON, D.C. } 20234\end{array}$} & $\begin{array}{l}\text { 11. Contract/Grant No. } \\
\text { DNA-EO-72-804 } \\
\text { Subtask No. PC-100-05 }\end{array}$ \\
\hline \multicolumn{2}{|c|}{$\begin{array}{l}\text { 12. Sponsoring Organization Name and Address } \\
\text { Defense Nuclear Agency, Washington, D.C. 20305. (Support } \\
\text { was also received from NBS Office of Standard Reference } \\
\text { Data). }\end{array}$} & $\begin{array}{l}\text { 13. Type of Report \& Period } \\
\text { Covered. } \\
\text { Interim report for } \\
\text { Reriod Apr = June } 1973 \\
\text { 14. Sponsoring Agency Code }\end{array}$ \\
\hline
\end{tabular}

15. SUPPLEMENTARY NOTES

16. ABSTRACT (A 200-word or less factual summary of most significant information. If document includes a significant bibliography or literature survey, mention it here.)

The Biggs and Lighthill photon cross section data set $(0.01 \mathrm{keV}$ to $>10 \mathrm{GeV}$, $z=1$ to 100), previously compared in this report series with the ENDF/B data set ( $1 \mathrm{keV}$ to $100 \mathrm{MeV}, 87$ elements with $Z=1$ to 94), has since been revised to provide explicit coherent and incoherent scattering cross sections, rather than the more approximate free-electron (Klein-Nishina) scattering cross section. In this report we present a comparison of this revised set with the ENDF/B set, again in the form of percent differences between the two data sets. These differences for total, coherent scattering, incoherent scattering, photoelectric absorption and pair production cross sections are tabulated for 87 elements with $Z=1-83,86,90,92$ and 94 at 41 energies between $1 \mathrm{keV}$ and $100 \mathrm{MeV}$. Coherent scattering differences below a few hundred $\mathrm{keV}$ are typically $1-5 \%$ and at higher energies are typically $10-30 \%$ for 1 ow $\mathrm{Z}$ and $2-10 \%$ for high $\mathrm{Z}$. The incoherent scattering differences are generally less than $1 \%$ above $10 \mathrm{keV}$ and typically $5-30 \%$ at $1 \mathrm{keV}$. These differences are primarily in regions of negligible scattering contribution to the total cross section, for which the differences are dominated by differences in the photoelectric absorption. The resulting differences in the total cross section are typically $1-5 \%$ except near $1 \mathrm{keV}$ where differences are as high as $26 \%$ for $\mathrm{Z}=68$ and 69 . In addition to the table of percent differences we include tables of the cross section values in barns/ atom for these two sets, since they have not been generally available in this form.

17. KEY "WORDS (AIphabetical order, separated by semicolons)

Attenuation coefficients; comparison; cross sections; photoeffect; photons; $x$-rays 18. AVAILABILITY STATEMENT

TI UnL IMITED.

X] FOR OFFICIAL DISTRIBUTION. DO NOT RELEASE TO NTIS.

\begin{tabular}{|c|c|}
\hline $\begin{array}{c}\text { 19. SECURITY CLASS } \\
\text { (THIS REPORT) } \\
\text { X } \\
\text { UNCL ASSIFIED }\end{array}$ & 90 \\
\hline $\begin{array}{c}\text { 20. SECURITY CLASS } \\
\text { (THIS PAGE) } \\
\text { UNCL ASSIFIED }\end{array}$ & 22. Price \\
\hline
\end{tabular}


